THE COLLEGE

BRIXTON HILL. 


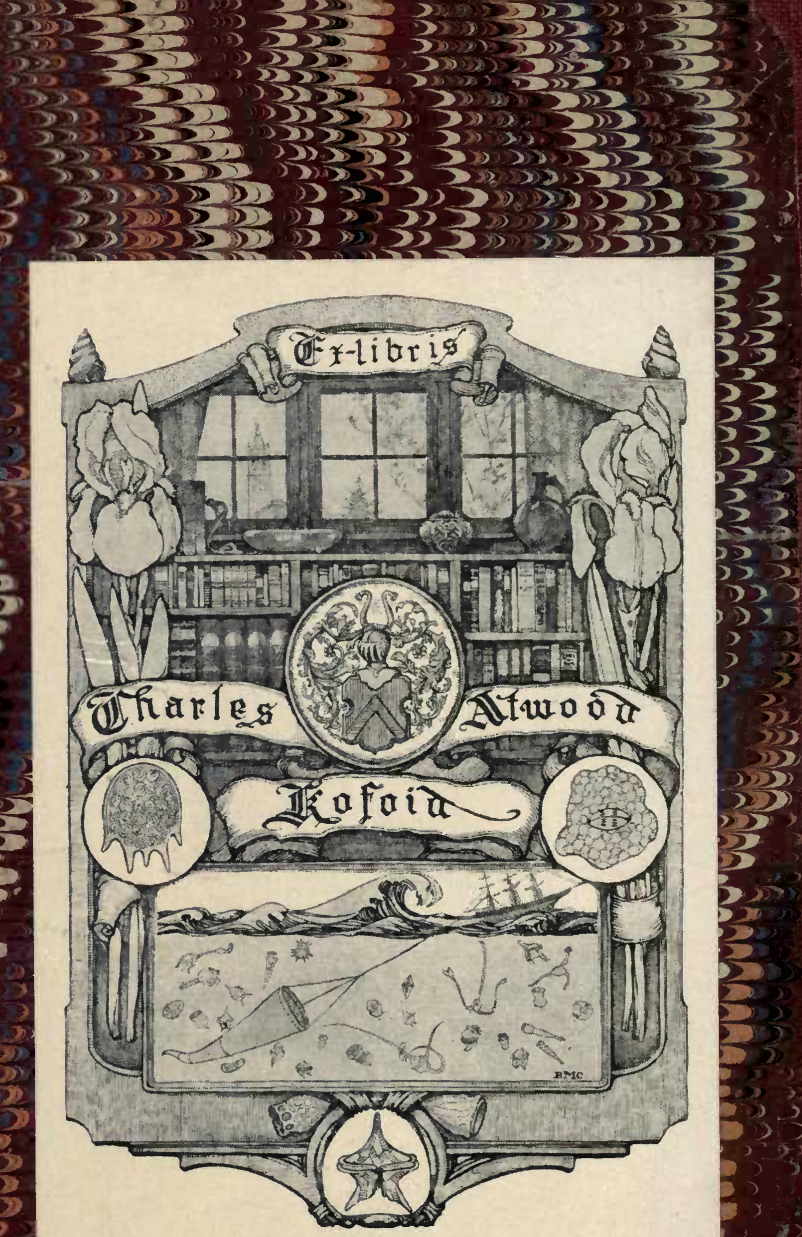



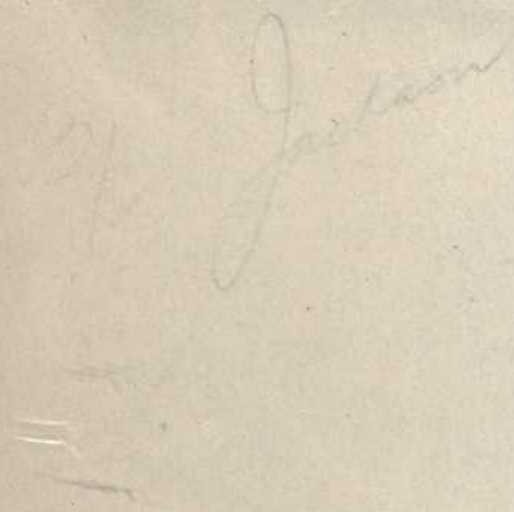



\section{ANIMAL PHYSIOLOGY.}

BY

WILLIAM B. CARPENTER, M.D.

F.R.S. F.G.S. F.L.S.

REGISTRAR OF THE UNIVERSITY OF LONDON.

Alew exdition,

THOROUGHLY REVISED, AND PARTLY RE-WRITTEN.

\section{LONDON :}

H. G. BOHN, YORK STREET, COVENT GARDEN. 


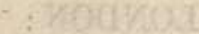

LONDON :

R. CLAY, PRINTER, BREAD STREET HILL. 


$$
(x+2)
$$

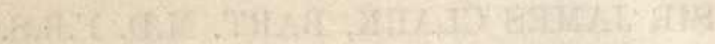
ate 1.7.

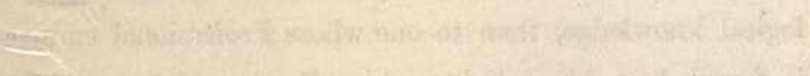

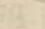

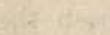

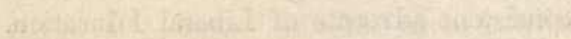

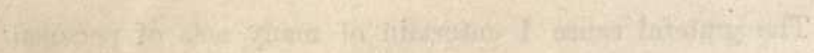

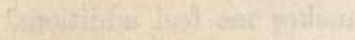




\section{P R E F A C E.}

THe issue of the present Volume may be considered as an attempt to supply what the Author has long considered to be a deficiency in the literature of this country,-that, namely, of an Educational Treatise on Animal Physiology, which should at the same time communicate to its readers the facts of greatest importance as regards their practical bearing, and present these in such a form as to place the learner in possession of the essential principles of Physiological Science.

The Author has followed the general plan of the Treatise on Animal Physiology contributed by Professor Milne-Edwards, one of the most eminent Naturalists in France (in which country it is not thought beneath the dignity of men of the highest scientific reputation to write elementary books for the instruction of the beginner), to the "Cours Élémentaire d'Histoire Naturelle" adopted by the Freneh Government as the text-book of instruction in the Colleges connected with the University of Paris, which requires from every Candidate for its Degree of "Bachelor of Sciences" a competent knowledge both of Animal and of Vegetable Physiology. He has also had at his disposal the admirable series of Illustrations prepared for that work, which, as a whole, are unsurpassed either in beauty or in exactness.

In carrying-out this plan, however, the Author has entirely followed his own judgment; and has made so much more use 


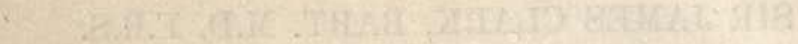

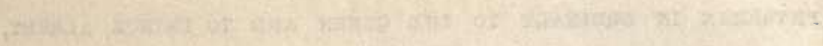

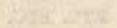

2

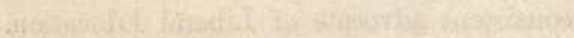

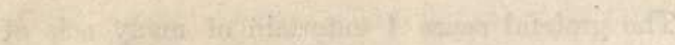
7.

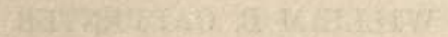




\section{P R E F A C E.}

The issue of the present Volume may be considered as an attempt to supply what the Author has long considered to be a deficiency in the literature of this country,-that, namely, of an Educational Treatise on Animal Physiology, which should at the same time communicate to its readers the facts of greatest importance as regards their practical bearing, and present these in such a form as to place the learner in possession of the essential principles of Physiological Science.

The Author has followed the general plan of the Treatise on Animal Physiology contributed by Professor Milne-Edwards, one of the most eminent Naturalists in France (in which country it is not thought beneath the dignity of men of the highest scientific reputation to write elementary books for the instruction of the beginner), to the "Cours Élémentaire d'Histoire Naturelle" adopted by the French Government as the text-book of instruction in the Colleges connected with the University of Paris, which requires from every Candidate for its Degree of "Bachelor of Sciences" a competent knowledge both of Animal and of Vegetable Physiology. He has also had at his disposal the admirable series of Illustrations prepared for that work, which, as a whole, are unsurpassed either in beauty or in exactness.

In carrying-out this plan, however, the Author has entirely followed his own judgment; and has made so much more use 
of his own materials than of those supplied by the treatise of Professor Milne-Edwards, that the work may be regarded as almost entirely original. The present Edition, too, has undergone very considerable modifications; the first chapter, which now contains a complete outline of the Elementary Tissues of the Animal Body, and the last, in which a comprehensive sketch is given of the principal phenomena of Reproduction and Development throughout the Animal Kingdom, having been entirely re-written and illustrated with numerous additional figures. In order to make room for the large amount of new matter now introduced (not less than one-fifth of the entire volume), the second chapter, containing a General View of the Animal Kingdom, has been much abridged; - a change the Author has the less regretted being obliged to make, since there are now before the public several excellent Elementary Treatises on Zoology, which had no existence at the time this volume originally appeared.

Everyone who desires to see the study of Physiology duly appreciated as a branch of General Education, must feel gratified at the progress which has been made of late years in the public recognition of its value. The University of London led the way, by the introduction of Animal Physiology into the programme of study to which all Candidates for its Degree of Bachelor of Arts are required to conform. The Universities of Oxford and Cambridge have since admitted it as one of the subjects which Candidates may select for their Bachelor of Arts Examination, and in which they may obtain Honours. And in many of the large Public Educational Institutions with which this country is now so abundantly furnished, it forms a part of the regular course of instruction.

It has been the Author's steady aim, not merely to adapt his treatise to the wants of those who wish to acquire a general knowledge of the principal facts and doctrines of Physiological Science, but also to render it suitable to that 
which he considers a far more important purpose of the study, -namely, the culture and discipline of the Mind itself. Having been satisfied, by no inconsiderable experience of different modes of Education, that Natural Science, if judiciously taught, is second in value to no other subject as an educational means, and that it may be made to call forth a more varied and wholesome exercise of the mental powers than almost any other taken singly, - he has kept this purpose constantly in view; and he trusts that the experience of intelligent Instructors will be found so far to concur with his own, that the study of Physiology may be still more generally introduced into Popular Education. It can only be by the general diffusion of sound information on this subject, that the Public Mind ean be led to understand the difference between Rational Medicine, and that Empiricism which now presents itself under so many different forms; that it can appreciate the true value of measures of Sanitary Reform, the efficiency of which must depend upon the amount of support they receive from an intelligent public opinion; and that it can be preserved from those Epidemic Delusions, whose prevalence, from time to time, is not less injurious to the minds of which they lay hold, than is that of Epidemic Diseases to the bodies of those who suffer from them.

$\mathrm{He}$ has only further to add that, whilst keeping in view the most important practical applications of the Science of Physiology, he has nat thought it desirable to pursue these too far ; since they constitute the details of the Art of preserving Health, which is founded upon it, and which may be much better studied in a distinct form, when this outline of the Science has been mastered. And, for the same reason, he has adverted but slightly to those inferences respecting the Infinite Power, Wisdom, and Goodness, of the Great First Cause, which are more obvious, although, perhaps, not really more clear and valid, in this Science, than in any other. 


\section{viii}

PREFACE.

Believing, as he does, that such inferences are more satisfactorily based upon the general manifestations of Lav and Order, than upon individual instances of Design, he has thought it the legitimate object of this treatise to lay the foundation for them, by developing, so far as might be, the Principles of Physiology,-leaving it to special treatises on Natural Theology, to build-up the applications.

UNIVERSITY HALL, LoNdON,

Jan. 1859. 


\section{CONTENTS.}

PAGE

INTRODUCTION

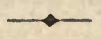

\section{$\bullet$}

CHAPTER I.

On the Vital Operations of Animals, and the Instruments bY Which they are Performed . . . . . . . . . 17

Chemical constitution of the animal body - • . 31 STRUCTURE OF THE PRIMARY TISSUES . . . . . . 36

CHAPTER II.

General View of the Animal Kingdom . . . . . . 84

Vertebrata . . . . . . . . . . . : . 84

MAMMALS . . . . . . . . . . . . . 89

BIRDS . . . . . . . . . . . . . . 92

REPTILES . . . . . . . . . . . . . . . 93

BAtrachia . . . . . . . . . . . . 97

FISHES . . . . . . . . . . . . 100

Articulata . . . . . . . . . . . 102

INSECTS . . . . . . . . . . . . 104

ARACHNIDA . . . . . . . . . . 105

CRUSTACEA ............... 106

CIRRHIPEDA . . . . . . . . . . . . . . 109

MYRIAPODA . . . . . . . . . . . . . 110

ANNELIDA . . . . . . . . . . . . $i b$.

ENTOZOA . . . . . . . . . . . . . 111

Mollusca . . . . . . . . . . . . . 112

CEPHALOPODA . . . . . . . . . . . . . 116

PTEROPODA . . . . . . . . . . . . 117

GASTEROPODA . . . . . . . . . . . 118

CONCHIFERA ............. . . $i b$.

TUNICATA . . . . . . . . . . . . 121

POLYZOA . . . . . . . . . . . 122 
CHAPTER II.-Continued.

RADIATA

ECHINODERMATA . . . . . . . . . . . 125

ACALEPHE . . . . . . . . . . . . 128

POLYPIFERA . . . . . . . . . . . . 129

Protozoa . . . . . . . . . . . . . 135

RHIZOPODA . . . . . . . . . . . 136

INFUSORIA . . . . . . . . . . . . . . 139

PORIFERA . . . . . . . . . . . 140

CHAPTER III.

Nature and Souroes of Animal Food

CHAPTER IV.

Digestion ANd Ábsorption . . . . . . . . . . 162

PREHENSION OF FOOD . . . . . . . . . 163

MASTICATION . . . . . . . . . . 166

INSALIVATION . . . . . . . . . . . 176

DEGLUTITION . . . . . . . . . . . . 178

DIGESTIVE APPARATUS . . . . . . . . . . 181

GASTRIC DIGESTION :-CHYMIFICATION . . . . . 188

INTESTINAL DIGESTION :-CHYLIFICATION . . . . . 193

DEFECATION . . . . . . . . . . . . . 195

ABSORPTION OF NUTRITIVE MATERIAL . . . . . 196

SANGUIFICATION •.............. . . 199

\section{CHAPTER V.}

Of the Bloon, and its Circulation . . . . . . 202

PROPERTIES OF THE BLOOD . . . . . . . . 203

CIRCULATION OF THE BLOOD . . . . . . . . . . 216

CIRCULATING APPARATUS OF THE HIGHER ANIMALS . . 222

FORCES THAT MOVE THE BLOOD . . . . . . . . 232

COURSE OF THE BLOOD IN THE DIFFERENT CLASSES OF

ANIMALS . . . . . . . . . . 240 
CHAPTER VI

Of Respiration

NATURE OF THE CHANGES ESSENTIALLY CONSTITUTING RESPIRATION . • . . • . . . . . . . 259 STRUCTURE AND ACTIONS OF THE RESPIRATORY APPARATUS 265

CHAPTER VII.

OF ExCREMION AND SECRETION . . . . . . . . . . 292

GENERAL PURPOSES OF THE EXCRETING PROCESSES . . $i b$. NATURE OF THE SECRETING PROCESS.-STRUCTURE OF THE SECRETING ORGANS. . . . . . . . . . 298 CHARACTERS OF PARTICULAR SECRETIONS . . . . . 304

\section{CHAPTER VIII.}

General Review of the Nutritive Operations.-Formation OF the Tissues . . . . . . . . . . . . . 316

GENERAL REVIEW OF THE NUTRITIVE OPERATIONS . . ib. FORMATION OF THE TISSUES . . . . . . . . 317 REPAIR OF INJURIES. • . . . . . . . . . 323

\section{CHAPTER IX.}

ON the Evolution of Light, Heat, and Electricity by Animals . • . . . . . . . . . . . 327

ANIMAL LUMTNOUSNESS . • • . . . . . . . $i b$. ANIMAL HEAT . . . . . . . . . . . . . . 332 ANIMAL ELECTRICITY . . . . . . . . . . 340

\section{CHAPTER $\mathrm{X}$.}

Functions of the Nervous System.

STRUCTURE AND ACTIONS OF THE NERVOUS SYSTEM IN THE PRINOIPAL CLASSES OF ANIMALS . . . . . 350 FUNCTIONS OF THE SPINAL CORD.-REFLEX ACTION • . 374 FUNCTIONS OF THE GANGLIA OF SPECIAL SENSE.-CONSENSUAL ACTIONS . . . . . . . . . . 380 FUNCTION OF THE CEREBELLUM.-COMBINATION OF MUSCULAR ACTIONS . . . . . . . . . . 384 FUNCTION OF THE CEREBRUM. - INTELLIGENCE AND WILL. 385 


\section{CHAPTER XI.}

On Sensation, and the Organs of the Senses . . • • 387

SENSE OF TOUCH . . . . . . . . . . . . 390

SENSE OF TASTE . . . . . . . . . . . . . 395

SENSE OF SMELL . . . . . . . . . . . . . 398

SENSE OF HEARING . . . . . . . . . . . . . 401

SENSE OF SIGHT . . . . . . . . . . . . . 413

\section{CHAPTER XII.}

Of Animal Motion, and its Instrdments . . . . . 443

CONTRACTILE TISSUES.-MUSCULAR CONTRACTILITY . . 444 APPLICATIONS OF MUSCULAR POWER.-BONES AND JOINTS. 453 MOTOR APPARATUS OF MAN.—SKELETON AND MUSCLES • 464 OF THE ATTITUDES OF THE BODT, AND THE VARIOUS KINDS OF LOCOMOTION • • • • • • • • • • • • . 489

\section{CHAPTER XIII.}

Of the Production of Sodnds: Voice and Speech . . . 513

CHAPTER XIV.

OF INSTINCT AND INTELLIGENCE . . . . . . . . . 525

MANIFESTATIONS OF INTELLIGENCE . . . . 546

CHAPTER XV.

Of Reproduction . . . . . . . . . . . . . 552

GEMMIPAROUS OR NON-SEXUAL REPRODUCTION . . . 553

SEXUAL REPRODUCTION, OR GENERATION . . . . 557 


\section{ANIMAL PHYSIOLOGY.}

\section{INTRODUCTION.}

Tre importance of the study of Animal Physiology, as a branch of General Education, can scarcely be over-estimated; and it is remarkable that it is not more generally appreciated. It might have been supposed that curiosity alone would have led the mind of Man to the eager study of those wonderful actions by which his body is constructed and maintained; and that a knowledge of those laws, the observance of which is necessary for the due performance of these actions, - in other words, for the maintenance of his health,-would have been an object of universal pursuit. That it has not hitherto been so, may be attributed to several causes. The very familiarity of the occurrences is one of these. We are much more apt to seek for explanations of phenomena that rarely present themselves, than of those which we daily witness. The Comet excites the world's curiosity, whilst the movements of the sun, moon, and planets are regarded as things of course. We almost daily see vast numbers of animals of different tribes, in active life around us ; their origin, growth, movements, decline, death, and reproduction, are continually taking place under our eyes; and there seems to common apprehension nothing to explain, where everything is so apparent. And of Man too, the ordinary vital actions are so familiar, that the study of their conditions appears superfluous. To be born, to grow, to be subject to occasional disease, to decline, to die, is his lot in common with other animals; and what knowledge can avail (it may be asked) to avert the doom imposed on him by his Creator? 
In reply to this it is sufficient to state, that millions annually perish from a neglect of the conditions which Divine wisdom has appointed as requisite for the preservation of the body from fatal disease ; and that millions more are constantly suffering various degrees of pain and weakness, that might have been prevented by a simple attention to those principles which it is the province of Physiology to unfold. From the moment of his birth, the infant is so completely subjected to the influence of the circumstances in which he is placed, that the future development of his frame may be said to be governed by them; and thus it aepends, in great part, upon the care with which he is tended, and the knowledge by which that care is guided, whether he shall grow up in health and vigour of body and mind; or shall become weakly, fretful, and selfwilled, a source of constant discomfort to himself and to others; or shall form one of that vast proportion, whose lot it is to be removed from this world before infancy has expanded into childhood. The due supply of warmth, food, and air are the principal points then to be attended to; and on every one of these the greatest errors of management prevail. Thousands and tens of thousands of infants annually perish during the few first days of infancy, from exposure to cold, which their feeble frames are not yet able to resist; and at a later period, when the infant has greater power of sustaining its own temperature, and is consequently not so liable to suffer from this cause, the seeds of fature disease are sown, by inattention to the simple physiological principles, which should regulate its clothing in accordance with the cold or heat of the atmosphere around. Nor is less injury done by inattention to the due regulation of the diet, as to the quantity and quality of the food, and the times at which it should be given; the rules for which, simple and easy as they are, are continually transgressed through ignorance or carelessness. And, lastly, one of the most fertile sources of infantile disease, is the want of a due supply of pure and wholesome air; the effects of which are sure to manifest themselves in some way or other, though often obscurely and at a remote period. It is physiologically impossible for human beings to grow up in a sound and healthy state of body and mind, in the midst of a close, ill-ventilated atmosphere. Those that are least able to resist its baneful influence, are carried off by the dis- 
eases of infancy and childhood; and those whose native vigour of constitution enables them to struggle through these, become the victims, in later years, of diseases which cut short their term of life, or deprive them of a large part of that enjoyment which health alone can bring.

Nor is the effect of these injurious causes confined to infancy, though most strikingly manifested at that period. "The child is father to the man," in body as well as in mind; but the vigorous health of the adult is too often wasted and destroyed by excesses, whether in sensual indulgence, in bodily labour, or in mental exertion, to which the very feeling of buoyancy and energy often acts as the incentive; and the strength which, carefully husbanded and sustained, might have kept the body and mind in activity and enjoyment to the full amount of its allotted period of "threescore years and ten," is too frequently dissipated in early manhood. Or, again, the want of the necessary conditions for the support of life,- the warmth, food, and air, on which the body depends for its continued sustenance, no less than for its early development,--may cause its early dissolution, even where the individual is guiltless of having impaired its vigour by his own transgressions.

These statements are not theoretical merely: they are based upon facts drawn from observations carried on upon the most extensive scale. Wherever we find those conditions, which the Physiologist asserts to be most favourable to the preservation of the health of the body, most completely fulfilled, there do sickness and mortality least prevail. A few facts will place this subject in a striking light. "The average mortality of infants among rich and poor in this country (and with little variation throughout Europe) is about one in every four and a-half before the end of the first year of existence. So directly, however, is infant life influenced by good or bad management, that, about a century ago, the workhouses of London presented the astounding result of twenty-three deaths in every twentyfour infants under the age of one year. For a long time this frightful devastation was allowed to go on, as beyond the reach of human remedy. But when at last an improved system of management was adopted in consequence of a parliamentary inquiry having taken place, the proportion of deaths was speedily reduced from 2,600 to 450 in a year. Here, then, B 2. 
was a total of 2,150 instances of loss of life, occurring yearly in a single institution, chargeable, not against any unalterable decrees of Providence, as some are disposed to contend as an excuse for their own negligence; but against the ignorance, indifference, or cruelty of man. And what a lesson of vigilance and inquiry ought not such occurrences to convey, when, even now, with all our boasted improvements, every tenth infant still perishes within a month of its birth !" I

The effect of attention to cleanliness and ventilation in the reduction of an excessive infantile mortality, has been equally shown in the experience of the Dublin Lying-in Hospital. At the conclusion of 1782 , it was found that out of 17,650 infants born alive, no fewer than 2,944, or one in every six, had died within the first fortnight. By the more efficient ventilation of the wards, the proportion of deaths during the first fortnight was at once reduced to 419 out of 8,033 , or but little more than one in twenty; and it has subsequently been still further diminished.

In the island of St. Kilda, the most northern of the Hebrides, according to the statement of a gentleman who visited it in 1838, as many as eight out of every ten children die between the eighth and twelfth day of their existence; in consequence of which terrible mortality, the population of the island is diminishing rather than increasing. This is due, not to anything injurious in the position or atmosphere of the island ; for its "air is good, and the water excellent:" but to the "filth in which the inhabitants live, and the noxious effluvia which pervade their houses." The huts are small, lowroofed, and without windows ; and are used during the winter as stores for the collection of manure, which is carefully laid out upon the floor, and trodden under foot, till it accumulates to the depth of several feet. The clergyman, who lives exactly as those around him do, in every respect, except as regards the condition of his house, has reared a family of four children, all of whom are well and healthy; whereas, according to the average mortality around him, at least three out of the four would have been dead within the first fortnight.

It is not a little remarkable that a recent sanitary inquiry carried out by order of the Danish government, into the con-

${ }^{1}$ Dr. A. Combe on the Physiological and Moral Management of Infancy. 
dition of the Icelandic population, should have disclosed the existence of almost precisely similar habits of life among them, with almost precisely the same results. The dwellings of the great bulk of the peasantry seem as if constructed for the express purpose of poisoning the air which they contain. They are small and low, without any direct provision for ventilation, the door serving alike as window and chimney; the walls and roof let in the rain, which the floor, chiefly composed of hardened sheep's-dung, sucks up; the same room generally serves for all the uses of the whole family, and not only for the human part of it, but frequently also for the sheep, which are thus housed during the severest part of the winter. The fuel employed in this country chiefly consists of cow-dung and sheep's-dung, caked and dried; and near the sea-coast, of the bones and refuse of fish and seafowl; producing a stench, which to those unaccustomed to it is completely insupportable. In addition to this, the people are noted for their extreme want of personal cleanliness; the same garments (chiefly of black flannel) being worn for months without having even been taken off at night. Although the Icelanders enjoy an almost complete exemption from many diseases (such as consumption) which are very fatal elsewhere, and the number of births is fully equal to the usual average, the population of the island does not increase, and in some parts actually diminishes. This result is in great measure due, as at St. Kilda, to the very high rate of infantile mortality; a large proportion of. all the infants born being carried off before they are a fortnight old. It is in the little island of Westmannoe, and the opposite parts of the coast of Iceland, where the bird-fuel is used all the year round, instead of (as elsewhere) during a few months only, that the rate is the highest; the average mortality for many years having been sixty-four out of every hundred, or nearly two out of three, of all the infants born in these localities.

But it is yet more remarkable that the imamediate cause of the high rate of infantile mortality should have been preeisely the same in the Workhouses of London, the Lying-in Hospital of Dublin, and the close filthy huts of the peasantry of Iceland and St. Kilda; for it was almost entirely referrible to one single disease, "Trismus nascentium," or, "Lock-jaw of the New-born;" and this disease has diminished in exact 
proportion to the improvement of the places it previously infested, in respect to ventilation and cleanliness. Thus, it is so rare for a case of it now to occur in London, that many practitioners of large experience have never seen the disease. In the Dublin Lying-in Hospital, the number of deaths from it has been reduced to three or four yearly. And there cannot be a reasonable doubt, that, by due attention to the same conditions, it might be exterminated from Iceland and from St. Kilda. There is scarcely, in fact, a disease incident to humanity, which is more completely preventible than this; and yet the annual sacrifice of life which it formerly caused in our own country alone, might have been reckoned by tens of thousands.

Although the peculiar suseeptibiltty of the constitution of children, gives to foul air and other causes of disease a much more destructive influence over them, than the like causes have over persons more advanced in life, yet it is now well ascertained that the rate of mortality among different classes of the community varies in a degree which bears a very close relation to the nature of the conditions under which they live. Thus, whilst the annual average number of deaths in the whole of England and Wales is about 22 out of every thousand persons living, there are localities in which the annual average exceeds 50 in a thousand, and others in which it falls as low as 11 in a thousand. And it is not a little remarkable, that the difference is almost entirely referrible to the mortality produced by Fevers and allied diseases, which, as experience has now fully demonstrated, are absolutely preventible by due attention to the ordinary conditions of health.

As the population of England and Wales may at present be estimated at about twenty millions, and its actual mortality at about 440,000 , what may be termed its inevitable mortalityarising from diseases that would not be directly affected by sanitary improvements-would be only one half, or 220,000; so that the same number of lives may be considered to be annually sacrificed by the public neglect of the means of preserving them,- the deaths from typhus alone being no fewer than 50,000. But as it is scarcely to be supposed that every part of our population could be placed in conditions as favourable as those which prevail where the rate of mortality is the lowest, we may take 13 per thousand as the average to 
which it may be safely affirmed, on the basis of actual experience, that the annual mortality may be reduced, by such efficient sanitary measures as render the dwellings of the mass of the population fit for human habitation; this would give an annual mortality for England and Wales of 260,000, showing a saving of 180,000 lives annually in that one portion of the British empire. And it must be remembered that this amount of mortality represents a vastly greater amount of sickness, since, for every death, there are numerous cases of severe illness; so that it would be scarcely too much to affirm that at least a million out of the whole number of such cases annually occurring, are preventible, like the 180,000 deaths, by adequate provisions for the supply of pure. air and water, and by efficient sewerage for the removal of decomposing matters. It cannot be doubted that, even in a mere pecuniary point of view, the expense of such arrangements would be amply compensated by the prevention of a vast amount of that loss of productive labour of various lkinds, which is at present due to disease ; and, considered on the large scale, as a question of social economy, the importance of sunitary legislation can scarcely be over-rated. But much cannot be expected to be done in this direction, until such an intelligent public opinion shall have been created, by the general diffusion of sound physiological information, as shall be sufficiently forcible to bear down the self-interested opposition of those, who do not see that the value of their property will be permanently increased at least in proportion to the amount of money judiciously expended upon it.

A more remarkable illustration of what is to be effected by sanitary improvements can scarcely be adduced, than that which is presented by the comparison between the locality termed "the Potteries," in the immediate vicinity of Kensington, and the "Model Lodging-houses," which have been erected in various parts of the Metropolis. The site of the group of dwellings constituting the former is far from being insalubrious in itself, and rows of handsome houses are rising up in its immediate neighbourhood; but the condition of these dwellings is most filthy. A few years ago, as many as 3,000 pigs were kept in this locality (the number has since been somewhat diminished); and the boiling of fat and other offal, which is carried on by some of the pig-feeders, some- 
times taints the air for a mile round. Very few of the tenements have any water-supply; the wells are useless, or worse than useless, through the contamination of their water with putrescent liquid which filters down into them; and the drainage of the dwellings both for men and pigs is almost entirely superficial, being chiefly discharged into a stagnant piece of water called the "Ocean," which is covered with a filthy slime and bubbles with poisonous gases, and very commonly has dead dogs or cats floating on its surface. It is difficult to conceive anything more horribly offensive than the rears of some of the houses, whose yards are filled with ordure and other filth collected for manure, which is here stored for weeks, or even months, until an opportunity occurs for selling it. And even the public ways are generally covered with black putrescent mire. Now, during ten months of the year 1852, when no epidemic prevailed, as many as forty deaths occurred in the Potteries, out of a population of about one thousand,- the mortality being thus at the rate of 48 per thousand annually; and no fewer than four-fifths of these deaths occurred at, or beneath, five years of age. In the first ten months of 1849 , when cholera was prevalent, the number of deaths was fifty, or about one in twenty of the whole population, twenty-one of these being due to cholera and diarrhœea, and twenty-nine to typhus and other diseases. - On the other hand, in the whole population of the "Model Lodging-houses," amounting to 1,343 , only seven deaths took place in the whole twelve months of 1852, or at the rate of scarcely more than 5 per thousand; and although they contain a large proportion of children, yet only half the number of deaths occurred below ten years old. During the prevalence of the cholera-epidemic, no cases of that disease occurred among them, although it was raging in their various neighbourhoods; and from the time that their drainage has been rendered thoroughly efficient, no case of fever has presented itself among their inmates.

The experience of Cholera-epidemics is peculiarly valuable, on account of the marked tendency of this disease to search out and expose defects, which have continued to produce other diseases year after year, without having been suspected as the causes of them. The greatest severity in each visitation has shown itself in identical localities, provided those 
remained in the same foul state as at first; whilst new localities have been affected, just in proportion to the degree in which they have participated in the same conditions; and those originally attacked have escaped, wherever they had adopted the requisite means of purification. Thus, at Newcastle-onTyne and Gateshead, the first outbreak occurred in the very same streets, and even in the same houses, in the three visitations of 1831, 1848, and 1853. An outbreak which occurred in 1853, at Luton, in Bedfordshire,- where, out of a population of 126 persons, inhabiting twenty-five houses, no fewer than fifty-four attacks of choleraic disease, fifteen of them fatal, took place within three weeks, - was most distinctly traceable to defect of sewerage, which had been previously manifesting its malign influence on the general health of the town. And the fearful pestilence which devastated the neighbourhood of Golden Square (London) in the autumn of 1854, was no less distinctly traceable to the contamination of the pump-water by the bursting of a sewer into the well. On the other hand, Exeter and Nottingham, which suffered severely in the first epidemic, escaped comparatively unharmed in the subsequent visitations; and this result is plainly due to the sanitary improvements which had been made in the interval. In 1832 there perished of the epidemic in Exeter, as many as 402, out of a population of 28,000 , or no fewer than one in seventy; and a vast amount of suffering, with a heavy expense, was entailed upon the town. In 1848-9, on the other hand, out of a population of about 32,600 , there were but 44 deaths, or less than one in seven hundred; and upwards of one-half of these occurred in a single parish, that lies very low, and in the midst of putrid exhalations from the city drains. In Nottingham, with a population of 50,000 , there were 296 fatal cases of cholera in 1832, nearly all of these being in the lower part of the town, which was ill-drained, extremely filthy, and densely populated; but in 1848-9, though the population had increased to 58,000 , the number of deaths from cholera was no more than 18 , all of these occurring in localities, which, in spite of what had been done, retained much of their previous filth.

The foregoing are only samples of a vast number of ouses which might be adduced, in proof of the absolute preventibility of Cholera, and of other diseases of the same class. It 
may be well to subjoin a few additional facts, derived from the cholera-experience of $1848-9$, which, from its general diffusion, tested, in a very remarkable degree, the relative healthfulness of different provincial towns, and of different metropolitan districts. Thus, among the whole population of the ten towns of Exeter, Derby, Cheltenham, Leicester, Nottingham, Rochdale, Norwich, Preston, Halifax, and Birmingham, amounting to 657,000 , there were no more than 238 deaths from cholera; whilst, in an equal population inhabiting the towns of Newcastle-under-Lyne, Plymouth, Brighton, Merthyr Tydvil, Portsea, Tynemouth, Wigan, Hull, Wolverhampton, and Leeds, the number of deaths was no fewer than 10,415, or forty-three times as great. So again, in twenty-five Metropolitan districts, chiefly on the north side of the Thames, having a total population of about 310,000 , the number of deaths from cholera was only 389 ; whilst in twenty-two districts, almost entirely on the south side of the river, the number of deaths, out of a population of almost exactly the same amount, was 5,932, or more than twelve times as great. In no instance is there the least difficulty in accounting for these contrasts. They all point to the same general conclusion; that, namely, of the immense influence which is exercised over human health by the purity of the air that is breathed, and of the water that is drunk; and it is because these two conditions are in a great degree capable of public regulation, that legislative interference has so much in its power, and is so imperatively called for by the interests of humanity, which speak solemnly and distinctly to all who claim the rights of property in the foul "plague-spots" which deface our country, of their bounden duty to render them not unfit for human occupation.

But although the magnitude of the evils resulting from the neglect of the conditions of Public Health, gives to this subject the first claim on our consideration, yet it is not the less important that every individual should acquire as much knowledge of the constitution of his body, and of the right means of keeping it in working order, as will save him from seriously damaging either himself or other people by his ignorance of such matters. It is less than ten years since a fearful sacrifice of life occurred among the deck-passengers on board the Irish steamer "Londonderry," who were ordered 
below by the Captain on account of the stormy character of the weather, and on whom the hatches were closed down, although the cabin which was crowded by them had scarcely any other means of ventilation. Out of 150 of these unfortunates, no fewer than 70 died of suffocation before the morning, - a catastrophe only second to that which occurred in the "Black Hole of Calcutta," in which 123 out of 146 died during one night's confinement in a room eighteen feet square, provided with only two small windows. Yet the Captain of the "Londonderry" was acquitted of all blame; since he had done what seemed to him best for the welfare of his passengers, the result being due simply to his astounding ignorance of the fact that men cannot live without having air to breathe. Not a year passes without the occurrence of numerous deaths from the like cause; and yet these are really insignificant, when compared with the vast amount of disease which is constantly attributable to inattention, on the part of individuals, to those simple means of securing an adequate supply of air which are within the reach of every one. And when we bear in mind that the respiratory function is only one of the processes whose due performance has to be provided for, and that the regulation of the food and drink, of the excretions, of clothing and temperature, of exercise (bodily and mental) and repose, and of the reproductive functions, all fall within rules which it is the province of Physiology to prescribe, we see how vain it is to expect that the body can be maintained in health, without some acquaintance with that science, or at least with the rules which it lays down. For, although it is quite true that man has within himself certain instincts which afford him a considerable measure of guidance in all these particulars,hunger and thirst, for example, leading him to take the sustenance which his body requires, weariness tempting him to needed repose, and so on,- - yet it is no less certain that in a state of artificial civilisation these instincts are so often overborne by acquired tastes, or by the pressure of other circumstances, that they cannot alone be safely relied on. Hence it is all the more important that the rules for preserving health should be based on an intelligent knowledge of Physiological principles; otherwise, like the natural instincts, they are likely to be put aside as occasion prompts ; whereas, in proportion as 
the individual is possessed of their rationale, will he be likely to shape his conduct in accordance with them.

The general principles of Physiological science, again, will be likely to be thoroughly apprehended, in proportion as they are based on an extended recognition of the phenomena which they comprehend. Every physiologist is now satisfied that the life or vital actions of no one species of animal can be correctly understood, unless compared with those of other tribes of different conformation. Hence, for the student of physiology to confine himself to the observation of what takes place in Man alone, would be as absurd as for the astronomer to restrict himself to the observation of a single planet, or for the chemist to endeavour to determine the properties of a metal by the study of those of that one only. There is not a single species of animal, that does not present us with a set of facts which we should never learn but by observing it; and many of the facts ascertained by the observation of the simplest and most common animals, throw great light upon the great object of all our inquiries, the Physiology of Man. For though in him are combined, in a most wonderful and unequalled manner, the various faculties which separately exhibit themselves in various other animals, he is not the most favourable subject for observing their action; for the obvious reason that his machinery (so to speak) is rendered too complex, on account of the multitude of operations it has to perform : so that we often have to look to the lowest and simplest animals for the explanation of what is obscure in man, their actions being less numerous, and the conditions which they require being more easily ascertained.

The diffusion of Animal life is only one degree less extensive than that of regetable existence. As animals cannot, like plants, obtain their support directly from the elements around, they cannot maintain life, where life of some kind has not preceded them. But vegetation of the humblest character is often sufficient to maintain animals of the highest class. Thus the lichen that grows beneath the snows of Lapland, is, for many months in the year, the only food of the rein-deer; and thus contributes to the support of human races, which depend almost solely upon this useful animal for their existence. No extremes of temperature in our atmosphere seem inconsistent with animal life. In the little pools 
formed by the temporary influence of the sun upon the surface of the arctic snows, animalcules have been found in a state of activity; and the ocean of those inhospitable regions is tenanted, not only by the whales and other monsters which we think of as their chief inhabitants, whose massive forms are only to be encountered "few and far between," but by the shoals of smaller fishes and inferior animals of various kinds upon which they feed, and through vast fleets of which the mariner sails for many miles together.

On the other hand, even the hottest and most arid portions of the sandy deserts of Africa and Asia are inhabited by animals of various kinds, provided that vegetables can find sustenance there. The humble and toilsome ants make these their food, and become in turn the prey of the cunning antlion and of the agile lizard; and these tyrants are in their turn kept under by the voracity of the birds which are adapted to prey upon them. The waters of the tropical ocean never acquire any high temperature, owing to the constant interchange which is taking place between them and those of colder regiens; but in the hot springs of various parts of the world, we have examples of the compatibility of even the heat of almost boiling water with the preservation of animal life. Thus in a hot spring at Manilla which raises the thermometer to $187^{\circ}$, and in another in Barbary whose usual temperature is $172^{\circ}$, fishes have been seen to flourish. Fishes have been thrown up in very hot water from the crater of a volcano, which, from their lively condition, was apparently their natural residence. Small caterpillars have been found in hot springs of the temperature of $205^{\circ}$; and small black beetles, which died when placed in cold water, in the hot sulphur baths of Albano. Intestinal worms within the body of a carp have been seen alive after the boiling of the fish for eating; and the inhabitants of some little snail-shells, which seemed to have been dried up within them, have been caused to revive by placing the shells in hot water for the purpose of cleaning them.

The lofty heights of the atmosphere, and the dark and rayless depths of the ocean, are tenanted by animals of beautiful organisation and wonderful powers. Vast flights of butterflies, the emblems of summer and sunshine, may sometimes be seen above the highest peaks of the Alps, almost 
touching with their fragile wings the hard surface of the never-melting snow. The gigantic condor or vulture of the Andes has been seen to soar on its widely-expanded wings far above the highest peak of Chimborazo, where the barometer would have sunk below ten inches. The existence of marine fishes has been ascertained at a depth of from 500 to 600 fathoms ; and in the deep recesses of those caverns in Styria and Carniola, which are inhabited by the curious Proteus (ZooL. \$ 532), numerous species of insects are found, all of which, however, like the Proteus, are blind.

Having thus glanced at some of those facts which demonstrate the practical importance of the study of Physiology, and having indicated the mode in which that study should be pursued, it remains to offer a few observations upon its value with reference to the culture and discipline of the mind itself. One of its great advantages is, that it not only calls forth, in a degree second to no other, both the observing and the reasoning powers; but that it offers so much that is attractive by its novelty to those who enter upon it seriously, and make it an object of regular pursuit. For it affords abundant opportunities, even to the beginner, of adding to the common stock of information respecting the structure and habits of the vast number of living beings that people our globe. The immense variety of the objects which come under the investigation of the physiologist, so far from discouraging the learner, should have the effect of stimulating his exertions, by opening to him new fields for productive cultivation. Of by far the larger part of the organised creation, little is certainly known. Of no single species, - of none of our commonest native animals, - not even of Man himself,-can our knowledge be regarded as anything but imperfect. Of the meanest and simplest forms of animal life, we know perhaps even less than we do of the more elevated and complex; and it cannot be doubted that phenomena of the most surprising nature yet remain to be discovered by patient observation of their actions. It was not until very recently, that the existence of a most extraordinary series of metamorphoses, more wonderful than those of the insect, has been discovered in the jelly-fish of our seas, in the barnacles that 
attach themselves to floating pieces of timber, and in the crabs, lobsters, and shrimps of our shores. The very best accounts we have, of the structure, habits, and economy of the lower tribes of animals, have been furnished to us by individuals who did not think it beneath them to devote many years to the study of a single species; and as there are very few which have been thus fully investigated, there is ample opportunity for every one to suit his own taste in the choice of an object.

And none but those who have tried the experiment, can form an estimate of the pleasure which the study of Nature is capable of affording to its votaries. There is a simple pleasure in the acquisition of knowledge, worth to many far more than the acquisition of wealth. There is a pleasure in looking in upon its growing stores, and watching the expansion of the mind which embraces it, far above that which the miser feels in the grovelling contemplation of his hard-sought pelf. There is a pleasure in making it useful to others, comparable at least to that which the man of generous benevolence feels in ministering to their relief with his purse or his sympathy. There is a pleasure in the contemplation of beauty and harmony, wherever presented to us. And are not all these pleasures increased, when we are made aware,-as in the study of Nature we soon become,- - that the sources of them are never-ending, and that our enjoyment of them becomes more-intense in proportion to the comprehensiveness of our knowledge? And does not the feeling that we are not looking upon the inventions or contrivances of a skilful human artificer, but studying the wonders of a Creative Design infinitely more skilful, immeasurably heighten all these sources of gratification? If it is not every one who can feel all these motives, cannot every one feel the force of some?

There is certainly no science which more constantly and forcibly brings before the mind the power, the wisdom, and the goodness of the Creator. For whilst the Astronomer has to seek for the proofs of these attributes in the motions and adjustments of a universe, whose nearest member is at a distance which imagination can scarcely realize, the Physiologist finds them in the meanest worm that we tread beneath our feet, or in the humblest zoophyte dashed by the waves 
upon our shores, no less than in the gigantic whale, or massive elephant. And the wonderful diversity which exists amongst the several tribes of animals, presents us with a continual variety in the mode in which these adjustments are made, that prevents us from ever growing weary in the search.

But it is not only in affording us such interesting objects of regular study, that the bounty of Nature is exhibited. Perhaps it is even more keenly felt by the mind which, harassed by the cares of the world, or vexed by its disappointments, or fatigued by severer studies, seeks refuge in her calm retirement, and allows her sober gladness to exert its cheering and tranquillizing influence on the spirit.

"With tender ministrations, thou, O Nature, Healest thy wandering and distracted child; Thou pourest on him thy soft influences, Thy sunny hues, fair forms, and breathing sweets, The melody of woods, and winds, and waters.Till he relent, and can no more endure To be a jarring and dissonant thing Amidst the general voice and minstrelsy,But bursting into tears wins back his way, His angry spirit healed and harmonized By the benignant touch of love and mercy."

COLERIDGR 


\section{CHAPTER I.}

OF THE VITAL OPERATIONS OF ANIMAIS, AND THE INSTRUMENTS BY WHICH THEY ARE PERFORMED.

1. Lrviva beings, whether belonging to the Animal or to the Vegetable kingdom, are distinguished from the masses of inert matter of which the Mineral kingdom is made up, by peculiarities of form and size, of structure, of elementary composition, and of actions.-Wherever a definite form is exhibited by Mineral substances, it is bounded by plane surfaces, straight lines, and angles, and is the effect of the process of crystallization, in which particles of like nature arrange themselves on a determinate plan, so as to produce a regular aggregation; and there is, probably, no Inorganic element or combination which is not capable of assuming such a form, if placed in circumstances adapted to the manifestation of its tendency to do so. The number of different crystalline forms is by no means large ; and as many substances crystallize in several dissimilar forms, whilst crystals resembling one another in form often have a great diversity of composition, there is no constant correspondence between the crystalline forms and the essential nature of the greater number of mineral substances. If that peculiar arrangement of the molecules which constitutes crystallization should be wanting, so that simple cohesive attraction is exercised in bringing them together, without any general control over their direction, an indefinite or shapeless figure is the result. With this indefiniteness of form, there is an absence of any limit whatever in regard to size: a crystal may go on increasing continuously, so long as there is new material supplied; but this new material is deposited upon its surface merely, and its addition involves no interstitial change ; the older particles, which were first deposited, and which continue to form the nucleus of the crystal, remaining just as they were. In Organized bodies, on the other haud, we meet with convex surfaces and rounded outlines, and with a general absence of angularity; and the simplest grades, both of Animal and of 
Vegetable life, present themselves under a shape which approaches more or less closely to the globular. From the highest to the lowest, each species has a certain characteristic form, by which it is distinguished ; this form, however, often presents marked diversities at different periods of life, and it is also liable to vary within certain limits among the individuals of which the species is composed. The size of Organized structures, like their form, is restrained within tolerably definite limits, which may nevertheless vary to a certain extent among the individuals of the same species. These limits are most obvious in the higher animals, whilst they seem almost to disappear among certain members both of the Animal and the Vegetable kingdoms, which tend to increase themselves almost indefinitely by a process of gemmation or budding, so as to produce aggregations of enormous size. Such aggregations, however, being formed by the repetition of similar parts, which can maintain their existence when detached from one another, may, in some sense, be regarded as clusters of distinct organisms, rather than as single individuals. Such is the case, for example, with the wide-spreading forest-tree, and with those enormous masses of coral of which reefs and islands are composed in the Polynesian Archipelago. For every separate leaf-bud of the tree, like every single polype of the coral, if detached from its stock, can, under favourable circumstances, perform all the functions of life, and can develop itself into a new fabric resembling that from which it was separated.

2. The differences between Organized and Inorganic bodies, in regard to their structure, are much more important than those which relate to their external configuration. . Every particle of a mineral substance, in which there has not been a mere mixture of components, exhibits the same properties as those possessed by the whole ; the minutest atom of carbonate of lime, for instance, has all the properties of a crystal of calc-spar, were it as large as a mountain. Hence it is the essential nature of an Inorganic body that each of its particles possesses a separate individuality, and has no relation but that of juxtaposition to the other particles associated with itself in one mass.-The Organized structure, on the other hand, receives its designation from being made up of a greater or less number of dissimilar parts or organs; each of these 
being the instrument of some special action or function, which it performs under certain conditions ; and the concurrence of all these actions being necessary to the maintenance of the structure in its normal or regular state. Hence there. is a relation of mutual dependence among the parts of an Organized fabric, which is quite distinct from that of mere proximity; and this relation is most intimate, not in the case of those beings which have the greatest multiplication of parts, but among those in which there is the greatest dissimilarity among the actions of the several organs. Thus it has been just shown that among Plants and Zoophytes, a small fraction of an organism may live independently of the rest; the necessary condition being that it shall either itself contain all the organs essential to life, or shall be capable of producing them,-as when the leaf-bud develops rootlets for its nutrition. This "vegetative repetition," and consequent capacity of sustaining the loss of large portions of the fabric, still shows itself in animals much higher in the scale than Zoophytes; thus it is not uncommon to meet with Star-fish in which not only one or two, out of the five similar arms, but even three or four, have been lost, without the destruction of the animal's life ; and this is the more remarkable, as these arms are not simply members for locomotion or prehension, but are really divisions of the body, containing prolongations of the stomach. In like manner, many of the Worm tribes, whose bodies show a longitudinal repetition of similar parts, can lose a large number of their joints without sustaining any considerable damage. In the bodies of the higher animals, however, where there are few or no such repetitions (save in the two lateral halves of the body), and where there is, consequently, a greater diversity in character and function between the different organs, the mutual dependence of their actions upon one another is much more intimate, and the loss of a single part is much more likely to endanger the existence of the whole. Such structures are said to be more highly organized than those of the lower classes; the principle of " division of labour" being carried much further in them, a much greater variety of objects being attained, and a much higher perfection in the accomplishment of them being thus provided for. Thus the individuality of a plant or a zoophyte may be said to reside in each of its multiplied parts ; 
whilst that of one of the higher animals resides in the sum of all its organs.

3. The very simplest Organized fabric is further distinguished from Inorganic bodies by marked differences in regard to intimate structure and consistence. Inorganic substances can scarcely be regarded as possessing a structure, since their perfection consists in their homogeneousness and their solidity. It is the essential character of Organized fabrics, on the other hand, that they are formed by a combination of solid and liquid components, so intimately combined and arranged as to impart a heterogeneous character to almost every portion of their substance; and in all the parts which are most actively concerned in the vital operations, softness of texture seems an essential condition,those parts only being so consolidated as to acquire anything comparable to the density of mineral bodies, which are destined to possess the simply physical property of resistance, so as to be subservient either to support, to protection, or to mechanical movement. A comparison between the pulpy portion of the leaves of Plants and the heartwood of the stem, between the membranous tissues of the Coral-polypes and the stony masses which they form, between the firm shell of the Crab or the Oyster and the substance of the included body, or between the solid bones of Man and the flesh which clothes them, will serve to illustrate this principle. It is in such solidified portions of the Organized fabric, that the greatest resemblance exists to Inorganic bodies; but even these portions all pass through the condition of soft tissue, the consolidation of which is effected by the deposit of some hardening material (generally carbonate or phosphate of lime), in its interstices. - It is by the reaction which is continually taking place between the solid and the liquid parts of Organized structures, that their integrity is maintained. For we shall find it to be a result of their peculiar composition, that they are prone to continual decay; and this decay would speedily destroy them altogether, if it were not compensated by new formation. The materials for their reproduction must always be presented to the tissues in a liquid state, and all the dead and decomposing matter must be reduced to the same form, in order that it may be carried off; so that the intermingling or mutual penetration of solids and liquids, in 
the minutest parts of Organized bodies, is a necessary condition of their existence.

4. Organized structures are further distinguished from Inorganic masses by the peculiarity of their chemical constitution. This peculiarity does not consist, however, in the presence of any elementary substances which are not found elsewhere; for all the elements of which Organized bodies are composed, exist abundantly in the world around. This, indeed, is a necessary consequence of the mode in which they are built up ; for that which the parent communicates in giving origin to a new being, is not the structure itself, but the capacity to form that structure from materials supplied to it; and it is by progressively converting these materials to its own use, that the germ develops itself into the complete fabric.-Now out of about seventy simple or elementary substances which are known to occur in the Mineral world, not above twenty present themselves as constituents of Vegetable and Animal fabrics; and many of these occur there in extremely minute proportion. Some of them, indeed, appear to be introduced merely to answer certain chemical or mechanical purposes; and the composition of the parts which possess the highest vital endowments is extremely uniform. They are nearly all formed at the expense of certain "organic compounds," which are made up of the four elementary substances, oxygen, hydrogen, carbon, and nitrogen ; and these elements appear to be united,-not as in the case of inorganic compounds, two by two, or after the binary method,- but all four together, so as to form a compound atom of great complexity. Thus common nitre is regarded as a binary compound of nitric acid and potass, since it can be decomposed into those two constituents and can be re-formed by their union; and in the same manner, its nitric acid is a binary compound of nitrogen and oxygen, whilst its potass is a binary compound of potassium and oxygen. But neither albumen nor gelatine, which are the principal materials of the animal tissues, can be resolved into any two other substances, by the union of which it can be re-formed; and when once it has been decomposed by chemical agencies, no means known to the chemist can reproduce it. Albumen can, in fact, be generated only by the living Plant, at the expense of the carbon, hydrogen, oxygen, and nitrogen, which it draws from the elements around; and 
gelatine can only be formed in the animal body by a metamorphosis of the albumen which it derives from the Plant. The peculiar mode in which the elements of these substances are held together, renders them very prone to decomposition; so that Organized bodies, when no longer alive, rapidly pass into decay, unless they are secluded from the contact of oxygen, or are kept at a very low temperature. Such decay, however, is continually taking place during life, and would make itself obvious if its products were not carried out of the system as fast as they are generated within it. It essentially consists in the resolution of the four principal components of organic compounds-carbon, hydrogen, oxygen, and nitrogen, in combination with oxygen drawn from the atmosphere-into the three binary compounds, water, carboric acid, and ammonia, which thus restore to the Inorganic world the original materials of Organized fabrics, in the very forms from which those materials were first derived by the agency of the growing Plant. (See Veater. Physiol.)

5. It is, however, by their peculiar actions, that living Organisms are most completely differentiated from the inert bodies of which the Mineral kingdom is composed. There can be no doubt that of many of the changes which take place during the life of an Organized being, a large proportion (especially in the Animal kingdom) are effected by the direct agency of physical and chemical forces; and there is no reason to believe that these forces have any other.operation in the living body, than they would have out of it under similar circumstances. Thus the propulsion of the blood by the heart, through the large vessels, is a purely mechanical phenomenon; as is also the movement of the limbs by the lever-action of the forces brought to bear on their bones. So, again, the digestive operations which take place in the stomach are of a purely chemical nature ; and the interchange of gases between the air and the blood, which takes place in the act of respiration, must be regarded in the same light.But after every possible allowance has been made for the operation of physical and chemical forces in the living organism, there still remain a large number of phenomena which cannot be in the least explained by them, and which must be regarded as the result of an agency that differs from these as they differ from each other; and this agency, which 
is recognised by the effects it produces -in the same manner as we recognise heat or electricity by their effects-may be conveniently designated vital force. ${ }^{1}$ Thus, to revert to our previous illustrations, the mechanical power employed in the propulsion of the blood, or in the movements of the limbs, is evolved by muscular contraction, a phenomenon altogether peculiar to the living muscle ; and the muscle derives its property of contractility from the previous development of its peculiar tissue in the act of nutrition. So the solvent fluids by which the digestion of food is accomplished, are separated from the blood by an act of secretion, which can only be performed by a glandular apparatus in the living walls of the alimentary canal. And the materials for the nutrition of the muscular tissue, and for the secretion of the digestive solvent, as of all the other acts of nutrition and secretion which are continually going on in the living body, are derived from the blood, - a liquid which possesses properties very different (as we shall hereafter see) from any mere mixture of chemical compounds, and which is prepared by actions totally beyond the power of the chemist to imitate,the laboratory of the living organism being requisite for their performance.

6. The whole assemblage of vital actions which is performed by the living Animal, may be arranged under two principal groups; one of them consisting of those which are directly concerned in the development and maintenance of its Organized fabric ; the other including all those by which it is brought into conscious relation with the world around. The former group includes the acts of digestion, absorption, and assimilation, by which the nutritive materials are prepared for becoming part of the living fabric ; the circulation of the assimilated materials through the body; their conversion, by the act of nutrition, into the solid textures; the formation of various secretions, having various purposes to serve in the economy; the removal, by the acts of respiration

1 The Author has elsewhere given his reasons for the belief, that Vital force bears the same "correlation" to the Physical and Chemical forces, as the latter bear to each other; but the discussion of this subject is not suited to an elementary treatise; and the essential peculiarity of the manifestations of vital force in the phenomena of life, requires that it should be treated as belonging to a distinct category. 
and excretion, of the effete matters with which the blood becomes charged by the decomposition continually going on in the body; the maintenance of animal-heat by the same process; and the act of reproduction, whereby the race is perpetuated, in spite of the limited duration of the individual. The foregoing, which are for the most part common to the Animal and the Plant, are termed Organic Functions, or Functions of Vegetative Life. But, in addition to these, it is the characteristic of Animals generally, that they are sensible to impressions made by surrounding objects, so that they possess some consciousness of what is going on about them; and that they also possess the power of re-acting on those objects by movements of their own, so as to change either their own places, or the places of surrounding objects in relation to themselves. These two functions, sensibility and the power of spontaneous motion, being peculiar to animals, are distinguished as Animal Functions, or Functions of Animal Life. In the higher. animals, they are the most important and characteristic phenomena of their existence; so that it would seem as if the whole assemblage of organic functions had no other destination in them, than to build up and keep in order the apparatus by which the functions of animal life are performed. But this state of things is entirely reversed among those lower tribes of animals which border most closely on the Vegetable kingdom; for we find that among such, the manifestations of sensibility and power of spontaneous movement are so feeble, that it may be doubted whether these attributes are really present in them; and even in higher orders, there are many in which the proper animal powers are in such a low grade of development, that they appear as if they were destined merely to minister to the organic functions.

7. Thus, although the characteristic difference between the Animal and the Vegetable kingdom, taking each as a whole, may be truly said to consist in the possession by the former of endowments which do not exist in the latter, this does not express the essential difference between Animals and Plants; since, while there are many tribes among the former in which the proper animal powers are reduced to so low a degree as to prevent it from being certainly affirmed that they are present at all, there are many tribes among the lower plants which exhibit a power of spontaneous movement fully as 
great as that which exists among the lowest animals; so that no positive line can be drawn between the two kingdoms on the basis of this distinction alone. There is another very important physiological difference, however, between the two kingdoms, which seems to afford an adequate means of settling the true place of those tribes whose position would otherwise be doubtful. This lies in the nature of their food, and the source from which it is obtained. For although it.is now known that the primary tissues of plants are originally formed of the same albuminous material as are those of animals (the cellulose layers which constitute the great bulk of the vegetable fabric being a subsequent deposit), yet this material is generated in the Plant by the combination of the elements which it obtains from the carbonic acid, water, and ammonia of the soil or of the atmosphere; whilst the Animal is destitute of all power of thus forming it for itself, and is hence entirely dependent upon the plant for its supplies of nutriment. Thus, whilst the very humblest forms of Vegetation, in common with the highest, are found to have the power of decomposing carbonic acid under the influence of sunlight, setting free its oxygen and retaining its carbon, the humblest forms of Animal life, in common with the highest, derive their nutriment either directly from plants, or from the bodies of other animals which have subsisted on vegetable food, whilst they produce a converse change in the atmosphere by their respiration, absorbing from it oxygen, and giving forth to it carbonic acid. This criterion will serve, it is believed, to distinguish the very lowest forms of Animal life from those humble forms of Vegetation which they most closely resemble in the simplicity of their organization (§ 128); and its application will generally be found to be very easy. There is now no longer any doubt that a large proportion of the beings formerly ranked as Animalcules, are really to be regarded as Plants, notwithstanding that they possess a power of active and apparently spontaneous movement, far greater than that of many unquestionable animals. And generally it may be said that the presence of a bright-green or bright-red colour in any of these simple organisms, where it is not derived from coloured substances taken in as food, affords a strong probability of their vegetable character; these colours being produced in the 
course of that series of chemical changes, by which, under the influence of light, the living plant can unite, inorganic elements into organic compounds.

8. Not only do Animals differ from Plants in the nature and sources of their aliment, but also in the mode in which it is taken into their bodies; and this difference is related alike to the character of the food of animals, and to the general conditions of animal existence. For the Plant extends its roots through the soil in search of liquid, and spreads out its leaves to the air for the purpose of imbibing some of its gaseous ingredients. But the Animal could not so exist, and be at the same time endowed with the power of moving from place to place; nor could it appropriate solid nutriment, if it were not provided with some peculiar means of receiving and preparing this. For these purposes, animals (with few exceptions) are provided with an internal cavity or stomach into which the food is received from time to time, in which it can be carried about in the general movements of the body, and within which it can be prepared for being received by absorption into the current of nutrient liquid which circulates through the body. This stomach is nothing else than a bag formed by the prolongation of the external covering of the body into its interior ( $\$ 36)$; its cavity receives the food introduced into it by the mouth; its walls pour out or secrete a fluid which acts upon the food in such a manner as to dissolve it; and through its walls are absorbed those portions of the food which are fit to be employed as nutriment, while the remainder is cast forth from the cavity, either by the aperture which first admitted it, or by a distinct orifice. The exceptional cases, in which no stomach exists, chiefly occur in one particular tribe of animals, the Entozoa ( $\$ 105)$, which live either in the intestinal canal or in the substance of the tissues of other animals, and which are supported by the nutrient juices of these; such an organ obviously not being required by creatures which have no power of locomotion, and which can imbibe liquids already prepared for their use, through the whole of the soft surface of their bodies. But there is a large tribe of very simple animals, the Rhizopoda (§ 129), in which, notwithstanding the absence of any regular stomach, the food is received into the very substance of the jelly-like particle of which the body consists; a mouth and 
stomach being extemporized, as it were, on each occasion that aliment is ingested; and an anal orifice being extemporized in like manner, when the indigestible residue has to be cast forth. All true Animalcules (\$ 133) have a proper mouth, into which food is drawn by the current created by the cilia ( $\$ 45)$ wherewith it is fringed; and this mouth leads to the general cavity of the body, within which the food is subjected to the digestive process. In Zoophytes ( $\$ 121)$ which possess a proper stomach, this organ forms so large a part of the animal, that its entire body may be almost said to consist of the stomach and of the prehensile appendages by which it draws in its food. But in all the higher tribes, the stomach, with the alimentary canal proceeding from it, are suspended freely within the general cavity of the body; and we shall find that the space that surrounds these viscera is extremely important in the economy of all but vertebrated animals, as being a sort of reservoir into which the nutrient materials prepared by the digestive process first transude, and from which it is carried into the remoter parts of the system. In vertebrated animals, this cavity - called in them the peritoneal cavity, from its being lined with a serous membrane (\$ 28), termed the peritoneum -is not subservient to the same purposes ; the nutrient materials being taken up from the walls of the digestive cavity, both by. the blood-vessels and by special absorbents, and being by them carried into the current of the circulation. It is obvious that until they have found their way, through one or other of these channels, into the general system, the nutrient materials introduced as food into the stomach of an animal are not within its body, properly so called, any more than a fluid is within a plant when it bathes the exterior of its roots, or within an entozoon when in contact with the soft surface of its integument. In each case, the absorption of the fluid is first requisite; and it is with this that its application to the requirements of the living body really commences.

9. But further, when we compare together, not the lowest, but the highest members of the Vegetable and Animal kingdoms respectively - those in which their respective attributes are most characteristically displayed,-we find that they present such differences as to render it quite impossible to confound the one with the other. Although it is easy even 
for the scientific naturalist to mistake a Protophyte (or one of the simplest forms of vegetation) for an Animalcule, and although Zoophytes are continually ranked in the popular mind with the Plants they so much resemble in form, no one is in any danger of confounding the Oak and the Elephant, the Palm and the Whale. For among the higher Animals, not only the principal organs, but the greater part of their elementary parts or tissues, are formed upon a plan entirely different from that which prevails in Plants. All the arrangements of their organism or corporeal edifice are made for the purpose of enabling them to perform, in the most advantageous manner possible, those peculiar functions with which they have been endowed, - to receive sensations, - to feel, think, and will,-and to move in accordance with the directions of the instinct or the judgment. For these purposes we find a peculiar apparatus, termed the Nervous system, adapted. This apparatus consists of a vast number of fibres, spread out over the surface of the body, and especially collected in certain parts, called Organs of Sense (such as the eye, nose, ear, tongue, lips, and points of the fingers). These have the peculiar property of receiving impressions which are made upon their extremities, and of conveying them to the central masses of nervous matter (known in the higher animals as the Brain and Spinal Cord.), by the instrumentality of which they are communicated to the mind.

10. From the Nervous centres, other cords proceed to the various Muscles, by which the body is moved. These muscles, commonly known as "flesh," are composed of a tissue which has the power of contracting suddenly and forcibly, when peculiar stimuli are applied to it. In this respect, it bears a resemblance to the contractile tissues by which the movements of plants are produced (VEget. PHYs. $§ 390$ ); but it differs from them in being thrown into action, not only by stimuli that are applied directly to itself, but by an influence conveyed through the nervous system. Thus, in an animal recently dead, we may excite any muscles to contraction, by sending a current of electricity into the nerves supplying them; and in a living animal we may do the same by simply touching those nerves. But the stimulus which these nerves ordinarily convey, originates in an act of the mind, which is connected in some mysterious and inscrutable manner with 
the central masses of the nervous system. Thus, we desire to perform a certain movement or set of movements; this desire leads to an act of volition or will; and the will causes a certain force or motor impulse to issue from the brain and travel along the nerves, so as to produce the desired motion, by exciting contractions in the muscles that perform it. Or, again, a certain sensation calls forth an emotion, which prompts a certain muscular movement, and may even cause it to take place against the will,-as when a strong sense of the ludicrous produces laughter, in spite of our desire (owing to the unfitness of the time and place) to restrain it; for the emotion, like the act of volition, produces a change in the nervous centres, which causes a motor impulse to travel along the nerves, and thus calls the muscles into contraction. And it seems to be in the same manner that those instinctive actions are produced, which, although few in adult Man when compared with those resulting from his will, predominate in his infant state, and through the whole life of the lower animals (Chap. xIv.). We shall also find that the nervous and muscular systems of animals are concerned in a class of actions with which the mind has no necessary connexion; these automatic actions, such as those of swallowing (§ 195) and breathing ( $\$ 340)$, having for their object to assist in the performance of the organic functions, and to protect the body from danger.

11. In the higher Animals, then, the presence of this Nervo-Muscular apparatus is an essential and obvious distinction between their structure and that of Plants; and we find that it constitutes a large part of the bulk of the body. Thus the whole interior of the skull of Man is occupied by his brain ; his limbs are composed of the muscles, and of the bones which support them and which are put in motion by them; and it is only in the interior of his trunk, that we find organs corresponding with those which form the entire fabric of the Plant. These organs of Nutrition have for their main purpose, to supply the wants of the organs of animal life ; every exercise of which is accompanied by a certain decay or wear of their structure, and which consequently require to be continually nourished and repaired, by the materials provided by what may be termed the regetative organs. But in the lower of tribes of Animals, we do not find the animal functions to possess this predominance. In fact, among the 
many which are fixed to one spot during nearly their whole lives, and which grow and extend themselves like plants, the movements of the body are but few in number, and trifling as to their variety; these movements are only destined to assist in the performance of the organic functions, as by bringing food to the mouth, and water to the respiratory organs; and the nervo-muscular apparatus by which they are effected, bears so small a proportion to the organs of nutrition, as to seem like a mere appendage to them, and is sometimes altogether undiscoverable. This is the case, for example, in the lowest kinds of shell-fish, such as the Oyster, and in the Coral-polypes.

12. Hence we perceive, as we descend the Animal scale, a nearer and nearer approach to the character of Plants; and this we shall find to be the case, not only in the general arrangement of the organs, but also in the nature of the elementary tissues of which these are composed. For in the higher animals, the whole organism is constructed in such a manner as to admit a free motion in its individual parts. The different portions of the skeleton or hard framework are connected with each other by flexible ligaments, which are adapted to resist a very powerful strain; the muscles are attached to these by fibrous cords or tendons, which, also, can support a vast weight; and the several muscles and other parts, which need to be mutually connected, but also require a certain power of moving independently of one another, are bound together by a very elastic loosely-arranged tissue, consisting of fibres crossing and interlacing in every direction, the interstices between which are filled with fluid. Now to these fibrous tissues, there is nothing analogous in plants, because no freedom of motion is required, or even permitted, among their parts ; and we find them bearing a less and less proportion to the whole, as we descend the animal scale. On the other hand, we find the various forms of true cellular tissue, such as predominate in plants (VEGET. Prys. Chap. IIr.), becoming more and more abundant, as we pass from the highest to the lowest animals, and having more and more important duties to fulfil. But even in the highest Animals, as will hereafter appear, they are the immediate instruments of the most important among the organic functions, just as they are in Plants. 


\section{Chemical Constitution of the Animal Body.}

13. By far the larger proportion of the Animal fabric is formed at the expense of the substance termed Albumen; the composition and properties of which, therefore, claim our first attention. The fundamental importance of albumen in the animal economy, is shown by the fact that it constitutes, with fat, and a small proportion of certain mineral ingredients, the whole of that mass of nutrient material stored up in the eggs of oviparous animals, which, being appropriated by the germ to the building up of its fabric, is converted by it into the bones, muscles, nerves, tendons, ligaments, glands, membranes, \&c. of the embryo. We find it also constituting a large proportion of the solid matter of the blood and other nutrient fluids of the adult animal ; and it is the fundamental form to which the various azotized substances employed as food ( $\$ 153$ )-such as animal flesh, or the gluten of breadare first reduced by the act of digestion. It is composed of 49 carbon, 36 hydrogen, 14 oxygen, 6 nitrogen, with a minute proportion of sulphur; it is generally blended, also, with more or less of fatty matter, and with saline and earthy substances.

14. Albumen may exist in two states,-the soluble and insoluble. In the animal fluids it exists in its soluble form ; and is united (as an acid to its base) with about $1 \frac{1}{2}$ per cent. of soda, forming an albuminate of soda. It is not altered by being dried at a low temperature, but still retains its power of being completely dissolved in water. When a considerable quantity of it exists in a fluid (as in the white of the egg), it gives to it a glairy tenacious character; but it is nearly tasteless. When such a fluid is exposed to a temperature of about $150^{\circ}$, a coagulation or 'setting' takes place, as in the familiar process of boiling an egg. But if the albumen be present in smaller quantity, the fluid does not form a consistent mass, but only becomes turbid ; and this only after being boiled. Albumen which has been dried at a low temperature, however, may be heated to the boiling point of water, without passing into the insoluble condition; a fact which is of peculiar interest in relation to the power which the Tardigrada (Zoox. § 841) possess, of sustaining a very high temperature without the loss of their vitality, when 
their bodies have been completely dried up in the first instance. No trace of organization can be detected in coagulated albumen, which seems to be composed only of a mass of granules; and in this respect it differs in an important degree from fibrin-as we shall presently see. Albumen may also be made to coagulate readily by the action of acids, especially the nitric (aqua-fortis); so that a very small quantity of it may be detected in water, by the turbidity produced by adding to it a drop or two of nitric acid, and then heating it. Now, when thus coagulated, albumen cannot be dissolved again by any ordinary process; but its solution may be accomplished by rubbing it in a mortar with a caustic alkali, potass or soda. From this solution it may be precipitated again on the addition of an acid in sufficient quantity to neutralise the alkali. Albumen is distinguished, then, by its peculiar property of coagulating on the application of heat, or on being treated with certain acids.

15. Nearly allied to albumen is the substance termed Casein, which replaces it in milk; and this is specially worthy of notice here, because it is the sole form in which the young Mammal receives albuminous nourishment during the period of suckling, in which it draws its sustenance from its parent. Like albumen, this substance may exist in two forms, the soluble, and the insoluble or coagulated; and the presence of a small quantity of free alkali seems essential to its continuance in the soluble form. Casein differs from albumen, however, in this, that it does not coagulate by heat, and that it is precipitated from its solution by organic acids, such as the acetic and lactic, which have no coagulating action on albumen. It is further remarkable for the facility with which its coagulation is effected by the contact of certain animal membranes; as we see when a small piece of rennet (which is the dried stomach of the calf) is put into a large pan of milk in the process of cheese-making, the ' curd' which then separates being composed of casein entangling the oily particles of the milk. In the coagulated state, casein differs but very little from albumen, and is readily converted into it by the gastric fluid. It is remarkable for its power of dissolving the earthy phosphates, as much as 6 per cent. of phosphate of lime being usually obtainable from it; and it is in this combination, that the large quantity of bone-earth 
required for the consolidation of the skeleton of the young animal, is introduced into its system. A substance resembling casein is obtainable from the serum of the blood, especially in pregnant females; and also from the serous fluid which occupies the interstices of the tissues. It is found, also, mingled with albumen, in the yolk of the egg, forming a compound which (before its true character was known) has been distinguished as vitellin. Now as all the liquids containing casein have it for their special function to supply formative materials to rapidly-growing tissues, we may with much probability regard it as still more closely related to them than is albumen itself. It differs from albumen but little, if at all, in the ultimate proportions of its elements (\$13).

16. The substance of which muscles are composed, has been commonly considered to be Fibrin ( $(17)$; but it differs essentially from fibrin in its properties, and is now distinguished as Syntonin. Its chief peculiarity is its solubility in very dilute muriatic acid ( 1 part to 100 of water), and its precipitation in the form of a jelly when the acid is neutralised; this jelly treated with dilute alkalies forms a solution which coagulates by heat; and thus it seems to be reduced nearly to the condition of albumen. This is, in fact, very much what takes place in the act of digestion of flesh-meat; the muscle-substance being first dissolved by the muriatic or other acid of the gastric fluid, and the solution being then rendered alkaline by the mixture of bile and other secretions in the small intestine.

17. In the blood and other nutrient fluids of the animal body, there is found a substance which is so closely related to albumen in its ultimate chemical composition, as not to be distinguishable from it with any certainty; but which, though fluid whilst circulating in the living vessels, coagulates spontaneously after having been for a short time withdrawn from them, the coagulum or clot being distinguished from that of albumen or fibrin by the fibrillar arrangement of its particles, which indicates an incipient organization. This substance, termed Fibrin, may be obtained in a separate form, by stirring fresh-drawn blood with a stick, to which it adheres in threads. In this condition it possesses the softness and elasticity which characterise the flesh of animals, and contains about three-fourths of its weight of water. It may bo 
deprived of this water by drying, and then becomes a hard and brittle substance ; but, like dried flesh, it imbibes water again when moistened, and recovers its original softness and elasticity. From the recent experiments of Dr. Richardson, it appears that the coagulation of blood-fibrin depends upon the escape of ammonia, being accelerated by such conditions as favour the liberation of this gas, and retarded or prevented by such as cause its retention in the liquid; whilst, even after the clot has been formed, it may be dissolved by ammonia, forming again when that gas is set free. Fibrin differs from syntonin or muscle-substance in not being dissolved by very dilute muriatic acid, but being merely caused to swell up into a gelatinous mass, which contracts again when more acid is added. It combines with the earthy phosphates, of which as much as $2 \frac{1}{2}$ per cent. is sometimes found in the ash left by its combustion.

18. There can be no doubt that fibrin is formed in the blood and in the other fluids in which it presents itself, at the expense of albumen. What is its precise destination, cannot as yet be clearly specified; but there are several circumstances which point to the conclusion that it is to be regarded as a transitional stage in the metamorphosis of albumen into the simple fibrous tissues ( $\$ 23$.) Thus, when the ordinary clot of blood is examined microscopically, it is found to consist, not, like an albuminous coagulum, of a homogeneous mass of granules, but of a network of imperfectly-formed fibres, enclosing the red corpuscles in its interstices. A much more distinct network of the same kind may be seen in the colourless coagulum formed by the liquid which may be skimmed off the surface of the blood drawn from persons suffering under any severe inflammation; such blood coagulates slowly, and its red corpuscles and the fluid in which they float have an unusual tendency to separate from each other; and the fibrin previously dissolved in the latter sets into definite fibres, which continue for some days to increase in firmness. It is a liquid of the same kind, charged with fibrin in a peculiarly " plastic" condition, that is poured forth for the formation of new tissue when the reparative processes are at work for the healing of a wound or the reunion of divided parts ; and it is by a plug of coagulated fibrin, which gradually comes to present a more and more 
distinctly fibrous structure, that the mouths of divided bloodvessels are closed up, when the flow of blood from them spontaneously stops. In all such cases, the fibrous network, if formed out of connexion with a living body, passes after a time into decay ; but if it be formed in apposition with living parts, blood-vessels gradually extend into it from these, its nutrition is maintained and improved, and it progressively comes to present the ordinary characters of the simple fibrous tissues $(\S 22)$.

19. Although the tissues most actively concerned in carrying on the vital operations, retain for the most part the composition "of albumen, yet that very large proportion of the fabric of the higher animals whose offices are essentially mechanical, has a very different chemical constitution. If we boil down either their bones, their skin, or their internal membranes, we shall get a considerable quantity of the substance scientifically termed Gelatin, familiarly glue. Though consisting of the same elements as albumen, its composition is simpler, because these elements are united in smaller proportions; the atom or combining equivalent of gelatin being made up of 13 Carbon, 10 Hydrogen, 5 Oxygen, 2 Nitrogen. The distinctive character of gelatin consists in its solubility in warm water, its coagulation on cooling into a uniform jelly which can be liquefied again by warmth, and its formation of a peculiar insoluble compound with tannin. Gelatin is very sparingly soluble in cold water, though made to swell up and soften by prolonged contact with it. A solution of only one part of gelatin in 100 of hot water is sufficiently strong for the whole to form a consistent jelly on cooling. The reaction of gelatin with tannin is so decided, that the presence of only one part in 5000 of water is at once detected by infusion of galls ; and it is in this action that the process of tanning consists, - the gelatinous fibre of the skin, which would speedily pass into decay, being converted into a comparatively unchangeable substance. The different tissues which have gelatin for their base, yield it to boiling water with different degrees of facility; this diversity apparently depending in some degree upon the definiteness of their organization. Thus the "sound" or air-bladder of the cod, sturgeon, and other fish, which, when dried and cut into strips, is known as isinglass, is very readily acted on; the 
same is the case with the animal substance of bones from which the earthy matter has been removed; and in each case the fibrous texture of the living tissue is but very imperfectly developed. For the extraction of gelatin from the skin, the ligaments, the tendons, and various internal membranes, whose fibrous texture is more pronounced ( $\$ 29)$, a much longer action of boiling water is required.

20. A peculiar modification of gelatin, which presents itself in Cartilage (or gristle), is distinguished as Chondrin. This requires longer boiling than gelatin for its solution in water; as is seen when a knuckle of veal or of mutton is cooked, the tendons and ligaments about the joint being almost reduced to pulp, whilst the cartilages are scarcely at all softened. The essential properties of chondrin are nearly the same as those of gelatin, and its composition seems nearly identical; but it is thrown down from its solution by muriatic and acetic acids and some other reagents, which do not disturb a solution of gelatin.

21. It is not yet fully known how the material of the gelatinous tissues is produced in the animal body. There can be no doubt of its being producible from albumen; since we find it in large proportion in the tissues of animals that have never received gelatin into their bodies in any shape. And although carnivorous animals will receive it as part of their aliment, yet there is strong, reason to believe that the gelatin which is thus supplied to them does not really serve to nourish their bodies, but that it is speedily decomposed and got rid of ( $\$ 159)$. It may be considered as quite certain that the albuminous tissues cannot be formed by the metamorphosis of gelatin; whilst conversely, looking to the fact that in the egg and in milk no gelatin is provided for the young animal, although the gelatinous tissues form a yet larger proportion of its body than they do in the adult, we seem entitled to question whether it is possible that these tissues can be formed in any other way than at the expense of the albuminous constituents of the blood.

\section{Structure of the Primary Tissues.}

22. In considering the structure of the "primary tissues," of which the various organs of animals are composed, it will be convenient first to treat of those which are subservient 
merely to the physical actions of the framework; as, for example, by holding its parts together, by communicating motion, or by giving them mechanical support and protection.-The several parts of the body, even to the very minute divisions of its organs, are held together by what may be termed, in contradistinction to Muscular and Nervous fibre, the simple fibrous tissues; and these are merely endowed, like ordinary cords, with the power of resisting tension or strain, either without themselves yielding to it at all, or with a certain amount of elasticity, which enables them first to yield to a certain degree, and then to recover their previous state. These two qualities are characteristic of two distinct forms of simple fibrous tissue, the white and the yellow.

23. The White fibrous tissue presents itself under various forms, being sometimes composed of fibres so minute as to be scarcely distinguishable, but more commonly presenting itself under the aspect of flattened bands, which are but imperfectly divided into fibres, and have more or less of a wavy aspect (fig. 1). This tissue is resolved, by long boiling, into gelatine; and when treated with acetic acid, it swells up and becomes

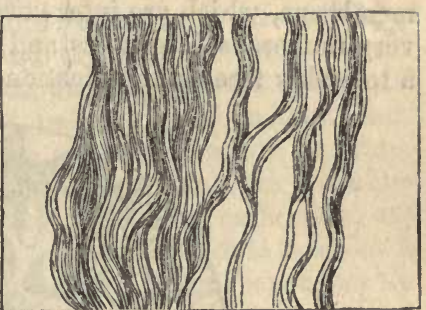

Fig. 1.-White Fibrues Tissue. transparent, by which peculiarity it can be readily distinguished from the other kind, to be next described. The Yellow fibrous tissue presents itself in the form of long, separate,clearly defined fibres, which sometimes branch, and which break short off when overstrained, their extremities being disposed to curl up (fig. 2). They are, for the most part, between 1-5,000th and 1-10,000th of an inch in diameter; but they are often

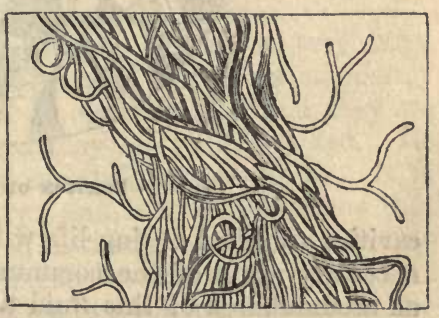

Fig. 2.-Yellow Fibrous Tissue. met with both larger and smaller. This kind of tissue undergoes but very little change from long boiling, and it is 
not acted on by acetic acid. It is but little prone to decomposition, and will exhibit its peculiar elasticity long after it has been separated from the body, provided it be kept moist. - These two forms of tissue exist separately in certain parts of the fabric, but they are much more frequently combined; and the proportion of the yellow elastic tissue which exists in any such combination, may be readily determined under the microscope by the use of acetic acid, which renders all the white fibrous structure so transparent, that the yellow fibres are seen completely isolated in the midst of it.

24. One of the tissues which is composed of such an admixture of white and yellow (or non-elastic and elastic) fibres, is the one which was formerly called "cellular," but which is now more correctly designated as Areolar. ${ }^{1}$ This is composed of a mesh-work of fibres, and of bands of fibrous membrane, which are interwoven in such a manner as to leave very numerous interstices and cavities amongst them, having a tolerably free communication with each other (fig. 3). These

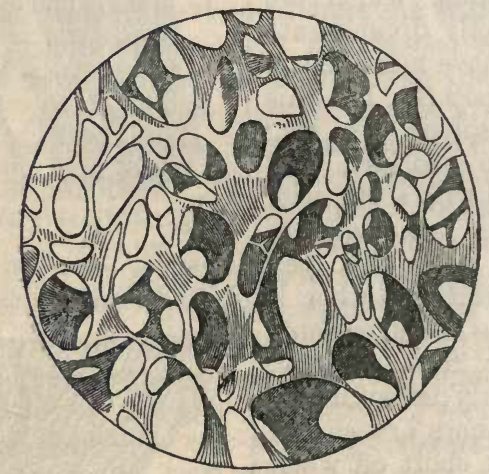

Fig. 3.-Portion of Areolar Tissuz.

cavities are filled during life with a serous fluid ; ${ }^{2}$ and it is a necessary result of the communication between them, that if an accumulation of this fluid takes place to an undue extent,

1 From the Latin areola, a small open space.

2 A fluid resembling the serum of the blood, diluted with water (§ 236). 
as in dropsy, it descends by gravity to the lowest situation. Hence, the legs swell more frequently than any other parts. In its natural state, this tissue possesses considerable elasticity; hence, when we press upon any soft part, and force out the fluid beneath into the tissue around, the original state returns as soon as the pressure is removed. But in dropsy, it appears as if the elasticity of the fibres were impaired or destroyed by their being over-stretched; for when we press with the finger upon a dropsical part, a pit remains for some time after the finger has been removed.

25. This Areolar tissue is diffused through almost the whole fabric of the adult animal, and enters into the composition of almost every organ. It binds together the minute parts of which the muscles are composed; it lies amongst the muscles themselves, connecting them together, but yet permitting them sufficient freedom of motion; it exists in large amount between the muscles and the skin; it forms sheaths to the blood-vessels and nerves, and so connects them with the muscles that they shall not be strained or suddenly bent by the movements of the latter; and it enters into the structure of almost every one of the organs which are contained in the cavity of the trunk, uniting its parts to each other, and keeping the whole in its place. But it is a great mistake to assert, as it was formerly common to do, that it penetrates the harder organs, such as bone, teeth, and cartilage. Its purpose obviously is to allow a certain amount of motion among the parts it unites ; and we find that the more free this motion is required to be, the larger is the proportion borne by the yellow or elastic fibres, to the white or non-elastic.

26. Although the Areolar tissue contains a very large number of blood-vessels and nerves, yet it does so merely because it furnishes the bed or channel in which they are conducted to the parts where they are really wanted. Its own vitality is low, and its sensibility very slight. It is quickly reproduced after injury ; and it is by its means that losses of substance are repaired in tissues of a more elaborate kind, which are not so easily regenerated.

27. The continuity or connectedness of this tissue over the whole surface of the body, admits air to pass readily from one part to another; and the inflation or blowing-up of its cavities with air, which has sometimes happened accidentally, 
and has sometimes been purposely effected, does not produce: any disorder in the general functions of the body. In blowing the nose violently, some part of the membrane lining its cavity has occasionally given way, so as to allow air to pass into the areolar tissue of the face, and especially into that contained in the eyelids, which is particularly loose ; an enormous swelling of these parts then takes place, presenting a very frightful appearance, but not attended with the least danger, and subsiding of itself in a few days. This swelling presents a character to the touch quite different from that which would be occasioned by a similar distension with liquid; for it gives somewhat of the crackling feel that is occasioned by pressing on a blown bladder. A similar inflation of the areolar tissue of the body has sometimes occurred from the formation of an aperture, by disease or injury, in the walls of the lungs or air-passages, and the consequent escape of air during the act of breathing: in one remarkable case of this kind, the skin of the whole body was so tightly distended with air as to resemble a drum. It is intentionally practised by butchers, who "blow up" the areolar tissue of their veal, in order to increase its plumpness of aspect; and the inflation of the areolar tissue of the head, in the living state, has been sometimes practised by impostors, in order to excite commiseration.

28. Fibres and shreds of fibro-membrane, resembling those of which areolar tissue is composed, may be so interwoven as to form a continuous sheet of membrane, having a smooth and glistening surface; and in this manner are produced the Serous Membranes that line the different cavities in which the viscera (or organs contained within the skull, the chest, and the abdomen) are lodged. The peculiar manner in which these membranes are arranged, will be explained hereafter (§ 43). One of their surfaces is always free or unattached, whilst the other is in contact with the outer wall of the cavity; and from the free surface, which is covered with a layer of flattened epithelium-cells (fig. 10), a serous fluid is exhaled, which adds to its smoothness. It is by an accumulation of this fluid, that dropsies of the cavities are produced,such as water on the brain, or in the chest.

29. By the union of fibres of a stronger kind, those firmer tissues are produced, which are employed wherever a greater 
strain has to be borne. This is the case with the Ligaments, which bind together the bones at the joints, the Tendons, by which the muscles are usually attached to the bones, and the tough Fibrous Membranes that envelope and protect many of the most important viscera. In these any considerable amount of elasticity would be misplaced; and we consequently find that they are chietly or entirely composed of the white fibrous tissue. Whenever an elastic ligament is required, however, we find the white replaced by yellow. One of the best examples of this is seen in the ligament of the neck of many quadrupeds, commonly known as the paxywaxy; which is given to the large herbivorous quadrupeds, such as the ox, to assist them in supporting their heavy heads with as little exertion as possible; whilst carnivorous quadrupeds, such as the lion and tiger, are endowed with it to give them additional power of carrying away heavy burdens in their mouths. In Man we scarcely find a trace of it. This yellow fibrous tissue is found, moreover, in the walls of the arteries ( $\$ 248)$, to which it gives their peculiar elasticity; and it also forms the vocal cords of the larynx (\$ 681). It is by the same kind of elastic ligament that the claws of the Feline tribe are drawn back into their sheaths when not in use, being projected (when required) by muscular action; and that the two pieces of the shell of Bivalve Mollusks are united at the hinge, and are at the same time kept apart for the admission of water between them, except when the animal forcibly draws them together by its adductor muscle (§ 113).

30. All these fibrous tissues, then, are concerned in actions purely mechanical; and there is nothing in their properties which is so distinct from those of inorganic substances, as to require to be considered as vital. We may consider them, therefore, as among the lowest forms of animal tissue; and accordingly we find that, when the higher forms degenerate or waste away, these appear in their place. Such a degeneration may take place simply from want of use. Thus if, from palsy or want of power of the nerves, the muscles of the legs are disused for several years, they will lose their peculiar property of contractility $(\$ 5)$; and it will be found that scarcely any true muscular structure remains, but that it is replaced by some form of fibrous tissue. Or again, if the 
front of the eye be so injured by accident or disease, that light cannot pass through it to make its impression on the nerve, that nerve, being thrown into disuse, will gradually degenerate into fibrous tissue. Moreover, this change may take place as a part of the regular actions of life ; for there are certain organs in the young animal previous to birth, which are not required afterwards; and these degenerate in like manner, gradually wasting away, and leaving only traces behind them, - tubes shrivelling into fibrous ligaments, and glandular structures remaining only as areolar tissue.

31. Along every free surface of the body, both external and internal, is spread out a delicate structureless layer, which is termed the Basement or Primary Membrane. This forms the outer layer of the True Skin, lying between it and the Epidermis or scarf-skin ( $\$ 37)$; in the same manner it underlies the Epithelial layer of the Mucous membranes which line the open cavities of the body ( $\$ 39$ ), and of the Serous membranes which line its closed cavities (\$ 43); and it occupies the same position in the walls of the bloodvessels, gland-ducts, and other tubes. It is difficult to separate it, in any of these parts, from the tissues with which it is in contact; and its characters may be well studied by dissolving the calcareous part of an oyster or mussel-shell in dilute acid, when it will be found that layers of a thin transparent membrane are left, which have been thrown off at each act of shell-formation, from the surface of the mantle. This elementary membrane, like that which forms the walls of cells (\$32), is remarkable for the readiness with which

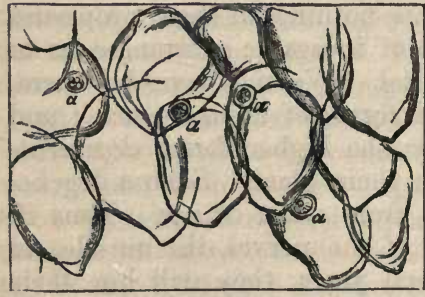

Fig. 4.-Nucleated CeLls; $a$ a, nuclei. it is permeated by fluid, although no visible pores can be seen in it.

32. A considerable part of the fabric of even the highest Animal is formed, like the entire organism of the Plant, of Cells, either unchanged or in some way metamorphosed. A cell is a minute bag or vesicle, formed of a structureless membrane, and having its cavity filled with fluid of some kind. In some part of its interior, most commonly adhering 
to its wall, there is usually to be observed a solid collection of granular matter, which is termed the nucleus (fig. 4, a a). The typical form of the cell is globular or oval (fig. 5); but when a number of cells are in contact with each other, and are pressed together, their sides become. flattened; so that when they are cut across no intervals are seen between them, but their walls are everywhere in contact (fig. 6), just as in

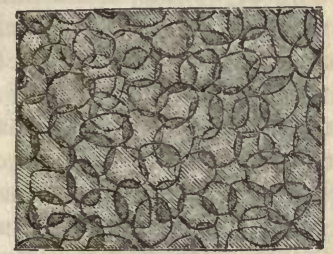

Fig. 5.

Rounded Celus in Cartilage OF BAT'S EAR.

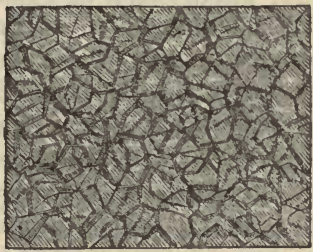

Fig. 6.

Polygonal Cells from CarTILAGE IN MOUSE'S EAR.

the section of a regetable pith. The chemical composition of the nucleus differs from that of the cell-wall ; for whilst the latter is dissolved by acetic acid, the former (like the yellow elastic tissue, with which its substance appears to have some relationship) is unchanged by it. When the formation of a cell is complete, and it is not destined to reproduce its kind, the nucleus frequently disappears; this is the case, for example, with the red corpuscles of the blood of Mammalia (§ 229), and also with Fat-cells ( $\$ 46)$.

33. New cells may originate in one of two very distinct modes ; either from a pre-existing cell, or by an entirely new production in the midst of an organizable fluid or blastema. The most remarkable example of the first process is presented in the early development of the germ, which entirely consists of an aggregation of cells, every one of which undergoes successive subdivisions into two, so that the total number in the germ-mass is repeatedly doubled (Chap. xv.). Tho same method of multiplication by binary subdivision may be seen to continue throughout life in Cartilage-cells ( $\$ 47$ ), the growth of which almost exactly repeats the history of the growth of the lowest forms of Sea-weeds. The process of subdivision seems to commence in the nucleus, which begins to separate itself into two equal parts, and each of these draws 


\section{MULTIPLICATION AND NEW PRODUCTION OF CELLS.}

around it a portion of the contents of the cell; so that the cell-wall, which is at first merely doubled inwards by a sort of hour-glass contraction, at last forms a complete partition between the two halves of the original cavity. The process may be repeated either in the same or in a transverse direction, so as to produce four cells, which may be either arranged in a single line 0000 or may form a cluster 88; and another subdivision of each cell will, of comrse, again double the entire number. In other cases, however, the nucleus appears to break up at once into several fragments, each of which may draw around it a portion of the contents of the parent-cell, which becomes invested by a cell-wall of its own; and thus the cavity of the parent-cell may at once become filled with a whole brood of young cells, without any successive subdivision. Generally speaking, the former method seems to prevail in structures which, like Cartilage, have a comparatively permanent destination; whilst the latter is followed in cases in which the cells thus formed are destined only for a transitory existence. This is the case especially in Cancerous structures, which are particularly distinguished by their proneness to the rapid production of cells within cells.

34. The production of new cells in the midst of an organizable blastema or formative fluid, such as is poured out from the blood for the reparation of an injury, is a very different process. This blastema, when first effused, is an apparently homogeneous semi-fluid substance ; as it solidifies, however, it becomes dimly shaded by minute dots, and as it is acquiring further consistence, some of these dots seem to aggregate, so as to form little round or oval clusters, bearing a strong resemblance to cell-nuclei. These bodies appear to be the centres of the further changes which take place in the blastema; for if it be about to undergo development into a fibrous tissue ( $\$ 18)$, they seem to be the centres from which the fibrillation spreads; whilst, if a cellular structure is to be generated, it is from them that the cells take their origin. The first stage of the latter process appears to consist in the accumulation of the substance which the cell is to include, about each nucleus, and around this the cell-membrane is subsequently developed. It is in this mode that the development of new structures, for the filling up of losses of substance, is provided for; and it appears, from recent 
inquiries, that the blastema will resolve itself into fibres or into cells, according as the wound is completely secluded from the air, or is exposed to it. It is under the former condition that losses of substance are most rapidly and most completely repaired; whilst it is under the latter that inflammation is most likely to arise, in consequence of the bad effect produced by the contact of air with the raw surface; the process of healing, when thus interfered with, going on less favourably as well as more slowly.

35. The very simplest and most independent condition of the animal Cell, is probably to be found in the nutritive fluids of the body; in which we meet with floating cells that are completely isolated from each other, and which are consequently just as self-sustaining as are the separate vesicles of the Yeast-plant, of the Red Snow, or of other simple cellular Plants. These cells are of two classes. In the blood of animals generally, and in the chyle and lymph of Vertebrata, we find a larger or smaller proportion of colourless corpuscles, which are usually nearly spherical in form, and which exhibit various stages of development into cells, being sometimes little else than collections of granules, without any distinct enveloping membrane, whilst, in other instances, there is a distinct cell-wall, cell-cavity, and nucleus. These bodies, if watched under a sufficiently powerful microscope, may often be seen to undergo very curious changes of form, resembling those of the A moeba (\$129). Besides the foregoing, however, the blood of Vertebrated animals contains a far larger proportion of red corpuscles, which are flattened disks, sometimes circular but more commonly oval, having pellucid and colourless walls, but having their cavities filled with a peculiar coloured fluid. As these will be more fully described hereafter ( $\$ 229)$, it is not requisite to do more than notice them here as constituting a most important part of the animal organism, probably not less than a twelfth part of the entire weight of Man and the higher animals, being thus composed of nothing else than these isolated cells. ${ }^{1}$

36. Next in independence to the cells or corpuscles floating in the animal fluids, are those which cover the free

1 The entire weight of the blood of Man seems to be about one-sixth part of that of the body; and the moist corpuscles constitute about half the entire weight of the blood. 
membranous surfaces of the body, and which form the Epidermis, or superficial layer of the skin, and the Epithelium of the internal membranes. And it will be convenient here to consider the entire structure of the Skin, the Mucous Membranes, and the Serous Membranes, which are complex fabrics, chiefly made up of the elementary tissues already described.-These membranes may each be considered as composed of three principal parts, namely, the superficial layer or layers of cells, the basement-membrane whereon the cells lie, and the subjacent texture covered by this, which consists of fibrous tissue compactly interwoven and traversed by blood-vessels, nerves, absorbents, and also containing glands of various kinds. The Skin and Mucous Membrane may, in fact, be regarded as belonging to one and the same type; for they are continuous with each other wherever one of the open cavities of the body communicates with the surface, as at the mouth, nostrils, and anus; and in the Hydra (\$121) it has been experimentally found that the membranous layer covering the body may be made to change places with that which lines the stomach, without any sensible disturbance in the functions of either. The difference between the two essentially consists in this; that the Skin, being destined especially for the reception of sensations, and for the protection of the soft parts beneath, is more copiously furnished with nerves than with blood-vessels, and has its surface covered by a firm, dry cuticle; whilst the Mucous Membrane, ministering especially to the organic functions, is comparatively little supplied with nerves, but is abundantly furnished with blood-vessels, and in certain parts with absorbents, whilst its cellular layer is soft and easily permeable by liquids. Both in the skin and in mucous membrane we find a multitude of minute glands, for the separation of particular fluids from the blood; the nature of these differs with the locality.

37. The fibrous mesh-work of the Cutis or True-Skin is continuous with that of the Areolar tissue which lies immediately beneath it; so that the two textures are not separated one from the other by any definite boundary (as the examination of a vertical section (fig. 7) clearly proves), but are distinguishable only by the compactness of the one, as contrasted with the looseness of the other. The outer surface of the Cutis usually presents numerous minute elevations or papillae 
(fig. $7, i$ ), which are commonly arranged in rows; of these, some are organs of touch, being furnished with sensory nerves that end upon a peculiar cushion-like organ in their interior ( $\$ 490)$; but into others no nerves can be traced, so that, as these are copiously supplied with blood-vessels, it is probable that they minister to the nutrition of the epidermis.

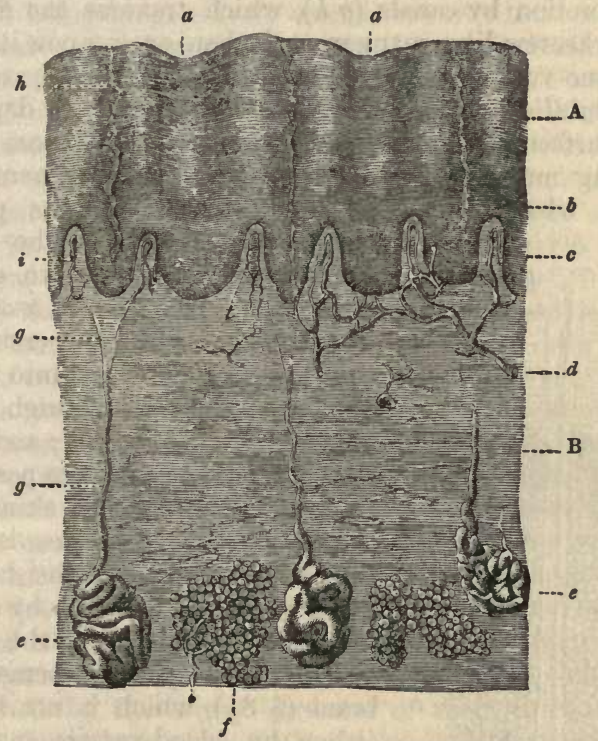

Fig. 7.-Vertical Section of THE SkIN,

Showing the different structures which it contains. A, Epidermis; $a \boldsymbol{a}$, its outer surface; $a-b$, its horny layer; $b-c$ its inner soft layer, dipping down into the hollow between the papillæ; B, Cutis; $d$, arterial twig supplying its vascular papillæ; $e$ e, perspiratory glandulæ; $f$, cluster of fat-cells; $g g$, perspiratory duct, traversing the true skin; $h$, its continuation through the epidermis; $i i$, tactile papillæ, with their nerves.

This is the more probable from the fact that we find these vascular papillæ very large and full of blood-vessels in the interior of corns, warts, and other such productions, formed by a "hypertrophy" or over-nutrition of the epidermis in particular spots ; and also in situations in which the ordinary epidermis is very thick, as it is on the black pads of the foot of the dog or cat. And a highly vascular structure of the 
same kind is found in the matrix or receptacle of the growing roots of nails, hoofs, horns, \&c. which are only modified forms of epidermis. Imbedded in the substance of the cutis wo find, in most situations, the perspiratory glands (fig. 7, e e), by which the watery fluid that is continually being exhaled from the skin, is separated from the blood ( $\$ 371)$; these send forth their secretion by canals $(g h)$, which traverse the epidermis in a corkscrew-like manner, and then open upon its surface by oblique valvular orifices. In the Cutis, also, are lodged the hair-follicles $(\$ 38)$, which are really pits or depressions of its surface, with a vascular papilla at the bottom of each, supplying nutriment for the abundant development of the cells in which the hair originates, as will be presently described. Wherever the hair-fol-

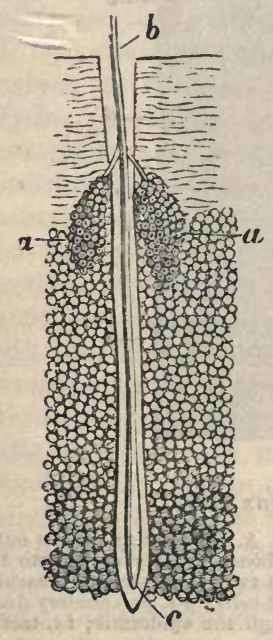

Fig. 8. licles occur, there do we also find sebaceous follicles (fig. 8, $a$ a); these are peculiar glandulæ, secreting fatty matter, which is poured into the haircanal, so as to come through it to the surface of the epidermis ; and the use of this secretion, which is particularly abundant in the dark skins of the natives of warm climates, is to prevent the cuticle and the hair from being too much dried up by exposure to air.-The surface of the Cutis is covered by a layer of basement-membrane ( $\$ 31$ ), which is not traversed either by blood-vessels, nerves, or absorbents; so that none of these pass into the epidermis which lies on its outer side.

38. The Epidermis, otherwise termed the cuticle, or "scarf-skin," is composed of numerous layers of $\mathrm{nu}$ -

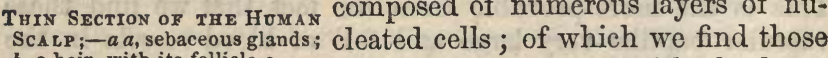
$b, a$ hair, with its follicle $c$. in immediate contact with the basement-membrane to be nearly spherical ; those a little removed from it to be rendered polygonal by the mutual pressure of their sides; those nearer the outer surface to be flattened, and this in an increased degree, as we pass from within 
outwards, until we arrive at layers composed entirely of dry flat scales, which show but little indication of ever having been cells. There is no doubt, however, that all these forms are but different stages of the existence of one and the same set of epidermic cells; these taking their origin in the formative fluid exuded on the surface of the basement-membrane, and being progressively carried towards the surface by the successive development of new layers beneath them, whilst the layers above them are thrown off, or are worn away; and at the same time undergoing a change of form, in the first instance from mutual pressure, and afterwards from the loss of their contained fluid. At the same time they are rendered more firm in texture, by the formation of a horny secretion in their interior; so that the outer layers of epidermis form a consistent membrane, which is raised from the surface of the Cutis when fluid infiltrates between them (as when the hand has been long soaked in water), or is poured out by the vessels of the latter (as when a blister is applied); whilst the soft internal layers remain in contact with the basement-membrane.-The number of layers varies greatly in different parts, being usually found to be greatest where there is most pressure or friction, as if the irritation determined an increased supply of blood to the spot, and thus favoured an augmented development of epidermic cells. Thus, on the soles of the feet, particularly at the heel and the ball of the great toe, the Epidermis is extremely thick; and the palms of the hands of the labouring man are distinguished by the horny hardness of their thick cuticle. - It was formerly supposed that a special layer of a soft spongy tissue, termed the rete mucosum, intervenes between the Cutis and the Epidermis; and that this was the special seat of the colour of the skin in the dark races. It is now well ascertained, however, that this supposed rete consists of nothing else than the newly-forming soft layers of the true epidermis; and that the colouring matter is diffused through the epidermic cells, so as to tinge the entire thickness of the cuticle, although its presence is particularly obvious in the deeper layers.-The Nails may be considered as nothing more than an altered form of Epidermis; when examined near their origin, they are found to consist of cells which gradually dry into scales that remain 
coherent; and when thin sections are treated by a dilute solution of soda, these scales swell out again (as do also those of the cuticle) into globular cells. A new production is continually taking place in the groove of the skin in which the root of the nail is imbedded, and also from the whole of the surface beneath it; the former adds to the length of the nail; the latter to its thickness.-The structure of Hairs is essentially the same. The base of each is formed of a "bulb," which consists of a mass of epidermic cells developed from the vascular papilla at the bottom of the hair follicle (fig. $8, c)$; and as this narrows into the "shaft" of the hair, a difference shows itself between the cortical or outer layer, and the medullary or pith-like substance of the interior. The former, which is continuous with the outer layers of the epidermis, is composed of flattened scales, arranged in an imbricated (tile-like) manner, so that the surface of the hair is usually marked by transverse jagged lines ; the latter consists of cells which frequently retain their spheroidal form, like the inner layers of the epidermis; but in the human hair these cells are elongated into fibres. It is very seldom that there is any canal in the interior of the Hair, although irregular spaces are not unfrequently left by the drying-up of the fluid contents of the cells. The structure of Quills is essentially the same as that of hairs on a large scale; and we there see the difference very distinctly marked between the cortical portion which forms the "barrel" of the quill, and the medullary portion which forms the white pith-like substance of the stem of the feather. The Scales, where they are really epidermic appendages, as is the case in serpents and lizards, are formed upon the same pattern; and we have a good example of the detachment of the entire epidermis at once (reminding us of the casting of the shell of the crab and lobster) in the "sloughing" of the suake.

39. The Mucous Membranes form a sort of internal skin, lining those cavities of the body which open on its surface; and the elements of which they are composed are essentially the same, though combined and arranged in a different manner, in accordance with their difference of function. The principal part of the thickness of every ordinary mucous membrane is made up, as in the skin, by the consolidation of areolar tissue, the fibres of which are continuous with those 
of the ordinary areolar tissue on which the membrane rests ; this layer is copiously furnished with blood-vessels, but it is seldom supplied with many nerves. Thus the mucous membrane lining the stomach possesses in health so little sensibility, that we are not aware of the contact of the substances taken in as food, unless they are of an acrid character, or of a temperature very different from that of the body; and though the mucous membrane lining the air-passages is very susceptible of certain kinds of irritation, yet it has but little ordinary sensibility in the state of health, except near the entrance to the windpipe. The large supply of blood which these membranes receive, has reference to their active participation in the functions of secretion and absorption. One secretion is common to all, that of the mucus by which they are covered; this serves to protect them from the irritation that would otherwise be produced by the contact of solid or liquid substances, or even of air, with their free surfaces; and we see the results of its deficiency, in the inflammation which attacks the membrane, sometimes proceeding to its entire destruction, when from any cause the secretion is checked, as it sometimes is by injuries of the nerves supplying the part.

40. In every mucous membrane, as in the skin, the fibrous texture is bounded on the free surface by basement-membrane, beyond which no blood-vessels pass. And the surface of the basement-membrane is covered by cells, arranged either in a single layer or in multiple layers, constituting the Epithelium. This, although answering to the Epidermis in structure and position, has a very different character ; for its cells neither dry up nor become horny; nor do they adhere in such a manner as to form a continuous membrane, except in the interior of the mouth and cesophagus (gullet), where the epithelium is endowed with somewhat of the firmness of cuticle, in order to resist the abrading contact of hard substances. The epithelium cells of mucous membranes are commonly somewhat flattened; but in some situations, as on the villi of the intestinal canal (fig. $9, d$ ), they have more of a cylindrical, or rather conical shape, their smaller extremities being in contact with the basement-membrane. The epithelial cells are frequently cast off, like the epidermic, especially from the parts that are most concerned in secretion; 
and they are as continually replaced by newly-formed cells, which are produced on the surface of the basement-membrane, at the expense of the fluid that transudes through it from the blood-vessels copiously distributed to its under surface.

41. Mucous membrane may either exist in the condition of a simple expanded surface, or may have a much more complex arrangement, by which its surface is greatly increased. The simple mucous membrane, such as that which lines the nose and air-passages, is found, for the most part, where no absorption has to be performed, and where only a moderate amount of secretion is necessary. But where it is to absorb as well as to secrete, it is usually involuted or folded upon itself, in such a manner as to form a series of little projections, and also a number of minute pits (fig. 9). These pro-

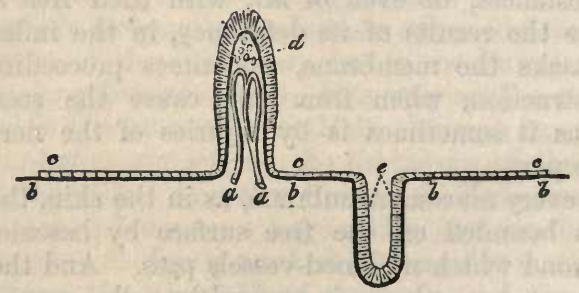

Fig. 9. Diagram representing the Mucous Membrane op the Intestinal Canal.

$a \boldsymbol{a}$, absorbent vessels; $b b$, basement membrane; $c c$, epithelium-cells of level surface of membrane; $d d$, cylindrical epithelium-cells of villus; $e e$, secreting cells of follicle.

jections sometimes have the form of long folds; in other instances they are narrow filaments, crowded together so as almost to resemble the pile of velvet. In either case, the absorbent surface is vastly increased; but chiefly so by these filaments, which are termed villi, and act as so many little rootlets. On the other hand, it is in the pits or follicles, that the production of the fluid which is to be separated or secreted from the blood, chiefly takes place.- Not only are the flat expanded surfaces of the mucous membrane covered with epithelium cells, but the villi also are sheathed by them; and the secreting follicles are lined by the same. 
The cells covering the villi (fig. $9, d$ ) perform the important function of selecting and absorbing certain nutritious elements of the food, which they communicate to the absorbent vessels in the interior of the villi. On the other hand, the epithelium-cells of the follicles $(e)$ seem to be the real agents in the secreting process; drawing from the blood, as materials for their own growth, certain elements contained in it; and falling off, when mature, so as to discharge these substances as the product of secretion, giving place to a fresh crop or generation of cells, which go through a series of changes precisely similar to the preceding.

42. Now these follicles are the simplest types or examples of all the Glandular structures, by which certain products are separated from the blood, some to be cast forth from the body as unfit to be retained in it, and some to answer particular purposes in the system. In all of them the structure ultimately consists of such follicles, sometimes swollen into rounded vesicles, and sometimes extended into long and narrow tubes. Each follicle, vesicle, or tube, is composed of a layer of basement-membrane, lined with epithelium-cells, and surrounded on the outside with minutely distributed blood-vessels ; and it seems to be by the peculiar powers of these cells, that the products of the secreting action, whether bile, saliva, fatty matter, or gastric fluid, are formed (see Chap. vrr.).- Hence we see that the act of Secretion is, in animals as in plants, really performed by cells. It is necessary to bear in mind, however, that a simple transudation of the watery parts of the blood may take place without any proper secreting action, in the dead as in the living body; it is in this manner that the serous fluid of areolar tissue and serous membrane is poured out, and that the watery portion of the urine is separated.

43. The Serous Membranes which line the closed cavities of the body, though composed of the same elements as the skin and mucous membranes, have a much simpler structure, and can scarcely be said to minister directly to any important vital function. The tissue of which Serous membrane is principally composed, scarcely differs, except in its greater density, from the laxer areolar tissue whereby the membrane is attached to the walls which it covers like plaster ; it is but sparingly supplied either with blood-vessels or absorbents; and 
it contains very few nerves. The smooth surface of the membrane forms one unbroken plane, being neither raised into villi, nor depressed into follicles ; and its basement-membrane is covered with a single layer of flat epithelium-cells, which are closely applied to it and to each other,

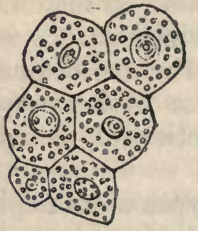

Fig. 10. like the pieces of a pavement (fig. 10). It is with such a membrane that every one of those great cavities is lined, which contains important viscera ; and it is also continued on to the outer surface of these viscera, so as to afford them an external coating over every part save that by which they are attached. Thus the heart is suspended

Patement EptrheLTUM CELLS OF SEROUS freely, by the large vessels proceeding from MEMBRANE.

its summit, within a bag or sac of fibrous membrane peculiar to itself, which is termed the pericardium. The cavity of this bag is completely lined by the serous membrane (fig. 11, $p^{\prime}$ ), which closely embraces

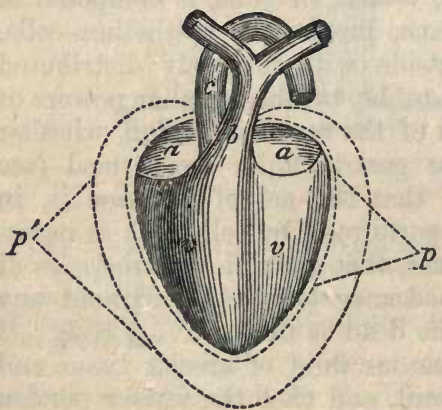

Fig. 11.-Diagram of the Pericardium. $a$, auricles; $v v$, ventricles; $b$, pulmonary artery ; $c$, aorta ; $p p^{\prime}$, pericardium. the vessels, and which then bends down over the surface of the heart, so as to enclose it in the envelope $p$. Hence it will be seen that this membrane, whilst including the heart, and allowing it to communicate with its vessels, forms a completely shut sac; and it may be likened to a common double cotton or woollen night-cap, which has a similar cavity between its two layer's, the head being really on the cutside of this, whilst seeming to be within the envelope. The two layers of the pericardium, though separated in the diagram for the sake of distinctness, are really in mutual contact, save when separated by the interposition of fluid poured out in disease. Each of the lungs, in like manner, is suspended in a closed sac of its own, termed the pleura; and the surface of the lung is covered by a serous membrane, which is reflected over the wall of the pleural cavity. 
A similar arrangement exists in the great cavity of the abdomen; but the number and the complex relations of the viscera which this contains, give to the disposition of its serous membrane, termed the peritoneum, a peculiar complication. The cavity of the skull also is lined by a serous membrane, termed the arachnoid, and this is prolonged over the surface of the brain, and enters its lateral ventricles ( $\$ 458)$. The chief purpose of these membranes appears to be to facilitate the movements of the included organs, by forming smooth surfaces which shall freely glide over each other; this is evidently of great importance, where such constantlymoving organs as the heart and lungs are concerned. Their surfaces are kept constantly moist with a serous fluid which exudes from the blood; but in the state of health this fluid does not accumulate in their carities, being absorbed as fast as it is poured out. Various forms of dropsy, however,such as "water on the brain," "water on the chest," and "ascites," or dropsy of the abdomen-are the result of the increased outpouring of fluid into the serous cavities of the arachnoid, the pericardium, the pleura, and the peritoneum respectively.

44. Nearly allied to the Serous membranes are the Synovial, which form closed sacs in the interior of joints, covering the ends of the cartilages, and then lining the fibrous capsule which passes from one bone to the other. The mode of their arrangement will be understood from the accompanying diagram; in which $a$ a represent the extremities of the two bones which are jointed together, $b b$ the layers of cartilage with which they are severally covered, and the dotted line $c c$ the synovial membrane, which is seen to form the sac or bag $c^{\prime} c^{\prime}$, whilst at the points $c c c c$ it is reflected upon the cartilages of the joints. In point of fact, however, the Synovial membrane is not ordinarily traceable as a distinct layer over the surface of these

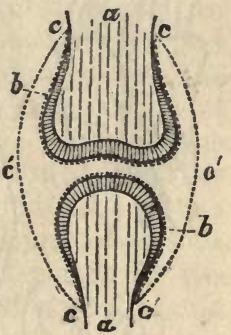

Fig. 12.

Diagram of The 8TRUCTURE OF A JOINT.

a a, extremities of the bones, covered with cartilage ; $b \vec{b}$, layer of cartilage closely covered with synovial membrane ; $c c^{\prime} c$, reflected layer of 8ynovial membrane forming synovial capsule. cartilages, but seems to have become incorporated with them; for though in the embryo its presence may be distinctly proved 
by the continuity of its blood-vessels over the entire cartilage, yet these are found to retreat gradually as the joint is brought into use, until at last they only form a circle round the border of the cartilage. Some of the Synovial membranes, as that of the knee-joint, are furnished with little fringe-like projections, somewhat resembling the villi of mucous membranes $(\$ 41)$; these are extremely vascular, and are furnished with an epithelium which very readily falls off; and there is a strong probability that they are concerned in the secretion of the synovial fluid, which is much denser than the ordinary serous transudation, having from 6 to 8 per cent. of additional albumen, and presenting a glairy appearance like that of white of egg. It is interesting to see that the same purpose may thus be served by the extension of the membrane in either direction, either outwards into a villous filament, or inwards into a follicle; the function being determined in each case rather by the

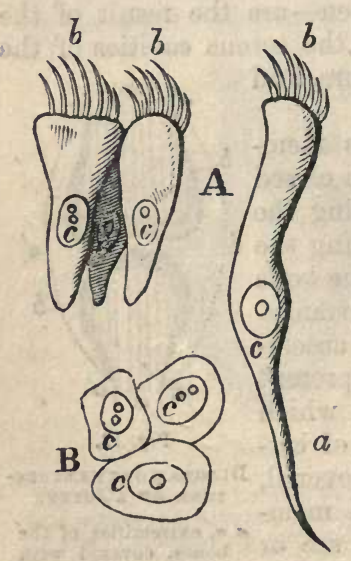

Fig. 13.-Ciniated Epitheltum CeLLs; as seen sideways at $A$, and in transverse section at $\mathbf{B}$; their cilia are seen at $b$, their nuclei at $c$; at $a$ is shown one of these cells unusually elongated. attributes of the cells, and by the supply of blood, than by the form which the secreting surface may happen to present.

45. The cells of Epithelium, whether flattened or cylindrical, are observed to be furnished in particular situations with a fringe of delicate filaments, which are termed cilia. These, although of extreme minuteness, are organs of great importance in the animal economy, on account of the extraordinary motor powers with which they are endowed. The form of the cilia is usually a little flattened, and tapering gradually from the base to the point. Their size is extremely variable; the largest that have been observed being about 1-500th of an inch in length, and the smallest 1-13,000th. When in motion, each filament appears to bend from its root to its point, returning again to its original state, like the stalks of 
corn when depressed by the wind; and if a number be affected in succession with this motion, the appearance of progressive waves following one another is produced, as when a corn-field is agitated by repeated gusts. When the ciliary motion is taking place in full activity, however, nothing can be distinguished save the whirl of particles in the surrounding liquid; and it is only when the rate of movement slackens, that the shape and size of the individual filaments, and the manner in which their stroke is made, can be made out. The motion of the cilia is not only quite independent (in all the higher animals at least) of the will of the animal, but is also independent even of the life of the rest of the body; being seen to continue after the death of the animal, and even going on with perfect regularity in parts separated from the body. Thus, isolated epithelium-cells have been seen to swim about actively in water, by the agency of their cilia, for some hours after their detachment from the mucous membrane of the nose; and the regular movement of cilia has been noticed fifteen days after death, in the body of a tortoise in which putrefaction was already far advanced. In the gills of the River Mussel, which are amongst the best objects for the study of this most curious phenomenon, the movement endures with similar pertinacity. - The purpose of this remarkable agency is obviously to propel fluids over the surfaces which are furnished with cilia. We find it taking the most important share in the functions of life among the lowest classes of animals. Thus, in Animalcules of various kinds, the cilia are the sole instruments, not merely for the production of those currents in the water which may bring them the requisite supplies of air and food, but also for propelling their own bodies through the liquid. In most Zoophytes, and in the inferior Mollusks, which pass their lives with little or no change from one spot to another, the motion of the cilia lining the alimentary canal and clothing the gills (where such have a special existence), draws into the mouth the minute currents which serve as food, and also renews the layer of water in contact with the respiratory surface. The gills of Fishes are not furnished with cilia, another provision being made by muscular action for continually driving fresh streams of water over them; but the motion may be very well seen upon the gills of the young 
Tadpole or larva of the Water Newt, which hang down as fringes on either side of the neck. In the higher air-breathing animals, the function of the cilia is much more limited. They clothe the mucous membrane which lines the air-passages; and their function appears to be, in that and other cases, to prevent the accumulation of the secretion with which the membrane is kept moist, by keeping up a continual onward movement of it towards the outlet of the passage. In some other cases, however, we find the ducts of secreting organs furnished with cilia, whose action is obviously to assist in carrying the products of secretion towards their outlet.

46. Passing on, now, to those tissues of animals of which cells constitute the permanent components, instead of being successively thrown off and replaced as they are in the Epidermis and Epithelium, we may first notice the Adipose tissue, or $F a t$, in which the oily and fatty matters of the body are for the most part contained. This tissue is composed of minute cells or vesicles (fig. 14), having no communication with each other, but lying side by side in the meshes of the

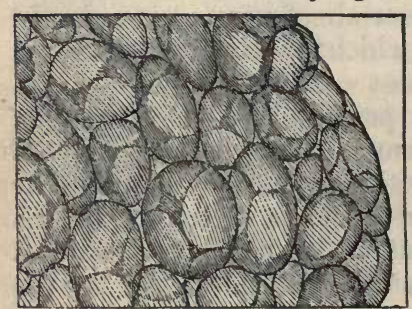

Fig. 14.-FAT Cexts, HighLY MAGNIFIED. areolar tissue, which serves to hold them together, and through which also the bloodvessels find their way to them. From the fluid in these vessels, the fatty matter is separated in the first place by the secreting action of the cells ; and it is prevented from making its way through the very thin walls of the cells, by the simple expedient of keeping these constantly moist with a watery fluid, the blood. ${ }^{1}$ The blood-vessels have also the power of taking back the fatty matter again into the circulation, when it is wanted for other purposes in the economy. These deposits of fatty matter answer several important objects. They often assist the action of moving parts, by giving them support without interfering with their free motions; thus the eye rests on a sort of cushion of fat, on which it can freely turn, and through which the muscles

1 Thus oil will not pass into blotting-paper, if this have been previously moistened with water. 
pass that keep it in play. It also affords, by its power of resisting the passage of heat, a warm covering to animals that are destined to live in cold climates; and it is in these that we find it accumulated to the largest amount. Further, being deposited when nourishment is abundant, it serves as a store of combustive material, which may be taken back into the system, and made use of in time of need. The causes which peculiarly contribute to the production of fat, will be considered hereafter $(\$ 162)$.

47. Another tissue of which cells form the principal part, is that termed Cartilage or gristle. Its simplest state is that of a mass of firm substance, composed of chondrin ( $(20)$, through which are scattered a number of cells, at a greater or less distance from one another. In the simple cellular cartilages, such as those which cover the ends of the bones where they glide over one another so as to form moveable joints, no trace of structure can

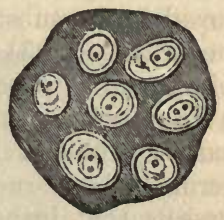
be seen in the intervening substance. Fig. 15. - SEcrios op But in cartilages which have to resist not only pressure but also extension or strain, we find the space between the cells partly occupied by fibres, which resemble those of ligaments; and such are termed fibro-cartilages. They are found in Man between the vertebræ of which the spinal column is made up (§ 71); and also uniting the bones of the pelvis (\$645). Sometimes, where elasticity is required, the fibres are those of the yellow fibrous tissue $(\S 23)$; this is the case with the cartilage which forms the external ear. Cartilage is not penetrated by blood-vessels, at least in its natural state. The blood is brought to its surface by a set of vessels which bulge out into dilatations or swellings upon it, so that a large quantity of fluid comes into the immediate neighbourhood of the cartilage, being only separated from it by the thin walls of the vessels; and it appears that this fluid, or so much of it as is required, is absorbed by the nearest cells, and transmitted by them to the cells in the interior, so that the whole substance is nourished. This is precisely the mode in which the interior of the large sea-weeds (whose tissue consists of cells imbedded in a gelatinous substance, and therefore bears 
a close resemblance to animal cartilage) obtains its nourishment from the surrounding fluid.

48. The permanent Cartilages seem to undergo very little change from time to time. Their wear is slow; and, being purely mechanical, it is confined to the surface. It is replaced by the materials absorbed from the blood, which are employed in the development of new cells, - sometimes within the old ones, sometimes in the space between them. When a portion of eartilage has been destroyed, however, by disease or injury, it is not renewed by true cartilaginous structure, but by what seems a condensed areolar tissue. Although cartilage does not usually contain vessels, yet these may be rapidly developed in its substance, by a process which will be described hereafter ( $\$ 393)$, when it becomes inflamed. This may be often seen to take place. The front of the eye is formed by a transparent lamina of a substance somerwhat resembling cartilage, which bulges like a wateh-glass: this, which is termed the cornea ( $\$ 533)$, is properly nourished only by vessels that bring blood to its edge, where it is connected with the tough membrane that forms the white of the eye. But when the cornea becomes inflamed, minute vessels may be seen to spread over it, proceeding from its circular edge towards its centre; and at last some of these often become of considerable size. Under proper treatment, however, these vessels gradually shrink and disappear; and the cornea becomes nearly as transparent as before.

49. Many parts exist in the state of Cartilage in the young animal, which are afterwards to become Bone; and it has been commonly believed that all bone has its origin in a cartilaginous structure. This, however, is not the fact, as will be presently shown. Before attempting to explain the formation of Bone, it will be desirable to describe its structure. When we cut through a fully formed bone, such as that of the thigh, we find that the shaft or elongated portion is a hollow eylinder; of which the walls are formed by what appears to be solid bone; whilst the interior is filled, in the living state, by an oily substance laid up in cells, and termed marrow. Towards the extremities, however, the structure of bone is very different. The outside wall becomes thinner; and the interior, instead of forming one large cavity, is divided into a vast number of small chambers, like, those 
of areolar tissue, by thin bony partitions, which cross each other in every direction, forming what is called the "cancellated" structure. These chambers or cancelli are filled with marrow; like the central cavity, with which they communicate. In the flat bones, moreover, - such as those of the head-we find that the two surfaces are composed of dense plates of bone, like that which forms the shaft of the long bones; but that between them there is a layer of cancellated structure, filled in like manner with marrow. But when we examine with the microscope a thin section of even the densest bony matter, we find it traversed by a network of minute canals, continuous with the central cavity. These canals usually run, in the shafts of long bones, in the direction of their length; and are connected, every here and there, by cross branches (fig. 16). They are termed the Haversian canals, after the name of their discoverer, Havers.-The lining membrane of the large central cavity is copiously supplied with bloodvessels ; and this sends off prolongations into the cancelli at the extremities of the bone, and into the Haversian canals. Thus blood is conveyed into the interior of the bone; but no vessels can be traced absolutely into its texture, so that all the spaces which lie between the Haversian canals are as destitute of vessels as

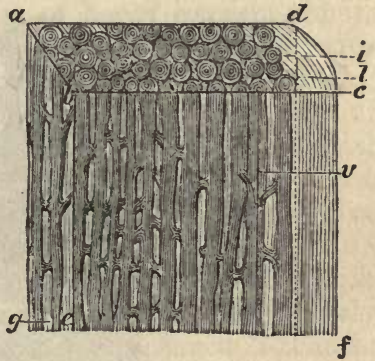

Fig. 16.-Diagram RePRESENTING THE STRUCTURE OP A PORTION OP THE SHAFT OF A LONG BONE.

$a \quad b \quad c \quad d$, the surface as seen in transverse section; $b$ e $f c$, surface seen in longitudinal section; $i, \mathrm{Ha}$ versian systems cut across, each having an Haversian canal in its centre; $g v$, Haversian systems cut longitudinally, $l$, lamellæ near the surface of bone, destitute of Haversian systems.

is healthy cartilage. These spaces are provided with nutriment by the following very remarkable arrangement.

50. When we cut across the shaft of a long bone, and examine a thin section with a microscope, we of course see the open extremities of the Haversian canals (fig. 17, a); just as we see the cut ends of the ducts and vessels of wood, when we make a transverse section of a stem. Around each of these apertures, the bony matter is arranged in concentric rings, which are marked out and divided 
by circles of little dark spots; and when these spots are examined with a higher magnifying power, it is seen that they are small flattened cavities, from which proceed a number of extremely minute tubules (A). These tubules pass out

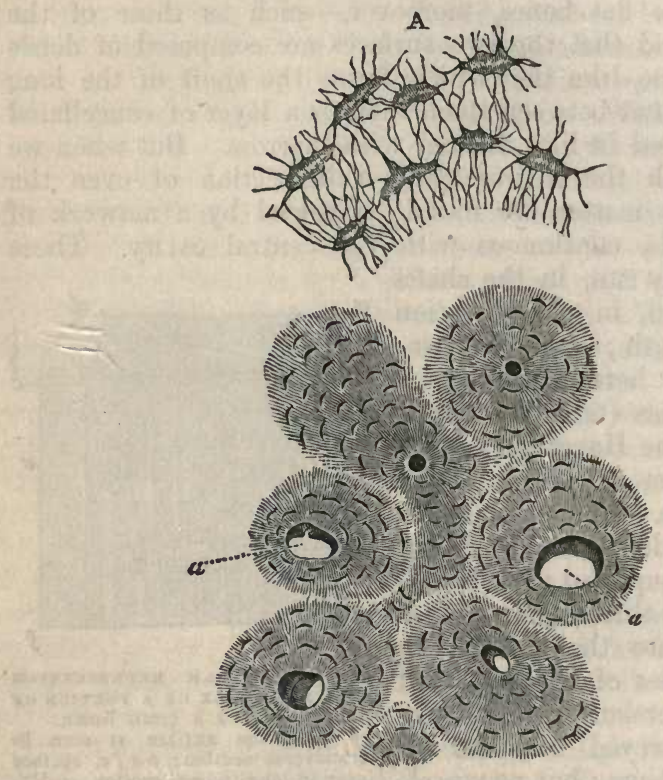

Fig. 17. -Transverse Section of Bone.

Showing the concentric rings round $a \boldsymbol{a}$, the Haversian canals. At A are seen some of the cavities with their radiating tubes, more highly magnified.

from the two flat sides of each cavity; one set passes inwards, towards the centre of the ring, and the other outwards, towards the ring that next surrounds them. These minute tubuli, which are far smaller than the smallest blood-vessels, may thus be traced into every part of the substance of the bone; and those proceeding from different rings are so connected with each other, that a communication is established between the innermost and the outermost circles. The tubuli which open upon the sides of the Haversian canals, are thus enabled to take up the nourishment with which they are 
supplied by the blood-vessels, and to transmit it to the outer circles, or those furthest removed from those vessels; and in this manner, a much more active nutrition takes place in bone than that which is performed in cartilage. It has been proved by various experiments, that the substance of bone is undergoing continual change; and it is owing to the comparative activity of its nutritive processes, that bone is so readily and perfectly repaired, when it has been broken by violense or has been injured by disease.

51. But the peculiarity of Bone consists, not so much in this remarkable arrangement of its organic structure, as in its solidity and firmness. This is given to it by the union of a large quantity of mineral matter with the organic substance of its tissue. The mineral matter of bones consists almost entirely of two compounds of Lime; the carbonate, with which we are familiar in the form of limestone and chalk; and the phosphate, which is seldom found as an ingredient of rocks or soils, except where it has been derived from animal remains. The latter greatly predominates, at least in the bones of the higher animals. We may easily separate the animal and the mineral portions of the bony tissue. If we soak a small bone for some time in muriatic acid much diluted with water, the compounds of lime are entirely removed from it, and the organic substance remains; the latter is now quite flexible, and almost transparent, so that the distribution of its vessels (if they have been previously injected with colouring matter) may be distinctly seen. On the other hand, if we subject a bone to strong heat, the animal portion will be burnt out, and the earthy matter will remain. The form of the bone will be still retained; but the cohesion between the earthy particles is so slight, that the least touch will break them asunder. Thus we see that the hardness of bone, or power of resisting pressure, is given by the earthy matter; whilst its tenacity, or power of holding together, depends upon the animal portion. Although the animal substance which remains after the solution of the mineral matter, has been commonly described as Cartilage, yet it is not so in reality; for it consists not of chondrin, but of gelatin; and instead of being made up of an aggregation of cells united by an intervening substance, it may be torn into layers of an indistinctly-fibrous matting. In fact, 
it corresponds closely with the white fibrous tissue ( $\$ 23)$, both in structure and composition; and so far from this view of its nature being inconsistent with the history of the formation of bone, it will be found to be in entire harmony with it. The proportion which the mineral bears to the animal substance of bone is very constant, when the proper-osseous tissue alone is taken into account; being almost exactly two of the former to one of the latter, or $66 \frac{2}{3}$ per cent. to $33 \frac{1}{3}$ per cent. But when the composition of entire bones, including the contents of the Haversian canals and cancelli, is compared, the proportion of mineral to animal matter is found to vary greatly in different classes of animals, in the same animal at different ages, and even in different bones of the same individual; the mineral matter predominating in bones of a compact texture, and the animal in those whose substance is more spongy.

52. In the first development of the embryo, a sort of mould of cartilage is laid down for the greater part of the bones; though, in the case of the flat bones, this mould is generally limited to the central portion, the place of their marginal part being occupied by a fibrous membrane only. The process of ossification, or bone-formation, commences with the deposit of calcareous matter in the intercellular substance of the cartilage, so as to form a sort of network, in the interspaces of which are seen the remains of the cartilage-cells. The tissue thus formed can scarcely be considered as true bone, for it contains neither lacunce nor canaliculi. Before long, however, it undergoes very important changes; for many of the partitions are removed, so that the minute chambers which they separated coalesce into larger ones; and thus are formed the cancelli of the spongy substance, and the Haversian canals of the more compact. These are at first much larger than they are subsequently to become; for they are gradually narrowed by deposits of true bony tissue, which successively take place upon their interior walls, at the expense of the materials supplied by the blood brought thither by their contained vessels; and it is by this formation of concentric layers around the cavities of the Haversian canals, that the appearance of concentric rings is produced, which we have just seen to be presented by transverse sections of long bones. In old bones the Haversian canals are 80 nearly filled by these deposits, that there is barely room 
for the blood-vessels to pass along them. And it is through their complete blocking up, by a continuance of the same growth, that the supply of blood is cut off from the interior of the bone which forms the antlers of the deer, so that they die and fall off; their shedding and renewal being an annual process. ${ }^{1}$ - Whilst the formation of the Haversian canals and cancelli is being effected by the partial removal of the first formed partitions, a complete cavity is formed in the centre of the shaft of every long bone (at least in Mammals and Birds), by the entire removal of the solid tissue. This cavity is at first not much larger than one of the Haversian canals ; but as the bone grows in diameter by additions to the exterior of its shaft, so is the cavity in its interior augmented by the removal (by absorption) of the first-formed bone; and this double process continues until the bone has attained its full diameter. The formation of new bone on the exterior of the shaft seems to be the result of the consolidation-of the fibrous tissue of the periosteum (or membrane covering the bone) by calcareous deposit; the lacunæ being probably the cavities of cells which were entangled in the fibres, and the canaliculi being outgrowths from these; and new fibrous tissue being formed on the outside of the periosteum, to replace that which has been taken into the bone. Thus it comes to pass, that after a time none of the bone first formed in its cartilaginous mould any longer remains, the whole of it having been removed by absorption; since the central cavity of the perfect bone is much larger than the entire cartilaginous shaft in which it originated. And thus it also comes to pass, that (as gelatin is the basis of fibrous tissue) bones yield gelatin, not chondrin, upon being long boiled.The increase of the shaft in length, however, is the result of a different process. In all bones of any considerable dimensions, the process of ossification commences in more than one point at a time. In the long bohes, there are usually three such points; one for the shaft, and the others for the two

1 It is commonly stated that the death of the anclers is due to the formation of a bony ring at their base, which cuts off the supply of blood from the "velvet" which covers then: ; but though this may contribute to produce the effect, it is by no means the sole cause, as the interior of the antlers is supplied with blood from the vessels of the bone from which they sprout, and not from those of the "velvet" only. 
extremities. Long after the ossification of the shaft and of the extremities has been completed, these parts remain separated from each other by the interposition of a thin layer of unconsolidated cartilage ; so that, although the bone appears firm and complete, its three portions fall apart, if it be macerated sufficiently long in water for the cartilage to decay. Now it is by the progressive consolidation of the cartilage at these two junctions, and by the continual formation of new cartilage as the old is taken into the bone, that the length of the shaft continues to increase up to adult age; and then, its full size having been attained, the whole thickness of the intervening layer of cartilage is replaced by bone, so that the shaft and extremities become firmly consolidated. - The general history of the formation of the flat bones is nearly the same. In these, when they are large, or have projecting out-growths, there are several centres of ossification; and although the first ossification takes place in the substance of cartilage, yet the subsequent growth seems to be effected mainly by the consolidation of fibrous membrane.

53. The foregoing description applies chiefly to those higher and more complete forms of Bone, which are found in Birds and Mammals. In Reptiles and Fishes, the process of ossification is stopped short, as it were, at an early period; and thus the texture of their bones resembles that which we find the skeleton to present in the earlier life of the higher animals. - The long bones of Reptiles (with one remarkable exception in the Pterodactylus, $\S 669$, which is adapted to the life of a Bird) have no one central cavity, but are penetrated by numerous large Haversian canals, like those of very young bone; and various pieces remain separate in them throughout life, which, originating in distinct centres of ossification, subsequently coalesce in Birds and Mammals. This permanent separation is still more remarkable in the bones of Fishes; and it is consequently in them that we can best study the real composition of the skeleton,-every piece which originates in a distinct centre of ossification, being, in the eye of the philosophical anatomist, a separate bone. Further, there is a large group of Fishes in which the skeleton retains the cartilaginous character through life; a certain quantity of mineral matter being deposited in the 
cartilage, but its conversion into true bony structure never taking place. In a few, not even a firm cartilage is produced; and all the trace of a skeleton is a cylinder formed of hexagonal cells, resembling those of the pith of plants, which takes the place that is generally occupied by the "bodies" of the vertebræ (\$ 71). Such a cylinder, which is termed the chorda dorsalis, precedes the formation of the vertebral column in other vertebrated animals ( $\$ 757)$. In the curious Amphioxus (Zool. $\S 642$ ), even this is wanting; and the only rudiment of the bony skeleton is to be found in the fibrous sheath that surrounds the nervous centres, and sends off prolongations between the successive transverse bands of muscles, which are attached to these, as they are in other fishes to the ribs and the spines of the vertebræ.

54. In connexion with the structure of Bone, it will be convenient to describe that of Teeth, although the general description of the form and development of these organs will be more appropriately given in connexion with the account of their instrumental uses ( $\$ \S 181-183)$. The principal part of the substance of all teeth is made up of a solid tissue, which has been appropriately called Dentine. Of this substance, one variety, which is peculiarly close in texture, and susceptible of a high polish, is familiarly known as ivory. The more perfect forms of dentine, such as present themselves in Man and the Mammalia generally, consist of a.hard transparent substance formed by the union of animal matter and calcareous salts (chiefly phosphate of lime), in the proportion of about 28 of the former to 72 of the latter; the mineral matter thus bearing a somewhat larger ratio to the organic, than it does in $^{2}$ bone. This dentinal substance is traversed by minute tubuli of about 1-10,000th of an inch in diameter, which appear as dark lines, generally very close to-

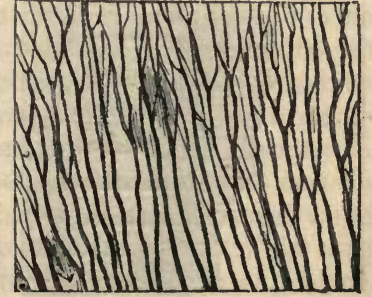

Fig. 18. gether; these pass in a radiating Portrox or Denrre (highly magnimanner from the central cavity fied), showing its tubular structure. of the tooth, diverging from each other as they approach its exterior; but when seen in only a small part of their 
course, they appear to be nearly parallel (fig. 18), though usually more or less wavy. They occasionally divide into two branches, which continue to run, at a little distance from one another, in the same parallel direction; and they also frequently give off small lateral branches, which again send off smaller ones. In some animals the tubuli may be traced at their extremities into minute cavities analogous to the lacunæ of bone; and the lateral branchlets also occasionally terminate in similar cavities. Thus the whole tooth may be likened, in some degree, to a single Haversian system in bone ; the central cavity, which is lined by a vascular membrane, representing the Haversian canal, while the radiating tubuli of the former correspond with the radiating canaliculi of the latter; the chief difference lying in the absence of lacunæ along the course of the radiating tubes. In a large proportion of Fishes, however, there is no single central cavity, but the whole tooth is traversed by a system of medullary canals, not only resembling the Haversian, but actually continuous with those of the bone on which the tooth is implanted; and as each of these is the centre of a distinct system of radiating tubuli, the resemblance of their dentine to bone is very close. A somewhat similar condition of the dentine (obviously a lower or less specialized form of this substance) presents itself in certain Reptiles and Mammals. In the Teeth of Man and most other Mammals, and in those of many Reptiles and some Fishes, we find two other sub-

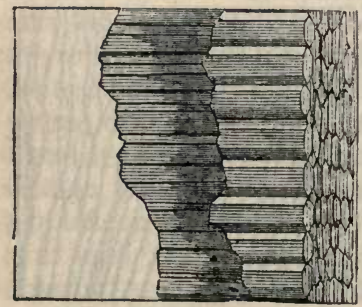

Fig. 19. stances, one of them harder and the other softer than dentine. The former, which is called Enamel, consists of long prismatic cells, which pass from one surface to the other of the thin layer formed by this substance over the crown, or sometimes in the interior of the tooth ( $\$ 182)$. These prisms are usually hexagonal in form, as is seen in Porrrox of ENAMEL (highly magni- transverse section (fig. 19); and
fied), showing its component prisms.

their course is usually more or less wavy. In teeth which have to sustain an extraordinary amount of compression (as is especially the case with those of 
the Rodentia), the enamel-prisms cross and interlace with one another, in such a manner as to prevent that seraration which would readily occur if the direction of all of them were the same. Of all the tissues of the animal body, the Enamel is the most remarkable for the predominance of mineral ingredients; these amount to no fewer than 98 parts in 100 , leaving when removed only 2 per cent. of organic matter. The softer component of Teeth, known as the Cementum, or Crusta petrosa, possesses the essential characters of true bone; but when only a thin layer of it is present, we do not find it traversed by medullary canals, its system of lacunæ and canaliculi being then in relation to the nearest vascular surface,-as is the case also with very thin laminæ of ordinary bone, such as we find in the seapula (blade-bone) of a Mouse.

55. We come, lastly, to the two tissues which are of the highest importance in the Animal fabric, and to which all the rest are merely subsidiary; namely, the Muscular and the Nervous. It is through the instrumentality of these, that all the actions are performed which essentially constitute Animal life; for the nervous apparatus is the medium by which the consciousness of the individual is affected by what takes place around him, or within his own body, and by which, in his turn, he originates movements in his body, and through it in things external to it; whilst the muscles are, so to speak, the servants of the nerves, doing, with a force of their own, the work which the nerves direct. The relation between the two may be likened to that of the rider and his horse, or of the engine-driver and his locomotive; for the nerves can put forth no motor power by themselves; whilst, on the other hand, the muscles (with certain exceptions) remain inert except when stimulated to contract by the agency of the nerves. The muscles use the tendons and the framework of bones, joints, \&c., for the mechanical appiication of their power, as will be shown hereafter (Chap. XII.); but these parts of the fabric have not the slightest power of originating motion by themselves. Hence, all Animal Force takes its rise in one or other of these two tissues; and we shall find that the special purpose of the whole apparatus of Organic life, is, by providing materials for their nutrition and renovation, to build them up in the first instance, and then 
to keep them in working order. For every development of animal force involves a change of state of the Nervo-muscular substance : a certain amount of it ceasing to exist as living tissue, and passing into the condition of dead matter; and its elements resolving themselves, under the influence of the free oxygen brought to them by the blood, into new combinations, which are carried forth from the body as quickly as possible. Consequently, if the Nervo-muscular tissues be not renewed as rapidly as they are used up, their powers must speedily fail from the progressive loss of their substance. In this particular they are on a different footing from the other elementary parts of the organism; for although each of these seems to have a certain term of life, the length of which is in some degree related inversely to its functional activity,those which live the fastest having the. shortest individual duration, and vice versâ,- - there are none which are called upon to give forth their whole vital energy in one effort, and which may thus have their existence as parts of the living organism terminated at any moment by a demand for their peculiar power.

56. Muscular Fibre presents itself under two forms, which are ordinarily very distinct from each other; although it is probable that they may ultimately prove to be but modifications of one and the same. The first, which is known as the striated fibre, is that of which all those muscles are composed, which constitute what is commonly designated as "flesh" or the "lean" of meat. If any "joint" of meat be even cursorily examined, it will be seen that its whole substance is made up of distinct masses; held loosely together by areolar tissue; and these masses, which are known as "muscles," are easily isolated from each other by dissection. Every such Muscle is formed by the union of a number of bundles, having a generally parallel arrangement, which are closely bound together by areolar tissue, and are themselves composed of bundles still more minute, united in a similar manner. These, again, may be separated in the same way; and at last we come to the primitive fibres of which this tissue is composed. Each of these primitive fibres terminates at either extremity in tendinous fibre, which unites with other fibres to form the tendinous cords or bands, that are attached to the points of the skeleton which the muscle 
has to bring together. The muscular fibre itself consists of a delicate membranous tube, enclosing a great number of fibrillae, or extremely minute fibrils, which are not capable of further division (fig. 20). The peculiar transverse marking

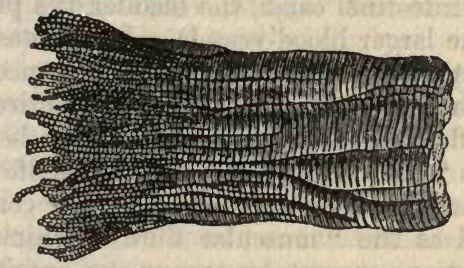

Fig. 20.-Striated Muscular Fibre separating into Fibrille.

or striation by which this form of muscular fibre is characterised, is found, when the fibre is separated into its fibrillæ, to be due to the peculiar markings which every fibril presents. These markings, consisting of alternate light and dark spaces, give to the fibril a beaded appearance ; but this is only an optical deception, since its form is in reality cylindrical, or nearly so. It is easy to see how the correspondence of the light and dark spaces respectively, throughout the whole bundle of the fibril, will give rise to the banded appearance which the entire fibre presents. The form and diameter of the fibres vary considerably, both in different tribes, and in different parts of the same animal. In the higher classes, their form usually approaches a cylinder; but the parts which press against one another are somewhat flattened, so that it is more or less prismatic. In Insects, on the other hand, the fibrillæ are arranged in flat bands, so that the fibre often consists of but a single layer of them. The diameter of the fibres in Man averages about 1-400th of an inch, and does not differ very widely in either direction; in the cold-blooded Vertebrata, however, the average size is greater, and the extremes are also wider; the diameter of the fibres varying in the Frog from 1-100th to 1-1000th of an inch, and in the Skate from 1-65th to 1-300th of an inch. The diameter of the fibrils is nearly the same in all classes, seldom departing much from 1-10,000th of an inch; and the average distance of the dark striæ from each other is nearly the same. 
57. The other form of Muscular Fibre, which, from the absence of transverse striation, is distinguished as smooth or non-striated, is found not in large masses, but in thin layers, forming part of the wall of various hollow organs, such as the stomach and intestinal canal, the bladder, the principal glandducts, and the larger blood-vessels. In all these situations it is so exclusively concerned in the performance of the vegetative or nutritive functions, and it is so entirely withdrawn from the influence of the will, that it has been frequently designated as "the muscular fibre of organic life ;" the striated fibre, of which the voluntary muscles are composed, being distinguished as the "muscular fibre of animal life." But these designations are not by any means consistent with the facts of the case ; for in a large proportion of the Molluscous classes, the muscles of animal life are composed of nonstriated fibre, whilst the heart of Man and of other Vertebrata, though a muscle of organic life, is made up of striated fibre. In fact, the employment of the one or of the other kind of fibre would seem to be chiefly determined by the kind of contraction which is required from it (\$ 59). The non-striated fibres are arranged, like those of the other muscles, in a parallel manner into bands or bundles; but

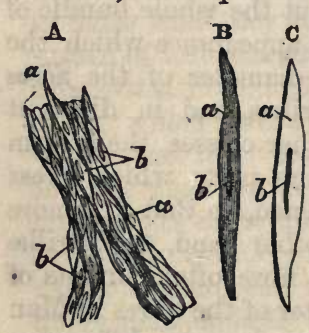

Fig. 21.

A, Portion of a band composed of non-striated muscular fibre, showing, $a \boldsymbol{a}$, the spindle-shaped cells, and, $b b$, the elongated nuclei; $B$, a single cell isolated, and more highly magnified; $C$, a similar cell treated with acetic acid. these bundles, instead of being themselves grouped into larger ones having a like parallel arrangement, are generally interwoven into a kind of network, having no fixed points of attachment. The form of the indiviaual fibres is much more variable than that of the striated kind, being often very much flattened out; and hence their general dimensions cannot well be estimated. By macerating a portion of this kind of tissue in dilute nitric acid, each fibre may be resolver! into bundles of long spindle-shaped bodies, which, containing elongated staff-shaped nuclei (fig. 21), may be regarded as cells, although it is difficult to distinguish their walls from their contents. This form of muscular tissue is commonly mingled with a large quantity of the ordinary fibrous 
structure; and we find it dispersed in small quantity through the latter in the skin, to which (especially in particular regions) it gives a contractility that is manifested under the influence of cold or of mental emotions, and thus produces that general roughness and rigidity of the surface which is known as cutis anserina, or "goose's skin."

58. Under the influence of certain exciting causes, or stimuli (Chap. XII.), striated muscular fibres suddenly and forcibly contract. Their two ends approach one another, and their strix become closer; but they bulge out in the middle to a corresponding degree. This causes a like change in the bundles which are made up of these fibres; and thus the whole muscle, when shortened by the drawing together of its two ends, is greatly enlarged in diameter, especially towards its middle. Of this any one may convince himself, by bending his forearm upon the arm (as when the hand is brought to the mouth), and feeling the fleshy mass upon the front of the latter. The muscle, in fact, does not in the least degree change its own bulk in the act of contraction; for its enlargement in diameter is exactly equivalent to the shortening of the distance between its extremities. The contraction of a muscular fibre is ordinarily followed, after a short interval, by its relaxation; of this we have a remarkable illustration in the contractions excited by the electric stimulus. But relaxation of individual fibres is not incompatible with the continuance of the state of contraction of the muscle as a whole. For it appears that when an ordinary muscle is thrown into contraction, all its fibres do not usually contract together, but only a small part of them; and that, as long as its contraction is maintained so as to exert a constant force, a continual interchange is taking place in the action of the fibres by which this is kept up-those which have been shortened becoming slack, and being replaced (as it were) by others, which pass into the contracted state for a time, and then relax again, being succeeded by another set. Now as the ends of those fibres which are actually in a relaxed condition, are brought near together by the contraction of the rest, the fibre is thrown out of the straight line, and assumes a wavy or zigzag form, which was formerly supposed to be the state of contraction, but is now known to be otherwise. This peculiar arrangement gives place to the straight form, either when the 
fibre passes into the state of contraction, or when, by the relaxation of the whole muscle, its ends are separated again to their full extent.

59. Now the alternate contraction and relaxation, which is thus made to produce a continued contraction in ordinary muscles, elsewhere occasions a different effect. Thus in the heart, all the fibres of the ventricles seem to contract together and all to relax together, - those of the auricles contracting whilst the others are relaxing, and vice vers $a$; - and in this way the alternate contractions and dilatations of that most important organ are continually kept up. Again, in the muscular coat of the intestinal canal, we observe the contraction of each part to be almost immediately followed by its relaxation; but the peculiarity of its movement is, that the contraction is propagated on (as it were) to the succeeding part, which in its turn contracts and then relaxes, producing the same action in the part that follows it,- and so on along the whole canal. This peristaltic motion ( $\$ 215$ ), as it is called, is obviously adapted to propel the contents of the intestinal tube from one extremity of it to the other; just as the peculiar action of the heart is adapted to receive and propel the blood alternately, or as the mode of contraction of the ordinary muscles enables them to keep up a continued strain for a great length of time. It is much less rapid and energetic than the action of the heart; for it is the characteristic of the non-striated fibre, that its contraction follows much less closely on the application of the stimulus, and is much less rapidly succeeded by relaxation, than that of the striated fibre.

60. The Nervous tissue consists of two distinct structures, of one of which the trunks of the nerves are entirely made up, whilst the other enters largely into the composition of the ganglia or centres of action (\$61). The former, termed the white or fibrous tissue, consists of straight fibres, lying side by side, and bound together by areolar tissue into bundles (fig. 22); these, again, are united with others into a larger group ; and by the union of a considerable number of such groups, the nervous trunks are formed, which are distributed through the body, especially to the skin and muscles. Nervous Fibre, like muscular, presents itself in the higher animals under two forms, of which one may be considered as more completely developed than the other; these are known 
as the tubular and the gelatinous. The " tubular" fibres are so named because each possesses a distinct tubular sheath of a delicate structureless membrane (fig. 22, A), which encloses the proper nerve-substance, and isolates it completely from the

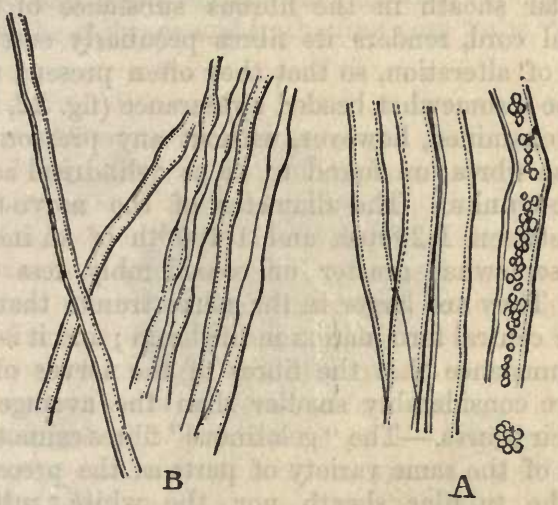

Fig. 22.-Structure of NeRVE-TuBes.

Tubular Nerve-fibres ; A, from a nerve-trunk; B, from the substance of the brain.

blood-vessels and other surrounding structures; this tube does not either branch or unite with others, and there is reason to believe it to be continuous from the origin to the termination of the nerve-trunk. Within the tube is a hollow cylinder of a material known (after its discoverer) as the "white substance of Schwann ;" and this encloses a sort of central pith, which is transparent and semi-fluid in the living state, but undergoes a kind of coagulation into a granular substance after death, and under the influence of chemical re-agents. There is reason to believe that this central pith or "axis-cylinder" is the essential component of the nervous fibre, and that the hollow cylinder which surrounds it serves only to isolate it more completely; for we not unfrequently see the former to be alone continued, both the tubular sheath and the white substance stopping short; and this at either extremity of the fibre, where it separates itself from those with which it is bound up in the nerve-trunk. The proper form of the fibre seems always to be truly cylindrical; though 
it is very liable to be altered by manipulation, a small excess of pressure in one part forcing the contents of the tube towards some other where they are more free to distend it, and thus producing a swelling. The greater delicacy of the tubular sheath in the fibrous substance of the brain and spinal cord, renders its fibres peculiarly susceptible of this kind of alteration, so that they often present under the microscope a somewhat beaded appearance (fig. 22, B); when carefully examined, however, without any previous disturbance, these fibres are found to be as cylindrical as those of the nerve-trunks. The diameter of the nerve-tubules is usually between 1-2000th and 1-4000th of an inch; but it may be somewhat greater or considerably less than this average. They are larger in the.nerve-trunks than they are near their central termination in the brain ; and it is a remarkable circumstance that the fibres of the nerves of "special sense" are considerably smaller than the average in every part of their course.-The "gelatinous" fibres cannot be shown to consist of the same variety of parts as the preceding; for neither the tubular sheath nor the white substance of Schwann can be distinguished in them. They are flattened, soft, and apparently homogeneous, sometimes showing a disposition to split into very delicate fibrillæ; being of a yellowish-grey colour, they are sometimes designated the grey fibres. Their diameter averages between the 1-4000th and 1-6000th of an inch. As these "gelatinous" fibres form a considerable proportion of the trunks of the Sympathetic system of nerves ( $(461)$, they have been supposed to belong properly to it, and to minister exclusively to the organic functions, like the non-striated muscular fibre ( $\$ 57)$; but,"there is no doubt that this is an incorrect notion, and that even the fibres of the ordinary nerve-trunks may present the "gelatinous" aspect, probably from incompleteness of development.

61. In the central organs of the Nervous system-namely, the brain and spinal cord of the Vertebrata, the ganglia or knot-like swellings on the nervous cords' which take their place in the lower animals, and similar ganglia belonging to the Sympathetic system - we find a form of nervous tissue altogether distinct from the preceding; which, from its consisting of large cells or vesicles, is generally known as the 
vesicular. These nerve-vesicles, sometimes known as ganglion-globules, may be regarded as originally spherical, or nearly so, in form (fig. $23, a$ ); but they often present one or more prolonged extensions; and as these when single resemble tails, and when multiple are like the rays proceeding from a star, the cells are said in the first case to be "caudate,"

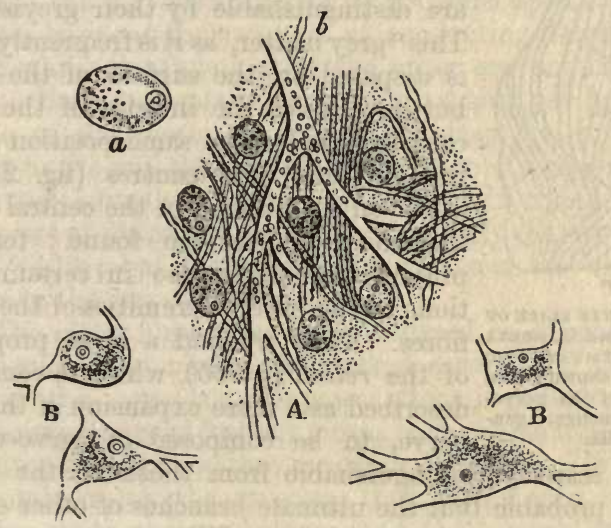

Fig. 23.-Vesicular Nerve-substancr.

A, combination of Ganglion-cells (of which one is shown separately at $a$, more highly magnified), and Nerve-fibres in the grey substance of the brain, which is also traversed by a capillary vessel, $b ; \mathrm{B}$ B, Ganglionic cells with caudate prolongations.

and in the second to be stellate (B). These prolongations have been traced into continuity, in some instances, with the axis-cylinders of nerve-tubes, whilst in other cases they seem to unite with those proceeding from other vesicles. It is not by any means certain, however, that the nerve-tubes thus connect themselves with the nerve-vesicles in all instances; since it frequently appears as if the former passed in among the latter, without coming into direct continuity with them. Sometimes a ganglion-cell seems to lie in the course of a tubular fibre, which enlarges to envelope it, and then contracts again to its former dimensions. There can be no reasonable doubt, however, that in some way or other the nerve-fibres and the nerve-vesicles come into some kind of communication in the ganglionic centres. The vesicles are 
filled with a finely-granular substance, which extends into their prolongations; and in the warm-blooded Vertebrata they

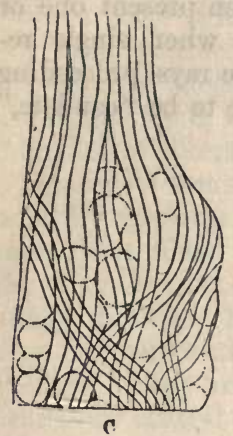

Fig. 24.-THIN SLICE oF ONE OF THE GANGLIA OF THE SYMPATHETIC System, showing the passage of the nervefibres amongst ganglionic cells.

that are scarcely distinguishable from those of the brain; and it is probable that the ultimate branches of other sensory nerves have some such termination. Wherever we meet with vesicular substance, we find it imbedded in a minute network of blood-vessels; and a copious supply of oxygenated blood is requisite to the due performance of its actions.

62. There can be no doubt that the special office of the Nerve-fibres is to convey the influence of the changes which are effected in one part of the system, to other and remote parts; just as the wires of a galvanic battery conduct the electric influence from the instrument which excites it, to some distant point where it is to be applied to some use. The effects of such changes in the state of the Nervous System are propagated in two opposite directions;- the impressions made upon the skin and other parts possessed of sensibility, being conveyed towards a portion of the nervous centres called the sensorium, and there giving rise to sensations; - and the influence of the emotions or volitions to which these sensations give rise ( $\$ 7)$, being propagated from the central organs to the muscles, which they excite to contraction. And by the discoveries of Sir C. Bell, hereafter to. 
be described it has been fully proved that these opposite changes are conducted by two different sets of fibres;-one conveying to the central organs those which originate in the circumference; - and the other conveying to the circumference those which originate in the centre ( $\$ 451)$. The transmission of these changes is completely interrupted by division of the nervous trunk, or by pressure upon it; and it sometimes happens that one set of conducting fibres is thus affected, whilst the functions of the other are not impaired; so that a limb may retain its sensibility and yet be totally destitute of the power of motion, or may be completely obedient to the will though totally destitute of sensibility. In Vertebrated animals, we find some nerves in which there is only one set of fibres, so that the trunk is only sensory or only motor $(\$ 459)$; but in general, the two sets are bound up together in the same sheath.

63. The motor fibres may be considered as originating in the vesicular substance of the central organs, and as terminating in the muscles; the power which is generated in the former being conveyed by their means to the apparatus through which it operates to produce mechanical motion. When the nerve-trunks reach the muscles, they divide into branches which penetrate their substance, and these again subdivide and ramify minutely, so that at last the fibres may often be observed running singly, passing amongst the muscular fibres, but not appearing to penetrate their tubular sheaths. These terminal fibres seem often to double back upon themselves, so as to form loops, either re-entering the branch from which they issued, or connecting themselves with some neighbouring branch; so that the ultimate distribution of the motor nerves in the muscular substance, is a sort of plexus or network. The sensory fibres, on the other hand, may be considered as originating in the sensory surfaces, such as the skin, the interior of the nose, the lining membrane of the cavities of the internal ear, the retina of the eye, \&c.; and as passing towards the central organs, conveying to these the impressions they have received, which impressions may either affect the consciousness, or may excite respondent movements, or may act in both modes, through the instrumentality of the vesicular substance to which they are transmitted. The immediate dependence of the functional activity of this 
substance upon the supply of blood which it receives, is shown by the fact, that if this supply be temporarily cut off, either by failure of the heart's action (as in fainting), or by pressure on the blood-vessels which convey it, immediate insensibility, with loss of all power of motion, is the result. And the same is the case with regard to the organs of sense; for if the circulation through them be interrupted, no sensory impression can be made upon the nerve-fibres which originate in them, as we see when the movement of blood in a limb is suspended by pressure upon its artery.

64. The foregoing constitute the principal tissues among the higher animals, in which the principle of division of labour is most fully carried out, every component part having its own peculiar structure and its own special action. As we descend in the scale, we find these distinctions less and less obvious, so that when we come down to Zoophytes ( $\$ 121$ ), we meet with but little differentiation either in the textures or in the actions of the several parts of the body; the whole substance of these animals being composed of a tissue, which very closely resembles that which is first formed in higher animals for the reparation of wounds, having the appearance of a solidified blastema ( $\$ 34$ ), with nuclear particles, in various phases of development into cells and fibres, more or less thickly scattered through it; and this substance being everywhere contractile, and everywhere (at least in many instances) equally capable of participating in the functions of nutrition and reproduction. And when we pass still lower, to that simplest type of animal life, which is presented to us in the Rhizopods ( $\$ 129)$, we do not meet with even this amount of definite structure, but find the entire substance of their bodies composed of an apparently homogeneous jelly, which, like the more organized tissue of the Zoophytes, is everywhere contractile, and which has also the power of performing every operation required for its growth and maintenance as a living being. In such creatures there is not the slightest vestige of a Nervous system; and it remains a question whether, in consequence of this deficiency, they are altogether destitute of consciousness, or whether this endowment is diffused, as it were, through the whole substance of their bodies.

65. Every component part of the fabric must be regarded 
as having a life of its own, which it maintains by drawing to itself the nutrient material supplied by the circulating current; but as the continuance of its vital activity is dependent upon the continuance of its nutrition, the life of no tissue can be prolonged for any considerable period after the circulation has ceased. But after the movement of the blood has come to an end, though the body as a whole is dead, its parts may remain alive for a certain time, and may perform their functions, so long as they are supplied with the necessary materials. Thus, various secretious, the growth of hair, and muscular movements, have been observed to take place in dead bodies. But they cannot continue, because the necessary conditions are withheld by the stoppage of the circulation, - a function which thus binds, as it were, into one whole the scattered elements, and causes the different operations to minister one to another. As every component part has an independent life, so has it a limited duration, quite irrespective of that of the organism as a whole. Thus the cells which float separately in the blood, seem to be continually undergoing change,-dying, and giving place to new ones. We have seen that the cells of the epidermis and of some parts of the epithelium are being constantly thrown off and renewed. The duration of the cells of fat and cartilage appears to be much greater; in fact, we have no precise knowledge of their term of life. That of the bony tissue is probably greater still; yet there is adequate evidence that it is by no means indeterminate. But that of the muscular and nervous tissues seems to depend almost entirely on the use that is made of them. Thus we may justly say,-however startling the assertion may seem,-that death and decay are continually going on in every living animal body, and are essential to the activity of its functions.

66. Many animals are reduced to a state of apparent death by dryness, by cold, or by exclusion of the air. A curious example of the first kind is furnished by the Tardigrada (ZooLOGY, § 841); some species of which may not only be completely dried up, but may even be exposed in that state to a temperature much exceeding that of boiling water, without losing the power of recovery when moistened. A similar power of revival after being dried up is possessed by the common Wheel Animalcule, and probably also by tho 
eggs of many minute Entomostracous Crustacea (Zoology, §§ $883,931)$. It is unquestionable that many Fishes, especially those of fresh-water lakes, will revive on being thawed after having been completely frozen; and the same has been ascertained of certain Caterpillars. The Snail, when retiring for the winter, seals the orifice of its shell with an impervious lid; and in this cavity it may remain shut up for years, until re-excited to activity by warmth and moisture. Animals in such states of torpidity strongly resemble seeds that are prevented from germinating, apparently for unlimited periods, by being kept at a moderate temperature, and excluded from the influence of air and moisture, which, with adequate warmth, would call them into active growth, but which, at a lower temperature, would occasion their decomposition. There are no positive facts which enable us to say how long Animals may remain in a parallel condition; but there seems no reason why it might not be indefinitely prolonged.

67. The death of the body, then, does not consist in the mere suspension of its vital activity; for so long as that activity may be renewed when the requisite conditions are supplied, so long must the organism be considered as alive, however death-like its condition may seem. Among warmblooded animals, such a suspension, if complete, cannot be endured for more than a very brief period, without the extinction of life; for the substance of their tissues is so prone to decomposition, that it speedily passes into decay unless prevented from doing so either by a reduction of temperature, or by complete drying-up, or by entire seclusion. from air; and although each of these methods, practised upon animal substances already dead, may prevent the occurrence of decomposition for almost unlimited periods, yet neither can be applied to the living tissues of any of the higher animals, without occasioning the entire loss of their vitality, as we see (in regard to cold) in the loss of members by "frost-bite." Such parts die, because not only is their vital activity suspended, but their vital properties are annihilated. Their death, however, does not necessarily involve that of the organism as a whole; since the stoppage of their function may not disarrange the general train of vital operatione, or their duty can be discharged by other organs. And among many of the lower animals, we find that there is a provision 
for their replacement by ordinary acts of growth ; and that even when the body has been so severely injured that the organic functions are seriously disturbed for a time (as when a Hydra is divided into two or more pieces, § 122), the vitality of the individual parts is sufficiently enduring, and their reparative powers sufficiently energetic, to enable them to reproduce all that is wanting for the completion of the organism, and for the renewal of its ordinary actions. Among the higher animals, the death of the organism at large may be said to take place when the circulation finally ceases; since, as we have just seen, every individual part must ere long lose its peculiar functional activity, and the entire body be subject to decay.

68. From what has been stated, it will be seen that Life cannot be regarded as a condition in which decay is resisted; for an incessant decay is taking place in every living organism as a necessary condition of its vital activity, being only checked when that activity is itself suspended. But it is a condition in which, by the wonderful harmony and mutual adaptation of the operations of the different parts, the reparative action of the Organic Functions is made to countervail the destructive action involved in the exercise of the Animal Faculties; whilst the latter, in their turn, serve to furnish the conditions requisite for the maintenance of the former. So long as all these actions go on with regularity and completeness, so long the whole body lives; but if any one of the more important among them be interrupted, the stoppage of the whole is the result. This relation of mutual dependence is most intimate in the higher animals; in which, by the differentiation of the several tissues and organs, and the specialization of their functions, the division of labour is carried to its greatest extent, so that no part can entirely fulfil the duty of any other. On the other hand, it is among those lowest forms of animal life, in which there is the greatest multiplication of similar parts, and the greatest diffusion of the same endowments amongst them all, that we find the dependence of the several parts of the organism upon each other to be the slightest, and severe injuries to be tolerated with the least general disturbance. 


\section{CHAPTER II.}

GENERAL VIEW OF THE ANIMAL KINGDOM.

69. WHEN we examine the Animal Kingdom as a whole, it. is easy to distinguish in it four general plans or types of structure, by which, with almost infinite variations in detail, the formation of the several beings that compose it has been guided. As specimens of these four plans or types, we may name four animals which are familiar to almost every one,the Dog, the Lobster, the Snail, and the Star-fish. The differences by which these types are distinguished, are manifested in the arrangement of the different organs of the body ; and particularly in the form of the nervous system and its instruments. It has been already stated $(\S 4)$ that the power of feeling, and of spontaneous motion, is that which peculiarly distinguishes the Animal from the Plant; and as these powers are possessed in very different degrees, and exercised in very different modes, by the various tribes of animals, - whilst the operations of nutrition are performed, as in plants, in a much more uniform manner, - they afford us a satisfactory means of separating these tribes from one another. For the nervous system is the organ to which these powers are due; and we find it presenting forms so different in the four great divisions already alluded to, that we can at once distinguish them by this alone, even where (as sometimes happens) there may be such a blending, in a particular animal, of the general characters of two of them, as to lead us to hesitate in assigning its precise place in the animal kingdom.

70. The highest of these four divisions is that denominated Vertebrata, or Vertebrated Animals; it receives its name from the structure characteristic of it,-the possession of a jointed back-bone or vertebral column,- which will be presently described. This is the group to which Man belongs; and all the animals it contains bear a greater or less resem blance to him in structure. We notice in regard to their external form, that they are alike on the two sides of their body; every part having its fellow on the other side. This 
"bi-lateral symmetry" extends to the arrangement of those internal parts which are connected with the functions of animal life; namely, the nervous system, the organs of sense, and the muscular apparatus. But it does not always extend to the organs of nutrition, which are unequally disposed on the two sides : thus, in Man, the heart and stomach are on the left side, and the liver on the right, while the lungs are much larger on the right side than on the left. But in many of the lower Vertebrata, there is an almost perfect symmetry in the disposition of these organs, as there is also in the early embryo of those in which this symmetry is subsequently departed from; so that it may be truly said that this symmetry is characteristic of the Vertebrate type, although for special purposes it is frequently superseded.

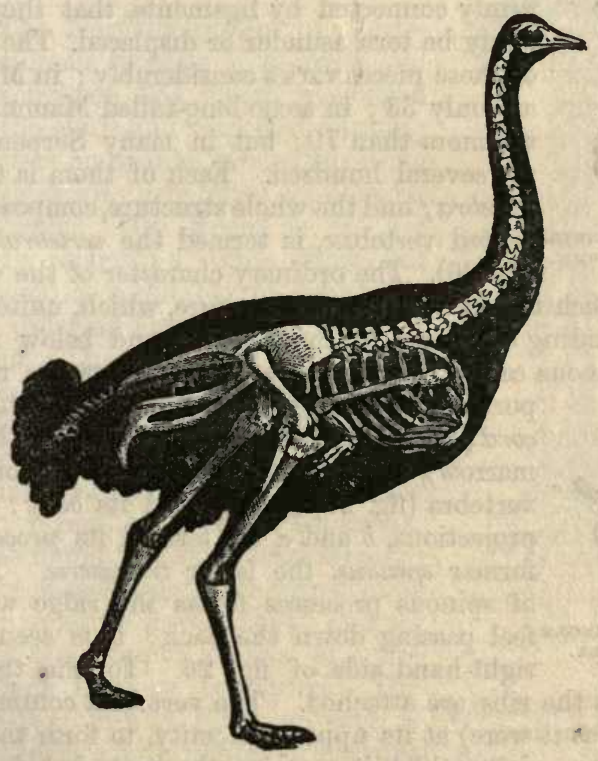

Fig. 25.-SKELETON OF THE OSTRICH.

71. In all Vertebrated animals, the skeleton is chiefly internal (fig. 25); and consists of bones, which are capable of 
growing, and of being reproduced after injury, like any other part of the living tissue ; being copiously supplied with bloodvessels, which penetrate into their interior. These bones give support, and afford points of attachment, to the soft parts, in the limbs (where they exist) as well as in the trunk; but the former are not unfrequently wanting, as in Serpents : and we must look in the trunk, therefore, for that peculiar arrange-

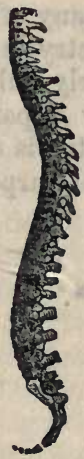
ment which is characteristic of this division of the Animal Kingdom. The back-bone, as it is commonly termed, is found in all Vertebrated animals; though in a few among them (the lowest Fishes) it is very imperfect ( $\$ 53$ ). It consists of several pieces jointed together, so as to possess great flexibility; whilst they are so firmly connected by ligaments, that they cannot easily be torn asunder or displaced. The number of these pieces varies considerably ; in Man there are only 33 ; in some long-tailed Mammals there are more than 70; but in many Serpents there are several hundred. Each of them is termed a vertebra; and the whole structure, composed of the Fig. 26-VERTE- united vertebræ, is termed the vertebral column BRAL CoLUMN. (fig. 26). The ordinary character of the vertebræ is, that each is perforated by an aperture, which, united to the corresponding apertures of those above and below it, forms a continuous canal; and in this canal, one of the most im-

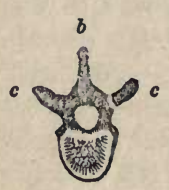

a portant parts of the nervous system, the spinal cord (commonly but erroneously termed the spinal marrow), is contained. The solid portion of the vertebra (fig. $27, a$ ) is termed its body; and the projections, $b$ and $c$, are termed its processes, the former spinous, the latter transverse. The row of spinous processes forms the ridge which we Fig. 27.-SINGLE feel passing down the back; it is seen on the
VERTEBRA. right-hand side of fig. 26 . To the transverse processes the ribs are attached. The vertebral column is expanded (as it were) at its upper extremity, to form the skull; in the large cavity which it contains, the brain is lodged; and its bones are so arranged as to give protection to the organs of sense also. At the opposite extremity we see it contracted into the tail; which is composed of a series of vertebræ 
resembling those of the back, but simpler in their form, and not possessing a cavity for the spinal cord. We commonly find that in those animals in which the skull is very large, the tail is short; and that where the tail is very long or powerful, the head is small. Thus in man and in the apes, the head is large, and there is no external appearance of a tail ; but there are some very imperfect vertebræ at the lower end of the spinal column, which constitute the rudiment of it. In the long-tailed monkeys and in the kangaroo (whose tail is like a third hindleg), the head is comparatively small. But this rule does not hold good universally.

72. The Nervous system of Vertebrated animals consists of a Brain and Spinal Cord (fig. 28), which are lodged within the skull and vertebral column; and of nervous trunks proceeding from these, which are distributed to all parts of the body. The Brain is not (as commonly reputed) a single organ, but is composed of a number of ganglionic masses, differing considerably in their functions. Thus each of the nerves of special sense (smell, sight, hearing, and taste) has its own proper centre; and there is another of considerable size, which seems to perform the same office in regard to common sensation. These are found in Vertebrata generally ; and their proportionate size corresponds with the relative development and activity of the several organs of sense with which they are connected. The bulk of the brain of Man, however, is made up by two large masses of nervous

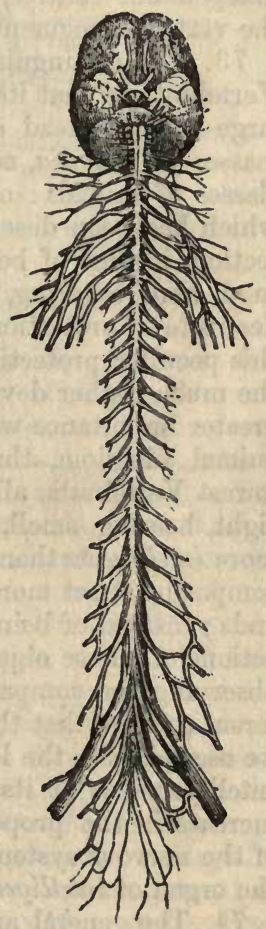
matter, which are known as the Cerebral Hemispheres; these, as will be shown hereafter (chap. x.), are so small in the brains of Fishes as to be scarcely distinguishable; and their relative size 
and complexity of structure increase as we ascend the scale, in pretty close accordance with the increase of the intelligence or reasoning faculty. There is also another large nervous mass, called the Cerebellum; the function of which seems to consist in the regulation of the more complex movements. The Spinal Cord is made up of a longitudinal succession of independent centres, of which one corresponds with each of the vertebral segments of the body.

73. The distinguishing feature of the Nervous system in Vertebrata is, that its several centres are thus united into one large mass, instead of forming a nuinber of separate small masses or ganglia, as we shall find that they do in the lower classes of animals : and that it is inclosed in the bony casing which has been described as peculiarly destined for its protection, instead of being enveloped with all the other organs in a hard covering, as in the Lobster, or of being entirely destitute of protection, as in the Slug. That it should receive this peculiar protection is quite necessary, in consequence of the much higher development which it attains, and the much greater importance which it possesses, in this division of the animal kingdom, than in any other. In all but the very lowest Vertebrata, all five kinds of sensation exist; - namely, sight, hearing, smell, taste, and touch. We find in this group more intelligence than in any other; that is to say, the animals composing it act more with a designed adaptation of means to ends; instead of being impelled by a blind instinct to perform actions of whose objects they are not aware. And we find, by observing and comparing the structure and actions of the different groups, that the intelligence gains upon the instinct, as we ascend from the lowest Fishes towards Man, in whom the intelligence is at its highest; whilst we observe a similar increase in the proportion which the brain bears to the rest of the nervous system. Hence we conclude, that the brain is the organ of intelligence, or of the reasoning faculties.

74. The general arrangement of the other organs in Vertebrated animals, is shown in fig. 29. At $m$ is seen the mouth, forming the entrance to the digestive cavity, of which the termination is at the opposite extremity of the body; $i, i$, is the intestinal canal, and $l$, the liver : these organs occupy the part of the body which is called the abdomen or belly. The mouth also opens, however, into the windpipe, or trachea, $t$, 
which conducts air into the lungs, $p$; these organs, with the heart, $h$, are contained in the portion. of the trunk called the

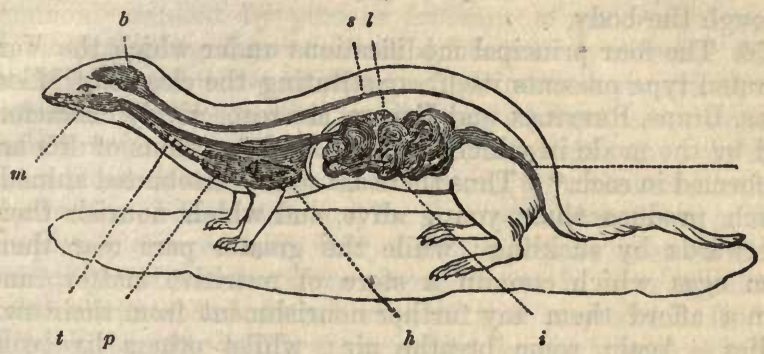

Fig. 29.-Diagram, showiNg the position of the prINcIPal ORgaNs iN VERTEBRATA.

thorax, or chest. At $b$ is seen the position of the brain; and at $s$ that of the spinal cord.

75. The foregoing characters apply, with greater or less modification as to details, to the classes of Mammals (commonly termed Quadrupeds), Birds, Reptiles, and Fishes; and these further agree in the following points, all of which, therefore, enter into our idea of a Vertebrated animal. The number of limbs or members never exceeds four; and of these, two, or even all four, may be absent. In all the classes just named, four is the general number; and the absence of two or more is the exception. Thus in Mammals, we find all four present in every tribe save that of Whales, which want the hinder pair; though the upper or anterior pair may take the form of arms, wings, legs, or fins, according to the element which the animal is formed to inhabit. In Birds we find the posterior pair invariably present in the form of legs ; whilst the anterior pair, though almost always developed into wings, is absent in a few instances. In Reptiles we find considerable variety ; all four members are present in the Turtle tribe, and in most Lizards, as well as in the Frog tribe; but they are entirely absent in the whole tribe of Serpents ; and there are Lizards which have only one pair. And in Fishes, we usually find two pairs, constituting the pectoral and ventral fins ; but one or both pairs are sometimes absent, as in the Eel, Lamprey, \&c. We have further to remark, in regard to the general characters of Vertebrated animals, that, 
with one exception, they have all red blood ( $\$ 226)$; and that they possess a complex apparatus for circulating this through the body.

76. The four principal modifications under which the Vertebrated type presents itself, constituting the classes of MAMinals, Birds, Reptiles, and Fishes, are respectively characterised by the mode in which the principal functions of life are performed in each.* Thus there are some Vertebrated animals which produce their young alive, and which nourish them afterwards by suckling; while the greater part rear them from eggs which contain a store of nutritive matter, and do not afford them any further nourishment from their own bodies. Again, some breathe air; whilst others live constantly in water, and have no direct communication with the atmosphere. Some, moreover, have the power of keeping up a high temperature, so that their bodies always feel warm to the touch; whilst the temperature of others varies with that of the atmosphere, so that their bodies give a feeling of coldness : the former are termed narm-blooded-the latter cold-blooded. There is a like difference in their mode of life; some of them being destined to live on the surface of the earth, whilst others are chiefly inhabitants of the air, and others again are the tenants of the ocean.

77. Mammats are distinguished from all other Vertebrata by the first of the characters just adverted to; being the only animals that produce their young alive, and nourish them afterwards by suckling. Like Birds and Reptiles, they breathe air by means of lungs; and, in common with Birds, they are warm-blooded and have a complete double circulation of their blood, carried on by a heart with four cavities. They are for the most part quadruped (that is, four-footed), and are destined to live upon the surface of the earth; but Man, and the Apes that approach nearest to him, are biped, having the power of walking on two limbs, and of using the others for different purposes; whilst the Bat tribe have the two arms converted into wings, which enable them to fly through the air like birds (for which the older naturalists mistook

* Many Zoologists range the Frogs and their allies in a separate class, under the name of AMPHIBIA; but when looked at from a physiological point of view, the author does not see that they require to be separated from the true Reptiles. 
them); and the Whale tribe are adapted in their general form to lead the life of fishes (among which they are still commonly ranked by persons ignorant of natural history). Notwithstanding these marked differences in external form, there is a great correspondence as to internal structure; for bats and whales, as well as ordinary quadrupeds, produce their young alive, and suckle them afterwards ; they are also warm-blooded, breathing air, and having an active circulation. The bodies of Mammals are, for the most part, more or less completely covered with hair, which serves to keep in their warmth ; and this is seldom absent, except in such as inhabit warm climates and do not require this provision. In the Whales, the same end is answered by the thick layer of oil in the substance of the skin, constituting the blubber; and Man is left to form a protective covering for his body by the exercise of his own ingenuity. The general arrangement of the

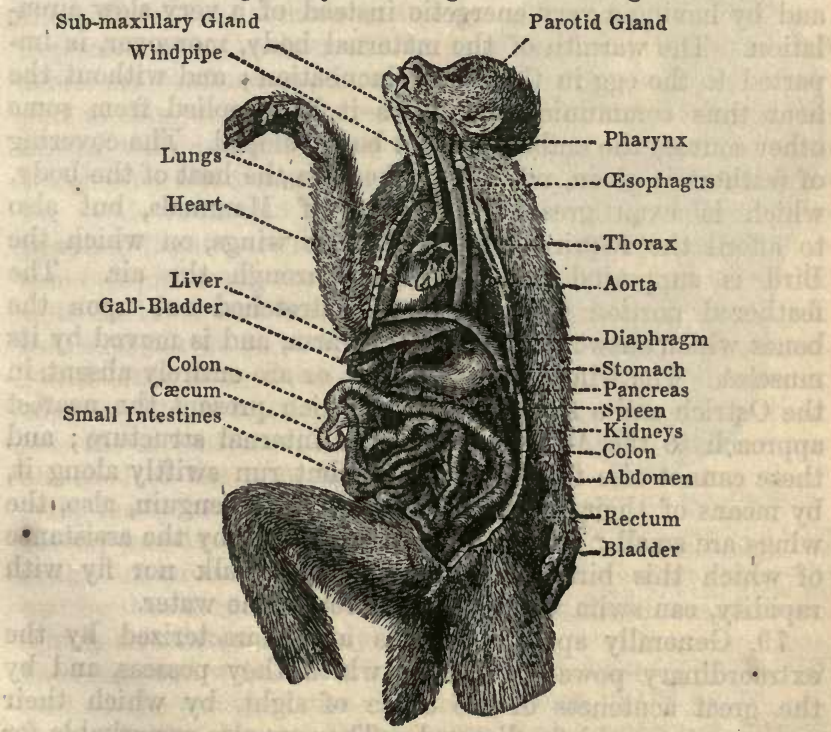

Fig. 30.-IXTERIOR OF A MONKEY.

internal organs of Mammals will be seen from the accompanying figure of the body of a Monkey, laid open in such 
a manner as to exhibit the chief of them. The cavity of the trunk is completely divided, by the muscular partition termed the diaphragm, into two portions-the thorax, containing the heart and lungs; and the abdomen, containing the digestive apparatus. It is chiefly by the alternate contraction and relaxation of this muscle, that the act of breathing is performed in Mammals, as will be explained hereafter ( $\$ 331)$.

78. In Birds there is a much closer conformity to one general plan than we find among Mammals. The covering of feathers, by which we ordinarily distinguish the members of this class, prevails universally ; and there is no wide departure from the typical form. This class belongs to the oviparous division of the Vertebrata; the young being reared from eggs. But it is distinguished from Reptiles, which are also oviparous and air-breathing, by being warm-blooded; and by having a very energetic instead of a very slow circulation. The warmth of the maternal body, moreover, is imparted to the egg in the act of incubation; and without the heat thus communicated (unless it be supplied from some other source) the embryo cannot be developed. The covering of feathers is given, not only to keep in the heat of the body, which is even greater than that of Mammals, but also to afford the required surface for the wings, on which the Bird is supported and propelled through the air. The feathered portion of the wings is stretched out upon the bones which answer to those of our arm, and is moved by its muscles. The wings are very small, or are entirely absent, in the Ostrich and a few other birds, which present the nearest approach to the Mammalia in their internal structure; and these cannot rise from the ground, but run swiftly along it, by means of their powerful legs. In the Penguin, also, the wings are small; and they are used as fins, by the assistanee of which this bird, which can neither walk nor fly with rapidity, can swim very quickly through the water.

79. Generally speaking, Birds are characterized by the extraordinary power of motion which they possess, and by the great acuteness of the sense of sight, by which their movements are chiefly directed. They are also remarkable for their instinctive actions, which are chiefly related to their care of their young, for whom they usually construct a protective 
nest. The nutritive functions are performed with extraordinary activity in Birds, that the means may be supplied for the maintenance of their locomotive activity. Their blood is particularly rich in red particles, and its heat is usually considerably above that of Mammals. Its circulation is very energetically carried on; and although the lungs themselves are constructed upon a type inferior to that of Mammals, and the mechanism of respiration is less complete, yet, by an extension of the respiratory organs through the whole fabric, the aeration of the blood is carried on with unequalled energy ( $\$ 326)$.

80. The arrangement of the organs contained in the cavity of the trunk of Birds differs from that which has been described in Mammals, chiefly in this, - that there is usually no diaphragm to separate the chest from the abdomen, and that although the lungs themselves are confined to the upper part of this cavity, they are connected with a series of air-sacs which are distributed through the whole of it. In the aceompanying figure, which represents the internal organs of the Ostrich, the heart is seen at $a$, the stomach at $b$, and the intestinal tube at $c$. The windpipe, $d$, opens into the lungs, $e$, which are themselves small, and are attached to the ribs, instead of lying freely in the cavity of the chest; but the space they would otherwise have occupied is filled up by the large air-cells, $f, f$, which communicate freely with

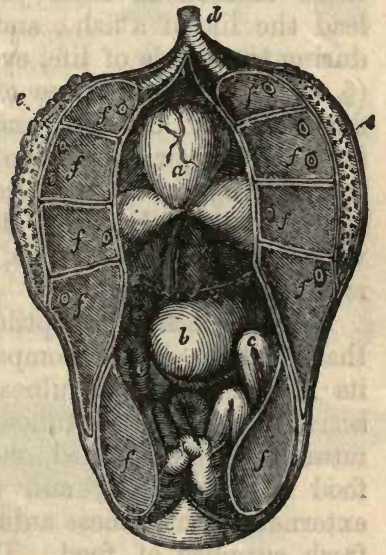

Fig. 31.-LUNGs of THE Ostrich.

$a$, the heart; $b$, the stomach ; $c c$, the intestines; $d$, the trachea; $e$, the lungs; $f f f$, air-cells, in which are also seen the tubes by which these aircells communicate with the lings. the lungs and with each other, and which even occupy a large part of the cavity of the abdomen, as seen in the figure.

81. In the class of REPTILES we find a variety of form so remarkable, that, if we were influenced by this alone, we should scarcely regard the animals it contains as belonging to 
the same group; yet the structure of the internal organs, on which classification is founded, is essentially alike in all; and their physiological condition presents no important dissimilarity. Four obviously different tribes, Turtles, Lizards, Serpents, and Frogs, are brought together by the following characters. They are all oviparous, in this respect agreeing with Birds and Fishes ; but they are cold-blooded, and have not a complete apparatus for the double circulation of the blood, in which respect they differ from Birds; and they breathe air by means of lungs, instead of breathing water by gills, in which respect they differ from Fishes. But by the lowest group, that of Frogs and their allies, this class is united to that of Fishes in a most remarkable manner; for these animals in their young state breathe by gills, and lead the life of a fish; and some of them retain their gills during the whole of life, even after the lungs are developed ( $\S 87)$. The first three of the tribes just mentioned undergo no such change : and they further agree in this, that they breathe air during the whole of their lives, coming forth from the egg in the same condition as that in which they are subsequently to live, and also in having their bodies covered with horny scales or plates, whilst the skin of the Frog tribe is soft and unprotected.

82. The class of Reptiles presents a marked contrast to that of Birds, in the comparative slowness and feebleness of its movements, the dulness of its sensibility, and the inactivity of its organic functions. As there is no fixed temperature to be maintained, one important source of demand for food is withdrawn; and when not excited to activity by external warmth, these animals may pass long periods without fresh supplies of food. Their blood is very poor in red corpuscles, and its circulation is comparatively languid. A reduction of the temperature of their bodies to within a few degrees of freezing point, induces complete torpidity, which continues until they are roused by a renewal of warmth.

83. The Turtle tribe is peculiarly distinguished by the inclosure of the body in a bony covering; of which the upper arched portion (termed the carapace) is formed by the coalescence of the ribs with a set of bony plates developed in the substance of the skin; whilst the lower flat plate (termed the plastron), which is often incomplete, is 
formed by an expansion of the sternum or breast-bone, which is spread out sideways, instead of being raised into a projecting keel as in Birds. The carapace and plastron are covered with large horny plates, variously arranged in the different species, and constituting what is commonly called tortoise-shell. These plates are often very beautifully disposed, forming a kind of tesselated pavement; as in the common Tortoise (fig. 32), which is often preserved alive in our gardens.

84. In the tribe of Lizards, the body has no such covering; but these animals, having more activity than the tortoises (which are proverbially slow), are enabled

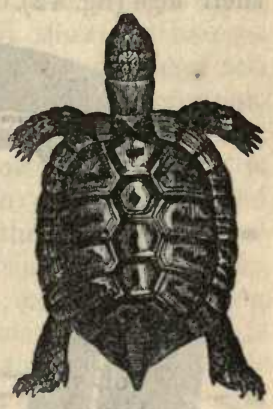

Fig. 32.-TORTOISE. to make their escape from danger, whilst the latter are obliged to trust to their bony casing for protection from it. In their general form, Lizards approach Mammals, being four-footed, and living for the most part on land; but they differ from them not only in their essential reptilian characters, but also in several others of less consequence. Their bodies are usually covered with scales, which lap over one another like the tiles of a roof; but in the Crocodile tribe, many parts of

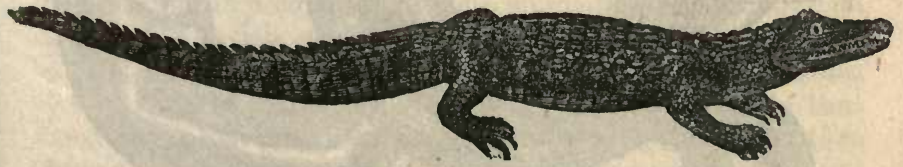

Fig. 33.-Crocodile.

the surface are covered with large knotted horny plates, that meet at their edges like the scales of tortoise-shell, and afford an almost impenetrable covering. Although some of the Lizard tribe spend a large part of their time in water, they all breathe air; but, as their respiration is very inactive, they can remain for long periods beneath the surface, without being obliged to come up to breathe.

85. The tribe of Serpents may be regarded as lizards without feet; their spinal column is immensely prolonged; and 
their ribs are also very numerous; and they are able to crawl upon the points of these, using them almost as Centipedes do their legs (fig. 42). But in general the movement of their

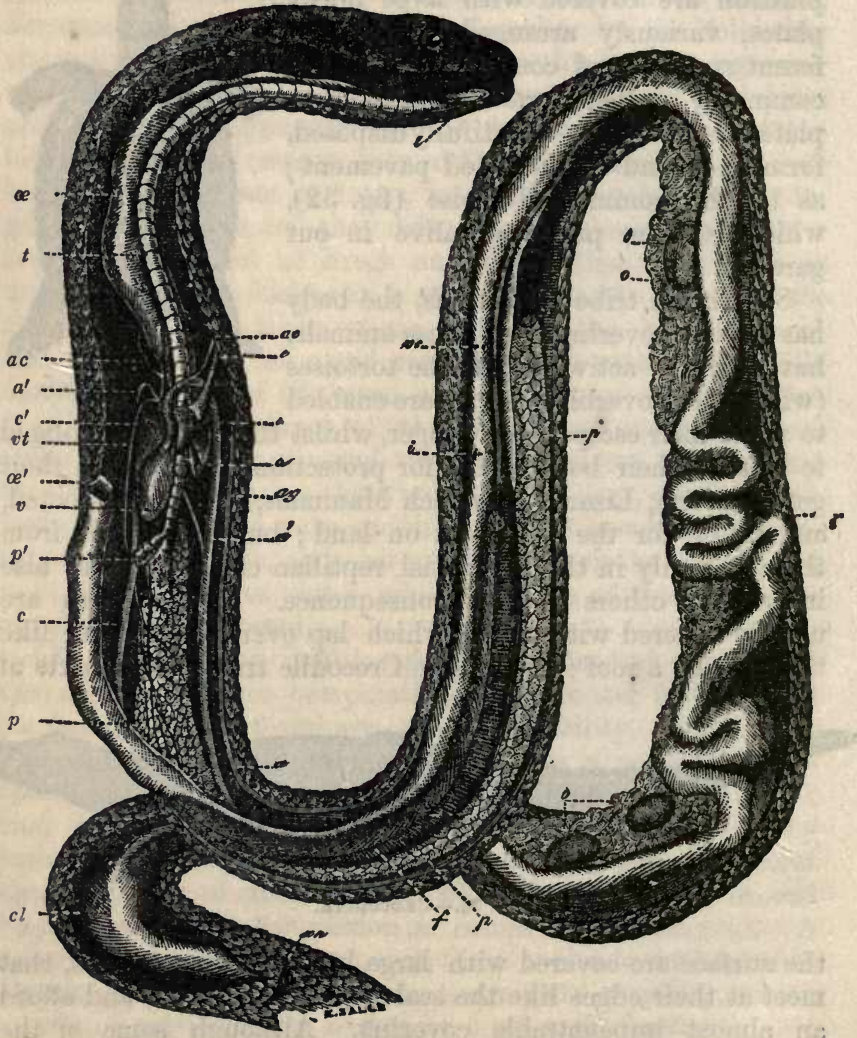

Fig. 34.--ANatomi of a Coluber

bodies is accomplished by their being drawn-up into folds; and then straightened so as to project the head. The prolonged form of the body in Serpents occasions a curious variation in the arrangement of the principal organs, which is shown in the accompanying figure. The csophagus or 
gullet, $\infty$, which leads from the mouth to the stomach, is a long and very wide canal, being even larger than the stomach at its commencement; a portion of it is removed at oe', in order to show the heart, \&c., which would otherwise be concealed by it. The stomach, $i$, is long and narrow; and the intestinal tube, $i^{\prime}$, after making a few turns or convolutions, passes backwards in a straight line, to terminate in the cloaca, $c l$, which opens externally by the orifice, an. The liver, $f$, is also much lengthened. From the mouth also proceeds the long windpipe, $t t$, which conveys air to the lungs, or rather to the single lung; for the lung on the left side, $p^{\prime}$, is scarcely at all developed, whilst that on the right, $p$, extends along a great part of the body. At $o$ is seen the ovarium, in which the eggs, $o^{\prime} o^{\prime}$, are produced; and this also is very much lengthened, extending from the cloaca a good way up the body, so as nearly to meet the lung. The other references are to the parts of the heart, and the principal vessels; the structure and arrangement of which will be explained hereafter ( $(284)$.

86. The Batrachia, or animals of the Frog tribe, are readily distinguished from all the preceding, by their soft naked skins ; even when the form of the body, as in the common Salamander or Water-Newt, resembles that of the lizards. They are also remarkable for the metamorphosis which they undergo in the early part of their lives; for they come forth from the egg in a condition which is, in all essential particulars, that of a fish, and undergo a gradual series of changes, by which their form and structure become assimilated to those of the true reptiles. This change is most complete in the Frogs and Toads; the early form of which is known as the tadpole. The principal stages of this change are represented in figs. 35 to 39 ; in which, however, the relative sizes are not preserved, the tadpoles being much larger in proportion (for the sake of displaying their form and the gradual development of their legs) than the complete frog. Soon after the young tadpole has come forth from the egg, it presents the form which is shown in fig. 35 ; its head and trunk are large, and the latter is prolonged into a flattened. tail, by which the little animal swims freely through the water. There is not the least appearance of limbs or members. It breathes by gills, which are long fringes, hanging 
loosely in the water on either side of the head. At a later period, however, these gills, which are merely temporary, disappear; and the breathing is carried on by another set, which are situated behind the head, and are covered in by a fold of skin; the water gains access to these by passing through the mouth, exactly as in Fishes. The form is then that which is represented in fig. 36 . In a short time afterwards, the animal still breathing by its gills, the hind-legs begin to sprout forth, as it were, at the base of the tail; this

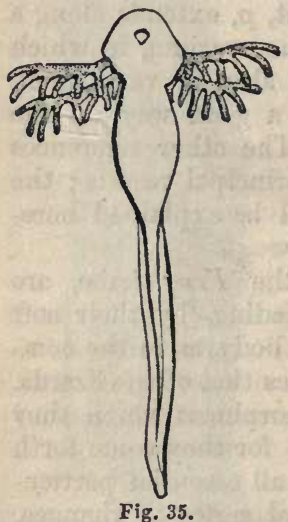

Fig. 35.

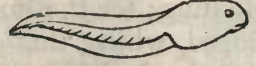

Fig. 36.

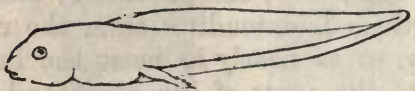

Fig. 37.

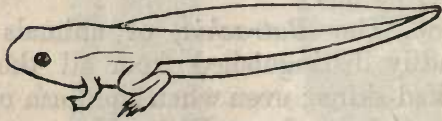

Fig. 38.
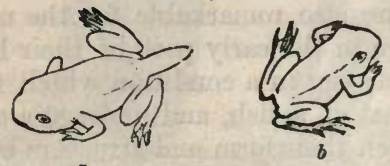

Fig. 39 .

stage is shown in fig. 37. At a still later period, the forelegs begin to be developed, as seen in fig. 38 ; and from that time they are nourished at the expense of the tail, which gradually disappears, as seen in fig. $39, a, b$. During this period, other important changes are taking place in the interior of the body; the chief of which are the development of the lungs and the gradual disuse of the gills, so that the animal becomes fitted to live on land and breathe air, and is no longer capable of remaining long under water without coming to the surface to respire.

87. The metamorphosis in other members of the group is 
less complete than in the Frog, being checked at a less advanced stage. Thus in the common Water-Newt, the tail is retained during the whole of life, and the animal continues to be an inhabitant of the water, though breathing air alone. There are some very curious animals, however, in which the change is stopped, as it were, at a much earlier period, so that the gills also are retained; and in these, the lungs are suffciently developed to enable the animals to breathe air, so that they can live either on land or in water. Such Batrachia are scientifically known as perennibranchiate, this term expressing the persistency of their gills. In fig. 40 is represented

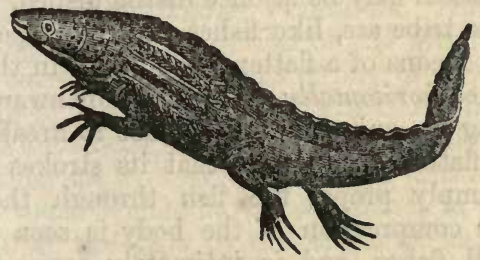

Fig. 40.-Aхоцотц.

an animal of this kind, the Axolotl, which inhabits some of the lakes of Mexico. And in fig. 41 is shown the form of a still more remarkable animal, the Lepidosiren, or mud-fish, recently

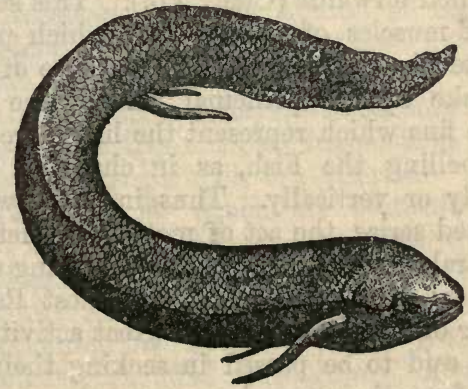

Fig. 41.-Lepidosiren.

brought from th3 rivers of Africa, the metamorphosis of which appears to be checked at a still earlier period, so that it is very difficult to decide whether it should be regarded as 
a Fish or as a Reptile, so complete is the mixture of characters which it presents.

88. The class of Fishes is distinguished from all other Vertebrata, by the adaptation of the animals composing it to breathe by means of water in their adult state, so as to be capable of living in that element only. Like Reptiles, they are oviparous and cold-blooded; and in these characters they differ completely from the Whales and other Mammals, which are, like them, inhabitants of the great deep, but which are warm-blooded, viviparous, and air-breathing animals. There is a simple external character, by which the members of the two classes may be at once distinguished. The animals of the Whale tribe are, like fishes, chiefiy propelled through the water by means of a flattened tail ; but in the former the tail is flattened horizontally, so that its downward stroke may serve to bring the animal to the surface to breathe; whilst in Fishes it is flattened vertically, that its strokes from side to side may simply propel the fish through the water. A flattening or compression of the body is seen more or less in almost all fishes, and is intimately connected with the nature of their motion through the element they inhabit ; as it serves the double purpose of diminishing the resistance which is offered to their progress, and of increasing the extent of the oar-like surface, by the lateral stroke of which the body is propelled forwards (Chap. xII.). This stroke is given by a series of muscles of great power, which pass from the prolonged extensions of one vertebra to those of another, and altogether make up the principal part of the bulk of the animal. The fins which represent the limbs are not so much used in propelling the Fish, as in changing its direction either laterally or vertically. Thus in the lowest group of the Vertebrated series, the act of motion is chiefly performed by the vertebral column itself, instead of being committed to the limbs, as in Mammals, Birds, and most Reptiles. The larger number of Fishes swim with great activity ; and their lives may be said to be passed in seeking their subsistence and in flying from their enemies.

89. Fishes are for the most part very voracious, and their food consists in great part of the members of their own class. In seeking it, they appear to be chiefly guided by the sight; for their eyes are usually large and highly developed, while 
the other organs of sense are formed upon a very inferior type. They swallow it without much division in the mouth; but it seems to undergo rapid digestion. The blood of some Fish, whose muscular activity is peculiarly great, is rich in red corpuscles, and of a temperature not much lower than that of Mammals; but, generally speaking, it contains much less solid matter than that of the warm-blooded Vertebrata, and its temperature follows that of the surrounding medium.

90. Although Fishes breathe by gills instead of by lungs, these gills are connected with the mouth, so that the water which passes over them is received into it, in the same manner as the air is in the higher Vertebrata. This is a character which distinguishes the position of the gills of fishes from that of the corresponding organs of any of the inferior tribes. They are lodged in a cavity on each side of the throat; and this cavity opens outwardly, either by one large valve-like aperture on either side, or by several; through these apertures the streams of water which have been taken in by the mouth, and forced over the gills by the action of its muscles, make their exit.

91. All Fishes are oviparous; and the number of eggs which they produce is generally prodigious. It is very seldom that after the eggs have been deposited and fertilized, the parents take any further concern in regard to them; though there are a few instances in which a kind of nest is made, and others in which the egg is retained and hatched within the body, so that the young comes forth alive. This last is the case with the Sharks and Rays, which, notwithstanding that their skeleton is cartilaginous, are higher than Fishes generally in several other parts of their organization.

92. All the animals which are destitute of a vertebral column are called Invertebrata; and this division into the Vertebrated and Invertebrated groups was formerly regarded as the first step in the classification of the animal kingdom. But it was pointed out by Cuvier, that in the Invertebrated division are comprehended three groups, of which the members differ as much from one another as they do from Vertebrated animals; and that each of these ought, therefore, to rank with the first, as a primary division. This is evident to those who are but slightly acquainted with the structure of the animals already named $(\S 69)$ as characteristic speci- 
mens of these divisions; and it will become more apparent as we proceed.

93. In the second division, that of Articulata, or Articulated (jointed) animals, we find a conformation very different from that which has been just described. The exterior of the body is still perfectly symmetrical, as in the Vertebrata; and the interior is even more symmetrical; for the organs that represent the heart and lungs are equally disposed on the two sides of the central line of the body. But the skeleton, instead of being internal, is external; and is composed of a series of pieces jointed together, which form a casing that includes the whole body. In general, these pieces are very similar to each other; so that the whole body appears like the repetition of a number of similar parts, as we see in the Centipede (fig. 42). The limbs are usually very numerous, where they exist at all ; and they have a jointed covering, like that of the body. But in the lower tribes of this group, such as Leeches and Worms, the limbs or members are but slightly developed, or are altogether absent; and in the highest, which approach most nearly to the Vertebrata in their general organization, the number of members is much reduced,-although it is never less than six. The hard matter of which the Fig. 42. external skeleton is composed, undergoes little CENTIPEDE. or no change when it is once fully formed ; and, in order to accommodate it to the increasing size of the animal, this covering is thrown off and renewed at intervals during the period of growth.

94. The nervous system consists of a series of separate ganglia, which are arranged in a cord or chain along the central line of the body. There is usually a pair of large ganglia in the head, bearing a resemblance (in their peculiar connexion with the eyes) to the ganglionic centres of the optic nerves in Vertebrata; and there is commonly one for each segment or division of the body, from which the nerves pass to supply its muscles, as they do from the spinal cord of Vertebrata. The cord which connects these ganglia is double, 
and the ganglia themselves are composed of two halves, which have little connexion with each other. The chain thus formed (fig. 43) passes along the under-side of the trunk of the animal (as seen at $g$, fig. 44), not on what seems its back; and by the presence of this double chain of ganglia an Articulated animal may bo distinguished, even when, in its general structure, it should seem to belong to the group of Mollusea ( $\$ 102$ ).

95. The general arrangement of the organs in the Articulata is shown in the accompanying figure of a Crayfish. The mouth, situated on a projecting head, opens into $s$, the stomach, from which passes backwards the intestinal tube, $i, i$, to terminate at the

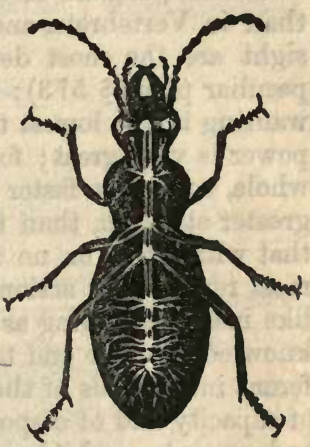

Fig. 43.-Nervous SysteM OF AN INSECT. opposite extremity of the body. The upper part of the tube is surrounded by the liver, $f$, which is here very large. In the head are seen the ganglia, $c$; and along the under-side of the body is seen the chain of ganglia,

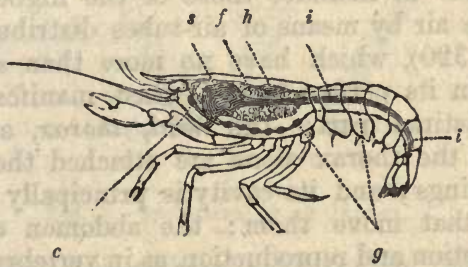

Fig. 44.-Diagran sHowing THE position of the PHINCIPAL ORgans in the Articulata.

g. The blood is nearly colourless, and is usually impelled through the body not by a single organ or heart, but by a succession of contractile cavities, one for each segment, which open into one other longitudinally, forming what is known as the dorsal vessel; in the Cray-fish and its allies, however, one part of this, $h$, is specially enlarged, so as in great degree to serve as a heart for the system generally. The respiratory organs are not connected with the mouth; and are not usually 
restricted to one part of the body, but are diffused either on its outside or through its substance.

96. The organs of sense, in this group, are less numerous than in Vertebrata, and are inferior in perfection; those of sight are the most developed, and are formed upon a very peculiar plan ( $\$ 573)$; but all organs of special sense appear wanting in the lowest tribes. Yet we find that the muscular power is very great; for the animals of this group, taken as a whole, can move faster in proportion to their size, and possess greater strength, than those of any other. We observe, too, that with little or no intelligence, they are prompted to the most remarkable actions by instinct alone. They seem to act like machines, doing as they are prompted, without choice, or knowledge of the end to be gained ; and consequently the different individuals of the same species have not that difference of capacity and of disposition, which we see in animals whose endowments are higher.

97. In the highest division of the Articulated series, we easily recognise, as forms quite distinct from each other, the Insects, the Spiders, the Crustaceous animals (crabs, lobsters, \&c.), and the Centipedes. The class of Insects is distinguished, for the most part, by the presence of wings; but to this there are exceptions. It includes those of the higher Articulata, which breathe air by means of air-tubes distributed through the body ( $\$ 320)$, which have no more than six legs, and whose body, in its perfect form at least, manifests a division into three distinct parts-the head, thorax, and abdomen (fig. 45). To the thorax alone are attached the six legs, as well as the wings; and its cavity is principally occupied by the muscles that move them: the abdomen contains the organs of digestion and reproduction, as in vertebrated animals. In the greater part of this class, the young animal comes forth

- from the egg in a condition very different from that which it is ultimately to possess; and it undergoes a complete metamorphosis, the larva which the egg produces bearing a close resemblance in form to the lower Articulata, and only attaining the condition of the imago or perfect insect by passing again into a state of inactivity, during which the store of nutriment which it has acquired is applied to the development of new organs. This pupa or chrysalis condition may be considered as a sort of postponed completion of the embryonic 
life, which was interrupted at a very early period. In some tribes, however, the general form is the same from the first, and the wings are the only parts deficient; these gradually

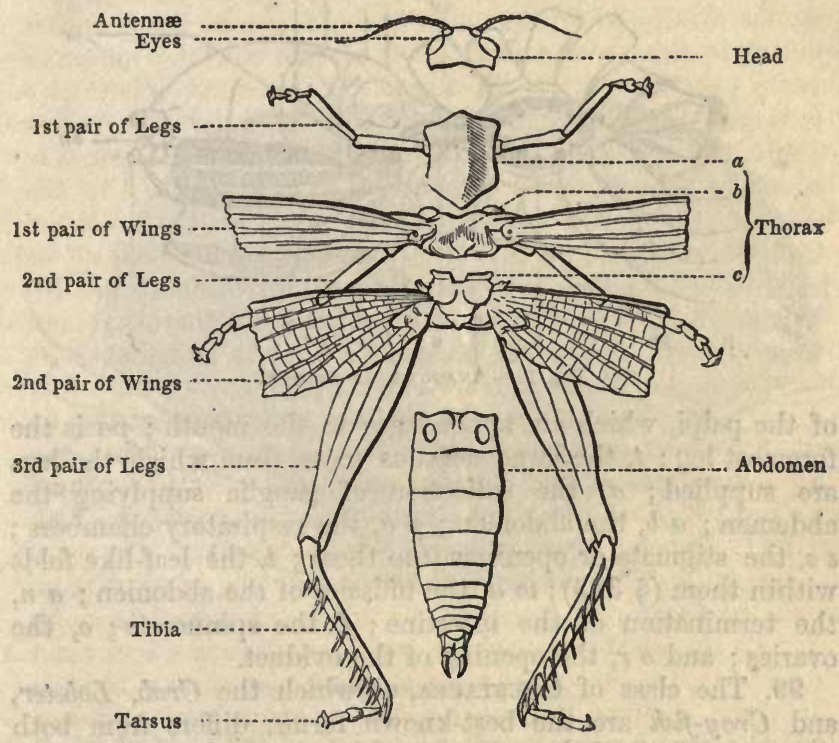

Fig. 45.-SKELETON OF AN INSECT.

make their appearance, and the insect is then complete. Such is the case with the Grasshopper and Cricket; and a change of this kind is termed an incomplete metamorphosis.

98. The animals of the class ARACHNIDA, which includes the spiders, scorpions, and mites, are, like Insects, articulated, breathing air, and possessing legs, but the number of these legs is never less than eight; there is an entire absence of wings, and the head is united with the thorax, so that the body seems to be formed of two principal divisions, - the cephalo-thorax (as it is termed), and the abdomen. In fig. 46 we have a representation of the arrangement of the parts con. 
tained in these cavities. At $c t$ is seen the cephalo-thorax opened from below, and giving attachment to the legs; at $m$ is shown the place of the mandibles or jaws; at $p$ is seen one

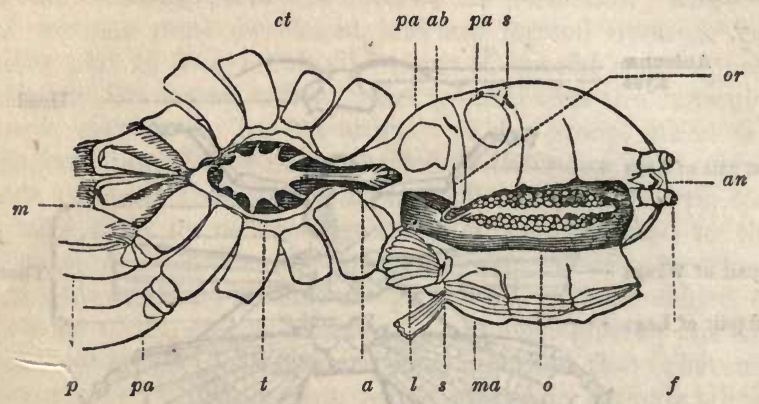

Fig. 46.-ANATOMX of SPIDER.

of the palpi, which are appendages to the mouth; $p a$ is the foremost leg; $t$, the large nervous mass, from which the legs are supplied; $a$, the collection of ganglia supplying the abdomen ; $a b$, the abdomen ; $p a$, the respiratory chambers ; $s s$, the stigmata or openings into these; $l$, the leaf-like folds within them ( $\$ 323) ; m a$, the muscles of the abdomen; $a n$, the termination of the intestine; $f$, the spinnerets; $o$, the ovaries; and $o r$, the opening of the oviduct.

99. The class of Crustacea, of which the Crab, Lobster, and Cray-fish are the best-known forms, differs from both the preceding, in being adapted to breathe by means of gills, and thus to reside in or near water, instead of inhabiting the air. Moreover, the body is inclosed in a hard covering, which generally contains a good deal of carbonate of lime, and which is thrown off at regular intervals. This covering also incloses the members, which are never less than ten in number, and are frequently more numerous. There is great variety of form among the animals of this group, which is altogether one of great interest.-In the Crab tribe, the head, thorax, and abdomen are all drawn together, as it were, into one mass ; and the general arrangement of the organs it contains is exhibited in the succeeding figure, which shows them nearly as they are found to lie, when the upper part of the shell, or carapace, is removed. At $t$ there is left a portion of 
the membrane which lines the carapace and covers in the viscera. On the central line, at $c$, is seen the heart, which in the Crustacea is large and powerful in its action; from it there passes forwards the artery $a 0$, which supplies the eyes

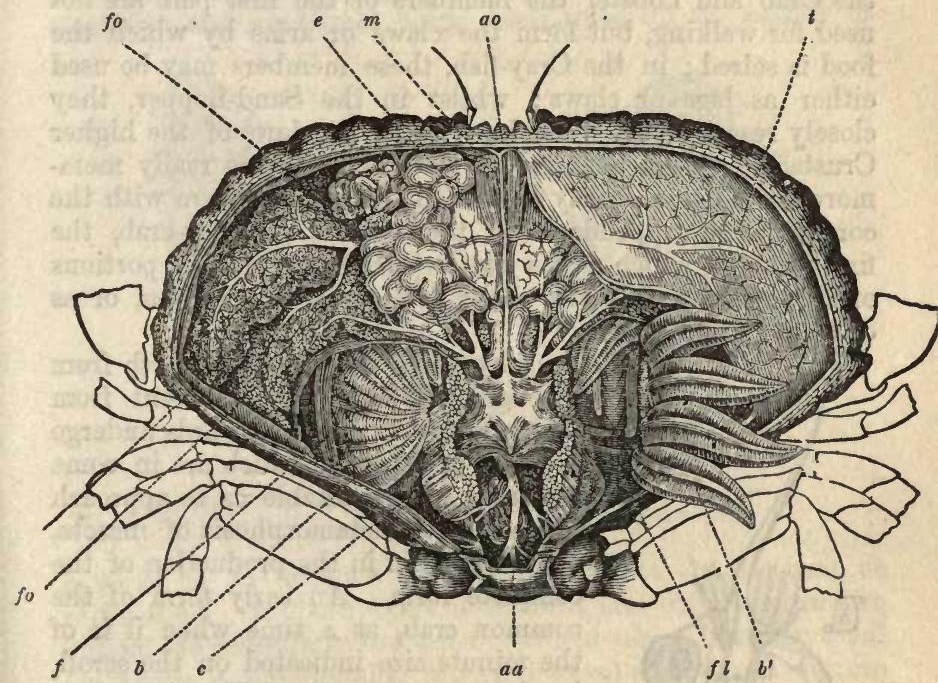

Fig. 47.-ANatomy or A Cina.

and the front of the body; whilst the artery $a a$ passes to the lower and hinder parts; at $b$ are seen the gills of the left side in their natural position; whilst at $b^{\prime}$ are seen those of the right side, turned back to show their under-surface, and to disclose the lower portion of the shell, $f l$. At $e$ is seen the stomach, situated close behind the mouth; and at $m$ are pointed out its powerful muscles, by the action of which the food is ground down. The bulky ovary is seen on either side of the stomach; and the space between this and the edge of the shell is occupied by the very large liver, $f o$.

100. In most of the Crustacea, however, the body is more: prolonged. In some, as the Lobster, there is an indication of a division of the body into three parts, representing the head, thorax, and abdomen of insects ; whilst in others, as the Sand- 
hopper, the rings or segments are almost as similar to each other as they are in the centipede tribe. There is no class in which we find the same parts exhibiting so great a variety of forms, and rendered subservient to so many uses. Thus in the Crab and Lobster the members of the first pair are not used for walking, but form the claws or arms by which the food is seized; in the Cray-fish, these members may be used either as legs or claws; whilst in the Sand-hopper, they closely resemble the other legs. And the jaws of the higher Crustacea, of which there are several pairs, are really metamorphosed legs; as may be seen by comparing them with the corresponding appendages of the Limulus or king-crab, the first joints of which act as jaws, whilst the remaining portions of these members serve either as legs for locomotion, or as claws for prehension.

101. Most of the Crustacea, like insects, come forth from

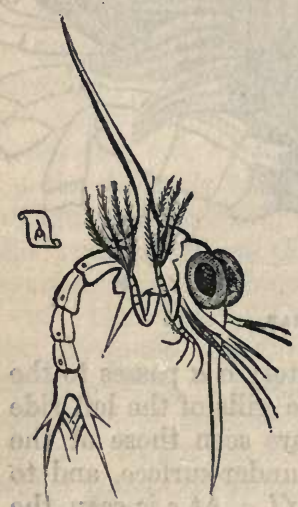
the egg in a state very different from their adult form ; and afterwards undergo a series of changes, which are in some instances so remarkable as to approach the complete metamorphosis of insects, and which end in the production of the complete form. An early form of the common crab, at a time when it is of the minute size indicated on the scroll, is shown in fig. 48. The immature Crustacea of different tribes bear much more resemblance to each other, than do the forms into which they are ultimately to be developed; and the differences they afterwards present are chiefly due to a variety in the amount

Fig. 48. - ZOEA, OR LARVAL
FORM OF THE CRAB. undergo.

102. It is one of the most remarkable results of modern zoological research, that in immediate connexion with the class of Crustacea, if not as actual members of it, we have to place a group of animals which were for some time associated with the Mollusca; their bodies being inclosed in shells, which do not fit closely around them, nor give more than a general protection to their members. This group is 
the Barnacle tribe, forming the class CIRRHIPEDA, or tendrilfooted animals. They agree with the lower Mollusca, in being fixed to one spot during all but the earliest period of their lives; the shell being sometimes attached by a long nembranous or leathery tube, as that of the Barnacle (fig. 49); and sometimes being itself fixed on the surface of

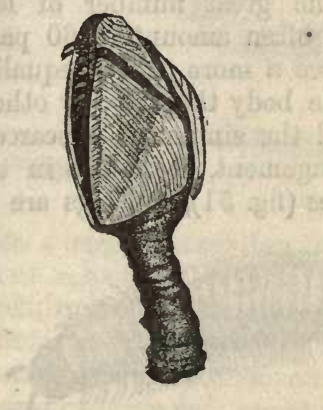

Fig. 49. - SHELL OF THE BARNACLE.

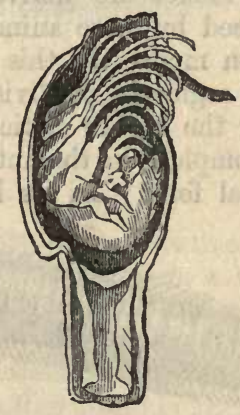

Fig. 50.-BODY OF THE BARNACLE.

a rock, or on another shell, as is that of the Balanus or acorn-shell. In both cases, the form and structure of the animal are essentially the same. When taken from the shell (in which it lies doubled up, as it were) and spread out, its articulated nature is evidenced by its division into segments, and by the regularity of the arrangement of their tendril-like appendages. These are not formad like legs, since they could be made no use of, the animal being incapable of moving from place to place; but they serve to produce currents in the surrounding water, by which food is brought to the mouth, and the blood is submitted to the influence of a fresh supply of air. The nervous system of this group is formed precisely upon the plan of that of the Articulata generally (\$94): and if any doubt could have remained as to its true place in the series, it is removed by the knowledge of the fact, that the animals composing it bear a strong resemblance in their early condition to some of the lower Crustacea, possessing eyes and legs, and swimming freely about; and that they attain their adult form by passing 
through a series of metamorphoses, in which they lose their eyes and legs, and become fixed for the remainder of their lives.

103. We now pass back to another class of the higher group of Articulata, adapted to breathe air and to inhabit the land,-the Mrriapoda or Centipede tribe (fig. 42). Botb these names are derived from the great number of legs possessed by these animals, which often amount to 60 pairs or even more. In this class we see a more perfect equality of the segments or divisions of the body than in any others among the higher Articulata; and the similarity is scarcely less complete in the internal arrangement, than it is in the external form. In its lower tribes (fig. 51), the legs are so

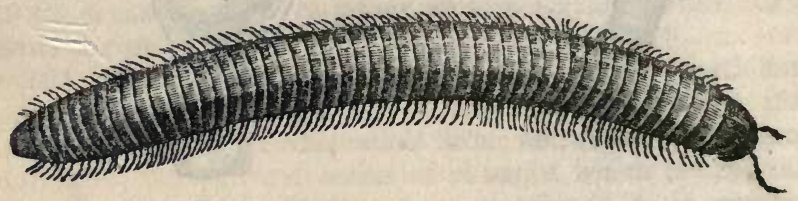

Fig. 51.-Iunus.

weak as scarcely to be able to sustain the body, which moves, therefore, partly in the manner of that of a worm. The animals of this class undergo no proper metamorphosis; but there is a considerable addition to the number of their segments and legs after they have come forth from the egg.

104. We now pass to the lower division of Articulata, in which the body possesses no jointed members ; and the animals belonging to this group are for the most part included in the class of ANNELIDA, the Leech and Worm tribe. We here find the body enveloped, - not in a hard casing, formed of distinct pieces united by a flexible membrane,-but in a skin which is altogether flexible, and which gives little indication of a division into segments. This class includes several distinct tribes, which all agree, however, in the long worm-like form of the body, and in the similarity of the different ganglia or their nervous system. The Earth-worm and its allies are adapted to live on land and to breathe air; but the greater number of Annelids are purely.aquatic; and these breathe by gills, which form tufts that are disposed on various parts of the body. In the Nereis, or Sea-centipede (fig. 52), these 
tufts are arranged regularly on the several segments, and the animal can swim by the motion that it gives them; besides these, it has a kind of bristle-shaped appendage, that seems

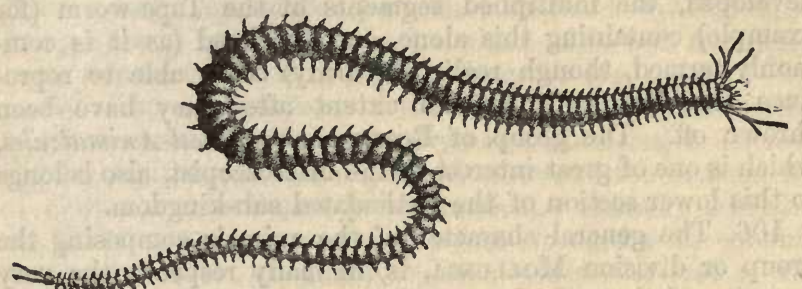

Fig. 52.-NEREIs.

like a rudimentary leg, which assists it in crawling. But there are others of these marine-worms, that form a tubular shell, in which they reside during the greatest part of their lives; and in these the gills, if disposed along the body, would have been removed from the access of water ; they are therefore arranged round the head, often forming (as in the Serpuloe, fig. 145) tufts of great brilliancy and elegance.

105. Below the Annelida are other worm-like tribes of yet greater simplicity of conformation, but still presenting the same general plan of structure. Of one of these the common Leech may be taken as an example; of another, the Tape-

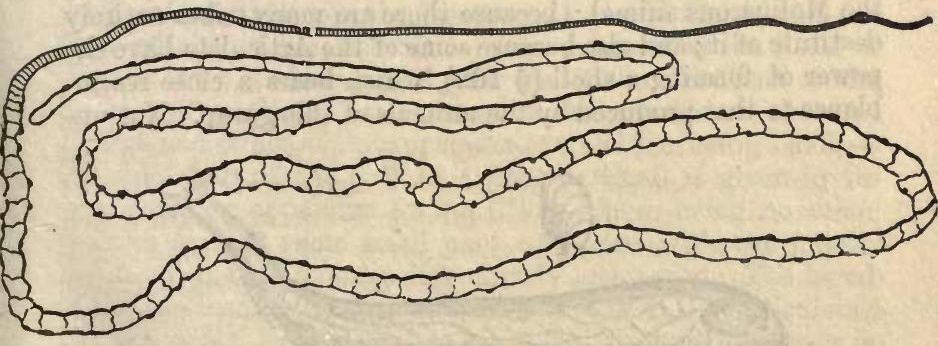

Fig. 53.-TAPE-พORM.

worm (fig. 53). This last belongs to a group termed ENTOZOA, from the circumstance that they inhabit the bodies of other animals. They are remarkable for the very low development of their digestive apparatus, - their nourishment being appa- 
rently imbibed through the whole surface of their bodies from the juices in the midst of which they live; whilst, on the other hand, their reproductive apparatus is enormously developed, the multiplied segments of the Tape-worm (for example) containing this alone, and the head (as it is commonly termed, though really the body) being able to reproduce these to an indefinite extent after they have been thrown off. The group of Rotifera, or Wheel-Animalcules, which is one of great interest to the Microscopist, also belongs to this lower section of the Articulated sub-kingdom.

106. The general character of the animals composing the group or division Moluusca, is, in many respects, the very opposite of that which prevails in the Articulated animals. The body is soft (whence the name of the group is derived), neither possessing an internal skeleton, nor any proper external skeleton. In some of the most characteristic specimens of the group, such as the Slug, there is no hard frame-work or skeleton whatever, the body being alike destitute of support and protection. In most Mollusks, however, the body has the power of forming a shelly covering, which serves for its protection; but this does not give any assistance in its movements by affording fixed points for the attachment of the muscles ; in fact, when the animal puts itself in motion, it is obliged to make its locomotive organs project beyond the shell. We must not regard the shell as an essential part of the Molluscous animal ; because there are many tribes entirely destitute of it; and also because some of the Articulata have the power of forming a shell ( $\$ 102)$, which bears a close resemblance to that produced by the animals of this group. Not un-

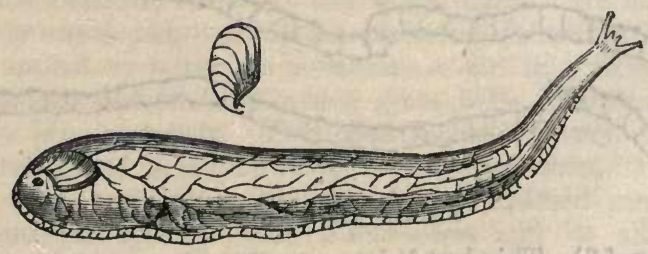

Fig. 54.-TESTACELIA.

frequently we see that, of two animals whose general structure is almost exactly the same,-as that of the Snail and Slug,- 
one possesses a shell into which it can withdraw its whole body for the sake of protection, whilst the other has none; and several intermediate forms exist; in which the shell bears a larger or smaller proportion to the body, sometimes being able to contain nearly the whole of it, and sometimes being a mere rudiment, as in the Testacella (fig. 54).

107. The external form of the body of Mollusks is subject to great variation; and generally has a good deal to do with the degree in which the organs of sense and the instruments of motion are developed in the particular animal. For these are almost always symmetrical, being arranged with equality on the two sides of a middle line; whilst the rest of the body, containing the organs of nutrition, is often unequal on the two sides. But in the lower Mollusca, which have little or no power of moving from place to place, even this degree of symmetry is altogether lost. Few of the Mollusca have any powers of active movement; in fact, the term sluggishness, derived from a characteristic member of the group, very well expresses their general habit. The Gasteropods, which may be regarded as the types of the whole series, crawl upon a fleshy disk, by the successive contractions and relaxations of which they advance slowly along the surface over which they move ; this kind of action is easily studied, by causing a Snail or Slug to crawl upon a piece of glass, and by looking through this at the under side of its foot. Hence, there is a great contrast between the inertness of the Mollusca, and the high activity of the Articulata. This contrast shows itself in the structure of their bodies ; for whilst the chief part of the interior of an Insect is made up of the muscles which move its legs and wings, - the apparatus of nutrition being small,the chief part of the bulk of a Slug or Snail is given by its very complex apparatus for nutrition-there being no other muscles (except some small ones connected with the mouth and head) than the fleshy disk already mentioned. The blood of the Mollusca is nearly colourless, as it is in the Articulata; but the organ by which it is circulated through the bolly is much more powerful and complete, bearing more resemblance to the heart of Vertebrated animals. The skin is usually thick and spongy in its texture; having muscular fibres interwoven in its substance, so that it can contract or extend itself in any part; and having the power of exuding shelly matter 
from its surface, in those species which form such a protection. This envelope, which is called the mantle, is very loosely applied round the parts which it contains; and it frequently extends itself into folds or duplicatures, which wrap round the gills, and sometimes meet and adhere so as to inclose them within a cavity of their own. In the Cuttlefish, the water within this cavity is renewed from time to time by the muscular movements of its walls; but usually a current of fluid is kept up over the surface of the gills, by the action of the cilia ( $\$ 45)$ with which they are covered.

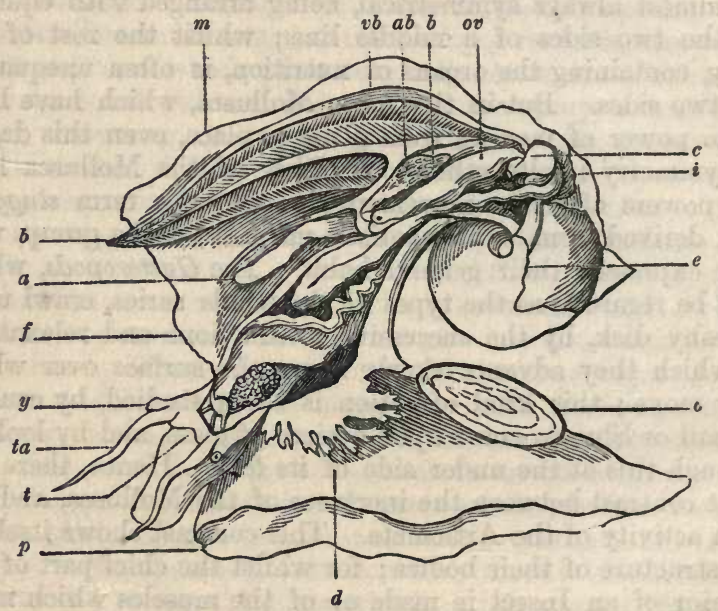

Fig. 55.-Anatomy of Turbo Pica.

108. The accompanying figure of the interior of a Turbo will show the very large size of the digestive apparatus, and of the other organs of nutrition. The muscular disk or foot is seen at $p$; and this carries the operculum $o$, which serves to close the mouth of the shell when the body of the animal is drawn within it. At $t$ is shown the proboscis, on either side of which are the tentacula or feelers, $t a$, bearing the eyes at $y$. Just behind the tentacula is seen the large cephalic ganglion, sending nerves to the eyes; and behind this again are the salivary glands. The mantle, $m$, is opened and folded back to show the respiratory cavity, in which lie the gills 
$b b$; to this cavity, water has access by means of a wide slit, of which the edge, $f$, of the mantle forms one part of the border, whilst at $d$ is seen a fringed membrane that forms another part. At $c$ is seen the heart, which receives the blood from the gills by $v b$, the branchial vein, and then transmits it to the body generally; at e, far up in the spire, are the stomach and liver; at $a$, the anal orifice of the intestine within the branchial cavity, and at ov the oviduct, which opens in the same situation.

109. Thus it is seen that, - whilst the body of an Articulated animal may be compared to that of a man in whom the apparatus of nutrition (contained in the chest and abdomen) is of the smallest possible size, but whose limbs are strong, and his movements agile, - the body of a Mollusk resembles that of a man "whose god is his belly," his digestive apparatus becoming enormously developed, whilst his limbs are feeble, and his movements heavy. Such varieties, in a greater or less degree, are continually presenting themselves to our notice.

110. The nervous system of the Mollusca generally consists of a single ganglion or pair of ganglia, which are placed in the head, or (when that is deficient) in the neighbourhood of the mouth; and of two or more separate ganglia, which are found in different parts of the body, and are connected with the preceding by nervous cords. The former correspond to those contained in the head of Insects; but of the latter, one only is connected with the foot or organ of motion, the remainder having for their function to regulate the action of the gills, and to perform other movements connected with the operations of nutrition. In fig. 56 is represented one of the simpler forms of this nervous system, that of the Pecten or Scallop-shell; $\mathbf{A} \mathbf{A}$ are the ganglia near the mouth, from which the organs of sense

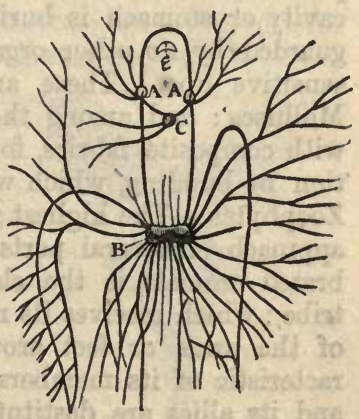

Fig. 56. -Nervous SYSTEM or Prcten. are supplied; $\mathrm{B}$ is the ganglion connected with the gills; and $c$ is that from which power is given to the foot. The 
two first lie wide apart, but are connected by an arched band that passes over the gullet, $e$. The organs of sense in the higher forms of Mollusca are more developed than those of motion. They serve to direct the animal to its food, and to warn it of danger; but there seems an absence, in all save the highest species, of that ready and acute sensibility which is so remarkable in the preceding groups; and the variety of impressions which they can receive appears to be but small. In no instance has a special organ of smell been certainly discovered; the organ of hearing is always imperfect, and frequently absent altogether; and the eyes are very often wanting. In the lower Mollusca there are no certain indications of the existence of any organs of special sense ; and there is probably but a limited amount of general sensibility.

111. As the Articulata are divided into two subordinate groups, according to the presence or absence of articulated limbs or members, so may we arrange the Mollusca in two subdivisions, according to the presence or absence of a distinct head, that is, a projecting part of the body, containing the mouth or entrance to the digestive cavity, and also bearing the organs of sense which guide the animal in the discovery and selection of its food. In the higher Mollusca, there is a distinct head, furnished with eyes, and sometimes with imperfect ears ; but in the lower, the entrance to the digestive cavity or stomach is buried deep among other parts, and is guarded by no other organs of sense than the tentacula or sensitive lips. These are termed acephalous, or headless Mollusca : and among the lowest of them ( $\$ 114)$, we meet with composite fabrics, formed by the process of multiplication by budding, which was formerly regarded as peculiar to Zoophytes. - The highest group of Mollusca, in regard to the approach of several parts of its structure to that of Vertebrated animals, is the class of CEPHaLOPODA, or Cuttle-fish tribe : which receives its name from the peculiar arrangement of the arms or feet around the mouth, which is the characteristic of its members (fig. 57). The common Cuttle-fish and its allies are destitute of any external protection; but they usually have a flat shel, commonly known as the cuttlefish bone, inclosed in a fold of the mantle, and lying along the back. In the Calamary, this is horny in its texture, and is sufficiently flexible to offer no resistance to the action of 
the fin-like tail, by which the animal is propelled through the water very much in the manner of a fish. The Pearly Nautilus is the only type now existing of an inferior order of

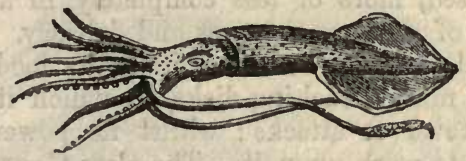

Fig. 57.-CАLAMARY.

Cephalopods, which approaches the Gasteropods in many parts of its organization. The body is inclosed in the last chamber of a shell (usually spiral in form), the cavity of which is divided by numerous transverse partitions ; and such shells, the fossilized remains of very numerous forms of this group that existed in the ancient seas, constitute the nautilites, ammonites, belemnites, \&c., which abound in many rocks (fig. 58). The Cuttlefish are animals of considerable activity; their mouth is furnished with a horny beak, strongly resem-

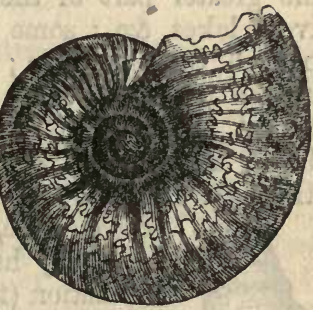

Fig. 58.-Aммолтте. bling that of the parrot; and their arms are provided with a series of very curiously constructed suckers, by the action of which they can take a very firm hold of anything which they desire to grasp.

112. The class of PTEROPODA, or wing-footed Mollusks, consists of but few species, and the animals which it contains are all of them of small size; but the individuals are often very numerous, whole fleets of them being sometimes seen covering the ocean, especially in the Arctic and Antarctic regions, where they constitute one of the

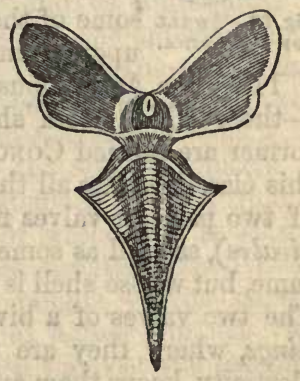

Fig. 59.-HтAгzA. principal articles of food to the Whale. The general form of the body usually differs but little from that represented in fig. 59 . 
On either side, a little behind the head, the mantle is extended into a fin-like expansion, by the aid of which the animal can swim through the water. The hinder part of the body is usually inclosed, more or less completely, in a shell, which is commonly of extreme thinness and delicacy. The head is not furnished with long arms, to grasp the food; but it has a number of minute sucking disks, by which it can lay firm hold of whatever it attacks : whilst its powerful rasp-like tongue is set to work upon it.-The class GASTEROPODA contains those animals which, like the Snail and Slug, crawl upon a fleshy disk on the under side of their bodies; and the number of distinct forms which it includes is very large. The greater part of them are inhabitants of the sea-shore, rivers, lakes, \&c. ; some have the power of swimming freely through the open sea; and the proportion of those that breathe air and live on land, is comparatively small. The general structure of the animals of this group has been already described ( $\$ 108)$. Some of them form shells, whilst others are destitute of them. The shells are composed of a

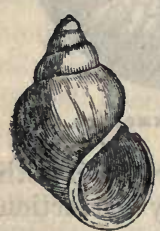
single piece, or are univalve, except in one tribe ; and they have usually more or less of a spiral formation (fig. 60). The animals of this class all possess a distinct head; and this is generally furnished with eyes, as well as with tentacula. They have often a powerful masticating apparatus, and are voracious in their habits; Fig. 60.-Shers some of them feed upon vegetable matter, others or Paldina. upon animals.

113. The Acephalous Mollusca are divided into two groups, - those which form shells, and those which do not. The former are termed CONCHIFERA, or shell-bearing animals ; and this class includes all the Mollusca that form a shell composed of two parts or valves fitted together (which shell is termed bivalve), as well as some others whose general structure is the same, but whose shell is formed in several pieces, or multivalve. The two valves of a bivalve shell (fig. 61) are connected by a hinge, where they are united by a ligament, which, by its elasticity, keeps them apart while it holds them together. This is their usual condition when the animal is alive; and in this manner the water which is required for their respiration, and also to convey their supply of food, has free access to the internal 
parts. But when any alarm or irritation causes the animal to close its shell, it does so by means of a muscle (sometimes

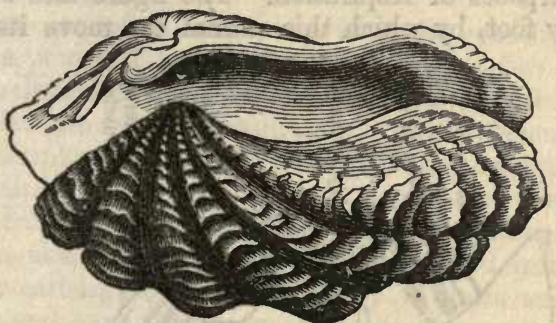

Fig. 61.-Shell of TRIDACNE.

single, sometimes double), which stretches across from one valve to the other, and which, by contracting, draws them together. Each valve is lined by an extended fold or lobe of the mantle. In the higher tribes of the class, these lobes are united along their edges, leaving apertures for the ingress and egress of water (which are sometimes prolonged into tubes, fig. 150), and another for the foot. But in the Oyster and its allies, which have no foot, or a very small one, the mantle-lobes are quite disunited. The accompanying diagram (fig. 62) gives a general idea of the arrangement of the organs in one of the higher acephalous Mollusca, the Mactra, which is among those having two muscles for the drawing together of the valves. The upper end, as represented in this figure, is that which is considered as the anterior end or front of the animal, being that nearest which the mouth lies; and the posterior extremity (the lowest in the figure) is that at which the intestinal canal terminates, and at which the respiratory tubes are formed. Near the anterior muscle, we find the mouth, or entrance to the stomach ; it is furnished with four riband-shaped tentacula, of which one is seen in the figure; and these seem to possess peculiar sensitiveness. Near the mouth lie the anterior ganglia of the nervous system, which represent the brain of higher animals; and these are connected by long cords with the posterior ganglion, which lies near the posterior muscle. The stomach, intestines, and liver occupy the central portion of the cavity of the shell; and the intestinal tube is seen to pass backwards, 
terminating near one of the canals or siphons, which also carries out the water that has been taken in through the other. for the purposes of respiration. The figure also shows the large fleshy foot, by which this animal can move itself along

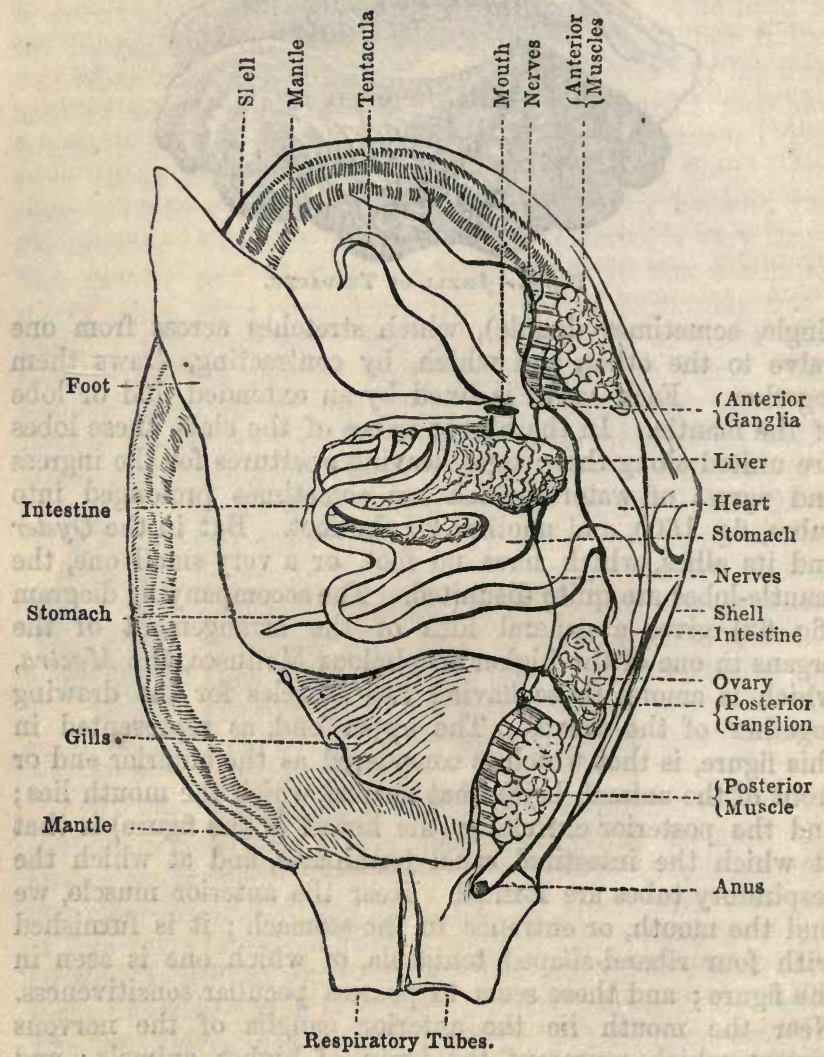

Fig. 62.-ANatomi of Mactra.

the ground, or bore into sand or mud. The heart and circulating system are less complete than in the Gasteropoda; but are far higher in character (as are most of the other parts 
of the nutritive apparatus) than the corresponding parts in Articulated animals, in which the apparatus for locomotion so much predominates.

114. The group" of Acephalous Mollusks which are destitute of the power: of forming a shell, includes two classes, of which one does not depart widely from the general Molluscan type, whilst the other presents so strong a general resemblance to Zoophytes, that until recently it has been universally ranked with it. The first of these classes receives its name Tunicata from the circumstance that the mantle, instead of secreting a shell, is very commonly condensed into a tough leathery or cartilaginous tunic. Many of these animals live separately, and have the power of freely moving through the water. Others are associated in compound masses, of which, however, the individuals are not connected by any internal union. But others form really composite structures, like those of Zoophytes ( $\$ 124)$; each individual being able to live by itself alone, but being connected by a stem and ressels with the rest. The general structure of the individuals is the same, however, in the single and in the composite animals of this class, and may be understood from the accom-

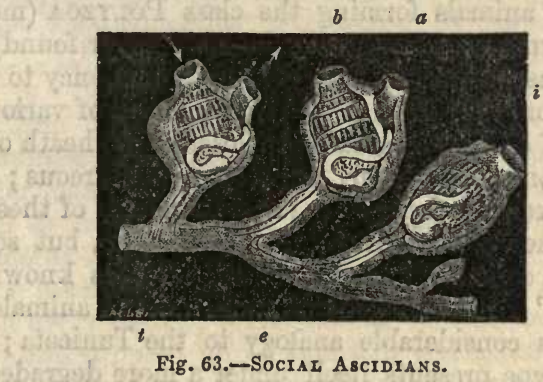

panying figure (fig. 63). The cavity of the mantle possesses, as in the former instance, two orifices; by one of which, $b$, a current of water is continually entering, whilst by the other, $a$, it is as continually flowing out. These orifices lead into a large chamber, the lining of which, folded in various ways, constitutes the gills; and at the bottom of this chamber lie the stomach, $e$, and the intestinal canal, $i$, which terminates near the aperture for the exit of the water. All these parts 
are covered with cilia, by the action of which a continual stream is made to flow over the gills and to enter the stomach; and the minute particles which the water brings with it, and which are adapted to serve as food, are retained and digested in the stomach. Even these animals, fixed to one spot during all but the early part of their lives, and presenting but very slight indications of sensibility, possess a regular heart and system of vessels; and these vessels form part of the stem, $t$, by which the compound species are connected. A single nervous ganglion is found between the two orifices; this seems to receive sensory fibres from tentacula situated around the oral orifice, and to transmit motor filaments to the muscular coat which underlies the outer tunic, so that any irritation applied to the former occasions a contraction of the latter, which tends to expel the offending particle.-This class is one of particular interest to the naturalist, since we see in it the tendency to the formation of compound structures, by a process resembling that of the budding of plants, which is essentially characteristic of Zoophytes; this tendency, however, is more fully manifested in the succeeding class.

115. The animals forming the class PolyzoA (more commonly known as Bryozoa) are seldom or never found solitary; since, in consequence of their universal tendency to multiply by gemmation, they form clusters or colonies of various kinds. The body of each individual is inclosed in a sheath or "cell," which is sometimes horny, sometimes calcareous; and the composite skeleton formed by the aggregation of these, which has sometimes a branching or leaf-like form, but sometimes possess the compactness of a stony coral, is known as the "polyzoary." In their general structure the animals of this class possess considerable analogy to the Tunicata; but the Molluscan type presents itself under a more degraded aspect, no vestige of a heart or of blood-vessels being here discernible, and the general structure being so simplified as to manifest no great degree of elevation above that of Polypes. The typical structure of these animals may be understood from that of the Bowerbankia (fig. 64), which is one of those whose cells are not in contact with each other, but grow forth at intervals from a creeping stem. The mouth, $a$, is situated in the midst of a circle of arms fringed with cilia; these 
arms do not serve, however, like those of polypes, to grasp the food; but the vibration of their cilia produces a powerful

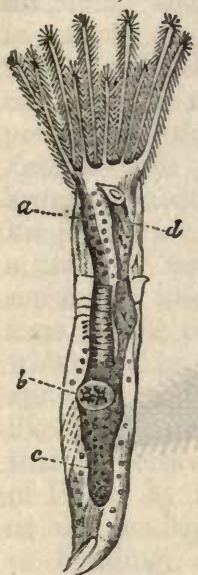

Fig. 64 . current which brings both food and oxygen. The mouth leads by a large funnel-shaped csophagus or gullet, to a gizzard, $b$; in which the particles of food that enter it are ground down, by the action of its muscular walls and of the tooth-like processes that line it. Below this gizzard is the true digestive stomach, $c$, around which the rudiment of a liver may be traced; and from this stomach there passes upwards an intestinal tube, which terminates by a distinct orifice at $d$, on the outside of the circle of arms. The digestive apparatus is evidently formed, therefore, upon a much higher plan in these animals than it is in the true polypes, which have no true anal orifice. The Molluscan character of these animals is further shown by the presence of a single nervous ganglion, situated vetween the two orifices, Bowerвasкia. as in the Tunicata; this acts upon a complex
a, œsophagus; $b$, giz- apparatus of muscles, by which the animal zard ; $c$, stomach;
$d$, orifice of intes- can be either drawn into its cell or projected tine. forth from it, with great rapidity.

116. The fourth subdivision, that of RADIATA, includes those animals which have the parts of the body arranged in a circular manner around a common centre, so as to present a radiated or rayed aspect. This arrangement is well seen in the common Star-fish (fig. 65), which has five such rays, all having a precisely similar structure, and thus repeating each other in every respect. The mouth of this animal is in the centre ; and it opens into a stomach, which occupies the central disk, and sends prolongations into the rays. The nervous system is, in like manner, composed of a repetition of similar parts. A plan of it is seen in fig. 66 ; where $a$ shows the position of the mouth, which is surrounded by a ring or nervous cord, having five ganglia, corresponding to the five arms. From each of these ganglia proceeds a branch along its arm, terminating in a little organ at its extremity, which is believed to be an imperfectly-developed eye. No other organs of special sense can be detected in any of these ani- 
mals; and it is on!y in a few that $\epsilon$ ven these imperfect eyes can be discovered. In the inferior Radiata, not the slightest

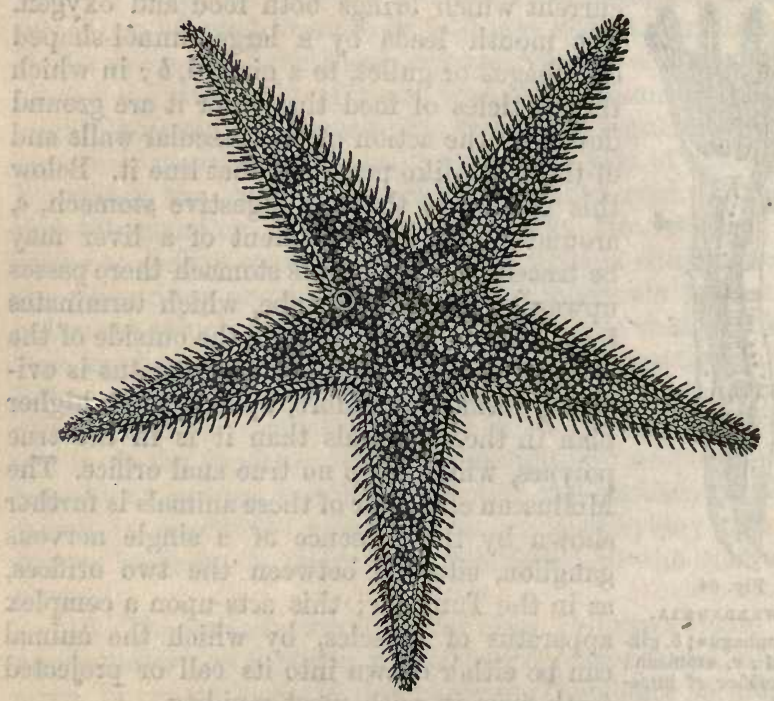

Fig. 65.-SHeLI of StaR Frsh.

traces of a nervous system have yet been discovered; and it is very doubtful whether any such structure exists in

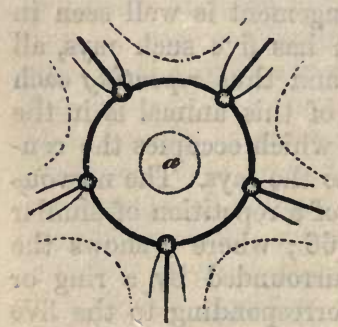

them. It is only among the higher Radiata that any locomotive power exists; and this is usually so feeble that the animals remain in the same locality during the greater portion of their lives. Generally speaking, there is a period in the history of each species, in which there is a more active movement, that serves to prevent the accumulation of indi-

Fig. 66.-NERVOUS SYSTEM OF STAR FISH. viduals in one spot; but this movement is of a purely automatic character, rather resembling that of the "zoospores" of plants, than the intentional change of place of the higher animals. 
117. The circular arrangement of the organs of Radiated animals is a striking point of resemblance to the Vegetable kingdom; and it has frequently caused mistakes to be made in regard to the Sea-Anemones and other large polypes, which, when their mouths are open and their arms spread out, look so much like the blossoms of some of the Composite tribe of plants, as to have received the name of animal flowers. But there is yet a stronger analogy between the lower members of the Radiated group and the Vegetable kingdom; for among the former, as in the latter, we find a union of many individuals, which are capable of existing separately, into one compound structure, having a plant-like form. This is the nature of the stem of Coral (fig. 76); which is, in fact, the skeleton of one of these compound systems, consisting of a number of polypes united by a jellylike flesh; just as the woody stem of a tree is the skeleton that supports a vast number of buds, each of which is capable of living by itself. This aggregation results from the indefinite multiplication of parts by the process of gemmation - or budding, and from the persistence of the connexion between these parts, notwithstanding that, if separated, they can maintain an independent existence. To the treelike fabrics thus produced, the name Zoophytes (animal plants) is commonly given; and ordinary observers often find it difficult to get rid of the idea of their vegetable origin. The animals that formed them are, of course, fixed to one spot during all but the earliest periods of life; and the amount of movement which they perform, for the purpose of obtaining and securing their food, is very little greater than that which is witnessed in the Sensitive plant and Venus's fly-trap.

118. The class of Echinodermata receives its name from the prickly character of its covering, which is evident enough in the Echinus or Sea-Urchin, and in the Star-fish; but there are other animals, sufficiently resembling these in general structure to be united in the same class, which have a body entirely soft,-namely, the Holothuria (fig. 67), commonly termed Sea-Cucumbers. This class ranks as the highest among the Radiata, in regard to general complexity of structure. The skeleton of the Sea-Urchin, Star-fish, and other animals resembling them, is a box-like shell or "test," formed 
of a great number of pieces, very regularly arranged and united together (fig. $69, \mathrm{e}$ ); but these pieces are for the most

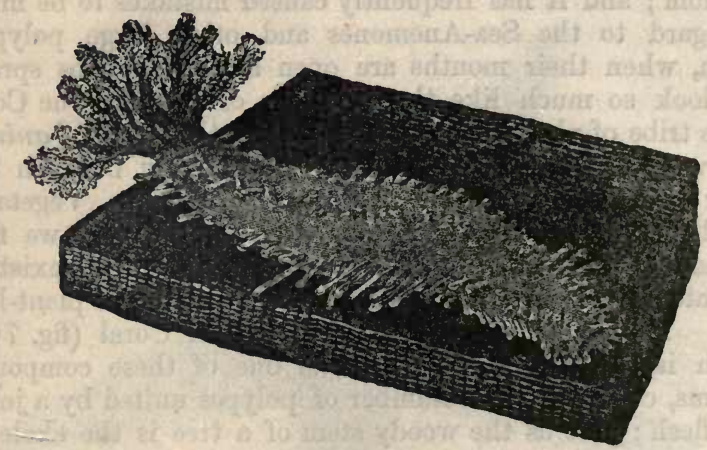

Fig. 67.-HoLothuRIA.

part only repetitions of one another; and the different portions have not that variety of uses which we see in higher

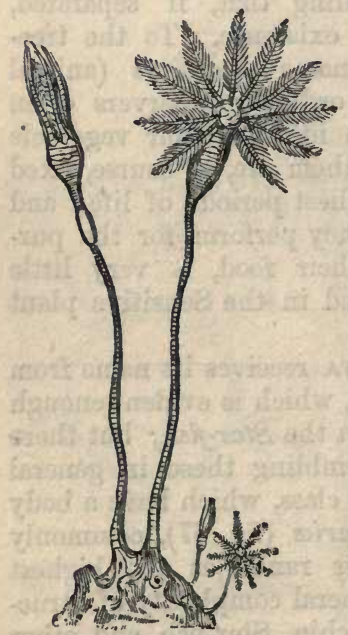

Fig. 68.-Encrinite. animals. With the exception of the tribe of Encrinites or lily-like animals (fig. 68), of which there are very few now existing, but which were very abundant in former ages, all the animals belonging to this class are unattached, and are capable of moving freely from place to place. Their motions are very sluggish, however, and are principally effected by means of an immense number of minute tubular feet (fig. 68, c), furnished with suckers at their extremities, which can be projected from almost any part of the body. These are seen in rows on the under side of each arm of the Star-fish; they are put forth through rows of very minute apertures in the shell of the SeaUrchin (commonly termed the Sea- 
Egg); and they are also arranged in rows on the surface of the body of the Holothuria, as seen in fig. 67 .

119. The radiated arrangement is very evident in the whole bodies of the Star-Fish (fig. 65), and Echinus or SeaUrchin (fig. 69); but in the Holothuria (fig. 67) it is nearly confined to the parts about the mouth; which, however, exhibit it so completely, that such an animal cannot be mistaken for one of the Articulated series, even though, as sometimes happens, the body is prolonged into a worm-like form. The digestive apparatus in this class has usually a high degree of complexity, as will be seen by the accompany-

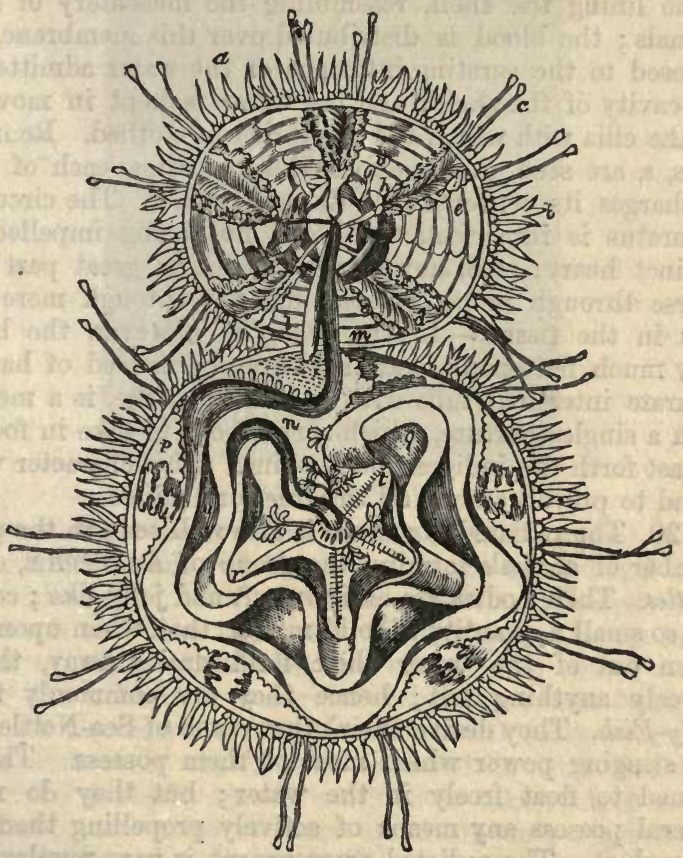

Fig. 69.-INTERIOR of EchINUS.

ing figure (fig. 69), which shows the interior of an Echinus, whose globular shell has been sawn across its equator, so as 
to allow of the separation of its two halves. The mouth, $k$, situated at one of the poles of the shell, is surrounded by a very curious apparatus of jaws and teeth (fig. 69), which forms what is termed the "lantern;" from the mouth commences the long narrow œesophagus, $m$, that leads to the stomach, $n$, which is merely a dilated portion of the alimentary tube; the continuation of this, $0, q, r$, forms the intestinal canal, which winds once round the shell, and then doubles back and winds in the opposite direction, terminating at the anal orifice, $s$, which is situated at the opposite pole. The intestine is held in its place by a double fold of the membrane lining the shell, resembling the mesentery of higher animals; the blood is distributed over this membrane, to be exposed to the aerating influence of the water admitted into the cavity of the shell; and the water is kept in movement by the cilia with which the membrane is clothed. Round the anus, $s$, are seen the five branching ovaries, each of which discharges its contents by a distinct orifice. The circulating apparatus is imperfect, the blood not being impelled by a distinct heart; still, however, it moves in great part of its course through proper vessels, and not through mere channels in the tissue. - In the Star-fish, however, the body is very much flattened; and the stomach, instead of having a separate intestinal tube with a distinct orifice, is a mere bag with a single aperture, which serves both to take in food and to cast forth the indigestible remains. This character will be found to prevail among all the inferior Radiata.

120. The radiated structure is also well seen in the greater number of animals forming the group of ACALEPHA, or Seci= Nettles. Their bodies are entirely soft and jelly-like ; containing so small a quantity of solid matter, that, when upon being taken out of the water their fluid drains away, there is scarcely anything left; hence they are commonly termed $J e l l y-F i s h$. They derive their other name of Sea-Nettles from the stinging power which most of them possess. They are formed to float freely in the water; but they do not in general possess any means of actively propelling themselves through it. The radiated arrangement is very regularly pre. served in some of this group, whilst it is less evident in others. The accompanying figure (fig. 70) represents one of the Medusa tribe, as seen floating in water. The umbrella- 
shaped disc above contains the stomach, which is placed in the centre, and which opens by a single orifice or mouth, directed downwards. Around the stomach are four chambers, in which the eggs are prepared. The mouth is surrounded by four large tentacula, which bring to it the necessary supply of

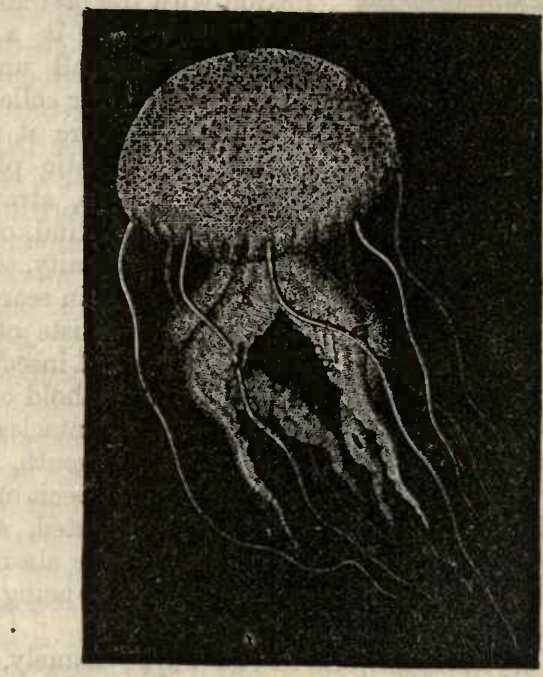

Fig. 70.-Peragia.

food; and other tentacula are seen, in this species, to be hanging from the edge of the disc. In the edge of this disc, the nutritious fluid, which flows in channels prolonged from the stomach and excavated out of the soft tissues, seems to be exposed to the influence of the surrounding water; but nothing like a heart or a regular circulation exists.-Recent discoveries in regard to the developmental history of the Medusce and their allies, have rendered it very doubtful whether the Acalephoe should continue to take rank as a distinct class ; since many of them constitute only a particular phase in the life of the Hydroid Zoophytes ( 124 ).

121. The class of PoLYPIFERA, or coral-forming animals, commonly known as Zoophytes, includes two principal tribes, which differ from one another in structure to such a degree as to 
require separate notice. The group of Hydrozoa, or Hydroid Zoophytes, so named from the little Hydra, or fresh-water

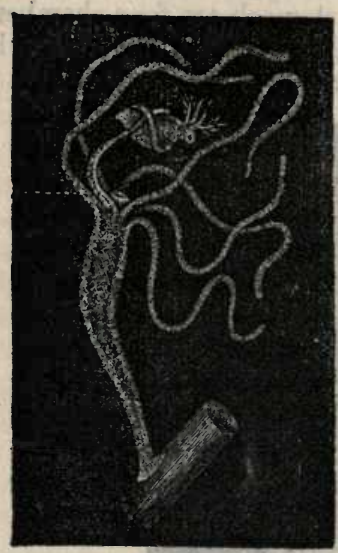

Fig. 71.-HYDRA, OR FRESH-WATER PoLXPE. polype, which may be regarded as its type, will be first described on account of its near connexion with the preceding. The Hydra (fig. 71) is a solitary polype, not at all uncommon in ponds or other collections of fresh water, where it is found attached to aquatic plants, or to floating sticks, straws, \&c., by means of a kind of sucker at its lower extremity, stretching out its tentacles in search of its food, which consists of minute aquatic worms and insects. These are securely laid hold of by one or more of the tentacles, and are drawn into the mouth, $a$, which leads to the stomach or general cavity of the body, in which they are digested, and from the walls of which the nutritious portions are absorbed, the portions of the food which are not capable of being digested being cast out through the mouth.

122. The Hydra multiplies in two ways; namely, by gemmation or budding, and by a proper generative process. Little bud-like processes are developed from various parts of the walls of the stomach, which gradually assume the form of the parent, possessing a mouth surrounded by tentacles, and a digestive cavity which is at first in connexion with that of the parent; the communication is gradually cut off, however, by the closure of the canal of the footstalk of the young polype; and ere long the footstalk itself separates, and the young polype henceforth leads an entirely independent life. Not unfrequently, however, the young polype itself puts forth buds before its separation; and as many as nineteen young Hydræ, in different stages of development, have been seen to be thus connected with one and the same stock. Another very curious endowment of the Hydra depends upon the same facility of developing the whole structure from any part of it; 
for into whatever number of parts its body may be cut up, each, under favourable circumstances, can give origin to a new and entire polype, so that thirty or forty individuals may thus be produced by the division of one.

123. The proper generative process, here reduced to its utmost simplicity, consists in the development of a germ-cell and of sperm-cells in the substance of the wall of the stomach, the former being produced near the footstalk, the latter just beneath the arms. The egg which is evolved from the former, being fertilized by the products set free from the latter, gives origin to a young Hydra, which resembles its parent. The two reproductive processes, however, are performed under very different conditions; for whilst multiplication by gemmation is favoured by warmth and a copious supply of food, the true generative process seems to be brought about by a lowering of the temperature, and to have for its object the perpetuation of the race through the winter, the egg being capable of enduring a degree of cold which would be fatal to the polype itself.

124. The group of $H y$ drozoa is for the most part made up of composite fabrics more or less resembling the Campanularia (fig. 72), which may be likened to a Hydra whose buds do not detach themselves, but remain in connexion with the stock that produced them; the whole plant-like struc-

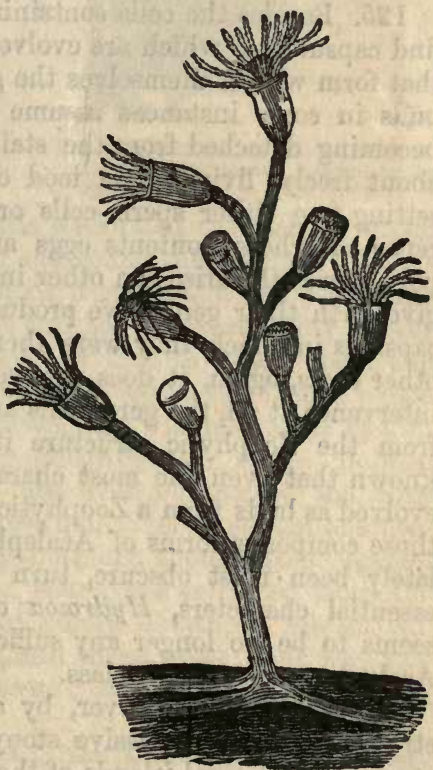

Fig. 72.-CAMPANULARIA. ture, moreover, being strengthened by the consolidation of its external layer into a horny sheath, which retains its form after the destruction of the soft parts. Thus each comes to consist of a stem and branches, on the sides or ends of which 
are a number of little cells or bell-shaped chambers, with their mouths upwards, every one of them containing a polype that bears a strong resemblance to the Hydra. Each of these polypes is capable of living independently of the rest, obtains its nourishment by means of its own arms, and digests it in its own stomach; but all are connected by a canal that passes along the stem and branches, in which a kind of circulation takes place, that strongly reminds us of that of the compound Tunicata (§ 114). This plant-like structure extends itself by budding; new branches are formed from those previously existing; and these are enlarged at a certain point into cells, in which after a time polypes make their appearance.

125. Besides the cells containing the polypes, however, we find capsules in which are evolved buds of a different nature, that form within themselves the generative products. These buds-in some instances assume the form of Medusoe, and, becoming detached from the stalk that put them forth, swim about freely, living upon food obtained by themselves, and setting free either sperm-cells or germ-cells, by the concurrence of whose contents eggs are formed, from which new polype-growths arise. In other instances the Medusoid bodies give forth their generative products, without ever leaving the capsules in which they were themselves developed. And in other cases, again, it does not seem that any Medusoid form intervenes at all, the germ-cells and sperm-cells being evolved from the Zoophytic structure itself. But since it is also known that even the most characteristic Medusan forms are evolved as buds from a Zoophytic stock (Chap. xv.), and since those composite forms of Acalephæ whose structure has until lately been most obscure, turn out to be, as regards their essential characters, Hydrozoa organized for floating, there seems to be no longer any sufficient ground for ranking the Acalephæ as a separate class.

126. It is not, however, by animals of this very simple structure, that the massive stony fabrics are built up, which constitute the coral islands of the Pacific Ocean, and of which a large portion of our limestone rocks seems to be composed. These are constructed by animals belonging to the group of Anthozoa, and formed upon the same general plan with the Sea-Anemone, - a plan which is higher than that of the Hydra, inasmuch as we find the interior of the body containing other 
cavities around the stomach, which are destined to prepare the generative products. In fig. 73 , we have a representation of the Sea-Anemone, as seen from above; showing its mouth in the centre, surrounded by its numerous radiating tentacula; these are often brightly coloured, and give to the animal the appearance of a beautiful flower. In fig. 74, a similar animal is represented, cut open to show its interior.

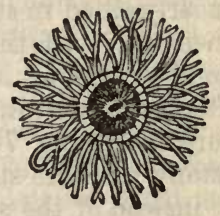

Fig. 73.-SEA-ANEMONE, seen from above.

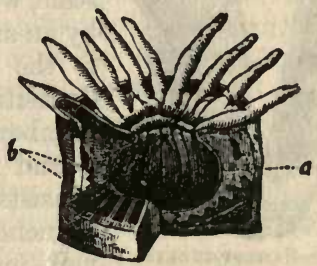

Fig. 74.-Section of Sea-ANemone. $a$, cavity of stomach; $b$, surrounding chambers.

The mouth is seen to open into a rounded stomach, $a$, which has no other orifice outwards; and round this stomach there is a series of radiating membranous partitions, which divide the space intervening between it and the outer covering of the body into numerous chambers, $b$. Within these chambers, and attached to their partition-walls, are found the bodies which are commonly designated ovaries, but which contain spermcells or germ-cells according to the sex. It is doubtful whether these two products are ever formed by the same individual, as they are in the Hydra. The Sea-Anemone does not usually multiply itself by budding, though some species. do so; but large numbers of young are produced from the eggs, which are fertilized and partly developed whilst still within the ovarian chambers, and these make their way into the stomach through an aperture at its deepest point, and finally escape by the mouth.

127. The Sea-Anemone itself, like the Hydra, is a solitary animal, capable of shifting its place at will; and it forms no stony skeleton or support. But there are other animals of the same general structure, which have the power of depositing stony matter in the membrane of their base or foot, and in the membranous partitions between the chambers; and this stony deposit forms a Coral or Madrepore, such as is shown 
in the accompanying figure (fig. 75). The particular arrangement of the radiating plates of the Madrepore (shown at the

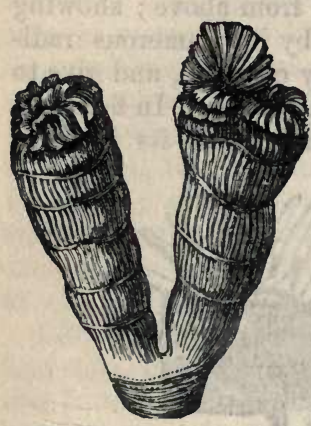

top of each stem) is the result of the form of the soft structures by which it was deposited; and wherever we see a structure of this kind in coral, whether upon a large or a small scale, we may infer that it was formed by an animal nearly allied in structure to the Sea-Anemone. Of the stone depositing coral-animals, a large number are often associated in a compound structure, as in fig. 76 ; this consists of a stony tree-like stem and branches; but instead of the soft aniFig. 75.-Caryophyltis. mal matter being contained in its interior, as in the Hydrozoa, it usually forms a kind of flesh

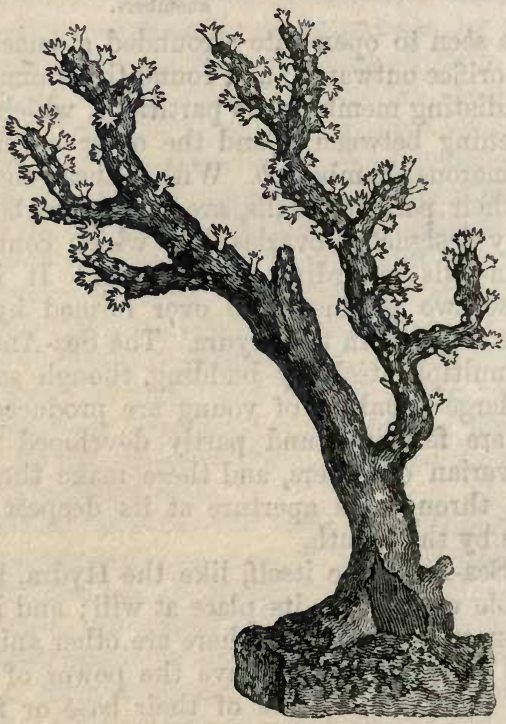

Fig. 76.-STEM or CoraL.

that clothes the surface, and connects together the different 
polypes ; and new branches, are formed either by the subdivision of the polypes, or by gemmation from the connecting substance.

128. When we pass from Zoophytes to animals of still simpler organization, we lose all trace of definite symmetry, and find ourselves amid forms which cannot be referred to any particular plan of growth. These, moreover, are for the most part distinguished by an extreme simplicity of structure; no such differentiation of parts exhibiting itself among them, as is shown in the "organs" of even the simplest Zoophyte or Worm. Hence they are appropriately designated Protozon. They may, in fact, be considered as essentially consisting of homogeneous particles of a jelly-like substance, to which the name of Sarcode has been given; and the chief modification this undergoes, consists in the consolidation of certain parts of it by the deposit of horny, calcareous, or siliceous matter, so as to form a skeleton. This may take place on the outer surface only, so as to form shells very like those of Mollusks in miniature, as we see among Foraminifera (fig. 78); or it may occur in the midst of the fleshy substance, so as to form an internal network, such as presents itself in the Sponge. The endowments of the "sarcode" are very extraordinary; and will be best understood by observation of the life-history of one of those simplest Protozoa, in which the whole body consists of but a minute particle of it.

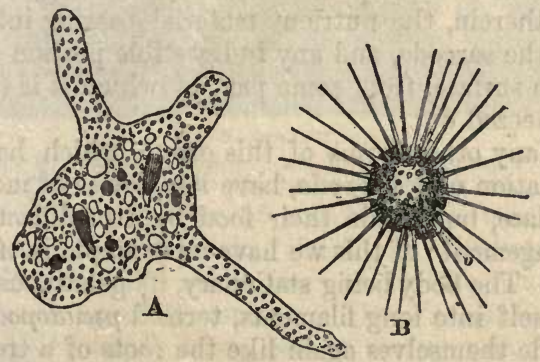

Fig. 77.-Rнгооора :-A, Amœba; B, Actinophrys.

129. Such an example is afforded by the $A$ moeba (fig. $77 . \mathrm{A}$ ), - a creature frequently to be met with in great abundance in fresh and stagnant waters, vegetable infusions, \&c. Its 
organization is so low, that there is not even that distinct differentiation into containing and contained parts which is necessary to constitute a cell $(\$ 32)$; for although the superficial layer of the sarcode possesses more consistence than the interior, it is nevertheless obvious that it has not the tenacity of a membrane, since (as will be presently seen) it does not oppose the passage of solid particles into the interior. However inert this creature may seem when first glanced at, its possession of vital activity is soon made apparent by the movements which it executes and the changes of form it undergoes; these being, in fact, parts of one and the same set of actions. For the shapeless mass puts forth one or more finger-like prolongations, which are simply extensions of its gelatinous substance in those particular directions; and a continuation of the same action, first distending the prolongation, and then, as it were, carrying the whole body into it, causes the entire mass to change its place. After a short time another prolongation is put forth, either in the same or in some different direction; and the body is again absorbed into it, so as to shift its place still more. It is by means of this movement that the creature obtains its supplies of food; for when, in the course of its progress, it meets with a particle appropriate for its nutriment, its gelatinous body spreads itself over this, so as to envelope it completely ; and the substance (sometimes animal, sometimes vegetable), thus taken into this extemporized stomach, undergoes a sort or digestion therein, the nutrient material passing into the substance of the sarcode, and any indigestible portion making its way to the surface, from some part of which it is (as it were) finally squeezed out.

130. Many other forms of this group, which has received the designation of Rhizopoda, have less power of moving from place to place, but obtain their food by a modification of the same arrangement: of this we have an example in Actinophrys (fig. 77 в). The body being stationary, its gelatinous substance extends itself into long filaments, ternicl pseudopodia: these often divide themselves again like the roots of a tree (whence the designation of the group), so as to form threads of extreme tenuity; and sometimes these threads meet again and coalesce, so as to form a sort of irregular network. When any minute animal or vegetable organism happens to come in contact 
with one of these threads, it is usually held by adhesion to it, and the filament forthwith begins to retract itself; as it shortens, the surrounding filaments also apply themselves to the captive particle, bending their points together, so as gradually to inclose it, and themselves retracting until the prey is brought to the surface of the body; and the substance of the threads being itself drawn into that of the body, the entrapped particle is embedded along with this, and undergoes digestion in the surrounding sarcode, any indigestible particle being subsequently extruded from the surface of the body, just as in the Amoeba. The reproduction of these creatures, so far as is yet known, is effected by self-division, like that of the Infusoria ( $\$ 135)$; but there is reason to believe that a "conjugation," or reunion of two individuals, sometimes occurs, and that this is to be looked on as representing the sexual propagation of higher animals.

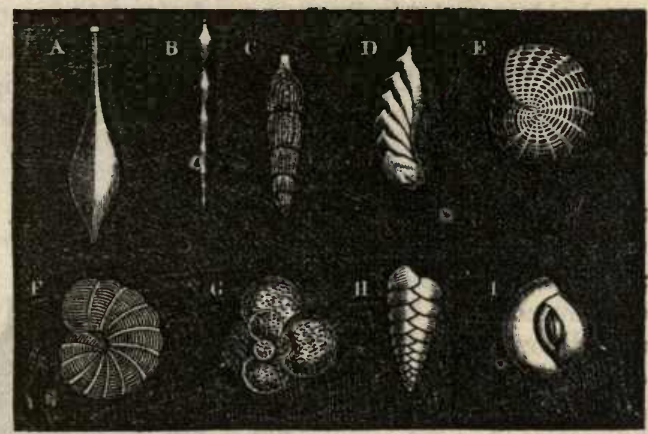

Fig. 78.-FORAMINITERA.

A, Oolina ; B, C, Nodosaria ; D, Cristellaria ; E, Polystomella ; F, Dendritina, G, Globigerina; H, Textularia; I, Quinqueloculina.

131. This Rhizopod type of animal life is manifested in two groups of great interest, which are characterised by the possession of hard shells, formed by the consolidation of the external layer of sarcode. The Foraminifera have calcareous shells, which often bear a strong resemblance to those of Nautili, \&c. in miniature (fig. 78), but which really have an entirely different relation to the animals that form them. For whilst the Nautilus occupies only the last or outer chamber of its shell, the chambers previously formed 
being empty and deserted, each chamber of the Rotalia, or any other Foraminiferous shell, is occupied by a segment of sarcode, which is to a great degree independent of the rest, and is only connected with those on either side of it by delicate threads of the same substance; and the extension of the shell is due to the formation of an additional segment of sarcode on the outside of the last-formed chamber. Each segment has usually the power of putting forth its own "pseudopodia" through minute apertures in the shell, and thus of drawing in its own nourishment through these; but even when (as sometimes happens) these food-collecting threads are put forth from the last chamber alone, the nutriment there obtained is transmitted to the segments within by percolation through the substance of the sarcode, and not through any tubular canal. - The accumulation of the shells of Foraminifera in some parts of the existing sea-bottom is very remarkable; and similar accumulations in past ages have formed no unimportant part of the crust of the eartha large part of the Chalk-formation having had its origin in them, as well as nearly the whole of the Nummulitic limestone by which it was succeeded.

132. But animals whose essential structure seems to be nearly the same, may form siliceous instead of calcareous shells; and thus are produced those beautiful organisms, known under the name of Polycystina (fig. 79), which are occasionally found in the existing seas, but whose remains are met with under a far greater variety of forms in certain of the newer marine deposits. There is not in these the same tendency to form composite structures by the multiplication

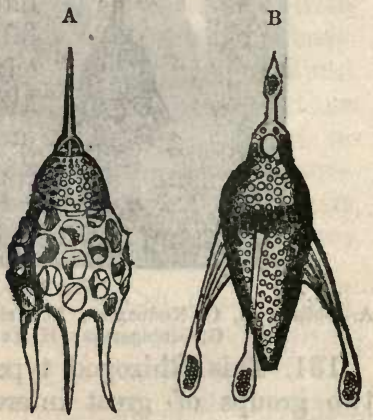

Fig. 79.-Pourcxstiva.

A, Podocyrtis; B, Rhopalocanium. of segments, as in the Foraminifera ; but the complication of the individual form is often much greater. Yet, however complex the form, the essential composition of these creatures seems to retain the same attribute of simplicity, which cannot be conceived capable of further reduction. 
133. The Animalcules to which the name of INFusoria may be properly restricted (the Rotifera, or Wheel-Animalcules, $\S 105$, whose organization is much higher, together with many organisms whose true nature is vegetable, being excluded), present an advance upon the simplicity of the Rhizopoda in this, - that whilst their bodies consist for the most part of sarcode, and present scarcely anything that can be termed a distinction of organs, their external surface is condensed into a membrane too firm to admit either of indefinite extension into pseudopodia, or of the passage of alimentary particles through it; and consequently the form of the body, although not insusceptible of being temporarily changed by pressure, possesses a considerable degree of constancy for each species (fig. 80). $\Delta$ mouth, or definite aperture for the ingestion of food, is provided; with an additional orifice in some instances, through which indigestible or effete matters may be discharged from the interior. Into this mouth, ali-

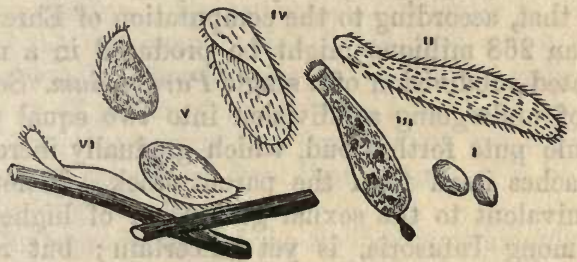

Fig. 80.-Infosory ANimaLcules.

I. Monads; Ir. Trachelis anas; Irr. Enchelis, discharging fæcal matter; Iv. Paramocium; v. Kolpoda; vr. Trachelis fasciolaris.

mentary particles are drawn by the agency of the cilia with which some part of the surface of the body is provided; these cilia being always so disposed as to serve at the same time for the general locomotion of the animalcule, and for the production of currents that shall bring food to its interior.

134. Although most Infusoria move freely through the water in which they live, yet certain kinds of them attach themselves by footstalks to marine plants or other floating bodies, during at least a part of their lives; and in this condition bear no slight resemblance to Zoophytes, though of far simpler organization. It is in these sessile forms that the agency of the cilia in creating currents which bring food to 
the mouth, becomes most conspicuous. The alimentary particles introduced into the mouth commonly have to pass down a short canal before they enter the general cavity of the body; and within this cavity a number of minute particles are commonly aggregated into a sort of little pellet, as may be well seen when Infusoria are fed with carmine or indigo. One after another of these pellets being thus introduced into the interior, which is occupied by a soft sarcode, each seems to push onwards its predecessors ; and a kind of circulation is thus occasioned in the contents of the cavity. The pellets that first entered make their way out after a time (their nutritive materials having been yielded up), generally by a distinct anal orifice, sometimes, however, by any part of the surface indifferently, and sometimes by the mouth.

135. The multiplication of Infusoria ordinarily takes place by spontaneous fission, precisely after the manner of the multiplication of ordinary cells $(\S 33)$. This process, under favourable circumstances, may be performed with such rapidity, that, according to the computation of Ehrenberg, no fewer than 268 millions might be produced in a month by the repeated subdivision of a single Paramecium. Sometimes, instead of undergoing subdivision into two equal parts, the Animalcule puts forth a bud, which gradually increases, and then detaches itself from the parent stock. Whether anything equivalent to the sexual generation of higher animals occurs among Infusoria, is yet uncertain; but recent researches afford a probability in the affirmative.

136. In the tribe of Porifera, or Sponges, we seem to have the connecting link between Protozoa and Zoophytes. For their animality does not lie so much in the individual particles, as in those aggregations whioh begin to shadow forth that distinction into organs which is carried out more completely among Zoophytes : and there is a large section of the last-named group, in which the polypes are connected together, not by a regular stony or horny stem, but by a sponge-like mass; while the extension of the fabric is provided for by the budding out of this spongy portion of it, the orifices of whose canals after a time become furnished with polype-mouths. The true Sponge (fig. 81) consists of a fleshy substance, composed of an aggregation of particles of sarcode, supported upon a skeleton which usually consists of a net- 
work of horny fibres, strengthened by spicules of mineral matter, sometimes calcareous, but more commonly siliceous. The entire mass is traversed by a great number of canals, which may be said to commence in the small pores upon its surface, and which discharge themselves into the wide canals that terminate in the large orifices, or vents, that usually project more or less from the surface of the Sponge. Through this system of canals, there is continually taking place, during the living state of the animal, a circulation of water, which is drawn in from without through the minute pores, then passes into the large canals, and is ejected in a constant stream from

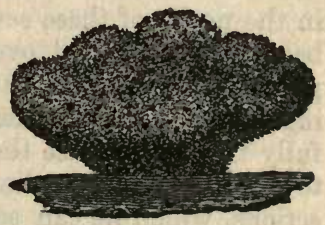

Fig. 81.-SPONGE. the vents. The immediate cause of this movement seems to lie in the vibration of cilia so extremely minute that their existence can only be detected by the most careful microscopic examination. Its purpose is evidently to convey to the animal the nutriment which it requires, and to carry off the matter which it has to reject. No distinct indications of sensation, or of power of locomotion, have been seen in the Sponge: but changes in the form of its projecting rents may be seen to take place from time to time, if it be watched sufficiently long.

137. The reproduction of the Sponge is commonly effected by the budding forth of little particles of sarcode, from the layer which lines the larger canals; these become furnished with cilia, and, when detached and carried out by the current that issues from the vents, swim freely about for some time; so as, before fixing themselves and beginning to develope into Sponges, to spread the race through distant localities. But it appears that Sponges are also reproduced by a true sexual process; "sperm-cells" and "germ-cells" being produced (as in the Hydra, § 123) in different parts of the organism, and a true embryo taking its origin in the action of the contents of the former upon those of the latter. 
138. We thus conclude our general survey of the Animal Kingdom; which, it is hoped, will be found to answer the purpose for which it was designed,-that of giving such an amount of preparatory knowledge respecting the principal types of animal structure, as may enable even the beginner to comprehend what will hereafter be stated of their physiological actions. It has not been attempted to observe any proportion in the notice of these several types ; the higher forms having been slightly passed over, because the details of their vital phenomena will constitute the principal subject of the following pages; whilst some among the lower have been more fully treated, because the ordinary reader cannot be expected to have even that outline-acquaintance with their nature and actions, which he can scarcely help possessing in the case of animals that are familiar to him.

\section{CHAPTER III.}

NATURE AND SOURCES OF ANIMAL FOOD.

139. BeFore we examine the nature of the process by which the food of animals is prepared for absorption into their bodies, it will be desirable to consider the characters of the aliment itself, and the purposes to which it is to be appropriated. The term food or aliment may be applied to all those substances which, when introduced into the living body, serve as materials for its growth, or for the repair of the losses which it is continually sustaining ( $\$ 55)$. When animals are deprived of these materials, we see their bodies progressively diminishing in bulk, their strength decreases, and death at last takes place, after sufferings more or less prolonged. In warm-blooded animals, however, a yet more urgent demand for food is created by the requirements of the heat-producing process; and many substances are fitted to supply this, which cannot serve for the nourishment of the tissues.

140. The demand of the body for food is made known by a peculiar sensation, which has its seat in the stomach, namely, hunger. It is increased by mental and bodily exercise, and 
by everything which augments the general energy of the system; whilst, on the contrary, everything which tends to retard the operations of life, such as bodily and mental inactivity, sleep, or depression of spirits, tends also to render the demand for food less imperious. Thus, cold-blooded animals, particularly Reptiles, can sustain a very prolonged abstinence, when the general activity of their functions is kept down by a low temperature; and hybernating Mammals, which pass the winter in a state of torpidity, require no food during the continuance of their lethargy. But with this exception, warm-blooded animals require a constant supply of nutriment, not merely for the maintenance of their proper heat, but also for the repair of the waste resulting from that continuous activity which the uniform temperature of their own bodies enables them to keep up. This is the case with Man and the Mammalia generally, and still more with Birds, whose temperature is higher, and whose movements are more active and energetic. It is also more the case with young animals than with adults; since in the former the changes in the tissues, in consequence of the increase they are undergoing, take place with much more rapidity than in the latter, the bulk of whose bodies remains stationary. Hence, if children, young persons, and adults be shut up together, and deprived of food, the younger will usually perish first, and the adults will survive the longest. The Italian poet Dante has given a terrible picture of such an occurrence, in his history of the imprisonment of Count Ugolino and his children.

141. The difference in the demand for food between the young growing animal and that which has arrived at maturity, is very remarkable in the case of Insects. There are no animals more voracious than the larva or caterpillar; and there are none that can sustain abstinence, with little diminution of their activity, better than the imago or perfect insect. The larvæ of the Flesh-fly, produced from the eggs laid in carrion, are said to increase in weight 200 times in the course of 24 hours; and their voracity is so great as to have caused Linnæus to assert, that three individuals and their immediate progeny (each female giving birth to at least 20,000 young, and a few days sufficing for the production of a third generation) would devour the carcase of a horse with greater celerity than a lion. The larva of the Silk-worm 
weighs, when hatched, about 1-100th of a grain; previously to its first metamorphosis it increases to 95 grains, or 9,500 times its original weight. The comparative weight of the full-grown caterpillar of the Goat-moth to that of the young one just crept out of the egg, is said to be as 72,000 to 1 . For this enormous increase a very constant supply of material is necessary, and many larvæ perish if left unsupplied with food for a single day. On the other hand, a black beetle (Melasoma) has been known to live seven months, pinned down to a board; and another beetle (Scarabæus) has been kept three years without food,-and this without manifesting any inconvenience or loss of activity. There are many perfect insects which never eat after their last change, but die as soon as they have performed their part in the propagation of the race.

142. The nature of the food of animals is as various as the conformation of their different tribes. It always consists, however, of substances that have previously undergone organization. There are some apparent exceptions to this, in the case of animals which seem to derive their support, in part at least, from mineral matter. Thus, the Spatangus (an animal allied to the Echinus, $§ 119$ ) fills its stomach with sand; but it really derives its nourishment from the minute animals which this contains. The Earthworm and some kinds of Beetles are known to swallow earth; but only to obtain from it the remains of vegetable matter that are mixed with it. By some races of Man, too, what seems to be mineral matter is mixed with other articles of food, and is said to be nutritious; this may be beneficial, in part, by giving bulk to the aliment, and thus exciting the action of the stomach ( $\$ 205)$; but it has been found, in one case at least, that the supposed earth consists of the remains of animalcules, and contains no inconsiderable portion of organic matter.

143. There are many instances in which, no obvious supplies of food being afforded, the mode of sustenance is obscure ; and it has been frequently supposed that, in such cases, the animals are sustained by air and water alone. But it will always be found that, where food is taken in no other way, a supply of the microscopic forms of animal or vegetable life is introduced by ciliary action ( $\$ 45)$; and it is on these, indeed, that a large proportion of the lower forms of aquatic animals depend entirely for their support. 
144. The first division of aliments is naturally into those which are derived from the Animal and Vegetable kingdoms respectively. Wherever plants exist, we find animals adapted to make use of the nutritious products they furnish, and to restrain their luxuriance within due limits. Thus among Mammals, the Dugong (an animal having the general form and structure of the whale, but adapted to a vegetable diet) browses upon the sea-weeds that grow beneath the surface of the tropical ocean; the Hippopotamus roots up with his tusks the plants growing in the beds of the African rivers, and fills his huge paunch, not only with these, but with the decaying vegetable matter which he finds in the same situation; the Antelopes, Deer, Oxen, and other Ruminants, crop the herbage of the plains and meadows ; the Giraffe is enabled by his enormous height to feed upon the tender shoots which are above the reach of ordinary quadrupeds ; the Sloths, living entirely in trees, and hanging from their branches, strip them completely of their leaves; the Squirrels extract the kernels of the hard nuts and seeds; the Monkeys devour the soft pulpy fruits; the Boar grubs up the roots and seeds buried under the soil ; the Reindeer subsists during a large part of the year upon a lichen that grows beneath the snow; and the Chamois finds a sufficient supply in the scanty vegetation of Alpine heights. Not less is this the case among ${ }^{\cdot}$ Birds ; but in the classes of Reptiles and Fishes, the number of vegetable-feeders, and consequently the variety of their food, is much less.

145. Among Insects, a very large proportion derive their food entirely from Plants, and many from particular tribes of plants only; so that, if from any cause these should fail, the race may for a time disappear. There is probably not a species of plant which does not furnish nutriment for one or more tribes of insects, either in their larva state or their perfect condition; and in this manner it is prevented from multiplying to the exclusion of others. Thus, on the Oak no less than two hundred kinds of caterpillars have been estimated to feed; and the Nettle, which scarcely any beast will touch, supports fifty different species of insects,-but for which check it would speedily annihilate all the plants in its neighbourhood. The habits and economy of the different races existing on the same plant, are as various as their structure. 
Some feed only upon the outside of the leaves; some upon the internal tissue; others upon the flowers or on the fruit; a few will eat nothing but the bark ; while many derive their nourishment only from the woody substance of the trunk.

146. The excessive multiplication of certain tribes of Insects has sometimes had the effect of devastating an entire country. Thus the "plague of locusts" is not unfrequently repeated in tropical countries, and is dreaded by the inhabitants even more than an earthquake. These insects are of such extreme voracity that no green thing escapes them; and when their numbers are so increased that they fly in masses which look like dark clouds, and cover the ground where they alight for miles together, it may be easily conceived that the devastation they create must produce incalculable injury. The north of Africa and the west of Asia are the countries most infested by these pests. It is related by Augustin, that a plague, induced partly by the famine they had created, and partly by the stench occasioned by their dead bodies, carried off 800,000 inhabitants from the kingdom of Numidia and the adjacent parts. They occasionally attack the south of Europe. It is recorded that Italy was devastated by them in the year 591 ; and that a prodigious number both of men and beasts perished from similar causes,-no less than 30,000 persons in the kingdom of Venice alone. These tremendous swarms usually advance towards the sea; and being there checked, and having completely exhausted the country behind them, they themselves die of famine, or are blown into the sea by a gale. In 1784 and 1797, they devastated Southern Africa; and it is stated by Mr. Barrow (in his Travels in that country) that they covered a surface of 2,000 square miles; that, when cast into the sea by a strong wind from the north-east, and washed upon the beach, they formed a line fifty miles long, and produced a barrier along the coast three or four feet high ; and that, when the wind again changed, the stench created by the putrefaction of their bodies was perceived at a distance of 150 miles inland. A similar event occurred in the Barbary States in 1799 , and was followed, as in the other cases, by a plague.

147. We have occasionally an example of similar devastation in our own country, though on a smaller scale. Thus, a few years ago, the turnip-crops of some parts of England 
were almost entirely destroyed by the larvæ of an insect called the "turnip-fly." The parent insects were seen buzzing over the fields, and depositing their eggs in the plants, which they do not themselves employ as food; and in a few days all the soft portions of the leaves were destroyed, and nothing but the skeletons and stalks were left.- Some kinds of timber occasionally suffer to no less an extent from the devastations of insects, whose operations are confined to the wood, and do not manifest themselves externally, until the tree is seen to languish and at last to die. The pine-forests of the Hartz mountains in Germany have been several times almost destroyed by the ravages of a single species of beetle, less than a quarter of an inch in length. The eggs are deposited beneath the bark; and the larvæ, when hatched, devour the sapwood and inner bark (the parts most concerned in the functions of vegetation) in their neighbourhood. It was estimated that, in the year 1783, a million and a half of pines were destroyed by this insect in the Hartz alone ; and other forests in Germany were suffering at the same time. The wonder is increased, when it is stated that as many as 80,000 larvæ are sometimes found on a single tree.

148. But every class in the Animal Kingdom has its carnivorous tribes, which are adapted to restrain the too rapid increase of the vegetable-feeders (by which a scarcity of their food would soon be created), or to remove from the earth the decomposing bodies that might otherwise be a source of disease or annoyance. The herblvorous races, being for the most part very prolific, would very rapidly increase to such an extent as to produce an absolute famine, if not kept in check by the races appointed to limit their multiplication. Thus, the myriads of Insects which find their subsistence in our forest-trees, if allowed to increase without restraint, would soon destroy the life that supports them, and must then all perish together; but another tribe (that of the insectivorous Birds, as the woodpecker) is adapted to derive its sulsistence from them, and thus to keep their numbers within salutary bounds. Their occasional multiplication to the enormous extent mentioned in the preceding paragraphs, is probably due in general to the absence of the races that should keep them in check. This may occur from accidental causes, or may be produced by the interference of Man. Thus, 2 set of L 2 
ignorant farmers have imagined that a neighbouring rookery was injurious to them, because they saw the rooks hovering over the newly-sown corn-fields, and seeming to pick the grains out of the ground ; and having extirpated the rookery, they have found in the course of a year or two that they have done themselves an immense injury, - the roots of their corn and grasses being devoured by the grubs of cockchafers and other insects, the multiplication of which was before prevented by the rooks, whose natural food they are.

149. On the other hand, by an intelligent application of this principle, the excessive multiplication of insects has been prevented where it had already commenced. Thus, no means of extirpating the larvæ of the turnip-fly was found so successful, as turning into the fields a number of ducks, which quickly removed them from the plants. And in the island of Mauritius, the increase of locusts, which had been accidentally introduced there, and which were becoming quite a pest, was checked by the introduction from India of a species of bird, the grakle, which feeds upon them.

150. Of the carnivorous tribes themselves, however, the increase might be so great as to destroy all the sources of their food, were it not that they are kept in check by others, larger and more powerful than themselves, which, not being prolific, are not likely ever to gain too great a power. Thus, among birds, the eagles, falcons, and hawks rear only two or three young every year, whilst many of the smaller birds produce and bring up four or five times that number.-The following is a curious instance of the system of checks and counterchecks, by which the "balance of power" is maintained amongst the different races. A particular species of moth having the fir-cone assigned to it for the deposition of its eggs, the young caterpillars, coming out of the shell, consume the cone and superfluous seed; but, lest the destruction should be too great, another insect of the ichneumon kind lays its eggs in the caterpillar, inserting its long tail in the openings of the cone until it touches the included insect, its own body being too large to enter. Thus it fixes upon the caterpillar its minute egg, which, when hatched, destroys it.

151. The peculiarity of the agency of Insects, in the economy of nature, has been justly remarked to consist in their power of very rapid multiplication, in order to accomplish a 
certain object, and then in their as rapidly dying off. In this respect they resemble the Fungi among plants. (Botany, § 789.)

152. There are great variations in the degree of power possessed by animals of different species to sustain abstinence from food, which appear to be related to their respective habits of life ; such as most easily obtain a constant supply of food being immediately dependent upon it, and vice vers $\hat{Q}$. Thus, among the larvæ of Insects, those that feed upon vegetables or dead animal matter (in the neighbourhood of which their eggs are usually deposited by the parent) speedily die if placed out of reach of their aliment; whilst those that lie in wait for living prey, the supply of which is uncertain, are able to endure a protracted abstinence, even to the extent of ten weeks, without injury. Again, carnivorous Birds and Mammals are generally able to exist for some time without food; their natural habits leading them to glut themselves upon the carcase of the animal they have destroyed, in such a manner as to prevent them from requiring any new supply for some time : thus the wild cat has been kept twenty days without food, the dog has lived for thirty-six days in the same circumstances, and the eagle for a similar period. But some herbivorous animals, such as the camel and the antelope, whose habits are such as to keep them out of the reach of food for several days together, are able to endure a similar abstinence; whilst among the insectivorous Mammals, which naturally take food often, and but little at a time, the power of abstinence is much less, - the mole, for instance, perishing in confinement, if not fed once a day, or even more frequently.

153. We have next to consider the different substances used as food, in regard to their chemical composition; and to inquire for what purposes in the nutrition of the body they are respectively destined. The Vegetalle tissues are chiefly made up of the three components, oxygen, hydrogen, and carbon; the oxygen and hydrogen having the same proportions as in water. Their composition being thus nearly the same as that of starch, gum, and sugar (into which, indeed, they may for the most part be converted by a simple chemical process), alimentary substances of this kind form a natural group to which we may give the name of Saccharine (sugary). - But in many vegetable substances used as food, there is a considerable quantity of oily matter, stored up in cells; and the same kind 
of matter constitutes the principal part of the fat of animals. Of these oily and fatty matters, also, the chemical elements, oxygen, hydrogen, and carbon, are the only ingredients ; but they are combined in proportions different from the last, the two latter predominating considerably. Hence they constitute another group of alimentary materials, to which the term Oleaginous may be given.-Lastly, most Vegetables contain, in greater or less amount, certain compounds which consist of the four elements, oxygen, hydrogen, carbon, and nitrogen, of which the animal tissues are composed. These compounds exist most largely in the corn-grains, and also in the seeds of the pea and bean tribe; but there are few vegetable substances used as food by animals, that do not contain them in some small amount. The gluten of wheat, the legumin of peas, and other vegetable substances of this kind, together with the flesh of animals, the composition of which ( $\$ 13)$ is identical with theirs, are united into a third group, to which the name Albuminous is given.-We cannot properly include in this group, however, the gelatinous portions of the animal tissues, which exist largely in gristle, bone, the skin, and other parts; because gelatin (the substance that forms glue), though it agrees with albumen in being made up of the four ingredients just named, differs from it extremely in the proportions of those elements $(\S 19)$; so that, although there is good reason to believe that gelatin may be formed out of albumen, it does not seem that any albuminous compound can be formed out of gelatin. Hence we must consider the gelatinous compounds separately.

154. Of these four groups, the last two are distinguished as azotized compounds, or substances that contain azote or nitrogen; whilst the first two are spoken of as non-azotized, being destitute of this element. The distinction is a very important one ; and must be kept steadily in view in considering the ultimate destination of each kind of food. It is obvious from what has been already stated as to the composition of the animal tissues ( $\S 13-21)$, that azotized compounds must supply the chief materials for their nutrition and re-formation. The non-azotized substances must be for the most part destined, unless converted into azotized compounds within the living body, either to be simply deposited in its interstices, or to be thrown off from it again without ever actually forming part of its organised structure. 
155. Now, in regard to the non-azotized, or the saccharine and oleaginous groups of alimentary substances, it appears to be an established fact, that none of the higher animals can be permanently supported upon them alone. Thus, dogs that have been fed on sugar and starch only, do not survive long; and it is evident, before their death, that their tissues are gradually undergoing decay. It has been thought that such results might be partly explained upon the fact, that animals fed upon one simple substance soon become disgusted with it, and will even refuse it altogether; but the experiments have been repeated with a combination of various non-azotized substances, and the same result has occurred. Still it is too much to affirm, as some have done, that these substances do not contribute in any degree to the nutrition of the animal tissues; since there is ample evidence that the presence of fattymatter in the blood is a condition essential to the production of newly forming tissue; and we find that either oleaginous substances, or substances belonging to the saccharine group which can be readily converted into fat within the body, constitute an important part of the food of Man, and of animals generally. ${ }^{1}$

156. That such a conversion can take place, has been demonstrated by experiments carefully conducted upon bees, which have been found to generate wax when fed upon sugar only; and also upon cows, which give off in their milk so much larger a quantity of butter than can be produced at the expense of the fat contained in their food, that there is no other mode of accounting for its presence, than by regarding it as generated from the starchy portion of their diet. And the fattening power of starehy and saccharine articles of diet is well known to breeders of cattle; though the articles which contain oily matter in addition seem to possess a higher value in this respect.

157. But if these non-azotized compounds, which exist so largely in the food of herbivorous animals, are not destined to form any other permanent part of the animal organism than the oleaginous contents of the fat-cells ( $\$ 46)$, the question again arises, - what becomes of them? It is not enough

1 The value of cod-liver oil, which is now so extensively used in the treatment of diseases of imperfect nutrition; seems to depend upon the readiness with which it can be digested and assimilated, so as to furnish the supply of fat required by the formative processes. 
to say that they are deposited as fat; since it is only when a large quantity of them is taken in, that there is any increase in the quantity of fat already in the body. We shall hereafter see that they are used up in the process of respiration, one great object of which is, to produce a certain amount of heat, sufficient to keep up the temperature of the body, in warm-blooded animals, to a high standard. We might almost say with truth, that a great part of the oleaginous and saccharine principles is burned within the body, for this purpose. The process will be hereafter considered more in detail $(\$ \S 412,413)$; and at present we need only stop to remark upon the adaptation between the food provided for animals in different climates, and the amount of heat which it is necessary for them to produce. Thus the bears, and seals, and whales, from which the Esquimaux and the Greenlander derive their support, have an enormous quantity of fat in their massive bodies : this fat is as much esteemed as an article of food amongst these people, as it would be thought repulsive by the inhabitants of southern climates; and by the large quantity of it they consume, they are able to support the bitterness of an Arctic winter, without appearing to suffer more from the extreme cold than do the residents in more temperate climes during their winter. On the other hand, the antelopes, deer, and wild cattle, which form a large proportion of the animal food of savage or half-cultivated nations inhabiting tropical regions, possess very little fat; and the comparatively small supply of carbon and hydrogen, of which the combustion is required to keep up the bodily temperature of the inhabitants of those regions, is derived from the flesh of these animals, in the manner that will be presently explained.

158. The application of the substances forming the albuminous group, to the support of the animal body, by affording the materials for the nutrition and re-formation of its tissues, needs little explanation. The proportions of the four ingredients of which they are all composed, are so nearly the same, that no essential difference appears to exist among them ; and it is a matter of little consequence, except as far as the gratification of the palate is concerned, whether we feed upon the flesh of animals (syntonin, $\$ 16$ ), upon the white of egg (albumen, § 13), the curd of milk (casein, § 15), the grain of wheat (gluten), or the seed of the pea (legumin). All these 
substances are reduced in the stomach to the form of albumen; which is the raw material out of which the various fabrics of the body are constructed. But the rule holds good with regard to these also, that by being made to feed constantly on the same substance,-boiled white of egg for instance, or meat deprived of the principle that gives it flavour, - an animal may be effectually starved; its disgust at the food being such, that even if it be swallowed it is not digested. It is very interesting to remark that, in the only instance in which Nature has provided a single article of food for the support of the animal body, she has mingled articles from all the three preceding groups. This is the case in Milk; which contains a considerable quantity of the albuminous substance, casein, that forms its curd; a good deal of oily matter, the butter; and no inconsiderable amount of sugar, which is dissolved in the whey. The proportions of these vary in different Mammalia, being related as it would seem to the habits of the young animal thus sustained, while they depend in part upon the nature of the food supplied to the animal that forms the milk; but the three substances are thus combined in every instance.

159. But although the greater part of the organised tissues of animals have a composition nearly allied to that of albumen, many of them also contain a large quantity of gelatin ( $\$ 19)$. It seems certain that this gelatin may be produced out of albuminous substances; since in animals that are supported on these alone, the nutrition of the gelatinous tissues does not seem to be impaired. But it appears equally certain, that gelatin cannot be applied to the nutrition of the albuminous tissues. Many series of experiments have been made on this subject, with a view of determining how far gelatin-soup made from crushed bones (such as that which long constituted a principal article of diet in the hospitals of Paris) is adequate for the support of the body in health. The result of these has been uniformly the same, - namely, that although gelatin may be advantageously mixed with albumen, fibrin, gluten, \&c., and those other ingredients which exist in meat-soup and bread, yet that, when taken alone, it has little (if any) more power of sustaining life, than sugar or starch possesses. Although it might have been thought likely that gelatin employed as food might be applied within the body to the nutrition of its gelatinous tissues, yet there 
is strong reason to believe that these, like the albuminous, are formed at the expense of the albuminous matter of the blood, and that gelatin thus introduced undergoes a rapid decomposition, yielding up a considerable part of its carbon and hydrogen to the combustive process, which is the only function to which it affords any substantial pabulum. Consequently the current idea regarding the nutritive value of jellies of various kinds, has little or no real foundation.

160. It has been already stated $(\S 68)$ that all the living tissues of the body are continually undergoing a sort of death and decay; and that they do this the more rapidly, in proportion as they are called upon for the discharge of their functions. The need of material capable of replacing that which has been lost, is consequently the chief source of the constant demand for aliment. Even in young, actively growing animals, the quantity required for the increase of their bodies constitutes but a very small proportion of that which is taken in ; of the remainder, a part is at once rejected as indigestible; and the rest is appropriated to the repair of the waste which is continually going on. This waste is much greater in young animals than in adults; for all their vital processes are more actively and energetically performed : their movements are quicker in proportion to their size ; and injuries are more speedily repaired. To remove the products of this decomposition is the special object of the various processes of excretion; and among these, the respiration, by which a large quantity of carbon and hydrogen is carried off in the form of carbonic acid and water, is of the most constant importance, on account of the heat which it thus enables the animal body to maintain. This temperature, in Carnivorous animals, appears to be sufficiently kept up by the combustion of the carbon and hydrogen set free by the decay (or metamorphosis, as it may be termed) of their tissues; but this combustion goes on with much more rapidity, in consequence of their almost unceasing activity, than it does in the Herbivorous animals, which lead comparatively inactive lives. Every one who has visited a menagerie must have noticed the continual restlessness of the Tigers, Leopards, Hyenas, \&c., which keep pacing from one end of their narrow cages to the other ; and it would seem as if this restlessness were a natural instinct, impelling them to use muscular exer- 
tion sufficient for the metamorphosis of an adequate amount of tissue, that enough carbon and hydrogen may be set free for the support of the respiratory process. And we see a corresponding activity in the Human hunters of the swift-footed antelope and agile deer, which answers a similar purpose ; and which is remarkably contrasted with the stupid inertness of the inhabitants of the frigid zone, that is only occasionally interrupted by the necessity of securing the supplies of food afforded by the massive tenants of their seas.

161. The nutrition of the Carnivorous races may, then, be thus described. The bodies of the animals upon which they feed, contain flesh, fat, \&c., in nearly the same proportion as their own; and all, or nearly all, the aliment they consume, goes to supply the waste in the fabric of their own bodies, being converted into its various forms of tissue. After having remained in this condition for a certain time, varying according to the use that is made of them, these tissues undergo another metamorphosis, which ends in restoring them to the condition of inorganic matter; and thus give back to the mineral world the materials which were drawn from it by plants. Of these materials, part are burned off, as it were, within the body, by union with the oxygen of the air taken in through the lungs, from which organs they are discharged in the form of carbonic acid and water: the remainder are carried off in the liquid form by other channels. Hence we may briefly express the destination of their food in the following manner :-

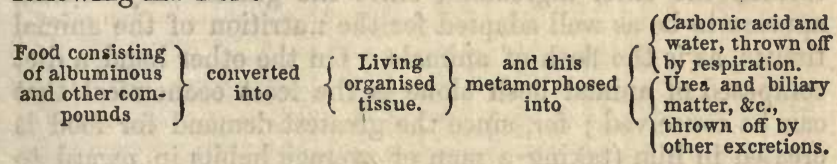

162. But in regard to the Herbivorous animals, the case is different. They perspire much more abundantly, and their temperature is thus continually kept down ( $\$ 372)$. They consequently require a more active combustion, to develop sufficient bodily heat; and the materials for this are supplied, as we have seen, by the non-azotized constituents of their food, rather than by the metamorphosis of their own tissues, which takes place with much less rapidity than in the carnivorous tribes. Hence we may thus express the 
destination of this part of their food ; that of the albuminous matters, here much smaller in amount, being the same as in the preceding case :-

Starch, oil, and partly (Fatty and ) but chiefly (Carbonic acid and water, $\left.\begin{array}{c}\text { other non-azo- } \\ \text { tized compounds }\end{array}\right\} \begin{gathered}\text { converted } \\ \text { into }\end{gathered}\left\{\begin{array}{c}\begin{array}{c}\text { other animal } \\ \text { tissues, }\end{array} \\ \begin{array}{l}\text { thrown off } \\ \text { directly as as }\end{array}\end{array}\left\{\begin{array}{l}\text { disengaged by the respi- } \\ \text { ratory process. }\end{array}\right.\right.$

The proportion of the food deposited as fat, will depend in part upon the surplus which remains, after the necessary supply of materials has been afforded to the respiratory process. Hence, the same quantity of food being taken, the quantity of fat will be increased by causes that check the perspiration, and otherwise prevent the temperature of the body from being lowered, so that there is need of less combustion within the body to keep up its heat. This is consistent with the teachings of experience respecting the fattening of cattle ; for it is well known that this may be accomplished much sooner, if the animals are shut up in a warm dwelling and are covered with cloths, than if they are freely exposed in the open air.

163. Now the condition of Man may be regarded as intermediate between these two extremes. The construction of his digestive apparatus, as well as his own instinctive propensities, point to a mixed diet as that which is best suited to his wants. It does not appear that a diet composed of ordinary vegetables only, is favourable to the full development of either his bodily or his mental powers; but this cannot be said in regard to a diet of which the corn-grains furnish the chief ingredient, since the gluten they contain appears to be as well adapted for the nutrition of the animal tissues, as is the flesh of animals. On the other hand, a diet composed of animal flesh alone is the least economical that can be conceived; for, since the greatest demand for food is created in him (taking a man of average habits in regard to activity and to the climate under which he lives) by the necessity for a supply of carbon and hydrogen to support his respiration, this want may be most advantageously fulfilled by the employment of a certain quantity of non-azotized food, in which these ingredients predominate. Thus it has been calculated that, since fifteen pounds of flesh contain no more carbon than four pounds of starch, a savage with one animal and an equal weight of starch, could support life for the same length of time during which another restricted to animal 
food would require five such animals, in order to procure the carbon necessary for respiration. Hence we see the immense advantage as to economy of food, which a fixed agricultural population possess over the wandering tribes of hunters which still people a large part both of the Old and New Continents.

164. The following Table exhibits the proportions of albuminous, starchy or saccharine, fatty, and saline substances, contained in various articles ordinarily used as food by Man; together with the proportion which water bears in each case to the solid constituents of the food, which becomes a most important element of consideration when the nutritive value of different kinds of food is compared :-

\begin{tabular}{|c|c|c|c|c|c|c|c|c|}
\hline Substances, 100 parts. & 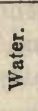 & 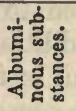 & 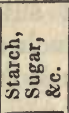 & 离 & 营 & 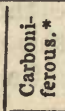 & 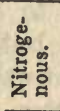 & 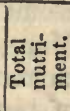 \\
\hline Human Milk ............... & 89 & 3.5 & 4.2 & 3.0 & 0.2 & 11.4 & 3.5 & 14.9 \\
\hline Cow's Milk .................. & 86 & 4.5 & 5.0 & 4.1 & 0.7 & 14.8 & 4.5 & 19.3 \\
\hline Skimmed Milk ............ & 87 & 4.5 & 5.0 & 2.7 & 0.7 & 11.5 & 4.5 & 16.0 \\
\hline Butter Milk ............... & 87 & 4.5 & 5.0 & 0.5 & 0.7 & 6.0 & 4.5 & 10.5 \\
\hline Beef and Mutton .......... & 73 & 19.0 & ... & 5.0 & 2.0 & 12.0 & 19.0 & 31.0 \\
\hline Veal & 77 & 19.0 & $\ldots$ & 1.0 & 0.6 & 2.4 & 19.0 & 21.4 \\
\hline Poultry......................... & 74 & 21.0 & ... & 3.0 & 1.2 & 7.2 & 21.0 & 282 \\
\hline Bacon & 20 & 0.8 & $\ldots$ & 70.0 & 1.3 & 168.0 & 0.8 & 168.8 \\
\hline Cheese (Cheddar) .......... & 36 & 29.0 & $\cdots$ & 30.0 & 4.5 & 72.0 & 29.0 & 101.0 \\
\hline ," (Skimmed).......... & 44 & 45.0 & $\cdots$ & 6.0 & 5.0 & 14.4 & 45.0 & 59.4 \\
\hline 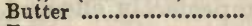 & 15 & $\because$ & 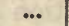 & 83.0 & 2.0 & 199.0 & & 199.0 \\
\hline 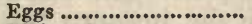 & 74 & 14.0 & $\ldots$ & 10.5 & 1.5 & 25.0 & 14.0 & 39.0 \\
\hline White of Egg ................ & 78 & 20.0 & $\cdots$ & 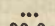 & 1.6 & $\ldots$ & 20.0 & 20.0 \\
\hline Yolk of Egg ................. & 52 & 16.0 & ... & 30.0 & 1.3 & 72.0 & 16.0 & 88.0 \\
\hline White Fish.................... & 79 & 19.0 & $\ldots$ & 1.0 & 1.2 & 2.4 & 19.0 & 21.4 \\
\hline Salmon $. . . . . . . . . . . \ldots \ldots \ldots . .$. & 78 & 17.0 & $\ldots$ & 4.0 & 1.4 & 9.6 & 17.0 & 26.6 \\
\hline 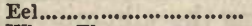 & 80 & 10.0 & & 8.0 & 1.3 & 19.2 & 10.0 & 29.2 \\
\hline Wheat Flour ................ & 15 & 11.0 & 70.0 & 2.0 & 1.7 & 74.8 & 11.0 & 858 \\
\hline Barley-meal.................... & 15 & 10.0 & 70.0 & 2.4 & 2.0 & 75.8 & 10.0 & 85.8 \\
\hline Oat-meal .......................... & 15 & 12.0 & 62.0 & 6.0 & 3.0 & 76.4 & 12.0 & 88.4 \\
\hline Rye-meal ........................ & 15 & 9.0 & 66.0 & 2.0 & 1.8 & 70.8 & 9.0 & 79.8 \\
\hline Indian-meal ............... & 14 & 9.0 & 65.0 & 8.0 & 1.7 & 84.2 & 9.0 & 93.2 \\
\hline Rice & 14 & 7.0 & 76.0 & 0.3 & 0.3 & 76.7 & 7.0 & 83.7 \\
\hline Haricots ......................... & 19 & 23.0 & 45.0 & 3.0 & 3.6 & 52.2 & 23.0 & 75.2 \\
\hline 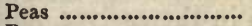 & 13 & 22.0 & 58.0 & 2.0 & 3.0 & 62.8 & 22.0 & 84.8 \\
\hline Beans....................... & 14 & 24.0 & 44.0 & 1.4 & 3.6 & 47.4 & 24.0 & 71.4 \\
\hline Lentils ........................... & 14 & 29.0 & 44.0 & 1.5 & 2.3 & 47.6 & 29.0 & 76.6 \\
\hline Wheat-bread ................ & 44 & 9.0 & 49.0 . & 1.0 & 2.3 & 51.4 & 9.0 & 60.4 \\
\hline Rye-bread .................... & 48 & 5.3 & $46.0^{\circ}$ & 1.0 & 1.4 & 48.4 & 5.3 & 53.7 \\
\hline Potatoes ....................... & 74 & 2.0 & 23.0 & 0.2 & 0.7 & 23.5 & 2.0 & 25.5 \\
\hline Green Vegetables .......... & 86 & 2.0 & 4.0 & 0.5 & 0.7 & 5.0 & 2.0 & 7.0 \\
\hline Arrow-root ....................... & 18 & $\cdots$ & 82.0 & $\ldots$ & $\ldots$ & 82.0 & $\ldots$ & 82.0 \\
\hline
\end{tabular}

* The value of the Fat is stated in this column according to its heating equivalent of starch, which is larger in the ratio of 24 to 1 . Hence, in the last column, the proportion of nutriment in aliments containing fat, comes to be greater than the weight of their solids would indicate. 
Those articles of food in which the nitrogenous compounds predominate, are especially fitted for the maintenance of the solid fabric of the body ; whilst those in which the carbonaceous compounds are in largest excess, are those which are most effective as supplying materials for the combustive process. Conspicuous among the former are the various kinds of animal flesh, as also the white of eggs ; whilst among the latter the most noticeable are bacon and butter, rice and potatoes, the former consisting almost wholly of fat, the latter being chiefly composed of starch. Of all single articles of food, good wheaten bread, in which the proportion of nitrogenous to carbonaceous components is about as 5.7 to 1 , seems to be the one best suited to the ordinary wants of Man ; but this acquires much additional value from the concurrent use of a moderate amount of fatty matter in the form of butter.

165. If the more highly azotized forms of food be employed exclusively, a great excess of them must be consumed to supply the carbon needed for respiration; whilst if the more carbonaceous kinds of food be used as the sole sustenance, unless the quantity ingested be large enough to afford the requisite supply of azotized material for the maintenance of the tissues, their nutrition must be imperfectly effected, and the strength must fail. Not only in the instance just cited, but in a variety of others, the instincts of mankind have led to such a combination of different articles of diet, as includes in their appropriate proportions the albuminous, the saccharine, and the oleaginous principles. Thus with meat we eat potatoes; and with the white meats which are deficient in fat, we eat bacon. Wè use melted butter with most kinds of fish, or fry them in oil ; whilst the herring, the salmon, and the eel, are usually fat enough in themselves, and are dressed and eaten alone. A similar adjustment is made when we mix eggs and butter with sago, tapioca, and rice; when we add oil and the yolk of an egg to salad; when we boil rice with milk, and combine cheese with maccaroni. Bacon and greens, and pork and pease-pudding, again, are combinations founded in taste, which approve themselves to the judgment; as is also the Irish dish termed kolcannon, consisting of potatoes and cabbage, with a little bacon or fat pork. So are the mixture so common in Ireland and Alsace, of butter- 
milk or curdled-milk with potatoes ; and the combination of rice and fat, which is the staple of the diet of many Eastern nations. Even the morsel of butter or the bit of cheese which the English labourer eats with his hard-earned bread, are not matters of luxury, but have a positive importance ; and the existence of these tastes and habits shows how by long experience Man has at last learned to adjust the composition of his food, so as best to maintain the health and vigour of his body. With a difference of requirement comes a difference of tastes. Thus men who are going through a very laborious course of exertion, prefer meat to bread or vegetables, feeling it to be more sustaining to their strength. On the other hand, those who are continuously exposed to the severity of an Arctic winter, eat with relish large masses of fat, on which they would look with disgust under other circumstances. The quantity of work which a man can do, and his power of sustaining extreme cold, both depend in great part, as has now been abundantly proved, upon the adequacy of the sustenance he takes : the demand, in the first case, being for albuminous material to supply the waste of his tissues; whilst in the second it is for combustive material suitable to generate heat in large measure,-a purpose which is far more efficiently answered by oleaginous substances, than by those of a starchy or saccharine nature. Experience further shows that the healthy condition of the blood of Man can only be maintained by the use of fresh vegetables as part of his ordinary diet. When these are withdrawn for any length of time, the disease known as Scurvy is certain to appear, unless lemon-juice or some other efficacious antiscorbutic be employed as a substitute. This is a fact of the utmost importance in provisioning ships for long voyages; the tendency to scurvy being increased by confinement and insufficient ventilation, and by the exclusive use of salt provisions.

166. Besides these organic substances, there are certain Mineral ingredients, which may be said to constitute a part of the food of Animals ; being necessary to their support, in the same manner as other mineral substances are necessary to the support of Plants. Of this kind are common salt, and also phosphorus, sulphur, lime, and iron, either in combination or separate. - The uses of Salt are very numerous and 
important. It exists largely in the blood, and in the various animal fluids which are secreted from it; and it is also an essential ingredient of most of the solid tissues. Its presence obviously tends to prevent that spontaneous decomposition to which organic substances are liable. Phosphorus is chiefly required to be united with fatty matter, to serve as the material of the nervous tissue; and to be combined with oxygen and lime, to form the bone-earth by which the bones are consolidated. Sulphur exists in small quantities in several animal tissues; but its part seems by no means so important as that performed by phosphorus. Lime is required for the consolidation of the bones, and for the production of the shells and other hard parts that form the skeletons of the Invertebrata. Of the limestone rocks of which a great part of the crust of our globe is composed, a very large proportion is made up of the remains of animals that formerly existed in the ocean. Thus some almost entirely consist of masses of Coral, others of beds of Shells, and others of the coverings of minute Foraminifera ( $\$ 131)$. To these mineral ingredients we may also add Iron, which is a very important element in the red blood of Vertebrated animals.

167. These substances are contained, more or less abundantly, in most articles generally used as food; and where they are deficient, the animal suffers in consequence, if they be not supplied in any other way. Common Salt exists, in no inconsiderable quantity, in the flesh and fluids of animals, in milk, and in the egg : it is not so abundant, however, in plants ; and the deficiency is usually supplied to herbivorous animals by some other means. Thus salt is purposely mingled with the food of domesticated animals ; and in most parts of the world inhabited by wild cattle, there are spots where it exists in the soil, and to which they resort to obtain it ; such are the "buffalo-licks" of North America. Phosphorus exists also in the yolk and white of the egg, and in milk, - the substances on which the young animal subsists during the period of its most rapid growth; it abounds not only in many animal substances used as food, but also (in the state of phosphate of lime or bone-earth) in the seeds of many plants, especially the grasses ; and in smaller quantities it is found in the ashes of almost every plant. When flesh, bread, fruit, and husks of grain, are used as the chief articles 
of food, more phosphorus is taken into the body than it requires ; and the excess has to be carried out in the excretions. Sulphur is derived alike from vegetable and animal substances. It exists in flesh, eggs, and milk; also in the azotized compounds of plants ; and (in the form of sulphate of lime) in most of the river and spring water that we drink. Iron is found in the yolk of egg, and in milk, as well as in animal flesh; it also exists, in small quantities, in most vegetable substances used as food by man,- such as potatoes, cabbage, peas, cucumbers, mustard, \&c. ; and probably in most articles from which other animals derive their support.

168. Lime is one of the most universally diffused of all mineral bodies; there being very few animal or vegetable substances in which it does not exist. It is most commonly taken in, among the higher animals, combined with phosphoric acid, so as to form bone-earth, in which state it exists largely in the seeds of most grasses. A considerable quantity of lime exists, moreover, in the state of carbonate and sulphate, in all hard water.

169. When an unusual demand exists for lime, however, for a particular purpose, an increased supply must be afforded. Thus a hen preparing to lay, is impelled by her instinct to eat chalk, mortar, or some other substance containing the carbonate of lime which is required for the consolidation of the shell; and if this be withheld, the egg is soft, its covering being composed of animal matter alone, not consolidated by the deposit of earthy particles. The thickness of the shells of aquatic Mollusks depends greatly upon the quantity of lime in the surrounding water. Those which inhabit the sea, find in its waters as much as they require; but those that dwell in fresh-water lakes, which contain but a small quantity of lime, form very thin shells ; whilst, on the other hand, those that inhabit lakes in which, from peculiar local causes, the water is loaded with calcareous matter, form shells of remarkable thickness.

170. The mode in which the Crustacea, whose calcareous shell is periodically thrown off ( $(99)$, are able to renew it with rapidity, is very curious. There is laid up in the walls of their stomachs a considerable supply of calcareous matter, in little concretions, which are commonly known as "crabs" eyes." When the shell is cast, this matter is taken up by 
the blood, and is thrown out from the surface, mingled with animal matter. This hardens in a day or two, and the new covering is complete. The concretions in the stomach are then found to have disappeared; but they are gradually replaced, before the supply of lime they contain is again required.

\section{CHAPTER IV \\ LIGESTION AND ABSORPTION.}

171. Having now considered the nature of the food of Animals, and the sources from which it is obtained, we have next to consider the process by which the aliment is received into their bodies, and prepared to form a part of their own fabric. This process, termed Digestion, is naturally divided, among the higher animals at least, into various stages. In the first place, there is the prehension or laying hold of the aliment, and its introduction into the mouth or entrance to the digestive cavity. In the mouth it usually undergoes a preparation; which consists partly in its being cut, ground, or crushed, by mechanical action, into minute pieces; and partly in the working-up of these pieces with a fluid that is poured into the mouth, - the saliva. These two processes are termed mastication and insalivation; similar processes are performed, in some animals, in a part of the digestive tube intermediate between the mouth and the stomach, and even in the latter itself. The stomach is usually situated at some distance from the mouth, and is connected with a tube called the oesophagus or gullet; and the passage of the food into this, constituting the act of swallowing, is termed deglutition. The food, having arrived in the stomach, is acted-upon by a peculiar fluid which it contains, and much of its alimentary portion is dissolved, so that a pulpy mass is formed which is termed chyme; hence this process, which is the first stage of digestion properly so called, is termed chymification or the manufacture of chyme. The chyme, which passes into the intestines, is further acted-on by secretions that are poured into them ; and a certain nutritive combination of albuminous 
and fatty matters, termed chyle, is separated from the matters that are to be thrown off: this process, which is the second stage of true digestion, is termed chylification. The rejected portions of the food, with secretions poured into the alimentary. canal, find their way out through the intestinal tube; and are voided at its terminal orifice by the act of defecation. And lastly, the nutritive materials are taken up by absorption into vessels that are distributed upon the walls of the digestive cavity, and undergo a gradual change, by which they are converted into blood. These two processes are called absorption and sanguification (or manufacture of blood). Each of the foregoing stages will now be separately considered.

\section{Prehension of Food.}

172. The introduction of aliment within the entrance to the digestive cavity is accomplished in various methods in different animals. In the Mammalia in general, the aperture of whose mouth is guarded by fleshy lips, these, with the jaws and teeth, are the chief instruments of this operation. But in Man and the Monkey tribe the division of labour is carried further; the food being laid hold of by the anterior members, or hands, and by them carried to the mouth. Where the hand has the power of grasping, and especially where the thumb can be opposed to the fingers, the action of a single member is sufficient; but there are several animals which, like the Squirrel, use both limbs conjointly to hold their food, the extremity not having itself the power of grasping. The Ant-eaters, Woodpeckers, Chameleons, and other insect-eating animals, obtain their food by means of a long extensible tongue; this either serving to transfix the insect, or being covered with a viscid saliva which glues it to the surface. The Giraffe uses its long tongue to lay hold of the young shoots on which it browses; and the Elephant employs its trunk, which is nothing else than a prolonged nose, for every kind of prehension (fig. 82). Many of the Invertebrata are furnished with little appendages round their mouths by

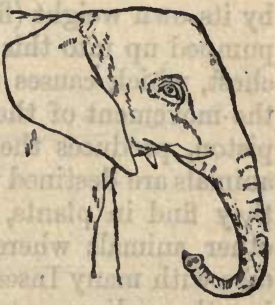

Fig. 82

HEAD OP ELEPHANT. which the food is conveyed into them; such are the palpi M 2 
of Insects, of which a pair is attached to each jaw (fig. 84); the tentacula of Mollusks, which are sometimes extremely prolonged, as in the Cuttle-fish tribe (fig. 85); and the similar organs of the polypes (fig. 71).

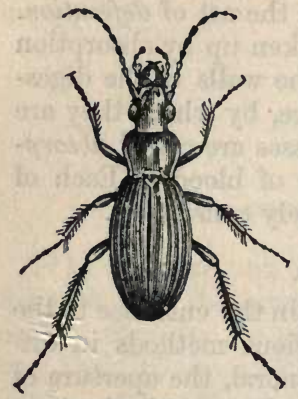

Fig. 83.-CARABUS.

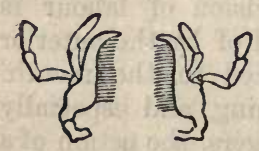

Fig. 84. JAws OF THE SAME INSECT.

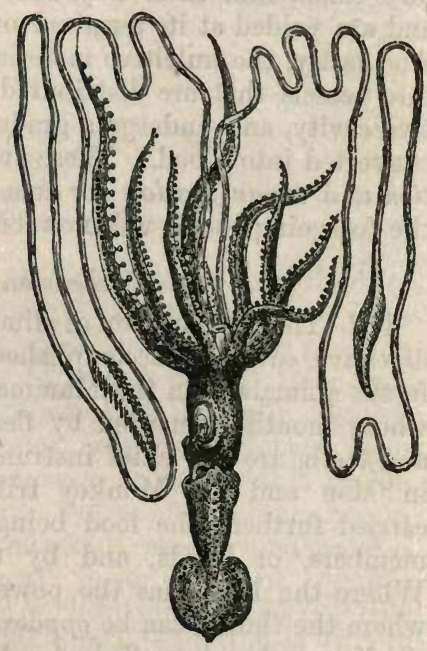

Fig. 85.-LoLigopsis.

173. The reception of liquids is accomplished in two ways. Sometimes the liquid is made to fall into the mouth, simply by its own weight (fig. 86); in other instances it is drawn or pumped up into this cavity, - either by the expansion of the chest, which causes a rush of air towards the lungs, - or by the movement of the tongue, which, being drawn back like a piston, produces the action of sucking. Some of the lower animals are destined to be entirely supported by liquids which they find in plants, or which they draw from the bodies of other animals whereon they live as parasites. This is the case with many Insects ; and their mouth, instead of presenting the ordinary structure, is formed into a sort of tube or trunk, very much extended, through which the juices are drawn up according to the wants of the animal. Such a conformation exists in the butterfly and moth tribe, whose 
trunk, when not in use, is coiled up in a spiral beneath the head; as is shown in fig. 87, representing the head of a
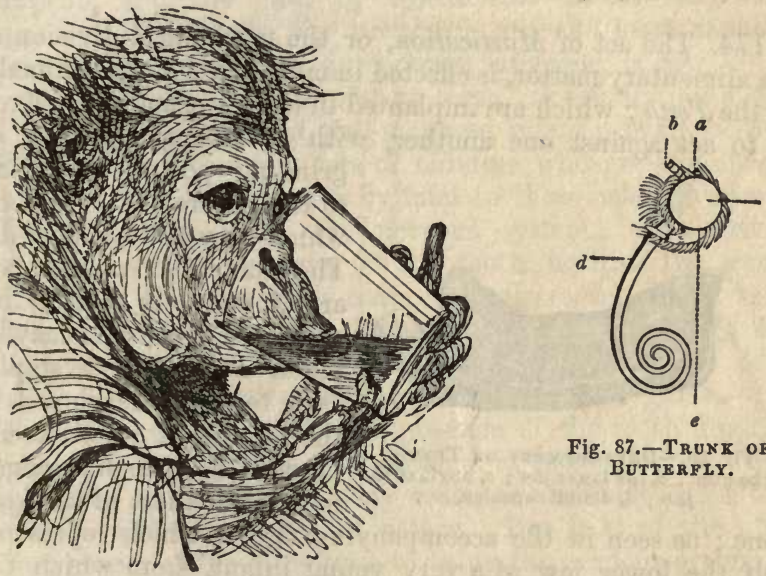

Fig. 87. -TRUNK OP A BUTTERFIY.

Fig. 86. CHIMPANZEE DRINKING.

Butterfly, $a$, of which the eye is seen at $c$, the base of the antennæ at $b$, the palpi at $e$, and the trunk at $d$. In some of the Fly tribe, the trunk attains a length several times greater than that of the body, as shown in fig. 88 , representing a

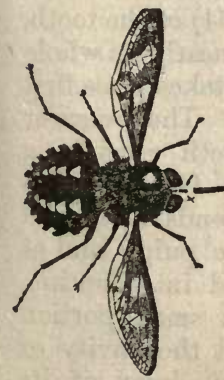

Fig. 88.-NEMESTRINA LONGIROSTRIS.

dipterous (two-winged) insect from the Cape of Good Hope, which sucks the juices of a single kind of flower, the length of whose tube just equals that of its long proboscis. 


\section{Mastication.}

174. The act of Mastication, or the mechanical division of the alimentary matter, is effected in most of the higher animals, by the Teeth; which are implanted in the jaws, and are so fixed as to act against one another, with a cutting, crushing, or

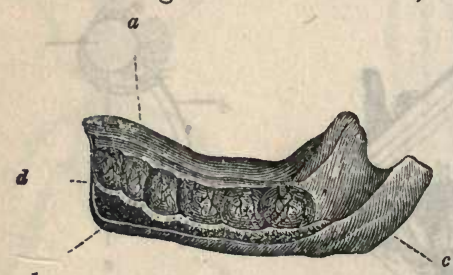

$b$

Fig. 89.-Development of TeEth.

$a$, the gum; $b$, the lower jaw ; $c$, angle of the jaw ; $d$, dental capsules. grinding power, according to the nature of the food on which they have to operate. The manner in which they are formed is worthy of note. In Man, who may be taken as a fair example, each tooth is developed in the interior of a little membranous sac, which is lodged in the thickness of the jaw-

bone; as seen in the accompanying figure, which represents half the lower jaw of a very young infant, from which the outside has been removed. This sac, which is named the dental capsule ( $a$, fig. 90), is composed of two membranes, abundantly furnished with blood-vessels; and it encloses in its interior a little bud-like protuberance, $b$, in which ramify a great number of nervous filaments and minute ressels, $c$. The matter composing this little body, which is termed the pulp, is gradually converted into the dentine ( $\$ 54)$ of the tooth, ${ }_{d} \quad{ }_{d}$ which in Man constitutes nearly its whole structure; this conversion takes place first at its highest points, $d, d$. The crown or upper portion of the tooth receives a covering of enamel ( $\$ 54)$. Gradually the process of conversion extends more and more to the interior of the pulp; and at last the whole is changed into dentine,

Fig. 90.-DENTAL Capsule. with the exception of a small portion that still remains, occupying what is termed the cavity of the tooth, which is frequently laid open by decay of its external wall. The fang of the tooth, which is the part last formed, receives an envelope of cementum (\$ 54), which invests it up to the part at which the enamel begins. As the 
root of the tooth is developed, the crown is gradually pushed upwards, so as to press against the upper portion of the capsule and the gum by which this is covered. These parts yield slowly to the pressure; and the tooth makes its way to the surface; or, in common language, is cut.

175. The process of "cutting teeth" is usually not a severe one in the healthy and well-managed infant; but it occasions the death of vast numbers of children who are injudiciously treated; and it is especially fatal to those who have a tendency to disease of the nervous system. The irritation caused by the pressure of the tooth against the gum, is liable to excite, in such cases, convulsive actions of various kinds, on the principles hereafter to be explained ( $\$ 473)$; and, as the removal of the source of irritation is of the most urgent importance, the lancing of the gums,- - doing that in an instant which the pressure of the tooth might not accomplish for days, - is a measure of most obvious utility ; however unnecessary it may seem, in ordinary cases, to interfere with the course of nature. But it is of the utmost importance at the same time to bring the nervous system into a less excitable condition; and no measure is commonly more efficacious in this respect, than removal into a fresh and pure atmosphere.

176. At the same time that the development of the tooth is thus taking place, the bone of the jaw is becoming hardened, and closes round its root, forming a complete socket. This partly interrupts the passage of vessels and nerves to the tooth, which, when once fully formed, seems to acquire no further growth, and to possess but little power of repairing injuries occasioned by disease or accident. Hence a tooth which is broken or decayed, is not restored as a bone would be. Still, however, its root or fang is penetrated by a small nerve and artery, which are distributed to the membrane that lines the cavity; and it is to the action of air upon the former, when the cavity is laid open by decay, that the pain of tooth-ache is chiefly due. The remedies which are most effectual in removing this pain, such as kreosote, nitric acid, or a heated wire, are those which destroy the vital power of the nerve.

177. But there are teeth, in many animals, which never cease to grow, and in which the central cavity is always filled 
with pulp. Such have no proper root; for additional matter is being continually formed at their base, and thus the whole tooth is pushed upwards. This is the case with the Elephant's tusks; and also with the large teeth that occupy the front of the jaw in Rabbits, Squirrels, Rats, and other gnawing ani-

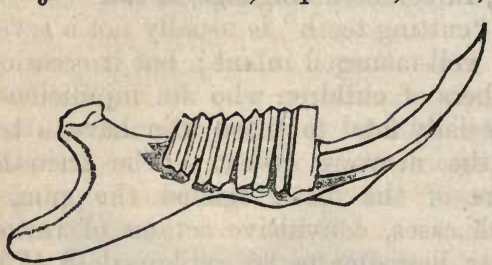

Fig. 91. Jaw and TeEth or Rabit. mals (fig. 91). The upper edges of these teeth are being constantly worn away by use : and they are kept up to their proper level by the growth of the tooth from below. But it sometimes happens that one of these teeth is broken off; and the one opposite to it in the other jaw is then thrown into disuse. It continues, however, to grow up from below; but, not being worn down at the top, its length increases greatly, so that it may become a source of great inconvenience to the animal.

178. The teeth are but passive instruments in the act of mastication. They are put in movement by the jaws in which they are fixed; and these are made to act against each other by various muscles. The upper jaw is usually fixed to the head; and has not, therefore, any power of moving independently of it. But the lower jaw is connected with the skull by a regular joint on either side; and is so moved by the muscles attached to it, as to cut, crush, or grind the food, according to the nature of the teeth.

179. There is considerable variety, in different animals, as to the extent of motion which the lower jaw possesses. In the purely Carnivorous quadrupeds, it has merely a hinge-like action, that of opening and shutting ; and by the sharpness of the edges of the molar teeth, it is thus rendered a powerful cutting instrument. But in the Herb: rorous animals, which have to grind or triturate their food letween the roughened surfaces of their molars, such a limited motion would be of no avail; and we accordingly notice, if we watch an ox or a horse whilst masticating its food, that the lower jaw has considerable power of motion from side to side. On the other hand, in the Rodents, or gnawing animals furnished with 
two large front teeth, the lower jaw has no power of moving from side to side, but is rapidly drawn backwards and forwards ; and, as the ridges of the molar teeth are arranged in the opposite direction, they become very powerful filing instruments, by which the toughest vegetable substances are quickly reduced.

180. In the Human jaw, there is a moderate power of motion in all these different directions; and it is furnished with all the muscles by which they are effected in the different animals that perform them; but these are not so large or strong. The most powerful of the muscles of the lower jaw, in all animals, is that by which it is drawn up against the upper, so as to close the mouth. This arises from the side of the skull in the region of the temple, and is hence. called the temporal muscle. It covers at its origin a large surface of bone; but its fibres approach one another as they descend, and pass under a bony arch (which may be felt between the cheek and the ear), to attach themselves to a process or projection of the lower jaw (a, fig. 92), about an inch in front of the joint. As the distance from the fulcrum of the point $a$, at which the power is applied, is thus much less than that of the front of the jaw $b$, where chiefly the resistance is encountered, the power of the muscle is applied at a mechanical disadvantage; and, to overcome a given resistance, the muscle must itself be several times more powerful. Thus the Tiger and Lion, which

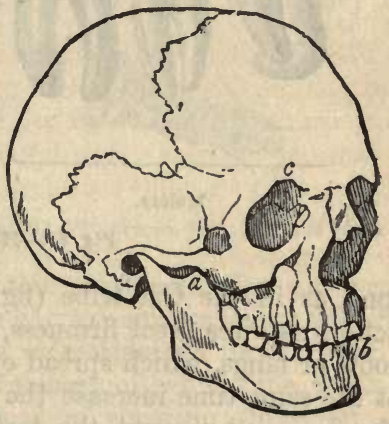

Fig. 92.- HUNAN SKUL. can lift and carry away the bodies of animals weighing several hundred pounds, must possess temporal muscles that shall contract with a force of two thousand, or even more.

181. In Man, as in most of the other Mammalia, there are three kinds of teeth, adapted for different purposes. The first terminate in a thin cutting edge, and are intended simply to divide the food introduced into the mouth ; these are termed incisor teeth (fig. 93). Others have more of a conical form, 
and in many animals (especially those of carnivorous habits) project far beyond the former; they are adapted not to cut the food, but, by being deeply fixed in it, to enable the animal to tear it asunder: these are termed canine teeth. The teeth of the third kind have large irregular flattened surfaces, and are adapted to bruise and grind the food; these are called molar (or mill-like) teeth. The manner in which these different teeth are implanted in the jaw, varies with the form of their crowns, and is in accordance with their several uses. The incisors, whose action tends as much to bury them in their sockets as to draw them forth, have but a single root or fang of no great length. The canine teeth, on which there is often considerable strain, penetrate the jaw more deeply than the incisors; especially when they are large and

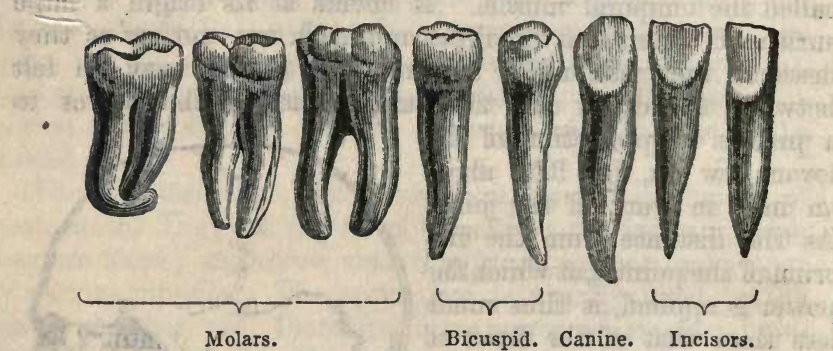

Fig. 93.-Human TeEth.

long, as in the Cat tribe (fig. 94). And the molars, whose action requires great firmness, have two, three, or even four roots or fangs, which spread out from each other; and these at the same time increase the solidity of their attachment to the jaw, and prevent the teeth from being forced into their sockets by any amount of pressure.

182. The arrangement of the dental apparatus varies, in different Mammalia, according to the nature of the aliment on which they are destined to feed; and this correspondence is so exact, that the anatomist can generally determine by the simple inspection of the teeth of an animal, not only the nature of its food, but the general structure of the body, and even its ordinary habits. Thus, in those that feed exclusively on animal flesh, the molar teeth are so compressed as to form 
cutting edges, which work against each other like the blades of a pair of scissors (fig. 94); whilst in animals that live on insects, these teeth are raised into conical points, which lock
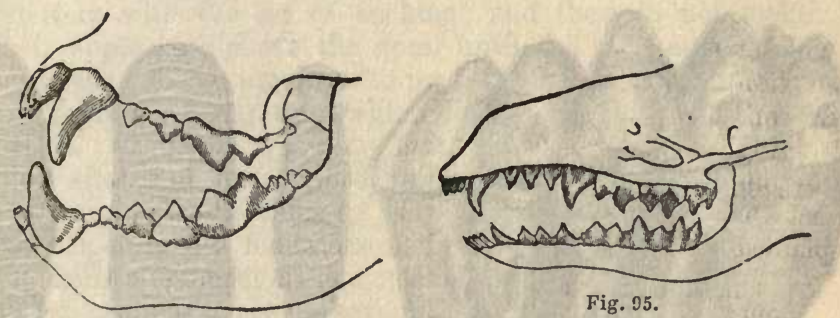

Fig. 94-Teeth of Carivorous Animat. TeEth of Insectivorons animal.

into corresponding depressions in the teeth of the opposite jaw (fig. 95). When the nourishment of the animal consists principally of soft fruits, these teeth are simply raised into rounded elevations (figs. 97, 98); and when they are

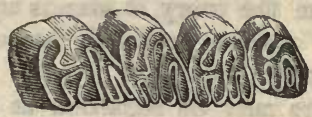

Fig. 96.

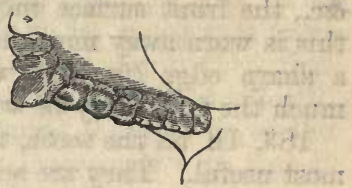

Teeth of Herbivorous Animar. Fig. 97.-Teeth of Frugivorods Animal.

destined to grind harder vegetable substances, they are terminated by a large flat and roughened surface (figs. 96, 99). The roughness of this surface is maintained by the peculiar arrangement of the three substances of which the tooth is composed. The enamel, instead of covering its crown, is arranged in upright plates, which are dispersed through the tooth; and the space between them is filled up by plates of ivory and of cementum ( $\S 54)$. These last, being softer than the enamel, are worn down the soonest; and thus the plates of enamel are left constantly projecting, so as to form a rough surface admirably adapted to the grinding action which the tooth is destined to perform. The mode in which these plates are disposed, affords a most characteristic distinction. between the two species of Elephant at present existing, 
namely, the African and the Indian; as also between each of these and the great extinct species known as the Mammoth (fig, 99). In the great gnawing teeth of the Rabbit,

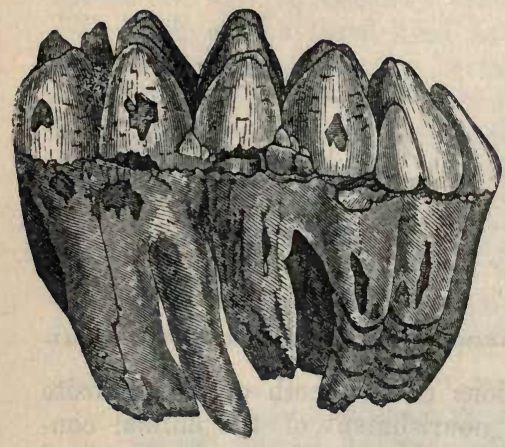

Fig. 98.-Molar Tooth of Mastodon.

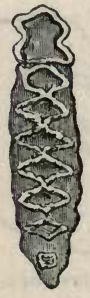

1.

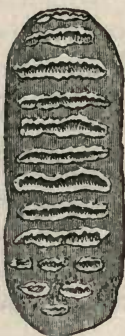

2.

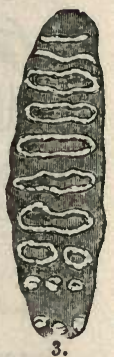

3.

Fig. 99.-Molar TeEth of ElephaNts. 1, African Elephant; 2, Indian Elephant ; 3, Mammoth.

\&c., the front surface only is covered with enamel ; and as this is worn away more slowly than the ivory, it stands up as a sharp edge (fig. 91), which is always retained, however much the tooth may be worn away.

183. Of all the teeth, the molars may be regarded as the most useful. They are seldom absent in the Mammalia ; and their office is usually essential to the proper digestion of the food. Animal flesh (the most easily digested of all substances) needs but to be cut in small pieces; but the hard envelopes

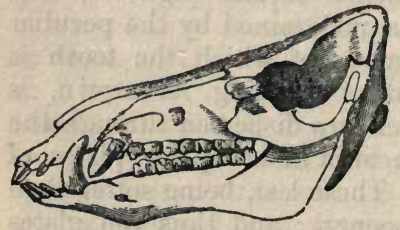

Fig. 100.-SkuLL OF BoAr. of beetles and other insects must be broken up; and the tough woody structure of the grasses, and the dense coverings of the seeds and fruits on which the herbivorous animals are supported, must be ground down. The incisors and canines are chiefly employed among Carnivorous animals for the purpose of seizing their living prey, and are never deficient in them; but they are less required in Herbivorous animals; and either or both kinds are not unfrequently deficient. Sometimes, however, they are not 
only present in the latter, but are largely developed, serving as weapons of attack and defence; as in the Boar (fig. 100).

184. In the Mammalia in general, as in Man, the teeth are not much developed at the time of birth, that they may not interfere with the act of sucking; and they do not make their appearance above the gum, until the time approaches when the young animal has to prepare its own food, instead of simply receiving that which has been prepared by its parent. The teeth which are first formed are destined to be shed after a certain period, and to be replaced by others. They are called milk-teeth; and in Man they are twenty in number,-namely, four incisors in the front of each jaw, and two canines and four molars on each side. These begin to fall out at about the age of seven years; previously to which, however, the first of the permanent molars appears above the gum, behind those of the first set. The incisors and canines of the first set are replaced by incisors and canines respectively; but the molars of the first set are replaced by teeth like small molars, having only two fangs; these are called false molars, or, more properly, bicuspid teeth (fig. 93). The second of the true molars does not make its appearance until all the milk-teeth have been shed; since it is only then that the jaw becomes long enough to hold any additional teeth. The third does not usually come up until the growth of the jaw is completed; and as this time corresponds with that at which the mind as well as the body is matured, they are commonly known as wise or wisdom teeth. There are then thirty-two teeth in all, or sixteen in each jaw ; - namely, four incisors, two canines, four bicuspid, and six true molars. - In extreme old age, these teeth fall out like those of the first set; but they are not replaced by others, and their sockets are gradually obliterated.

185. There are a few Mammalia which do not possess teeth. This is the case with the common Whale, in which they are replaced by an entirely different structure. From the upper jaw (fig. 102) there hang down into the mouth a number of plates of a fibrous substance (fig. 101), to which we give the name of whalebone, though it is really analogous to the gum of other animals. The fibres of these plates are separate at their free extremities, and are matted (as it were) together, so as to form a kind of sieve. Through this sieve the Whale 
174 ABSENCE OF TEETH IN WHALE, ANT-EATER, ETC.

draws water in enormous quantities, whenever it is in want of food; and in this manner it strains out, as it were, the minute gelatinous animals upon which it lives, from the water of the seas it inhabits. The water thus taken in is expelled from the nostrils or blow-holes, which are situated at the top
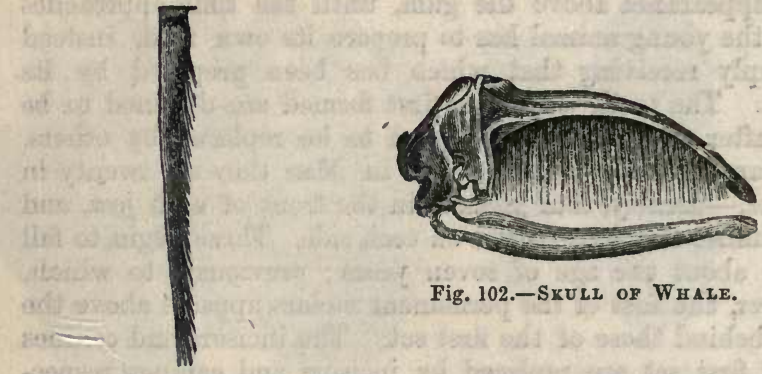

Fig. 102.-SxULL OF WHALE.

Fig. 101.-WHA LEBONE.

of the head. Most of the Whale tribe have short fringes of this kind in the roof of the mouth; but in none, except the Baloena, or Greenland Whale, is it long enough to make it worth separating; all the other species having teeth; either in one or both jaws. - It is a curious fact, that the rudiments of teeth may be discovered in both jaws of the young Greenland whale, although they are never to be developed. And the rudiments of incisor teeth in the upper jaw, and of canine teeth in both jaws, may also be discovered in the young of the Ruminant quadrupeds (oxen, sheep, \&c.), though they never show themselves above the gum.

186. The Ant-eaters, also, are destitute of teeth, and usually obtain their food by means of their long extensible tongues,

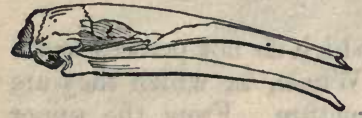

Fig. 103.-SKULL of the ANT-Eater. which are covered with a viscid saliva; this being pushed into the midst of an ant-hill, and then drawn into the mouth, brings into it a large number of these insects, which are sufficiently bruised between the toothless jaws (fig. 103). Lastly, may be mentioned as a curious exception to the general rules respecting the teeth of Mammalia, the remarkable Ornithorhyncus of New Holland (ZooLOGy, § 317), which feeds, 
like the duck, upon the water-insects, shell-fish, and aquatic plants, that it obtains from the mud, into which it is continually plunging its singular bill; and its jaws, entirely destitute of teeth, are furnished with horny ridges, by which it can in some degree masticate its food.

187. Among Birds, there is an entire absence of teeth; and the mechanical division and the reduction of food is performed in the stomach, in the manner hereafter to be mentioned $(\$ 200)$. The mouths of almost all Reptiles, excepting the Turtle tribe, are furnished with numerous teeth (fig. 104); but these are not

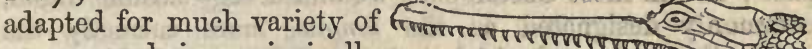
purposes, being principally destined to prevent the escape of the prey which the animals have secured; Fig. 104.-HEAd of Gaviat. (Crocodile and their shape is conse-

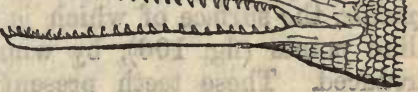
quently nearly uniform, being for the most part simply conical. There are some Lizards, however, which are herbivorous ; and these have large rough teeth, somewhat resembling the molars of Mammalia. The Iguanodon, an animal of this tribe, attained a gigantic size in past ages of the world.

188. In Fishes, the teeth are commonly very numerous (fig. 105), but they have for their object only to separate and retain

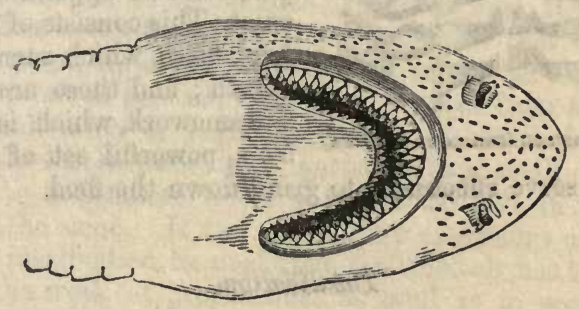

Fig. 105.-HEAd OF SHARK.

their food; and there is little variety in their form. Frequently they have no bony attachment, being only held by the gum, as in the Shark; and they are consequently often torn away, but they are as readily replaced. Sometimes, bəw- 
ever, the tooth seems like a continuation of the bone of the jaw, not being in any way separated from it, and the tubular structure of the latter being continued into it without any interruption. The teeth of fishes are often set, not only upon the proper jaw-bones, but upon the surface of the palate, and even in the pharynx or swallow.

189. In the Invertebrata there are generally no proper teeth; in the Articulated and sometimes in the Molluscous series, however, we meet with firm horny jaws, which are often furnished with projections that answer the same purpose ; and in most Gasteropods we find a very curious organ, commonly designated as the tongue, more correctly the palate, the surface of which is beset with innumerable toothlike points (fig. 106), by whose rasping action the food is reduced. These teeth present great varieties of form and

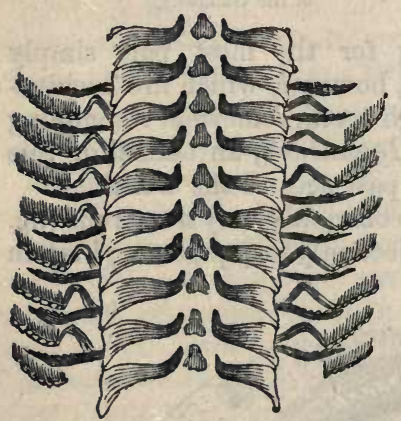

Fig.106.-Dental Organ of Nerita. arrangement in the different genera and species of this group; and these varieties appear to bear some relation to the nature of the food on which the animals respectively live. It is remarkable that in an animal so low in the scale as the Echinus or Sea-Urchin (\$119), a very complex dental apparatus should exist. This consists of five long hard teeth, which surround the mouth; and these are fixed in a framework which is worked by a powerful set of muscles, and thus serve effectually to grind down the food.

\section{Insalivation.}

190. The act of mastication is connected with another; which is also of great importance in preparing for the subsequent process of digestion. This is the blending of the saliva with the food, during its reduction between the teeth, -an act which is termed insalivation. The saliva is separated from the blood, by glands which are situated in the neigh- 
bourhood of the mouth; of these there are three pair in Man, two beneath the tongue (fig. 107), and one in the cheek, each pouring-in its secretion by a separate canal. The salivary fluid is principally composed of water, in which a small quantity of animal matter and some saline substances (chiefly common salt) are dissolved; the whole amount of these, however, is not more than 1 part in 100 . The secretion of saliva is not constantly going on ; but the fluid is formed as it is wanted. The stimulus by which the gland is set in action may be simply the motion of the jaws ; thus, on first waking in the morning, the mouth is usually dry, but it is soon rendered moist by the movements which take place in speaking. The contact of solid substances with the membrane lining the mouth appears also to excite the flow; hence dryness of the mouth may often be remedied for a time, when no water is at hand, by taking a pebble into its interior, and moving this from side to side. There are certain substances, however, whose presence in the mouth has a special influence in provoking an increased secretion of saliva; and every one knows, too, that the simple idea of savoury food will excite an increased flow, making the "mouth water" as it is popularly termed. These are instances of the power of the nervous system, through which such impressions are conveyed, over the act of secretion.

191. In the case of farinaceous or starchy food, the admixture of saliva occasions the commencement of that chernical change in which its digestion consists, namely, its conversion into sugar; but in general, the benefit derived from this process of insalivation is just that which is obtained by the chemist, when he bruises in a mortar, with a small quantity of fluid, the substances he is about to dissolve in a larger amount of the same. If the preliminary operations of mastication and insalivation be neglected, the stomach has to do the whole of the work of preparation, as well as to accomplish the digestion; thus more is thrown upon it than it is adapted to bear; it becomes over-worked, and manifests its fatigue by not being able to discharge even its own proper duty. Thus the digestive function is seriously impaired, and the general health becomes deranged in consequence. A malady of this kind is very prevalent in the United States; and is almost universally attributed by medical men, in part at least, to the 
general habit of very rapidly eating or rather "bolting" the meals. There is another evil attendant on this practice, - that much more food is swallowed than is necessary to supply the wants of the system ; for the sense of hunger is not so readily abated by food which has not been prepared for digestion; and thus the feeling of satiety is not produced, until the stomach has already received a larger supply than it is well able to dispose of. Imperfect mastication of the food is very apt to occur, in persons who are losing their teeth by old age or decay; and where these are not replaced by artificial means, the next best remedy is to cut the food into very small portions, before it is taken into the mouth, and to masticate it there as thoroughly as possible.

\section{Deglutition.}

192. In the Mammalia, the cavity of the mouth is guarded behind by a sort of moveable curtain, which is known as the veil of the palate (fig. 107); and this hangs down during

\section{Veil of the palate}

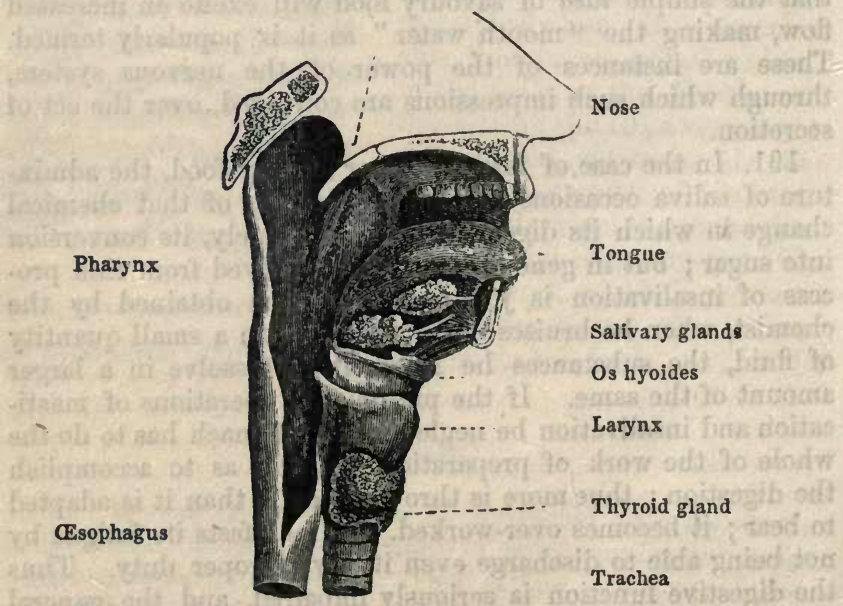

Fig. 107.-Perpendicular Section of the Mouth and Throat.

mastication, in such a manner as to prevent any of the food from passing backwards. This partition, which does not exist 
in Birds and other animals that do not masticate their food, hangs from the arch and sides of the palate, so as to touch the tongue by its lower border; but it can be lifted in such a manner as to give the food free passage beneath it, into the top of the gullet. When mastication is completed, the food is collected on the back of the tongue into a kind of ball; and this, being carried backwards by the action of its muscles, presses against the partition just mentioned, and causes it to open. The food thus passes into a sort of funnel, formed by the expansion of the top of the oesophagus or gullet; this cavity, termed the pharynx, communicates above with the nostrils, and in front with the larynx, which is at the top of the trachea or windpipe. The oesophagus is a long and narrow tube, which descends from the pharynx to the stomach, lying just in front of the vertebral column, and behind the heart and lungs. It is surrounded by muscular fibres, disposed in various ways; by the action of which the food that has once passed into the pharynx is propelled downwards to the stomach.

193. But in order to reach this tube, the alimentary ball must pass over the glottis or aperture of the larynx. With a view to prevent its falling-in, the larynx is drawn, in the very act of swallowing, beneath the base of the tongue; and this action presses down a little valve-like flap, the epiglottis, upon the aperture, so as in general effectually to prevent any solid or fluid particles from entering it. But it sometimes happens that, if the breath be drawn-in at the moment of swallowing, a small particle of the food, or a drop of fluid, is drawn into the glottis; and this action (commonly termed "passing the wrong way,") excites a violent coughing, the object of which is to drive up the particle, and to prevent it from finding its way into the lower part of the windpipe. It may also happen that a larger substance may slip backwards, by its own weight, into the glottis, when there was no intention of swallowing, and when the larynx was consequently not drawn forwards beneath the tongue. The presence of such a substance in the windpipe excites a violent and frequently almost suffocating cough (\$ 342$)$; the effect of which is sometimes to drive it up through the glottis, and thus to get rid of the source of irritation.

194. The act of swallowing is itself involuntary, and may N 2 
be even made to take place against the will. This may seem contrary to every one's daily experience ; but it is nevertheless true. The movement by which the food is carried back, beneath the arch of the palate, into the pharynx, is effected by the will ; but when the food has arrived there, it is laid hold of, as it were, by the muscles of the pharynx, and is then carried down involuntarily. It has several times happened, that a feather, with which the back of the mouth was being tickled to excite vomiting, having been introduced rather too far, has been thus grasped by the pharynx, and has been swallowed. Moreover, we cannot perform the act of swallowing, without carrying something backwards upon the tongue; and it is the contact of this something, even if it be only a little saliva, with the membrane lining the pharynx, that produces the muscular movement in question.

195. This action is one of the kind now denominated reflex (§ 430). It is produced through the nervous system ; for if the nerves supplying the part be divided, it will not take place. But it does not depend upon the Brain; for it may be performed after the brain has been removed, or when its power has been destroyed by a blow. It is caused by the conveyance to the top of the Spinal Cord, of the impression made on the lining of the pharynx ; this impression, brought thither through one set of nerves, excites in the spinal cord a motor impulse; which, being transmitted thence through another set of nerves, calls the muscles into action.

196. This action is, therefore, necessarily connected with the impression, so long as this portion of the spinal cord, and the nerves proceeding from it, are capable of performing their functions : and it is one of those to which we may give the name of instinctive, to distinguish it from those which are effected by an effort of the Will, intentionally directed to accomplish a certain purpose. It may even take place without the animal being aware of the contact of any substance to be swallowed with the lining of the pharynx; for there is good reason to believe that when the brain has been destroyed, or paralyzed by a blow, all sensibility is destroyed ; and we have also sufficient reason to consider it as suspended in profound sleep or apoplexy, in which states swallowing is still performed. In the severest cases of apoplexy, however, the power of swallowing is lost; and this is a symptom of great 
danger, since it shows that not the brain alone, but the upper part of the spinal cord, is suffering from the pressure; and that the movements of respiration, which depend upon a similar action of the nervous system ( $(340)$, will probably soon cease, so that death must ensue.

\section{Digestive Apparatus.}

197. The food, thus propelled downwards by the action of the muscles of the pharynx and of the oesophagus (gullet),

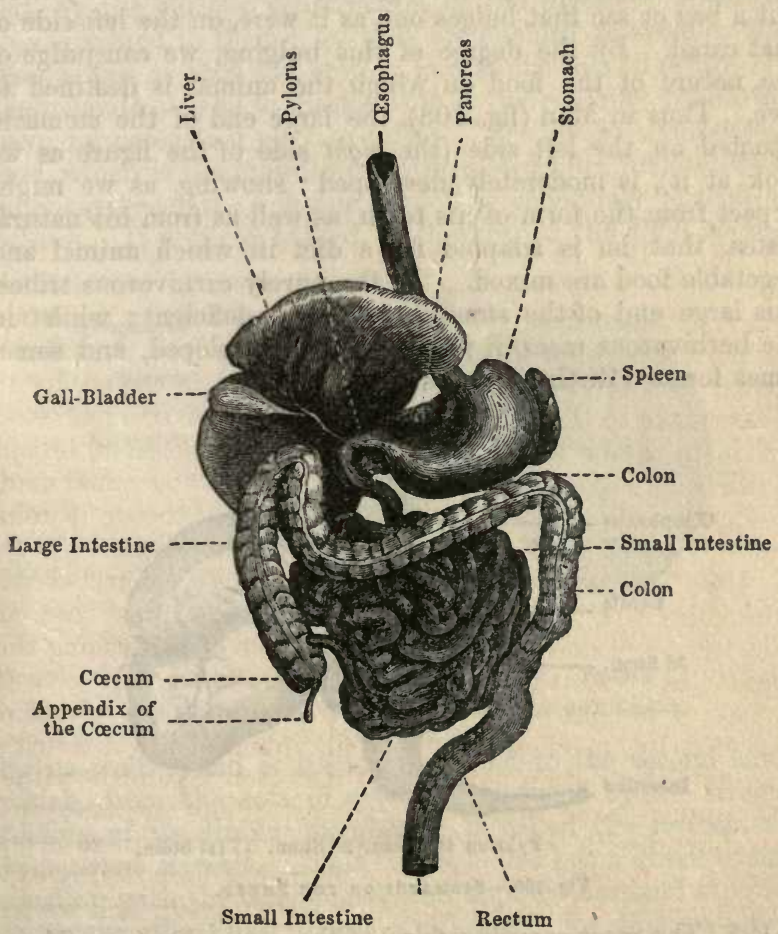

Fig. 108.-Digestive Apparatus of Max.

arrives, in Man and the Mammalia, at the stomach; which is a large membranous bag, placed across the upper part of the 
abdomen (fig. 108). The form of this stomach varies much, according to the nature of the aliment to be digested. Where the food is animal flesh, which is easily dissolved, the stomach is small, and appears like a mere enlargement of the alimentary tube; this is the case in the Cat tribe, for example. In Herbivorous animals, on the contrary, the stomach is very large, the food being delayed there a long time on account of the difficulty with which it is digested; and the principal part of its cavity is not a simple enlargement of the alimentary tube, but a bag or sac that bulges out, as it were, on the left side of that canal. By the degree of this bulging, we can judge of the nature of the food on which the animal is destined to live. Thus in Man (fig. 108), the large end of the stomach, situated on the left side (the right side of the figure as we look at it), is moderately developed; showing, as we might expect from the form of his teeth, as well as from his natural tastes, that he is adapted for a diet in which animal and vegetable food are mixed. In the purely carnivorous tribes, this large end of the stomach is almost deficient; whilst in the herbivorous races, it is enormously developed, and sometimes forms a distinct pouch.

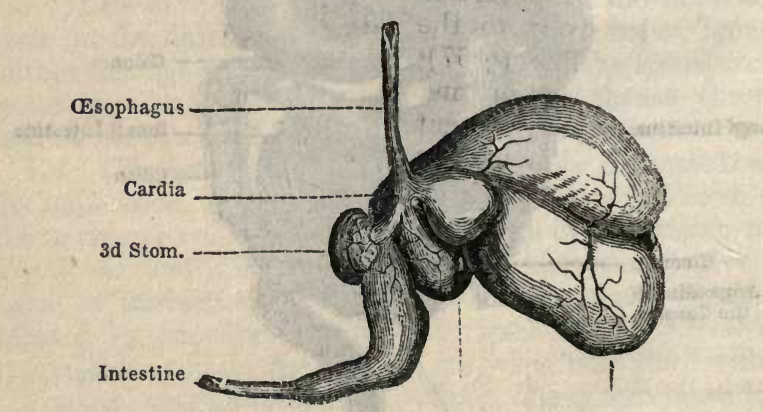

Pylorus 4th Stom. 2d Stom. 1st Stom.

Fig. 109.-StoMACHS of THE ShFeP.

198. The most complex form of the stomach among Mammals, is that which we find in the animals that ruminate or chew the cud. It possesses, in fact, no less than four distinct cavities, through all of which the food has to pass during the 
process of digestion. The external appearance of the stomach of the Sheep is seen in fig. 109; and its interior is displayed in fig. 110. The food of the Ruminant animals is not chewed by them before it is first swallowed. In their wild state, they are peculiarly exposed to the attacks of their carnivorous enemies, when they come down from their rocky heights to browse upon the rich pastures of the valleys. If they were then obliged to masticate every mouthful, they would be subjected to long-continued danger at every meal; but, by the curious construction of the digestive apparatus, this is spared to them; for they are enabled to swallow their food as fast as they can crop it, and afterwards to return it to their mouths, so as to masticate it at their leisure, when they have retreated to a place of safety. The crude unmasticated food, which is brought-down by the œsophagus, first enters the large cavity on the left side, which is commonly termed the paunch. It is there soaked, as it were, in the fluid secreted

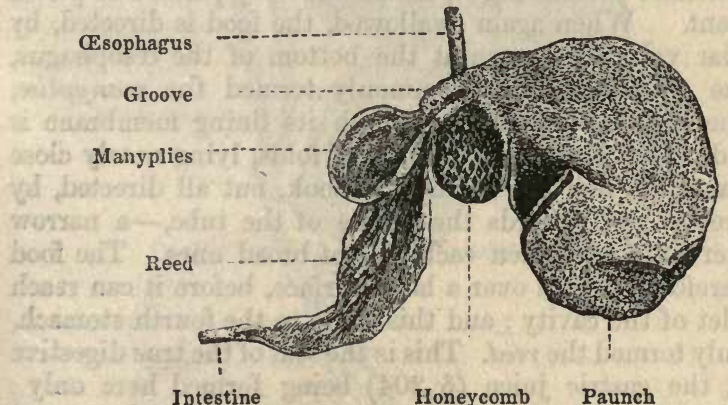

Fig. 110.-SEction of THE STOMACHS of THE SHEEP.

by its walls; and is then transmitted to the second cavity, which, from the sort of network produced by the irregular folding of its lining membrane, is called the reticulum or honey-comb stomach. This stomach also has a direct communication with the œsophagus, and appears destined especially to receive the fluid that is swallowed; for this passes immediately into it, without going into the first stomach at all. The folds of its lining membrane present a large surface, through which fluid may be absorbed into the system. It is. 
here that we find the curious arrangement of water-cells in the stomach of the Camel, by which that animal is enabled to retain a supply of water for several days. These cells correspond with the little pits which are seen in the honey-comb stomach of the Sheep, but are much deeper, and their orifices may be closed by the action of a set of muscular fibres which pass in every direction round each, so as to form a net-work including these orifices in its meshes.

199. After the food has been macerated in the fluids of the first and second stomachs, it is returned to the mouth by a reversed peristaltic action of the oesophagus, which brings it up as a succession of globular pellets, that are formed by compression in a sort of mould at the lower end of the oesophagus. These pellets are subjected within the mouth to mastication and insalivation; and the food is then ready for the real process of digestion. It is this mastication which is commonly known as the "chewing of the cud;" and the animal, whilst performing it, seems the very picture of placid enjoyment. When again swallowed, the food is directed, by a peculiar valvular groove at the bottom of the œsophagus, into the third stomach, commonly termed the manyplies, from the peculiar manner in which its lining membrane is arranged. This presents a number of folds, lying nearly close to one another, like the leaves of a book, but all directed, by their free edges, towards the centre of the tube, - a narrow fold intervening between each pair of broad ones. The food has, therefore, to pass over a large surface, before it can reach the outlet of the cavity; and this leads to the fourth stomach, commonly termed the reed. This is the seat of the true digestive process, the gastric juice ( $\$ 204)$ being formed here only; and it is from this that the rennet is taken, which is used in making cheese to cause the milk to coagulate or curdle. In the sucking animal, the milk passes directly into this fourth stomach, without entering either the first or second stomachs, and without being delayed in the third, the folds of which adhere together so as to form a narrow undivided tube. The paunch is at that time comparatively small, being of less size than the reed; and its dimensions increase, as soon as the young animal begins to distend it by swallowing solid regetable matter.

200. In the digestive apparatus of Birds, we find a con- 
siderable modification of form, resulting from the fact that, as these animals do not masticate their food, they require some

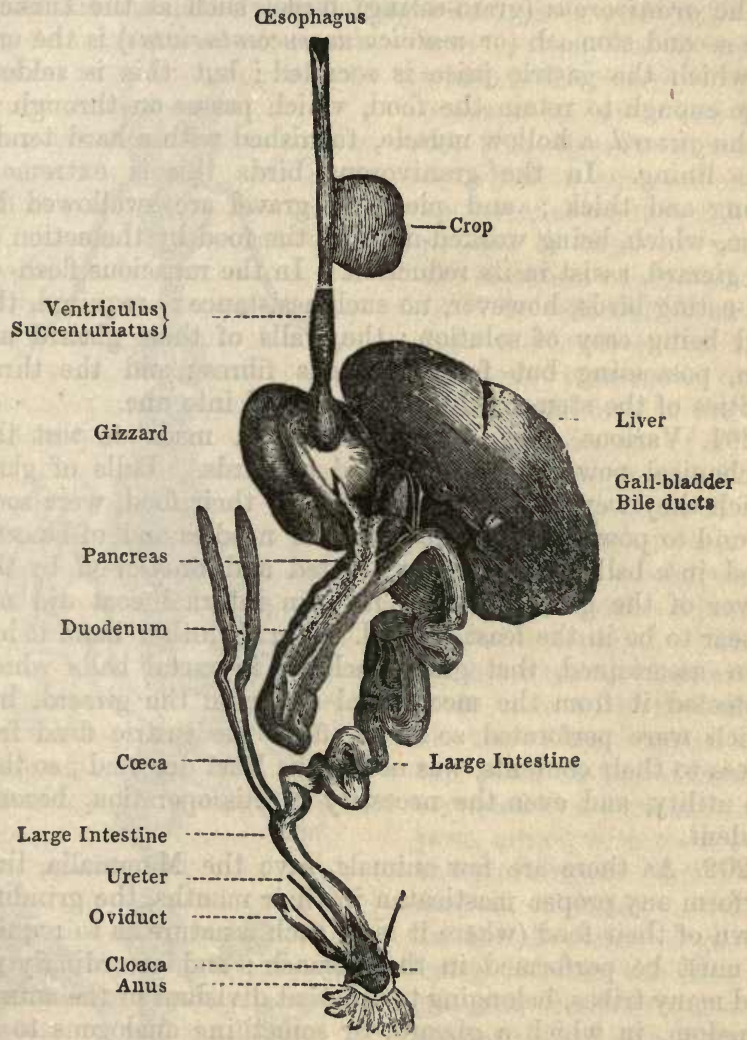

Fig. 111.-Digestive Apparatus of Fown.

other means of reducing it. This means is provided for them in their stomach. In the tribes whose food is of such a nature as to require being moistened before it is rubbed down, and especially in those which feed upon grains, the oesophagus has a pouch-like dilatation, termed the crop or craw (fig. 111); in this it is retainer, and exposed to tise action 
of fluid secreted by its walls, just as it is in the paunch of ruminant quadrupeds. This crop is of enormous size in some of the granivorous (grain-eating) birds, such as the Turkey. The second stomach (or ventriculus succenturiatus) is the one in which the gastric juice is secreted; but this is seldom large enough to retain the food, which passes-on through it to the gizzard, a hollow muscle, furnished with a hard tendinous lining. In the granivorous birds this is extremely strong and thick; and pieces of gravel are swallowed by them, which, being worked-up with the food by the action of the gizzard, assist in its reduction. In the rapacious flesh- or fish-eating birds, however, no such assistance is required, the food being easy of solution; the walls of their gizzard are thin, possessing but few tendinous fibres; and the three cavities of the stomach are almost united into one.

201. Various experiments have been made to test the mechanical powers of the gizzard of Birds. Balls of glass which they were made to swallow with their food, were soon ground to powder; and the points of needles and of lancets, fixed in a ball of lead, were blunted and broken-off by the power of the gizzard, whilst its own internal coat did not appear to be in the least injured. On the other hand it has been ascertained, that grain enclosed in metal balls which protected it from the mechanical action of the gizzard, but which were perforated so as to afford the gastric fluid free access to their contents, was not in the least digested; so that the utility, and even the necessity of this operation, become evident.

202. As there are few animals, save the Mammalia, that perform any proper masticaton in their mouths, the grinding down of their food (where it is of such a nature as to require it) must be performed in the stomach ; and accordingly we find many tribes, belonging to different divisions of the animal kingdom, in which a gizzard, or something analogous to it, exists. It is possessed by almost all Cephalopods, and by many of the Gasteropods. In the walls of the stomach of some of these last, there is a considerable amount of mineral matter deposited, intermixed with the hard tendinous fibres of which they chiefly consist. A powerful gizzard is also found in many Insects, but here it is placed above the digestive stomach (fig. 112, c). The accompanying figure exhibits 
the alimentary canal of a Beetle, from its commencement ta its termination. At $a$ is seen the head, bearing the jaws, $\&$ c. ; from this the gullet passes straight backwards, and is dilated into a crop at $b$, below

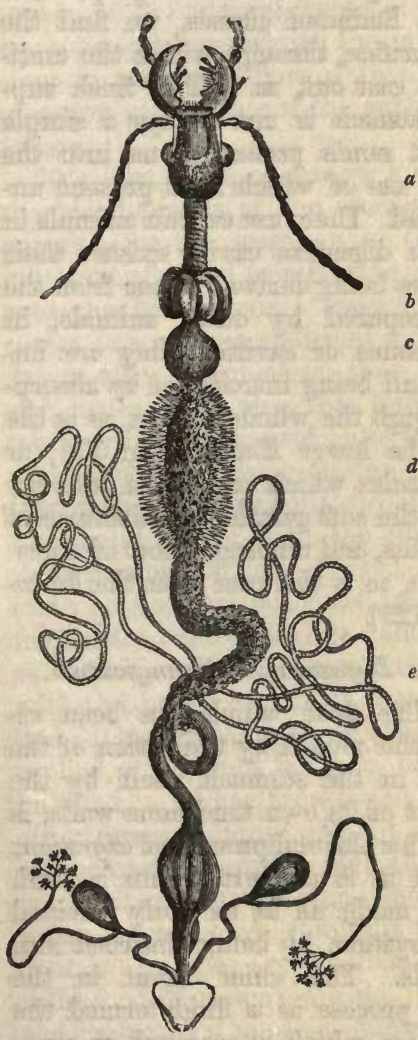

Fig. 112.-Digestive Apparatos of BEETLE. which is the gizzard, $c$. This opens at its lower end into the true digestive stomach, $d$; which is surrounded by an immense number of little

a follicles or bags, by which the secretion of the gastric juice is effected (\$204). Into the lower end of this, the long vessels, e, open,

- which constitute in Insects ths only rudiment of a liver (§ 358). In many of the Crustacea, the walls of the d stomach are beset with regular rows of teeth, which are moved by the action of powerful muscles. These teeth are cast or shed at the same time with the shell. In the Wheel-Animalcules, the place of the gizzard is occupied by a curious pair of jaws, armed with teeth; by the working of which, the food is effectually crushed. In the Bryozoa, a gizzard exists between the oesophagus and the true digestive stomach ; and the stomach itself is surrounded by the little follicles which secrete the bile, and pour it into that cavity ( $\$ 115$ ).

203. In animals which subsist exclusively on flesh, however, no such complicated apparatus exists. Thus in Serpents (fig. 34 ), the stomach is but a slight dilatation of the alimen- 
tary tube; and it is not easy to say where it commences and terminates. In Spiders and Scorpions, too, which live upon the juices they suck from other animals, the alimentary tube is very simple; and it is scarcely dilated into a proper stomach. And in most of the Radiated classes, we find the stomach to possess only one orifice, through which the undigested residue of the food is cast out, as well as fresh supplies taken in. But this stomach is not always a simple bag; thus in the Star-fish it sends prolongations into the rays, the use of which is at present un-

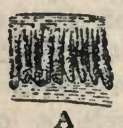

A

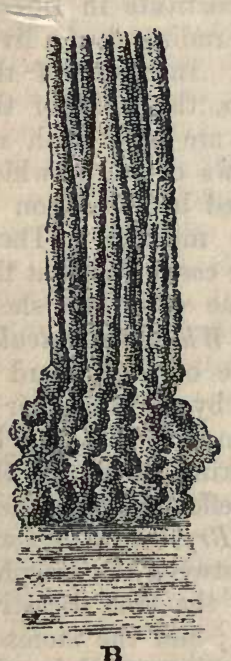

$\mathbf{R}$ Fig. 113. determined. There are certain animals in which no digestive cavity exists: their sustenance being derived either from the juices prepared by other animals, in whose tissues or cavities they are imbedded, and being introduced by absorption through the whole surface, as is the case in the lower Entozoa (fig. 53); or from particles which are drawn into the midst of the soft gelatinous substance of their bodies, and undergo a sort of diges tion there, as is the case with the Rhizopoda (\$ 129).

\section{Gastric Digestion:-Chymification.}

204. The food which has been reduced in the mouth by the action of the teeth, or in the stomach itself by the movement of its own tendinous walls, is prepared for the real process of digestion; by which it is converted into a fluid, and thus made fit to be truly received into the system, by being absorbed into its vessels. The chief agent in the digestive process is a fluid termed the as seen in a vertical section rated from the blood by a vast number of the Stomach, magnified of little bags or follicles (fig. 113), imthree diameters at $\mathbf{A}$. and
twenty diameters at $\mathbf{B}$. bedded in the walls of the stomach. When the cavity is empty, this fluid is secreted in very small quantities; but, like the salivary secretion, it is poured out 
in abundance when the lining membrane is stimulated by the contact of food, especially solid food. Only a limited quantity is secreted at any one time; and this quantity is just that which is sufficient to dissolve fond enough for the supply of the natural wants of the system. The contact of any solid substances with the interior of the stomach, is suffcient to produce a flow of this fluid into its cavity; but the secretion soon ceases if the substance be not of an alimentary nature.

205. The sense of hunger appears due to the distension of the blood-vessels of the stomach, which takes place in preparation for the secretion of the gastric fluid. This determination of blood towards the stomach seems to occur whenever the body needs a fresh supply of nourishment ; and it ceases as soon as a sufficient amount of gastric fluid has been drawn off. Hence it is, that hunger is relieved by eating; and hence it is, also, that hunger is for a time relieved by taking solid substances into the stomach, even though they contain no nourishing matter. It is from having experienced this, that savage nations are in the habit of mixing indigestible solid matter with the fluids that sometimes constitute their principal articles of food. Thus the Kamschatdales mix earth or saw-dust with the train-oil on which alone they are frequently reduced to live ; and the Veddahs, or wild hunters of Ceylon, mix the pounded fibres of soft or decayed wood with the honey on which they feed when meat is not to be had. One of them being asked the reason of the practice, replied, "I cannot tell you, but I know that the belly must be filled." It has been found by experiment, that soups and other forms of liquid aliment are not alone fit for the support of the system, even though they may contain a large amount of nutritious matter; and the medical man well knows, that many persons have stomachs too weak and irritable to retain "slops" (as they are commonly termed), who can yet digest solid food of a simple kind. All these instances show, that the contact of a solid substance with the walls of the stomach, is the proper stimulus or excitement to the secretion of the gastric fluid.

206. This fluid, when poured upon the food, is thoroughly mixed-up with it by a peculiar movement of the walls of the stomach, which is continually bringing fresh portions of the 
alinentary mass into contact with its sides, so that the whole is after a time equally 'exposed to the influence of the gastric secretion. If this movement were not to take place, only the outside of the mass would be digested, and the central portion would remain but little affected.

207. The nature of the gastric fluid, and the mode of its operation upon the food, have been studied by withdrawing a portion of it from the stomach, and by observing its properties and actions out of the body. A sufficient quantity for this purpose cannot be easily procured. Spallanzani, an Italian physiologist of the last century, contrived to obtain it, by causing birds and other animals to swallow sponges to which pieces of thread were attached; these, when they had remained long enough in the stomach to cause a secretion of the gastric juice, were drawn up again ; and the fluid they had absorbed was pressed out into vessels, in which its properties could be examined. More recently, however, an advantageous opportunity has presented itself for obtaining supplies of gastric fluid in a less objectionable manner. A young man, named Alexis St. Martin, received a very severe wound in his left side, by the bursting of a gun; and although this wound laid open the cavity of his stomach, he recovered his health completely, and subsequently married and had a family. There remained, however, an aperture in his stomach, which would not close up; and through this orifice, which was usually covered by a bandage, the contents of the stomach could be drawn out. The gastric juice was obtained by introducing an India-rubber tube into the stomach when it was empty, and by moving it about within the cavity; the contact of the tube then excited the follicles to secretion (on the principle already mentioned, § 204); and the fluid thus poured into the stomach was drawn off through the tube.

208. The Gastric Juice is very like saliva in its appearance, but it is distinctly acid to the taste ; and it is found, by chemical examination, to contain a considerable quantity of muriatic acid * in an uncombined state. Besides this, it contains a considerable quantity of a peculiar animal substance which seems like altered albumen, and which has been designated pepsin; as well as other ingredients of less importance.

* Muriatic acid is commonly known as spirit of salt. 
This fluid possesses the power of dissolving albuminous substances of various kinds, when these are submitted to its action at the constant temperature of $100^{\circ}$ (which is about that of the stomach), and are frequently shaken-up with it. The solution appears to be in all respects as perfect as that which naturally takes place in the stomach, but requires a longer time. It does not seem, however, that the gastric juice has a special solvent power for any other than albuminous substances. Gelatinous and saccharine matters are taken-up by it, as by other watery fluids; but neither starchy nor oleaginous substances undergo any other change by its action, than consists in the separation of their particles by the solution of the membranes and fibres which held them together. There is every reason to believe that what is true of artificial is true of natural digestion; and that so far from the whole operation being performed in the stomach, as was formerly supposed, gastric digestion is limited to the solution of the albuminous, gelatinous, and saccharine constituents of the food.

209. With regard to the precise mode in which the gastric fluid acts in dissolving albuminous substances, there is yet some uncertainty; although there can be no longer any reasonable doubt, that the operation is of a purely chemical nature. An artificial gastric fluid, capable of effecting all that can be done by that which is secreted in the living stomach, may be made, by macerating (or soaking) a portion of the membrane lining the stomach of a pig, or of the fourth stomach of a calf (even after it has been washed and dried) in water, which dissolves a portion of the pepsin; and by then acidulating this solution with muriatic or acetic acid. It has been proved that both the acid and the pepsin are essential to the process of solution; for the acidulated fluid without the animal matter acts extremely slowly upon pieces of meat, hard-boiled egg, \&c., submitted to it; and water in which the stomach has been macerated, but which contains no acid, will not act at all. But the acidulated water alone will readily dissolve the substances just mentioned, at a higher temperature; and thus it appears that the acid is the real solvent; and that the pepsin has for its office to produce some change in the albuminous substances, by which they are more readily dissolved. The recent inquiries of Liebig and other 
Chemists, render it probable that this change is of the nature of fermentation.

210. It is a fact of great practical importance, that a certain quantity of the gastric fluid can act only upon a limited amount of alimentary matter; so that, if more food be taken into the stomach than the gastric fluid can dissolve, it remains there undigested. Now it has been already mentioned, that the quantity of the gastric fluid secreted at any one time, is proportional, not to the amount of food in the stomach, but to the wants of the system ; so that, if more food be swallowed than is required to repair the waste of the body, it lies for some time unchanged in the stomach, and becomes a source of irritation which prevents the due discharge of its functions; and the evil goes on increasing with every addition to the contents of the cavity. This may not be felt by the individual at the time; but it leaves permanent effects, which manifest themselves sooner or later in derangement of the general health. The habit of taking more food than is really necessary, and of irritating the stomach by stimulating substances or fluids (such as pepper, mustard, spirits, \&c.), is a fertile source of disease. The injurious effects of these are manifested by the thirst which is the consequence of their use, and which is a call (as it were) on the part of the stomach, to prevent their irritating action by diluting them with water.

211. By the solution of its albuminous portion, and the separation of its other component particles, the food is reduced in the stomach to a kind of pulp, which is termed chyme. The consistence of this will of course vary according to the nature of the food, and the quantity of fluid in the stomach ; but in general it is grayish; semi-fluid, and uniform throughout. When the food has been of a rich character, the aspect of the chyme resembles that of cream; but when the food has consisted of farinaceous substances (rice, potatoes, \&c.), the chyme is more like gruel. At the point where the stomach opens into the intestinal canal, which is called the pylorus, there is a kind of valve, which permits the chyme to pass as fast as it is formed, but closes against the portions of the food which are yet solid and undigested; and thus the chyme escapes from the stomach in successive waves, slowly at first, but afterwards more rapidly, as the digestive process approaches its completion. 


\section{Intestinal Digestion; Chylification.}

212. The process of digestion is by no means completed in the stomach ; for much of the matter which escapes from it in the chyme, is destined to undergo a further change whilst passing through the intestinal canal ; especially in the herbivorous tribes, whose food, being less digestible than that of the carnivorous races, requires to be longer delayed in the intestinal canal, in order that it may yield up its nutritious portion. Hence we find this canal of enormous extent in most animals whose food is vegetable, being in the Sheep about twenty-eight times the length of the body ; in the purely carnivorous animals, on the other hand, it is comparatively short, being in the Lion only about three times the length of the body, while in the Serpent it runs almost straight from one extremity to the other; and in animals which live on a mixed diet, it is of medium length, being in Man about six times as long as his body. The intestinal tube is usually distinguished into the small and the large intestine ; of which the small is the first portion, and the large the second. The former, as shown in fig. 108, is disposed in a convoluted or twisted manner, so that a great extent of it may be packed within a small compass; it usually forms about three-fourths of the whole length of the canal. It is held in its place by a serous membrane termed the peritoneum, which forms an immense number of folds that suspend it (as it were) from the vertebral column; but these still allow it a considerable power of movement.

213. Soon after passing from the stomach into the intestinal canal, the food is mingled with three secretions, which have an important influence on the changes it is further to undergo ; these are the Bile, the Pancreatic fluid, and the Intestinal juice. The two former are prepared by two large glandular masses, the Liver and the Pancreas (or sweetbread), which, in all the higher animals, are completely detached from the alimentary canal, and send their secretions into it through special ducts; the latter, like the gastric juice, is formed in little follicles lodged in the wall of the canal itself. The peculiar matter which forms the chief solid constituent of bile, is essentially a soap formed by the union of two resinoid acids, with soda as a base ( $(364)$. The composition of the 
pancreatic fluid closely corresponds with that of saliva, which it much resembles in appearance. The intestinal juice, like the gastric, is a nearly colourless, somewhat viscid fluid, containing an organic compound not far removed from albumen; but it differs from the gastric juice in being alkaline instead of acid. The relative offices of these three fluids have not yet been determined with certainty ; but there appears good reason to believe: (1) that the bile, by its alkalinity, neutralizes the acidity which the chyme derives from the gastric juice, and that this neutralization favours the metamorphosis of starch into sugar, which has been almost suspended in the stomach; (2) that the bile aids the pancreatic fluid in reducing the oleaginous particles to the condition of an emulsion, that is, in bringing them into a state of very minute division, in which they remain suspended in the albuminous solution; (3) that the pancreatic fluid aids the salivary matter which was swallowed with the food, in the transformation of starch into sugar; (4) that the intestinal juice has a solvent power for albuminous substances which is scarcely inferior to that of the gastric juice, with a power of converting starch into sugar which is scarcely inferior to that of saliva or pancreatic fluid. The fluid of the Small Intestine, compounded of the salivary, gastric, intestinal, biliary, and pancreatic secretions, appears to possess a far greater digestive power than that of the stomach, being capable of dissolving, or at any rate of reducing to an absorbable condition, nutritious substances of every class. This process goes on during the passage of the alimentary mass along the small intestine; and the nutritious materials are progressively withdrawn by absorption, partly into the blood-vessels, which appear to receive whatever are in a state of perfect solution ( $\$ 218)$, and partly into the lacteal absorbents, which take up nothing but that peculiar emulsion of albumen and fatty matter which is termed chyle ( $\$ 222)$.

214. At the extremity of the Small Intestine, there is a kind of pouch, called the coecum; which in some animals seems almost like a second stomach, and which is furnished with one or more little appendages, termed coeca. * This is very small in Man, and does not seem to perform any important

* The word crecum is used in Anatomy to denote a tube closed at one extremity. 
function; but in most herbivorous animals it is larger (as in the Monkey, fig. 30); and it is found to secrete an acid fluid, which resembles the gastric juice, and which may have for its office to perform a second digestion upon the substances which have escaped the first. These coeca are sometimes very large in the intestinal canal of Birds (fig. 111).From the coecum, the Large Intestine ascends as high as the liver, crosses the upper part of the abdomen, and then descends again, as shown in fig. 108 ; this portion is termed the colon; and it terminates in the rectum, which forms the extremity of the intestinal tube.

215. The alimentary mass is propelled along the first part of the intestinal canal,--and the residue left after the absorption of the nutritive materials is carried along the continuation of it,-by the contraction of its muscular coat, producing what is termed the peristaltic motion of the bowels. The fibres of this muscular coat are chiefly arranged in a ring-like manner around the tube; so that, when they contract, they narrow the diameter of the tube. They are stimulated to contract by the contact of the solid or liquid matter passing through it (Chap. XII.); and their contraction forces this matter .onwards, into the succeeding portion of the tube. This contracts in its turn, so as to propel its contents further; and thus the mass is gradually driven from one extremity of the canal to the other. The peristaltic movement does not seem to depend (as do the contractions of the muscles concerned in swallowing, § 195) upon the nervous system; for it will take place after the intestinal tube has been completely separated from the principal nervous centres; and also after the death of the animal, if this have been produced by a sudden cause. Thus, if a Rabbit be killed by a smart blow at the top of the neck, and the abdomen be immediately opened, the peristaltic movement will be seen in vigorous action, especially if the animal have eaten a full meal an hour or two previously.

\section{Defecation.}

216. In passing through the large intestine, the undigested residue is still more completely deprived of the nutritive matter it may contain; and its fluid portion is absorbed, so that it becomes more solid. It is allowed to accumulate in the rectum, until its bulk occasions inconvenient pressure upon 
the surrounding parts ; and it is kept-in by a circular muscle or sphincter, which surrounds the outlet of the alimentary canal. But when the accumulation has taken place beyond this amount, it excites a reflex action ( $\$ 195)$ in the muscles that surround the abdomen; and these make pressure sufficient to overcome the resistance of the sphincter, and to force out the contents of the rectum.

\section{Absorption of Nutritive Material.}

217. We have only now to inquire into the mode, by which the nutritive matter extracted from the food is taken-up from the alimentary canal and applied to the nutrition of the body. In all Vertebrated animals, there exists a special set of vessels termed Absorbents; of which those forming one division,

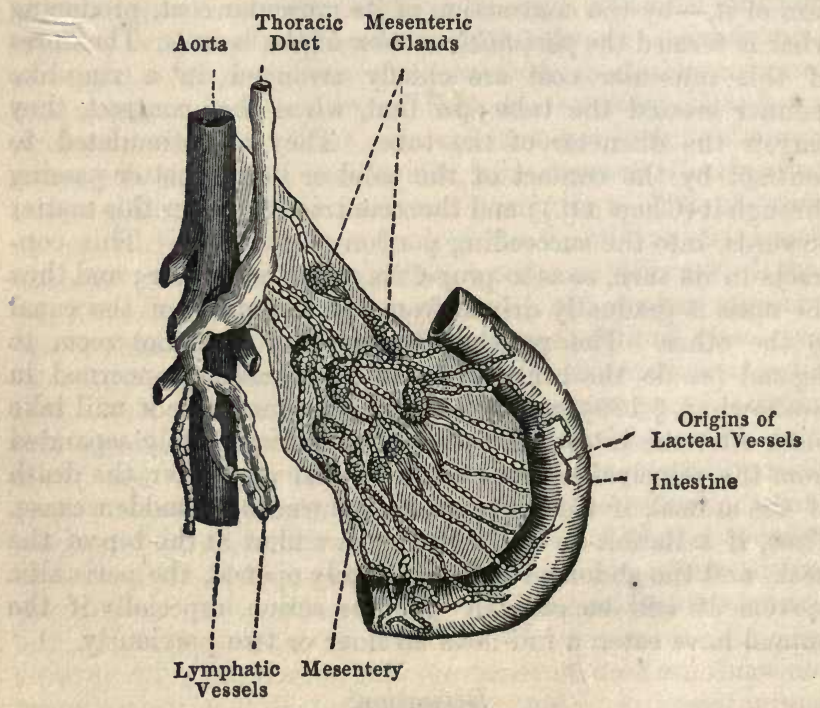

Fig. 114.-ChYLE-vessers.

known as Lacteals, from the milk-like character of their contents, originate in the numberless villi or minute projections with which the mucous membrane that lines the small intestine is covered ( $\$ 41)$. During the act of digestion, the 
epithelium-cells, which clothe the extremity of each villus (fig. 9), become distended with an opalescent fluid, the chyle (\$222), which they select from the contents of the small intestine; and this is subsequently given up by them to a lacteal tube, which, without any open mouth, commences in the midst of each villus. The vessels which thus originate, unite into minute trunks, and these again into larger ones ; and these pass between the two layers of the mesentery (or fold of peritoneum by which the intestines are suspended, § 212) towards the lower part of the spinal column: where they deliver their contents into a sort of reservoir, which thus becomes the receptacle for all the chyle that has been collected from the alimentary canal (fig. 114). In traversing the mesentery, the lacteals of the higher animals pass through little knot-like bodies of a peculiar nature, which are called mesenteric glands. These appear to afford the means for the performance, within a more concentrated space, of the assimilating action which is carried on during the passage of the ehyle through the lacteal system; for in Reptiles, in which these glands do not exist, the absorbent vessels are much more extended and spread out than they are in Birds and Mammals.

218. Near the surface of each of these villi, moreover, lies a minute network of Blood-vessels; and there is now no longer any doubt that these receive, by simple imbibition,* any substances, whether alimentary or otherwise, which exist in a state of perfect solution in the contents of the intestinal canal. For a great variety of such substances have been detected, by chemical analysis, in the blood which is returned from the walls of the intestines by the mesenteric veins; whilst it is seldom that anything is found in the lacteals, save the proper constituents of chyle. It is through this channel that poisonous substances are taken into the circulation; and these may be absorbed from the walls of the stomach (on which there are no villi or lacteals), without ever passing from it into the intestinal tube. Hence it is a great

* That tendency-called Endosmose-which thinner liquids have to pass-towards and mix-with such as are more viscid, even through an intervening membrane, seems to be the physical cause (as experi? ment indicates) of this imbibition; which is greatly promoted by the movement of blood in the vessels. 
mistake to characterise the lacteals (with the lymphatics) as Absorbents in any exclusive sense ; the fact being that their function is limited to a special selective absorption, whilst the more general action is performed by the blood-vessels.

219. But the reservoir above-mentioned receives, not only the lacteal vessels that bring nutritious matter from the intestinal tube, but also lymphatics, which are absorbent vessels of similar character, that originate in every part of the body. These, also, pass through a set of (so-called) glands, in their way towards this receptacle ; and the structure of these glands, of which many are seated in the neck, some in the arm-pit, others in the groin, \&c., is exactly the same as that of the mesenteric glands. The fluid they convey, which resembles very dilute liquor sanguinis ( $\$ 229)$, seems evidently destined to be again applied to the purposes of nutrition. There is some obscurity as to its source; but it seems probable that it may partly consist of the residual fluid, which, having escaped from the blood-vessels into the tissues, and having furnished the latter with the materials of their nutrition, is now to be returned to the former; and partly of those particles of the body, which, though they have lost their vitality in the course of the change it is continually undergoing, have not undergone a degree of decay that unfits them for serving, like the dead bodies of other animals, as a material for reconstruction by the organizing process. The lymphatics, being copiously distributed in the true Skin, absorb substances which are introduced into its tissue; and if these substances be of an irritating nature, they may occasion an inflammatory action in the absorbents and their glands. Thus when poisoned wounds in the hand have been received, as in opening the bodies of men or animals that have died of particular diseases, the effect is usually manifested at first by heat and pain in the arm, along which the inflamed absorbents can be traced as hard cords; and the glands in the arm-pit swell and become tender.

220. The lymphatics do not appear destined, however, to absorb from the surface of the skin ; this function being performed by the blood-vessels which are distributed abundantly in its substance. It is a fact now well established, that when the quantity of fluid in the body has been greatly reduced, absorption of water through the skin may take place to a 
considerable amount. Thus there is a case recorded by Dr. Currie, of a patient who suffered under obstruction of the gullet, of such a kind that no nutriment, either solid or fluid, could be received into the stomach; and who was supported for some weeks by immersion of his body in milk and water, and by the introduction of nutritive liquids into the lower end of the intestine. During this time, his weight did not diminish ; and it was calculated by Dr. Currie, that from one to two pints of fluid must have been daily absorbed through the skin. The patient's thirst, which had been very troublesome previously to the adoption of this plan, was removed by the bath, in which he experienced the most refreshing sensations.- It is well known that shipwrecked sailors and others, who are suffering from thirst owing to the want of fresh water, find it greatly alleviated, or altogether relieved, by dipping their clothes into the sea, and putting them on whilst. still wet.

221. From the receptacle into which the chyle, and a considerable proportion of the contents of the lymphatics, are delivered, a tube passes upwards in front of the spine (fig. 114); and this tube, called the Thoracic Duct, conveys these nutritious fluids to the point where they are to be delivered into the current of blood. This delivery takes place at the angle where two great veins unite, - a point at which there is less resistance than in any other part of their walls. These veins are the Jugular, which brings the blood from the neck, and the Subclavian, which conveys it from the arm, of the right side (fig. 122); on the left side there is a smaller duct, which receives some of the lymphatics of the left side, and opens into the blood-vessels at a corresponding point between the left jugular and subclavian veins.

\section{Sanguification.}

222. The Chyle of Vertebrated animals, as taken-up by the lacteals, may be regarded as blood in an early stage of its formation, with a large excess of fatty matter. It contains about 90 parts of water in 100 ; about $3 \frac{1}{2}$ parts of albumen, and the same of fat; and about 3 parts of other animal and saline matter. Its appearance and characters differ, according to the part of the lacteal system from which it is drawn. If obtained near the surface of the intestines, before it has passed 
through the glands, it is entirely destitute of that power of spontaneously coagulating, or clotting, which is so remarkable in blood: and when examined with a microscope, it is seen to present a number of oily globules of various sizes ; together with an immense number of very minute particles or molecules, which also seem of a fatty nature ; and to these last, whose diameter is between 1-24,000th and 1-36,000th of an inch, the milky whiteness which characterises chyle appears principally due. But the chyle drawn from the lacteals, after they have passed through the mesenteric glands, possesses the power of coagulating slightly; hence it would seem that some of its albumen has undergone a transformation into fibrin (§ 17). At the same time, a great increase is observed in the number of certain floating corpuscles, which are occasionally to be noticed in the first chyle, but which are very abundant in the fluid drawn from the glands and from the lacteals that have passed through them; of these, which bear a strong resemblance to the colourless corpuscles of the blood (\$234), the average diameter is about 1-4,600th of an inch.By the time that the chyle reaches the central receptacle, its power of coagulating has still further increased; so that its resemblance to blood, except in regard to colour, is much stronger. The proportion of fibrin and albumen which it contains, is much greater than that which existed in the first chyle, whilst the amount of oily matter is less.

223. There can be little doubt that the change which the chyle undergoes in its passage through the lacteals, is partly due to the influence of the living walls of these vessels upon the fluid in contact with them, and partly to that of the colourless corpuscles which float in the fluid, and which form the principal constituents of the absorbent glands. The whole apparatus, indeed, may be looked upon as one great Assimilating Gland, having for its function to make blood out of crude nutriment; provided-for in the higher Vertebrata by the convolution of the lacteals in the mesenteric glands, and in the lower, by the simple extension of the vessels themselves. It is probable that, by being brought into very close neighbourhood with the blood in these glands, the chyle may be made to undergo some further change ; although, as each fluid is contained in its own tubes, which do not communicate, there can be no proper intermixture. 
224. There are certain glandular bodies, disposed in various parts of the system, which seem to discharge a similar office; withdrawing the raw material (so to speak) from the general current of the circulation, and returning it again in a state of higher elaboration. Such are the Spleen, the Thyroid and Thymus glands, and the Supra-Renal capsules. Besides these, the Liver probably exerts an assimilating action upon the crude materials which are made to pass through its substance, almost immediately after having been received into the blood-current, and before they are allowed to pass into the general circulation; the whole of the blood returned by the gastric and mesenteric veins from the walls of the alimentary canal, being conveyed through the liver by the portal system, in its way to the heart (\$ 267).

225. In the Invertebrated animals, neither lacteals nor lymphatics exist; and the blood-vessels, whose absorbent powers are to a certain extent restricted in the higher animals, have to perform the functions of these. There are animals, however, which are destitute not only of lacteal and lymphatic vessels, but even of blood-vessels; and in these, as in the Cellular Plants, there is but little transmission of fluid from one part of the body to the other; for every portion, both of the internal surface (or lining of the stomach), and of the external surface which is bathed in the surrounding fluid (for most of these animals are aquatic), seems equally to possess the power of absorption ; and the parts to whose nourishment the fluid thus received into the body is to be appropriated, are in the immediate neighbourhood of those which have absorbed it. This is the case, for example, in the Hydra and Sea-Anemone, and, more or less, in all the Polypes ; as well as in the lower Worms. Between these, therefore, and the Cellular Plants, a remarkable analogy exists in regard to the mode in which the nutriment is absorbed and applied ; the difference being, that the Animal possesses a digestive cavity, lined by an inward extension of the external surface, which does not exist in Plants ( $\S 8)$. And it is upon the walls of this cavity, that the absorbent vessels of the higher Animals (whether lacteals or blood-vessels) are distributed, collecting the nourishment in contact with them; just as the roots of a Plant, spread through the soil, draw up that which it contains. But among those lowsst animals in which the digestive cavity altogether 
disappears ( $\$ 203)$, the function of absorption is not in any way limited ; since every part seems to have the power of receiving from without, and of assimilating to its own substance, the nutrient materials which it needs.

\section{CHAPTER V. \\ OF THE BLOOD, AND ITS CIRCULATION.}

226. The processes that have been already explained, have for their object to prepare the nutritious fluid, which supplies the materials for the growth of the several parts of the body, and which is conveyed through them by the apparatus to be presently described. In Man and the higher animals, this fluid, which is known as the Blood, has a red colour, and contains a large quantity of solid matter. The redness of the blood has been mentioned as a distinctive character of the Vertebrated classes ( $\$ 75$ ); it exists in Mammalia, Birds, Reptiles, and Fishes, and in these alone. In the Molluscous classes, as also in most of the Articulated, the nutritious fluid is nearly colourless ; and it will hereafter appear that this fluid bears, in some respects, a stronger resemblance to the chyle and lymph of the Vertebrata, than to their blood (§ 234). There is an apparent exception in the case of certain marine Worms, the fluid circulating in whose vessels has a reddish hue; this does not depend, however, upon the presence of any red particles, such as are characteristic of the blood of Vertebrata ( $\$ 229$ ), but upon a reddish tinge in the fluid itself, which does not seem altogether to answer to the character of blood (\$ 294).

227. The blood of all the higher animals exists in two different states. When it is drawn from a slight scratch or other wound of the skin, it is of a bright red hue; whilst that which is drawn in bleeding from the arm, is of a dark purple. The former is termed arterial blood, because it is contained, for the most part, in the tubes which are called Arteries, and which are conveying it from the heart to the tissues it has to nourish. The latter is called venous blood, because it is drawn from the Veins, by which it is returned from the tissues to the heart, after having performed its part in them. Hence it 
is evident that this change of character has been produced during the passage of the blood through the tissues; and so important is the alteration, that the blood which has been subjected to it is not fit to pass again into the arteries of the body, until it has been renewed by exposure to air in the Lungs. In their vessels, the contrary change - of which the nature will be presently explained ( $(253)$-is effected, the dark hue of venous blood giving place to the bright red of the arterial fluid; this is again changed during the passage of the blood through the body, to be again restored in the lungs. The same is the case in regard to Fishes, whose gills perform the same function as the lungs of air-breathing Vertebrata. And among the Invertebrated classes, although the deterioration of the blood in its passage through the body is not made manifest by any change of colour, yet its renewal by exposure to air in the respiratory organs is not less requisite.

228. Hence the continual movement of the blood is necessary for two purposes in particular;-first, to convey the nutritive materials from the place where they are received and prepared, to that in which they are appropriated, and thus to afford to every organ a constant supply of the materials which it requires ; - and, second, to carry this fluid, at regular intervals, to certain organs by whose instrumentality it may be exposed to the influence of the air, so as to regain the qualities it has lost, and part with what it has taken-up to its prejudice. But there are many other objects fulfilled by it, which will unfold themselves as we proceed.

\section{Properties of the Blood.}

229. When the circulating blood of a red-blooded animal is examined with a microscope, it is seen to consist of two distinct parts; - a clear and nearly colourless fluid, to which the name of liquor sanguinis (or liquor of the blood) is given; and of an immense number of rounded particles floating in this fluid, which are often termed the globules of the blood. The shape and size of these particles are, for the most part, very uniform in animals of the same species; but in no instance are they globular; and it is better, therefore, to term them corpuscles. In Man and most other Mammals, they aro nearly flat discs, resembling pieces of money, but usually exhibiting a slight depression towards the centre (fig. 115). 
No nucleus can be distinguished in them, but they present a dark central spot, which is an optical effect of their bi-concave form; and this spot may be made to disappear by the addition
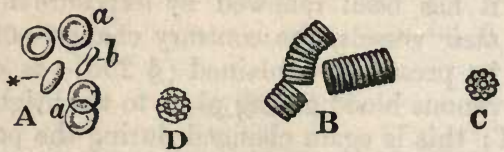

Fig. 115.-Red Corpusches of Human Blood.

Seen separately at $\Lambda, a d$ showing the front view, $b$ the profile or edge view, and * a three-quarter view; at B united with each other so as to form columns like pil es of money; at $c$ in a state of alteration such as exposure to air will producs; D shows a colourless corpuscle, or lymph-globule.

of water to the liquid in which they are suspended, the dises first becoming flat, then bulging-out on either side, and at last swelling so as to burst. The reason of this will be presently explained ( $\$ 231$ ). In MAN and MAMMaLs generally, the diameter of these blood-discs varies from about 1-2800th to $1-4000$ th of an inch; but in the small Musk-deer, it is less than 1-12,000th. In the Camel tribe, the discs are oval, as in the lower Vertebrata.

230. In Birds, Reptiles, and Fishes, the blood-particles present some curious differences from those of Mammalia. In the first place, they are much larger; their form, also, is oval instead of being round; and instead of being depressed in the centre, they bulge-out on each side. This bulging is

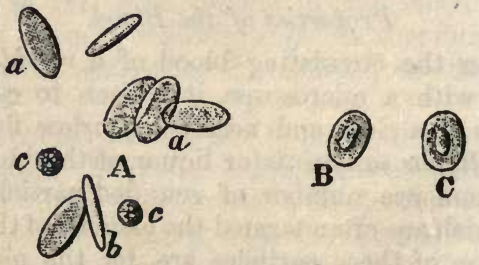

Fig. 116.-Blood Corpuscles of Pigeon.

At $A$ are seen the red corpuscles $a, b$, and the colourless, or lymph globules $c, c$; at $\mathrm{n}$, a red corpuscle treated with acetic acid; and at $\mathrm{c}$, the same treated with water, $s o$ as to render the nucleus more distinct.

evidently occasioned by the presence of a nucleus which is more solid than the rest; the nucleus, however, is not so well 
seen in the corpuscles of circulating or of freshly-drawn blood, as it is in that of blood which has been drawn for some little time; and it is best brought into view by treating the blood either with water or with acetic acid. The long diameter of the oval discs of BrRDs (fig. 116) varies from about 1-1700th to 1-2400th of an inch; and the short diameter from about
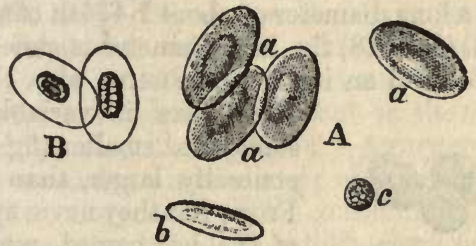

A

(2) $c$

Fig. 117.-Brood Corpuscres of FroG.

At $\mathbf{A}$ are seen the red corpuscles $a, b$, and the colourless corpuscle $c$; at $\mathbf{B}, \mathbf{a}$ red corpuscle treated with acetic acid.

1-300th to 1-4800th. Thus the discs, though much longer than those of Man, are not in general much broader. In ReptiLes,
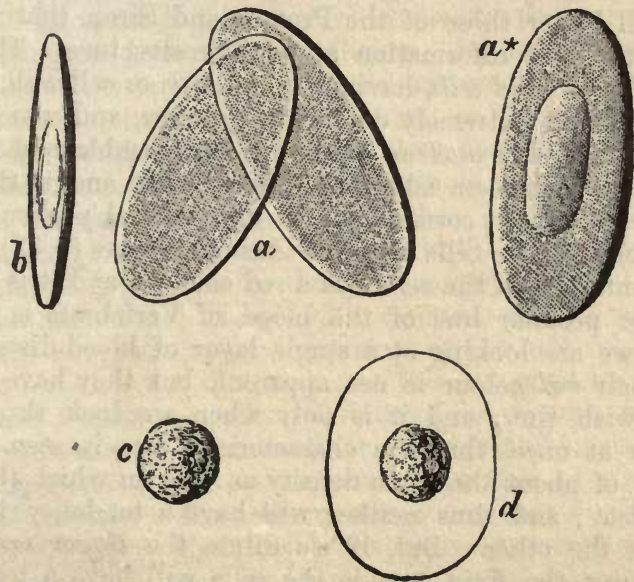

Fig. 118.-Brood Conpuscres of Proteus.

$a, b$, red corpuscles; $a *$, corpuscle showing the nucleus; $c$, colourless corpuscle: $d$, red corpuscle treated with water. 
there is considerable diversity as to the size of the dises ; but the largest particles are found in the group of Amphibia, and especially in those species which retain their gills through life. The oval dises of Frogs (fig. 117) have a long diameter of about 1-1000th of an inch, and a transverse diameter of about 1-1800th. Those of the perennibranchiate Amphibia ( $\$ 87$ ) may even be distinguished by the naked eye; those of the Siren having a long diameter of about 1-435th of an inch, whilst in the Proteus (fig. 118) the long diameter is stated occasionally to reach 1-337th of an inch. In Fishes, also, the size of the

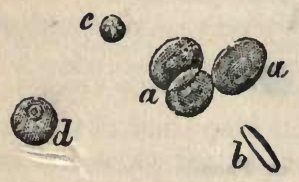

Fig. 119.-Brood Coxpuscres or ROACH.

$a, a, b$, red corpuscles ; $c$, coluurless corpuscle; $d$, red corpuscle treated with water. blood-discs is variable; they are sometimes smaller (fig. 119), though generally larger, than those of the Frog; but they never approach those of the last-named remarkable animals. Hence the great size of the blood-dises of the curious Lcpidosiren (fig. 41) is strongly indicative of the Reptilian affinities of that species.

231. It is by observing the large blood-dises of the Frog, and still better those of the Proteus and Siren, that we can obtain the best information as to their structure. They are evidently flattened cells, having an envelope or cell-wall, which consists of an extremely delicate membrane, and which con. tains a fluid. The nucleus consists of an assemblage of minute granules, which seem adherent to each other and to the wall of the cell ; and it corresponds, in all essential particulars, to the nuclei of the cells of other Animal tissues (\$ 32). The fluid contained in the cells has a red colour ; and it is to this that the peculiar hue of the blood of Vertebrata is owing. When we are looking at a single layer of blood-dises, however, their red colour is not apparent, but they have rather a yellowish tint; and it is only when we look through a number at once, that the characteristic hue is seen. The fluid is of about the same density as that in which the particles float; and thus neither will have a tendency to pass towards the other. But, if we dilute the liquor sanguinis with water, the fluid outside the cells will have a tendency to pass towards their interior, according to the law of Endosmose. The cells will in consequence be first distended, and 
will then burst; and their contents will be diffused through the surrounding fluid, whilst their membranous walls will subside to the bottom. On the other hand, if the liquor sanguinis be rendered denser than the fluid in the blooddiscs, as by the admixture of gum or syrup, the latter will pass towards it, and the cells will become still more flattened, and more or less completely emptied. The flexibility and elasticity of the blood-dises are well seen, in watching (with a microscope) its flow through the minute vessels; for if one of them meets with an accidental obstruction to its progress, its form becomes accommodated to that of the space left for it to pass, and it makes its way through a very small aperture, recovering its usual form immediately afterwards.

232. The Red Corpuscles differ considerably in chemical composition from the liquid in which they float. Of the solid residue obtained by drying, about one-eighth is formed by their cell-walls, the remainder being yielded by the cellcontents. The latter portion seems to consist chiefly of a mixture of two components, which have been named globulin and hoematin. The former is a colourless substance, nearly allied to albumen in composition, but differing from it in some of its reactions ; its most characteristic peculiarity, however, being its power of crystallizing. Its crystals, the form of which varies in different animals, are usually tinged deeply with hæmatin, from which they cannot easily be freed. The composition of hæmatin, to which alone the colour of the red corpuscles (and consequently of the whole mass of the blood) is due, is notably different from that of the albuminoid compounds ; the proportion of carbon to the other components being much greater, and a definite quantity of iron being an essential part of it. This iron, in a certain state of oxidation, has been supposed to be the source of the red colour; but such is certainly not the case; and this hue must be, like the colours of Plants, a peculiar attribute of the organic compound which presents it.-Besides their globulin and hæmatin, the red corpuscles contain a certain proportion of fatty and mineral matters. The former, which are united with phosphorus, are of a kind which are scarcely traceable in the liquor sanguinis; and the latter are remarkable as having potass for their principal base, whilst the base of the salts of the liquor sanguinis is chiefly soda. Hence it appears that 
the Red Corpuscles draw into themselves nearly the whole of the iron, phosphorus, and potass, which the chyle pours into the circulating current; and that they modify a large proportion of the solid matter of the blood, that which they contain being notably different in composition from that of the liquor sanguinis, which does not differ, save in the proportion of its components, from the liquid portion of Chyle or Lymph.

233. The proportion of Red Corpuscles to the whole mass of the blood varies greatly in different animals, and even in different states of the same animal. It is greatest in those which have the highest muscular vigour and activity, and which consume the largest quantity of oxygen by respiration; hence these particles are rather more numerous in the blood of Birds than in that of Mammals, and far more abundant in these last than in Reptiles or Fishes. Again, they are more numerous in Men of ruddy complexion, strong pulse, and active habits, than in those of pale skins, languid circulation, and comparatively feeble powers. In a healthy Man they seem to constitute about half the mass of the circulating blood; but they contain as much as three-fourths of its solid matter, the proportion of $d r y$ corpuscles being about 150 in 1000 parts of blood, whilst that of the other solid matters is about 50. A very marked decrease occasionally presents itself in disease ; the proportion of dry corpuscles being sometimes reduced as low as 27 . When too abundant, they produce what is known as the plethoric condition of the body, in which hæmorrhage from the bursting of a blood-vessel is liable to occur. Their number is effectually reduced by bleeding; and the aspect of those who have suffered from extreme loss of blood, gives sufficient evidence that the deficiency is not made-up for a long period. The most effectual means of restoration, in cases where the proportion of blood-corpuscles is too low, is a highly nutritious diet, with the administration of iron as a medicine; for this substance seems to have the power of hastening the reproduction of the corpuscles, being itself an essential ingredient in their contents; and there are facts which show its remarkable power of increasing their amount in proportion to the mass of the blood.

234. It appears that the red corpuscles, like other cells, have a certain allotted term of life; and as they are contirually dying, they must be as continually reproduced. The 
mode in which this reproduction is effected has not yet been clearly made out ; but there is strong reason to believe that the red corpuscles are developed from the corpuscles of the chyle and lymph ( $\$ 222)$ which are continually being poured into the circulating current, and of which isolated examples, known as the white or colourless corpuscles, are met with in every drop of blood that is examined under the microscope. The size of these is pretty much the same in all Vertebrata, their diameter being usually about 1-3000th of an inch. In the blood of Man and the Mammalia in general (fig. 115, D) they are not easily distinguished from the red particles; their diameter being nearly the same, while the colour of single dises of the two kinds is not very dissimilar. But in the lower Vertebrata, whose blood has large oval red particles, the difference between the two kinds is very obvious; and the resemblance which the colourless globuler (c, figs. 116-119) bear to those of the chyle and lymph, is very striking. Similar colourless particles exist, to a variable amount, in the nutritive fluid of Invertebrated animals ; so that in this, as in some other respects, that fluid bears a stronger resemblance to the chyle and lymph of the Vertebrata, than it does to their blood, which is characterised by the presence of the red particles.

235. Physiologists are now generally agreed, that one of the functions of the Red Corpuscles is to convey oxygen from the lungs to the tissues and organs through which the blood circulates, and to bring back the carbonic acid which is set free in these, so as to deliver it at the lungs. For although it is certain that the liquor sanguinis can also convey these gases, yet experiment shows that the red corpuscles can take up, bulk for bulk, a much larger proportion of them; and that the blood which is richest in these particles is, therefore, most fit to serve as the medium for the transmission between the respiratory organs and the body at large. Now it is in the nervo-muscular apparatus that there is the greatest demand for oxygen; for this apparatus is not capable of vigorous action, unless oxygen be freely supplied to it. The quantity of this it requires, however, depends upon the exercise of its powers; for when at rest, it needs little or no more than is made use of by the other tissues; but whilst in activity, it. needs a greatly-increased supply. The quantity of oxygen which the animal takes-in by its lungs, and the amount of 
carbonic acid which it gives-off by the same channel, vary, therefore, with the muscular exertion it makes. This variation is most easily observed and measured in Insects; and it is found in them to be enormous ( $\$ 308$ ). As, however, the blood of the Invertebrata does not contain these red particles, to which so important a function has been assigned, it may be asked, how the conveyance of oxygen to their tissues is provided for. The reply is very simple. In Insects, and other ARTrCULATA which have active powers of motion, the air is conveyed to the tissues, not through the medium of the blood, but directly through air-tubes which convey it to every part of the body (§321). And in the Moluuscous classes, as among the Crustacea also, the nervo-muscular system forms so subordinate a part of the general mass of the body, and its movements are so sluggish, that the quantity of oxygen which the fluid part of the blood conveys to them, is sufficient for their need.

236. Of the properties of the Liquor Sanguinis, whilst it is circulating in the vessels, the microscope tells us nothing; since it constantly remains in the state of a transparent fluid. But if the blood be withdrawn from the living body, it soon undergoes a very curious and important change. A large portion of it passes into the solid state, forming the crassamentum or clot; whilst there remains a transparent liquid of a yellowish hue, which is termed the serum. It is evident that the clot contains all the red particles; but it is easily proved that its coagulation is not due to them. For the blood of a Frog, or of any other animal having blood-discs sufficiently large, may be caused to pass through filtering-paper, which will retain and collect its blood-discs, allowing the liquor sanguinis to flow through it ; and this fluid will coagulate just as completely as if these particles were retained in it. Again, in certain conditions of the blood (generally resulting from disease), even when the coagulation is allowed to take place in the ordinary manner, the fibrin and the red particles separate from one another, - the latter gradually subsiding, whilst the former are left at the surface ; and the upper part of the clot is then nearly colourless, exhibiting what is commonly known as the buffy coat or crust; whilst the lower part of it includes the red particles, and has a very deep colour. The buffy coat, being composed almost exclu- 
sively of the fibrous network, is very firm in its texture, being sometimes almost leathery in its character; whilst the lower part of the clot, which is chiefly composed of the red particles, loosely bound together by scattered fibres, is very soft, and easily broken asunder. This effect may be also produced, by acting on healthy blood with certain substances which retard its coagulation, such as a strong solution of Glauber's salt; for if sufficient time is allowed, the red particles will subside in consequence of their greater specific gravity, leaving a colourless layer of fibrin above them.-It is of the liquor sanguinis, in a concentrated form, that those exudations consist, which are poured out from the blood for the repair of injuries, and which pass spontaneously into the condition of a simple form of tissue ( $\$ 393$ ).

237. When a very thin slice of the clot is examined with a microscope, it is found to be made up of a net-work of an imperfectly fibrous character, interlacing in every direction, and including the blood-dises in its meshes. These fibres are produced by the spontaneous change in the fibrin of the blood, from the fluid to the solid form. So long as the blood is circulating in the vessels of the living body, so long does its fibrin remain dissolved in the watery part of it; but so soon as it is withdrawn from these, and is allowed to remain at rest, it undergoes this remarkable change. If fresh-drawn blood be continually stirred with a stick or beaten with twigs, the fibrin coagulates in irregular strings, which adhere to the stick or twigs ; and it does not then include the red particles, which are left behind in the fluid. In this manner it may be completely separated from the other elements of the blood, which have not in themselves the least tendency to coagulate spontaneously. Although forming a large proportion of the substance of the clot, the fibrin, when dried, does not consti tute more than from 2 to 3 parts by weight in 1000 of blood. This proportion is augmented to 6,8 , or even 10 parts, in severe inflammatory diseases.

238. When the fibrin and the red particles have both been separated from the blood, there remains a fluid, the serum. in which a good deal of albumen is dissolved, together wivn fatty matter, and other organic substances; with the addition. of saline matter, of which a considerable proportion is chloride of sodium, or common salt. The proportion which the solid P 2 
matter of the serum bears to the whole mass of blood, in health, is about 53 parts in 1000 ; and of these about 40 parts are albumen, 8 parts saline matter, and 5 parts fat, with eertain ill-defined substances, of which some appear to be organic compounds that are undergoing metamorphosis into solid tissues, whilst others are the products of the decay of the tissues, which are being progressively withdrawn and eliminated by the excretory organs.।

239. The influence of the Blood as a whole upon the animal as well as on the nutritive functions, is easily proved. When an animal is bled largely, it is gradually weakened as the flow proceeds, and at last it seems to lose all consciousness and power of movement. If allowed to remain in this condition, it seldom or never recovers of itself. But if we inject into its veins, by small quantities at a time, blood similar to that which it has lost, the apparent corpse becomes as it were reanimated, and all its functions are completely re-established. The importance of the red particles is manifestly seen in the effect of this remarkable operation, which is called the transfusion of blood; for if, instead of blood freshly obtained from mother living animal, we inject serum without these particles, the effect is but little greater than if so much water were introduced, and the animal dies of the hæmorrhage. By this operation, practised on the Human subject, many valuable lives have been saved, that would otherwise have been destroyed by loss of blood. Again, if, by mechanical means, as by tying the principal blood-vessel going to any organ, we cause a permanent diminution to any considerable extent, in the quantity of blood with which it is supplied, a decrease in its size is soon apparent, and it may even shrink almost to nothing. On the other hand, we observe that, the more active the function of a part, the larger is the quantity of blood with which it is supplied. Thus, when the antlers of the Stag, which fall off every year, are being renewed, the arteries that supply the parts of the skull from which they spring, are greatly increased in size; but they shrink again, as soon as the growth of the horns is completed for that year. A similar increase takes place among animals that suckle their young, in the size of the arteries that supply the mammary glands,

"by which the milk is formed; and these also shrink, when this liquid is no longer required. 
240. The following appear to be the chief uses of the principal constituents of the Blood, considered separately, in the general economy:-The fibrin is the material which is most assimilated to the condition of the solid tissues, having the power of passing from the liquid state into a low and simple form of organization. It was formerly supposed to be the nutritive material at the expense of which the solid tissues generally are immediately produced; the muscular substance, in particular, being regarded as chemically identical with it. But there is now good reason to think that the greater part of the tissues form themselves at the expense of the albumen of the serum and perhaps of the globulin of the red corpuscles; and that the purpose of the fibrin is chiefly to give origin to those simple forms of fibrous or connective substance, the production of which is the first step in the reparation of injuries. Were it not for its power of coagulation, the slightest cut or scratch might become fatal, from the gradual draining-away of the blood; and such, in fact, has actually happened, in cases of disease in which the fibrin is deficient. The presence of fibrin also gives a degree of viscidity to the blood, which, as experiment proves, favours (instead of resisting, as might have been expected) its passage through capillary tubes; and thus, when there is a deficiency in this ingredient, local stagnations and obstructions in the circulation of the blood are very liable to occur. The albumen of the blood may be considered, like that of the egg, as the raw material, at the expense of which (in combination with fat) every other organic compound in the body is generated. It is, as we have seen, the substance to which all the tissueforming elements of the food are reduced in the process of digestion; and in this condition it seems to be continually appropriated by the acts of self-formation that are taking place, with varying rapidity, throughout the body, just as the albumen of the egg is appropriated by the self-formative operations of the embryo. There is strong reason to believe that a large proportion of the solid tissues regenerate themselves by the direct appropriation of this material; and if (as has been already stated to be probable) the simple fibrous tissues find their material in the fibrin, and the muscular substance in the globulin of the red corpuscles, it is from the albumen that these substances are themselves elaborated, both of them 
being, as it were, in process of organization. The albumen of the blood further serves to supply the albuminoid matters which are required as constituents of various secretions, especially those which are concerned in the digestive process, as the saliva, the gastric juice, and the pancreatic fluid. A large amount is daily drawn-off for the production of the peculiar ferments contained in these secretions, whose action upon the food is necessary for its reduction to the form in which alone it can be received into the circulating current. Hence the making of new blood involves a considerable expenditure of the old.

241. The liquid in which the fibrin and albumen are dissolved, has a considerable power of absorbing gases; and this is greatly increased by the presence of the saline matters which it holds in solution. Hence the liquor sanguinis not only sustains the nutrition of the body, but can also serve, to a considerable extent, as a medium of communication between the lungs and the tissues. In this kind of activity, however, it is completely surpassed by the red corpuscles (\$ 235). Independently of their use in ministering to the function of Respiration, there seems reason to believe that the red corpuscles are also subservient to that of Nutrition; for a certain conformity which exists between the organic and mineral substances they contain ( $\$ 232$ ), and the composition of Muscle and Nerve, taken in connexion with the manifest relation between their number and the activity of the Nervo-muscular apparatus, makes it probable that they have it for their especial office to prepare the materials which are to be used in its production and renewal of those tissues. The saline matter of the blood has many important offices: thus it furnishes the mineral ingredients which are requisite for the production of the tissues and secretions ; it helps to preserve the organic substances from decomposition; and, in conjunction with the albumen, it keeps up the density of the serum to the point at which it is equivalent to that of the contents of the red corpuscles, without which balance the condition of the latter would be seriously impaired (§ 231). Finally, the fatty matters of the blood are subservient to two very important functions - the maintenance of heat, and the formation of tissue. They maintain the combustive process, whenever there is a deficiency of more readily combustible material; and they also take part with 
albumen in the formation of all new tissue, its nuclear particles being always found to include fat-granules.

242. The presence of a due proportion of the foregoing substances in the blood is an essential condition of health; and we find it provided-for in the marvellous power which the blood, like any solid tissue, seems to have of making itself from the materials supplied to it, and of getting rid of what is superfluous or unsuitable. Thus an excess of albuminous matter in the food does not seem to produce more than a very limited increase in the quantity of albumen in the blood, the surplus being made to undergo changes within the body, which issue in its being removed by the excretory organs. An excess of any of the saline compounds is very speedily strained off (as it were) into the urine. And an excess of fatty matters is drawn off either by the formation of fat as a tissue, or by the augmented activity of the liver in producing bile. This conservative power is still more remarkably shown in the completeness with which the poisons that are generated in the body by the decay of its tissues, and which are received into the current of the circulation for the purpose of being conveyed to the several excreting organs, are drawn off from it, so as to leave the blood pure. Thus, carbonic acid is being continually produced in such large quantities, that its accumulation in the blood, even for five minutes, would be fatal ; yet by the aerating process to which the lungs are subservient, it is got rid of as fast as formed, so that the blood is restored to its previous purity. In like manner, the urea, which is one of the products of the wear and tear of the muscles consequent upon their use, is so perfectly and constantly eliminated by the kidneys, that its detection in the circulating current is a matter of difficulty, although we know that it must always be passing through this.

243. Thus the circulating current may be likened to a tidal river running through the midst of a large town, and supplying it with the water needed for the drink of its human and other inhabitants, as well as with that which is required for the various manufacturing and cleansing operations carried on within its precincts; the same stream also receives the drainage of the town, and consequently becomes charged with the products of animal and vegetable decomposition, and the foul refuse of manufactories; and as the flow 
of the tide brings back a large proportion of what is carried down at ebb, the waters speedily become so contaminated with hurtful and offensive matters as to be unfit for use, unless means be provided for getting rid of these as fast as they are poured in. The perfection with which this requirement is fulfilled in the Animal body, while it excites our admiration, should also incite us to imitation, so far as the art of Man can hope to imitate the works of the Divine Artificer.

\section{Circulation of the Blood.}

244. In some of the lower tribes of Animals, the blood appears to circulate in channels which are merely excavated in the substance of their tissues and organs. But among all the Vertebrata, and even in most of the Invertebrated classes, the movement of the blood takes place in a very complicated apparatus, which is composed, 1st, of a system of tubes or canals which serve to convey it through every part of the structure, and $2 \mathrm{~d}$, of a special organ for the purpose of giving motion to that liquid. These canals are known as the blood-vessels; and this special organ is the heart.

245. The Heart is the centre of the circulating apparatus. It is a kind of fleshy bag, communicating with the bloodvessels : and it alternately dilates to receive the blood, which is conveyed to it by one set of these ; and then contracts so as to force it out into another set of tubes. In this manner a continual current is kept up. All but the lowest animals have a heart, or something which represents it. Such an organ exists, not merely among all the Vertebrated classes, but in all the Mollusea, and in the higher Articulata. But, as will presently appear, there is a great diversity in its form, and in the complexity of its construction; for whilst, in its simplest condition, it possesses but one cavity, communicating with both sets of vessels, - it contains, in its highest forms, four different chambers, each of which has its own peculiar function.

246. The two sets of blood-vessels just adverted-to are, 1st, the Arteries, which convey the blood from the heart into the several parts and organs of the body; and $2 \mathrm{~d}$, the Veins, which collect the blood that has been distributed through these, and return it to the heart. The Arterial system, as it issues from the heart, consists of one or more large trunks, which divide into branches, very much in the manner of the 
stem of a tree; these branches again subdivide into others more numerous but smaller, and these again into twigs still more numerous and more minute; until almost every portion of the body is so penetrated with them, that not even a trifling scratch, cut, or prick, can be made, without wounding some one of these small divisions (fig. 120).-The Venous system presents a corresponding distribution, but it is destined for an opposite purpose ; and we must regard it as commencing in the tissues by the minuter canals, which run together like the

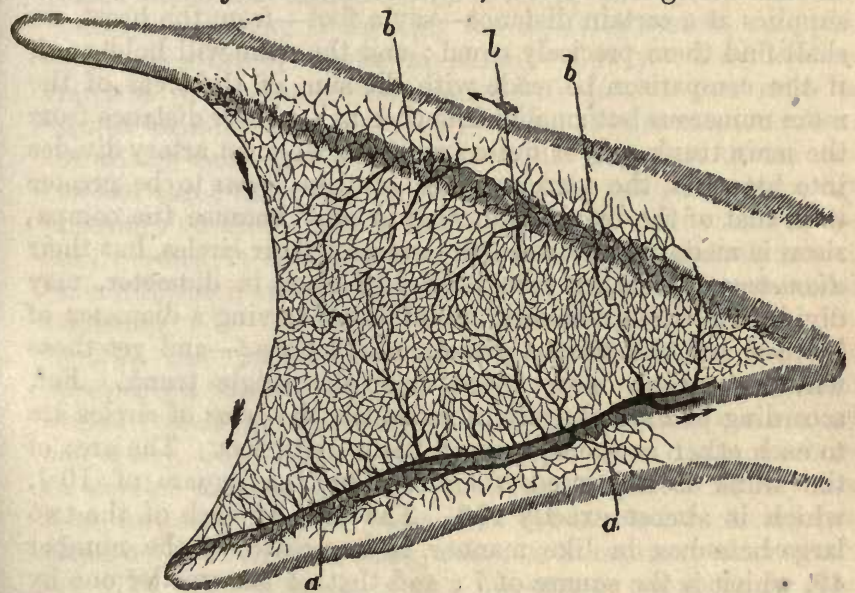

Fig. 120.-Distrubution of THE SMALLER BLOCD.VESEELS IN THE MEMBRANE BETWEEN TWO OP THE TOES OY THE HIND YOOT OP THE COMMON FROG; $a \boldsymbol{a}$, veins; $b$, arteries.

little rivulets that form the origin of a mighty river, or like the smallest fibres of which the roots of a tree are made up. The larger canals thus formed gradually unite with each other as they approach the heart, towards which they all tend, just as the various tributary streams pour their contents into one principal channel : and at last all the veins empty into the heart, by one or two large trunks, the blood which they have conveyed from the several parts of the body; just as all the tributaries which have arisen over a wide extent of country, pour into the ocean the water they have collected, by one mouth which is thus common to all of thern.

247. Although the number of the Arterial branches increases 
so vastly, as we proceed from their origin towards their termination, yet their capacity does not, at least in any considerable degree; - that is, the first or main trunk will allow as much fluid to pass through it in a certain time, as will the whole of the first set of branches into which it divides, or the still more numerous subordinate branches into which these diverge. Or, to put this fact in another form, if we cut across the main trunk, and compare the area, or space included within its circular walls, with the sum of the areas of all the branches it supplies at a certain distance-say a foot-from the heart, we shall find them precisely equal ; and the same will hold good, if the comparison be made with the sum of the areas of the more numerous but smaller branches at a greater distance from the main trunk. It is quite true that, when an artery divides into branches, the combined size of these seems to be greater than that of the trunk; but this is only because the comparison is made, not between the areas of their circles, but their diameters. Thus, an artery of 10.1 lines in diameter, may divide into three branches, two of them having a diameter of 7 lines, and the third a diameter of 2 lines;-and yet these will convey no more blood than the single trunk. For, according to a simple rule in geometry, the areas of circles are to each other as the squares of their diameters. The area of the trunk is expressed, therefore, by the square of $10 \cdot 1$, which is almost exactly 102. The area of each of the two large branches, in like manner, is expressed by the number 49 , which is the square of 7 ; and that of the smaller one by 4 , the square of 2 ; and the sum of these $(49+49+4)$ is exactly 102 , making the combined areas of the branches the same as that of the trunk. In like manner, one of the branches of 7 lines diameter might subdivide into two branches of a little less than 5 lines each; for, as the square of 5 is 25 , and twice that number is equal to 50 , the combined areas of the two branches of 5 lines each, exceed by very little the area of the trunk of 7 lines.-Hence it results, that the pressure of the blood upon the walls of the arteries will be everywhere almost exactly the same;-a conclusion which is confirmed by experiment.

248. There are certain differences in the structure and distribution of the Arteries and Veins, which it is desirable to mention. The Arteries receive the blood pressed out from 
the heart, and must be strong enough to resist the force of its contraction; otherwise, as there is a considerable impediment to its onward flow, produced by the minuteness of the tubes through which it has to pass, and the friction to which it is subjected against their sides, their walls would give way, and they would burst. They have, accordingly, a tough elastic fibrous coat, which contains also more or less of non-striated muscular fibre. On the other hand, the Veins receive the blood after the heart's power over it has been almost expended in forcing it through the capillary system, and when it is consequently moving much more slowly. They are very large in proportion to the arteries; so that, if we were to cut across a limb at any place, and to estimate the respective areas of all the veins and arteries, we should find that of the veins two or three times as great as that of the arteries. Hence the pressure on their walls is much less ; and their strength does not require to be so great. Accordingly we find their walls much thinner, and the tough elastic fibrous coat almost entirely wanting.

249. The difference in the force with which the blood presses on the walls of the arteries and veins, is seen when these vessels are wounded. If a small incision be made into an artery, the blood spouts from it to a great distance; but if a similar incision be made in a vein, the blood merely flows out, unless we stop its passage to the heart, by making pressure on the vein above the orifice, as in ordinary blood-letting (§ 277). Hence much greater pressure is requisite to check bleeding from an artery, than to stop bleeding from a vein; and it frequently happens that no amount of pressure can prevent the continued drain of blood from the former, so that it becomes necessary to stop the flow of blood through the artery altogether, by tying a ligature tightly round it.

250. The Arteries are for the most part so distributed, that their trunks lie at a considerable distance from the surface of the body, so as to be secluded from injury; and they are often specially protected by particular arrangements of the bony parts. Of the Veins, on the other hand, a large proportion lie near the surface, and they are consequently more liable to be injured; but, for the reason just stated, wounds in them are of comparatively little consequence.

251. The ultimate ramifications of the Arteries are conti- 
nuous with the commencing twigs of the Venous system. The communication is established by means of a set of extremely minute vessels, which are termed Capillaries.* These capillaries form a network, which is to be found in almost every

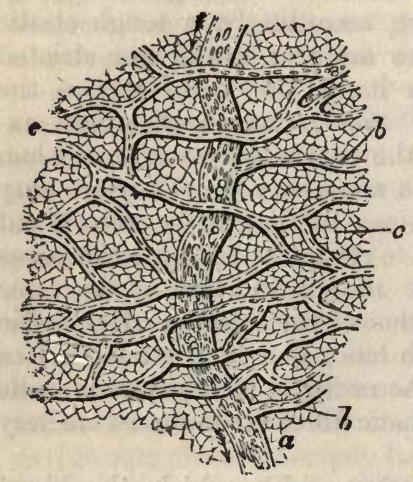

Fig. 121.-Portion of thi MEMBRaNe BETWEEN THE TOES OF THE HIND FOOT OP THE FROQ, more highly magnified than in fig. 120, showing the network of Capillaries that traverses it ; $a$, small venous trunk; $b \quad b$, branches communicating with the capillaries; $c$, intervening tissue covered with epithelium cells. part of the body (fig. 121). It is in them alone that the blood ministers to the operations of nutrition and secretion. Even the walls of the larger blood-vessels are incapable of directly imbibing nourishment from the blood which passes through them; but are supplied with minute branches, which proceed from neighbouring trunks, and form a capillary network in their substance. The diameter of the capillaries must of course bear a certain proportion to that of the blood-dises which have to pass through them: in Man they are commonly from about 1-2500th to 1-1600th of an inch in diameter. In the true capillaries, it would seem that only one row or file of these particles can pass at a time; but we frequently see vessels pasising across from the arteries to the veins, which will admit several rows. There seems, however, to be a considerable difference in the diameter of the same capillary at different times; a change sometimes taking place from causes which are not yet understood. $t$ The rate at which the blood moves

* From the Latin capilla, hair ; so named on account of their being, like hairs, of very minute size. Their diameter is really, however, far less than that of ordinary hairs.

+ The circulation of the blood in the Frog's foot, the tail of the Tadpole, the gilis of the larva of the Water-Newt, the yolk-bag of embryo Fish, and other appropriate subjects for the observation, is one of the most beautiful and interesting spectacles that the Microscope can open to us. Details of the various modes of exhibiting it will be found in the Author's treatise on "The Microscope and its Revelations," Chap, xviii. 
through the capillaries of a warm-blooded animal, has been determined by microscopic examination to be about 3-100ths of an inch per second. From the comparison of this rate with that of the flow of blood through the larger arteries, which has been found by experiment to be nearly 12 inches per second, it appears that the area of the capillary system must be nearly four hundred times as great as that of the vessels which supply it with blood.

252. Thus the Arterial and Venous systems communicate with each other at their opposite extremities; their large trunks through the medium of the heart; and their ultimate subdivisions through the capillaries. Hence we may consider this double apparatus of vessels as forming a complete circle, through which the blood flows in an uninterrupted stream, returning continually to its point of departure; and the term circulation is therefore strictly applicable.

253. But the conveyance of the nutritive fluid to the several organs of the body, for their support and maintenance, is not the only object to which its circulation has to minister. It is requisite that the blood should be continually exposed to the influence of the air, by which it may get rid of the carbonic acid with which it has become charged during its circulation in the system, and may take-in a fresh supply of oxygen to replace that which has been withdrawn from it. In order to effect this exposure, the blood is conveyed to a particular organ, in which it is made to pass through a special set of capillary vessels, that bring it into almost immediate contact with air. In the lower tribes, in which this aeration is (from various causes hereafter to be explained) much less constantly necessary than in the higher, we find the respiratory organ supplied by a branch from the general circulation; and the blood which has passed through it, and which has been subjected to the invigorating influence of the air, is mingled in the heart with that which has been deteriorated by circulating through the system, which is again supplied with this mixed, half-aerated blood. But in the highest classes, there is a distinct circle of vessels subservient to the respiratory function : namely, an arterial trunk issuing directly from the heart, and subdividing into branches which terminate in the capillary system of the respiratory organ; a set of capillaries, in which the aeration of the blood takes place; and a system of veins 
which collects the blood from these, and returns it to the heart. This circuit of the blood is sometimes called the lesser circulation; to distinguish it from that which it makes through the general system, which is called the greater circulation.

254. Although carbonic acid is one of the chief impurities with which the blood becomes charged during its circulation, in consequence of the changes of composition which are continually taking place in the living body, it is by no means the only one; and other organs are provided, besides the lungs, for removing the noxious matters from the current of the circulation as fast as they are introduced into it. Thus, in the course of its movement through the general system, the blood is made to pass through the liver, the kidneys, and the skin, each of which has its special purifying office ; these organs, however, have no such special circulation of their own as the respiratory apparatus of higher animals possesses, though the liver, as we shall hereafter see ( $\$ 267)$, is peculiarly supplied by a sort of offset from the general circulation, so that the blood from which its secretion is formed is venous instead of arterial, like that transmitted to the lungs.

255. The course which the blood takes, and the structure of the apparatus which is subservient to its movement, differ very greatly in the several classes of animals. The chief of these differences will be pointed out hereafter; and it will be preferable to commence with the highest and most complex form of the circulating system, such as we find in Man, that it may serve as a standard of comparison with which the rest may be contrasted.

\section{Circulating Apparatus of the Higher Animals.}

256. In Man, and those animals which approach him most nearly in structure, the heart is situated between the lungs in the cavity of the chest, which is termed by anatomists the thorax. Its form is somewhat conical ; the lower extremity tapering almost to a point, and the upper part being much larger. The lower end is quite unattached, and points rather forwards and to the left; during the contraction of the heart, it is tilted forwards, and strikes against the walls of the chest, between (in Man) the fifth and sixth ribs. It is from the large or upper extremity that the great vessels arise; and 
these, being attached to the neighbouring parts, serve to suspend the heart, as it were, in a cavity in which its movements may take place freely. This cavity is lined by a smooth serous membrane ( $(43)$, which, near its top, is

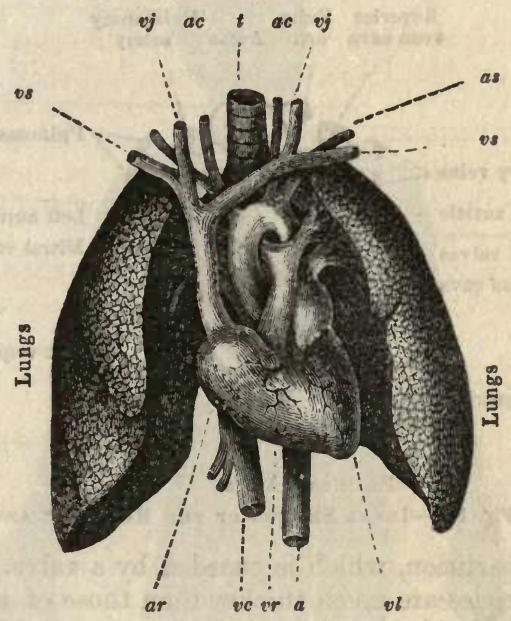

Fig. 122.-LUNGs, HEART, AND PRINCIPAT VESSELS DF MAN.

$a r$, right auricle; $v r$, right ventricle; $v l$, left ventricle; $a$, aorta; $v c$, rena cava; $a c$, carotid arteries; $v j$, jugular veins; $a s$, subclavian artery ; vs, subclavian veins ; $t$, trachea.

reflected downwards over the vessels, and covers the whole outer surface of the heart. Hence as the surface of the heart, and the lining of the cavity in which it works, are alike smooth, and are kept moist (in health) with a fluid secreted for the purpose, there is as little interruption as possible from friction in the working of this important machine.

257. The heart may be described as a hollow muscle, which, in Birds and Mammalia, as in Man, is divided into four distinct chambers. This division is effected by a strong vertical partition, that divides the entire heart into two halves, which are almost exactly similar to each other, excepting in the greater thickness of the walls on the left side ; and each of these halves (which do not communicate with one another) is again subdivided by a transverse partition, into two cavities, 
of which the upper one is termed the auricle, and the lower the ventricle. Thus we have the right and left auricles, and the right and left ventricles. Each auricle communicates with its corresponding ventricle, by an aperture in the

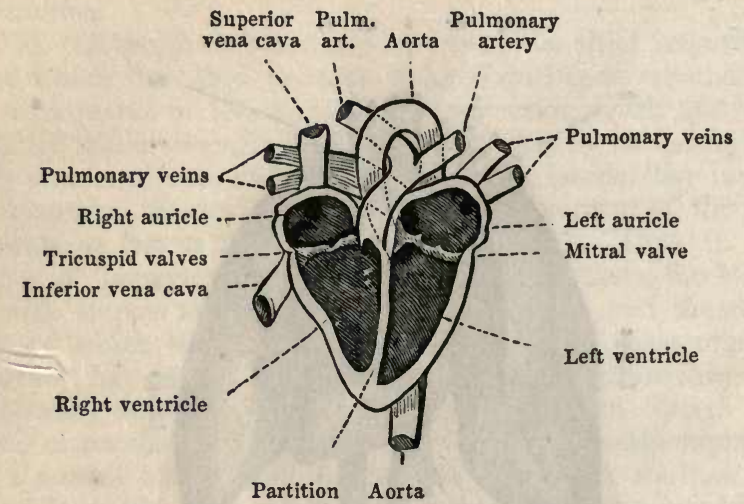

Fig. 123.-IDeat Section of the HumaN Heart.

transverse partition, which is guarded by a valve. The walls of the ventricles are much thicker than those of the auricles; and for this evident reason,- - that the ventricles have to propel the blood, by their contraction, through a system of remote vessels; whilst the auricles have only to transmit the fluid that has been poured into them by the veins, into the ventricles, which dilate themselves to receive it. The difference in the thickness of the walls of the left and the right ventricles is explainable on the same principle; for the left ventricle has to send the blood, by its contractile power, through the remotest parts of the body; whilst the right has only to transmit it through the lungs, which, being much nearer, require a far less amount of force for the circulation of the blood through them.

258. The arterial system of the greater circulation entirely springs from one large trunk, which is called the aorta (see figs. 122-124); this originates in the left ventricle, and is the only vessel which passes forth from that cavity. It first ascends towards the bottom of the neck; then forms what is termed the arch, a sudden curve, which gives it a downward 
direction; and then descends along the front of the spinal column, behind the heart, as far as the lower part of the

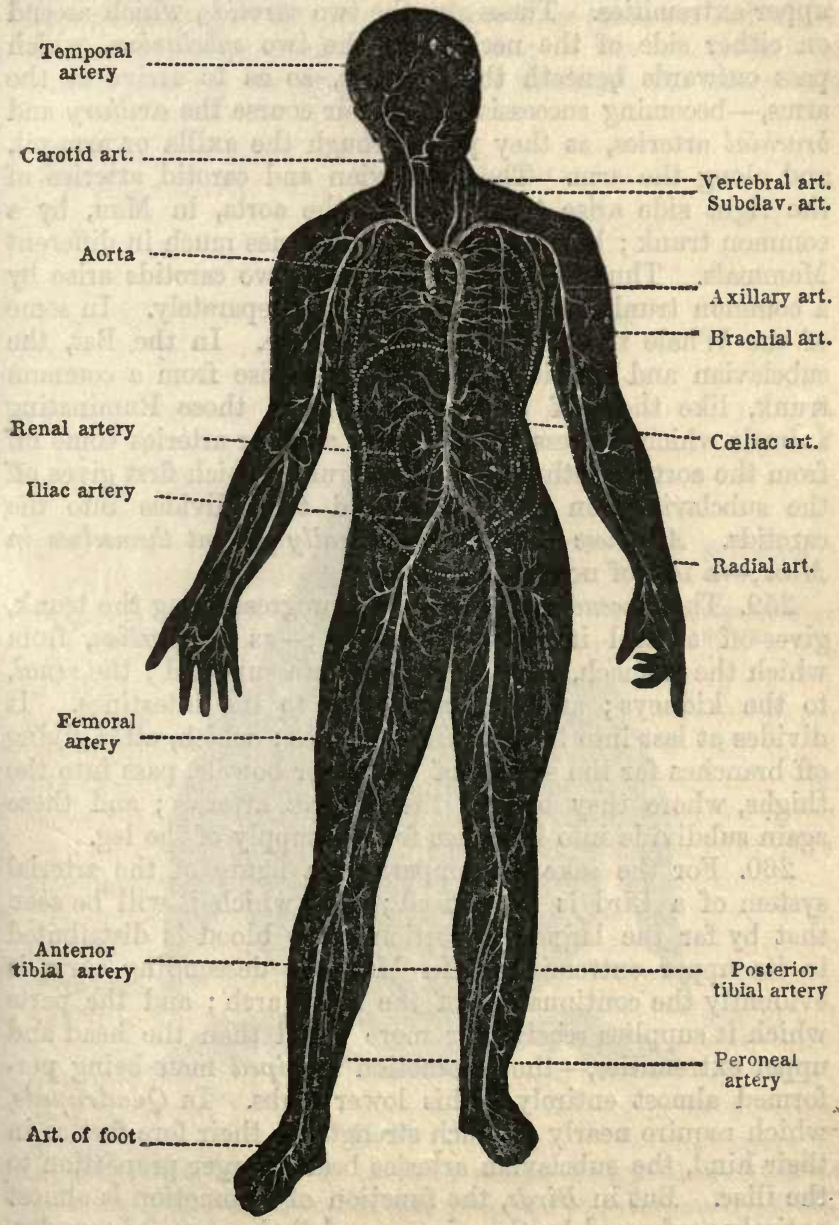

Fig. 124.-ARTERIAL SYSTEM OF MAN 
trunk, where it divides into two great branches, which proceed to the lower extremities. From the arch of the aorta are given off the arteries which supply the head and upper extremities. These are, the two carotids, which ascend on either side of the neck; and the two subclavian, which pass outwards beneath the clavicles, so as to arrive at the arms,-becoming successively in their course the axillary and brachial arteries, as they pass through the axilla or arm-pit, and along the arm. The subclavian and carotid arteries of the right side arise together from the aorta, in Man, by a common trunk; but this arrangement varies much in different Mammals. Thus in the Elephant, the two carotids arise by a common trunk, - the two subclavians separately. In some of the Whale tribe, all four are separate. In the Bat, the subclavian and carotid of the left side arise from a common trunk, like those of the right. And in those Ruminating animals which possess a long neck, all four arteries come off from the aorta together, by a large trunk, which first gives off the subclavians on either side, and then divides into the carotids. All these varieties occasionally present themselves in Man;-a fact of no small interest.

259. The descending aorta, in its progress along the trunk, gives-off several important branches;-as the coliac, from which the stomach, liver, and spleen are supplied; the renal, to the kidneys; and the mesenteric, to the intestines. It divides at last into the two iliac arteries; which, after giving off branches for the supply of the lower bowels, pass into the thighs, where they become the femoral arteries; and these again subdivide into branches for the supply of the leg.

260. For the sake of comparison, a figure of the arterial system of a Bird is introduced; from which it will be seen that by far the larger proportion of its blood is distributed to its upper extremities. In Man, the descending aorte is evidently the continuation of the aortic arch; and the parts which it supplies receive far more blood than the head and upper extremities, - the locomotion of biped man being performed almost entirely by his lower limbs. In Quadrupeds, which require nearly as much strength in their fore feet as in their hind, the subclavian arteries bear a larger proportion to the iliac. But in Birds, the function of locomotion is almost entirely performed by the wings; and their powerful muscles, 
which constitute the mass of flesh lying on the breast, are supplied with blood by the arteries of the upper extremities, which here possess a manifest predominance. The aorta, soon after its origin, subdivides into three large branches; of which the first two (one on either side giving-off the subclavian and carotid arteries) convey the blood to the head, the wings, and

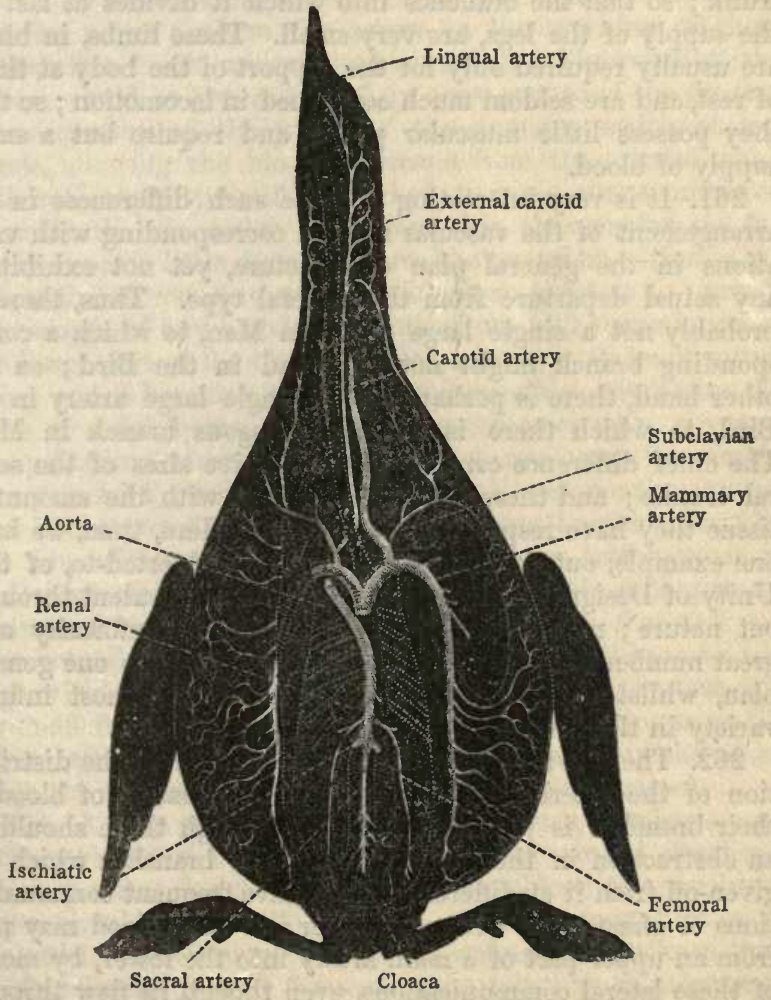

Fig. 125.-ARteriat System of Bird.

the muscles lying on the thorax; whilst the middle one curves backwards and downwards, and becomes the descending aorta. Now that which is here the continuation of the great side Q 2 
branch, is neither the carotid nor the subclavian, both of which are subordinate branches given-off from it; but it is the trunk which distributes the blood to the muscles of the breast, and which in Man is a subordinate branch of the subclavian artery (the mammary). The descending aorta is seen to lose itself almost entirely in supplying the viscera of the trunk; so that the branches into which it divides at last for the supply of the legs, are very small. These limbs, in birds, are usually required only for the support of the body at times of rest, and are seldom much concerned in locomotion ; so that they possess little muscular power, and require but a small supply of blood.

261. It is very interesting to trace such differences in the arrangement of the vascular system, corresponding with variations in the general plan of structure, yet not exhibiting any actual departure from the general type. Thus, there is probably not a single large artery in Man, to which a corresponding branch might not be found in the Bird; on the other hand, there is perhaps not a single large artery in the Bird, to which there is not an analogous branch in Man. The chief difference consists in the relative sizes of the several trunks; and these correspond closely with the amount of tissue they have respectively to supply. Here, then, we have one example, out of many that might be adverted-to, of that Unity of Design which we see everywhere prevalent throughout nature; manifesting itself in the close conformity of a great number of apparently-different structures to one general plan, whilst there is, at the same time, an almost infinite variety in the details.

262. There is a very interesting peculiarity in the distribution of the arteries, by which the due circulation of blood in their branches is provided for, even though there should be an obstruction in the main trunk. The branches which are given-off from it at different points, have frequent communications or anastomoses with each other; so that blood may pass from an upper part of a main artery into the lower, by means of these lateral communications, even though its flow through the trunk itself should be completely stopped.

263. These anastomoses are very numerous in the arteries of the limbs, and particularly about the joints ; and it is well that they are so; for, by relying on the maintenance of the 
circulation through them, the Surgeon is often able to save a limb, or even a life, which would otherwise be sacrificed. Arteries are liable to a peculiar disease, termed aneurism, which consists in a thinning-away, or rupture, of the tough fibrous coat, and a great dilatation of the other coats, so that a pulsating tumour is formed. This change takes place most frequently at the bend of the thigh, the ham, the shoulder, and the elbow; where the artery, in the working of these joints, often has to undergo sudden twists. The result of the disease would be generally fatal, in consequence of the gradual thinning-away of the walls of the tumour, which at last bursts, allowing the blood to escape from the arterial trunk with such rapidity as, if unchecked, to cause almost instantaneous death. In order to prevent this, the surgeon ties the artery at some little distance above the aneurism, - that is, he puts a thread round it, which is drawn so tight as to prevent the passage of any blood to the aneurism. The circulation in the lower part of the limb is at first retarded; its temperature falls ; and it becomes more or less insensible. But after the lapse of a few hours, the circulation becomes quite vigorous, the pulsations strong, the temperature rises, and the numbness passes off ; and as the main trunk still continues completely obstructed, this can only have been brought about by the flow of blood through the anastomoses, which must in that short period have undergone considerable enlargement. Examination of the vessels after death shows that this has been actually the case. Even the aorta has thus been tied in dogs, without causing death ; the anastomoses of the branches given-off from its upper part, with those proceeding from the lower, being sufficient to maintain the circulation in the latter, when the current through the main trunk is obstructed.

264. A very complex series of anastomoses, forming a complete network of large tubes, is found in several situations, where it seems desirable that the flow of blood to a particular organ should be retarded, whilst a large amount is to be allowed to pass through. Thus in animals which keep their heads near the ground for some time together, as in grazing, the arteries which supply the brain suddenly divide, on their entrance within the skull, into a great number of branches, by the anastomoses of which a complex network is formed; and from this network, by the reunion of its small vessels, 
originate the trunks which supply the brain in the usual manner. The object of this apparatus appears to be, to prevent the influence of gravitation from causing a too great rush of blood towards the brain, when the head is in a depending position; for the rapidity of its flow will be checked, as soon as it enters the network, and is distributed through its numerous canals. A similar conformation is found in the blood-ressels of the limbs of the Sloth, and of some other animals which resemble that animal in the sluggishness of their movements ; and its object is probably to prevent the muscles from receiving too rapid a supply of blood, which would give them what (for these animals) would be an undue energy of action; whilst, by the very same delay, their power of acting is greatly prolonged,-as we find it to be in Reptiles, whose circulation is languid ( $\$ 284$ ).

265. In the Whale tribe, and some other diving animals that breathe air, we find a curious distribution of the bloodvessels, which has reference to their peculiar habits. The intercostal arteries (which are sent-off from the aorta to the spaces between the ribs on each side) are enormously dilated, and are twisted into thousands of convolutions, which are bound together into a mass by elastic tissue. This mass, which is of considerable bulk, lies at the back of the chest, along both sides of the vertebral column; and it serves as a reservoir, in which a great quantity of arterial blood may be retained. The veins also have very large dilatations, which are capable of being distended, so as to hold a considerable amount of venous blood; and thus, while the animal is prevented from breathing by its submersion in the water, the circulation through the capillaries of the system is sustained, by the passage of the blood stored up (as it were) in the arterial system, into the venous reservoirs. If this provision did not exist, the whole circulation would come to a stand, in consequence of the obstruction it meets with in the lungs, when the breathing is stopped.

266. With regard to the Venous system, there is little to be added to what has been already stated ( $\$ \S 248-250)$ as to its general character and distribution. The large proportion which its capacity bears to that of the arterial system, is shown by the fact, that every main artery is accompanied by a vein (frequently by two) considerably larger than itself; and that the 
superficial veins, which lie just beneath the skin, are capable of conveying at least as much more. The veins of the body in general unite in two large trunks, the superior and inferior vena cava; which meet as they enter the right auricle of the heart (fig. 123). The superior vena cava is formed by the union of the veins which return the blood from the neck (the jugulars) with those which convey it from the arms (the subclavians), as shown in fig. 122; and the inferior cava ( $v c$, fig. 122) receives the blood from the trunk, the organs contained in the abdomen, and the lower extremities.

267. There is, however, an important peculiarity in the distribution of the veins of the Intestines, which should not pass unnoticed. Instead of delivering their blood at once into the inferior vena cava, these veins unite into a trunk, called the Vena Portoe (fig. 134), which enters the liver and subdivides into branches, whence a capillary network proceeds that permeates the whole of its mass. It is from the venous blood, as it traverses this network, that the secretion of bile is formed; and the blood which is brought by the hepatic artery serves chiefly to nourish the liver,-no bile being formed from it, until it has becomo venous. The blood is carried-off from this double set of capillaries by the hepatic vein, which conveys it into the inferior vena cava. In Fishes, not only the blood of the intestines, but that of the tail and posterior part of the body, enters this "portal" system, which is distributed to. their kidneys as well as to their liver. Thus all the blood which flows through the portal system, has to go through two sets of capillaries, between each period of its leaving the heart by the aorta, and its return to it by the vena cava.

268. We have yet to notice the lesser circulation, which is confined to the Lungs only. The venous blood which is returned to the heart by the venæ cavæ, enters the right auricle, and thence passes into the right ventricle. By the contraction of this last cavity, it is expelled through the pulmonary artery (fig. 123), which soon divides into two main trunks that proceed to the right and left lungs respectively. The right trunk again subdivides into three principal branches, which are distributed to the three lobes or divisions of the right lung; whilst the left divides into two branches, which are in like manner distributed to the two lobes of the left lung. The capillaries, into which these branches ultimately 
subdivide, are distributed upon the walls of the air-cells (fig. 162), and the character of the blood is in them converted, by exposure to the air, from the dark venous to the bright arterial. From this capillary network the pulmonary veins arise; and the branches of these unite into trunks, of which two proceed from each lung, to empty themselves into the left auricle (fig. 123). This auricle delivers the blood, now arterialized or aerated ( $(253)$, into the left ventricle, whence the aorta arises; and by the contraction of this cavity, it is delivered through that vessel to the system at large.-It will be observed that the vessel which proceeds from the heart to the lungs is called the pulmonary artery, although it carries dark or venous blood. This is because it conveys the blood from the heart towards the capillaries. And, for a similar reason, the vessels which return the blood from the capillaries to the heart are termed pulmonary veins, although they carry red or arterial blood.

\section{Forces that move the Blood.}

269. The mechanical action, by which the blood is caused to circulate in the vessels, is easily comprehended. The cavities of the heart, as already explained ( $\$ 245)$, contract and dilate alternately, by the alternate shortening and relaxation of the muscular fibres that form their walls (Chap. xIr.); and the force of their contraction is sufficient to propel the blood through the vessels which proceed from them. The two ventricles contract at the same moment; the auricles contract during the relaxation of the ventricles, and relax whilst the ventricles are contracting. The series of movements is therefore as follows :-The auricles being full of the blood which they have received from the venæ cavæ and pulmonary veins, discharge it by their contraction into the ventricles, which have just before emptied themselves into the aorta and pulmonary artery, and which now dilate to receive it. When filled by the contraction of the auricles, the ventricles contract in their turn, so as to propel their blood into the great vessels proceeding from them; and whilst they are doing this, the auricles again dilate to receive the blood from the venous system, after which the whole process goes-on as before. It is when the ventricles contract, that we feel the beat of the heart, which is caused by the striking of its lower extremity 
against the walls of the chest; and it is by the same action that the pulse in the arteries is produced ( $\$ 276$ ).

270. The combined actions of each auricle and its ventricle, may be illustrated by an apparatus like that represented in fig. 126. It consists of two pumps, $a$ and $b$, of which the pistons move up and down alternately; and these are connected with a pipe $c f$, in which there are two valves $d$ and $e$, opening in the direction of the arrow. The portion $c$ of the pipe represents the venous trunk by which the blood enters

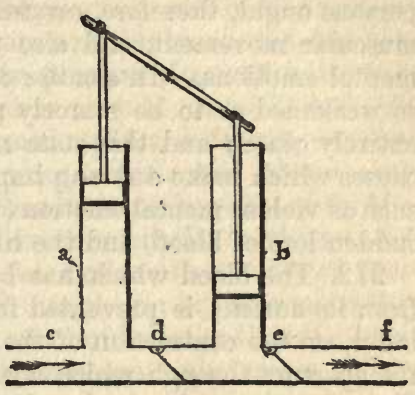

Fig. 126. the heart; the pump $a$ represents the auricle, and the raising of its piston enables the fluid to enter and fill it. When its piston is lowered, its fluid is forced through the valve $d$ into the pump $b$ (which represents the ventricle), whose piston rises at the same time to receive it; and when this piston is lowered in its turn, the fluid (being prevented from returning into $a$ by the closure of the valve $d$ ) is propelled through the valve $e$ into the pipe $f$, which may represent an arterial tube; whilst at the same time a fresh supply of blood is received into the pump $a$ by the raising of its piston.

271. The number of contractions of the heart ordinarily taking place in an adult man, is from 60 to 70 per minute. It is usually rather greater in women; and in children it is far higher, being from 130 to 140 in the new-born infant, and gradually diminishing during the period of infancy and childhood. It is rather greater in the standing than in the sitting posture, and in sitting than in lying down: it is increased by exercise, especially by ascending a steep hill or going upstairs, and also by any mental emotion. It is important to remember these facts, in reference to the management of those who are suffering under diseases of the heart or of the lungs, which prevent the ready passage of the blood through these organs ; for if more blood be brought to the heart by the great veins, than it can propel through the pulmonary arteries, a feeling of 
very great distress is experienced; and there may be even danger of rupture of the heart or large vessels, or of sudden cessation of the heart's action, causing instant death. Such persons ought, therefore, carefully to refrain from any violent muscular movement, and also to avoid giving way to strong mental emotions. - In syncope or fainting, the heart's action is so weakened as to be scarcely perceptible, though it does not entirely cease; and this state may be brought on by several causes which make a strong impression on the nervous system, such as violent mental emotion (whether joy, or grief, or terror), sudden loss of blood, and the like.

272. The blood which has been received by each ventricle from its auricle, is prevented from being driven back into the latter, on the contraction of the former, by a valve that guards the aperture through which it entered. This valve consists of a membranous fold, surrounding the borders of the aperture, and so connected with the neighbouring parts, as to yield when the blood passes from the auricle into the ventricle, but to be tightened so as completely to close the aperture when the blood presses in the contrary direction. The manner in which these valves act will be seen from fig. 127, which is a section of the right auricle with its ventricle. The auricle, $a$, receives its blood from the two venæ cavæ, $e, e$; and transmits it into the ventricle, $b$, by the orifice, $c$. On either side of this orifice are seen the membranous folds, which are kept in their places by the tendinous cords, $d$.

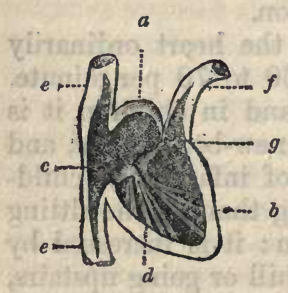

Fig. 127.-SEction of oNe SIDE OF THE HEART.

Now when the blood is passing from $a$ to $b$, these folds yield to the current; but when the cavity $b$ is filled and begins to contract, the blood presses against their under sides, so as to make them close against each other, as far as they are permitted to do by the tendinous cords. In this manner the aperture is completely shut, and no blood can flow back. A valve of this kind exists on each side of the heart; but there is a slight difference between the forms of the two, whence they have received different names. That on the right side has three pointed divisions, to which the tendinous cords are attached, and it is hence called the tricuspid valve; 
whilst that on the left side has only two, so as to bear some resemblance to a bishop's mitre, whence it is called the mitral valve.

273. The aorta and pulmonary artery are in like manner furnished with valves, which prevent the blood that has been forced into them by the contraction of the ventricles, from returning into those cavities when they begin to dilate again. These valves, however, are formed upon a different plan, and more resemble those of the veins, which will be presently described. They consist of three little pocket-shaped folds of the lining membrane of these arteries (similar to those at $b \vec{b}$, fig. 128), which are pressed flat against the walls of those tubes when the blood is forced into them; but as soon as they are filled, and the ventricles begin to dilate, so that the blood has a tendency to return, it presses upon the upper side of these pockets, and fills them out against one another, in such a manner as completely to close the entrance into the ventricle. The three little pocket-shaped folds, however, would not close the centre of the aperture, were it not that each of them has a little projection from its most prominent part, which meets with those of the others, and effects the requisite end. The situation of these valves (which are termed semilunar from their half-moon shape) is seen at $g$, fig. 127 , $f$ being the pulmonary artery.

274. The amount of blood sent-out from either ventricle at each contraction, in a middle-sized man, seldom exceeds 3 ounces; but the whole quantity of blood contained in the body is not less than $18 \mathrm{lbs}$ : : hence, it would require 96 contractions of the heart to propel the whole of this blood through the body, and these (at the ordinary rapidity) would occupy about $1 \frac{1}{3}$ minute. It has been calculated, from recent experiments, that the usual force of the heart in man would sustain a column of blood about 7 feet 2 inches high, the weight of which would be about 4 lbs. 3 oz. on every square inch. The backward pressure of this column upon the walls of the heart, or in other words, the force which they have to overcome in propelling the blood into the aorta, is estimated at about $13 \mathrm{lbs}$.

275. From the mode in which the blood is forced into the arterial system by a series of interrupted impulses, it might be supposed that its course would be a succession of distinct 
jets ; but this is prevented, so that the current is reduced to an equable stream by the time it reaches the capillaries, through the elasticity of the walls of the arteries. In order to comprehend how this acts, we may suppose a forcing-pump (§ 270) to propel its fluid, not into a hard unyielding tube of iron or lead, but into an elastic tube of india-rubber. The effect of each stroke of the pump will be partly expended in distending the tube, so as to make it contain an additional quantity of water ; and the suddenness of the jet at its opposite extremity will be diminished. In the interval of the stroke, the elasticity of the wall of the tube will cause it to contract again, and to force-out the added portion of its contents ; this it will not have completed by the time that the action of the pump is renewed; and in this manner, instead of an interrupted jet at the mouth of the tube, we shall have a continuous flow, which, if the tube be long enough, will become quite equable.* It is precisely in this manner that the elasticity of the arteries influences the flow of blood through them, by converting the interrupted impulses which the heart communicates to it, into a continued force of movement. In the large arteriès, these impulses are very evident; in the smaller branches they are less so, but they still manifest themselves by the jerking in the stream of blood proceeding from a wound in one of these vessels ; whilst in the capillaries, the. influence of the heart's interrupted impulses cannot usually be seen at all, the streams that pass through them being perfectly equable.

276. The phenomenon which we call the pulse, is nothing else than the change in the condition of the artery occasioned by the increased pressure of the fluid upon its walls, at the moment when the heart's contraction forces an additional quantity of blood into the arterial system. By the frequency and force of this change, we can judge of the power with which the blood is being propelled. But the pulse can only be well distinguished, when we can compress the artery against some resisting body, so that there is a partial obstruction to the flow of blood through it, which causes the distension to be more powerful ; the most convenient artery for this

* The same effect is obtained in an ordinary fire-or garden-engine, by the interposition of an air-vessel, in which the elasticity of compressed air is substituted for that of the wall of the pipe. 
purpose is the radial artery (fig. 124) at the wrist; but the carotid artery in the neck, and the temporal artery in the temple, may be felt, when it is desired to know the force of the circulation in the head; as may the arteries supplying other parts, when we wish to gain information respecting the organs they supply. An increased action in the organ, whether this be due to inflammation, or to a state of unusual activity of its function, causes an increase of size in the artery which supplies it; and thus the pulsation may be unusually strong in a particular trunk, when the heart's action and the general circulation are not in a state of excitement. For instance, a whitlow on the thumb will occasion its artery to beat almost as powerfully as the radial artery usually does; and excessive activity of the mind, prolonged for some hours, greatly increases the force of the pulsations in the carotid arteries, from which the brain is chiefly supplied.

277. When an artery is wounded, there is often great difficulty in controlling the flow of blood; for pressure can seldom be effectually applied in the situation of the wound; and the surgeon is generally obliged to tie the vessel above the orifice. As a temporary expedient, the loss of blood may be prevented by making firm pressure upon the artery above the wounded part, that is, nearer the heart; and many valuable lives have been saved by the exercise of presence of mind, guided by a little knowledge. The best means of keeping-up the requisite pressure, until the proper instrument (the tourniquet) can be applied, is to lay over the artery (the place of which may be found by its pulsation) a hard pad, made by tightly rolling or folding a piece of cloth; this pad. and the limb are then to be encircled by a bandage, by which the pressure is maintained; and this bandage may be tightened to any required degree, by twisting it with a ruler or a piece of stick. Thus a constant pressure may be exercised upon the artery, which will be generally sufficient to control the bleeding from it. But there are, unfortunately, many cases in which pressure of this kind cannot be applied; as for instance when the femoral artery is wounded high up in the thigh, or the carotid artery in the neck. And nothing else can then be done, but to compress the artery with the thumb, or with some round hard substance (such as the handle of an awl), until proper assistance can be obtained. 
278. The impulse of the heart, and the elasticity of the arteries, which together propel the blood through the capillary system, continue to act upon it after it is received into the veins; and are in fact the chief causes of its movement in them. If we interrupt the current of blood through an artery by making pressure upon it, and open the corresponding vein, the fluid will continue to flow from the latter, so long as the. artery contains blood enough to be forced into the vein by its own contraction; but as soon as it is emptied, the flow from the orifice in the vein will cease, even though the vein itself remains nearly full. If the pressure be then taken off the artery, there is an immediate renewal of the stream from the vein, which may be again checked by pressure on the artery. In the ordinary operation of bleeding, we cause the superficial veins of the arm to be distended, by tying a bandage round them above the point at which we would make the incision; and when an aperture is made, the blood spouts forth freely, being prevented by the bandage from returning to the heart. But if the bandage be too tight, so that the artery also is compressed, the blood will not flow freely from the vein; and the loosening of the bandage will then produce the desired effect. When a sufficient quantity of blood has been withdrawn, the bandage is removed; and the return-flow through the veins being now unobstructed, the stream from the orifice immediately diminishes so as to be very easily checked by pressure upon it, or may even cease altogether.

279. The veins contain a great

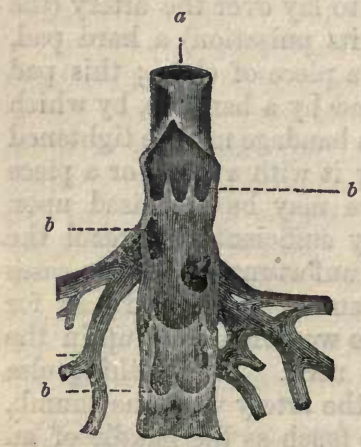

Fig. 128.-VEIN TAID OPEN, TO SHOW ITS VALVES. number of valves, which are formed, like the semilunar valves of the aorta ( $\$ 273$ ), by - a doubling of their lining membrane. Their situation may be known by the little dilatations which the veins exhibit at the points where they occur ; and which are yery obvious in the arm of a person not too fat, when it is encircled by a bandage that causes distension of the superficial veins. The structure of these valves is seen at $b b$, 
fig. 128; they consist of pocket-like folds of the lining membrane, which allow the blood free passage as it flows towards the heart, but check its reflux into the arteries. Hence it follows, that every time pressure is made upon the veins, it will force towards the heart a portion of the blood they contain, since this cannot be driven in a contrary direction. Now, from the manner in which the veins are distributed, some of them must be compressed by almost every muscular movement; these will become refilled as soon as the muscles relax; and they will be again pressed-on, when the movement is repeated. Hence a succession of muscular movements will act the part of a diffused heart, over the whole of the venous system, and will very much aid the flow of blood through its tubes. It is partly in this manner, that exercise increases the rapidity of the circulation. If the blood is brought to the heart by the great veins more rapidly than usual, the heart.must go through its operations more rapidly, in order to dispose of the fluid; and if these actions be impeded, great danger of their entire cessation may exist. Hence the importance of bodily tranquillity to those affected with diseases of the heart or lungs ( $\$ 271$ ).

280. Besides the aid thus afforded to the venous circulation, it is probable that there is another cause of the motion of the blood in them, which is independent of the action of the heart and of the arteries. Many facts lead to the belief that a new force is produced, while the blood is flowing through the capillary vessels, - a force which may, in some instances, maintain the circulation by itself alone. Thus in many of the lower animals, it seems as if the power of the heart were so unequal to the maintenance of the circulation, that this must partly depend upon some other influence; and even in the highest, there is evidence that the movement of blood in the capillaries may continue for a time, after the action of the heart and of the arteries has ceased to affect it.* This movement seems intimately connected with the changes to which the blood is subservient in the capillaries; for, if these be checked, not even the heart's action can propel the blood through them, although no mechanical

* For a full consideration of this question, see the Author's Principles of Comparative Physiology (4th edition), $\$ \S 247-251$; and Principles of Human Physiology (5th edition), \$§ 267-275. 
obstruction exists. Thus, when the admission of air to the lungs is prevented, the blood will not pass through the pulmonary capillaries, since it cannot undergo the change which ought to be performed there ; and it therefore accumulates in the pulmonary artery, the right side of the heart, and venous system; and if no relief be afforded by the admission of air into the lungs, the whole circulation is thus brought to a stand. This condition, which is termed Asphyxia, occurs in drowning, hanging, and other forms of suffocation ( $\$ 338$ ).

\section{Course of the Blood in the different Classes of Animals.}

281. The Circulation of the Blood takes place on the same general plan in all other Mammals, and in Birds, as in MAN. In all the animals included in these groups, the heart is composed of two halves quite distinct from each other; each possessing an auricle or receiving cavity, and a ventricle or propelling cavity. The course of their blood, which goes through a complete double circulation, is shown by the diagram (fig. 129). The vessels and cavities of the heart which contain venous blood are shaded; whilst those which convey arterial blood are left white: and this distinction is kept-up in the other figures. The direction of the blood is indicated by the arrows. Every drop of blood which has passed through the capillaries of the system, is transmitted to the lungs before it is allowed again to enter the aorta; and the whole mass of the blood passes twice through the heart, before any part of it is transmitted a second time to the vessels from which it was before returned.

282. The two sides of the heart do not possess, when that organ is perfectly formed, any communication with each other, except through the pulmonary vessels; and thus they might be regarded as two distinct organs, united for the sake of convenience. The right side of the heart, being placed at the origin of the pulmonary artery, and having for its office to propel the blood through the lungs so as to receive the influence of the air, may be called the respiratory heart: whilst the left side, which is placed at the origin of the aorta, and has to propel the blood to the body in general, may be called the systemic heart. The circulation would be performed precisely in the same manner, if these two organs 
DIFFERENT FORMS OF CIRCULATING APPARATUS. 241 Lesser circulation.

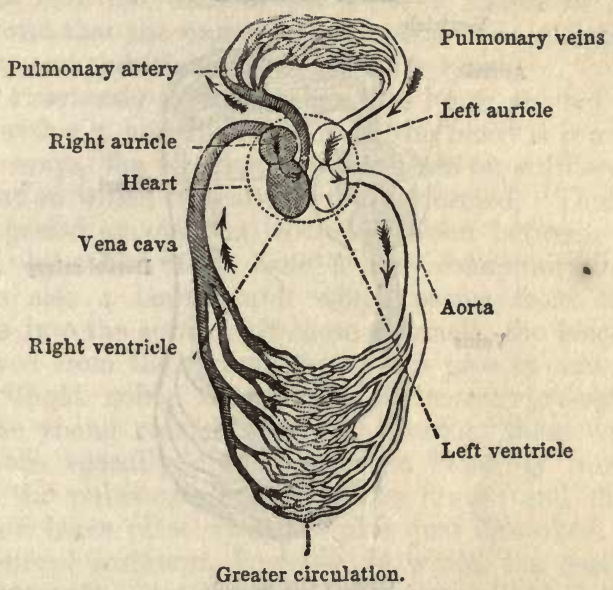

Fig. 129.-Diagram of the Circulation in Mammals and Birds.

were quite distinct from each other; and in fact they are almost so in the Dugong, one of the herbivorous Whales

Lesser circulation.

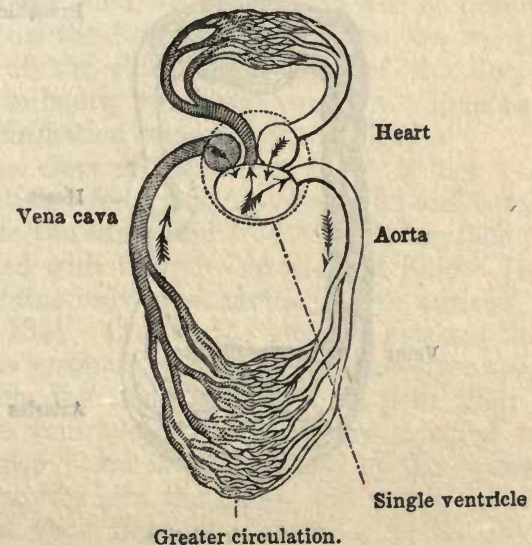

Greater circulation.

Fig. 130.-Diagram of the Circulation IN REPTILEB. $\mathbf{R}$ 
DIFFERENT FORMS OF CIRCULATING APPARATUS.

Lesser circulation.

Ventricle

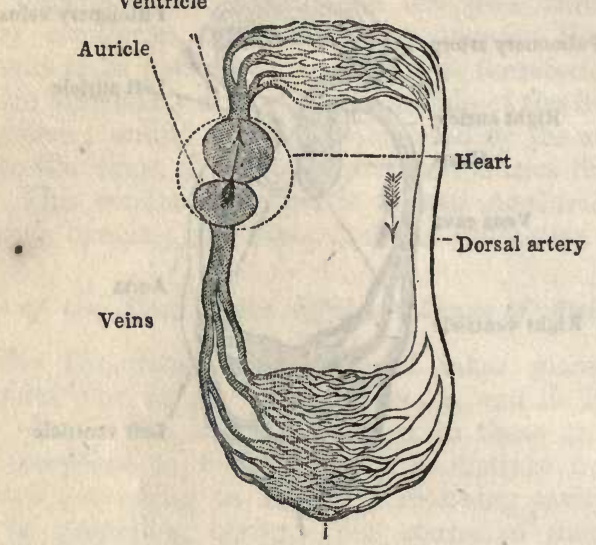

Greater circulation.

Fig. 131.-Diagram of the Circulatron in Fishes.

(ZooLOGY $\S 305)$. In the lower tribes of animals we shall Lesser Circulation.

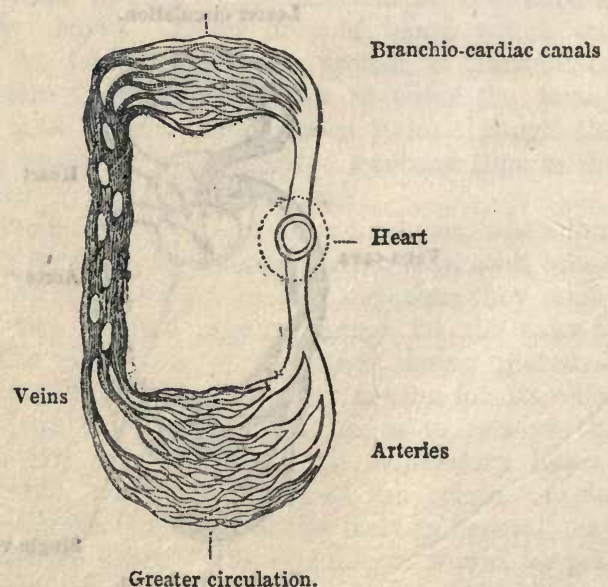

Fig. 132.-Diagram of the Crrculatron in Crustacea. 
presently find that there is but a single, instead of a double, heart; and that the organ which is absent is sometimes the systemic, and sometimes the respiratory heart.

283. Previously to birth, when the lungs are not yet distended with air, and the aeration of the blood is provided-for in other ways, the circulation takes place on a different plan from that on which it is afterwards performed. There exists at that period an opening in the partition between the two auricles, by which they have a free communication; and there is also a large trunk which passes from the right ventricle into the aorta. By these channels, the blood which is received from the systemic veins can pass at once into the aorta, without going through the pulmonary vessels. But when the young animal begins to breathe, these communications are speedily obliterated; the blood is transmitted through the pulmonary vessels to the lungs; and the whole circulation takes place upon the plan just described. There are occasional instances, however, in which the communication between the auricles remains open, so that the double circulation is never perfectly established; for a portion of the blood is allowed to pass from the right to the left side of the heart, without being aerated in the lungs, so that the blood which is sent to the system contains a mixture of venous with the proper arterial fluid, - a state which will be presently seen to be natural in the Reptile. Such cases are recognised by the blueness of the skin, the lividity of the lips, and the indisposition to bodily or mental exertion. Persons affected with this malformation seldom reach adult age.

284. In the class of REPTILES, there is not a complete double circulation; for a mixture of arterial and venous blood is sent alike to the lungs and to the general system; and no part is supplied with the pure arterialized fluid. In general the heart contains only three cavities,-two auricles and one ventricle (fig. 133). One of the auricles receives the venous blood from the system; whilst the other receives the arterialized blood from the lungs. Both these pour their contents into the same ventricle, where they are mingled together; and this mingled blood is transmitted, by the contraction of the ventricle, partly into the lungs, and partly into the aorta (fig. 130). In some Reptiles there is a partial division of the ventricle, so that the mixture of the arterial and venous 
blood is not complete; and whilst the blood transmitted to the lungs is chiefly that which has returned from the systemic veins, the blood which enters the aorta for the supply of the

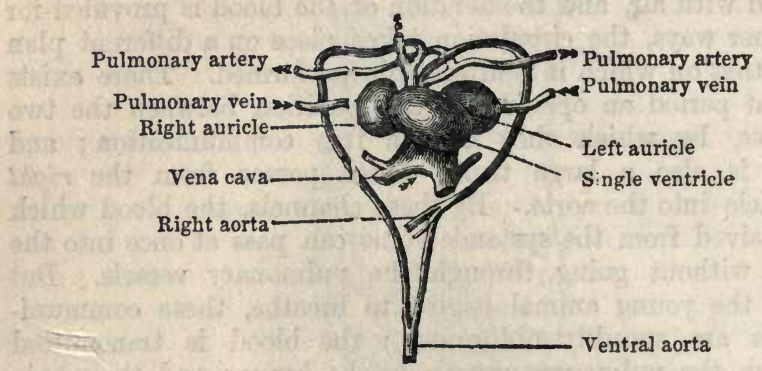

Fig. 133.-HEART OF TORTOIsE.

system is chiefly that which has returned from the lungs in an arterialized state. Hence such animals have a circulation which approaches very closely to that of Mammals and Birds ; and it is among them that we find the greatest vigour and activity in this generally inert and sluggish class.

285. The general arrangement of the blood-vessels in Reptiles is shown in fig. 134. It is seen that the aorta, soon after its origin, divides into three arches on either side; and that these, after sending off branches to the head and to the lungs, reunite into a single trunk, which corresponds exactly with the aorta of the higher animals. These arches are in fact the remains of a set of vessels, which will be found to be of the highest importance in Fishes, being there subservient to the aeration of the blood : in the true Reptiles, however, they are never concerned in this function, but they still remain, as if to show the unity of the plan on which this apparatus is formed. Precisely the same arrangement of the vessels may be seen in Birds and Mammalia, at an early stage of their development; but it afterwards undergoes considerable changes, by the obliteration of several of the arches ; for of the four pairs which may be seen at one period, a single branch only remains on either side; and one of these becomes the permanent arch of the aorta, whilst the other becomes the permanent pulmonary artery. 


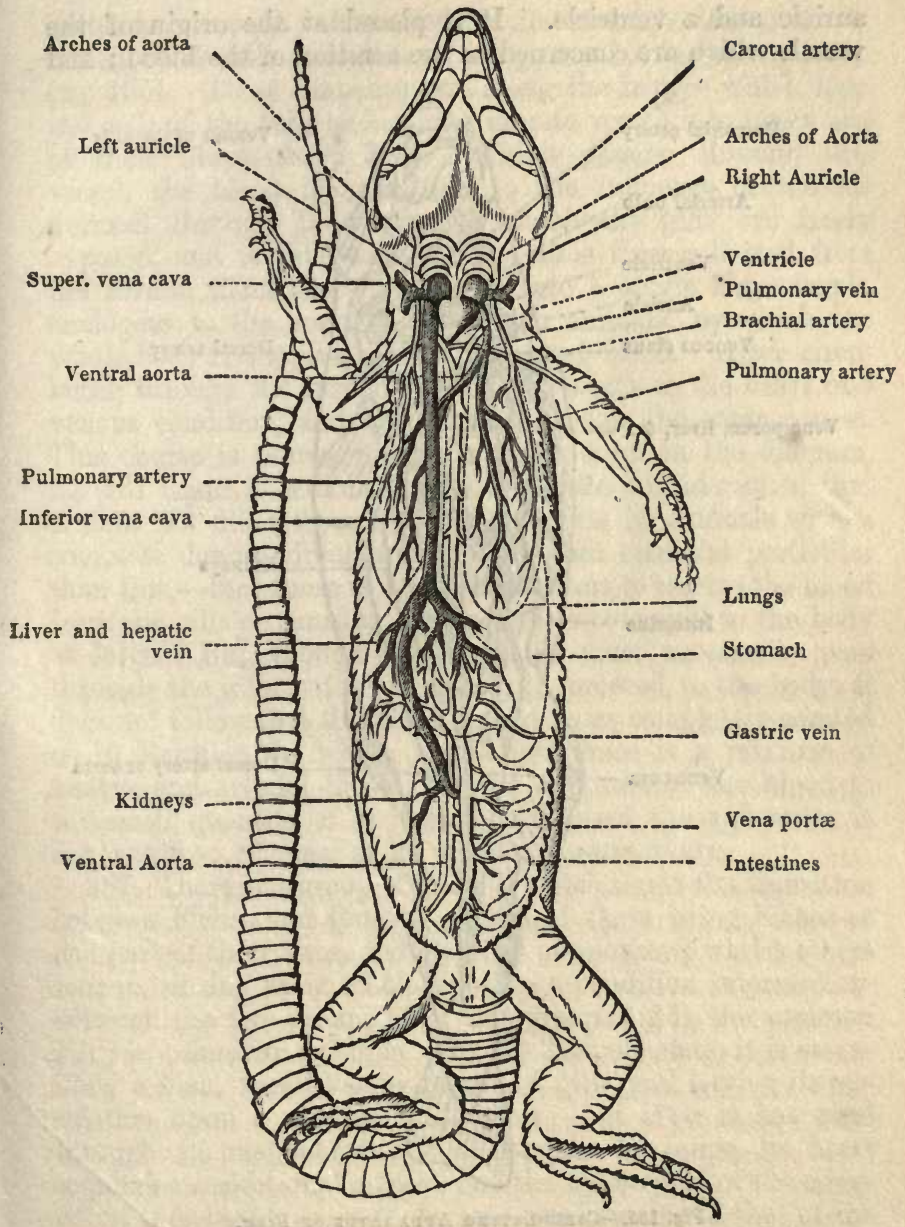

Fig. 134.-Circulating Apparatus of Lizakd.

286. In the class of FisHes, the circulating apparatus is still more simple. The heart only possesses two cavities, an 
auricle and a ventricle. It is placed at the origin of the vessels which are concerned in the aeration of the blood; and

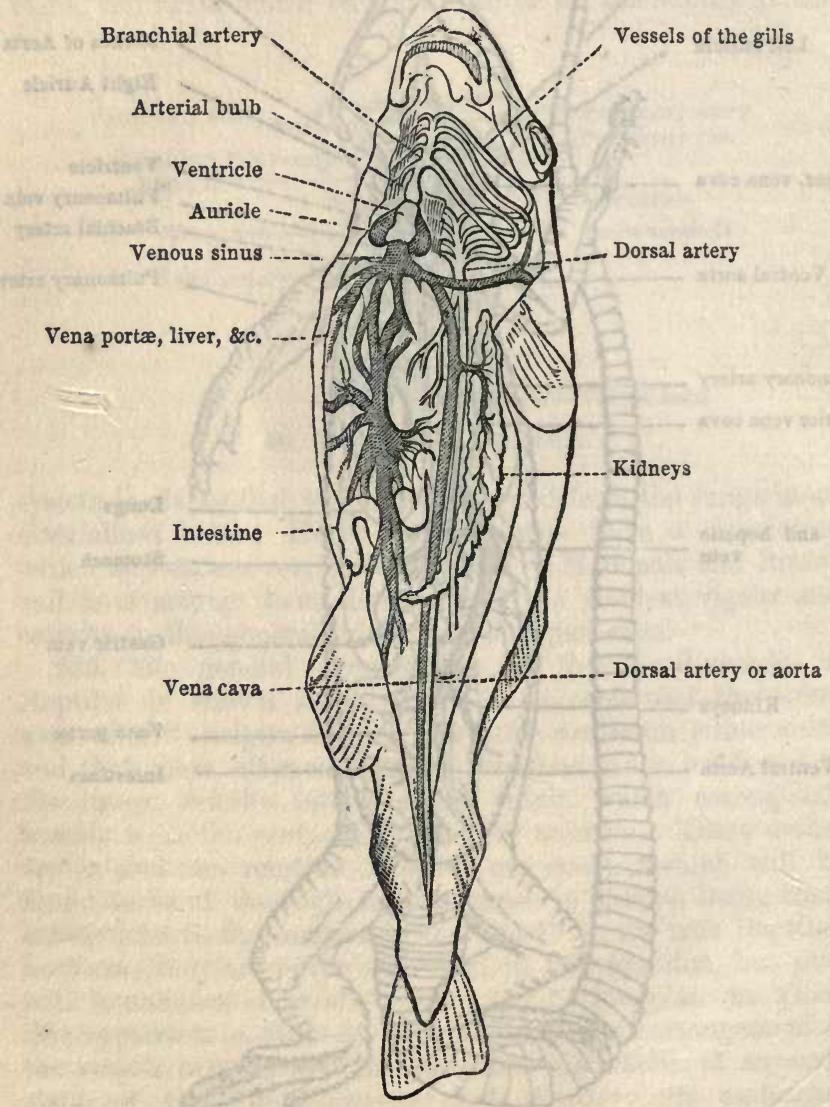

Fig. 135.-Circulating Apparatus of Fistr.

it receives and transmits venous blood only; hence it is analogous to the right side, or respiratory heart, of Birds and Mammalia. The venous blood, which is brought to it by the 
systemic veins, is transmitted by its ventricle into a trunk, which subdivides into four or five pairs of branches or arches (fig. 135). These branches run along the fringes which form the gills of the fish, and send a minute vessel into every one of their filaments ( $\$ 312$ ). Whilst passing through this vessel, the blood is submitted to the influence of the air diffused through the water, to which the gills are freely exposed, and is thus aerated; and it is then collected from the several filaments and fringes, into a single large trunk, analogous to the aorta of the higher animals, by which the whole body is supplied with arterialized blood. After circulating through the system, the blood returns to the heart in a venous condition, and again goes through the same course. This course is represented in a simple form in the diagram, fig. 131; and it will be seen, on a little consideration, that it does not differ from that which exists in Animals with a complete double circulation, in any other essential particular than this, - that there is no systemic heart to receive the blood from the gills or aerating organs, and to convey it to the body at large. But, though all the blood must necessarily pass through the gills before it can again proceed to the body, it does not follow that the blood should be as completely aerated as in Reptiles, in whose circulation there is a mixture of venous and arterial blood; for the exposure of the blood to the small quantity of air which is diffused through water is not nearly so effectual as its direct exposure to air.

287. There is a group of animals which forms the transition between Fishes and Reptiles; some of them being Fishes at one part of their lives, and Reptiles at another; whilst others remain, during their whole lives, in a condition intermediate between the two groups. Of this group ( $\$ 86)$, the common Frog is a familiar example. In the Tadpole state, it is essentially a Fish, breathing by means of gills, and having its circulation upon a corresponding plan; but after it has gone through its metamorphoses, it breathes by lungs, its heart acquires an additional auricle, and the whole plan of the circulation is changed, so as to become comformable to that of the true Reptile. This process takes place, not suddenly, but by progressive stages; and as these are extremely interesting, they will now be briefly described. In fig. 136 we have a representation of the circulating apparatus of the Tadpole in 
its fish-like condition. At $a$ is seen the large trunk which issues from the ventricle, forming a bulbous enlargement like that which is seen in the corresponding part of the Fish. From this enlargement proceed three trunks on each side, called the branchial arteries $\left(b r^{1}, b r^{2}, b r^{3}\right)$, which convey the blood to the gills or branchice; and after being aerated by passing through their filaments, the blood is collected by the bran-

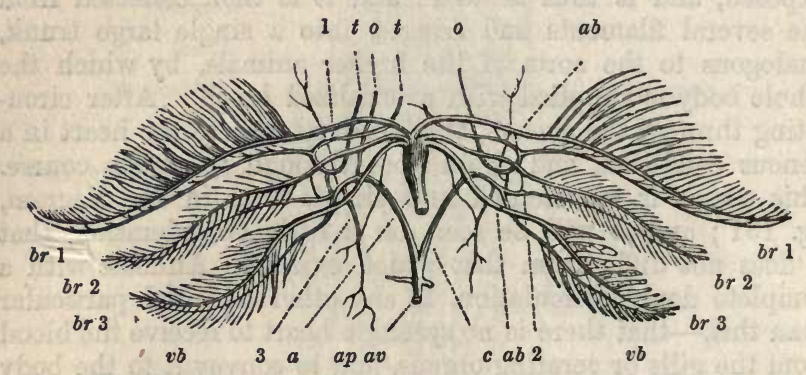

Fig. 136.-BLOOD-vessels of THE TADPOLE, IN PIRST STATE.

chial veins $(v b, v b)$. Of these, the first pair transmits its blood by the vessels $0, o, t$, (which are also formed in part by the recond pair) to the head and upper extremities; whilst the greater part of the blood of the second pair, with the whole of that of the third, is discharged into the trunk $c$ on either side. By the union of that vessel with its fellow, the trunk $a v$ is formed; which conveys the blood that has been aerated in the gills, to the general system, and is thus to be evidently regarded as the aorta. But we find here three small vessels $(1,2$, and 3$)$, which do not exist in the Fish ; and which establish a communication between the branchial arteries and the branchial veins, in such a manner, that the blood may pass from the former into the latter, without going through the filaments of the gills. These communicating vessels are very small in the Tadpole, and scarcely any blood passes through them; but it is chiefly by their enlargement, that the course of the blood is subsequently altered. There is also a fourth branch, $a p$, which proceeds to the lungs on either side; and as these organs are not yet developed, this pulmonary artery also is at first of very small size.

288. As the metamorphosis of the other parts proceeds, 
however, and the animal is being prepared for its new mode of life, the lungs are gradually developed, and the pulmonary arteries greatly increase in size; whilst the gills, on the other hand, do not continue to grow with the animal, but rather shrink, from the diminished supply of blood which they receive. For, during this period, the communicating branches

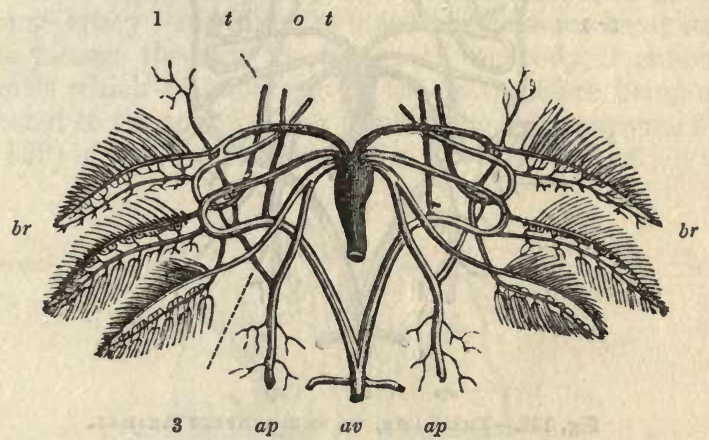

Fig. 137.-ThE SAME, in A MORE ADVANCED STATr.

increase in size; so that a considerable part of the blood which has been transmitted into the branchial arteries passes at once into the veins, and thence into the aorta, without being made to traverse the gills; its aeration being partly accomplished by the lungs. This state of things is seen in fig. 137 ; where $a p$, ap, are the enlarged pulmonary arteries; and where the. communicating branches are seen almost to form the natural continuations of the branchial arteries. A condition of this kind exists permanently in those Batrachia which retain their gills during their whole lives, and have the lungs imperfectly developed ( $\$ 87)$. When the metamorphosis is complete, the branchial vessels altogether disappear, but the arches still remain, as shown in fig. 138. The first of these arches supplies the vessels of the head, $t t$; which also, however, receive a branch $o$ from the second arch. The second arch, after giving off that branch, unites with its fellow to form the aortic trunk av. The third arch has completely shrivelled up. And the fourth arch or pulmonary artery has now attained its full size, and is become the sole channel through which the aeration of the blood is effected. 
289. Among the Invertebrated classes generally, the condition of the circulating apparatus differs from that which prevails

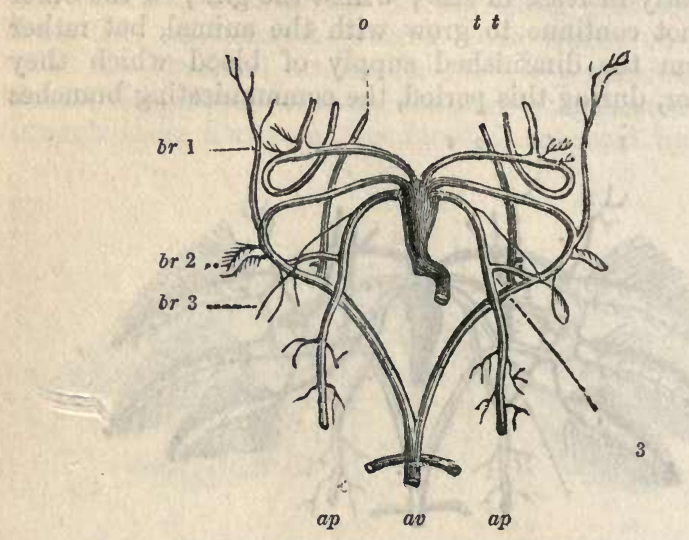

Fig. 138.-The Same, in the perfect ANimal.

throughout the Vertebrata, in one remarkable feature;namely, that whereas in the latter the blood moves in every part of its course through a set of closed vessels, it meanders in the former through a set of channels or sinuses excavated in the substance of the tissues, and communicating with the "general cavity of the body" in the midst of which the viscera lie. Generally speaking, it is in the venous system that the greatest deficiency exists; for the heart usually sends forth the blood by definite arterial trunks, which distribute it by its ramifications through the substance of the various parts of the body; and it is in its course from these to the respiratory organs that it is least restrained within definite boundaries. The degree of this imperfection differs considerably in the several groups of Invertebrata ; for whilst, in the highest Mollusca and Articulata, the vascular system is almost as complete as in Vertebrated animals, we find it gradually becoming less and less distinct as we descend, so that in the lower forms of both series it presents itself merely as an extension of the general cavity of the body, and is not furnished with any special organ of impulsion.

290. In the greater part of the Mollusca, the circulation 
takes place nearly on the same general plan as in Fishes; the heart having two cavities, and the whole of the blood traversing both the respiratory and the systemic vessels, between each time of its leaving the heart and returning to it again. But this heart is systemic, and not pulmonary ; for it receives the arterial blood from the gills, and transmits it to the great systemic artery; and after the blood has been rendered venous by its passage through the tissues of the body, it enters the channels which distribute it to the gills, before being again subjected to the action of the heart. The accompanying figure (fig. 139) of the circulation in the Doris (a kind of sea slug)

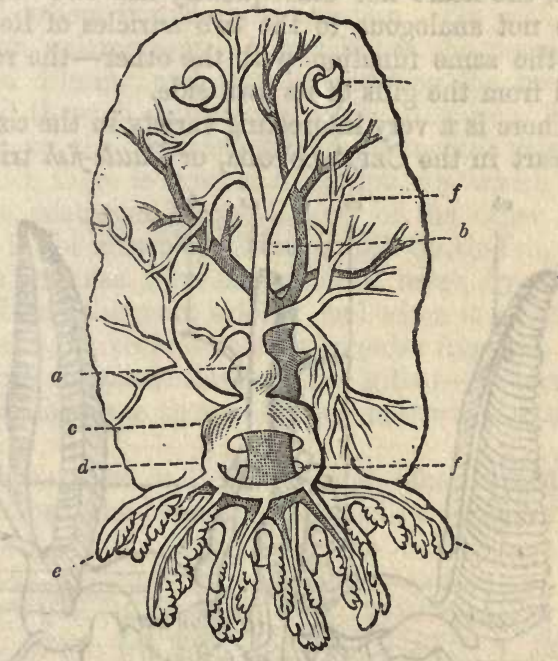

Fig. 139.-Circulativg Apparatus of Doris.

will serve to show the general distribution of the vessels in this group. The heart consists of the ventricle $a$, whence issues the main artery $b$; and of a single or double auricle $c$, in which terminate the veins, $d$, of the branchial apparatus $e$. The aerated blood which these convey to the heart, is transmitted by it, through the artery $b$, to the system at large ; and from this it is collected, in the state of venous blood, by the sinuses which terminate in the large trunk $f f$. By this trunk it 


\section{2}

CIRCULATION IN GASTEROPODA AND CEPHALOPODA.

is distributed to the gills $e$; and thence it returns to the heart, after having undergone aeration. Now if a second heart had been placed on the trunk $f f$, just as it is about to subdivide for the distribution of the blood to the gills, the circulation would have been analogous to that of Birds and Mammals. There is a great variety in the position of the gills in Molluscous animals, and a corresponding variety in the situation of the heart, which is usually placed near them. In the Doris the gills are arranged in a circular manner, round the termina tion of the intestinal canal ; but in many Mollusca they form straight rows of fringes on the two sides of the body. In these last, the heart not unfrequently has two auricles; but these are not analogous to the two auricles of Reptiles; for each has the same function with the other-the reception of the blood from the gills of its own side.

291. There is a very interesting variety in the conformation of the heart in the CEPHALOPODA, or Cuttle-fish tribe; which

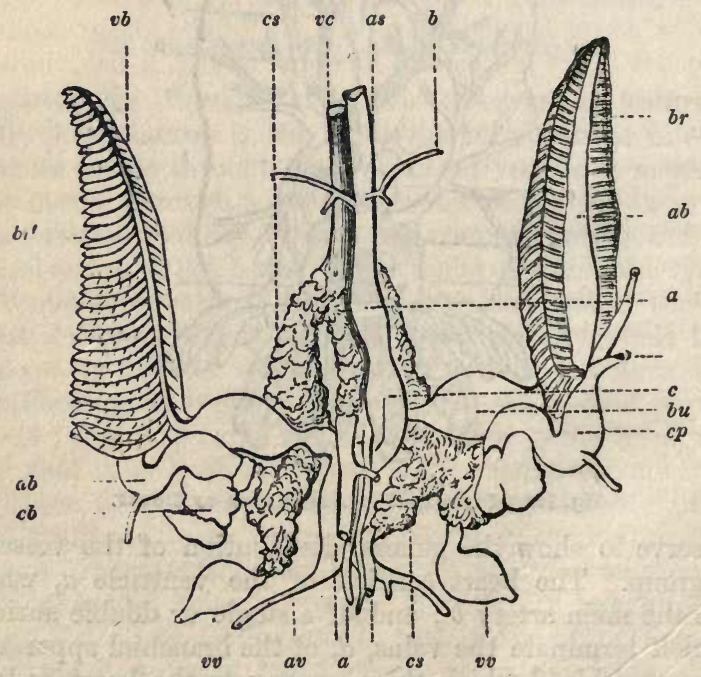

Fig. 140.-Circulating Apparatus of Cuttle-pish.

seems to form a connecting link between the plan of the circulation that prevails among the Mollusca in general, and that 
which we have seen in the class of Fismes. The auricle and ventricle of the heart are separated from each other; and whilst the latter remains in the position just described, the auricle occupies the place which the whole heart possesses in the class above. The course of the blood in these animals is shown in fig. 140 ; where $c$-represents the ventricle or systemic heart, from which arises the aorta $a, a, a s, a v$, that supplies the body with arterial blood. The venous blood is returned through the great vein $v c$, covered with a curious spongy mass $c s$, the use of which is not known; this also receives the blood from the intestinal veins $v v$; and it divides into two trunks which convey the blood to the gills or branchiæ ( $b r$ and $\left.b r^{\prime}\right)$, where it undergoes aeration. On each of these trunks is an enlargement, $c b$, which has the power of contracting and dilating, and thus of assisting the transmission of the blood through the arteries of the gills, $a b$. The blood is returned to the ventricle by the branchial veins, $v b$, on each of which there is another dilatation, $b u$, which might be regarded as analogous to the auricle of the other Mollusca, but that it is not muscular. Thus in the Cuttle-fish, the blood receives an impulse from the systemic heart, by which it is transmitted into the main artery; and when it returns by the systemic veins, it receives another impulse from the branchial hearts, before it passes through the gills; -an arrangement obviously analogous to that which we meet with in the highest Vertebrata.

292. In the Crab and Lobster, and other animals of the class Crustacea, the blood for the most part follows the same

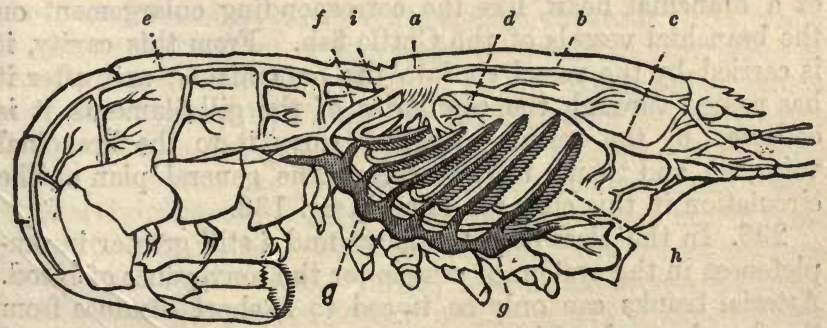

Fig. 141.-Circulating Apparatus of Lobster.

course as in the Mollusca, excepting that the heart contains but a single cavity. The arrangement of the circulating appa- 
ratus of a Lobster is seen in fig. 141, in which $\alpha$ is the heart; $b$ and $c$, the arteries to the eyes and antennæ; $d$, the hepatic artery; and $e$ and $f$, the arteries which supply the abdomen and thorax. The blood that has been propelled through these by the action of the heart, finds its way into the great venous sinus $g g$, which receives the fluid collected from all parts of the body; from this it passes to the gills, $h$; and thence it is returned to the heart by the branchial veins, $i$. Another view of a portion of the circulating apparatus is given in fig. 142, which represents a transverse section of it in the region

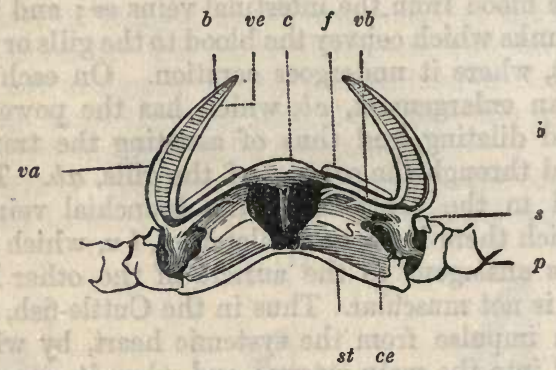

Fig. 142.-Branchial Circulation of Lobster.

of the heart, with one pair of gills. The heart is seen at c ; and from its under side proceeds one of the arterial trunks which convey the blood to the system. Returning thence, the blood enters the venous sinus $s$, which has an enlargement at the base of each gill; and this seems to act the part of a branchial heart, like the corresponding enlargement on the branchial vessels of the Cuttle-fish. From this cavity, it is carried by the vessel $v a$ into the branchiæ $b$; and after it has passed through the capillaries of the gill-filaments, it is collected by the vessels ve, which carry it to the branchial veins, $v b$, and thence to the heart. The general plan of the circulation in this class is shown in fig. 132.

293. In the class of INSECTS we find a still greater incompleteness in the system of vessels for the conveyance of blood. Arterial trunks can only be traced to a short distance from the dorsal vessel, which answers the purpose of a heart; and the nutritive fluid which they convey is delivered into the channels or sinuses that exist among the different organs. 
Nevertheless, it has a tolerably regular circulation; and the organ by which this movement is chiefly effected is a long tube, termed the dorsal vessel, which seems to propel it forwards, whilst two principal sinuses, one on either side, convey it backwards. The dorsal vessel, seen at $a$, is a membranous

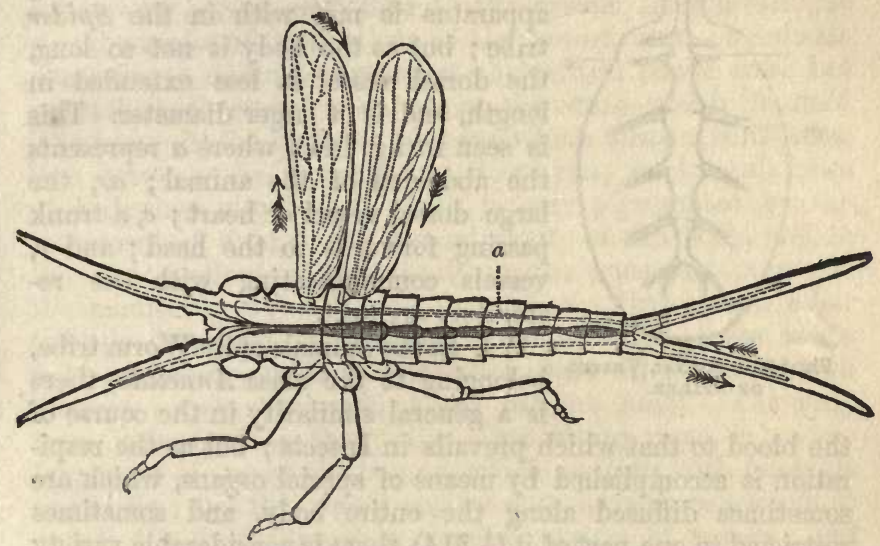

Fig. 143.-Circunatron IN INSECts.

tube lying along the back of the insect, and partly divided into several compartments by incomplete valvular partitions, which bear no inconsiderable resemblance to the valves of veins (\$ 279). By the successive contraction of these different portions, the blood which entered at the posterior extremity of the dorsal vessel is gradually propelled forwards; and when it arrives at the front of the body, it passes out by a series of canals, some of which convey it to the head, whilst others pass sideways and backwards for the supply of the body, with its appendages, the legs and wings. On returning from these parts, it re-enters the posterior end of the dorsal ressel. But, besides ministering to this general circulation, the several compartments of the dorsal vessel seem to act as independent hearts, each for its own segment; into which they send forth blood by minute arterial trunks, and from. which they receive it again by minute apertures furnished with valves. It is to be remarked that in Insects no special arrangement of vessels for the aeration of blood is required; since this aeration is 
accomplished by the conveyance of air, by means of minute air-tubes, into every part of the body, however small ( $(321)$;

a mode of respiration different from any that we notice elsewhere.-A very similar arrangement of the circulating apparatus is met with in the Spider tribe; but as the body is not so long, the dorsal vessel is less extended in length, and is of larger diameter. This is seen in fig. 144; where $\alpha$ represents the abdomen of the animal; ar, the large dorsal vessel or heart; $c$, a trunk passing forwards to the head; and $v$, vessels communicating with the respiratory organs.

294. In the animals of theWorm tribe,

Fig. 144. - Dorsal Vessex belonging to the class Annelida, there
op SPIDER. is a general similarity in the course of the blood to that which prevails in Insects; but as the respiration is accomplished by means of special organs, which are sometimes diffused along the entire body, and sometimes restricted to one part of it ( $\$ 314$ ), there is considerable variety in the provisions for submitting the blood to the influence of the air. In those which possess red blood (\$226), this, fluid can be seen to move in a closed system of vessels; whilst a colourless fluid containing numerous corpuscles flows through a set of canals prolonged from the general cavity of the body. It may be surmised that the two principal offices to which the circulation of the blood is subservient, are here separately performed; the red non-corpusculated fluid having for its office to aerate the tissues, whilst the colourless but corpusculated fluid serves for their nutrition.

295. A very curious departure from the normal type of the circulation presents itself in the class of Tunicata, the lowest of the Molluscous series ( $\$ 114$ ). The heart in these animals is much less perfectly formed than in the higher trives; though it still contains two cavities, one for receiving and the other for impelling the blood. The blood may be sometimes seen to flow in the direction customary among Mollusks; coming to the heart from the respiratory surface, and then going forth through an arterial trunk that conveys it into a 
system of channels excavated through the tissues, after passing through which it finds its way again to the respiratory surface, and thence to the heart. But after a certain duration of its flow in this direction, the current stops, and then re-commences in the contrary direction, proceeding first to the respiratory organs, and then to the system in general. It would seem as if in this, one of the lowest forms of animals possessing a distinct circulation, the central power were not yet sufficiently strong to determine the course which the fluid is to take. In the group of Bryozoa, which forms a connecting link between the Tunicata and Zoophytes, we lose all trace of a distinct circulation, which is only represented by the movement of fluid in the general cavity of the body, and in the prolongations of this cavity in the arms that surround the mouth (fig. 64).- In the Star-fish, Sea-Urchin, and other animals belonging to the class Echinodermata, there seems to be a regular circulation of nutritious fluid, carried on through distinct vessels, but without any definite heart The only trace, indeed, of anything like a propelling organ, is an enlargement of one of the trunks, which pulsates with tolerable regularity; but this would not seem to have force enough to propel the fluid through a complex system of vessels; and the circulation seems to be carried on chiefly by some force produced in the capillaries $(\$ 280)$.

296. The circulating apparatus of higher animals is only represented in Zoophytes, Medusae, and the lower Worms, by ramifying prolongations of the digestive cavity, which extend throughout the body, and are specially distributed to the respiratory surface, so as to subject the products of digestion to the aerating process. Thus, in the stony corals which are formed by animals constructed upon the general plan of the Sea Anemone ( $\$ 127)$, the gelatinous flesh that connects the polypes is traversed by a network of canals that open freely into the sides of their visceral cavities, of which they may be regarded as extensions ; whilst in the Campanularia (fig. 72) and other composite Hydraform Zoophytes, a like communication is established by a system of canals passing along the stem and branches, and becoming continuous with the base of each polype. In this system of canals, viewed under a sufficient magnifying power, a granular fluid may be seen to move, the direction of the flow being sometimes from the stem towards 
the polypes, and sometimes from the polypes towards the stem ; the rapidity and constancy of these currents depending apparently on the activity of the growth of the parts towards which they are directed. In the Medusoe we find the central stomach sending out prolongations towards the margin of the disk, where they frequently inosculate so as to form a network, which seems to have for its purpose to expose the product of digestion to the aerating action of the surrounding water ; and in this system of canals, also, a movement of fluid may be observed, which appears to depend upon the action of cilia in their interior. In all these cases, it is to be observed that the circulation of nutritive fluid is really effected by a modification of the digestive apparatus, instead of by an apparatus set apart for this sole purpose ; and the blending of the two functions is still more remarkably exhibited in the Sponge, the inosculating canals of which (\$136) may be regarded alike as constituting a ramifying digestive cavity, or as a simple form of circulating apparatus. The most correct method is perhaps to consider it as representing both these systems, which are here blended (as it were) into one ; the simplicity of structure characteristic of this type not admitting of the division of labour which we meet with in higher organisms.

\section{CHAPTER VI.}

\section{OF RESPIRATION.}

297. WE have seen that arterial blood, by its action on the living tissues, loses those qualities which rendered it fit for the maintenance of life; and that after having undergone this change, it recovers its original properties by exposure to air. This exposure is necessary, therefore, to the continued existence of Animals in general. If we place an animal under the receiver of an air-pump, and exhaust the air either partially or completely, a great disturbance soon shows itself in its various functions; shortly afterwards, the several actions of life cease to take place; and a state of apparent death comes on, which speedily becomes real, if air be not re-admitted. The influence of air is not less necessary to 
Plants than to Animals; for they also die when excluded from it: and thus its presence may be stated to be a general condition, necessary for the continuance of the life of all organised beings. - There is, however, a marked difference in the rapidity with which the deprivation of air occasions death in different animals ( $(310)$.

298. At first sight it might be thought that Animals which always live beneath the surface of the water, such as Fishes, Zoophytes, and many Mollusca, are removed from the influence of the air; and that they hence constitute an exception to this general law. But such is not the case; for the liquid which they inhabit has the power of absorbing, and of holding dissolved in it, a certain quantity of air, which they can easily separate from it, and which is sufficient for the maintenance of their life. They cannot exist in water which has been deprived of air (as by boiling, or by being placed under the exhausted receiver of an air-pump); for they then become insensible and die, just as do Mammalia and Birds when prevented from inhaling air in the ordinary manner.

299. The changes which result from the exposure of the blood or nutritious fluid of Animals to the air, either in the atmosphere, or through the medium of water, form a very important part of their vital actions ; and the changes themselves, together with the various mechanical operations by which they are effected, constitute the function of Respiration. The nature of these changes will be first explained; and the structure and operations of the organs by which they are performed will be afterwards described.

\section{Nature of the Changes essentially constituting Respiration.}

300. Atmospheric air, it has been stated, is necessary to the continued life of all animals ; but this fluid is not composed of one element alone. By the science of Chemistry, it is shown to be a mixture of three gases possessing very different properties. Besides the watery vapour with which the atmosphere is always more or less charged, the air contains 21 parts in 100 of oxygen, and 79 parts of nitrogen or azote; with about 1-5000th part of carbonic acid gas. The question immediately presents itself, therefore, whether these gases have the same action on animals; or, if their actions 
be different, to which of them specially belongs the property of thus contributing to the maintenance of life. This question may be decided by a few simple experiments. If we place a Bird or small Mammal in a jar filled with air, and cut off all communication with the atmosphere, it perishes by suffocation in a longer or shorter time; and the air in the vessel, which has thus lost the power of maintaining life, is found by chemical analysis to have lost the greater part of its oxygen. If we then place another animal in a jar filled with nitrogen gas, it perishes almost immediately; whilst if we place a third in pure oxygen, it breathes with greater activity than in air, and shows no sign of suffocation. It is then evident, that it is to the presence of oxygen that atmospheric air owes its vivifying properties.

301 . But the change produced in the atmosphere by animal respiration is not limited to this. The oxygen which disappears is replaced by carbonic acid; which, instead of being favourable to the maintenance of life, causes the death of animals which inhale it, even in small quantities. The exhalation of this substance is an action not less general in the Animal kingdom than the absorption of oxygen; and it is in these two changes that the act of respiration essentially consists.

302. The quantity of nitrogen or azote in the air that has been respired, varies but very little. There appears, however, to be a continual absorption of nitrogen by the blood, and as continual an exhalation of it. When the quantity exhaled and the amount absorbed are equal, or nearly so, no change manifests itself in the air which has been breathed; when the quantity absorbed is the greater, there is a diminution in that which the respired air contains; and when the quantity exhaled is the greater, there is a corresponding increase. An exhalation of nitrogen seems to be ordinarily taking place in warm-blooded animals, to an extent varying between 1-50th and 1.100th of the oxygen consumed; but when the same animais are partially or wholly deprived of food, an absorption of nitrogen usually occurs.

303. The differences in the character of the blood which are produced by its exposure to the air, have already been noticed ( $\$ 227)$; and we now see that they are essentially due to the absorption of oxygen, and the setting free of carbonic 
acid. These changes will take place out of the living body as well as in it ; provided that the blood can be exposed as completely to the influence of the atmosphere. When blood is drawn from a vein into a basin or cup, the dark hue of the surface is usually seen to undergo a rapid alteration, so as to present the arterial tint; but this is confined to the upper surface, because it alone is exposed to the influence of the atmosphere. The alteration takes place still more rapidly and completely if the blood be exposed to pure oxygen gas; but even then it is almost confined to the surface. It is not prevented, even though the direct communication between the blood and the gas be cut off by a membranous partition, as it is in the living animal; for if the blood be tied up in a bladder, the gas has still the power of penetrating to it, and of effecting the change in it ; and the change is manifested, not only by the alteration in the aspect of the blood, but by the disappearance of a certain quantity of oxygen, and its replacement by carbonic acid. Now if, by spreading out the blood in a very thin layer, we expose a much larger surface to the air, or if, by frequently shaking it, we continually change its surface, we render the change more complete. But still it is accomplished far less effectually than it is in the lungs or gills of a living animal ; for when it is passing through their capillaries, it is divided into an immense number of very minute streams, each of which is completely exposed to the influence of the air, and the combined surface of which is very great.

304. The question next arises, what becomes of the Oxygen which disappears, and what is the origin of the Carbonic acid which is thus produced by respiration? This question will now be considered.

305. When charcoal is burned in a vessel filled with air, the oxygen disappears, and is replaced by an equal bulk of carbonic acid: at the same time there occurs a considerable disengagement of heat. During respiration, the same phenomena occur : there is always an evident relation between the quantity of oxygen employed by an animal, and the amount of carbonic acid it produces (the latter being usually somewhat less than the former); and, as we shall see hereafter (Chap. Ix.), there is always a greater or less amount of heat produced. There exists, then, a great analogy between the principal phenomena of respiration, and those of the combus- 
tion of carbon; and this agreement in the results naturally leads to the belief that the causes of both are the same.-It is to be borne in mind, however, that the substitution of carbonic acid for oxygen is not the only change produced by respiration in the air; for there is nearly always a disappearance of oxygen (to an amount sometimes equal to onethird of that exhaled in combination with carbon), which is taken into the system to be applied to other uses (\$ 343).

306. It was at one time supposed that the oxygen of the inspired air combines, in the lungs themselves, with the carbon brought there in the blood; and thus produces the carbonic acid which is expired, occasioning at the same time the development of heat. But this theory is inconsistent with experiment; for it has been proved that the carbonic acid. is not formed in the lungs, but that it is brought to them in the venous blood of the pulmonary artery; and that their office is to disengage or get rid of it, whilst they at the same time introduce oxygen into the arterial blood. For in the first place, it can be shown that a considerable quantity of carbonic acid exists in venous blood, from which it may be removed by drawing it into a vessel filled with hydrogen or nitrogen, or by placing it under the vacuum of an air-pump ; it can also be shown that arterial blood contains a considerable quantity of oxygen. Again, if Frogs, Snails, or other cold-blooded animals, be confined for some time in an atmosphere of nitrogen or hydrogen (neither of which gases in itself exerts any injurious effect upon them), they will continue for some time to give off nearly as much carbonic acid as they would have done in common air; thus proving that the carbonic acid is not formed in the lungs by the union of carbon brought in the venous blood with the oxygen of the air, since here no oxygen was supplied; and showing that the carbonic acid must have been brought ready-formed. This process, however, could not be continued for any great length of time, even in cold-blooded animals; since a supply of oxygen is necessary to the performance of their various functions. And in warm-blooded animals, a constant supply of this element is so much more important, that they will die if cut off from it, even for a short time.

307. The quantity of oxygen thus taken in, and of carbonic 
acid thus disengaged, bears a very regular proportion to the amount of exertion which is made during the same time. Hence it is much greater in tribes whose habits are active, than in those which are inert; and it is also greater in any individual, during a day spent in active exercise, than it is in the same person during a day passed in repose. This obviously results from the fact, now established beyond all doubt, that every muscular contraction or production of muscular-force, and every production of nerve-force by which muscular contraction is usually called forth, involve, as their essential condition, the death and disintegration of proportionate amounts of muscular and nervous substances, which pass from the state of living tissues to that of dead matter; and for this operation, the presence of the oxygen in arterial blood is required. This oxygen combines with part of the materials thus set free as waste $(\$ 55)$, and converts them into the products that are thrown off by the various excretions. One of the chief of these products is carbonic acid, which is carried off by the lungs in the manner already described. Thus the presence of oxygen in the blood is essential to the development of nervo-muscular force; while the elements of the blood itself are required to re-form the tissues which have been thus destroyed.

308. It is among Birds and Insects that we find the greatest quantity of carbonic acid produced, in proportion to the size of the animals; and in both these classes we find extraordinary provisions for the energetic performance of this function ( $\S 321$ and 326). The greater energy of the respiration of Birds than that of Mammals, when compared with the greater number of the red corpuscles in their blood, gives an increased probability to the idea, that the red corpuscles are the chief carriers of oxygen from the lungs to the capillaries of the system, and of carbonic acid from the capillaries of the system to those of the lungs $(\S 235)$. The energetic respiration of Insects, though these corpuscles are absent, is fully accounted for by the peculiar manner in which the air enters every part of their bodies ( $\$ 321)$. In no case do we see the influence of muscular activity, on the amount of carbonic acid thrown off, more strongly manifested than in Insects. A humble-bee, while in a state of great excitement after its capture, made from 110 to 120 respiratory movements in a minute; after 
the lapse of an hour, they had sunk to 58 ; and they subsequently fell to 46 . In the first hour of its confinement it produced about 1-3rd of an inch of carbonic acid (a quantity many times greater, in proportion to its size, than that which Man would have set free in the same time); and during the whole twenty-four hours of the subsequent day, the insect produced a less amount than that which it then evolved in a single hour. In the Larva state, which is usually one of comparative inactivity, the respiration is not much above that of the Worm tribes ; and in the Chrysalis state of those which become completely inactive, the amount of respiration is still lower.

309. This chrysalis state, indeed, bears a strong resemblance to the condition of torpor in which many animals pass the winter. Reptiles, and most Invertebrata that inhabit the land, become (to all appearance) completely inanimate when the temperature is lowered below a certain point; yet retain the power of exhibiting all their usual actions when the temperature rises again. In this state, their circulation and respiration appear to cease entirely ; or, if these functions are carried on at all, they are performed with extreme feebleness ; and the animals may be prevented from reviving for a long time, without their vitality being permanently destroyed, if they be surrounded by an atmosphere sufficiently cold. Thus Serpents and Frogs have. been kept for three years in an ice-house, and have completely revived at the end of that period. Among Mammals there are several species which pass the winter in a state of torpidity; but this is less profound than the torpidity of cold-blooded animals, for the circulation and respiration never entirely cease, though they become very slow. There are various gradations between this condition and ordinary deep sleep. Thus some of the animals which hybernate or retire to winter quarters, lay up a supply of food in the autumn, and pass the cold season in a state differing but little from ordinary sleep, from which they occasionally awake, and satisfy their hunger. But others, like the Marmot, are inactive during the whole period, taking no food, and exhibiting scarcely any evidence of life, unless they are aroused. The consumption of oxygen and the production of carbonic acid, under such circumstances, are extremely slight, as might be anticipated from the languor of the circulation and the inactivity of the nervo-muscular 
system. But a very slight irritation is sufficient to produce respiratory movements; the heart's action is quickened; and the animal for a time shows an increase of its general activity.

310. Animals will in general bear deprivation of air well or badly, according as the respiration is more or less active. Thus a warm-blooded animal usually dies, if kept beneath water for more than a few minutes; though there are some which are enabled, by peculiar means, to sustain life much longer (§ 265). In cold-blooded animals, however, whose demand for oxygen is much less energetic, this treatment may be continued for a much longer time without the loss of life. Thus the common Water-Newt naturally passes a quarter of an hour or more beneath the surface, without coming up to breathe; and it may be kept down for many times that period without serious injury. And, as we might expect from their peculiar condition, warm-blooded animals, when hybernating, may be kept beneath water for an hour or more, without apparent suffering; although the same animals, in their active state, would not survive above three minutes. There is reason to believe that a similar condition may be produced in Man, by the influence of mental emotion, or of a blow on the head, at the moment of falling into the water; so that recovery is by no means hopeless, even though the individual may have been more than half an hour beneath the surface.

Structure and Actions of the Respiratory Apparatus.

311. In animals whose organization is most simple, the act of respiration is not performed by any organ expressly set apart for it ; but it is effected by all the parts of the body that are in contact with the element in which the animal lives, and from which they derive their necessary supply of oxygen. This is the case, for example, in the lower class of Animalcules, in the Polypes, Jelly-fish, Entozoa, and many other animals. Even in the higher classes, a considerable amount of respiratory action takes place through the skin, especially when it is soft and but little covered with hair, scales, \&c., as in Man, and in the Frog tribe ; but we almost invariably find in them a prolongation of this membrane, specially designed to enable the blood and the air to act upon each other, and having 
its structure modified for the advantageous performance of this function. This modification consists in the peculiar vascularity of this membrane, that is, in the large number of vessels that traverse its surface; and also in the thinness of the membrane itself, by which gases are enabled to permeate it the more readily. Moreover, we always find this membrane so arranged, that it exposes a very large surface to the air or water which comes into contact with it; and this surface may be immensely extended, without any increase in the size of the organ. Thus the small lungs of a Rabbit really expose a much larger respiratory surface to the air, than is afforded by the large air-sacs of a Turtle which are ten times their size. This is effected by the minuteness of the subdivision of the former into small cavities or air-cells, whilst the latter remain as almost undivided bags.

312. It is desirable to possess a distinct idea of the mode in which the surface is thus extended by subdivision. We may, for the purpose of explanation, compare the lung to a chamber, on the walls of which the blood is distributed, and to the interior of which the air is admitted. This chamber, for the sake of convenience of description, we shall suppose to

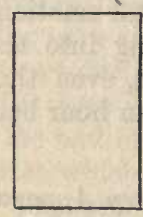

$\mathbf{A}$

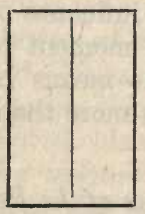

$\mathbf{B}$

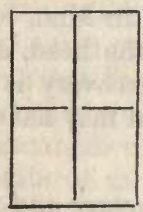

c

have two long and two short sides, as at $\mathrm{A}$. Now if a partition be built-up in the direction of its length, as at B, a new surface will be added, equal to that which the two sides previously exposed; since both the surfaces of this partition are supplied with blood, and are exposed to the air. Again, if another partition be built-up across the chamber, as at c, a new surface will be added, equal to that which the ends of the chamber previously exposed. And thus, by the subdivision of the first chamber into four smaller ones, the extent of surface has been doubled. Now if each of these small ones were divided in the same manner, the surface would again be doubled; and thus, by a continual process of subdivision, the 
surface may be increased to almost any extent compatible with the free access of air to the cavities, and of blood to the walls. In the same manner, where the respiratory membrane is prolonged outwardly, so as to form gills, which hang from the exterior of the body (as is the case in most aquatic animals), its surface is very much extended by disposing it in folds, and by dividing these folds into fringes of separate filaments. It has been calculated that, by this kind of arrangement, the gills of the Skate present a surface four times as great as that of the Human body.

313. The structure and arrangement of the Respiratory organs differ, according as they are destined to come in contact with air in the state of gas, or to act upon water in which a certain amount of air is dissolved. In the former case, we usually find the respiratory membrane (which is but a prolongation of the skin or general envelope) forming the wall of an internal cavity, - just in the same manner as the membrane, through which the act of absorption takes place in animals, is prolonged from the skin so as to form the wall of the digestive cavity (\$14). Such a cavity for the reception of air into the interior of the body, exists in all air-breathing animals; and in the Vertebrata it receives the name of lung. On the other hand, in animals that breathe by means of water, the respiratory surface is prolonged externally, so as to be evidently but an extension of the general surface,--just in the same manner as the roots of plants are prolonged into the soil around them. These prolongations, termed branchioe or gills, which may have various forms, carry the blood to meet the surrounding water, and to be acted-on by the air it contains ; and then return it to the body in a purified condition.

314. The form and arrangement of the gills vary greatly in the different classes of aquatic animals. Sometimes they simply consist of little leaf-like appendages, which have a texture rather more delicate than that of the rest of the skin, and which receive a larger quantity of blood. In other instances, they are composed of a number of branching tufts, which are more copiously supplied with vessels. Among the ANNELIDA we observe a great variety in the mode in which these tufts are disposed; and this is connected with the general habits of the animal. Thus in the Serpula (fig. 145), whose body is inclosed in a tube, the tufts are disposed 
around the head alone, and spread out widely into the semblance of a flower. In the Nereis (fig. 52) and its allies, they

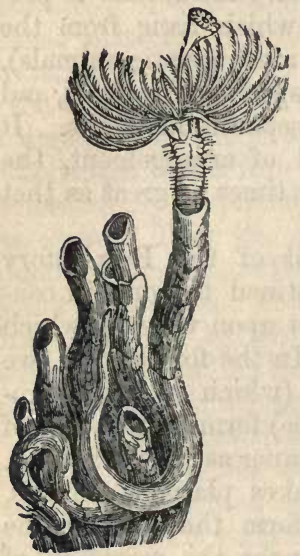

Fig. 145.

Gill-tufts of Serpula. are set upon nearly every division of the body, and are much smaller. Their usual arrangement in these marine worms may be seen in fig. 146, which represents one of the appendages of Eunice. The tuft of gills is shown at $b$; at $c$ is seen a bristleshaped filament, which may perhaps be regarded as the rudiment of a leg; and the projections to which the letters $t$ and $c i$ point, also seem connected with the movements of the animal. In the Arenicola (the lobworm of fishermen) we find the respiratory tufts disposed on certain segments only, and possessing more of an arborescent (treelike) form (fig. 147).

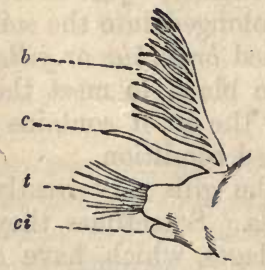

Fig. 146.

GiLl-tuft of Eunice.

315. A somewhat similar arrangement is seen in the larvæ of many aquatic INSECTS, which breathe by means of gills ; although all perfect Insects breathe air in the manner to be presently described. In fig. 148 is represented the larva of the Ephemera (Day-fly), which breathes by means

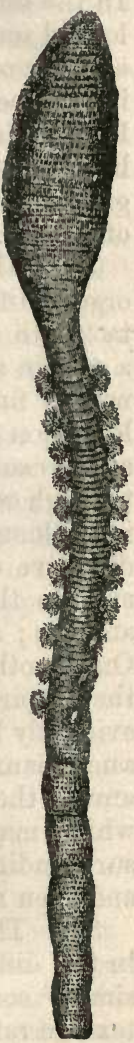

Fig. 147. Arentcola. of a series of gill-tufts disposed along the abdomen, and also prolonged as a tail. In the Crustacea, we usually find the gills presenting the form of flattened leaves or plates. In the lower tribes of the class, they project from the surface of the body; but in the higher, they are inclosed within a cavity, through which a stream of water is made con- 
stantly to flow, by mechanism adapted for the purpose. Their form and position in the Crab are shown at $b, b^{\prime}$, fig. 47 . Although these animals usually reside in the water, or only quit it occasionally, there are some species, known under the name of land-crabs, which have the power of living for some time at a distance from water. In order to prevent their gills from drying up, which would destroy their power of acting on the air, there is a kind of spongy structure in the gill-chamber, by which a fluid is secreted that keeps them constantly moist.

316. In the Moulusca we find the gills arranged in a great variety of modes. In the lowest class, the Tunicata, the respiratory membrane is merely the lining of the large chamber formed by the mantle (fig. 63), through which a stream of water is continually made to flow by ciliary action

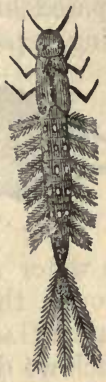

Fig. 148.

LARVA OF EPHEMERA. (\$ 319); and this surface is sometimes extended by the folding or plaiting of the membrane. In most of the CoNCHIFERA, however, we find four lamellae or folds of membrane

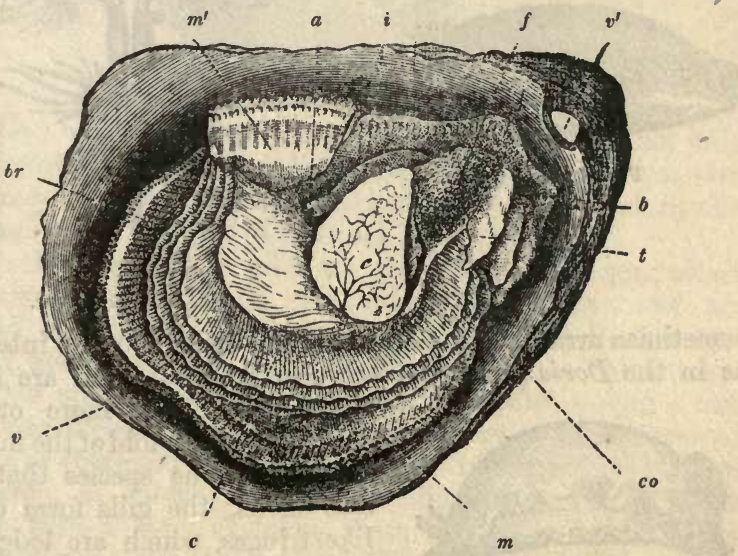

Fig. 149.-RESPIRATORY APPARATUS OP THE OTSTER.

$v$, one of the valves of the shell; $v^{\prime}$, its hinge; $m$, one of the lobes of the mantie; $m^{\prime}$, a portion of the other lobe folded back; $c$, muscles of the shell; $b r$, gills; $b$, mouth ; $t$, tentacula, or prolonged lips; $f$, liver; $i$, intestine; $a$, anus - co heart. 
(br, fig. 149), lying near the edge of the shell, and copiously supplied with blood-vessels. In the Oyster, these are freely exposed to the surrounding element, the lobes of the mantle being separated along their entire length; but where the

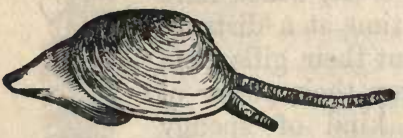

FH. 150.--TELLINA. mantle-lobes are united along their margin, so as to shut-in the gills, there are two orifices, often prolonged into tubes (as in the Tellina, fig. $150)$, through one of which the water is drawn-in for the purpose of respiration, whilst through the other it passes out, as in the Tunicata. In the aquatic GASTEROPODA there is scarcely any part of the body to which we do not find the gills attached in some species or other. In the naked marine species, which may be called Sea-slugs, they form fringes which are sometimes disposed along the sides of the body, as in the Tritonia and Glaucus (figs. 151, 152),

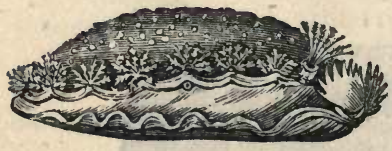

Fig. 151.-Tritonia.

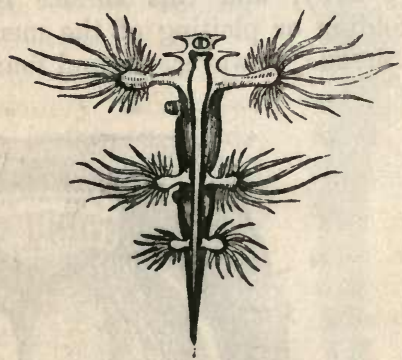

Fig. 152.-Graucus.

sometimes arranged in a circle around the end of the intestine, as in the Doris (fig. 153,-see also fig. 139); and are some-

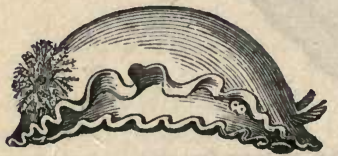

Fig. 153.-DoRrs. times covered-in, more or less completely, by a fold of the mantle. In most of the species that possess shells, the gills form comblike fringes, which are lodged in a cavity inclosed in the last turn of the spiral shell; and to this cavity the water is admitted, sometimes by a large opening, sometimes by a prolonged tube. In the CEPHALOPODA, we find 
the gills composed of a collection of little leaf-like folds, placed on a stalk (b, fig. 154); they are inclosed in a cavity which is covered-in by the mantle; and the walls of this cavity have the power of alternately dilating and contracting, so as to draw-in and expel water. It communicates with the exterior by two orifices, one of which, $o$, a wide slit, is for the entrance

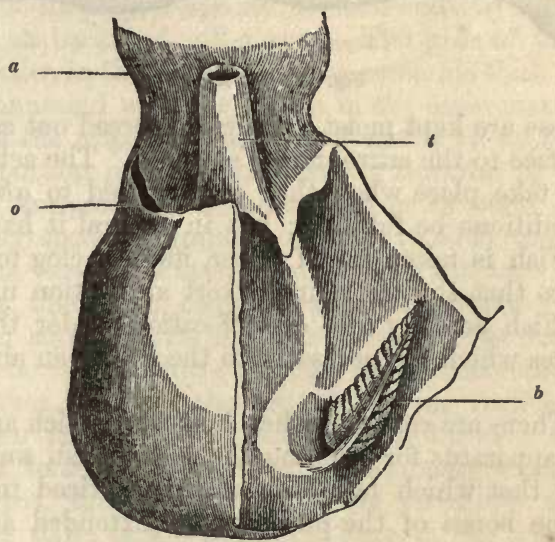

Fig. 154.-Gills of Poulp.

of water; whilst the other, $t$, is tube-like, and serves not only to carry-off the water that has passed over the gills, but also to convey away the excrements, and the fluid ejected by the ink-bag. This is called the funnel.

317. In Frshes, the gills are composed of fringes, which are disposed in rows on each side of the throat, and are covered by the skin. The cavity in which they lie has two sets of apertures; - one communicating with the throat, and the other opening on the outside. In the Fishes with a cartilaginous skeleton, we usually find as many of these external orifices as there are rows of gills ; thus in the Lamprey there are seven, as shown in the succeeding figure $(a)$. But in Fishes with a bony skeleton, there is usually but a single large orifice on either side; and this is covered with a large valve-like flap, which is termed the operculum or gill-cover. A continual stream of water is made to pass over the gills by the action of the mouth, which takes-in a large quantity of 
fluid, and then forces it through the apertures on each side of the throat, into the gill-cavities; and from these it passes out by the other orifices just described. Fishes, in common with other animals that breathe by gills, can only respire properly

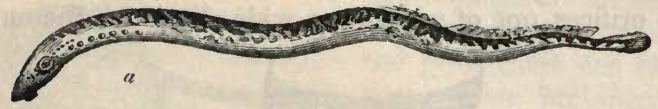

Fig. 155.-LAMPREX.

when these are kept moist, and are so spread out as to expose their surface to the surrounding element. The act of respiration can take place when they are exposed to air, provided these conditions be fulfilled; but in general it happens that, when a Fish is taken out of water, its gills clog together and dry up, so that the air cannot exert any action upon them; and the Fish actually dies of suffocation, under the very circumstances which are necessary to the life of an air-breathing animal.

318. There are certain Fishes, however, which are provided with an apparatus for keeping the gills moist, somewhat resembling that which has been already noticed in the landcrab. The bones of the pharynx are extended and twisted

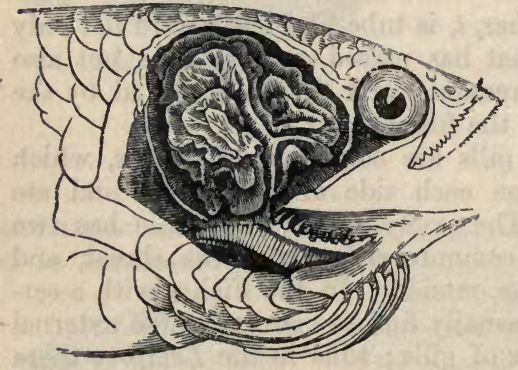

Fig. 156. Regpiratory ApParatus of ANABAs. in such a remarkable manner (fig. 156), as to form a number of small cavities ; these cavities the Fish can fill with water; and they form a reservoir of fluid, from which the gills may be supplied with a sufficient amount to keep them moist during some time. The gill-filaments themselves are so arranged that they do not clog together; and by this combination of contrivances, the species of Fish that are furnished with it can live for a long time out of water, so as to be able to journey for a considerable distance on land. Such a provision 
is especially desirable in tropical climates, where shallow lakes are often dried-up by continued drought, so that their inhabitants must perish, if they were not thus enabled to migrate. One of the most curious of these Fishes (most of which are inhabitants of India and China) is the Anabas or climbing-perch of Tranquebar; which climbs bushes and trees in search of its prey, a species of land-crab, by means of the spines on its back and gill-covers. - The gills of the Amphibious Reptiles, in their Tadpole state, resemble those of Fishes, and are connected with the mouth in the same manner.

319. In the respiratory actions of nearly all these animals, a very important part is performed by the cilia ( $\$ 45)$ which cover the surfaces of the gills. Even in such as do not possess any special respiratory organs ( $\$ 311)$, the action of the cilia is very important, in causing a constant change in the water that is in contact with their surface. Thus in Zoophytes, which are for the most part fixed to one spot, the action of the cilia produces currents in the surrounding water. On the other hand, in the actively-moving Animalcules, the same action propels their bodies rapidly through the water; though in some of them, which occasionally fix themselves like Polypes, the action of the cilia resembles theirs. In either case there is a continual change in the layer of water which is in immediate contact with the surface; and thus a constant supply of the air contained in the water is secured. A similar action goes-on, still more energetically, over the gilltufts of the Annelida; and this action continues after the death of the animal, or after the tuft has been separated from it, producing evident currents in the water in which it is placed. It is by the action of the cilia alone, that the continual current of water is kept-up through the respiratory chamber of the lower Mollusca; but this is superseded in Cephalopods and Fishes by the other means for sustaining this current which have been already noticed. Ciliary action may be well observed in the young Tadpole of the common Water-Newt, whose gills hang freely from the neck on either side; the cilia are themselves so minute that they cannot be readily distinguished; but the motion of the water produced by them may be at once perceived in a tolerable microscope, especially when small light particles, such as those of powdered charcoal, are diffused through it. 
320. In animals whose blood is made to act directly upon the air, we usually find a provision of some kind for introducing the air into the interior of the body. The simplest arrangement is that which we meet-with in the Snail and other terrestrial GASTEROPODS ; and it consists merely of a cavity ( $p$, fig. 157), resembling that in which the gills are disposed in the aquatic Mollusea, but having a free communication with

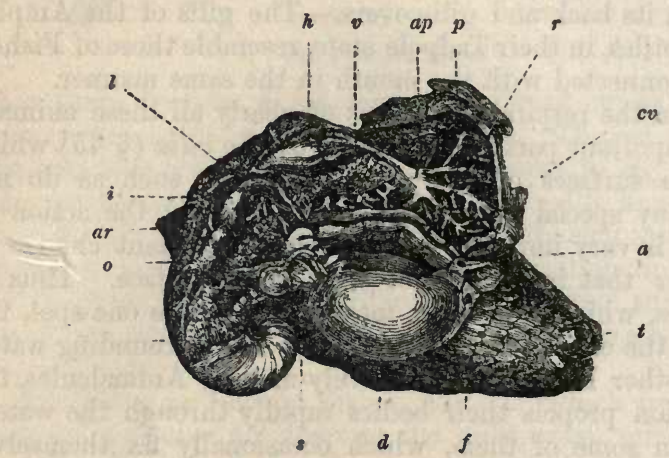

Fig. 157.-Axatomx of SNaIL.

$f$, muscular disc or foot; $t$, tentacula; $d$, diaphragm separating the respiratory cavity $p$ from other organs, but here turned back; $s$, stomach; $o$, ovary; ar, arterial trunk supplying the system; $i, r$, intestine; $l$, liver; $h$, heart; $a p$, vascular trunk spreading over the pulmonary cavity $p_{i} c v$, canal for excreting the viscid mucus secreted by the gland $v$.

the external air, and having the blood minutely distributed by vessels upon its walls.-In the Mrriapoda or Centipede tribe, in conformity with the general plan of Articulated structure ( $(93)$, we find a repetition of similar cavities along the body,

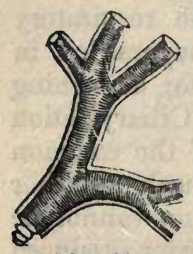

Fig. 158.

ATR-TUBE OF INSECr. one pair usually existing in each segment; and these open externally by small apertures, which are termed spiracles.

321. In INSECrs, the same general arrangementis modified in the mostremarkable manner. The spiracles do not open into distinct air-sacs, but into canals, which lead to two large tracheæ which run along the sides of the body, and are connected by several tubes that pass across itone usually for each segment. From these tracheæ others branch off, which again subdivide into more 
minute tubes; and, by the ramifications of these, even the minutest parts of the body are penetrated (fig. 159). These tubes are formed upon a similar plan with the air-vessels of Plants, having a spiral fibre winding inside their outer membranous coat (fig. 158); by the elasticity of which fibre, the tube is kept from being closed by pressure. In this manner the air is brought into contact with almost

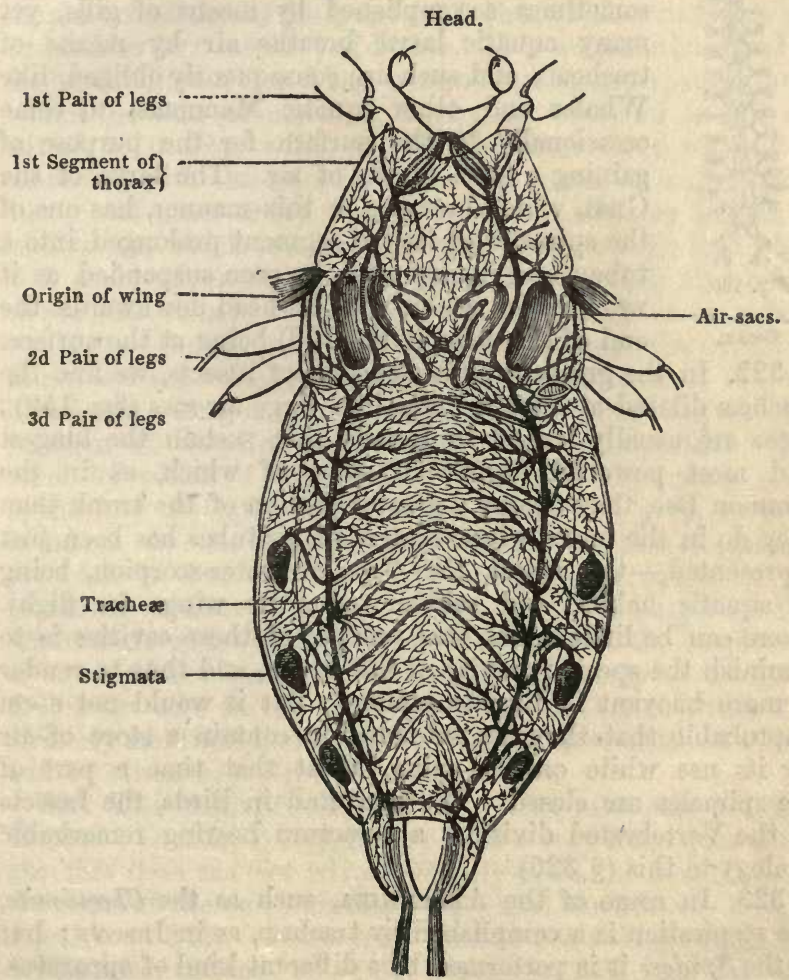

Fig. 159.-REspiratory Apparatus of INBEct (NEPA).

every portion of the tissue, and is enabled to act most energetically upon it; and thus the feeble circulation of these animals $(\$ 293)$ is in a great degree counterbalanced by 
the extraordinary activity of their respiration. There are no animals which consume so much oxygen, in proportion to their size, as Insects do when they are in motion ( $\$ 308)$; but when they are at rest, their respiration falls to the low standard of the tribes to which they bear the greatest general resemblance. Although, as we have seen, the respiration of aquatic larvæ is sometimes accomplished by means of gills, yet many aquatic larvæ breathe air by means of tracheæ; and such are eonsequently obliged, like Whales and other aquatic Mammals, to come occasionally to the surface for the purpose of gaining a fresh supply of air. The larva of the Gnat, which breathes in this manner, has one of the spiracles of its tail-segment prolonged into a tube; and it may often be seen suspended, as it were, in the water, with its head downwards, the Fig. 160. GNAT: end of this tube ( $t$, fig. 160) being at the surface.

322. In the greater number of perfect Insects, we find the tracheæ dilated at certain parts into large air-sacs (fig. 159); these are usually largest in Insects that sustain the longest and most powerful flight; in some of which, as in the common Bee, they occupy a greater portion of the trunk than they do in the insect whose system of air-tubes has been just represented,-this insect, the Nepa or water-scorpion, being of aquatic habits, and seldom using its wings for flight. There ean be little doubt that one use of these cavities is to diminish the specific gravity of the Insect, and thus to render it more buoyant in the atmosphere; but it would not seem improbable that they are intended to contain a store of air for its use while on the wing, as at that time a part of the spiracles are closed. We shall find in Birds, the Insects of the Vertebrated division, a structure bearing remarkable analogy to this ( $\$ 326)$.

323. In some of the ArACHNIDA, such as the Cheese-mite, the respiration is accomplished by tracheæ, as in Insects; but in the Spiders it is performed by a different kind of apparatus. Instead of opening into a system of prolonged tubes, each spiracle leads to a little chamber, the lining membrane of which is arranged in a number of folds that lie together like the leaves of a book; and thus a large surface is exposed 
to the air which is admitted through the spiracles. This arrangement is shown in fig. $46, l$.

324. Hitherto it has been seen that the respiratory apparatus is not connected with the mouth, which in the Invertebrated classes has the reception of food as its sole function. On this account, we cannot regard the air-sacs of Insects as bearing any real analogy to the lungs of Vertebrata. The simplest condition of the true lung is that which constitutes the air-bladder (or "sound") of Fishes. This we sometimes find in the condition of a closed bag, lying along the spine ; and its use cannot be that of assisting respiration, since air is not admitted to it from without. But in other cases we find it connected with the intestinal tube, by means of a short wide duct; and since many Fishes, as the Loach, are known to swallow air, which is highly charged with carbonic acid when it is again expelled, it seems probable that their air-bladder effects this change in precisely the same manner as a lung would do-the air being transmitted to it from the intestine. There are some Fishes in which the resemblance of the airbladder to a lung is more decided, and its connexion with the function of Respiration is evidently more important. The canal by which it communicates with the alimentary canal opens into the latter above the stomach, and even, in some instances, at the back of the mouth; so that a gradual approach is seen to the arrangement which exists in airbreathing animals. In these Fishes, as in the Amphibia that retain their gills ( $\$ 87)$, it would appear that the respiration is accomplished partly by the lungs, and partly by the gills ; this is the case in the curious Lepidosiren (fig. 41), which, as formerly mentioned, is regarded by some naturalists as a Fish, and by others as a Reptile.

325. The lungs of Reptiles are for the most part but little divided ; so that, although they hold a very large quantity of air, this does not act advantageously upon the blood, in consequence of the small surface over which the two are brought together (§ 312). In Serpents we find but a single lung, that of the other side not being developed (fig. 34); and this lung extends through nearly half the length of the body. Reptiles have no power of filling their lungs by a process resembling our inspiration, or drawing-in of air ; but they are obliged to swallow it, as it were, by the action of the mouth. The skin 
of Frogs is of great importance in their respiration-in fact, of almost as much consequence as their lungs. The necessity for more energetic respiration increases in these animals with the temperature, every rise in which excites them to greater activity. During the winter, which they pass beneath the water in a state of torpidity, the action of the water upon their skin is sufficient to aerate their blood. When the returning warmth of spring arouses them from their inaction, they need a larger amount of respiration, and come occasionally to the surface to take-in air by their lungs. And when summer comes on, the greater heat increases their need of respiration; and they quit their ditches and ponds, so as to allow the atmosphere to act upon their skin as well as upon their lungs. If they are prevented from doing so, they will die; and the same result follows if the skin be smeared with grease, so that the air cannot permeate it. Moreover, if the lungs be removed, and the animal be made to breathe by its skin alone, it may live for some time, if the temperature be not high. These facts show the great importance of the skin as a respiratory organ in Frogs.

326. The respiration of BIRDs is more active than that of

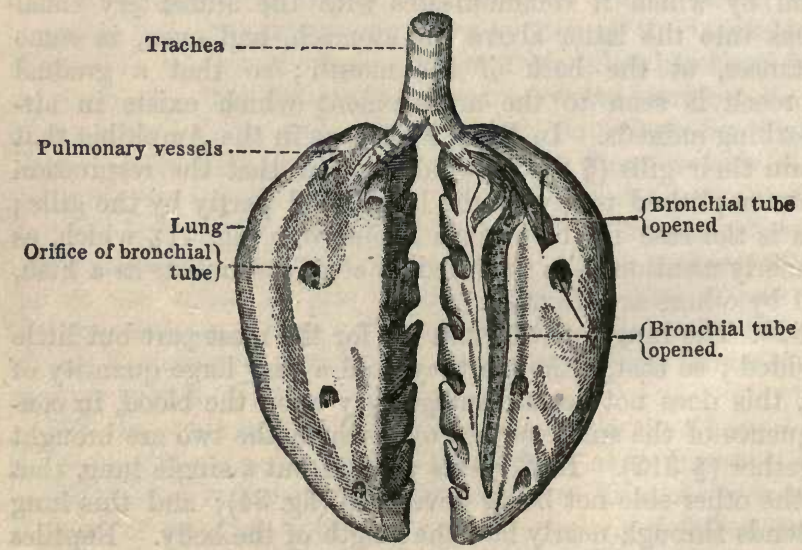

Fig. 161.-Air-tubes and Lungs of Birds.

any other Vertebrata; that is, they consume more oxygen, and form more carbonic acid, in proportion to their size. 
Their lungs, however, are not so minutely subdivided as are those of Mammals; but the surface over which the air can act upon the blood is immensely extended, by a provision which is peculiar to this class. The air introduced by the windpipe passes not only into the lungs properly so called, but into a series of large air-cells, which are disposed in various parts of the body, and which even send prolongations into the bones, especially in Birds of rapid and powerful flight, whose whole skeleton is thus traversed by air. The mode in which some of the bronchial tubes, or subdivisions of the windpipe, pass from the lungs to these air-cells, is shown in fig. 161. Now, by this arrangement, a much larger quantity of air is taken-in at once, and a much more extensive surface is exposed to its action, than could otherwise be provided for; and as the air which is received into the air-cells has to pass through the lungs, not only when it is taken-in, but when it is expelled again, its full influence upon the blood is secured.

327. Birds, like Reptiles, are destitute of the peculiar apparatus by which Mammals are enabled to fill their lungs with air; but it is introduced without any effort on their parts. For the cavity of their trunk is almost surrounded by the ribs and breast-bone; and the elasticity of the former keeps it generally in a state corresponding to that of our own lungs when we have taken-in a full breath. Thus the state of fullness is natural to the lungs and air-cells of Birds. When the animal wishes to renew their contents, however, it compresses the walls of the trunk, so as to diminish its cavity and to force out some of the air contained in the lungs, \&c. ; and when the pressure is removed, the cavity returns to its previous size by the elasticity of its walls, and a fresh supply of air is drawn into the lungs. The air-sacs answer the same purpose in Birds as in Insects, diminishing the specific gravity of the body, by increasing its size without adding to its weight, and thus rendering it more buoyant.

328. In Man and other Mammals, the lungs are confined to the upper portion of the cavity of the trunk, termed the thorax; which is separated from the abdomen by the diaphragm, a muscular partition, whose action in respiration is very important. (An imperfect diaphragm is found in some Birds, which approach most nearly to Mammals in their general structure.) The lungs are suspended, as it were, in 
this cavity, by their summit or apex; and are covered by a serous membrane termed the pleura, which also lines the thorax, being reflected from one surface to the other precisely in the manner of the pericardium $(\$ 43)$. Thus the pleura of the outer surface of the lung is continually in contact with that which forms the inner wall of the thorax; they are both kept moist by fluid secreted from them; and they are so smooth, as to glide over one another with the least possible friction. The lungs themselves are

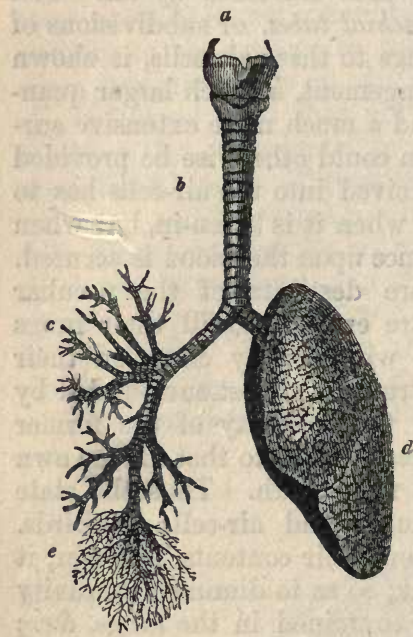
very minutely subdivided; and thus expose a vast extent of surface in proportion to their size. The air-cells of the human lung, into which the air is conveyed by the branches of the wind-pipe, and on the walls of which the blood is distributed, do not average above the 1-100th of an inch in diameter. In the accompanying figure is represented, on one side, the lung, $d$, presenting its natural appearance; and on the other, the ramifications of the airpassages or bronchial tubes, $c$, $e$, by which air is conveyed into every part of the lungs. The trachea or windpipe, b, opens into the pharynx or back of the mouth, by the larynx, a. The construction of this is especially destined to produce the voice, and will be explained under that head (Chap. xIII.); but it may be here mentioned that tire entrance from the pharynx into the larynx consists of a narrow slit, capable of being enlarged or closed by the separation or approximation of its lips, which form what is called the glottis. The aperture of the glottis is regulated by the muscular apparatus of the larynx; the actions of which are not under the direct control of the will, but are automatic, like those concerned in swallowing ( $\$ 194)$; and the purpose of this provision is to 
prevent the entrance of anything injurious into the windpipe. Thus if we attempt to breathe carbonic acid gas, which would produce an immediately fatal result if introduced into the lungs, the lips of this chink immediately close together, and so prevent its entrance. The contact of liquids or of solid substances, too, usually causes the closure of the aperture, so that they are prevented from finding their way into the windpipe; but this does not always happen, especially when the glottis is widely opened to allow the breath to be drawn-in (\$ 193).

329. The larynx, trachea, and bronchial tubes, to their minutest ramifications, in all air-breathing Vertebrata, are lined by a mucous membrane continued from the back of the throat; and this membrane, like the gills of aquatic animals, is covered with cilia, which are in continual vibration. It is obvious, however, that the purpose of this ciliary movement must be here different from that which is fulfilled by the same action on the surface of the gills ( $\$ 319)$; and it probably serves to get rid of the secretion which is being continually poured out from the surface of the mucous membrane, and which, if allowed to accumulate there, would clog up the aircells, and in time produce suffocation. The vibration of the cilia is observed to be always in one direction,-towards the outlet; and it is in this direction, therefore, that the fluid is gradually but regularly conveyed. The ciliary movement may be seen to take place on the surface of the mucous membrane of the nose ; but not on that of the pharynx, where it would be continually interrupted by the passage of food.

330. The constant renewal of the air in the lungs is provided for, in Mammals, by a peculiar mechanism, which accomplishes this purpose most effectually, though itself of the most simple character. We must recollect that the thorax in this class is an entirely closed cavity. It is bounded above and at the sides by the ribs (the space between which is filled up by muscles, \&c.), and below by the diaphragm, which here forms a complete partition between the thorax and abdomen. The whole of this cavity, with the exception of the space occupied by the heart and its large vessels (and also by the gullet, which runs down in front of the spine), is constantly filled-up by the lungs. Now the size of this cavity may be made to vary considerably; - in the first place, by the 
movement of the diaphragm; and in the second, by that of the ribs.

331. I. The diaphragm, in a state of rest or relaxation, forms a high arch, which rises into the interior of the chest, as at $g$, fig. 163 ; but when it contracts, it becomes much flatter (though always retaining some degree of convexity upwards), and thus adds considerably to the capacity of the lower part of the chest. The under side of the diaphragm is in contact with the liver and stomach, which, to a certain

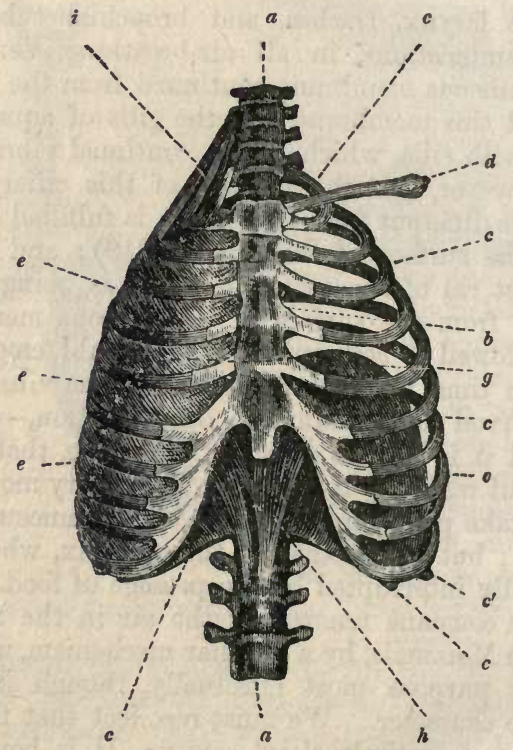

Fig. 163.-THoraX of MAN.

degree, rise and fall with it. It is obvious that, when the diaphragm descends, these organs, with the abdominal viscera in general, must be pushed downwards ; and as there can be no yielding in that direction, the abdomen is made to bulge forwards when the breath is drawn-in. On the other hand, when the contraction of the diaphragm ceases, the abdominal muscles press back the contents of the abdomen, force up the 
liver and stomach against the under side of the diaphragm, and cause it to rise to its former height.

332. II. The play of the ribs is rather more complicated. These bones, $c c$, and $c^{\prime} c^{\prime}$ (to the number of twelve on each side in Man), are attached at one end by a moveable joint to the spinal column, $a$; whilst at the other they are connected with the sternum (breast-bone) by an elastic cartilage. Now each rib, in the empty state of the chest, curves downwards in a considerable degree; and it may be elevated by a set of muscles, of which the highest, $i$, are attached to the vertebræ of the neck and to the first rib, whilst others, $e, e, e$ (termed intercostals), pass between the ribs. The cartilages also curve downwards in the opposite direction, from their points of attachment to the sternum. When the breath is drawn-in, the first rib is raised by the contraction of the muscles, $i$; and all the other ribs, which hang, as it were, from it, would of course be raised by this action to the same degree. But each of them is raised a little more than the one above it, by the contraction of its own intercostal muscle; and thus the lower ribs are raised very much more than the upper ones. Now by the raising of the ribs, they are brought more nearly into a horizontal line, as are also their cartilages; and since the combined length of the two is greater, the nearer they approach to a straight line, it follows that the raising of the ribs must throw them further out at the sides, and increase the projection of the sternum in front, thus considerably enlarging the capacity of the chest in these directions. When the movement of inspiration is finished, the ribs fall again, partly by their own weight, partly by the elasticity of their cartilages, and partly by the contraction of the abdominal muscles which are attached to their lower border.-For the full understanding of this description, it is desirable that the reader should examine the movements of his own or another person's chest, by placing his fingers upon different points of the ribs, and watching their changes of position during the drawing-in and the expulsion of the breath.

333. Now the cavity of the thorax is itself perfectly closed; so that, if it were not for the expansion of the lungs, a void or vacuum would be left when the diaphragm is drawn down and the ribs elevated. The atmosphere around presses to fill that vacuum; but this it can only 
do by entering the lungs through the windpipe, and inflating them (or blowing them out), so as to increase their size in. proportion to the increase of the space they have to fill. In this manner the lungs are made constantly to fill the cavity of the chest, however great may be the increase in the latter. But if we were to make an aperture through the walls of the chest, the air would rush directly into its cavity, when the movements of inspiration are performed, and the lung of that side ${ }^{1}$ would not be dilated. The same thing would happen if there were an aperture in the lung itself, allowing free communication between one of the larger bronchial tubes and the cavity of the chest ; for the air, although still drawn-in by the windpipe, would pass directly into the cavity of the chest, rather than dilate the lung, which would thus become entirely useless. Such an aperture is sometimes formed as the result of disease; and if the action of both lungs were thus prevented, death must immediately take place from suffocation.

334. The extent of the respiratory movements varies considerably; but in general it is only such as to change about the seventh part of the air contained in the lungs. (It may be generally noticed, that every fifth or sixth inspiration in Man is longer and fuller than the rest.) Their rate varies according to age, and to the state of the nervous system; being faster in infants and in young persons than in adults; and more rapid in states of mental excitement, or irritation of the bodily system, than in a tranquil condition. In a state of rest, from 14 to 18 inspirations take place every minute in an adult, and at each about 20 cubic inches of air are drawn-in; but both the depth and frequency of the inspirations are considerably increased by exercise. Taking an average alternation of activity and repose, it appears that about 360 cubic feet of air pass through the lungs every twenty-four hours, or 15 cubic feet every hour; and as the air which has once passed through the lungs contains about 1-24th part of carbonic acid, about 15 cubic feet of that gas, containing nearly 8 ounces of solid carbon, are thrown-off in the course of twenty-four hours.

335. Now carbonic acid, when diffused through the atmosphere to any considerable amount, is extremely injurious to

1 Each lung has its own cavity; the two being completely separated from each other by the pericardium ( $\$ 43$ ). 
animal life; for it prevents the due excretion by the lungs of that which has been formed within the body; and the latter consequently accumulates in the blood, and exercises a very depressing influence on the action of the various organs of the body, but particularly on that of the nervous system. The usual proportion is not above 1 part in 5000 ; and when this is increased to 1 part in 100, its injuxious effects begin to be felt by Man, in headache, languor, and general oppression. Now it is evident, from the statements in the last paragraph, that, as a man produces in twenty-four hours about 15 cubic feet of carbonic acid, if he were inclosed in a space containing 1500 cubic feet of air (such as would exist in a room 15 feet by 10 , and 10 feet high), he would in twenty-four hours communicate to its atmosphere from his lungs as much as 1 part in 100 of carbonic acid, provided that no interchange takes place between the air within and the air outside the chamber. The amount would be further increased by the carbonic acid tlrown off by the skin, the quantity of which has not yet been determined.

336. In practice, such an occurrence is seldom likely to take place; since in no chamber that is ever constructed, except for the sake of experiment, are the fittings so close as to prevent a certain interchange of the contained air with that on the outside. But the same injurious effect is often produced by the collection of a large number of persons for a shorter time, in a room insufficiently provided with the means of ventilation. It is evident that if twelve persons were to occupy such a chamber for two hours, they would produce the same effect with that occasioned by one person in twenty-four hours. Now we will suppose 1200 persons to remain in a church or assembly-room for two hours; they will jointly produce 1500 cubic feet of carbonic acid in that time. Let the dimensions of such a building be taken at 100 feet long, 50 broad, and 30 high; then its cubical content will be $(100 \times 50 \times 30) 150,000$ cubic feet. And thus an amount of carbonic acid, equal to 1-100th part of the whole, will be communicated to the air of such a building, in the short space of two hours, by the presence of 1200 people, if no provision be made for ventilating it. And the quantity will be greatly increased, and the injurious effects will be proportionably greater, if there be an additional consumption of 
oxygen, produced by the burning of gas-lights, lamps, or candles.

337. Hence we see the great importance of providing for free ventilation, wherever large assemblages of persons are collected together, even in buildings that seem quite adequate in point of size to receive them; and much of the weariness which is experienced after attendance on crowded assemblies of any kind, may be traced to this cause. In schools, factories, and other places where a large number of persons remain curing a considerable proportion of the twenty-four hours, it is impossible to give too much attention to the subject of ventilation; and as, the smaller the room, the larger will be the proportion of carbonic acid its atmosphere will contain, after a certain number of persons have been breathing in it for a given time, it is necessary to take the greatest precaution when several persons are collected in those narrow dwellings, in which, unfortunately, the poorer classes are compelled to reside. Even the want of food, of clothing, and of fuel, are less fertile sources of disease than insufficient ventilation; which particularly favours the spread of contagious diseases, on the one hand by keeping-in the poison, and thus concentrating it upon those who expose themselves to its influence; and, on the other, by obstructing the elimination of the waste matter from the system, the presence of which in the blood renders it peculiarly liable to be acted-on by all poisons having the nature of "ferments."

338. When the quantity of carbonic acid in the air accumulates beyond a certain point, it speedily produces suffocation and death. This is occasioned by the obstruction to the flow of blood through the capillaries of the lungs, which takes place when it is no longer able to get rid of the carionic acid with which it is charged, and to absorb oxygen in its stead. The general principle to which this stagnation may be referred has already been noticed ( $\$ 280)$. Now, as all the blood of the system, in warm-blooded animals, is sent through the lungs before it is again transmitted to the body, it follows that any such obstruction in the lungs must bring the whole circulation to a stand. The functions of the nervous system are directly dependent upon a constant supply of arterial blood (Chap. x.) ; and, accordingly, as this supply becomes progressively diminished in quantity and deteriorated in qua- 
lity, its actions first become irregular, producing violent convulsive movements, and at last, cease altogether, the animal becoming completely insensible. In this condition, which is termed Asphyxia, the pulmonary arteries, the right side of the heart, and the large veins which empty themselves into it, are gorged with dark blood; whilst the pulmonary veins, the left side of the heart, and the arteries of the system, are comparatively empty. Hence the action of the right side of the heart comes to an end, through a loss of power in its walls, occasioned by their being over-distended; whilst that of the left side ceases for want of the stimulus of the contact of blood, by which the muscular fibre is excited. If this state be allowed to continue, death is the consequence; but if the carbonic acid in the lungs be replaced by pure air, the flow of blood through their capillaries recommences,-the right side of the heart is unloaded and begins to act again,-arterial blood is sent to the left side, and excites it to renewed motion, -and the same being propelled by it to all parts of the body, their several functions are restored, the nervous system recovers its power of acting, and all goes on as before. These changes occur in exactly the same manner when a warmblooded animal is made to breathe nitrogen or hydrogen; since these gases do not perform that which it is the office of oxygen to effect, - the removal of carbon from the system, in the form of carbonic acid. And they also take place in a perfectly pure atmosphere, when the individual is prevented from receiving it into its lungs by an obstruction to its passage through the windpipe, such as that produced by hanging; strangulation, drowning, \&c. For the air in the lungs, not being renewed, speedily becomes charged with carbonic acid, to an extent that checks the circulation through their capillaries; and all the consequences of this follow as before.

339. The most efficient remedy in all such cases is evidently that suggested by the facts stated in the last paragraph,the renewal of the air in the lungs. But with this, other means should be combined; and the general directions ${ }^{1}$ of Dr. Marshall Hall, with the method of producing artificial

1 The instructions, though specially intended for the resuscitation of persons apparently drowned, are applicable with slight modification to other forms of Asphyxia. 
respiration suggested by Dr. H. R. Silvester, seem most likely to answer in practice :

Treat the patient instantly, on the spot, in the open air, exposing the face and chest to the breeze, except in severe weather.

I. To clear the Throat, place the patient gently on the face, with one wrist under the forehead-(all fluids and the tongue itself then fall forwards, leaving the entrance into the windpipe free). If there be breathing, wait and watch; if not, or if it fail, -

II. To excite Respiration, turn the patient well and instantly on his side, and excite the nostrils with snuff, or the throat with a feather, \&c., and dash cold water on the face previously rubbed warm. If there be no success, lose not a moment, but instantly,-

III. To imitate Respiration, lay the patient on his back, with the head and shoulders slightly elevated; then let the arms be raised and steadily extended upwards, by the sides of the head, so as to draw-up the shoulders. In this way, the ribs are drawn-up by the muscles passing to them from the shoulders, and the cavity of the chest is enlarged. If the arms be then carried-down to the sides of the body, the shoulders fall, the ribs are lowered, and the sides of the thorax approach one another, as in natural expiration,- an effect which may be increased by moderate pressure on the front and sides of the chest. By an alternation of these movements, an artificial Inspiration and Expiration will be effected, which, though imperfect, may restore life.

Iv. To induce Circulation and Warmth, meantime rub the limbs upwards, with firm grasping pressure and with energy, using handkerchiefs, \&c. (by this measure the blood is propelled along the veins towards the heart); let the limbs be thus warmed and dried, and then clothed, the bystanders supplying the requisite garments; avoiding the continuous warm bath, and the position on, or inclined-to, the back.

340 . The ordinary movements of respiration belong, like those of swallowing, to the class of reflex actions ( $\$ 430)$. We have seen that, in every such movement, a great number of muscles are called into play simultaneously; and this is effected by means of the stimulus which is sent to them from the spinal cord. But this stimulus does not originate there; 
for it is the consequence of impressions conveyed to the spinal cord, and especially to its upper end, by several nerves,--some originating in the lungs, and others in the general surface. The nerves originating in the lungs convey to the spinal cord the impression produced by the presence of venous blood in their capillaries: of this impression. we are not ordinarily conscious; but if we hold our breath for a few moments, we become aware of it; and it speedily becomes so distressing as to force us to breathe, even though we may try to resist it by an effort of the will. The impression conveyed by the nerves of the general surface is chiefly that produced by the application of cold to the skin. It is this which is the cause of the first inspiration in the new-born infant; which is not unfrequently prevented by the seclusion of its face (the part most capable of receiving this impression) from the influence of the air. Every one knows that, when the face is dipped into water, an inspiratory movement is strongly excited; and the same happens when a glass of water is dashed over the face. This simple remedy will often put a stop to hysterical laughter, by producing a long sighing inspiration. A still stronger tendency to draw-in the breath is experienced in the first dash of water over the body in the shower-bath. The respiratory movements, in the higher Animals, are placed under the control of the will, to a certain extent, because on them depend the production of sounds, and in Man the actions of speech ; but that they are quite independent of the will, and even of sensation, is shown by the fact that they will continue after the brain has been completely removed, provided the spinal cord and its. nerves are left without injury. In most of the Invertebrata they are connected with distinct ganglia, which minister to them alone. (See Chap. x.)

341. The actions of sighing, yawning, sobbing, langhing, coughing, and sneezing, are nothing else than simple modifications of the ordinary movements of respiration, excited either by mental emotions, or by some stimulus originating in the respiratory organs themselves. Sighing is nothing more than a very long-drawn inspiration, in which a larger quantity of air than usual is made to enter the lungs. This is continually taking place in a moderate degree, as already noticed (§ 334$)$; and we notice it particularly, when the attention is released after having been fixed upon an object which has excited it 
strongly, and which has prevented our feeling the insufficiency of the ordinary respiratory movements. Hence this action is only occasionally connected with mental emotion. Yawning is a still deeper inspiration, which is accompanied by a kind of spasmodic contraction of the muscles of the jaw, and also by a very great elevation of the ribs, in which the shoulders and arms partake. The purely involuntary character of this movement is sometimes seen in a remarkable manner in cases of palsy, in which the patient cannot raise his shoulder by an effort of the will, but does so in the act of yawning. Nevertheless the action may be performed by the will, though not completely; and it is one that is particularly excited by an involuntary tendency to imitation, as every one must have experienced who has ever been in company with a set of yawners. Sobbing is the consequence of a series of short convulsive contractions of the diaphragm; and it is usually accompanied by a closure of the glottis, so that no air really enters. In Hiccup, the same convulsive inspiratory movement oecurs, the glottis closing suddenly in the midst of it ; and the sound is occasioned by the impulse of the column ot air in motion against the glottis. In Laughing, a precisely reverse action takes place; the muscles of expiration are in convulsive movement, more or less violent, and send out the breath in a series of jerks, the glottis being open. This sometimes goes on until the diaphragm is more arched, and the chest more completely emptied of air, than it could be by an ordinary movement of expiration. The act of Crying, though occasioned by a contrary emotion, is, so far as the respiration is concerned, very nearly the same. We all know the effect of mixed emotions in producing something "between a laugh and a cry."

342. The purposes of the acts of coughing and sncezing are, in both instances, to expel substances from the air-passages, which are sources of irritation there; and this is accomplished in both by a violent expiratory effort, which sends forth a blast of air from the lungs.-Coughing occurs when the source of imitation is situated at the back of the mouth, in the trachea, or bronchial tubes. The irritation may be produced by acrid vapours, or by liquids or solids that have found their way into these passages, or by secretions which have been poured into them in unusual quantity as the result of 
disease ; and the latter will be the more likely to produce the effect, from the irritable state in which the lining membrane of the air-passages already is. The impression made upon this membrane is conveyed by the nerves spread out beneath its surface to the spinal cord; and the motor impulses are sent to the different muscles, which they combine in the act of coughing. This act consists, 1st, in a long inspiration, which fills the lungs; $2 \mathrm{~d}$, in the closure of the glottis at the moment when expiration commences; and $3 \mathrm{~d}$, in the bursting-open, as it were, of the glottis, by the violence of the expiratory movement, so that a sudden blast of air is forced up the air-passages, carrying before it anything that may offer an obstruction.-Sneezing differs from coughing in this, that the communication between the larynx and the mouth is partly or entirely closed, by the drawing-together of the sides of the veil of the palate over the back of the tongue; so that the blast of air is directed more or less completely through the nose, in such a way as to carry-off any source of irritation that may be present there.

343. Every one is aware that the air he breathes-forth contains a large quantity of vapour : this is not perceptible in a warm atmosphere, because the watery particles remain dissolved in it and do not affect its transparency; but in a cold atmosphere they are no longer held in solution, and consequently present the appearance of fog or steam. The quantity of fluid which thus passes off is by no means trifling, probably not less than from 16 to 20 ounces in the twentyfour hours; a portion of it undoubtedly proceeds from the moist lining of the mouth, throat, \&c., but the greater part is thrown-off by the lungs themselves. This fluid, when collected, is found to contain a good deal of decomposing organic matter, especially in cases in which the respiratory process has not been carried on with perfect freedom; such matter being oxydized and thrown-off under other forms, when the blood is duly aerated. Various substances of an odoriferous character, which have been taken into the blood, manifest their presence in this exhalation : thus turpentine, camphor, and alcohol, communicate their odour to the breath; and when the digestive system is out of order, the breath frequently acquires a disagreeable taint, from the reception of putrescent matters into the blood, and their exhalation through 
this channel.-Of the water of the blood, from which this exhalation is given-off, a small part is most probably formed by the direct union of the hydrogen contained in the food (especially when this is one of its predominating components, \$153) with the oxygen absorbed. For it has been found by careful experiment, that the proportion of inspired oxygen which disappears (not being contained in the carbonic acid expired, § 305), is much greater in animals that are fed on a flesh diet, than in those living on farinaceous food. Another portion of such oxygen probably unites with the sulphur and phosphorus of the food and tissues, to form sulphuric and phosphoric acids, which are excreted through the kidneys in combination with alkaline bases ( $\$ 367$ ).

344. Certain gases act as violent poisons, even when respired in very small proportion. Thus, a Bird is speedily killed by breathing air which contains no more than 1-1500th part of sulphuretted hydrogen; and a Dog will not live long in an atmosphere containing 1-800th part of this gas. The effects of carburetted hydrogen are similar; but a larger proportion is required to destroy life. Both these gases are given-off by decomposing animal and vegetable matter; the neighbourhood of which is consequently very injurious to health. Several cases of arsenical poisoning have occurred, from the accidental inhalation of a small quantity of arseniuretted hydrogen, the amount of arsenic contained in which must have been so minute as to be scarcely appreciable.

\section{CHAPTER VII.}

OF EXORETION AND SECRETION.

\section{General Purposes of the Excreting Processes.}

345. We have seen that the Blood, in the course of its circulation, not only deposits the materials that are converted into the several fabrics of which the body is composed, but also takes-up into itself the products of the decomposition which is continually going-on in its various parts ; and it is to replace this, that the constant Nutrition of the tissues is required. In order that the blood may retain its fitness for 
ne purposes to which it is destined, it is requisite that these roducts should be drawn-off from the current of the circulation, as constantly as they are received into it; and this is accomplished by the various processes of Excretion, which are continually taking place in different parts of the body. The uninterrupted performance of these is even more essential to the maintenance of life, than is an uninterrupted supply of nutritive materials ; for an animal may continue to exist for some time without the latter, but it speedily dies if either of the more important excretions be checked. We have a striking instance of this in the case of the Respiration, which may be regarded as a true function of Excretion, having for its object to set free Carbonic acid from the blood in a gaseous form,- - thereby contributing to the introduction of Oxygen into the blood, for the various important actions to which that element is subservient, especially the maintenance of Animal Heat. (Chap. IX.) The effects of the suspension of the respiratory process, even for a few minutes, in a warmlooded animal, have been shown (§338) to be certainly and speedily fatal ; and they are as certainly fatal in the end in cold-blooded animals, though a longer time is required to produce them.

346. The products of excretion are the same, as to their essential characters at least, through the whole Animal kingdom; and for this it is not difficult to find a reason. It will be remembered that the ultimate elements of the Animal tissues are four in number: oxygen, hydrogen, carbon, and nitrogen; and that the materials which make up the chief part of the fabric of different classes of animals-albumen, gelatin, fatty matter, \&c.-contain these elements united in constant proportions, from whatever source we obtain them. Hence we should expect to find the products of their decomposition also the same; and this is, for the most part, the case. Of these four ingredients, oxygen can never be said (in the healthy state at least) to be superfluous in the body; for a large and constant supply of it is required, to unite with the others and carry them off in their altered conditions. Thus, unless oxygen were continually introduced into the system, for the sake of uniting with the carbon that is to be thrown off by Respiration, that excretion must be checked; and it is required, in like manner, for uniting with hydrogen 


\section{NATURE AND OBJECTS OF EXCRETORY ACTIONS.}

to form water, and with compounds of nitrogen to form urea. Hence there is no need of an organ to carry off the superfluous oxygen; but an organ to introduce it is rather required; and this purpose, as we have seen, is answered by the Respiratory apparatus. But we find organs of excretion specially destined to carry off the carbon, hydrogen, and nitrogen, which are set free, under various forms, by the decomposition of the tissues. Thus the Respiratory organs, as we have seen, throw off carbon in the form of carbonic acid, and hydrogen which has been in like manner united with oxygen so as to form water. The Liver has for its office partly to separate these same elements from the blood in a different form, throwing them off in the condition of a peculiar fatty matter, which consists almost entirely of carbon and hydrogen. But it has another function of no less importance in animals whose respiration is active ; for by its agency the hydro-carbonaceous matter circulating in the blood is brought into a state in which it readily combines with oxygen to form carbonic acid and water; and thus the liver may be said to prepare the pabulum for the combustive process. Iastly, the Kidneys have for their chief object to throw off the azotized compounds which result from the decomposition of the tissues; these contain a very large proportion of azote or nitrogen, which is united with the other elements into the crystalline compounds, urea, and uric or lithic acid, the latter of which is usually thrown off in combination with soda or ammonia. And the kidneys further serve as the channel through which soluble matters of various kinds, which have found their way into the current of the circulation, and are foreign to the composition of the blood, are eliminated from it.

347. It is obvious that, when an animal has retained its usual weight for any length of time without change, the total weight of its excretions must be equivalent to the total weight of the solids and fluids it has taken-in. If these last have been no more in amount than was absolutely necessary for the maintenance of the body during that period, all the azotized portion of the food was first appropriated to the formation of the azotized tissues; whilst the non-azotized portion was used-up in maintaining the respiration ( $\$ 157)$. Consequently, no part of the food would pass at once into the biliary and urinary excretions; and these would have no other function than to 
separate or strain-off, as it were, the products of the decomposition of the tissues formed from it, when their term of life had expired ( $\$ 161)$. But it is certain that Man (as well as other animals which have in some degree learned his habits) frequently consumes much more food than is necessary for the supply of his wants ; and a little consideration will show, that the surplus must pass-off by these excretions, without ever forming part of the living fabric. For new muscular tissue is not formed in proportion to the quantity of aliment supplied, but in proportion to the demand created by the exercise of it ( $\$ 587)$; consequently, if more food be taken-in than is necessary to supply that demand, no use can be made of it. We never find that a Man becomes more fleshy by eating a great deal and taking little exercise ; indeed, the very contrary result happens, his flesh giving place to fat. But let him put his muscles to regular and vigorous exercise, and eat as much as his appetite demands, and they will then increase both in strength and bulk.

348. Hence, if more azotized food be taken-in, than is required to supply the waste of the muscular and other azotized tissues, the surplus must be carried-off by the organs of excretion-chiefly, indeed almost entirely, by the Kidneys. By throwing upon them more than their proper duty, they become disordered and unable to perform their functions; hence the materials which they ought to separate from the blood accumulate in it, and give rise to various diseases of a more or less serious character, which might have been almost certainly prevented by due regulation of the diet. The most common of these diseases among the higher classes are Gout and Gravel; both of these may be often traced to the same cause, - the accumulation in the blood of lithic acid, which results from the decomposition of the superfluous azotized food, and which the kidneys are not able to throw-off in the proper state, that is, dissolved in water. That these diseases are, comparatively speaking, rare among the lower classes, is at once accounted-for by the fact, that they do not take-in any superfluous azotized food;-all that they consume being appropriated to the maintenance of their tissues, and the kidneys having only to discharge their proper function of removing from the blood the products of the decomposition of these. 
349. Hence the radical cure of these diseases, in most persons who have a sufficiently vigorous constitution and firm resolution to adopt it, is abstinence from all azotized nutriment-whether contained in animal flesh, bread, or other articles of vegetable diet,- - save such as is required to supply the wants of the system. If such abstinence be carried too far, however, it will produce injurious instead of beneficial results, weakening the fabric, and impairing the digestive powers ; and if food be employed of a kind which is liable to produce lactic acid (the acid that appears in milk, when it turns sour), much disorder maystill remain, which must be avoided by using the kind of diet that is least liable to undergo this change.

350. Again, if more non-azotized food is taken into the system than can be got rid of by Respiration, it must either be deposited as fat, or it must be separated from the blood, and carried-off by the excretion of the Liver. But if too much work be thrown upon this organ, its function becomes disordered, from its inability to separate from the blood all that it should draw-off ; the injurious substances accumulate in the blood, therefore, producing various symptoms that are known under the general term of bilious; and to get rid of these, it becomes necessary to administer medicines (especially those of a mercurial character). which shall excite the liver to increased secretion. The constant use of these medicines has a very pernicious effect upon the constitution; and careful attention to the regulation of the diet, and especially the avoidance of a superfluity of oily or farinaceous matter, will generally answer the same end in a much better manner.

351 . That the materials of the Biliary and Urinary excretions pre-exist (like the carbonic acid thrown-off by respiration) in the blood, in forms which, if not identical, are at any rate closely allied to those under which they present themselves in the bile and urine, has now been fully proved. The quantity of them present in the circulating fluid, however, is usually very small; for the simple and obvious reason that, if the excreting organs are in a state of healthy activity, these substances are drawn-off by them from the blood, as fast as they are introduced into it. But if the excretions be checked, they speedily accumulate in the blood, to such a degree as to be easily detected by the Chemist, and also to make their presence evident by their effects upon the animal functions, 
especially those of the nervous system. This sometimes happens in consequence of disease, and it may be imitated by experiment; for when the trunk of the blood-vessel conveying the blood to the liver or kidney is tied, the excretion is necessarily checked, and the same results take place as when the stoppage has depended on want of secreting power. The biliary and urinary matters have the effect of narcotic poisons upon the brain; when they have accumulated in the blood, their symptoms begin to manifest themselves; and these symptoms increase in intensity, as the amount of the substances becomes augmented, until death takes place.

352. Besides the Excretions, we find various Secretions elaborated in different parts of the bodies of animals, with a view not so much to the purification of their blood, as to the fulfilment of special purposes in their economy. These vary considerably in the different classes of animals; though some of them, being concerned in functions almost universally performed, are equally general in their range. Thus we find the Salivary and Gastric fluids poured into the mouth and stomach, for the reduction and solution of the food ( $\$ \$ 190$ and 204); and the Lachrymal secretion poured out upon the surface of the eye, for the purpose of washing it from impurities ( $\$ 541$ ): while the secretion of Milk for the nourishment of the young is limited to Mammals; and poisonous secretions are formed in Serpents and Insects, for the destruction of their prey or for means of defence. Any one of these may be checked, without rendering the blood impure by the accumulation of any substances that should be drawn-off from it; but its cessation may produce effects fully as injurious, by disordering the function to which it is subservient. Thus, if the salivary and gastric secretions were to cease, the reduction of the food could not be effected, and the animal must starve, though its stomach were filled with wholesome aliment.-It is to be observed, in regard to nearly all these secreted fluids, that they contain but a small quantity of solid matter, and that this matter seems to be formed from the albumen of the blood by a process of incipient decomposition, which gives it the character of a "ferment."

353. The various acts of Secretion and Excretion which are continually taking place in the living body, are, like those of Nutrition, completely removed from the influence of the will; 
but they are strongly affected by emotions of the mind. This has been already pointed out in regard to the Saliva ( $\$ 190)$; and it is equally evident in the case of the Lachrymal secretion (\$ 541). In these instances, however, the effect of the emotion is manifested upon the quantity only of the secretion; in the case of the secretion of Milk, not only the quantity but quality is greatly influenced by the mental state of the nurse. The more even her temper, and the more free from anxiety her mind, the better adapted will be her milk for the nourishment of her offspring. There are several instances now on record, in which it has been clearly shown, that the influence of violent passions in the mother has been so strongly exerted upon the secretion of milk, as almost instantaneously to communicate to it an absolutely poisonous character, which has occasioned the immediate death of the child. ${ }^{1}$ The influence of emotional states upon the Secretions is probably communicated by the Sympathetic system of Nerves ( $\$ 461)$, which is very minutely distributed, with the blood-vessels, to the several glands which form them.

\section{Nature of the Secreting Process.-Structure of the Secreting Organs.}

354. Notwithstanding the different characters of the products of Secretion and Excretion, and the variety of the purposes to which they are destined to be applied, the mode in which they are elaborated or separated from the blood is essentially the same in all. The process is performed, in the Animal, as in the Plant, by the agency of cells; and the variety of the structure of the different Glands, or secreting organs, has reference merely to the manner in which these, their essential parts, are arranged. The simplest condition of a secreting cell, in the animal body, is that in which it exists in Adipose or fatty tissue; which is composed, as formerly explained ( $\$ 46)$, of a mass of cells, bound together by areolar tissue that allows the blood-vessels to gain access to them. Every one of these cells has the power of secreting or separating fatty matter from the blood; and the secreted product remains stored-up in its cavity, as in Plants (VEGET.

I See the Author's Principles of Human Physiology, chap. Xv.; and Dr. A. Combe on the Management of Infancy, chap. $x$. 
PHYs. § 324)-not being poured forth, as it is in most other cases, by the subsequent bursting of the cell.

355. But when the secreting cells are disposed on the surface of a membrane, instead of being aggregated in a mass, it is obvious that, if they burst or dissolve-away, their contents will be poured into the cavity bounded by that membrane; and this is the mode in which secretion ordinarily takes place. Thus, the Mucous Membranes (\$ 39) are covered with epithelium-cells, which are continually being cast-off, and which are replaced as constantly by a fresh crop; and they form by their dissolution the glairy viscid substance termed mucus, which covers the whole surface of the membrane, and serves for its protection. In parts of the membrane where it is necessary that the secretion should be peculiarly abundant, we find its secreting surface greatly increased, by being prolonged into vast numbers of little pits or bags, termed follicles, which are lined with epithelium-cells, that resemble those of its general surface (see fig. 9). Such follicles are very abundant along the whole alimentary canal of Man; and the glandulæ in which the Gastric and Intestinal fluids are elaborated, are almost equally simple in their structure (\$ 204).

356. Now although the most important Secretions and Excretions are separated, in Man and the higher animals, by organs of a much more complex nature, yet in the lower we find them generated after the same simple fashion. Thus in the little Bowerbankia ( $\$$ $115)$, the bile is secreted by minute follicles which are lodged in the walls of the stomach (fig. 64, c) and pour their secretion separately into its cavity, having no communication with one another. In more complex forms of glan-

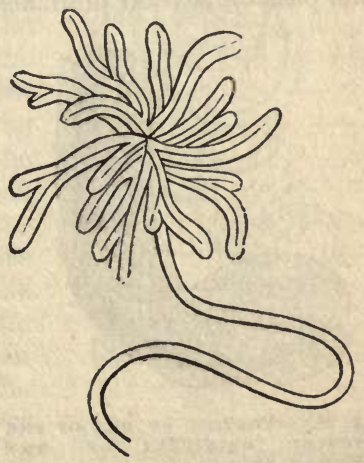

Fig. 164.-GLANDTHAT SECRETES THE ACRID FLUID DISCHARGED BY THE BOMBARDIER BEETLE. dular structure, however, several follicles open together into a tube, which discharges the product of their secretion 
(fig. 164); and thus the entire mass may be composed of numerous lobules, each having its own duct. Passing to still higher forms, we find all the ducts coalescing into a common trunk, so that the gland bears a strong resemblance to a bunch of grapes ; as is seen in fig. 165, which represents the structure

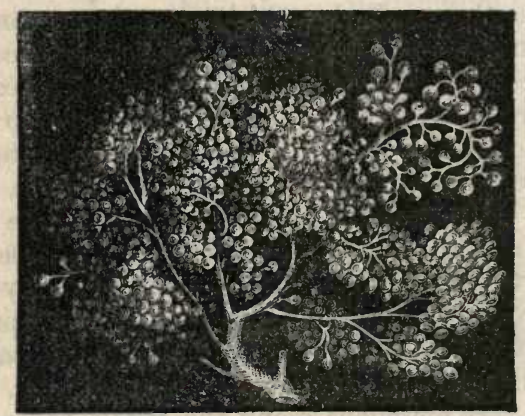

Fig. 165.-Ixtimate structure of a Composite Giann (the Parotid).

of the Parotid (one of the salivary glands) of Man. The main stalk is the duct into which all the others enter; from this pass off several branches, and these again give off smaller twigs, the extremities of which

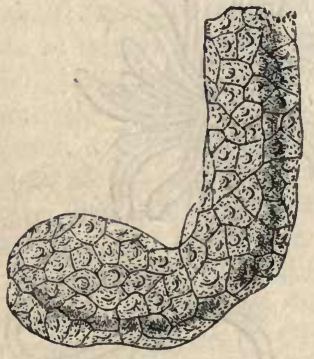

Fig. 166. - PORTION OP ONE OF THE TUBULT URINIFERI OF THE HUMAN KINNEY;

Showing its lining of flattened epithelium cells. enter the minute follicles in which the secretion is formed. These follicles are lined, as in their simple condition, with cells, which are the essential instruments in the production of the secretion; the fluid which they separate is poured, by the giving-way of their walls, into the small canals proceeding from the follicles, thence into the larger branches, and finally into the main trunk, by which it is carried into the situation where it is to be employed or from which it is to pass out. The Liver will be seen to possess a structure exactly resembling this, in the 
Crustacea, by referring to fig. 47 , fo ; and in the Mollusca it is nearly the same (figs. $157, l$, and $149, f$ ).

357 . The required extent of secreting surface is not unfrequently given, however, by the prolongation of the follicles into tubes, rather than by a great multiplication in their number. Of this we have a remarkable example in the Kidney of the higher animals ( $\$ 368$ ), which is entirely composed of such tubes, together with areolar tissue which binds them together, and the blood-vessels distributed amongst them. These tubes, like the follicles, are lined with epithelium-cells (fig. 166), which are the real instruments in the separation of their secreted product.

358. That there is nothing in the form of any secreting apparatus, however, which determines the peculiar nature of its secretion, is evident from this fact, - that, in glancing through the Animal series, we find the same secretion elaborated by glandular structures of every variety of form. Thus, we have seen that the bile is secreted, in the lowest animals in which we can distinguish it, by a number of distinct follicles, as simple in their structure as are those by which the mucous secretions are formed in the highest. Again, the bile is secreted in Insects, by a small number of long tubes, which open separately into the intestinal canal just below the stomach (fig. 112); and these tubes apparently differ in no respect from those that form the urinary secretion in the same animals, which open nearer the outlet of the intestinal canal. In fact, the distinct function of the latter was not known, until it was ascertained that uric acid is to be found in them. In fig. 167, which

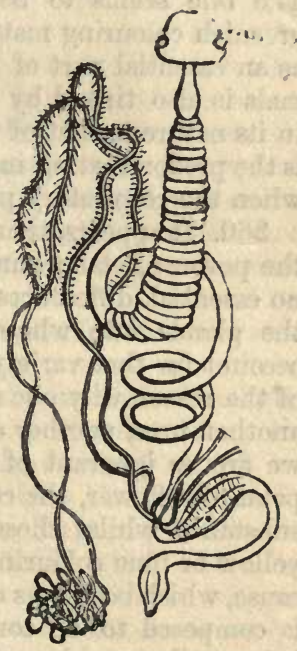

Fig. 167.

Altmentary Canal and HEPATIC TUBULES OF COCX: CHAFER.

represents the digestive apparatus of the Cockchafer, it is seen that the biliary vessels are only four in number, but are very long; and that, for a good part of their length, 
they are beset with a series of short tubes opening from them, by which the extent of secreting surface is much increased.-On the other hand, although the urinary secretion is generally formed by long tubes, yet in the Mollusca it is secreted by follicles, according to the general plan of their glandular structures.

359 . The secreting cells not unfrequently possess the potwer of elaborating a peculiar colouring matter, either separately, or along with the substances which seem more characteristic of the secretion. Thus the ink of the Cuttle-fish is in reality its urine, charged with a quantity of black matter formed in the pigment-cells (resembling those of the interior of the eye, §533) that line its ink-bag; and the corresponding secretion in other Mollusca is rendered purple by the same cause. The bile seems to be universally tinged with a yellow or greenish colouring matter, which may be regarded, therefore, as an essential part of the secretion; and the urine of Mammals is also tinged by a yellow pigment, which seems related in its nature to that of the bile. In all these pigments, carbon is the predominating ingredient ; and their amount is increased when the respiratory process is insufficiently performed.

360. It appears, then, that the different secreting cells have the power of elaborating a great variety of products ; and that no essential differences can be discovered in the structure of the glands into whose composition they enter, which can account for that variety. We are entirely ignorant, therefore, of the reason why one set of cells should secrete biliary matter, another urea, another a colouring substance, and so on ; but we are as ignorant of the reason why, in the parti-coloured petal of a flower, the cells of one portion should secrete a red substance, whilst those in immediate contact with it form a yellow or blue colouring matter; and we know as little of the cause, which occasions one set of the cells of which the embryo is composed to be converted into muscular tissue, another into cartilage, and so on.

361. One of the most curious points in the Physiology of Secretion, is the interchange which sometimes occurs in the functions of particular glands. When the operation of some one gland is checked or impaired by disease, it not unfrequently happens that another gland, or perhaps only a secreting surface, will perform its functions more or less perfectly; this 
happens most frequently in regard to the important Excretions, as if Nature had especially provided for their continued separation from the blood, that its purity may be unceasingly maintained. Thus the urinary secretion has been passed off from the surfaces of the skin, stomach, intestines, and nasal cavity, and also from the mammary gland; the colouring matter of the bile, when it accumulates in the blood (as in jaundice), is separated from it in the skin and conjunctival membrane of the eye ( $\$ 537)$; and milk has been poured forth from pustules on the skin, and from the salivary glands, kidneys, \&c. Such cases have been regarded as fabulous; but they rest upon good authority, and they are quite consistent with physiological principles.

362. Some of the main ducts or channels, through which the glands pour forth their secretions, are provided with enlargements or receptacles, which serve to retain and store up the fluid for a time, until it nay be desirable or convenient that it should be discharged. Thus, in most of the higher animals, the duct which conveys into the intestinal tube the bile secreted by the liver, is also connected with a receptacle termed the gall-bladder; the bile, as it is secreted, passes into this, and is retained there until it is

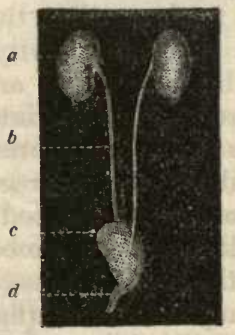

Fig. 168.-URINARY APPARATUS.

$a$, kidneys ; $b$, ureters; $c$, bladder; $d$, its canal, the urethra. wanted for assisting in the digestive process ( $\$ 213)$; when it is pressed out into the intestinal canal. It is a curious fact, that in most persons who die of starvation, the gall-bladder is found distended with bile; showing that the secretion has continued, although it has not been poured into the intestine for want of the stimulus occasioned by the presence of food in the latter. In many quadrupeds, especially those of the Ruminant tribe, the milk-ducts are in like manner dilated into a large receptacle, the udder, which retains the secretion as it is formed, until the period when it is needed. In all Mammals, and in some Reptiles, Mollusks, and Insects, but not in Birds or Fishes, we find the ureters, which convey away the urinary excretion from the kidneys, dilated at their lower extremity into a 
bladder (fig. 168), which serves to retain all the fluid that is poured forth by the gland during a considerable length of time, and thus prevents the necessity for its being continually passed out of the body.

\section{Characters of Particular Secretions.}

363. In nearly all animals, the Liver holds the first rank among Glands or secreting organs, in regard both to its size and to the obvious importance of its function. The principal varieties of its plan of structure in the Invertebrated classes having been already noticed ( $\$ 356)$, we shall here limit ourselves to a sketch of that peculiar arrangement of its elementary parts, which presents itself in Man and other Vertebrata. The position of this organ in the abdominal cavity is shown in fig. 30 . It is chiefly composed of a mass of cells of a flattened spheroidal form (fig. 169, B), the diameter of which is usually from 1-800th to 1-1600th of an inch; each cell presents a distinct nucleus, which is surrounded by yellow biliary matter in a finely granular condition; and in the midst of this there are usually one or two large fatty globules, or five or six small ones. The quantity of fat in the liver is very liable to increase, however, when there is a large amount of oily or fatty matter in the food, or when the respiratory function is not performed with sufficient activity.

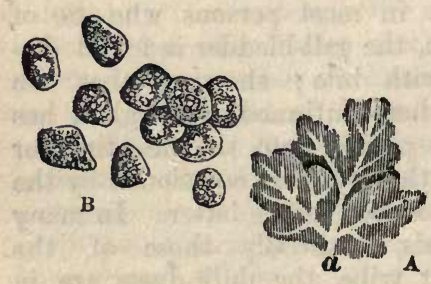

Fig. 169.-Portion of THE HUMAN Liver.

$\Delta$, Showing the manner in which the substance of its lobules is disposed around the branches of the hepatic vein $a$; B, cells of which the lobules are composed, more highly magnified.
The hepatic cells are clustered together into lobules of irregular form, but about the average size of a millet-seed; these lobules are disposed upon the ramifications of the hepatic vein (fig. $169, \mathrm{~A})$, like leaves upon the branches of a tree; and they are separated from one another by the peculiar distribution of the "portal" vessels and of the hepatic ducts. The Vena Portoe, it will be remembered, collects the blood that has been distributed to the alimentary canal, and conveys 
it to the liver, through which it is distributed by the subdivisions of this vessel, which acts the part of an artery ( $\$ 267)$. Its branches proceed to the surfaces of the lobules, amidst which they form by mutual inosculation a tolerably regular network (fig. 170, $b, b, b$ ); and from these branches a

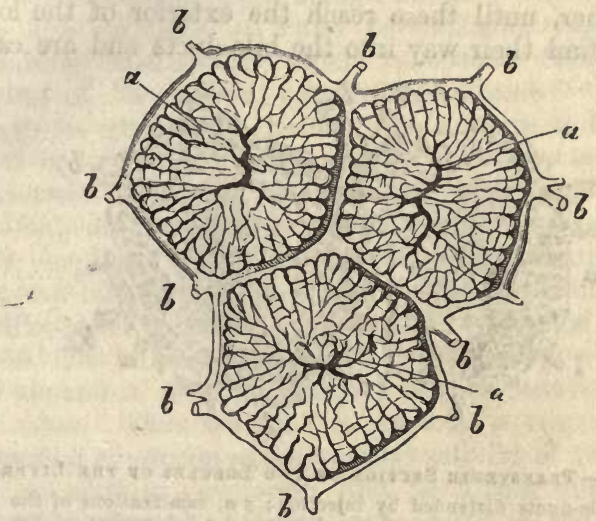

Fig. 170.-TRANSERSE SEctioN OF THRE LOBULES OP THE LTVER;

Showing the passage of the ramifications of the portal vessels from the network $b \quad b \quad b$, which surrounds the lobules, towards the centre of each lobule, near which they become continuous with the rootlets $a a a$ of the hepatic veins.

set of capillary twigs proceeds inwards towards the centre of each lobule, traversing in their course its aggregation of secreting cells. These capillaries finally terminate in the rootlets of the hepatic veins, which diverge from the centre of each lobule (fig. 170, $a, a, a$ ), and which collect the blood that has traversed its capillary system, to transmit it through larger trunks into the Vena Cava ( $\$ 266$ ), and thence to the heart. The liver is also supplied with arterial blood by the Hepatic artery; but this seems to have for its function rather to nourish the solid tissues of the organ, than to supply the materials for secretion. The bile-ducts, which convey away the fluid that is elaborated by the hepatic cells, appear to form a network which surrounds the lobules, connecting them together and sending branches towards the interior of each (fig. 171). It is still doubtful, however, whether they extend through the entire substance of the lobules, and whether the 
hepatic cells are really included within their extensions (as they are within the tubes or follicles of the liver of Invertebrata); or whether the cells lie outside the bile-ducts, in immediate contact with the capillary blood-vessels that traverse the lobule, filling up the entire space not occupied by them, and transmitting the products of their secretion from one to another, until these reach the exterior of the lobule, where they find their way into the bile-ducts and are carried

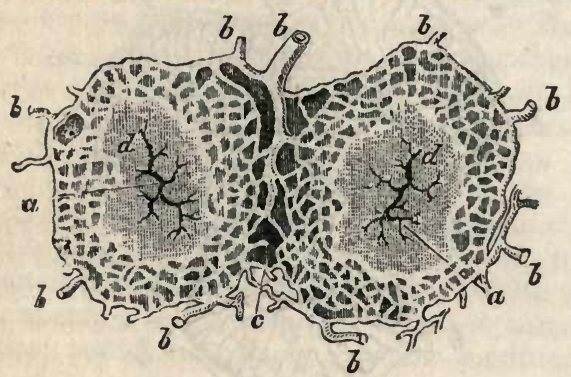

Fig. 171.-Transverse Section of two Lobuxes of THE Liver;

Showing the bile-ducts distended by injection; $a \boldsymbol{a}$, ramifications of the hepatic vein, occupying the centres of the lobules; $b \quad b \quad b$, branches of the hepatic ducts, which are largest in the space $c$, between the lobules, and which pass towards the centre through $d d$, the substance of the lobules.

off by them. - The bile may flow directly, as it is secreted, into the intestinal tube ( $\$ 213)$; but if digestion be not going on, so that its presence there is not required, it regurgitates into the gall-bladder (fig. 30 ), which stores it up until it is needed. In this reservoir it undergoes a certain degree of concentration by the removal of its watery part.

364. Bile is a yellowish (sometimes a greenish-yellow) fluid, somewhat viscid and oily-looking, and having a very bitter taste, followed by a sweetish after-taste. It is readily miscible with water, its solution frothing like one of soap; and it has the power, in common with soap, of dissolving oily matters; so that ox-gall is not unfrequently used to remove grease-spots from woollen stuffs. The basis of the principal ingredient of biliary matter, which constitutes about 5 parts in 100 of the secretion, is a fatty or resinoid acid which is termed the Cholic; this consists of 49 Carbon, 39 Hydrogen, and 9 Oxygen; and it forms, by "conjugation" with glycine (a 
sugary substance that is derivable from the decomposition of gelatin and albumen) and with taurine (a substance distinguished for the large proportion of sulphur it contains, -no less than 25 per cent), two other acids, the Glycocholic and the Taurocholic, which are mingled in different proportions in the bile of different animals, both being combined with soda as a base. Bile also contains a white crystallizable fatty substance resembling spermaceti, which is termed Cholesterin; this consists of 36 Carbon, 32 Hydrogen, and 1 Oxygen; and though its quantity in healthy bile appears to be very small, yet it occasionally increases to such an extent as to form the concretions known as "gall-stones," which, getting into the bile-duct, are transmitted along it with great pain and difficulty, or block it up altogether. The peculiar colouring matter of bile is quite distinct from the preceding substances; but like them it is extremely rich in carbon and hydrogen.

365. The bulk of the Liver, and the activity of the Respiratory apparatus, seem generally to bear an inverse ratio one to the other. Thus we find in Insects, a respiratory system possessing enormous extension and activity of function, and a liver so slightly developed, that for a long time it was not recognised as such. On the other hand, in the Mollusca, we find the respiration carried-on upon a lower plan, and with far less activity; but the liver is of enormous size, often making up a large part of the bulk of the body. Moreover, in the Crustacea, which are formed upon the same general plan with Insects, but which have an aquatic and therefore less energetic respiration, we find the liver very large, as in the Mollusca. In Reptiles and Fishes, again, whose respiration and temperature are low, the liver is comparatively larger than in Birds and Mammals, in which classes the respiration is more energetic, and the blood warm. In all these instances, however, the bulk of the liver depends in great part upon the accumulation of fat in its cells; and the secreting activity may be positively less in them, than it is in animals which have a comparatively small biliary apparatus.

366. The materials of the secretion of Bile are probably derived in part from the disintegration of the tissues, and in part more directly from the food. It is an interesting fact that the composition of bile and urine, taken together, corresponds closely with the composition of the blood; so that it $\times 2$ 
would appear as if the nutritive materials, in their ultimate metamorphosis, resolved themselves chiefly into these two excretory products. The greater part of the biliary matter poured into the intestinal canal seems to be ordinarily reabsorbed with the fatty matter of the food, and to be, like it, carried out of the system through the lungs in the form of carbonic acid and water; it being only when the bile has either been formed in excessive amount, or has been propelled along the intestinal tube with undue activity, that it is discharged in any quantity from the rectum, as in bilious diarrhoea. - The secreting action of the Liver, however, is by no means its sole mode of influencing the composition of the blood; for it has been shown by the recent researches of M. Bernard, that the blood which leaves the liver by the hepatic vein contains a peculiar substance of a saccharine nature, ${ }^{1}$ which does not exist in the blood brought to the organ by the portal vein. This substance appears to be elaborated by the converting power of the liver, either from materials supplied by the food, or from the products of the waste of the system; and it seems to be specially destined as a pabulum or fuel for the combustive process, being usually eliminated from the blood in the form of carbonic acid and water during its passage through the lungs, so as not to pass into the systemic circulation unless either its quantity be unusually great, or its elimination be interfered with by imperfect respiration. The liver seems also to form a peculiar fat, which is usually consumed in the same manner; but if the respiratory process be feeble, this fat accumulates in the cells of the liver itself.

367. The Urinary excretion has for its chief purpose to throw off those products, formed in a similar manner, which are highly charged with azote. The most important of its ingredients, in Man and the Mammalia, is the substance termed Urea, which has a crystalline form, and is very soluble in water. It contains 2 equivalents of Carbon, 4 of Hydrogen, 2 of Azote, and 2 of Oxygen; and it will be seen, by referring to the statement formerly given of the composition of albumen

1 This substance is spoken of by M. Bernard as sugar : it has been demonstrated, however, by the recent researches of Dr. Pavy, that the liver does not form sugar, but a substance that becomes sugar almost immediately upon contact with albuminous matters. 
(§ 13) and gelatin ( $\$ 19)$, that the amount of azote in proportion to that of the other elements is much greater in urea than it is in these substances, which form the materials of the animal tissues. The quantity of Urea which is daily excreted is very considerable, the average in an adult being about an ounce, and in a child of eight years old about half as much.-There is another compound which does not usually exist in large amount in the urine of the Mammalia, but which makes up a considerable part of the solid matter of this secretion in Birds and the lower Vertebrata; this is uric or lithic acid, which consists of 10 equivalents of Carbon, 4 of Hydrogen, 4 of Azote, and 6 of Oxygen. It is almost entirely insoluble in water, unless it be combined with soda or ammonia ; and in this state it ordinarily exists. When formed in too large quantity, however, it may be deposited in an insoluble form, constituting gravel ( $\$ 348$ ); and the same effect may result from the presence of some other acid, which, combining with the ammonia, precipitates or sets free the lithic acid. In the urine of herbivorous animals, uric acid is replaced by Hippuric acid, which contains a much larger proportion of carbon, its composition being 18 Carbon, 8 Hydrogen, 1 Nitrogen, and 5 Oxygen. Urine also contains a considerable quantity of Saline matter; part of which consists of what has been introduced into the system in the same form, and has to be got rid of as superfluous; whilst another part results from the conversion of the sulphur and phosphorus of the food into sulphuric and phosphoric acids by union with atmospheric oxygen ( $\$ 343)$, and from the combination of these acids with alkaline bases furnished by the food. The amount of alkaline phosphates contained in the urine may be considered as in some degree a measure of the expenditure of nervous tissue; whilst that of alkaline sulphates has some relation to the expenditure of muscular substance.

368. The Kidney, by which the secretion of Urine is eliminated from the blood, is an organ whose structure in the higher animals is very peculiar, although in the lower it is a mere aggregation of tubes or of follicles. . If we make a vertical section of the kidney of Man or any of the higher Mammalia (fig. 172, $\mathrm{A}$ ), we find that it seems composed of two different substances, one surrounding the other; to the outer; $a$, the name of cortical (bark-like) substance has been given; 
whilst the inner, $b$, is termed medullary (or pith-like). In the cortical substance, no definite arrangement can be de-

$\boldsymbol{A}$

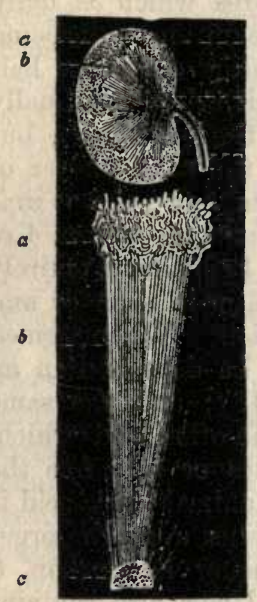
KIDNEY OF MAN.

A, vertical section of the kidney; $a$, cortical substance; $b$, tubular substance; c, calyX and pelvis ; $d$, ureter.

$B$, portion of the gland enlarged; $a$, extremity of the uriniferous tubes; $b$, straight portion; $c$, their termination in the calyx.
Fig. 172.-STRUCture of ThE

tected by the naked eye; it chiefly consists of a very intricate network of blood-vessels, surrounding the extremities of the tubes. But in the medullary substance we can trace a regular passage of minute tubes, from the circumference towards the centre. They commence in the midst of the network of blood-vessels (в, $a$ ), and then pass down in clusters, nearly in a straight direction, and slightly converging towards each other, until each cluster terminates in a little body, called the calyx or cup, which discharges the fluid it receives into the large cavity of the kidney, termed the pelvis or basin $(\mathrm{A}, \mathrm{C})$. From this it is conveyed away by the ureter $d$, which terminates in the bladder.

369. One of the most interesting circumstances in reference to the Urinary secretion, is the very large quantity of water which, in the higher animals, is got rid of through this channel, and the means by which it is drawn off. The kidneys seem to form a kind of regulating valve, by which the quantity of water in the system is kept to its proper amount. The exhalation from the Skin is liable to sustain great variations in its amount from the temperature of the air around; for when this is low, the exhalation is very much diminished; and when it is high, the quantity of fluid that passes off in this manner is increased ( $\$ 371)$. Hence, if there were not some other means of adjusting the quantity of fluid in the blood-vessels, it would be liable to continual and very injurious variation. This important function is performed by the kidneys, which allow such a quantity of water to pass into 
the urinary tubes, as may keep the pressure within the vessels very nearly at a uniform standard; an $\mathbf{3}_{2}$ a distinct and very curious provision exists for its separation. The extremity of many of the uriniferous tubes is made to include little knots or bunches of capillary vessels, which have extremely thin walls (fig. 173) ; and a vast number of such knots, which are termed "Malpighian bodies," after the name of their discoverer, are scattered through the cortical portion ( $(368)$ of the kidney. To these the blood brought to the organ by the renal artery is first conveyed; and the membranes that separate the interior of the capillary vessels from the cavity of the uriniferous tube, being of extreme thinness, water is

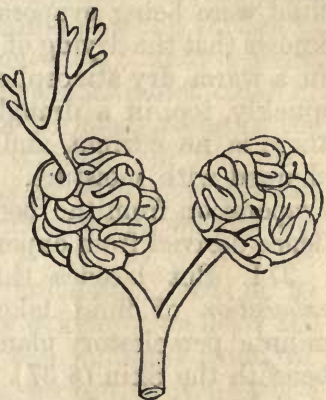

Fig. 173.-MALPIGHIAN BODTES OF THE KIDNEX. readily able to traverse them; and will do so in larger or smaller quantity, according as the pressure upon the walls of the capillaries is greater or less. The blood which has passed through these is next conducted to another set of capillaries, which form a network uyon the part of the tube that is lined by the secreting cells; and it is there subservient to the elaboration of the solid part of the secretion. Hence the quantity of water in the urinary secretion depends in part upon the amount exhaled from the skin,--being greatest when this is least, and vice versa, , - and in part upon the quantity which has been absorbed by the vessels. The quantity of solid matter in the secretion has but little to do with this ; for it depends upon the amount of waste of the muscular and other tissues that has been occasioned by their activity ( $\$ 367)$; and also upon the quantity of surplus aliment which has to be discharged through this channel, there being no other vent for it ( $\$ 348$ ).

370. Next to the excretions formed by the liver and the kidneys, that of the Skin probably ranks in importance. A large quantity of watery vapour is constantly passing-off from the whole surface of Man and other soft-skinned animals; 
and this amount is greatly increased under particular circumstances. A continual evaporation takes place from the surface of the skin, wherever it is not protected by hard scales or plates; and the amount of it will depend upon the warmth, dryness, and motion of the surrounding air, exactly as if the fluid were being evaporated from a damp cloth. Every one knows that the drying of a cloth is much more rapidly effected in a warm dry atmosphere, than in a cold moist one; more quickly, too, in a draught of air, than in a situation where there is no current, and where the air is consequently soon charged with moisture. That all these influences affect the evaporation from the bodies of Animals, there is ample evidence derived from experiment.

371. But besides this continual evaporation, a special exhalation of fluid takes place from the vast number of minute perspiratory glands imbedded in the fatty layer just beneath the Skin (\$ 37). Every one of these glandulæ, when straightened out, forms a tubule about a quarter of an inch in length ; and as it has been estimated that in a square inch of surface on the palm of the hand there are no fewer than 3528 of these glandulæ, the length of their tubing must be 882 inches or $73 \frac{1}{2}$ feet. The average number in other parts of the body may be estimated at about 2800 per square inch; and as the number of square inches of surface on a man of ordinary stature is about 2500 , the total number of perspiratory glandulæ must be not less than seven millions, and the length of their tubing nearly twenty-eight miles. The fluid which these perspiratory glands ordinarily exhale, is dissolved by the atmosphere, and carried off in the state of vapour, so as to pass away insensibly; but they are stimulated to increased action by the exposure of the body to heat, which causes them to pour forth their secretion in greater abundance than the air can carry off, and this consequently accumulates in drops upon the surface of the skin. The amount of perspiration may be considerably increased, without its becoming sensible, if the air be warm and dry, so as to carry off, in the form of vapour, the fluid which is poured out on the skin; but, on the other hand, a very slight increase in the ordinary amount immediately becomes sensible on a damp day, the air being already too much loaded with moisture to carry off this additional quantity. The distinction betyeen insensible and sensible 
perspiration, is not the same, therefore, with the difference between simple evaporation and exhalation from the skin; for a part of the latter is commonly insensible; and the degree in which it is so depends upon the amount of fluid exhaled, and the state of the surrounding atmosphere. If the fluid thus poured forth be allowed to remain upon the surface of the skin, it produces a very oppressing effect; most persons have experienced this, when walking in a mackintosh cloak or coat, on a damp day. The waterproof garment keeps in the perspiration, almost as effectually as it keeps out the rain; and consequently the air within it becomes loaded with fluid, and the skin remains in a most uncomfortable as well as prejudicial state of dampness.

372. The purpose of this watery exhalation, and of its increase under a high temperature, is evidently to keep the heat of the body as near as possible to a uniform standard. By the evaporation of fluid from the surface of the skin, a considerable quantity of heat is withdrawn from it, becoming latent (according to ordinary phraseology) in the change from fluid to vapour: of this we make use in applying cooling lotions to inflamed parts. The more rapid the evaporation, the greater is the amount of heat withdrawn in a given time; hence, if we pour, on separate parts of the back of the hand, small quantities of ether, alcohol, and water, we shall find that the spot from which the ether is evaporating feels the coldest, that which was covered by the alcohol less so, whilst the part moistened with water is comparatively but little chilled. The greater the amount of heat applied to the body, then, the more fluid is poured out by the perspiratory glands; and as the air can carry it off more readily in proportion to its own heat, the evaporation becomes more rapid, and its cooling effect more powerful. It is in this manner that the body is rendered capable of sustaining very high degrees of external heat, without suffering injury. Many instances are on record, of a heat of from $250^{\circ}$ to $280^{\circ}$ being endured in dry air for a considerable length of time, even by persons unaccustomed to a peculiarly high temperature; and individuals whose occupations are such as to require it, can sustain a much higher degree of heat, though perhaps not for any great length of time. Thus, the workmen of the late Sir F. Chantrey were accustomed to enter a furnace in which his 
moulds were dried, while the floor was red-hot, and a thermometer in the air stood at $350^{\circ}$; and Chabert, the "Fireking," was in the habit of entering an oven whose temperature was from $400^{\circ}$ to $600^{\circ}$. It is possible that these feats might be easily matched by many workmen, who are habitually exposed to high temperatures; such as those employed in iron-foundries, glass-houses, and gas-works.

373. That the power of sustaining a high temperature mainly depends upon the dryness of the atmosphere, is evident from what has just been stated; since, if the perspiration that is poured-forth upon the skin is not carried-off with sufficient rapidity, on account of the previous humidity of the air, the temperature of the body will not be sufficiently kept down. It has been found, from a considerable number of experiments, that when warm-blooded animals are placed in a hot atmosphere saturated with moisture, the temperature of their bodies is gradually raised $12^{\circ}$ or $13^{\circ}$ above the natural standard; and that the consequence is then inevitably fatal.

374. The amount of fluid exhaled from the skin and lungs ( $\$ 343)$ in twenty-four hours, probably averages about three or four pounds. The largest quantity ever noticed, except under extraordinary circumstances, was $5 \mathrm{lbs}$.; and the smallest, $1 \frac{2}{3} \mathrm{lbs}$. It contains a small quantity of solid animal matter, besides that of the other secretions of the skin which are mingled with it; and there is good reason to think that this excretion is of much importance, in carrying off certain substances which would be injurious if allowed to remain in the blood. That which is called the Hydrophatic system, proceeds upon the plan of increasing the cutaneous exhalation to a very large amount; and there seems much evidence, that certain deleterious matters, the presence of which in the blood gives rise to Gout, Rheumatism, \&c., are drawn off from it more speedily and certainly in this way, than in any other.

375. Besides the perspiratory glands, the skin contains others, which have special functions to perform. Thus in most parts which are liable to rub against each other, we find a considerable number of sebaceon follicles (fig. 8, $a a$ ), which secrete a fatty substance that keeps the skin soft and smooth. Besides these, the skin contains other follicles in particular parts, for secreting peculiar substances ; as, for instance, those which form the cerumen, a bitter waxy substance that is 
poured into the canal leading to the internal ear, for the purpose (it would seem) of preventing the entrance of insects.

376. The secretion of Milk is important, not so much to the parent who forms it, as to the offspring for whose nourishment it is destined. It does not seem to carry off from the system any injurious product of its decomposition ; for it bears a remarkable analogy to blood in the combination of substances which it contains; nevertheless it is found that, when this secretion is once fully established, it cannot be suddenly checked, without producing considerable disturbance of the general system. The structure of the Mammary gland closely resembles that of the parotid already described (fig. 165). It consists of a number of lobules, or small divisions, closely bound together by fibrous and areolar tissue ; to each of these proceeds a branch of the milk-ducts, together with numerous blood-vessels; and the ultimate ramifications of these ducts terminate in a multitude of little follicles, about the size (when distended with milk) of a hole pricked in paper by the point of a very fine pin.

377. The nature of the composition of Milk is made evident by the processes to which we commonly subject it. When it is allowed to stand for some time, its oleaginous part, forming the cream, rises to the top. This is still combined, however, with a certain quantity of albuminous matter, which forms a kind of envelope round each of the oil-globules; but in the process of churning, these envelopes are broken, and the oil-globules run together into a mass, forming butter. In ordinary butter a certain quantity of albuminous matter remains, which, from its tendency to decomposition, is liable to render the butter rancid; this may be got rid of by melting the butter at the temperature of $180^{\circ}$, when the albumen will fall to the bottom, leaving the butter pure and much less liable to change. In making cheese, we separate the albuminous portion, or casein, by adding an acid which coagulates it. The buttermilk and whey left behind after the separation of the other ingredients, contain a considerable quantity of sugar, and some saline matter. The proportion of these ingredients varies in different animals; and also in the same animal, according to the substances upon which it is fed, and the quantity of exercise it takes. The amount of casein seems to be greatest in the milk of the Cow, Goat, and Sheep; that of oleaginous matter in the 
milk of the Human female; and that of sugar in the milk of the Mare. The milk of the Cow, if a portion of its casein were removed, would resemble Human milk more nearly than any other; and it is therefore best for the nourishment of Infants, when the latter cannot be obtained. The important influence of Mental emotion on this secretion has already been noticed ( $\$ 353)$; and many more instances might be related, were not the ordinary facts in regard to it generally known.

\section{CHAPTER VIII.}

GENERAL REVIEW OF THE NUTRITIVE OPERATIONS-FORMATION OF THE TISSUES.

General Review of the Nutritive Operations.

378. Is the preceding Chapters (III. to v.) those processes have been described, by which the alimentary materials that constitute the raw material of the tissues, are converted into a fluid adapted for the Nutrition of the body; and we then (CHAPS. VI. and VII.) considered those functions, by which this fluid is kept free from the impurities it acquires during its circulation through the body, and is maintained in the state which alone can adapt it to the purposes it is destined to fulfil. These purposes may be regarded as fourfold. In the first place, the Blood is destined to supply the materials of the fabric of the body; which, as it is continually undergoing decay ( $\$ 68)$, requires the means of as constant a renovation. S'econdly, the Blood (in most animals at least) serves to convey to the tissues, the supply of oxygen which is required by them,--especially by the muscular and nervous tissues, - as a necessary stimulus to the performance of their functions. Thirdly, the Blood furnishes to the secreting organs the materials for the elaboration of the various fluids, which have special purposes to serve in the Animal economy,--such, for instance, as the Saliva, Gastric juice, Milk, \&c. And lastly, the Blood takes up, in the course of its circulation, the products of the waste or decomposition of the various tissues, which it conveys to the several organs, - the Lungs, Liver, 
Kidneys, \&c.,--destined to throw them off by Excretion. The greater number of these processes have already been treated of in more or less detail. Those included under the first head were considered, in a general form, in Chap. I. of this Treatiso. Those which are comprehended under the second head have been dwelt-on in Chaps. v. and VI.; and will be again noticed, when the actions of the Nervous and Muscular tissues are described. And the varied actions which are included under the third and fourth classes, have been discussed in the two Chapters which precede the present one. We have now to enter, in more detail, into the mode in which the circulating fluid is applied to the Nutrition and Formation of the Tissues.

\section{Formation of the Tissues.}

379. There is sufficient reason to believe that every living being is developed from a germ; no organized structure being able to take its origin (as some have supposed) in a chance combination of inorganic elements. All the facts relating to the production of Fungi and Animalcules, which have been imagined to favour this doctrine, may be satisfactorily explained in other ways (Veget. Phys. § 779 ; ZooL. \$ 1213). Now the first structure developed from this germ, in the Animal as in the Plant, is a simple cell; and the entire fabric subsequently formed, however complex and various in structure, may be considered as having had its origin in this cell. The cells of Animals, like those of Plants, multiply by the development of new cells within them; each of these becomes in its turn the parent of others; and thus, by a continuance of the same process, a mass consisting of any number may be produced from a single one. It is in this manner that the first development of the Animal embryo takes place, as will be shown hereafter (Chap. xv.). A globular mass, containing a large number of cells, is formed before any diversity of parts shows itself; and it is by the subsequent development, from this mass, of different sets of cells,-of which some are changed into cartilage, others into nerve, others into muscle, others into vessels, and so on,- that the several parts of the body are ultimately formed.

380. This process of differentiation is carried to very different degrees in the development of the several classes of 
animals ; for in some it is checked so early, that scarcely any distinction either of organs or of tissues ever shows itself; whilst in others it continues during a large proportion of the earlier period of life. It has na relation to growth, or simple increase of size; for this may take place by the multiplication of similar parts, giving rise, to that "vegetative repetition" which is so characteristic of the lower tribes of Animals, and which gives to many of them so strong a resemblance in general aspect to Plants; whilst, on the other hand, the developmental process by which higher forms of structure are evolved, sometimes takes place without any increase at all. It is in its degree of such differentiation, that what is called the lower or the higher organization of any living being essentially consists ; for whilst in the simplest forms of Animal structure every part is similar to every other, so that all the functions of life are performed in common by each, we find in Man (whose body may be regarded as presenting the highest type or example of this differentiating process) that no two parts are precisely similar, except those on the opposite sides of the body. This fact is occasionally manifested in a very singular manner, in the symmetry of disease; certain morbid poisons (as those of gout, and of several affections of the skin), which have a tendency to single out particular spots of the tissues whose nutrition they disturb, exhibiting their action in those parts of the two lateral halves of the body which precisely correspond with each other.

381. Now in the lowest grades of Animal structure, we find that the several tissues of the body can themselves appropriate from the products of digestion the nutrient materials they respectively require; so that, for their growth and maintenance, it is sufficient that these products should be carried into their neighbourbood by extensions of the digestive cavity (\$ 296). But in all the more highly-organized animals, it appears requisite that the nutrient material should pass through an intermediate stage of preparation, which is termed assimilation (or making-like); and this is effected by their introduction into the current of the circulation, and their mixture with the pre-existing blood, which, in virtue of its own vital powers, exerts upon them a converting action, which prepares them for being appropriated by the solid tissues.

382. When once the several forms of tissue have been 
developed, their nutrition is kept up by the supply of their respective materials which they derive from the blood. Each tissue draws from the circulating current that which it requires; and it is one of the most wonderful proofs of the skill with which the entire fabric has been planned and constructed, that the composition of the blood should be maintained at a nearly uniform standard, in spite of the continual change which is thus taking place in its actual components. It has been justly remarked, that each part of the body, by taking from the blood the peculiar substances which it needs for its own nutrition, does thereby act as an excretory organ, inasmuch as it removes from the blood that which, if retained in it, would be injurious to the nutrition of the body generally.

383. Hence it seems that such a mutual dependence must exist among the several parts and organs of the body, as eauses the evolution of one to supply the conditions requisite for the production of another; and this view is borne out by a great number of phenomena of very familiar occurrence, which show that a periodical change in one set of organs governs changes in others which at first sight might seem to have no relation to them. Thus the plumage of Birds, at the commencement of the breeding season, becomes (especially in the male) more highly coloured, besides being augmented by the growth of new feathers; but when the generative organs pass into their condition of periodical inactivity, the plumage begins at once to assume a paler and more sombre hue, and many of the feathers are usually cast, their nutrition being no longer kept up. So, again, it is no uncommon occurrence among Birds, for the female, after ceasing to lay, to assume the plumage of the male, and even to acquire other characteristic parts, as "spurs" in the fowl tribe. That, in these and similar instances, the development of organs is immediately determined by the presence or absence in the blood of the appropriate pabulum for the parts in question, and that its existence depends upon changes taking place in other parts, has been rendered still more probable by the results of experiments, which show that if the ordinary changes in one set of organs be prevented by their removal, those usually taking place in the others do not occur.

384. Though all the tissues derive the materials of their development from the blood which circulates in the vessels, 
yet there is considerable variety in the mode in which the supply is afforded; some tissues being furnished with blood much more copiously and directly than others, in consequence of the greater minuteness with which the capillaries are distributed through their substance. There are several, indeed, into which no blood-vessels enter, in their natural state; but which derive their nutriment by absorbing the liquor sanguinis that is brought into their neighbourhood. This is the case, for instance, with the Epidermis and Epithelium $(\$ \S 38,40)$; the cells of which are developed at the expense of the fluid which they absorb, through the basement membrane on which they lie, from the vessels of the skin or mucous membrane beneath it. In like manner, even the thick layer of Cartilage which covers the ends of most of the long bones, is destitute of blood-vessels ; and the small amount of nourishment it requires, is obtained by absorption from the vessels which surround it ( $\$ 47)$. This tissue undergoes very little change from time to time; and its growth takes place chiefly by addition of new matter to its surface ; consequently there is no necessity for any active circulation through its interior; and the transmission of nutritive fluid from one cell to another (as in the cellular tissue of Plants) is sufficient for its wants. Even in Bone, the blood-vessels are not very minutely distributed; for although there is a close network of capillary vessels on the membrane lining the Haversian canals and the cells of the cancellated structure ( $\$ 49)$, yet none of these pass into the actual substance of the bone. The simple Fibrous tissues are, for the most part, sparingly supplied with blood-vessels, as they are but little liable to decay or injury; though the Areolar tissue serves as the bed for the reception of the vessels which are on their passage to other tissues. Thus it is by its means that blood-vessels are conveyed into the Adipose tissue; for the ultimate elements of that tissue, namely, the fat-cells, are surrounded by capillary vessels, not entered by them. The same important purpose is answered by the areolar tissue that lies amongst the tubes which form the essential parts of the Nervous and Muscular tissues; for these tubes are not perforated by vessels, so that their contents must be nourished by fluid absorbed through their walls.

385. In no instance that we are acquainted with, in the 
higher animals at least, do the vessels directly pour the blood into any tissue for the purpose of nourishing it. Unless there have been an actual wound which has artificially opened the blood-vessels, no fluid can escape from them into the substance traversed by the capillaries, except by transuding the walls of the latter; and hence it would seem impossible that any of the floating cells contained in the blood can be deposited in the tissues and contribute to their development. The Liquor Sanguinis seems, therefore, to furnish all that is wanting for this purpose ; and it readily permeates the walls of the capillaries, the basement-membrane, and any other of the softer tissues, so as to arrive at the parts where it is to be applied. As it is withdrawn from the blood, it is continually being re-formed from the food; but if it be not supplied in sufficient quantity by the latter, the tissues are imperfectly nourished, and the strength of the body and the vigour of the mind are consequently alike impaired.

386. This imperfect nutrition seems to be the essential condition of one of the most destructive diseases to which the human frame is liable, - that commonly known as Consumption; which is, however, but one out of several diseases that may result from the same general defect of nutrition. If the liquor sanguinis be imperfectly elaborated, it is less fit to undergo organization; and, consequently, instead of being converted into living tissue, part of it is deposited, as an imperfectly organized mass, in the state known to the Medical man as Tubercle. Such deposits take place more frequently in the lungs than in any other part; and besides impeding the circulation and respiration, they produce irritation and inflammation, in the same manner as other substances imbedded in the tissues would do ; and so far from having, like many other aiseases, a natural tendency to cure, this malady, if unchecked, almost certainly leads to a fatal termination. Microscopic examination of tubercular matter shows that it consists of half-formed cells, fibres, \&c., together with a granular substance which seems to be little else than coagulated albumen. The only manner in which any curative means can be brought to bear upon this terrible scourge, is by attention to the constitutional state from which it results. This is sometimes hereditary; and is sometimes induced by insuffcient nutrition, obstructed respiration, habitual exposure to 
cold and damp, long-continued mental depression, \&c. The treatment of the Tubercular diathesis (as this state of constitution is termed) must be directed to the invigoration of the system by good food, active exercise, pure air, warm clothing, and cheerful occupation; and by the due employment of these means, at a sufficiently early period, many valuable lives may be saved which would have otherwise fallen a sacrifice. The value of cod-liver oil in the treatment of this disease, which is now a well-established fact, seems to depend upon the facility with which it is assimilated as a nutritive material. It is a remarkable fact that the inhabitants of Iceland, the greater part of whom live under conditions that might be expected to favour the development of tubercular disease, are singularly free from it; and the source of this exemption seems to consist in the very oleaginous nature of their diet. Consumption presents itself among the inhabitants of all climates; and the value of change to a patient who is affected with this malady, chiefly depends upon the opportunity which it affords him for abundant exercise in the open air, without injurious exposure to cold or damp.

387. From the foregoing facts it is evident, that the operations of Nutrition are due, on the one hand, to the independent properties of the several Tissues, which draw from the blood the materials of their continued growth and renewal; and, on the other, to the properties of the Blood, which supplies them with these materials. The blood, left to itself, could form no tissue more complex than a mere fibrous network: whilst, conversely, the various tissues of the body could not draw their nourishment directly from the products of digestion, and are consequently dependent upon the blood for their supply. We may illustrate the relation between the three states, - that of aliment, blood, and organized tissue,by comparing them with the three principal states which Cotton passes through in the progress of its manufacture,namely, the raw cotton, spun-yarn, and woven fabric. The spun-yarn could not of itself assume that particular arrangement which is given to it by the loom; and the loom could make nothing of the raw cotton, until it has been spun into yarn.

388. It is also evident, that the blood-vessels have no other purpose in the act of Nutrition, than to convey the circulating 
fluid into the neighbourhood of the part where it is to be employed ; and the blood, or at least its organizable portionthe liquor sanguinis - must quit the vessels before it can be employed in the development of new tissue. We might illusstrate this by the distribution of water-pipes through a city; they might pass into every house, nay, into every room, and yet the water must be drawn from the pipes before it can be applied to any required purpose. The spaces untraversed by vessels have been shown to be larger in some tissues, and smaller in others; the distribution of the capillaries being more minute, in proportion as the nutritive actions of the part go on more energetically. Now in the embryo, even of the most complex and perfect animals, there is a period when no blood-vessels exist, the whole mass being made-up of cells, every one of which lives for itself and by itself, absorbing nutriment from a common source, and not at all dependent upon its brethren. It is only when a diversity of structure begins to show itself,- - one part undergoing transformation into bone, another into muscle, and so on,--and when some portions of the fabric are cut-off from the direct supply of nourishment, - that vessels begin to show themselves. These are formed, like the ducts of Plants, by the breaking-down of the partitions between contiguous cells; they at first seem rather like passages or channels, than tubes with walls of their own; and this condition they retain in certain cases through life (§289).

\section{Repair of Injuries.}

389. Every animal possesses, in a greater or less degree, the power of not merely maintaining its organized fabric in its integrity, by the renewal of the parts which are from time to time passing into decay, but also of reproducing parts of that fabric which have been lost by disease or accident. This power seems greatest among the lowest tribes of Animals ; in many of which the entire organism can be reproduced from a small portion of it, as is the case with the Hydra (\$122), and with some species of Sea-Anemone (\$ 126). In the Star-fish, a far more highly-organized animal, the regenerative power is more limited, though it is still very remarkably manifested; for if one, two, or more of the rays be broken or cut off, they are gradually restored, provided the central disc be uninjured. 
Of certain kinds of Holothuria (fig. 67), which eject the entire mass of viscera under the influence of alarm, it has been observed that they not only continue to move about as if nothing had happened, but that, under favourable circumstances, they regenerate the whole of the digestive and reproductive apparatus thus parted-with.-Next to Zoophytes, there are no animals in which the regenerative power is known to manifest itself so strongly as the lower members of the Articulated series, such as the inferior Entozoa and the Turbellaria (ZooLOGY, \$ 924), among which last we find the Planaria almost rivalling the Hydra, although it is an animal of much more complex structure. The common Earthworm can reproduce either the head or any portion of the body of which it may have been deprived; but it cannot be multiplied by the division of its body into two or more parts (as asserted by some), since these parts, although they continue to move for a time, soon perish. There are Worms allied to it, however, in which the regenerative power is sufficient to produce the whole body from a separated fragment; and no fewer than twenty-six have thus been made to originate by the subdivision of a single individual. In the higher Articulata, such as Crustacea, Insects, and Spiders, the reparative capacity is limited to the restoration of limbs ; and even this would seem to be seldom preserved in perfect Insects, being restricted to the larval period of their lives. Little is known of the regenerative power possessed by the higher Mollusks; but it has been affirmed that the head of the Snail may be reproduced after being cut-off, provided the cephalic ganglion be not injured, and an adequate amount of heat be supplied.

390. It is only among the cold-blooded members of the Vertebrated series, that the reparative power extends to the renewal of entire organs; and this seems limited in Fishes to the reproduction of portions of the fins which have been lost by disease or accident. In Batrachia, however, it has been found that entire new legs, with perfect bones, nerves, muscles, \&c., may be reproduced after severe loss or injury of the original members; and even a perfect eye has been formed in place of one that had been removed. It is interesting to observe that the exercise of this reparative power essentially depends upon the temperature in which the animal is living; the reproduction of entire members apparently 
requiring a higher degree of the stimulus of Heat, than does their ordinary nutrition. In Lizards, an imperfect reproduction of the tail takes place when part of it has been broken off; but the newly-developed portion contains no perfect vertebræ, its centre being occupied by a cartilaginous column like that of the lowest fishes.-In the warm-blooded Vertebrata generally, the power of reproduction after loss or injury seems much more limited. We do not find that entire parts or members once destroyed, are completely renewed; though very extensive breaches of substance are often filled up. The tissues most readily reproduced are Bone, the Simple Fibres ( $\$ 22$ ), and the Membranes (such as the Skin, the Mucous and Serous membranes), of which these tissues form the basis. As a general rule, losses of substance in Glandular tissue, Muscle, and other parts of comparatively high organization, do not seem to be reproduced; but there is a curious exception to this in the case of Nervous tissue, which, with Blood-vessels, is very readily re-formed in the new growths by which losses of substance are repaired, as we often see in the rapid skinning-over of a large superficial wound. One of the most remarkable manifestations of reparative power in the Human body, is the re-formation of an entire bone, when the original one has been destroyed by disease. The new bony matter is thrown-out, sometimes within and sometimes around the dead shaft ; and when the latter has been removed, the new structure gradually assumes the regular form, and all the attachments of muscles, ligaments, \&c., become as complete as before. A much greater variety and complexity of actions are involved in this process, than in the reproduction of whole parts in the simpler animals; though its effects do not appear so striking. It appears that, in some individuals, this regenerating power is retained to a much greater degree than it is by the species at large; thus, there is a well-authenticated instance, in which a supernumerary thumb on a boy's hand was twice reproduced, after having been removed from the joint. In many cases in which the crystalline lens of the eye has been removed, in the operation for cataract, it has been afterwards regenerated; and there is evidence that, during embryonic life, the regeneration of lost parts may take place in a degree to which we have scarcely any parallel after birth; attempts being sometimes made at the re-formation of entire limbs, in 
place of such as are lost during the earlier periods of develop-. ment.

391. When an entirely new structure is to be formed,-as for the closure of a wound, the union of a broken bone, or the repair of any other injury, - the process is of a kind very much resembling the first development of the entire fabric. The neighbouring vessels pour out their liquor sanguinis, which is known to the Surgeon under the name of coagulable lymph; this fills up the open space, and forms a connecting medium between the separated parts. If this intervening layer be thin, the two sides of the wound may adhere so closely as to grow together without any perceptible interposition of new substance; this is what is called "healing by the first intention." But if the loss of substance has been too great to allow of such adhesion, the vacant space is filled by the gradual organization of the coagulable lymph; and this may take place in one of two very different modes, the determination being chiefly dependent on the condition of the wound as to seclusion from air or exposure to it.

392. The former of these conditions is by far the more favourable of the two; for the reparative material is usually developed gradually but surely into fibrous tissue, without any loss, and with very little irritation either in the part itself or in the system at large. This process seems to take place naturally in cold-blooded animals, even in open wounds; the contact of air not having that disturbing influence in them, which it exerts in warm-blooded animals. And Nature frequently endeavours to bring it about in the superficial wounds of warm-blooded animals, by the formation of a large scab, which protects the exposed surface; but this happens much less frequently in the Human subject than it does among the lower animals, the unnatural conditions in which a large proportion of the so-called civilised races habitually live (especially deficient purity of the air, continual excess in diet, and the frequent abuse of stimulants) being unfavourable to it. The performance of many operations which formerly left open wounds, in such a manner that the air may be effectually excluded by a valvular fold of skin, is one of the greatest improvements in modern Surgery.

393. In an open wound, on the other hand, which is healing by the process termed "granulation," the reparative 
material is rapidly developed into cells, amongst which bloodvessels speedily extend themselves. The formation of new. blood-ressels, in this and other cases, seems to commence in the giving-way of the walls of some of the previously-existing capillary loops, at particular spots, and in the escape of blood corpuscles in rows or files into the soft substance that surrounds them; thus channels or passages are excavated, which come into connexion with each other; and these channels, after a time, acquire proper walls, and become continuous with the vessels from which they originated,-to be in their turn the originators of a new series. The vitality of this new "granulation-tissue," however, is very low; and the part exposed to the air passes into the condition of pus (the yellow creamy fluid discharged from an open wound), which contains the same materials in a decomposing state. Thus there is a constant waste of organizable substance, the amount of which, in the case of an extensive wound, becomes a serious drain upon the system; at the same time, there is a much greater irritative disturbance both in the part itself and in the system generally; and the new tissue that is formed is of such low vitality that it subsequently wastes away, so as by its disappearance to leave a contracted cicatrix or scar.-The difference between the two modes of reparation now described is often one of life and death, especially in the case of large burns of the body in children.

\section{CHAPTER IX.}

ON THE EVOLUTION OF LIGHT, HEAT, AND ELECTRIOITY BY ANIMALS.

\section{Animal Luminousness}

394. A large proportion of the lower classes of aquatic Animals possess, in a greater or less degree, the power of emitting light. The phosphorescence of the sea, which has been observed in every zone, but more remarkably between the tropics, is due to this cause. When a vessel ploughs the ocean during the night, the waves-especially those in her wake, or those which have beaten against her sides-exhibit a diffused lustre, interspersed here and there by stars or ribands of more intense brilliancy. The uniform diffused 
light is chiefly emitted by innumerable minute animals, which abound in the waters of the surface; whilst the stars and ribands are due to larger animals, whose forms are thus brilliantly displayed. This interesting phenomenon, when it occurs on our own coasts, is chiefly produced by incalculable multitudes of a small creature, termed the Noctiluca, having a nearly globular form, and a size about equal to that of the head of a minute pin. When these cover the water, and a boat is rowed among them, every stroke of the oars produces a flash of light; and the ripple of the water upon the shore is marked by a brilliant line. If a person walk over sands that the tide has left, his footsteps will seem as if they had been impressed by some glowing body. And if a small quantity of the water be taken up and rubbed between the hands, they will remain luminous for some time. The transparency of the little animals to which these beautiful appearances are due, might cause them to be overlooked if they are not attentively sought; they somewhat resemble grains of boiled sago in their aspect, but are much softer. In the general simplicity of their structure, the Noctilucae appear to correspond rather with the Rhizopoda (§ 130) than with any other group; but they are distinguished by some remarkable peculiarities.

395. Of the larger luminous forms which are seen to float in the ocean-waters, a great proportion belong to the class Acalephac. The light emitted by these appears to be due to the peculiar chemical nature of the mucus secreted from their bodies; for this, when removed from them, retains its properties for some time, and may communicate them to water or milk, rendering those fluids luminous for some hours, particularly when they are warmed and agitated. It is probably from this source, that the diffused luminosity of the sea is partly derived. The secretion appears to be increased in amount, by anything that irritates or alarms the animals; and it is from this cause that the dashing of the waves against each other, the side of a ship, or the shore,- - or the tread of the foot upon the sand,- - or the compression of the animals between the fingers,-occasions a greater emission of light. But some of these causes may act, by bringing a fresh quantity of the phosphorescent secretion into contact with air, which seems necessary to maintain the kind of slow combustion on which the light depends. 
396. But the Noctilucæ and Acalephæ are by no means the only luminous animals which tenant the deep. Many Zoophytes appear to have this property in an inferior degree, and also some of the Echinodermata. Of the lowest class of Mollusks, the Tunicata, a very large proportion are luminous, especially those which float freely through the ocean, and which abound in the Mediterranean and tropical seas; the brilliancy of some of these can scarcely be surpassed. Among some of the Conchifera, also, the phenomenon has been observed; as well as in certain marine Annelida. Other marine animals of higher classes are possessed of similar properties; thus, many Crustacea, especially the minuter species, are known to emit light in brilliant jets; and the same may be said of a few Fishes: but it is probable that the luminosity attributed to many of the latter is due to the disturbance they make in the surrounding water, which excites its phosphorescence in the manner just explained. In all these, the general phenomena are analogous,-the luminous matter appearing to be a secretion from the surface of the animals, and to undergo a sort of slow combustion by combination with oxygen. Wherever it is presented by these animals, it is always most brilliant upon the surfaces concerned in respiration. The light continues for some days after death; but ceases at the commencement of putrefaction. ${ }^{1}$

397. In the class of INSECTs, there are several species which have considerable luminous power; and in these the emission of light is for the most part confined to a small part of the surface of the body, from which it issues with great brilliancy. The luminous Insects are most numerous among the Beetle tribe, and are nearly restricted to two families, the Elaterida, and the Lampyridoe. The former contains about 30 luminous species, which are known as fire-fies; these are all natives of the warmer parts of the New World. Their light proceeds from two minute but brilliant points, which are situated one on each side of the front of the thorax; and from another

1 There are certain cases, however, in which the production of Light, like that of Electricity ( $\$ 423$ ), appears to be a peculiar manifestation of Nervous power. There is strong reason to believe that Nerve-force may be directly metamorphosed (as it were) into these or other forms of physical and vital force, according to the principle of "Correlation" now generally admitted as regards the Physical Forces. 
beneath the hinder part of the thorax, which is only seen during flight. The light proceeding from these points is sufficiently intense to allow small print to be read in the profoundest darkness, if the insect be held in the fingers and be moved along the lines; and the natives of the countries where they are found (particularly in St. Domingo, where they are abundant) use them instead of candles in their houses, and tie them to their feet and heads, when travelling at night, to give light to their path through the forest. In all the luminous species of this family, the two sexes are equally phosphorescent.

398. The family Lampyridae contains about 200 species known to be luminous; the greater part of these are natives

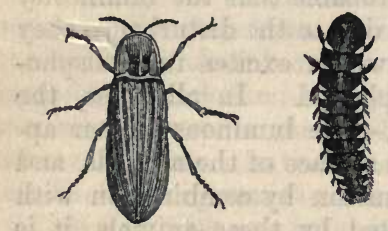

Fig. 174.-MALE AND FEMALE GLOWWORM. of America, whilst others are widely diffused through the old World. In most of these, the light is most strongly displayed by the female, which is usually destitute of wings, so that it might be mistaken for a larva. The species of our own country is known as the Glow-worm (fig. 174).

399. The light of the Glow-worm issues from the under surface of the last three abdominal rings. The luminous matter, which consists of little granules, is contained in minute sacs covered with a transparent horny lid; and this exhibits a number of flattened surfaces, so contrived as to diffuse the light in the most advantageous manner. The sacs are mostly composed of a close network of finely-divided airtubes ( $\$ 321$ ), which ramify through every part of the granular substance; and it appears that the access of air through these is a necessary condition of the phosphorescence. For if the aperture of the large trachea which supplies the luminous sac be closed, the light ceases; but if the sac be lifted from its place, without injuring the trachea, the light is not interrupted. All the luminous insects appear to have the power of extinguishing their light; and this they probably do when alarmed by approaching danger. The sudden extinction of the light is probably due to the animal's power of closing the aperture of the trachea. 
400. There are a few other Insects not included in these families, which are reputed to possess luminous powers; and of these the most remarkable are the Fulgorce, or Lanternflies (fig. 175); of which one species inhabits Guiana, whilst another is a native of China. These are insects of very remarkable form, having an extraordinary projection upon the head; and this is the part said to be luminous. The authority for the assertion, however, is doubtful;

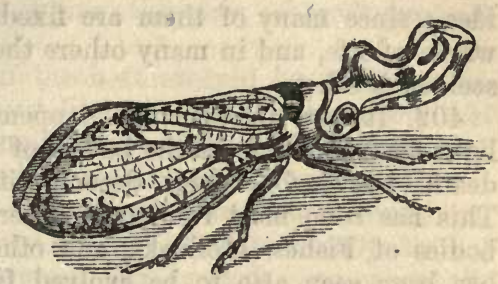

Fig. 175.-FuLgorA IANTERNARIA. and many Entomologists who have captured the insect, have denied the phosphorescent power imputed to it. But it is not impossible that the female only may possess it, and that it may only be manifested at one part of the year. One of the common English species of Centipede, which is found in dark, damp places, beneath stones, \&c., is slightly luminous; and the common Earthworm is also said to be so at the breeding season.

401. Of the particular objects of this provision in the Animal economy, little is known, and much has been conjectured. It is not requisite to suppose that its purposes are always the same; the circumstances of the different tribes which possess it being so different. The usual idea of its use in Insects, - that it enables the sexes of the nocturnal species to seek each other for the perpetuation of the race, - is probably the correct one. The light is more brilliant at the season of the exercise of the reproductive functions, than at any other; and is then exhibited by animals which do not manifest it at any other period. Moreover, it is well known that the male Glow-worm, - which ranges the air, whilst the female, being destitute of wings, is confined to the earth, -is attracted by any luminous object; as are also the Fire-flies, which may be most easily captured by carrying a torch or lantern into the open air: so that the poetical language in which this phosphorescence is described as "the lamp of love-the pharos - the telegraph of the night, which marks by its scintillations, in the silence of the night, the spot 
appointed for the lovers' rendezvous," would not seem so incorrect as the ideas of poets on subjects of Natural History too frequently are.-Regarding the uses of the luminosity of the lower marine tribes, it is more difficult to form a definite idea; since many of them are fixed to one spot during the whole of life, and in many others the sexes do not require to seek each other.

402. It not unfrequently happens, that an evolution of light takes place from the bodies of animals soon after their death, but before their decomposition has advanced far. This has been most frequently observed to proceed from the bodies of Fishes, Mollusks, and other marine tribes; but it has been seen also to be evolved from the surface of land animals, and even from the Human body. Indeed, some well-authenticated cases have been put on record, in which a considerable amount of light was given off from the faces of living individuals, who were near their end. All animal bodies contain a considerable quantity of phosphorus ( $\$ 166)$; and it is by no means impossible that some peculiar compound of this substance may be formed, during the early stages of decomposition, or even before death, which may, by its slow combustion, give rise to the luminous appearance. It appears that the whole substance of the body of the Fire-flies is phosphorescent ; for, according to an early historian of the West Indies, "many wanton wilde fellowes" rub their faces with the flesh of a killed fire-fly, "with purpose to meet their neighbours with a flaminge countenance."

\section{Animal Heat.}

403. One of the conditions necessary for the performance of Vital action, is a certain amount of warmth ; and we have seen that the animals which alone are capable of retaining their activity in the coldest extremes of temperature, are those which have the power of generating heat within themselves, and thus of keeping-up the temperature of their bodies to a high standard. Those which do not possess a power of this kind, are either rendered completely inactive, even by a comparatively moderate cold, or are altogether destroyed by it. Those which ordinarily do possess this power are destroyed even more rapidly by cold, if from any cause the production of heat within their bodies be interrupted; for they are the 
animals whose vital actions are the most varied and energetic, and in which an interruption to any one of them most speedily brings the rest to a stand. The inquiry into the amount of heat generated by different animals, and into the sources of its production, is one, therefore, of great practical importance.

404. Our knowledge of the heat evolved by the lower Invertebrated animals is very limited; but it is probable that in most of them the temperature of the body follows that of the element they inhabit, keeping a little above it for a time, if it be much lowered. Thus, when water containing Animalcules is frozen, they are not at once destroyed; but each lives for a time in a small uncongealed space, where the fluid seems to be kept from freezing by the heat liberated from its body. The temperature of Earthworms, Leeches, Snails, and Slugs, ascertained by introducing a thermometer into the midst of a heap of them, is usually about a degree or two above that of the atmosphere; and they also have the power of resisting for a time the influence of a degree of cold, which would otherwise immediately freeze their bodies.

405. In the cold-blooded Vertebrata, also, the heat of the body is almost entirely dependent upon that of the surrounding element. Thus most FisHes are incapable of maintaining a temperature more than two or three degrees higher than that of the water in which they live; and the warmth of their bodies consequently rises and falls with that of the sea, river, or lake they may inhabit. There are, however, a few marine Fishes which have the power of maintaining a temperature 10 or 12 degrees higher than that of the sea; and these are peculiar for the activity of their circulation, and for the deep colour of their blood, which possesses red particles ( $\$ 229$ ) enough to give to the muscles a dark red colour, like that of meat. The Thunny, a fish which abounds in the Mediterranean, where there are extensive fisheries for it, is one of this group.- It is to be remembered that the animals of this class are less liable to suffer from seasonal alternations of temperature, than are those which inhabit the air. In climates subject to the greatest atmospheric changes, the heat of the sea is comparatively uniform throughout the year, and that of deep lakes and rivers is but little altered. Many have the power of migrating from situations where they might other- 
wise have suffered from cold, into deep waters; and those species which are confined to shallow lakes and ponds, and which are thus liable to be frozen during the winter, are frequently endowed with sufficient tenacity of life, to enable them to recover after a process which is fatal to animals much lower in the scale. Fishes are occasionally found imbedded in the ice of the Arctic seas; and some of these have been known to revive when thawed.

406. In RePtiLes, the power of maintaining an uniform temperature is somewhat greater; being especially shown when the external temperature is reduced very low. Thus when the air is between $60^{\circ}$ and $70^{\circ}$, the body of a Reptile will be nearly of the same heat; but when the air is between $40^{\circ}$ and $50^{\circ}$, it may be several degrees higher. Frogs and other aquatic Reptiles have a remarkable power of sustaining a temperature above that of freezing, when the water around is not only congealed, but is cooled down far below the freezing-point. Thus in ice of $21^{\circ}$, the body of an edible frog has been found to be $37 \frac{1}{2}^{\circ}$; and even in ice of $9^{\circ}$, the animal has maintained a temperature of $33^{\circ}$. In these cases, as in Animalcules, the water in immediate contact with the body remains fluid, so long as the animal can generate heat; but at last it is congealed, and the body also is completely frozen. But it is certain that Frogs, like Fishes, may be brought to life again, after the fluids of their bodies have been so completely congealed that their limbs become quite brittle ; it is not known, however, whether this may happen with other Reptiles. It would appear that among Reptiles, as among Fishes, some of the more active species have the power of maintaining their bodies at a temperature considerably higher than that of the atmosphere; thus in some of the more agile of the Lizard tribes, the high temperature of $86^{\circ}$ has been noticed, when the external air was but $71^{\circ}$.

407. The only classes of animals in which a constantly elevated temperature is kept up, are Birds and Mammals. The bodily heat of the former varies from $100^{\circ}$ to $112^{\circ}$; the first being that of the Gull, the last that of the Swallow. In general we find that the temperature is the highest in species of rapid and powerful flight; and least in those which inhabit the earth. Birds that are much in the water have a special provision for retaining within their bodies the heat which 
would otherwise be too rapidly conducted away; their bodies being clothed with a thick and soft down, which is rendered impenetrable to fluid by an oily secretion applied with the bill. The temperature of MammaLs generally seems to range from $96^{\circ}$ to $104^{\circ}$; but that of the $\mathrm{Bat}$, and probably of other hybernating species, appears to be frequently much below the lower of these limits, even when the animals are in their ordinary activity. The mean or average heat of the body of Man is about $100^{\circ}$; but it has been observed as low as $96 \frac{1}{2}^{\circ}$ when the temperature of the air was $60^{\circ}$, and as high as $102^{\circ}$ when the air was at $82^{\circ}$. As a variation of $5 \frac{1}{2}^{\circ}$ may occur when the range of the external temperature of the air is only from $60^{\circ}$ to $82^{\circ}$, it is probable that observations made in cold climates will show that the temperature of the body may be still further lowered, when that of the air around is much depressed. But it seems that, in Man, as in other animals, the lower the temperature of the air around, the greater is his power of generating heat within his body, to keep up the necessary standard; and no observations yet made indicate that the temperature of the body ever falls below $95^{\circ}$ in health.

408. The young of warm-blooded animals have usually less power of maintaining an independent heat than adults. The embryo, whether in the egg, or within the body of the parent, is dependent upon external sources for the heat necessary to its full development. The contents of the egg, when lying under the body of its parent, are so situated, that the germspot ( $\$ 756)$ is brought into the nearest neighbourhood of the source of warmth. It is not usually until some weeks after the hatching of Birds, or the birth of Mammals, that the young animals have the power of maintaining an independent temperature. Thus young Sparrows, taken from the nest a week after they were hatched, were found to have a temperature of from $95^{\circ}$ to $97^{\circ}$; but this fell in one hour to $66 \frac{1}{2}^{\circ}$, the temperature of the atmosphere being at the same time $62 \frac{1}{2}^{\circ}$; and the rapid cooling was proved not to be due to the want of feathers alone. There are some birds, however, which can run about and pick up their food the moment they are hatched: these come into the world in a more advanced condition than the rest, and can maintain their temperature with little or no assistance. We find the same 
to be the case among Mammals. There are some species (such as the Guinea-pig) whose young are able from birth to walk and run, and to take the same food with the mother; and these have from the first the power of maintaining a steady temperature when the air around is not very cold. But, in general, the young of Mammals are much less advanced at the time of birth, being not unfrequently born blind as well as helpless; and they require considerable assistance, in keeping up their heat, from the parent or nurse. Thus the temperature of new-born puppies, removed from the mother, will rapidly sink to between $2^{\circ}$ and $3^{\circ}$ above that of the air.

409. These facts are of extreme practical importance, in regard to the treatment of the Human infant. Though not destitute of sight, at its entrance into the world, like the young of the Cat, Dog, or Rabbit, it is equally helpless, and dependent upon its parent not only for support but for warmth. And as the Human body is longer in arriving at its full development than is any other, so is it necessary that this assistance should be longer afforded. This assistance is the more necessary in the case of infants born prematurely; and it should be kept up during the years of childhood, gradually diminishing with age. It is too frequently neglected, by those who are well able to afford it, under the erroneous idea of hardening the constitution; and the want of it, consequent upon poverty, is one of the most fertile sources of the great mortality among children of the poorer classes. This is easily proved by the proportional number of deaths which take place in different parts of the year, at different ages. During the first month of infant life, the winter mortality is nearly double that of the summer; though there is very little difference between the two seasons in the mortality of adults. But in old age the difference again manifests itself to the same amount as in infants ; for old persons are almost equally deficient in the power of maintaining heat; they complain that their "blood is chill," and suffer greatly from exposure to cold.

410. The class of INsects presents us with some very interesting phenomena. In the larva and pupa states, the temperature of the body is never more than from $\frac{1}{2}^{\circ}$ to $4^{\circ}$ above that of the surrounding medium; but, in many tribes, the temperature of the perfect Insect rises so high, when it is 
in a state of activity, that it might be at such times called a warm-blooded animal; though in the states of abstinence, sleep, and inactivity, its temperature falls again nearly to that of the atmosphere. A single Humble-bee, inclosed in a phial of the capacity of 3 cubic inches, had its temperature speedily raised by violent excitement, from that of rest $\left(2^{\circ}\right.$ or $3^{\circ}$ above that of the atmosphere) to $9^{\circ}$ above that of the external air; and communicated to the air in the phial as much as $4^{\circ}$ of heat within five minutes. In another similar experiment, the temperature of the air in the phial was raised nearly $6^{\circ}$ in eight minutes. It is among the active Butterflies, and the Hymenopterous insects (Bee and Wasp tribe), which pass nearly the whole of their active condition on the wing, that we find the highest temperature; and next to them are the more active of the Beetle tribe. Those of the latter which seldom leave the ground, have little power of producing heat.

411. The greatest manifestation of this power is shown among Insects which live in societies; most of which belong to the order Hymenoptera. It has been seen that the body of a Humble-bee, in a state of activity, has a temperature of about $9^{\circ}$ above that of the atmosphere; but its nest has been found to have an ordinary temperature of from $14^{\circ}$ to $16^{\circ}$ above: the air, and from $17^{\circ}$ to $19^{\circ}$ above that of the chalk bank in. which it was formed. The production of heat is increased. to a most extraordinary degree, when the pupce are about to. come-forth from their cells as perfect Bees, requiring a higher temperature for their complete development. This is furnished by a set of Bees termed Nurse-bees, which are seen crowding upon the cells and clinging to them, for the purpose of communicating to them their warmth; being themselves evidently very much excited, and respiring rapidly, even at the rate of 130 or 140 inspirations per minute. In one instance, the thermometer introduced amongst seven nursingbees stood at $92 \frac{1}{2}^{\circ}$, whilst the temperature of the external air was but $70^{\circ}$. In Hive-bees, whose societies are large, this process occasions a still more remarkable elevation of temperature; for a thermometer introduced into a hive during May has been seen to rise to $96^{\circ}$ or $98^{\circ}$, when the range of atmospheric temperature was between $56^{\circ}$ and $58^{\circ}$. In September, when the bees are becoming stationary, the temperature of the hive is but a few degrees above that of the air. It was formerly 
supposed that Bees do not become torpid during the winter; but this is now known to be a mistake. Bees, like other Insects, pass the winter in a state of hybernation; but their torpidity is never so profound as to prevent their being aroused by moderate excitement. The temperature of a hive is usually from $5^{\circ}$ to $20^{\circ}$ above that of the atmosphere ; being kept at or above the freezing-point, when the air is far below it. Under such circumstances, their power of generating heat is most remarkable. In one instance, the temperature of a hive, of which the inmates were aroused by tapping on its outside, was raised to $102^{\circ}$; whilst a thermometer in a similar hive that had not been disturbed, was only $48 \frac{1}{2}^{\circ}$; and the temperature of the air was $34 \frac{1}{2}^{\circ}$.

412. The evolution of Heat in the Animal body may now be stated with tolerable certainty to depend for the most part on the union, by a process resembling ordinary combustion, of the carbon and hydrogen which it contains, with oxygen taken-in from the air in the process of Respiration. It has been elsewhere shown that, even in Plants, this union, when it takes place with sufficient rapidity, is accompanied by the disengagement of a considerable amount of heat (VEget. Phys, § 381); and in all those Animals which can maintain an elevated temperature, we find a provision for this union, both in regard to the constant supply of carbon and hydrogen from the body, and to the introduction of oxygen from the air. The supply of carbon and hydrogen may be derived (as already shown, $\S 157)$, either directly from the food, a large proportion of which is thus consumed in many animals without ever forming part of the tissues of the body; or it may be the result of the waste of the tissues, especially of the muscular, consequent upon their active employment ( $\$ 160)$, and converted into a substance peculiarly adapted for combustion by the agency of the liver ( $\$ 366)$. Or, again, it may be derived from the store laid-up in the system in the form of fat; which seems destined to afford the requisite supply, when other sources fail. Thus, when food is withheld, or when disease prevents its reception, the fat in the body rapidly diminishes; being burnt off, as it were, to keep up the temperature of the system. This is the case, too, during hybernation; the animals which undergo this change usually accumulating a considerable amount of fat in the autumn, and 
being observed to come forth from their winter quarters, with the return of spring, in a very lean condition.

413. The consumption of oxygen and the production of carbonic acid are found to bear, in every animal, a very close relation to the amount of heat liberated at the time. Thus in warm-blooded animals, the respiratory function is much more active than in the cold-blooded; but when the former are reduced to the state of cold-blooded animals, as occurs in hybernation ( $\S 309)$, their respiration is proportionately low. On the other hand, whenever the temperature of an animal is quickly raised by any extraordinary stimulus, above that which it was previously maintaining, it is always by means of increased activity of the respiratory movements, and augmented consumption of oxygen. Thus during the incubation of Bees ( $\$ 411)$, the insect, by accelerating its respiration, causes the evolution of heat and the consumption of oxygen to take place at least twenty times as rapidly as when in a state of repose. The same takes place when a hybernating animal is roused; and it is remarkable that even extreme cold will effect this for a time; but the animal, if exposed for too long a period to a very low temperature, will not be able to resist its influence, and will perish.

414. Although the combustion of carbon and hydrogen within the Animal body is undoubtedly the chief source of the production of heat, yet it must not be left out of view that there are other chemical changes in the system, which also contribute to it, though in a minor degree ( $\$ 343)$. Of this kind are the oxidation of the sulphur and phosphorus which enter the body in the organic compounds used as food, and which, being united by a combustive process with oxygen, pass out of the system in the urine, in the form of sulphuric and phosphoric acids, combined with alkaline bases $(\S 367)$.

415. Besides all these sources, it seems probable from various considerations, that Heat may occasionally be generated, like light and electricity, by the direct agency of the Nervous system; as one of the modes of force into which nervous power may be metamorphosed. Of course, in any such generation of heat, there must be the same consumption of nervous tissue, as would occur if its equivalent of nerve-force had been. manifested. 


\section{Animal Electricity.}

416. Almost all chemical changes are attended with some alteration in the electric state of the bodies concerned; and when we consider the number and variety of these changes in the living animal body, it is not surprising that disturbances of its electric equilibrium should be continually occurring. But these, when slight, can only be detected by very refined means of observation; and it is only when they become considerable, that they attract notice. Some individuals exhibit electric phenomena much more frequently and powerfully than others; and cases are occasionally recorded in the Human subject, in which there has been a most decided production of electricity, which manifested itself in sparks whenever the individual was insulated.

417. The sparks and crackling noise, however, which are occasionally observed on pulling off articles of dress that have been worn next the skin, especially in dry weather, are partly due to the friction of these materials with the surface and with each other ; the production of electricity being greatly influenced by their nature. Thus, if a black and a white silk stocking be worn, one over the other, on the same leg, the manifestation of electricity when they are drawn off, especially after a dry frosty day, is most decided; but this would also be the case if they were simply rubbed together, without any connexion with the body.

418. In most animals with a soft fur, sparks may be produced by rubbing it, especially in dry weather ; this is familiar to most persons in the case of the domestic Cat. But the electricity thus produced seems occasionally to accumulate in the animal, as in the Leyden jar, so as to produce a shock. If a cat be taken into the lap, in dry weather, and the left. hand be applied to the breast, whilst with the right the back be stroked, at first only a few sparks are obtained from the hair; but after continuing to stroke for some time, a smart shock is received, which is often felt above the wrists of both the arms. The animal evidently itself experiences the shock, for it runs off with terror, and will seldom submit itself to another experiment.

419. But there are certain animals which are capable of producing and accumulating electricity in large quantities, by 
means of organs specially adapted for the purpose ; and of discharging it at will, with considerable violence. It is remarkable that all these belong to the class of Fishes $;^{1}$ and that they should differ alike in their general conformation, and in their geographical distribution. Thus, the two species of Torpedo, belonging to the Ray tribe, are found on most of the coasts of the Atlantic and Mediterranean; sometimes so abundantly, as to be a staple article of food. The Gymnotus, or Electric Eel, is confined to the rivers of South America. The Malapterurus (commonly known as the Silurus) which approaches more nearly to the Salmon tribe, occurs in the Niger, the Senegal, and the Nile; and there are two other less known Fishes, said to possess electric properties, which inhabit the Indian seas.

420. Of all these, the Gymnotus (fig. 176) is the one which possesses the electric power in the most extraordinary degree. It is an eel-like fish, having nothing remarkable in its external appearance; its usual length is from six to eight feet, but it is said occasionally to attain the length of twenty feet. This fish will attack and paralyse horses, as well as kill small animals; and the discharges of the larger individuals sometimes prove sufficient to deprive even Men of sense and motion. This power is employed by the fish to defend itself against its enemies; and even, it is said, to destroy its prey (which consists of other fishes) at some distance; the shock being conveyed by water,

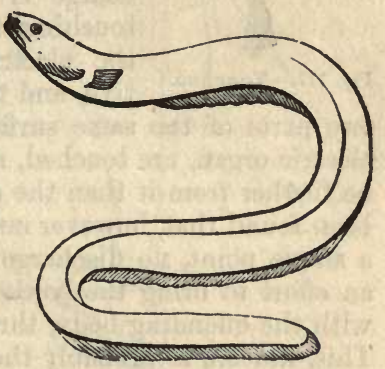

Fig. 176.-Grmnotus. as a lightning-conductor conveys to the earth the effects of the electric discharge of the clouds. The first shocks are usually feeble; but as the animal becomes more irritated, their power increases. After a considerable number of powerful discharges, the energy is exhausted, and is not recovered for some time; and this circumstance is taken advantage of in South America, both to obtain the fishes (which afford

1 Certain Insects and Mollusks have been said to possess electrical properties; but no special electric organ has been discovered in them. 
excellent food), and to make the rivers they infest passable to travellers. A number of wild horses are collected in the neighbourhood, and are driven into the water; the Gymnoti attack these, and speedily stun them, or even destroy their lives by repeated shocks; but their own powers of defence and injury are exhausted in the same degree, and they then become an easy prey to their captors.

421. The shock of the Torpedo (fig. 177) is less powerful; but it is sufficient to benumb the hand that touches it. From

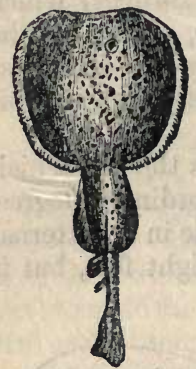

Fig. 177.-TORPEDO. its proximity to European shores, this fish has been made the subject of observation and experiment more completely than the other; and some curious results have been attained. It seems essential to the proper reception of the shock, that two parts of the body should be touched at the same time; and that these two should be in different electrical states. The most energetic discharge is procured from the Torpedo, by touching its back and belly simultaneously; the electricity of the back being positive, and that of the belly negative. When two parts of the same surface, at an equal distance from the electric organ, are touched, no effect is produced; but if one be further from it than the other, a discharge occurs. It has been found that, however much a Torpedo is irritated through a single point, no discharge takes place; but the fish makes an effort to bring the border of the other surface in contact with the offending body, through which a shock is then sent. This, indeed, is probably the usual manner in which its discharge is effected. If the fish be placed between two plates of metal, the edges of which are in contact, no shock is perceived by the hands placed upon them, since the metal is a better conductor than the human body; but if the plates be separated, and, while they are still in contact with the opposite sides of the body, the hands be applied to them, the discharge is at once rendered perceptible, and may be passed through a line formed by the moistened hands of two or more persons. In the same manner, also, a visible spark may be produced; but this is less easily obtained from the Torpedo than from the Gymnotus.-As to the uses of the electrical organs to the 
Fishes which possess them, no definite information can be given. It is doubtful to what extent they are employed in obtaining food; since it is known that the Gymnotus eats very few of the fishes which it kills by its discharge; and that Torpedos kept in captivity do not seem disposed to exercise their powers on small fishesplaced in the water with them. The chief use of their electrical power appears to be, to serve as a means of defence against their enemies.

422. The electric organs of the Torpedo (fig. 178) are of flattened shape, and occupy the front and sides of the body, forming two large masses, which extend backwards and outwards from each side of the head. They are composed of two layers of membrane, the space between which is divided by vertical partitions into hex- $c$, brain; $m e$, spinal cord; $o$, eye and optic nerve; $e$, agonal cells, $e$, like those of a honey-

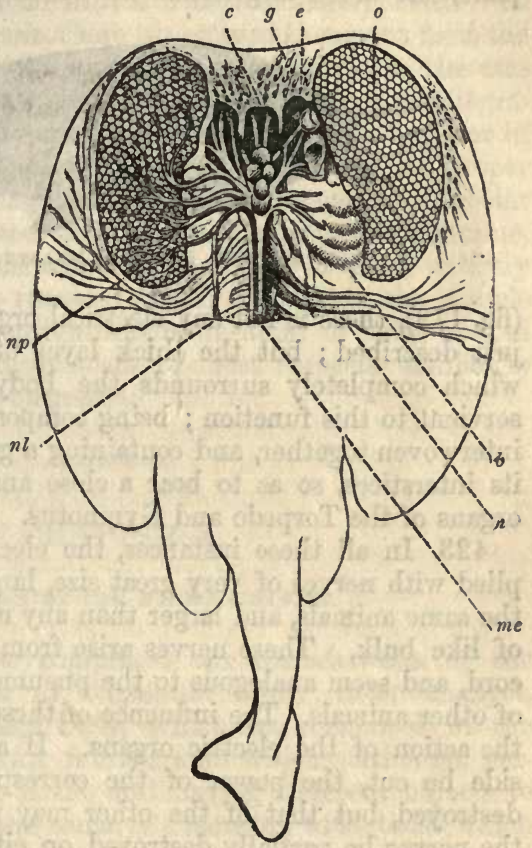

Fig. 178.-Electric Apparatus of Torpedo. electric organs; $n l$, lateral nerve; $n$, spinal nerves.

comb, the ends of which are directed towards the two surfaces of the body. These cells-which are filled with a whitish soft pulp, somewhat resembling the substance of the brain, but containing more water-are again subdivided horizontally by little membranous partitions; and all these partitions are profusely supplied with vessels and nerves.-The electrical 
organs of the Gymnotus are essentially the same in structure, but differ in shape in accordance with the conformation of the animal; they occupy one-third of its whole bulk, and run nearly along its entire length, being arranged in two distinct pairs, one much larger than the other. In the Malapterurus

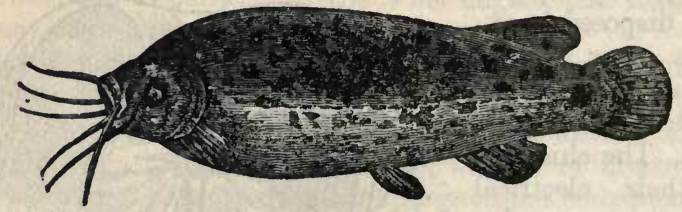

Fig. 179.- Electric Malapterurus.

(fig. 179), there is not any electrical organ so definite as those just described; but the thick layer of dense areolar tissue which completely surrounds the body, appears to be subservient to this function; being composed of tendinous fibres interwoven together, and containing a gelatinous substance in its interstices, so as to bear a close analogy with the special organs of the Torpedo and Gymnotus.

423. In all these instances, the electrical organs are supplied with nerves of very great size, larger than any others in the same animals, and larger than any nerves in other animals of like bulk. These nerves arise from the top of the spinal cord, and seem analogous to the pneumogastric nerve ( $\$ 458)$ of other animals. The influence of these nerves is essential to the action of the electric organs. If all the trunks on one side be cut, the power of the corresponding organ will be destroyed, but that of the other may remain uninjured. If the nerves be partially destroyed on either or both sides, the power is retained by the portions of the organs which are still connected with the brain by the trunks that remain. Even slices of the organ entirely separated from the body, except by a nervous fibre, may exhibit electrical properties. Discharges may be produced by irritating the part of the nervous centres from which the trunks proceed (so long, at least, as they are entire), or by irritating the trunks themselves. In all these respects, there is a strong analogy between the action of the nerves on the electric organs and on the muscles (Chap. xIr.); and it may be safely affirmed that the Nervous force develops 
Electricity by its action on the electrical organs, just as it produces Motion by its action on the muscles.

424. It is another interesting point of analogy between the action of Muscles and that of Electrical organs, that the former, like the latter, is attended with a change of electric state. In any fresh vigorous muscle, there is a continual current from the interior to exterior, which appears to depend upon the fact that the actions connected with the nutrition and disintegration of its tissue go on more energetically in the interior of the muscle, than they do near its surface, where the proper muscular fibres are mingled with a large proportion of areolar and tendinous substance. During the contraction of a muscle, this current is diminished in intensity, or is even entirely suspended; but it is renewed again, so soon as the muscle relaxes.-An electric current has been found to exist also in Nerves; and its conditions are in most respects similar to those of the muscular current.

\section{CHAPTER X.}

\section{FONCTYONS OF THE NERVOUS STSTEM.}

425. We have now completed our consideration of the Functions of Organic or Vegetative Life; those changes, namely, in the Animal body, which are concerned in the maintenance of its own fabric; and which, although performed in a different mode, and having different objects to fulfil, are essentially the same in character with those which take place in Plants. The first and most striking difference of mode results, as we have seen, from the nature of the food of Animals, which requires that they should possess a cavity for its reception, and a chemical and mechanical apparatus for its digestion (or reduction to the fluid form), in order that it may be prepared for absorption into the vessels. In regard to the absorption of the aliment, and its circulation through the system, there is but little essential difference between Plants and the lower Animals; but in the higher tribes of the latter, we find that a muscular organ having the action of a forcingpump is appended to the system of tubes in which the fluid 
circulates, in order to drive it through them with the requisite certainty and energy. The respiration of Animals, again, is essentially the same with that of Plants; the chief difference being that, in order to secure the active performance of this important function, the higher Animals are provided with a complex apparatus of nerves and muscles, by which the air or water in contact with the aerating surface is continually renewed. And in regard to the functions of secretion and excretion, we have seen that, though there is a wide difference in the form of the organs by which they are executed, they are the same in essential structure; and that the difference in their mode of operation consists chiefly in this, that their products in the Animal are destined to be carried out of the body, instead of being retained within it, as in Plants.

426. In regard to the immediate objects of these functions, also, there is but little essential difference; for in both instances it is the conversion of alimentary materials into living organized tissue. But the ultimate purpose of this tissue is far from being the same in the two kingdoms. Nearly all the nourishment taken-in by Plants is applied to the extension of their own fabric; and hence there is scarcely any limit to the size they may attain. There is very little waste or decay of structure in them, the parts once formed (with the exception of the leaves and flowers) continuing to exist for an indefinite time; this is a consequence of the simply physical nature of the functions of the woody structure, which has for its chief object to give support to the softer parts, and to serve as the channel for the movement of the fluid that passes towards and from them. - The case is very different in regard to Animals. With the exception of those inert tribes which may be compared with Plants in their mode of life, we find that the whole structure is formed for motion; and that every act of motion involves a waste or decay of the fabric which executes it. An energetic performance of the nutritive actions is required, therefore, in the more active Animals, simply to make good the loss which thus takes place; we find, too, that their size is restrained within certain limits ; so that, instead of the nourishment taken into the body being applied, as in Plants, to the formation of new parts, it is employed for the most part in the simple repair of the old. Thus we may say that, whilst the ultimate object of Vegetable Life is to build up a vast 
fabric of organized structure, the highest purpose of the Organic Life of Animals is to construct, and to maintain in a state fit for use, the mechanism which is to serve as the instrument of their Functions of Animal Life,-enabling them to receive sensations, and to execute spontaneous movements, in accordance with their instincts, emotions, or will.

427. This mechanism consists of two kinds of structure,the Nervous and Muscular,-which have entirely different offices to perform. The Nervous system is that which is the actual instrument of the Mind. Through its means, the individual becomes conscious of what is passing around him; its operations are connected, in a manner we are totally unable to explain, with all his thoughts, feelings, desires, reasonings, and determinations; and it communicates the influence of these to his muscles, exciting them to the operations which he determines to execute. But of itself it cannot produce any movement, or give rise to any action; any more than the expansive force of steam could set a mill in motion, without the machinery of the Steam-engine for it to act upon. The Muscular System is the apparatus by which the movements of the body are immediately accomplished; and these it effects by the peculiar power it possesses of contracting upon the application of certain stimuli, of which Nervous agency is the most powerful.

428. Although the presence of a Nervous System is the most distinguishing attribute of Animals, yet we do not encounter it by any means universally. For among certain of those classes which possess on other grounds a title to be ranked in the Animal kingdom, it seems beyond a doubt that no nervous system exists; and there are many others in which, if it be present at all, its condition is so rudimentary, that it can take little share in directing the general operations of the organism. The life of such beings, in fact, is chiefly vegetative in its nature; their movements are not dissimilar in kind to those that we witness in Plants; and their title to a place in the Animal kingdom chiefly rests upon the nature of their food, and the mode in which they appropriate.it $(\S \S 7,8)$. This is the case with the Protozoa generally ( $\$ \$ 128-137$ ), and in a less degree with Zoophytes ( $\$ \S 121-127$ ).

429. In proportion as we ascend the Animal series, however, we find the Nervous System presenting a greater and 
greater complexity of structure, and obviously acquiring higher and yet higher functions; so that in Vertebrated animals, and more especially in Man, it is evidently that portion of the organism to whose welfare everything else is brought-into subordination ( $\$ 73)$. And we observe this to be the case, not merely in virtue of its direct instrumentality as the organ of Mind, but also in the intimacy of its relation to the Organic functions, which are placed in great degree under its control. Thus we find that the inlets and outlets to the Digestive apparatus, the mechanism by which food is brought to the mouth and conveyed into the stomach, and that by which indigestible matters are voided from the large intestine, are subjected to its influence; although the act of digestion itself, and the passage of the aliment from one end of the canal to the other, are performed independently of it. So, again, the movements of Respiration, by which the air within the lungs is renewed as fast as it becomes vitiated, are not only effected through its instrumentality, but are placed, for the purposes of Vocalization, as far under the control of the Will as would be consistent with a due regard to the safety of life. Yet among many of the lower tribes of Animals, the ingestion of food and the aeration of the circulating fluid are provided-for by ciliary action alone $(\$ 45)$, in which we have every reason to believe that nervous agency has no participation whatever.

430. If, taking the Nervous System of Man as the highest type of this apparatus, we analyse in a general way the actions to which it is subservient, we find that they may be arranged under several distinct groups, which it is very important to consider apart, whether we are studying his psychical ${ }^{1}$ functions or those of the lower animals.-1. The simplest mode of its action is that in which an impression made upon an afferent nerve excites, through the ganglionic centre in which it terminates, an impulse in the motor nerve issuing from it, which, being transmitted by it to the muscular apparatus, calls forth a respondent movement. Of this action, which is called reflex, or "excito-motor," and which may be performed without any consciousness either of the impression or of the motion, we have already seen examples in the movements of Deglutition

1 This term is used to designate the sensorial and mental endowment of Animals, in the most comprehensive sense. 
( $\$ 194)$ and Respiration ( $(340)$. - 2. If the ganglionic centre to which the impression is conveyed, should be one through which the consciousness is necessarily affected, sensation becomes a necessary link in the circular chain; and the action. is distinguished as consensual, or "sensori-motor." The closing and opening of the pupil of the eye, in accordance with the amount of light that falls upon the retina, together with other remarkable adjustments which are involuntarily made in the working of that wonderful organ, are characteristic examples of this class. In the foregoing operations no mental change higher than simple consciousness of impressions-that is to say, Sensation, with which may be blended the simple feelings of pleasure and pain-is involved. Such would appear to be the condition of the Human infant on its first entrance into the world, before the self-education of its higher faculties has commenced; and such is probably the state of Invertebrated animals generally, whose instinctive actions seem to be referable to one or other of the foregoing classes.

431. But Sensation is the very lowest form of purely Mental action. When the outness or externality of the objects which give rise to our sensations has been recognised by perception, we begin to form ideas respecting their nature, qualities, \&c. ; and it is in the various processes of association, comparison, \&c., to which these ideas are subjected, that our Reasoning faculty consists. Now these processes may go on in great degree automatically, that is, without any control or guidance on our own part, as happens in the states of Dreaming, Reverie, and Abstraction; and they may express themselves in action, as we see in the movements of a Somnambulist, who may be said to be acting his dreams. This form (3) of Nervous activity, which may be termed ideo-motor, seems to be the ordinary mode in such of the lower animals as are governed by Intelligence rather than by instinct (Chap. xrv.); but it is abnormal and exceptional in Man.-With ideas are associated feelings of various kinds, which constitute Passions and Emations; and these (4), when strongly excited, may become direct springs of action, so powerful as even to master the control of the Will, producing emotional movements.

432. In the well-regulated mind of Man, however, the Will (5) possesses supreme direction over the whole current of thought, feeling, and action: regulating the succession of the 
ideas; keeping in check the passions and emotions, or, on the other hand, promoting their healthful activity by directing the attention to the objects of them ; and determining the movements which the reason prompts :-and the acquirement and right direction of such regulating power is the highest object of all Education.

433. It will be recollected that every form of Nervous System essentially consists of two kinds of nervous tissue,the tubular or fibrous, whose functions seem to be purely conductive ( $\$ \S 60,62)$, - and the vesicular or ganglionic, which seems to be the seat of all the changes to which this apparatus ministers, and the source of all its peculiar powers $(\$ \S 61,63)$. -The principal forms under which this apparatus presents itself in the several divisions of the Animal Kingdom, and the general nature of the functions to which it is subservient in each, will now be successively described in the ascending series, from Zoophytes up to Man.

\section{Structure and Actions of the Nervous System in the principal} Classes of Animals.

434. In most of the RADIATED classes, it is difficult to discover any distinct traces of a Nervous System; the general softness of their tissues being such, that it cannot be certainly distinguished amongst them. It clearly exists, however, in the highest group, the Echinodermata; and it presents an

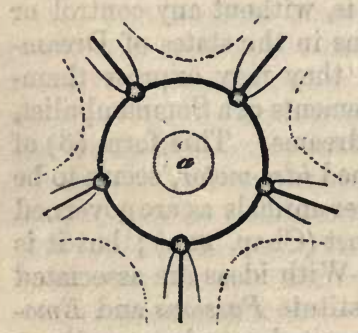

Fig. 180.-Nervous Sxstem of STAR-FISH.

$a$, position of the mouth. extremely simple form, which partakes of the general arrangement of parts in these animals. In the Star-fish, for instance, it forms a ring which surrounds the opening into the stomach (fig. 180); this ring consists of a nervous cord that forms communications between five ganglia, one of which is placed at the base of each ray. These ganglia appear to be all similar to each other in function. Every one of them sends a large trunk along its own ray; and two small branches to the organs in the central disk. The rays being all similar to each other in structure, it would appear that no one of these. 
ganglia can have any controlling power over the rest. All the rays have at their extremities what seem to be very imperfect eyes ; and so far as these can aid in directing the movements of the animal, it is obvious that they will do so towards all sides alike. Hence there is no one part which corresponds to the head of higher animals ; and the ganglia of the nervous system, like the parts they supply, are but repetitions of one another, and act independently of one another. Each would perform its own individual functions if separated from the rest; but, in the entire animal, they are brought into mutual relation by the circular cord, which passes from every one of the five ganglia to those on either side of it.-In Man, as well as in all the Vertebrated and Articulated animals, and in some of the Mollusca, there is a like repetition of the parts of the Nervous System on the two sides of the central line of the body; but the organs are only double, instead of being repeated five times. Still the two hemispheres of the brain, and the two halves of the spinal cord, in the Vertebrated animal, - and the two halves of the chain of ganglia, in the Articulated animal, - are as independent of one another as are the five separate ganglia of the Star-fish; and they are made to act in mutual harmony by similar uniting bands of nervous fibres, which are termed commissures.

435. In the nervous system of Mollusca, we do not meet with any such repetition of parts ; the body itself not presenting this character. In the lowest and simplest animals of this group, there exists only a single ganglion, which may be regarded as analogous to any one of the ganglia of the Star-fish ; but in the higher, we find the number of ganglia increased, in accordance with the increase of the functions which they have to perform. The simplest form of the nervous system in this class is seen in the accompanying figure (fig. 181), which represents one of the solitary Tunicata, the Ascidia. At $a$ is seen the orifice by which the water enters for sup- Fig. 181.-Nervoes Srsplying the stomach with food, and for aerat-

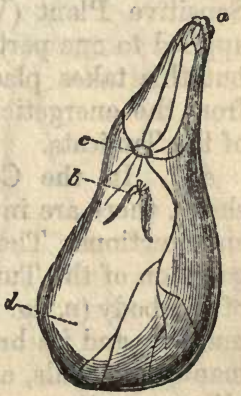
ing the blood ( $\$ 114)$; and at $b$ is the orifice by which the current of water passes out again, after it has served these purposes. 
Between these orifices is the single ganglion $c$, which sends filaments to both of them, and other branches which spread over the surface of the mantle $d$. These animals are for the most part fixed to one spot during nearly the whole of their existence; and they show but little sign of life, beyond the continual entrance and exit of the currents already adverted to. When any substance is drawn-in by the current, however, the entrance of which would be injurious, it excites a general contraction of the mantle; and this causes a jet of water to issue from both orifices, which carries the offending body to a distance. And in the same manner, if the exterior of the body be touched, the mantle suddenly and violently contracts.

436. These are the only actions, which, so far as we know, the nervous system of these animals is destined to perform. They scarcely exhibit any traces of eyes or other organs of special sense; and the only parts that appear peculiarly sensitive, are the small tentacula which guard the orifice $a$. It would seem as if the irritation caused by the contact of any hard substance with these, or with the general surface of the animal, caused a reflex contraction of the mantle, having for its result the getting-rid of the source of the irritation. Such a movement could only be performed by the aid of a Nervous system, which has the power of receiving impressions, and of immediately exciting even the most distant parts of the body to act in accordance with them. In the Venus's Fly-trap and Sensitive Plant (VEGET. PhYs., §§ 214, 391), an irritation applied to one part is the occasion of a movement in another; but this takes place slowly, and in a manner very different from the energetic and immediate contraction of the mantle of the Tunicata.

437. In the CoNCHIFERA, or animals inhabiting bivalve shells, there are invariably at least two ganglia, having different functions. The larger of these, corresponding to the single ganglion of the Tunicata, is situated towards the posterior end of the body (в, fig. 182), in the neighbourhood of the posterior muscle; and its branches are distributed to that muscle, the mantle, the gills, and the siphons. But we find another ganglion, or rather pair of ganglia (AA), situated near the front of the body, either upon or at the sides of the œsophagus, and connected by a commissural band that arches over it; these ganglia receive nerves from very sensitive tentacula which 
guard the mouth; and they evidently correspond, both in position and functions, to the sensory ganglia of higher animals, whilst the posterior ganglion has for its office to regulate the respiratory movements. In the Pecten, however, as in other Conchifera which possess a foot (fig. 62), we find an additional ganglion (c), the pedal, connected with the cephalic ganglia, and sending nervetrunks to that organ. There is good reason to believe that, whilst the cephalic ganglia alone are the instruments of sensation, so that they exert a general

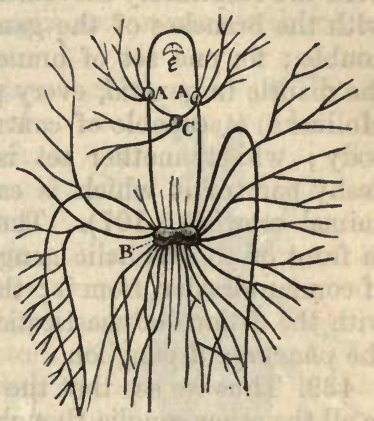
coutrol and direction over the Fig. 182.-Nervous System or Pecten. movements of the animal, the pedal and branchial ganglia minister to the reflex actions

A A, cephalic ganglia ; B, branchial ganglion; C, pedal ganglion; e. œsophagus.

( $\$ 433$ ) of the organs which they supply.

438. A similar arrangement is found in the higher Mollusks, among which the ganglia are more numerous, in accordance with the greater variety of functions to be performed. Of this we have an example in the Aplysia, a sort of sea-slug somewhat resembling those formerly alluded to $(\S 316)$. In proportion as we ascend the scale, we find the cephalic ganglia rising higher and higher on the sides of the cesophagus ; and in the Aplysia they meet on the central line above it, forming the single mass ( $\Delta$, fig. 183), which receives the nerves of the eyes, tentacula, \&c., and sends branches of communication to the other ganglia. The branches which it sends backwards are three on each

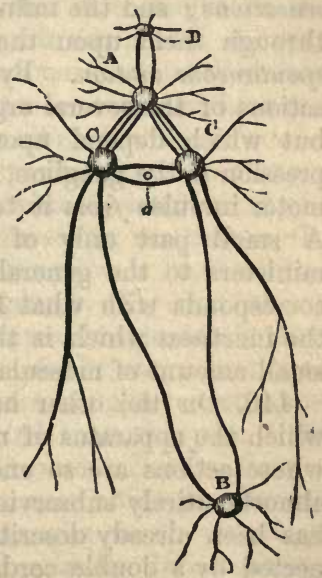

Fig. 183.-Nervous SxateM OF APLYSIA. 
side. Of these, one passes through the ganglionic masses $\mathrm{cc}$, to communicate with the ganglion $\mathrm{B}$, which is the one connected with the respiratory movements. The others are distributed with the branches of the ganglia cc, the function of which is double; for one set of branches from each is distributed to the mantle in general, every part of which (in these shell-less Mollusks) is capable of contracting and giving motion to the body; whilst another set is distributed to that thick and fleshy part of it which is called its foot, and on which the animal crawls ( $\$ 107)$. There is another ganglion, $\mathrm{D}$, lying in front of the cephalic ganglion, and also receiving branches of communication from it ; this ganglion is specially connected with the actions of mastication and swallowing, and is called the pharyngeal ganglion.

439. Thus we see that the cephalic ganglion sends branches to all the other ganglia, though these having different functions, do not communicate with each other; and thus every part has two sets of nervous connexions, one with the cephalic ganglia, and the other with its own ganglion. By the former, the animal becomes conscious of impressions made upon it, these impressions being converted in the cephalic ganglia into sensations; and the influence of its conscious power is exerted through them upon the several parts of its body, causing spontaneous motion. By the latter are produced those reflex actions of the several organs, which do not require sensation, but which depend upon the simple conveyance of an impression to the ganglion, and the transmission of the resultant motor impulse from it to the muscles supplied by its nerves. A small part only of the Nervous System of Mollusks ministers to the general movements of the body; and this corresponds with what has been elsewhere stated ( $\$ 107)$ of the inertness which is their general characteristic, and of the small amount of muscular structure which they possess.

440. On the other hand, in the Articulated classes, in which the apparatus of movement is so highly developed, and whose actions are so energetic, we find the Nervous System almost entirely subservient to this function. Its usual form has been already described ( $\$ 94)$ as a chain of ganglia connected by a double cord, which commences in the head, and passes backwards through the body. In general, we find a ganglion (or rather a pair of ganglia united on the middle 
line) in each segment; hence in the ANnelida and MrriaPODA, the ganglia are very numerous; but they are proportionably small. In INSECTs (fig. 184), the number of segments, and consequently of ganglia, never exceeds thirteen; and the ganglia are larger. - Whatever be the number of the ganglia, they are usually but repetitions of one another, the functions of each segment being the same with those of the rest. The nerves proceeding from them are chiefly distributed to the muscles of the legs ; or, where legs do not exist (as in the Leech), to the muscles that give motion to the body. This is the case in the larva of the Insect, as in the Centipede or Nereis; but in the perfect Insect the case is different ; for the apparatus of locomotion is confined to the thorax (\$97), and

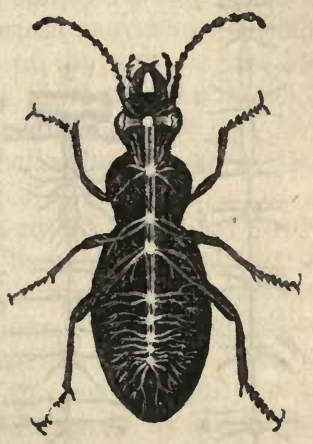

Fig. 184.-NERVOUS SYSTEM OR AN INSECT. the segments of the abdomen have no members. We accordingly find that the ganglia of the thorax, from which the legs and wings are supplied, are very much increased in size, and are sometimes concentrated into one mass; whilst those of the abdomen are very small, one or two of them oscasionally disappearing altogether.

441. A good example of this curious change in the nervous system of Insects, is seen in the Sphinx ligustri, or Privet Hawk-Moth, as shown in the succeeding diagrams. In fig. 185 the nervous system of the Caterpillar is represented; this consists of a pair of cephalic ganglia (1); from which proceeds, on each side, a cord of communication to the first ganglion of the trunk (2), and thence to the other ganglia (3 -13). No difference is seen in these ganglia, except that the last two are more closely connected than the rest. The cephalic ganglia, with their cords of communication, form a ring, through which the cesophagus passes; they are situated above it; but the whole chain of ganglia of the trunk is situated beneath the alimentary canal.-In fig. 186 is shown the Nervous System of the perfect Insect; in which it is seen that the whole is considerably abbreviated (the body of $\triangle \triangle 2$ 
the Moth being much shorter than that of the Caterpillar), and that great changes have taken place in the relative sizes of the ganglia. The cephalic

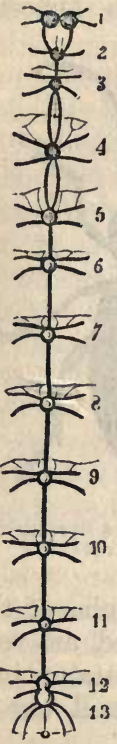

Fig. 185.-NerVOUB SYSTEM OF LARVA OF SPHINX LIGUSTRI.

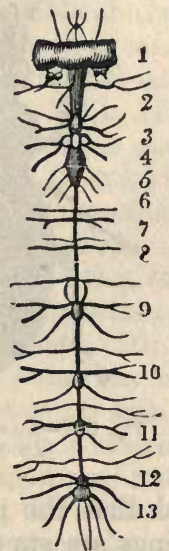

Fig. 186.-NERYOUS SYSTEM OF SPHINX IIGUSTRI. ganglia, being now connected with much more perfect eyes and other organs of sense, are greatly enlarged; the thoracic ganglia, from which the legs and wings are supplied, are enlarged and concentrated; whilst the abdominal ganglia are relatively diminished in size, the 7th and 8th being entirely wanting.

442. When the structure of the chain of ganglia is more particularly inquired into, it is found to consist of two distinct tracts; one of which is composed of nervous fibres only, and passes backwards from the cephalic ganglia, over the surface of all the ganglia of the trunk, giving off branches to the nerves that proceed from them; whilst the other connects the ganglia themselves. Hence, as in Mollusca, every part of the body has two sets of nervous connexions; one with the cephalic ganglia, the other with the ganglion of its own segment. Imprassions made upon it, being conveyed by the fibrous tract to the cephalic ganglia, become sensations; and by the influence of the conscious power, operating through these same ganglia, the general movements of the body are harmonised and directed. It is obvious that, as the motions of an animal are chiefly guided by its sight, the cephalic ganglia would have a governing influence over the rest, if only from their peculiar connexion with the eyes ; but there is good reason to believe that their functions are still more different from those of the ganglia of the trunk, and that sensation resides in them alone. The motions produced by 
the ganglia of the trunk, when separated from the head, are often very remarkable, and seem at first sight to indicate sensation and a guiding will; but, when they are carefully studied, it is found that striking differences are to be detected, by which their nature is found to be simply reflex,-a certain stimulus or irritation producing a certain movement, without any choice or guidance on the part of the animal, and probably without its consciousness. As there are no animals in which these reflex movements are more remarkable than they are in Centipedes and Insects, we shall pause to dwell upon them here in more detail.

443. If the head of a Centipede be cut off whilst the animal is in motion, the body will continue to move onwards by the action of the legs; and the same will take place if the body be divided into several distinct portions. After these actions have come to an end, they may be excited again by irritating any part of the nervous centres, or the cut extremity of the nervous cord. The body is moved forwards by the regular and successive action of the legs, as in the natural state ; but its movements are always forwards, never backwards ; and are only directed to one side when the direct movement is checked by an interposed obstacle. There is not the slightest indication of consciousness, either in direction of object, or in avoidance of danger. If the body be opposed in its progress, by an obstacle not more than one half its own height, it mounts over it and moves directly onwards, as in a natural state; but if the height of the obstacle be equal to its own, its progress is arrested, and the cut extremity of the body remains opposed to it, with the legs still moving. If, again, the nervous cord of a Centipede be divided in the middle of the trunk, so that the hinder legs are cut off from connexion with the cephalic ganglia, they will continue to move, but not in harmony with those in the fore part of the body,- -being completely withdrawn from any control on the part of the animal, though still capable of performing reflex movements by the influence of their own ganglia. Or, again, if the head of a Centipede be cut off, and, while it remains at rest, some irritating vapour (such as that of ammonia or muriatic acid) be caused to enter the air-tubes on one side of the trunk, the body will be immediately bent in the opposite direction, so as to withdraw itself as much as possible from the influence of 
the vapour: if the same irritation be applied on the other side, the reverse movement will take place; and the body may be caused to bend in two or three curves, by bringing the irritating vapour into the neighbourhood of different parts of either side. This movernent is evidently a reflex one, and serves to withdraw the entrances of the air-tubes from the source of irritation; just as the act of sneezing in higher animals causes the expulsion from the air-passages of any irritating matter, whether solid, liquid, or gaseous, which may have found its way into them; and we have no reason to regard the former as more voluntary than the latter, which we know to be purely reflex ( $(342)$.

444. Among Insects, we meet with reflex actions yet more curious. The Mantis religiosa (fig. 187) is remarkable for the

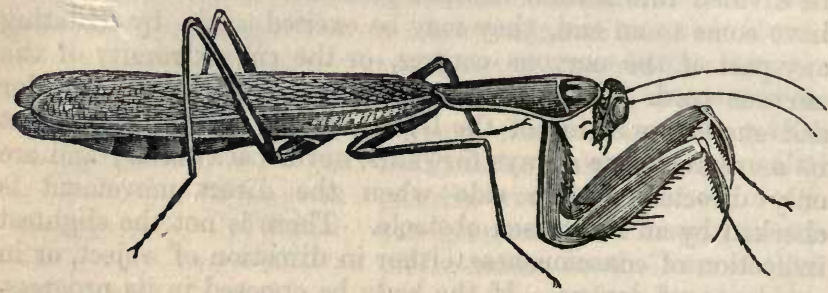

Fig. 187.-Maxtis Religiosa.

peculiar conformation of its first pair of legs, which serve as claws for seizing its prey; and also for the peculiar attitude which it assumes, especially when threatened or attacked. Supporting itself upon its two hinder pairs of legs, it rears up its head upon the long first segment of the thorax, elevating at the same time its large and powerful arms ; and the resemblance fancied to exist between this attitude and that of prayer, is the cause of the epithet religiosa having been given to it. Now if the first segment of the thorax, with its attached members, be removed, the posterior part of the body will still remain balanced upon the four legs which belong to it; resisting any attempts to overthrow it, recovering its position when disturbed, and performing the same agitated movements of the wings as when the unmutilated animal is excited. But it will remain quite at rest, so long as it is not irritated. On the other hand, the detached portion of the thorax which 
contains a ganglion, will, when separated from the head, set in motion its long arms, and impress their hooks on the fingers which hold it.-Again, a specimen of Dytiscus (a waterbeetle), from which the cephalic ganglia have been removed, executes the usual swimming motions when cast into water, with great energy and rapidity, striking all its comrades to one side by its violence; in these it will persist for half an hour, though so long as it lies on a dry surface it remains quiescent.

445. From these and similar facts, it appears that the ordinary moveruents of the legs and wings of Articulated animals are of a reflex nature, and are dependent upon the ganglia with which these organs are severally connected; whilst in the perfect animal they are harmonised, controlled, and directed by its conscious power, which acts through the cephalic ganglia and the trunks proceeding from it. When we come to compare the reflex movements of Insects with those of the higher animals, we shall perceive that there is no ground for supposing the ganglia of the trunk to be in themselves endowed with sensibility; so that, when the head is cut off, or the cephalic ganglia are removed, or their connexion with any part of the body is interrupted by division of their nervous cord, no sensation is felt, however much the movements it performs may seem at first to indicate this. (See § 467.)

446. From this account of the structure and uses of the chain of ganglia in the Articulata, it is obvious that these ganglia are so many repetitions of the pedal ganglia (or ganglion of the foot) of the Mollusca; and we have not yet had to notice any ganglia appropriated to other functions. In fig. 186 , however, is seen a small ganglion in front of the cephalic mass, which corresponds to the pharyngeal ganglion of the Aplysia (fig. 183, D); and we have now to describe an entirely distinct system of nerves, appropriated to the function of respiration. As the respiratory apparatus of Articulata, instead of being confined to one spot, like that of the Mollusca, is dispersed through the body ( $\$ \S 315$ and 320 ), the ganglia which minister to its actions are repeated in the several segments. There is, in fact, a chain of minute ganglia lying upon the larger cord, and sending off its nerves between those proceeding from the latter, as seen in fig. 185. These respiratory 
ganglia and their nerves are best seen in the front of the body, where the cords that pass between the ganglia diverge or separate from each other. This is shown

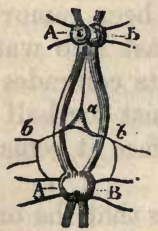

Fig.188.-Pontion OF THE NERVOUSSYSTEM OF INSECT;

Showing the respiraratory ganglia and nerves. on a larger scale in fig. 188; where $\mathrm{AB}$, A B, are two pairs of ganglia in the thoracic region, connected by two cords which diverge from one another; and between these. are seen the small respiratory ganglion $a$, and its branches $b$. These branches are distributed to the air-tubes and other parts of the respiratory apparatus, and communicate with those of the other system. We shall find that, even in the highest Vertebrata, there is a portion of the nervous centres which is set apart for the maintenance of the respiratory actions, and which may be regarded as the respiratory ganglion; though it is so closely connected with other parts of the mass as to seem but a part of it ( $\$ 450)$.

447. In the higher Invertebrata, among both the Articulated and the Molluscous classes, we find a tendency to the concentration of the ganglia into one or two masses,-carrying to a

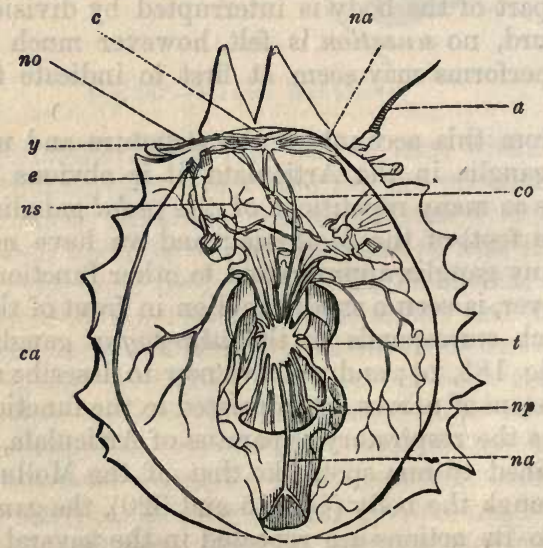

Fig. 189.-Nervous Sxstem of Crab (Maia).

$c a$, upper part of the shell laid open; $a$, antennæ; $y$, eyes; $e$, stomach; $c$, cephalic ganglion; no, optic nerves; co, œesophageal collar; ns, stomato-gastric nerves; $t$, thoracic ganglionic mass; $n p$, nerves of the legs, $n a$, abdominal nerve. 
greater extent that which has been already noticed in the perfect Insect ( $\$ 441)$. Thus in the Spider, the cephalo-thorax contains a single large ganglion ( $t$, fig. 46$)$, from which all the legs are supplied. The same is the case in the Crab, whose nervous system is represented in fig. 189. Besides this mass, $t$, however, which is situated beneath the alimentary canal, there is a single or double cephalic ganglion, $c$, which receives the nerves from the organs of sense, and sends backwards, to communicate with the mass $t$, a pair of cords that separate to give passage to the osophagus, round which they form a sort of collar co. And there are other small ganglia and nerves, connected with the operations of mastication and digestion, which are called stomato-gastric (from two Greek words, meaning the mouth and the stomach).

448. A similar concentration, though with a different arrangement of parts, is seen in the nervous system of the Poulp, one of the Coplialopoda ( $\$ 111)$. There is still a nervous collar through which the œesophagus passes ( $a$, fig. 190) ; but the organs of locomotion being the enlarged tentacula that surround the mouth, the nerves given off to them arise from ganglia that form part of the cephalic mass, $b, b$, instead of being located at a distance from it. At $o$ are seen the optic nerves, proceeding from distinct ganglia; and at $c$ is a heart-shaped ganglionic mass, which seems to bear more resemblance to the proper brain of higher animals, than does any that we elsewhere find in the Invertebrata. In front of this are two ganglia on the middle line, both of which belong to the stomato-gastric system, one supplying the lips and the other the pharynx. From the mass $g$, situated beneath the œsophagus, there pass backwards two cords $m m$, each of which has a ganglion $e$ upon its course, and from this are given off nerves to the general surface of the mantle; and also other two cords, which run backwards to supply the viscera, and especially the gills, - each passing through a long narrow ganglion $r$, before entering them. It would seem as if the ganglia $e$ and $r$ corresponded with the ganglia $c$ and $B$ in the Aplysia; but as if, in consequence of the great enlargement of the cephalic mass, they were proportionally reduced in size.

449. In the nervous system of Vertebrated animals, the ganglia are no longer scattered through the body, but are 
united into one continuous mass; and this mass, constituting the Brain and Spinal Cord, is inclosed within the bony

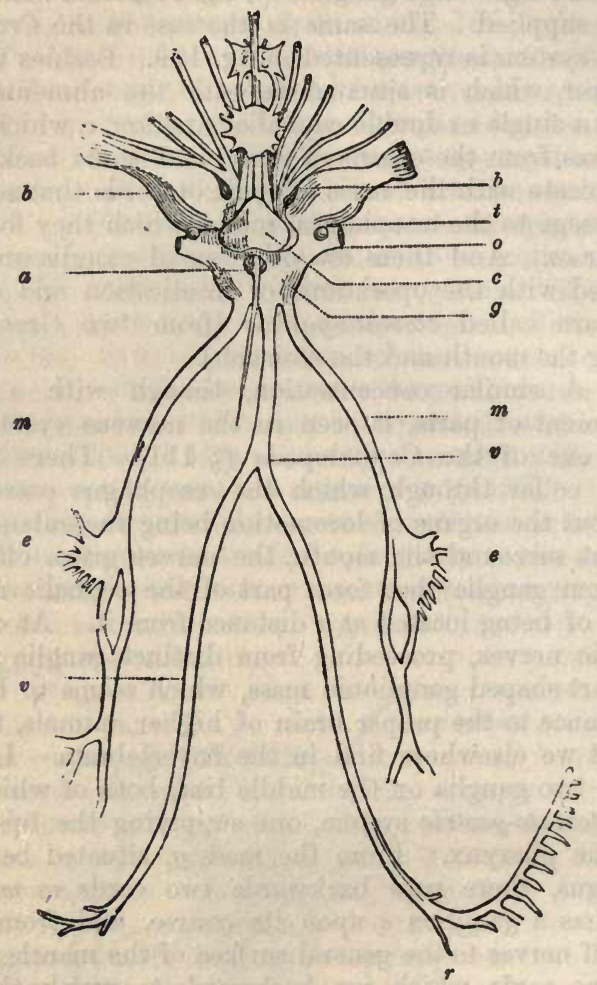

Fig. 190.-Nervous System OF Octopus (PoulP).

casing formed by the skull and vertebral column, in such a manner as to be protected by it from injuries to which it would otherwise be continually liable $(\$ \S 72,73)$. We have seen that among the Invertebrated classes the nervous system has no such peculiar defence, but lies among the other organs, sharing with them the protection afforded by the general hard envelope of the body. But in the Vertebrata, its development 
is so much higher, and its importance so much greater, that special care is taken to guard it from injury. - The term brain is commonly applied to the whole mass of nervous matter contained within the carity of the skull ; but this consists of several distinct parts, which have obviously different characters. The principal mass in Man and the higher Vertebrata is that which is termed the Cerebrum (fig. 195, a); this occupies all the front and upper part of the cavity of the skull, and is divided into two halves or hemispheres by a membranous partition which passes from back to front along the middle line. Beneath this, at the back part of the skull, is another mass, $b$, much smaller, but still of considerable size, termed the Cerebellum; and this also is divided into two hemispheres. At the base or under side of the cerebrum, and completely covered-in by it, are two pairs of ganglia ( 1 and $g$, fig. 196), which belong to the nerves of smell and sight. We shall presently find that these are, relatively speaking, much larger in the lower Vertebrata than in the higher.

450. The several masses of nervous matter contained in the skull, are connected with each other and with the spinal cord by bands of nerve-fibres and tracts of vesicular substance, which serve to bring the brain into connexion with the nervetrunks issuing from the spinal cord. But the Spinal Cord has also distinct properties of its own, analogous to those which have been shown to exist in the chain of ganglia in Insects. The upper part of it, which passes-up into the cavity of the skull, is termed the Medulla Oblongata ( $f^{\prime}$, fig. 197). This is connected with the nerves of respiration, mastication, and deglutition; and may be regarded as combining together the respiratory and the stomato-gastric systems of Invertebrata. The remainder of the spinal cord, which descends through the vertebral column, sends its nerves to the limbs and trunk; and may be regarded as analogous to the chain of ganglia by which the corresponding parts are supplied in Insects.

451. The nerves which issue from the Spinal Cord, all possess two sets of roots; one from the anterior portion of the cord, the other from its posterior portion (fig. 191). The fibres which come-off by these two sets of roots, soon unite into the trunk of the nerve, which thus possesses the properties common to both. It was the great discovery of Sir 
Charles Bell, that the posterior set of roots consists of those fibres that bring impressions from the body in general to the Spinal Cord; which impressions, if carried-on to the Brain,

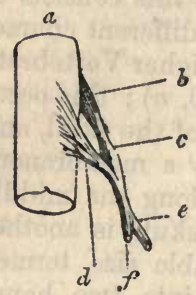

Fig. 191.-Pontron op The SPINat Cord,

Showing the origin of the nerves: $a$, spinal cord; $b$, posterior root; c, ganglion upon its course; $d$, anterior root; $e$, trunk formed by the union of both; $f$, branch.

become sensations. On the other hand, the anterior roots consist of fibres which convey motor influence from the Spinal Cord and Brain, to the muscles of the body. Thus if the spinal cord of an animal be laid bare, and the posterior set of roots be touched, acute pain is obviously produced; whilst, if the anterior roots be irritated, violent motions of the muscles supplied by that nerve are occasioned. Both these roots contain fibres that connect them with the brain as well as with the spinal cord; so that, through the same trunk, either of these centres may act upon the part. We shall presently find that there is good reason to believe the Brain to be the seat of sensibility and of voluntary power; whilst the Spinal Cord is the instrument of those reflex actions which take place automatically, as it were, without direction on the part of the animal, and which are concerned in the maintenance of the organic functions of the body, and in its preservation from injury.

452. The relative proportions which these different parts present, are very different in the several classes of Vertebrata. We find that among the lower, the Sensory Ganglia, or ganglionic centres immediately connected with the organs of sense (which are analogous to the cephalicganglia of the Invertebrata), are very large, and occupy a considerable part of the cavity of the skull ; whilst the Cerebrum and Cerebellum are comparatively small.-The Cerebrum increases, as we ascend the scale, in proportion to the development of the intelligence, and the predominance which it gradually acquires over blind undesigning instinct (Chap. xIv.). Its greatest development is seen in Man. - The Cerebellum seems to be connected with muscular motion, and to bear a proportion in size with the variety and complexity of the movements which the animal performs, serving to harmonise these and blend them together (§ 480). On the other hand, the Spinal Cord, and the nerves 
proceeding from it, are largest in those animals in which the brain is smallest.

453. It is in Fishes that we find the brain least developed, and the cerebral hemispheres bearing the smallest proportion to the other parts. On opening the skull, we usually observe four nervous masses (three of them in pairs) lying, one in front of the other, nearly in the same line with the spinal cord. Those of the first pair are olfactory ganglia, or the ganglia of the nerves of smell (fig. $192 \mathrm{~A}, \mathrm{ol}$ ). In the Shark, and some other Fishes, these are separated from the rest by peduncles or foot-stalks $(\mathrm{B}, \mathrm{ol})$; a fact of much interest, as explaining the arrangement which we find in $\operatorname{Man}(\$ 458)$. Behind these is a pair of ganglionic masses $(c h)$, of which the relative size varies considerably in different fishes (thus in the Cod they are much smaller than those which succeed them, whilst in the Shark they are much larger); these are the cerebral hemispheres. Behind these, again, are two large masses (op), the optic ganglia, in which the optic nerves termi-

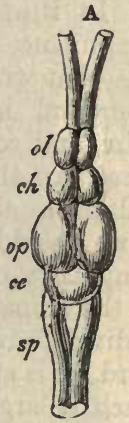

Fig. 192.-Brains of Frshes.

A, Cod ; B, Shark.
B

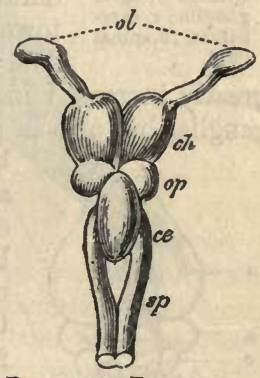
nate. And at the back of these, overlying the top of the spinal cord, is a single mass, the cerebellum (ce); this is seen to be much larger in the active rapacious Shark, the variety of whose movements is very great, than in the less energetic Cod. The spinal cord $(s p)$ is seen to be divided at the top by a fissure, which is most wide and deep beneath the cerebellum, where there is a complete opening between its two halves. This opening corresponds to that through which the osophagus passes in the Invertebrata ; but, as the whole nervous mass of Vertebrated animals is above the alimentary canal ( $\$ 74)$, it does not serve the same purpose in them; and in the higher classes the fissure is almost entirely closed by the union of the two halves of the cord on the central line.

454. In Reptiles we do not observe any considerable adrance in the character of the brain, beyond that of Fishes; 
save that the Cerebral hemispheres are usually larger, extending forwards so as to cover-in the Olfactive ganglia (fig. 193). The Cerebellum is generally smaller, as we should expect from

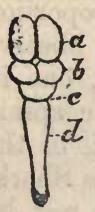

Fig. 193-BRAIN OY REPTILE.

$a$, cerebral hemispheres $b$, optlc ganglia; $c$, cerebellum; $d$,spinal cord.

the inertness of these animals, and the want of variety in their movements ( $\$ 480)$. The Spinal Cord is still very large, in proportion to the nervous masses contained in the skull; and, as we shall hereafter see, its power of keeping-up the movements of the body, after it has been cut-off from connexion with the brain, is very considerable.

455. In Birds, however, we find a considerable advance in the character of the brain, towards that which it presents in Mammalia. The Cerebral hemispheres ( $a$, fig. 194) are greatly increased in size, and cover-in, not only the olfactory ganglia, but also in great part the optic ganglia, $b$. The Cerebellum, $c$, also, is much more developed,

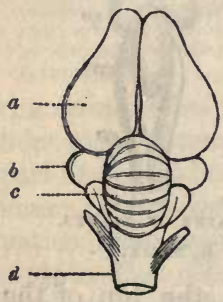

Fig. 194.-BRAlN or Ostrich. as we should expect from the number and complexity of the movements performed by the animals of this class; but it is still undivided into hemispheres. The Spinal Cord, $d$, is still of considerable size, and is much enlarged at the points from which the nerves of the wings and legs originate; in the species whose flight is most energetic, the enlargement is the greatest in the neighbourhood of the wings; but in those which, like the Ostrich, move principally by running on the ground, the posterior enlargement, from which the legs are supplied with nerves, is the more considerable.

456. In Mammals, we find the size of the Cerebral hemispheres very greatly increased, especially as we rise towards Man; whilst the olfactive and optic ganglia are proportionally diminished, and are completely covered-in by them. The surface of the cerebral hemispheres is no longer smooth, as in most of the lower classes, but is divided by furrows into a series of convolutions (fig. 196); by these, the surface over which the blood-vessels come into relation with the nervous matter is very greatly increased; and we find the 
NERVOUS SYSTEM OF MAN.

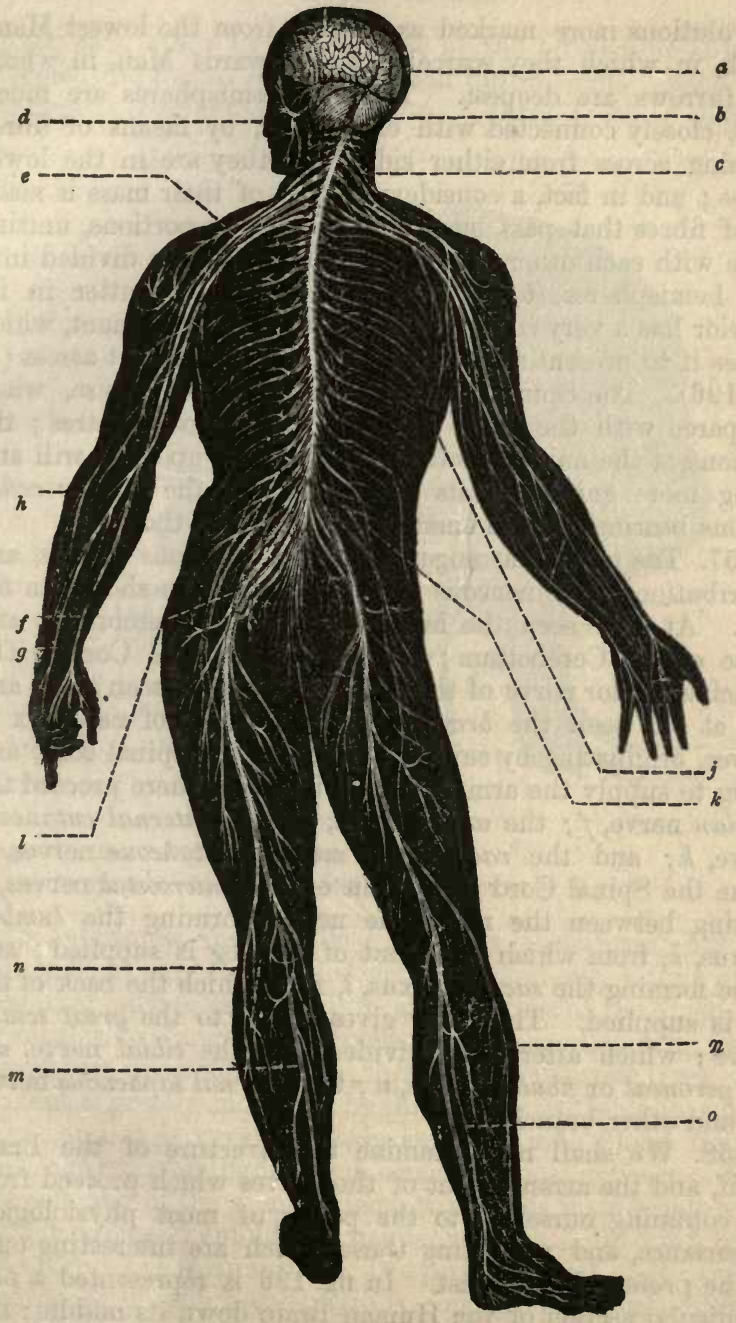

Fig. 195.- Nervous Ststem of Mar. 
convolutions more marked as we rise from the lowest Mammalia, in which they scarcely exist, towards Man, in whom the furrows are deepest. The two hemispheres are much more closely connected with each other, by means of fibres running across from either side, than they are in the lower tribes; and in fact, a considerable part of their mass is made up of fibres that pass among their different portions, uniting them with each other. The Cerebellum, also, is divided into two hemispheres (b, fig. 195); and the grey matter in its interior has a very complex and beautiful arrangement, which causes it to present a tree-like aspect when it is cut across $(d$, fig. 196). The Spinal Cord is much reduced in size, when compared with the other parts of the nervous centres; the motions of the animal now depending more upon its will and being more guided by its sensations, and the simply reflex actions bearing a much smaller proportion to the rest.

457. The general arrangement of the nervous centres, and distribution of the nervous trunks, of Man, are shown in fig. 195. At $a$ are seen the hemispheres of the Cerebrum; at $b$ those of the Cerebellum; and at $c$, the Spinal Cord. The principal motor nerve of the face (the facial) is seen at $d$; and and at $e$ is seen the brachial plexus, a sort of net-work of nerves, originating by several roots from the spinal cord, and going to supply the arm. From this plexus there proceed the median nerve, $f$; the ulnar nerve, $g$; the internal cutaneous nerve, $h$; and the radial and musculo-cutaneous nerves, $i$. From the Spinal Cord are given off the intercostal nerves, $j$, passing between the ribs; the nerves forming the lumbar plexus, $k$, from which the front of the leg is supplied; and those forming the sacral plexus, $l$, from which the back of the leg is supplied. The latter gives origin to the great sciatic nerve; which afterwards divides into the tibial nerve, $m$; the peroneal or fibular nerve, $n$; the external saphenous nerve, 0 ; and other branches.

458. We shall now examine the structure of the Brain itself, and the arrangement of the nerves which proceed from it; confining ourselves to the points of most physiological importance, and neglecting those which are interesting only to the professed anatomist. In fig. 196 is represented a perpendicular section of the Human Brain down its middle; the two hemispheres forming the Cerebrum having been separated 
from each other by the division of the broad fibrous band $f$, termed the corpus callosum, which unites them. Each hemisphere is considered as made up of three lobes or divisions,

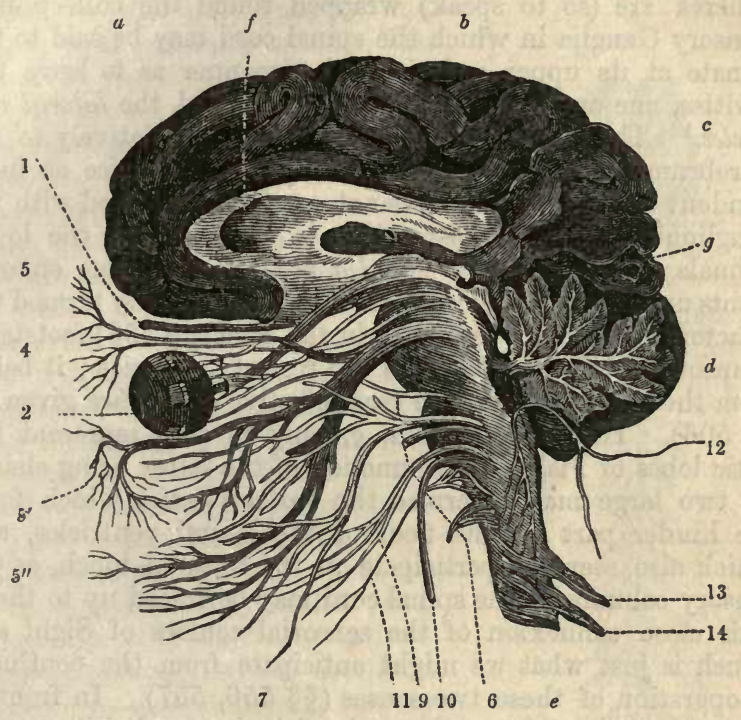

Fig. 196.--Section of ThE BraIN of MaN.

the anterior $a$, the middle $b$, and the posterior $c$; but these are not by any means distinctly marked-out, either on the external surface or in the internal structure of the organ. The vesicular or ganglionic nerve-substance is disposed for the most part upon the exterior, forming a continuous layer, whose extent is greatly increased by the convoluted folds in which it lies; and it is very copiously supplied with blood from the pia mater, a membrane which consists almost entirely of bloodvessels and of the areolar tissue that holds them together, and which so closely enfolds the hemispheres as to dip down into all the furrows of their surface. The principal part of the internal substance of each hemisphere is composed of nerve-fibres, of which some pass between its convolutions and the chain of ganglionic masses on which the cerebrum is superposed, others 
pass from each hemisphere to its fellow through the corpus callosum, whilst others again bring the different convolutions of the same hemisphere into mutual connexion. The hemispheres are (so to speak) wrapped round the collection of Sensory Ganglia in which the spinal cord may be said to terminate at its upper end, in such a manner as to leave two cavities, one on either side, which are called the lateral ventricles. $^{1}$ The Sensory Ganglia are so small relatively to the Cerebrum, that they would scarcely attract notice as independent centres, if they were not carefully compared with the ganglionic centres corresponding to them among the lower animals. The olfactory ganglia are mere bulbous enlargements upon the cords (1), which, though commonly termed the olfactory nerves, are really (as in the Shark, § 453 ) footstalks connecting these ganglia with the rest of the series; it being from these ganglia that the true olfactive nerves are given off (§ 506). The optic ganglia, $g$, only in part represent the optic lobes of Fishes; the function of the latter being shared by two large masses termed the thalami optici, which form the hinder part of the floor of the lateral ventricles, and which also seem to participate in the sense of touch, as the sensory columns of the spinal cord may be traced up to them. This close connexion of the sensorial centres of Sight and Touch is just what we might anticipate from the continual co-operation of these two senses $(\$ \S 556,557)$. In front of the optic thalami is another pair of large ganglionic masses, termed the corpora striata, which is in the like close relation with the motor columns of the spinal cord ; and it is chiefly from them and from the thalami optici, that the fibres proceeding to the surface of the Cerebral hemispheres radiate. The Cerebellum, which has no direct communication with the Cerebrum, but possesses independent connexions of its own with the upper part of the spinal cord, has its grey or vesicular and its white or fibrous substance so peculiarly disposed, as to present in section the appearance delineated at $d$, which is termed the arbor vita, or tree of life.

459. Of the nerves given off within the skull (figs.196,197),

1 There are other ventricles, which are merely spaces left on the middle plane by the imperfect coalescence of the two lateral columns of the nervous axis, like the openings formed by the divergence of the two halves of the nervous cord in Insects (fig. 188). 
the first pair are the olfactive, which proceed from the bulbs (1) of the olfactive peduncles, into the cavity of the nose. Next to these are the optic nerves (2), which may be partly traced to the optic ganglia, and partly to the thalami optici. The third (3), fourth (4), and sixth pairs (6), are nerves of motion only, and are distributed to the muscles of the eye. The ffth pair is for the most part a nerve of sensation only. Before leaving the skull, it divides into three great branches; of which the first (5) passes into the orbit (or cavity in which the eye is lodged), endows the parts contained in it with sensibility, and then comes out beneath the eyebrow, to be distributed to the forehead and temples; the second $\left(5^{\prime}\right)$ passes just beneath the orbit, and makes its way out upon the face, supplying the cheeks, nose, upper lip, \&c., which it endows with sensibility; whilst the third $\left(5^{\prime \prime}\right)$, which (like the spinal nerves) possesses a motor root also, supplies the muscles of mastication with the power of moving, and the parts about the mouth with sensibility. The seventh pair (7), or facial, is the general motor nerve of the face; and this does not endow the parts which it supplies with the least sensibility. Beneath the origin of this nerve is seen the cut extremity of another trunk, that of the auditory nerve (8), or

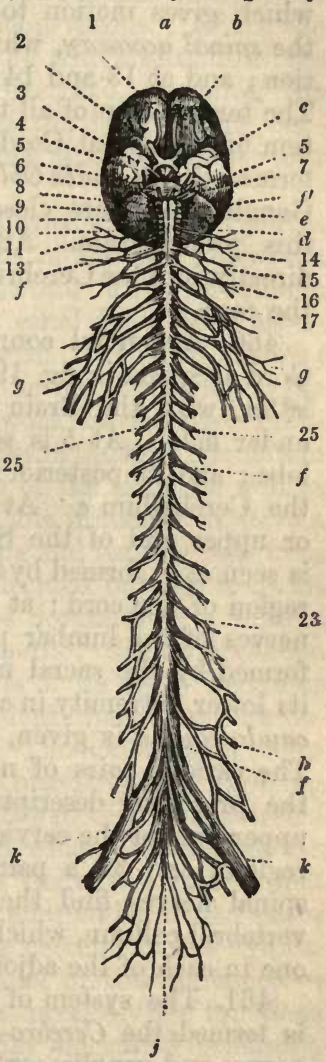

Fig. 197.-BRAIN AND SPINA] Cord of MAN. nerve of hearing. At 9 is seen the glosso-pharyngeal nerve, which supplies the back of the mouth and pharynx, and is concerned in the act of swallowing. Originating from the upper part of the spinal cord (or medulla bloongata) very near B B 2 
this, is the pneumogastric nerve, or par vagum (10), which supplies the lungs and air-passages, and also the heart and stomach. Below this, again, is the hypoglossal nerve (11), which gives motion to the tongue; at 12 is a nerve termed the spinal accessory, which is concerned in the acts of respiration; and at 13 and 14 are two of the regular spinal nerves. The termination of all these nerves is either in that prolongation of the Spinal Cord into the cavity of the skull, which is termed the Medulla Oblongata (fig. 197, $f^{\prime}$ ), or in the Sensory Ganglia which are closely connected with the upper part of this prolongation. Although some of them seem to pass directly into the Cerebrum, it is very doubtful if such is really the case.

460. A general connected view of the Brain and Spinal Cord is given in fig. 197 ; which represents the front of the latter, with the Brain $a$ turned back, so as to expose its under side. At $b$ is seen its anterior lobe; at $c$ its middle lobe ; and its posterior lobe $d$ is almost entirely concealed by the Cerebellum $e \cdot$ At $f^{\prime}$ is shown the Medulla Oblongata, or upper end of the Spinal Cord $f f$. The brachial plexus is seen at $g$, formed by the nerves that originate in the cervical region of the cord; at $h$ is the lumbar plexus formed by the nerves of the lumbar portion; and at $k$ is the sacral plexus formed by the sacral nerves. The spinal cord terminates at its lower extremity in a bundle of nerves $j$, to which the name cauda equina is given, from its resemblance to a horse's tail. The various pairs of nerves from 1 to 14 are the same as in the preceding description; 15 and 16 are nerves from the upper part of the cervical region; 25, a pair from the dorsal region; and 33, a pair from the lumbar region. All these spinal nerves find their way out through apertures in the vertebral column, which are formed by a union of two notches, one in each of the adjoining vertebræ.

461. The system of nerves which has been now described is termed the Cerebro-Spinal; but it is not the only set of nerves and ganglia contained within the bodies of Vertebrated animals. In front of the vertebral column there is a chain of oblong ganglia, which communicate with two large ganglia that lie among the intestines, and with several small ganglia in the head and other parts. They communicate also with the posterior roots of the spinal nerves, on which are another set 
of ganglia ( $c$, fig. 191), that seem to belong to the same system. The nerves proceeding from this system, which is called the Sympathetic, are distributed, not like those of the cerebrospinal, to the skin and muscles, but to the organs of digestion and secretion, and to the heart and blood-vessels. Hence the former system of nerves, being that by which sensations are received and spontaneous motions executed, is called the nervous system of animal life; whilst the latter, being connected with the nutritive processes alone, is termed the nervous system of organic life.

462. What is the nature of the influence which the Sympathetic system exerts over the functions of the parts to which it is distributed, is not yet clearly made out. The sympathetic nerves distributed to the alimentary canal have been ascertained to have the power of exciting its peristaltic actions; and those which are distributed with the blood-vessels (on the coats of which they form a minute net-work) have a direct influence over their calibre, producing changes in the local circulation in obedience to passions and emotions of the mind, as well as to states of other bodily organs. Of this influence we have a familiar example in the acts of blushing and turning pale from agitation of the feelings, and a more decided but less frequent one in the fainting which sometimes occurs from a sudden shock. It is doubtful, however, whether the Sympathetic system really possesses motor filaments of its own; its motor actions being certainly in part dependent upon filaments derived from the cerebro-spinal system. The action of its motor fibres upon the muscular coats of the blood-vessels supplying the glands, serves to regulate the quantity of the fluids secreted by these organs, especially in cases in which the demand for the secretion is intermittent; but as there is evidence that the quality of many secretions may be affected by mental states ( $\$ 353)$, it seems likely that the fibres peculiar to the Sympathetic system $(\$ 60)$ may be the channel of this influence.Although it is still impossible to define precisely the functions of the Sympathetic system, yet it may be stated generally, that in virtue of the two modes of action just explained, it seems to harmonise and blend together the various actions of Nutrition, Secretion, \&c., in such a manner as to bring them into conformity with each other, and with the condition of the organs of Animal life. 
463. We shall now consider, in more detail, the functions of the different parts of the Cerebro-Spinal System in Man and the higher animals; referring occasionally to the Invertebrated classes for illustrations which they can best afford. We shall commence by examining the functions of the Spinal Cord and Medulla Oblongata, which are the parts concerned in reflex action.

\section{Functions of the Spinal Cord.-Reflex Action.}

464. The Spinal Cord of Vertebrated Animals may be considered as a collection of ganglia, analogous to those of which the ganglionic cord of Articulata is composed; these ganglia being united, however, in an unbroken line, instead of being distinct from one another and brought into communication by cornecting cords. There is great difficulty in tracing-out the precise course of the nerve-fibres which form the white strands of the Spinal Cord; and it is doubtful how far any of them form a continuous connexion between the roots of the Spinal Nerves and the Brain. But there can be no doubt that such a connexion is established, either by the fibrous tracts or by the grey matter of the Spinal Cord; experiment having unequivocally shown that the latter participates with the former in this conducting power.

465. When the Cerebro-Spinal system is in full activity, its nerves convey impressions from every part of the body to the Brain, where they are communicated to the mind, - that is, the individual becomes conscious of them, or feels them as sensations. And by the fibres of the same system which pass in the contrary direction, the will acts upon the muscles so as to produce voluntary motion. Now the brain is not in constant action, even in a healthy person; it requires rest; and during profound sleep it is in a state of complete torpor. Yet we still see those movements continuing, which are essential to the maintenance of life; the breathing goes on uninterruptedly, liquid poured into the mouth is swallowed, and the position is changed when the body would be injured by remaining in it. The same is the case in apoplexy, in which the actions of the brain are suspended by pressure upon it. And the same will take place, also, in an animal from which the cerebrum has been removed; or in which its functions are completely suspended by a severe blow on the head. If 
the edge of the eyelid be touched with a straw, the lid immediately closes; if a candle be brought near the eye, the pupil contracts ( $\$ 534)$; if liquid be poured into the mouth, it is swallowed; if the foot be pinched or be burnt with a lighted taper, it is withdrawn; and, if the experiment be made upon a Frog, the animal will leap away as if to escape from the source of irritation. The respiratory movements, too, are kept up with regularity; so that there is no impediment to the continuance of the circulation, and the organic life of the animal may thus endure for some time. In one of the experiments made with the view of ascertaining the degree in which the activity of the Cerebrum is essential to the maintenance of life, a pigeon was kept alive (if alive it could be called) for some months after the removal of its cerebrum,--running when it was pushed, flying when it was thrown into the air, drinking when its beak was plunged in water, swallowing food which was put in its mouth, - though at all other times, when left to itself, appearing like an animal in profound sleep.

466. It is evident, therefore, that we are not to regard the Brain (according to the former opinion of Physiologists, and the belief which is still commonly entertained) as the only centre of nervous power, and as essential to the maintenance of the life of the body; and that we must attribute to the Spinal Cord no small amount of independent power. We might be disposed to infer, from the statements in the last paragraph, that an animal whose brain has been removed can still feel and judge and perform voluntary motions by means of the Spinal Cord; but this, again, would be putting a wrong interpretation upon the phenomena in question. It is observed that the motions performed by an animal in such circumstances are never spontaneous; they are always excited by a stimulus of some kind. Thus a decapitated Frog, after the first violent convulsive movements occasioned by the operation have passed away, remains at rest until it is touched; and then its leg, or even its whole body, will be thrown into sudden action, which immediately subsides again. In the same manner, the action of swallowing is not performed, except when it is excited by the contact of food or liquid (\$195); and even the respiratory movements, spontaneous as they seem to be, would not continue long, unless they were 
excited by the presence of venous blood in the vessels-especially in those of the lungs. These movements are all necessarily linked with the stimulus that excites them;-that is, the same stimulus will always produce the same movement, when the condition of the body is the same. Hence it is evident that the judgment and will are not concerned in producing them; but that they may be rather compared to the movements of an automaton, which are called-forth by touching certain springs.

467. The next question is, whether these movements can be performed without any feeling or sensation, on the part of the animal, of the cause that produces them. It is difficult to imagine that an animal, executing such regular and various actions, which so strongly resemble those it would execute in its complete state, and which are so perfectly adapted to their obvious purposes, can do so without consciousness ; and accordingly some Physiologists have regarded them as furnishing proof that the Spinal Cord possesses the property of sensibility, or, in other words, that an animal whose Brain has been removed can still feel. But this inference will not bear a close examination. Such movements take place, not only when the Brain has been removed and the Spinal Cord remains entire, but even when the Spinal Cord has been itself cut across into two or more portions. Thus if the head of a Frog be cut off, and its Spinal Cord be divided in the middle of the back, so that its fore-legs remain connected with the upper part, and the hind-legs with the lower, each pair of members may be excited to movement by a stimulus applied to itself; but the two pairs will not execute any consentaneous motions, as they will do when the Spinal Cord is undivided. Or, when the Spinal Cord is cut across without removal of the Brain, the lower limbs may be excited to movement, though completely paralysed to the will; whilst the upper remain under the control of the animal's sensation and conscious power.

468. Now although the Frog cannot tell us that it has no sensation in its lower limbs, we have very strong evidence to that effect; for cases are of no infrequent occurrence in Man, in which, the Spinal Cord having been injured in the middle of the back by disease or accident, there is not. only loss of voluntary control over the motions of the legs, 
but loss of sensation also. Further, in several cases of this kind, in which the injury was confined to a small portion of the cord, and the part below was not seriously disturbed, it has been noticed that motions may be excited in the limbs by stimuli applied directly to them,-as, for instance, by tickling the sole of the foot, pinching the skin, or applying a hot plate to its surface; and this without the least sensation, on the part of the patient, either of the cause of the movement, or of the movement itself; the nervous communication, which would otherwise have conveyed the impression to the brain and there given rise to sensation, being interrupted in the spinal cord.

469. By such cases, then, it appears to be clearly proved, that the actions performed by the Spinal Cord, when the Brain has been removed, or its power destroyed, or its communication with the part cut-off, do not depend upon Sensation; but upon a property peculiar to the Spinal Cord, by which impressions, made upon certain parts, necessarily excite motions of an automatic character. By other experiments it has been shown to be necessary for the exercise of this Reflex function (as it has been termed), that an impression should be conveyed by one set of nervous fibres, from the point where the stimulus is applied, to the Spinal Cord; and that a motor impulse, conveyed by another set of filaments, should issue from the Cord to the muscles. The excitor and motor filaments distributed to any part are commonly bound up in the same trunk, and are connected with the same part of the Spinal Cord; so that, if this portion or segment be completely separated from the rest, it may still execute the reflex movements of the parts to which its nerves are distributed; -just as a single segment of a Centipede will continue to move its legs, provided its own ganglion be entire ( $\$ 443)$.

470. 13ut in other instances it happens that we can more clearly distinguish between the excitor and the motor nerves, from their being distributed separately, and being connected with distinct portions of the spinal cord. Thus in the act of deglutition (\$195), the chief excitor nerve is the glosso-pharyngeal ( $\$ 459)$; this conveys the impression made by the contact of food with the pharynx, to the Medulla Oblongata; but it does not convey the motor influence to the muscles, this being accomplished by branches of another nerve, the 
pneumogastric. If the trunk of the glosso-pharyngeal nerve be pinched, an act of deglutition is made to take place; but if it be separated from the Medulla Oblongata, or the pneumogastric nerve be divided, or the Medulla Oblongata itself be destroyed, the movement can no longer be thus excited. Hence we see the necessity of the completeness of this nervous chain or circle-consisting of the nerve proceeding from the part stimulated to the ganglion, the ganglion itself, and the nerve proceeding from the ganglion to the muscles acted-on-in order that any such reflex movements may be produced.

471. The functions of the Spinal Cord appear to be wholly restricted to the performance of movements of this character. The proportion they bear to the motions which are dependent upon sensation and will, varies greatly in different animals; and it may be judged-of with tolerable accuracy, by comparing the relative sizes of the spinal cord and the brain. Thus in the lowest Fishes, the spinal cord seems the principal organ, and the brain an insignificant appendage to it. In Man, on the contrary, the spinal cord is so small in comparison with the brain, as to have been regarded (though incorrectly, as we have seen) in the light of a mere bundle of nerves proceeding from it. In the former, the ordinary movements of the body seem principally to depend upon the spinal cord, being only controlled and directed by the brain ; just as those of Articulated animals are chiefly dependent upon the ganglia of the trunk, being only guided by those of the head $(\$ 442)$. But in Man, those only are left to the spinal cord which are necessary for the maintenance of life; the ordinary motions of the body being for the most part voluntary. Still, as we have just now seen ( $\$ 468)$, reflex movements may be excited through the spinal cord, even in Man, when the influence of the will is cut off; and it is curious to observe, that the stimulus is most powerful when it acts upon the soles of the feet, and that it ceases to produce the same effect, when, by the restoration of the functions of the injured part of the cord, the power of the will over the limbs, and also their sensibility, are regained. There is much reason to believe that, when we are walking steadily onwards, and the mind is intently occupied with some train of thought which engrosses its whole attention, the individual movements 
of the limbs may be kept-up by reflex action, while their general direction is guided by visual sensation ( $\$ 479)$. And even when the mind is sufficiently on the alert to guide, direct, and control the motions of the limbs, their separate actions appear to be performed without any immediate exertion of the will ; and probably depend, therefore, rather upon the reflex function of the spinal cord, than upon the continual influence of the brain.

472. Besides the reflex movements of deglutition and respiration, which have been formerly considered (\$§ 195 and 340 ), and those of locomotion, on which we have now dwelt sufficiently, there are several others of a similar character, all of which have for their object the supply of the wants of the body, or its preservation from injury. Of these the only one which it is desirable here to notice is that of sucking, as performed by the young Mammiferous animal. In this operation there is a very complex union of the actions of different muscles, - those of respiration, together with those of the tongue and lips. So beautifully adapted is this combination to its designed purpose, that it could not be better contrived by the longest experience or the most careful study. Yet we find that the young Mammal commences to perform it without any experience or study, the instant that its lips touch the nipple of its parent. And that it is a reflex action, dependent upon the spinal cord alone for its performance, and requiring a stimulus to excite it, is proved by these remarkable facts ;that it has been performed by human infants which have been born destitute of brain, and which have lived for some hours; and also by puppies whose brain had been removed. These last not only sucked a moistened finger, when it was introduced between their lips, but also pushed out their feet, as the young animal naturally does against the dugs of the parent.

473. There are many irregular actions of the Spinal Cord, however, the careful study of which is of the highest importance to the Medical Man. It is probable that all convulsive movements are produced through its agency; these being for the most part of a reflex character, that is, dependent upon some stimulus or irritation which acts through the nervous circle described in $\$ 470$. Thus, convulsions are not unfrequent in children during the period of teething; and are then 
exicited by the irritation which results from the pressure of the, tooth as it rises against the unyielding gum (\$ 174). They are often occasioned, too, by the presence of indigestible or injurious substances, or of intestinal worms, in the alimentary canal ; and will cease as soon as this is properly cleared out. Again, in Tetanus or "lockjaw" resulting from a lacerated wound, the irritation of the injured nerve is the first cause of the convulsive action; and a similar local irritation is often the origin of Epileptic fits, in which the convulsion is accompanied by loss of consciousness. When these complaints prove fatal, it is usually by suffocation,- the muscles of respiration being fixed by the convulsive action, in such a manner that air cannot pass either in or out.

474. In other forms of convulsive disorders, however, the cause of irritation may directly affect the Spinal Cord, instead of being conveyed to it by the nerves from a distance. This seems to be the case, for example, in Hydrophobia; which terrible complaint is probably due to a poison introduced into the blood by the bite of the rabid animal, and conveyed by the circulating current to the nervous centres. So, when the poison termed Strychnia has found its way into the circulation, the whole Spinal Cord is thrown into such an excitable state, that the slightest stimulus produces the most violent convulsive movements, which succeed one another in extraordinary variety. And the teething-convulsions of infants often depend more upon a peculiar excitable state of the spinal cord, which results from atmospheric impurity, and is removed by change of air, than they do upon the irritation of the gums.-By knowing, as he now does, the part of the nervous system on which these convulsive disorders depend, the Physician is enabled to apply his remedies with much greater precision than heretofore, and to form a much more accurate estimate of the danger which attends them.

\section{Functions of the Ganglia of Special Sense.-Consensual Actions.}

475. It has been seen that the nerves of special sensethose of smell, sight, and hearing-terminate in ganglionic centres peculiar to themselves, which are lodged within the skull, and form part of what is commonly termed the brain, though distinct both from the Cerebrum and the Cerebellum. These Sensory Ganglia are almost the only representatives of 
the brain in the Invertebrated animals; and in Fishes they bear a very large proportion to the other parts, their relative size gradually diminishing as we ascend the scale towards Man. Now when we study the actions of these lower tribes of animals, we find that those which evidently depend upon sensation, especially the sense of sight, are very far from being of the same spontaneous or voluntary character as those which we perform. We judge of this by their unvarying nature,- the different individuals of the same species executing precisely the same movements, when the circumstances are the same,-and this evidently without any choice, or intention to fulfil a given purpose, but in direct respondence to an internal impulse. Of this we cannot have a more remarkable example than is to be found in the operations of Bees, Wasps, and other social Insects; which construct habitations for themselves upon plans which the most enlightened human intelligence could not surpass; yet which do so without hesitation, confusion, or interruption,- the different individuals of a community all labouring effectively for one common purpose, because their impulses are the same (Chapter XIV.)

476. In higher animals we may often notice the effect of similar promptings, by which the various species are guided in their choice of food, in the construction of their habitations, in their migrations, \&c. : but these are frequently modified to a certain degree by the intelligence which they possess. The closure of the pupil when the eye is exposed to a strong light, and its dilatation when the light diminishes ( $\$ 534)$, afford a very marked example of this "consensual" class of movements, which differ from the simply-reflex in requiring the stimulus of sensations, but which are, like them, quite independent both of the reason and of the will. A still more striking illustration, however, is furnished by the mode in which a little Fish, termed the Chcetodon rostratus, obtains its food. Its mouth is prolonged into a kind of beak or snout, through which it shoots drops of liquid at insects that may be hovering near the surface of the water, and rarely fails in bringing them down. Now, according to the laws of Optics, the insect, being above the water whilst the eye of the fish is beneath it, is not seen by it in its proper place; since the rays do not pass from the insect to the fish's eye in a straight line ( $\$ 528)$. 
The insect will in fact appear to the fish a little above the place which it really occupies; and the difference is not constant, but varies with every change in the relative positions of the fish and the insect. Yet the wonderful instinct with which the fish is endowed, leads it to make the due allowance in every case ; doing that at once, for which a long course of experience would be required by the most skilful Human marksman, under similar circumstances.

477. Though the Intelligence and Will of Man in a great degree supersede his consensual impulses, in the same manner as they hold in subordination his reflex movements $(\S 471)$, yet we have many indications of the direct operation of sensations in determining respondent movements. Of this kind are the start produced by a loud sound, particularly if unexpected; the closure of the eyes to a dazzling light, or on the sudden approach of a body that might injure them; the production of sneezing by a dazzling light; the provocation of laughter by tickling, or by some sight or sound to which no distinct ludicrous idea or emotion attaches itself ; and the excitement of vomiting by highly disagreeable sensations, as the sight of a loathsome object, an offensive smell, a nauseous taste, or by tickling the back of the mouth with a feather. ${ }^{1}$ None of these "consensual" movements can be excited without the consciousness of the subject of them; and this circumstance marks them out as belonging to a different category from the "reflex" movements performed through the instrumentality of the Spinal Cord.-In some convulsive disorders, the attacks are excited by causes that act through the organs of sense: thus, in Hydrophobia we observe the immediate influence of the sight or sound of liquids; and in many Hysteric subjects, the sight of a paroxysm in another individual is the most certain means of its induction in themselves.

478. But we may trace the agency of the Sensory Ganglia

1 This is the most ready way of exciting vomiting, when it is desired to free the stomach from poisons or unwholesome articles of food; but care must be taken not to apply the feather so low down as to cause it to be grasped by the muscles concerned in the act of swallowing; for its irritation, instead of producing vomiting, will then occasion an act of deglutition ( $(195)$, which may draw the feather from the hand of the operator, and carry it down into the stomach of the patient. 
in Man and the highest Vertebrata, not merely in their direct and independent operation on the Muscles, but also in the manner in which they participate in all voluntary action. For it is now well established, that the Will cannot bring about any definite movement, except under the guidance of sensations, derived either from the muscles themselves, or through some channel of information which indicates what the muscles are doing. It is for want of the guiding sensations afforded by the ear, that persons who are born deaf are also dumb, the will not being able to make use of the muscles concerned in vocalization; and where, by long training, some imperfect power of speech has been acquired, it has been gained by attention to the sensations arising from the muscular exertion of the organs themselves. It is by the guiding influence of the visual sensations, that the movements of the two eye-balls are made to correspond; and, in children born completely blind, it may be observed that the eyes roll about without any harmony, though a very slight perception of light is sufficient to bring their motions into consent. So, again, if the arm or the leg be so paralysed that its sensibility is lost whilst its muscles are still under the power of the will, that power can only be exerted to occasion movement by the assistance of the sight; a mother, for example, so affected, being only able to hold her infant upon her arm so long as she looks at it; and a man, whose legs are thus paralysed, being only able to sustain himself in standing or walking by constantly looking at his legs.

479. It seems to be obviously through the shorter channel afforded by the Sensory Ganglia, that those actions are performed, which, though originally directed by Intelligence and Will, come by frequent repetition to be so completely automatic as to resemble the instinctive actions of the lower animals. Thus it is within the experience of almost every one, that he occasionally walks through the streets with his mind intently and continuously engaged on some train of thought, without the least atten tion to, or even consciousness of, the direction he is taking; yet he avoids obstacles, and follows his accustomed course, obviously under the guidance of his visual sense, whilst the movements of his limbs are kept-up by reflex action ( $\$ 471)$; and on awaking, as it were, from his reverie, he may find that he has thus been automa- 
tically conducted to a place very different from that to which he had intended going. So, again, we may read aloud, or play on a musical instrument, without being at all aware of what we are about, the whole attention being absorbed by some engrossing thoughts or feelings within. And it seems to be in this manner that the movements of Somnambulists are guided; their Cerebrum being, as it were, cut-off from communication with the outer world, and their Sensory Ganglia acting independently of it.

\section{Function of the Cerebellum. - Combination of Muscular Actions.}

480. Much discussion has taken place of late years respecting the uses of the Cerebellum; and many experiments have been made to determine them. That it is in some way connected with the powers of motion, is now generally admitted. Its size in the different tribes of Vertebrated animals bears a pretty close correspondence with the variety and energy of the movements performed by them; being greatest in those animals which require the constant united effort of a large number of muscles to maintain their usual position, whilst it is least in those which require no muscular exertion for this purpose. Thus in animals that habitually rest and move upon four legs, there is but little occasion for any organ to combine and harmonize the actions of their several muscles; and in these the Cerebellum is small. But among the more active predaceous Fishes (as the Shark),-Birds of most powerful and varied flight (as the Swallow, which not only flies rapidly, but executes the most complicated evolutions in pursuit of its Insect prey with the greatest facility), - and Mammals which can maintain the erect position and use their extremities for other purposes than support and motion,-we find the Cerebellum of much greater size : whilst in Man, who surpasses all other animals in the number and variety of the combinations of muscular movement which he is capable of executing, it attains its largest dimensions and its greatest complexity of structure.

481. From experiments upon all classes of Vertebrated Animals, it has been found that, when the Cerebellum was removed, the power of walking, springing, flying, standing, or maintaining the equilibrium of the body, was destroyed. It did not seem that the animal had in any degree lost volun- 
tary power over its individual muscles; but it could not combine their actions for any general movement of the body. The reflex movements, such as those of respiration, remained unimpaired. When an animal in this state was laid on its back, it could not recover its former posture; but it moved its limbs or fluttered its wings, and evidently was not in a state of stupor. When placed in the erect position, it staggered and fell like a drunken man; not, however, without making efforts to maintain its balance.-Phrenologists, who attribute a different function to the Cerebellum, have attempted to put aside these results, on the ground that the severity of the operation was alone sufficient to produce them; but (as wo have already seen, $\S 465$ ) after a much more severe operation-the removal of the Cerebral Hemispheres, the Cerebellum being left untouched-the animal could stand, walk, fly, maintain its balance, and recover it when disturbed.

482. The motions of the body in the Invertebrated classes, being simple in their nature, and probably all of a reflex character (\$ 442), do not require a Cerebellum; and we do not find in them any nervous mass which clearly represents this organ.

\section{Functions of the Cerebrum.-Intelligence and Will.}

483. From the facts already stated, it is tolerably clear that the Cerebrum is the organ by which we reason upon the ideas that are excited by sensations, - by which we judge and decide upon our course of action, - and by which we put that decision into practice, by issuing a mandate (as it were), which, being conveyed by the nervous trunks proceeding from the brain to the muscles, excites the latter to contract. It is a common, but entirely erroneous idea, that Reason or Intelligence is peculiar to Man; and that the actions of the lower classes of Animals are entirely due to Instinct. There can be no doubt, however, that reasoning processes exactly resembling those of Man are performed by many Mammals, such as the Dog, the Horse, and the Elephant ; and it is probable that although we are best acquainted with these animals, on account of their tendency to associate with Man, there are others which have powers yet higher. We must admit that an animal reasons, when it profits by experience, and obviously adapts its actions to the ends it desires to gain, 00 
especially when it departs from its natural instincts to do this. Such is continually the case with the animals just mentioned, as will appear from some striking examples to be mentioned hereafter (Chap. XIV.). We perceive the presence of Intelligence also in the differences of character which we encounter among the various individuals of the same species; thus every one knows that there are stupid Dogs and clever Dogs, ill-tempered Dogs and good-tempered Dogs, as there are stupid Men and clever Men, ill-tempered Men and goodtempered Men. But no one could distinguish between a - stupid Bee and a clever Bee, or between a good-tempered Wasp and an ill-tempered Wasp; simply because all the actions of these animals are prompted by an unvarying instinct.

484. Among Birds, too, there are many manifestations of Intelligence, which constitute a remarkable distinction between their actions and those of Insects; though the instinctive tendencies of the two classes bear a close correspondence with each other. Their mode of life is nearly the same, so that Birds may be called the Insects of the Vertebrated series, whilst -Insects may be regarded as the Birds of the Articulated; and there are several curious points of analogy in the structure of their bodies. The usual arts which Birds exhibit in the construction of their habitations, in procuring their food, and in escaping from danger, must be regarded (like those of Insects) as instinctive; on account of the uniformity with which they are practised by different individuals of the same species, and the perfection with which they are exercised on the very first occasion, independently of all experience. But in the adaptation of their operations to particular circumstances, Birds display a variety and fertility of resource far surpassing that which is manifested by Insects; $\rightarrow$ as for instance, when they make trial of several means, and select that one which best answers the purpose ; or when they make an obvious improvement from year to year in the comforts of their dwelling; or when they are influenced in the choice of a situation by peculiar conditions, such as in a state of nature can scarcely be supposed to affect them. All these are obvious indications of an Intelligence which Insects do not possess ; that which is most wonderful in the actions of the latter (and there are none more wonderful) being the same in all the individuals of one species, being uninfluenced 
by education, and being performed under the direction of an Intelligence much higher than the boasted reasoning power of Man.

485. In the classes of RePTILES and Fishes, the manifestations of Intelligence are so slight as to be scarcely distinguishable. We find them capable of such an amount of education as enables them to recognise individuals from whom they have been accustomed to receive food; but they seem to have very little further power of profiting by experience; and we do not find that individuals ever shape-out for themselves a new course which can be regarded as purely rational. This very low grade of Intelligence obviously corresponds with the very rudimentary development of the Cerebrum in these classes ( $\$ 453,454)$.

The contrast between Instinct and Intelligence will be more fully displayed in a future Chapter; in which also a general account will be given of the Mental Operations to which the Cerebrum of Man is subservient.

\section{CHAPTER XI.}

ON SENSATION, AND THF ORGANS OF THE SENSES.

486. ALL save the very lowest kinds of Animals possess, there is good reason to believe, a consciousness of their own existence, first derived from a feeling of some of the changes taking place within themselves; and also a greater or less amount of sensibility to the condition of external things. How far any such endowment can be possessed by creatures which are destitute of a nervous system, and which are little else than particles of animated jelly, may be questioned. But there can be no reasonable doubt that where a nervous system exists, whatever consciousness any Animal may possess of that which is taking place within or around itself, is all derived from impressions made upon the extremities of certain of its nervous fibres; which, being conveyed by them to the central sensorium, are there felt ( $\$ 430)$. Of the mode in which the impression, hitherto a change of a material character, is there made to act upon the mind, we are absolutely ignorant; we only know the fact. Hence, although we comC C 2 
monly refer our various sensations to the parts at which the impressions are made,-as, for instance, when we say that we have a pain in the hand, or an ache in the leg,-we really use incorrect language ; for, though we may refer our sensations to the points where the impression was made on the nerve, they are really felt in the brain. This is evident from two facts ; first, that if the nervous communication of the part with the brain be interrupted, no impressions, however violent, can make themselves felt; and, second, that if the trunk of the nerve be irritated or pinched anywhere in its course, the pain which is felt is referred, not to the point injured, but to the surface to which these nerves are distributed. Hence the well-known fact that, for some time after the amputation of a limb, the patient feels pains which he refers to the fingers or toes that have been removed; this continues until the irritation of the cut extremities of the nervous trunks has subsided.

487. Among the lower tribes of Animals, it would seem probable that there is no other kind of sensibility than that which is termed general or common, and which exists, in a greater or less degree, in almost every part of the bodies of the higher. It is by this that we feel those impressions, made upon our bodies by the objects around us, or by actions taking place within, which produce the various modifications of pain, the sense of contact or resistance, the sense of variations of temperature, and others of a similar character. From what was formerly stated $(\$ 63)$ of the dependence of impressions made on the sensory nerves upon the action of the blood-vessels, it is obvious that no parts destitute of the latter can receive such impressions, or (in common language) can possess sensibility. Accordingly we find that the hair, nails, teeth, tendons, ligaments, fibrous membranes, cartilages, and bones, whose substance either contains no vessels, or but very few, are either completely incapable of receiving painful impressions, or have but very dull sensibility to them. On the other hand, the skin and other parts which usually receive such impressions, are extremely vascular; and it is interesting to observe that some of the tissues just mentioned, when new vessels form in them in consequence of diseased action, become acutely sensible. It does not necessarily follow, however, that parts should be sensible in a degree proportional to 
the amount of blood they contain; since this blood may be sent to them for other purposes. Thus, it is a condition necessary to the action of Muscles, that they should be eopiously supplied with blood ( $\$ 591)$; but they are not acutely sensible: and Glands, also, the substance of which has very little sensibility, receive a large amount of blood for their peculiar purposes.

488. But besides the general or common sensibility which is diffused over the greater part of the body of most animals, there are certain parts which are endowed with the property of receiving impressions of a peculiar or special kind, such as sounds or odours, which would have no influence upon the rest; and the sensations which these impressions excite, being of a kind very different from those already mentioned, arouse ideas in our minds such as we should never have formed without them. Thus, although we can gain a knowledge of the shape and position of objects by the touch, we could form no notion of their colour without sight, of their sounds without hearing, or of their odours without smell.

489. The nerves which convey these special impressions are not able to receive those of a "common" kind: thus, the Eye, however well fitted for seeing, would not feel the touch of the finger, if it were not supplied with branches from the 5 th pair, as well as by the optic nerve. Nor can the different nerves of special sensation be affected by impressions that are adapted to operate on others: thus, the ear cannot distinguish the slightest difference between a luminous and a dark object; nor could the eye distinguish a sounding body from a silent one, except by seeing its vibrations. But Electricity possesses the remarkable power, when transmitted along the several nerves of special sense, of exciting the sensations peculiar to each; and thus, by proper management, this single agent may be made to produce flashes of light, distinct sounds, a phosphoric odour, a peculiar taste, and a pricking feeling, in the same individual at one time. The inference which might hence be drawn - that Electricity and Nervous agency are identical-is nevertheless premature, as will be shown hereafter $(\S 585)$. 


\section{Sense of Touch.}

490. By the sense of Touch is usually understood that modification of the common sersibility ( $\$ 487$ ) of the body, of which the surface of the skin is the especial seat. In some animals, as in Man, nearly the whole exterior of the body is endowed with it in no inconsiderable degree; but in others, as in the larger number of Mammals, most Birds and Reptiles, and many Fishes, the greater part of the body is so covered by hairs, scales, or bony plates, as to be nearly insensible; and the faculty is restricted to particular portions of the surface, which often possess it in a very high degree. The sensory impressions, by which we receive the sensation of Touch, are made by the objects themselves upon the nerves which are distributed to the Skin; the general structure of which has been already described (§§ 36-38). Of the papillae which are thickly set upon many parts of its surface, some contain looped tufts of blood-vessels without nerves; and as these are found to be largest where the Epidermis is thickest (as, for example, in the pads on the under side of the Dog's foot), it seems obvious that they minister, not to sensation, but to the nutrition of that protective coating ( $\$ 492)$. But in other papillæ the blood-vessels are comparatively scanty, their interior being chiefly occupied by little cushions of condensed areolar substance to which the sensory nerves proceed ; and as their Epidermic coating is thin, and as the degree of sensibility of any part of the skin bears a close correspondence to the number of these papillæ which are included within a given area of its surface, it can scarcely be doubted that they are the special instruments of the sense of Touch.

491. The true skin, or Cutis ( $\$ 37$ ), from which alone leather is prepared, is thicker in most Mammals than in Man; but the thickness of the skin does not by any means involve (as is commonly supposed) deficient sensibility. Thus, in the Spermaceti Whale it has been observed that the sensory nerves, which are destined to be distributed on the skin, pass through the blubber without giving off any considerable branches, but spread out into a network of extreme minuteness as soon as they arrive near the surface. It is a fact well known to Whale-fishers, especially to those who pursue this species, that these animals have the power of 
communicating with each other at great distances. It has often been observed, for instance, that, when a straggler is attacked, at the distance of several miles from a "school," a number of its fellows bear down to its assistance in an' almost incredibly short space of time. It can scarcely be doubted that the communication is made through the medium of the vibrations of water, excited by the struggles of the animal, or perhaps by some peculiar movements specially adapted for this purpose, and propagated through the liquid to the immense surface of the skin of the distant Whales.

492. The sensibility of the true skin would be too great, if it were not protected by the Epidermis ( $\$ 38$ ), the thickness of which varies considerably, according as the part is to be endowed with acute sensibility, or to be protected from impressions of too strong a nature. Thus it is particularly thin at the ends of the fingers, and on the surface of the lips, which are used for feeling; but is thick on the palm of the hand, which is used for firmly grasping, and which would be continually suffering pain if its sensibility were too acute; and it is still thicker on the sole of the foot, especially on the heel and the ball of the great toe, where pressure has to be sustained.

493. Although the fingers of Man and of the Quadrumana, being endowed with peculiar sensibility, are their special organs of touch, yet we find that they cease to be so in most of the other Mammalia, whose extremities are adapted only for support and locomotion, and are terminated by hard claws or hoofs that completely envelop them. In many of these, we find the lips and tongue employed as the chief organs of touch ; in the Elephant, this sense is evidently possessed very acutely by the little finger-like projection at the end of its trunk; and in several other cases the vibrissce or whiskers are its special instruments, the bulbs of their long stiff hairs being copiously supplied with sensory nerves.

494. A curious modification of the sense of Touch appears to exist in Bats. It has been found that these animals, when deprived of sight and (as far as possible) of hearing and smelling also, still flew about with equal certainty and safety, avoiding every obstacle, passing through passages only just large enough to admit them, and flying through places with which they were previously unacquainted, without striking against the objects near which they passed. The same result happened 
when threads were stretched in various directions across the apartment. Hence some Naturalists were inclined to attribute to the Bat the possession of a sixth sense unknown to Man; but Cuvier correctly pointed out that this idea becomes unnecessary, if we attribute to the delicate membrane of the wings (as we are justified in doing) a high degree of tactile sensibility, so as to receive impressions from the pulses of the air that are produced by the action of the wings and modified by the neighbourhood of solid bodies.

495. The only idea communicated to our minds by the sense of Touch, when this is exercised in its simplest form, is that of resistance; and we cannot form a notion either of the size or shape of an object, or of the nature of its surface, by feeling it, unless we move the object over our own sensory organ, or move the latter over the former. By. the various degrees of resistance which we encounter, we estimate the hardness or softness of the body; and by the impressions made upon the papillæ, when they are moved over its surface, we form our idea of its smoothness or roughness. It is by attention to the muscular movements we execute, in passing our hands or fingers over its surface, that we acquire our ideas of its size and figure; and hence we perceive that the sense of touch, without the power of moving the tactile organ over the object, would have been of comparatively little use.

496. This sense is capable of improvement to a remarkable degree; as we see in persons who have become more dependent upon it in consequence of the loss of their sight. This doubtless results, in part, from the increased attention which is given to the sensations; and partly from the greater acuteness or impressibility of the organ itself, arising from the frequent use of it. Amongst other remarkable instances of this kind was that of Saunderson, who, though he lost his sight at two years old, acquired such a reputation as a mathematician, that he obtained a Professorship at Cambridge. He exhibited, in several ways, an extraordinary acuteness in his touch; but one of his. most remarkable faculties was the power of distinguishing genuine medals from imitations, which he could do more accurately than many connoisseurs in full possession of their senses.

497. The sense of temperature is of a different character from common tactile sensibility; and either may be lost, 
without the other being affected. It is rather of a comparative than of a positive kind ; that is, we form our estimate of temperature rather by comparing it with that to which our body (or the part of it employed to test the heat or cold) has been previously exposed, than by any knowledge which we derive through the sensation as to the actual degree of heat or cold to which the organ is exposed. Thus, if we plunge one hand into a basin of hot water, and the other into cold, and then transfer both of them to a basin of tepid water, this will feel cold to the hand which has been previously accustomed to the heat, and warm to the other. In the same manner, the temperature of Quito, which is situated half-way up a lofty mountain, is felt to be chilly by a person who has ascended from the burning plains at its base, whilst it seems intensely hot to another who has descended from its snow-capped summit ; the residents in the town at the same time regarding it as moderate,-neither hot nor cold. It is a curious circumstance, that a weak impression made on a large surface seems more powerful than a stronger impression made on a small surface; thus, if the fore-finger of one hand be immersed in water at $104^{\circ}$, and the whole of the other hand be plunged ip water at $102^{\circ}$, the cooler water will be thought the warmer; whence the well-known fact, that water in which a finger can be held will scald the whole hand.

498. Where any special organs of Touch exist in Invertebrated Animals, they are for the most part prolongations from the portion of the head near the mouth. This is the case with the arms of the Cuttle-fish, and with the tentacula of the lower Mollusca which are similar in position. Among Crustacea and Insects, the antennce or feelers (fig. 198, a, a) appear to be the special organs of touch. These are frequently

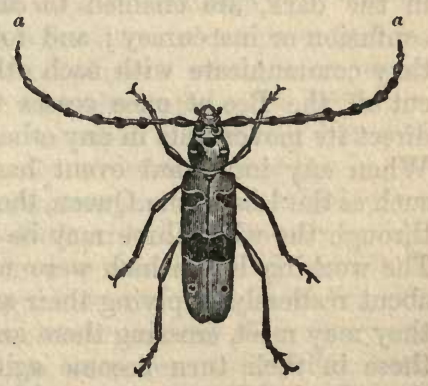

Fig. 198.-CAPRICORN-BeEtLE. very long, and present an extraordinary variety in their forms, of which some are depicted in fig. 199. They contain; for the most part, a large number of joints (in the Mole- 
Cricket above 100), and are very flexible. This flexibility enables them to be turned towards any object under examination by the Insect; and when the animal is walking, we see them constantly being applied to the surfaces of the bodies which it approaches, in a manner which leaves little doubt that they are used as organs of touch. It is no objection to this view, to say that, as their surfaces are hard, no delicate sensations can be received through them; for the slightest

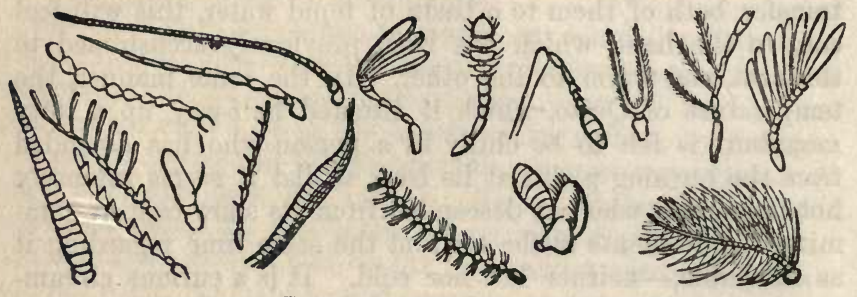

Fig. 199.-VARIOUSLY-FORMEd ANTENNE OP INSECTs.

contact of their firmest points with a hard substance, may produce a sense of resistance which will afford to the animal the information which it requires. The stick used by the blind man in feeling his way, serves a very similar purpose. - It appears to be by sensations received through their antennæ, that Bees and other Insects which naturally work in the dark, are enabled to carry-on their labours without confusion or inaccuracy; and to be by the same means, that they communicate with each other. When the antennæ are cut off, the Bee at once ceases to work, and seems unable to direct its movements in any other way than towards the light. When any important event has happened in a community, such as the loss of the Queen, the spreading of the intelligence through the whole hive may be watched by a close observer. The working bees which were near her are observed to run about restlessly, applying their antennæ to those of the others they may meet, crossing them and striking lightly with them; these in their turn become agitated and do the same; and thus the intelligence is speedily propagated throughout the hive. In the same manner, when two bees meet each other out of their hives, they seem to reconnoitre one another for some time by the movements of their antennæ; and often 
keep up these movements for a considerable period, as if carrying on a close conversation. That the Antennæ are delicate organs of Touch can, therefore, be scarcely questioned.

\section{Sense of Taste.}

499. The sense of Taste, like that of touch, is excited by the direct contact of particular substances with certain parts of the body; but it is of a much more refined nature than touch, inasmuch as it communicates to us a knowledge of properties which that sense would not reveal to us. All substances, however, do not make an impression on the organ of taste. Some of them have a strong savour, others a slight one, and others again are altogether insipid. The cause of these differences is not understood; but it may be remarked that, in general, bodies which cannot be dissolved in water have no savour; whilst most of those which are soluble have a taste more or less strong. Their solubility, in fact, seems to be one of the conditions requisite for their action on the organ of taste ; for when that organ is completely dry, it does not receive any sensation from solid bodies brought into contact with it, which may have the most powerful taste if reduced to a fluid form; and there are substances known, which, being perfectly insoluble in water, are insipid if applied to the tongue when it is covered as usual with a watery secretion; but which have a strong taste when they are dissolved in some other liquid, spirit of wine for instance.

500. The sense of Taste has for its chief purpose, to direct animals in their choice of food; hence its organ is always placed at the entrance to the digestive canal. In the higher animals, the tongue is the principal seat of it; but other parts of the mouth are also capable of receiving the impressions of certain savours. The mucous membrane which covers the tongue is copiously supplied with blood-vessels; and is thickly set, especially upon its upper surface, and towards the tip, with papilla, resembling in structure those of the skin, but larger. These papillæ, however, are not all sensory; for some, which are of conical form, are covered with a firm horny epithelium, and their function seems to be chiefly mechanical. These "conical" papillæ are very strongly developed in the tongues of many of the lower Mammalia, to which they impart a particular roughness; thus it is by their means that a Dog 
cleans of all its flesh the bone he licks, and that the Lion, by a single stroke of his tongue, can take off the skin from any part of the Human body. The tongue itself is made-up of muscular substance, which accomplishes the varied movements that are required in the acts of mastication and in the production of articulate sounds. It is supplied with nerves from the third division of the fifth pair, from the glossopharyngeal, and from the hypoglossal ( $\$ 459$ ). The last is the motor nerve of the tongue; the first is the one chiefly concerned in the conveyance of sensory impressions from the front and sides of the tongue; and the other (the glossopharyngeal) seems to have for its office to convey those impressions from the back of the tongue which excite the muscles of swallowing to action ( $(470)$, as well as those which produce the sensation of nausea and excite the act of vomiting. The gustative papillæ, which have a very thin epithelial covering, are for the most part supplied from the fifth pair ; and the branch of this proceeding to the tongue is known as the lingual nerve. When they are called into action by the contact of substances having a pleasant savour, they not unfrequently become very turgid, and rise-up from the surface of the mucous membrane; in this manner is produced the roughness that is felt on the surface of any portion of the tongue or inside of the cheek, against which a piece of barley-sugar or other similar substance has lain for some little time.

501. A considerable part of the impression produced by many substances, is received through the sense of Smell rather than by that of Taste. Of sthis any one may convince himself by closing the nostrils and breathing through the mouth only, whilst holding in the mouth, or even rubbing between the tongue and the palate, some aromatic substance ; its taste is then scarcely recognised, although it is immediately perceived when the nasal passages are re-opened, and its effluvia are drawn into them. There are many substances, however, whose taste, though not in the least dependent upon the action of the nose, is nevertheless of a powerful character; such are sugar, salt, quinine, and vinegar. Others, again, by irritating the mucous membrane, produce a sense of pungency allied to that which the same substances (strong acids, for instance, pepper, or mustard) will produce when applied to the skin for a sufficient length of time. Such sensations, 
therefore, are evidently of the same kind. with thase of Touch, differing from them only in the degree of sensibility of the organ through which they are received; and through these the sense of Taste is more nearly related to that of Touch, than is either of the other forms of special sensibility.

502. This sense has a very important function in most animals which possess it, - that of directing them in their ehoice of food. Most of the lower animals will instinctively reject the articles of food that would be pernicious to them; thus even the voracious Monkey will seldom touch fruits of a poisonous character, though their taste may be agreeable ; and animals whose digestive apparatus is adapted to one kind of food, will reject all others. It may be stated, as a general rule, that substances of which the taste is agreeable to us are useful and wholesome articles of food, and vice versa; but there are many signal exceptions to this. It is interesting to remark that in Man, when the reasoning powers are obscured by disease, his instincts in regard to food often manifest themselves strongly, and frequently constitute the best guide in its administration ; thus, there are many cases of fever in which the physician is in doubt whether wine will be injurious or beneficial, and in which he will usually find the disposition of the patient to reject it, or his readiness to receive it, to be his best guide. And in general it may be remarked that, in illness, the desire of the patient for food, or his disposition to take it, pretty certainly indicate the fitness or unfitness of the system to digest and appropriate it.

503. The tongue presents nearly the same structure among the Mammalia in general, as in Man ; but in Birds it is usually cartilaginous or horny in its texture, and destitute of nervous papillæ, so that the sense of taste cannot be very acute in any of those animals. Several of them use their tongues for other purposes, - the Woodpecker, for instance, to transfix insects, and the Parrot to keep steady the nut or seed which is being crushed between the jaws. In some Reptiles the tongue is large and fleshy; in others long and slender ; in others, again, it is almost entirely deficient: but in no instance does it seem to minister to any acute sense of taste. In Fishes it is for the most part absent. Many Invertebrated animals possess a tongue; but its uses are for the most part mechanical. Thus the tongue of the limpet is a 
powerful rasp (resembling that in fig. 107), by which it rubs down the sea-weeds on which it feeds; whilst the tongue of the Bee (fig. 289) forms a channel through which it draws-up the juices of flowers. In most Insects, the palpi, small jointed appendages in the neighbourhood of the mouth ( $\$ 172)$, seem to answer the purpose of an organ of taste; being observed to be in incessant motion whilst the animal is taking food, touching and examining it before it is introduced into the mouth.

\section{Sense of Smell.}

504. Certain bodies possess the property of exciting in us sensations of a peculiar nature, which cannot be perceived by the organs of taste or touch, and which seem to depend upon emanations that spread from them through the air, producing what we term odours. It appears probable that odours are, in reality, very finely-divided particles of the odoriferous substance; and this idea derives support from the fact that most volatile bodies are more or less odorous, whilst those which do not readily transform themselves into vapour, have little or no fragrancy in their natural state, but possess strong odorous properties as soon as they are converted into vapourby the aid of heat, for instance. The most powerful and penetrating odours are for the most part those of bodies already in a gaseous state, - such as sulphuretted and carburetted hydrogen; or of fluids which readily pass into the state of vapour, as the vegetable essential oils. But there are some solid substances, as musk, which are very strongly odorous; and which yet do not appear to diffuse themselves through the air in the state of vapour. The odoriferous particles of these must be of extreme minuteness ; for the substances do not seem to lose weight by freely imparting their peculiar scent to an unlimited quantity of air. Thus the experiment has been tried, of keeping a grain of musk freely exposed to the air of a room of which the doors and windows were constantly open, for a period of ten years ; the air, thus continually changed, was completely impregnated with the odour of musk; and yet at the end of that time, the particle was not found to have suffered any perceptible diminution in weight.

505. In order that we should become sensible of odours, it 
seems necessary that the odoriferous particles should come into actual contact with the membrane on which the nerve of smell is spread out. In this respect, the sense of Smell agrees with the senses of taste and touch; whilst it differs from those of sight and hearing, which take cognisance of changes that are produced by vibrations or undulations in the surrounding medium. It is, moreover, desirable that these odoriferous particles should be conveyed by the air, and not be diffused through fluid; for though it is necessary to the perfection of the sense of smell that the olfactory membrane should be kept moist, too great a quantity of fluid upon its surface deadens its peculiar sensibility, - as we find to be the case when we are suffering under an ordinary "cold." Hence it is only in air-breathing animals, that the sense of Smell can possess any considerable acuteness.

506. The most advantageous position of this organ is evidently at the commencement of the respiratory passages; so that the air which is being received into the lungs may pass through it and be tested (as it were) by its peculiar sensibility. In all the air-breathing Vertebrata we find a pair of cavities, the nasal fossae (fig. 200), which are situated between the mouth and the orbits. They possess two orifices, - the anterior nares, or nostrils $(b)$, usually opening upon the front of the face,-and the posterior nares, which open into the upper part of the pharynx $(c)$. The two cavities are separated from each other by a vertical partition, which passes backwards and

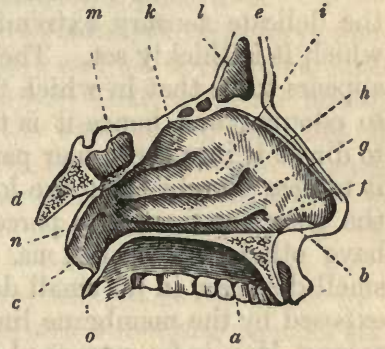

Fig.,200.-VERTicai SEction of THE Nasat Cavity.

$a$, mouth ; $b$, nostril; $c$, posterior opening ; $d$, portion of the base of the skull ; $e$, forehead; $f, h$, passages between the spongy bones, $g, i, k ; l$, frontal sinus; $m$, sphenoidal sinus; $n$,opening of Eustachian tube ; 0 , curtain of the palate. forwards on the middle line; their sides are formed by the various bones of the face, and by the cartilages of the nose; their extent is very considerable, especially in animals that have a prolonged muzzle. The interior of these cavities is lined by a delicate mucous membrane, whereon the Olfactory nerves, which enter through a multitude of minute orifices in 
the roof of the cavity, are distributed ; and the extent of its surface is increased, by its being folded over certain projections from the walls of the cavity, which are termed spongy bones. Of these there are three in Man $(g, i, k)$. Prolongations of this membrane are carried also into cavities hollowed out in the neighbouring bones, which are termed sinuses. Thus we have the frontal sinuses $l$, situated just above the nose, between the eyebrows; and the sphenoidal sinuses $m$, situated further back. There is also a large cavity hollowed out in the bone of the upper jaw on either side. The membrane which lines these is kept moist by its own secretion; and it is covered with vibratile cilia, the office of which seems to be to prevent that secretion from unduly accumulating, by conveying it over the surface of the membrane to the outlet.

507. The mechanism of the sense of Smell is very simple. When air charged with odoriferous particles passes over the membrane that lines the nose, some of these particles are delayed by the mucous secretion that covers it, and act upon the delicate sensory extremities of the olfactory nerve with which it is thickly set. The highest part of the nasal cavity appears to be that in which there is the most acute sensibility to odours ; and hence it is that when we snuff the air, so as to direct it into the upper part of the nose, instead of allowing it to pass simply along the lower portion from the anterior to the posterior nares, we perceive delicate odours which would have otherwise escaped us. The acuteness of the sense of smell depends, in no small degree, upon the extent of surface exposed by the membrane lining the nasal cavity; and in this respect Man is far surpassed by many of the lower Mammalia, especially among the Carnivora, Ruminantia, and some Pachydermata. The extreme delicacy of this sense in Deer, Antelopes, \&c., is well known, from its being a source of great difficulty to the hunter, who cannot advance near enough to attack them, except by stealing upon them in the direction contrary to that of the wind. In these animals it serves as the chief means by which they are warned of the proximity of their enemies; in the Carnivora, on the other hand, it serves to direct them to their prey. In general, however, it seems to have for its office to assist in directing animals to their food, and in warning them of the presence of noxious vapours. 
508. Besides receiving the Olfactory nerve, the mucous membrane of the nose is supplied by branches of the Fifth pair; this nerve endows it with common sensibility, and also receives the impressions produced by acrid or pungent vapours, which act upon it in the same way as the corresponding fluids do upon the tongie. Such vapours are felt by the irritation they produce, rather than smelt; and the impression they occasion gives rise to the reflex action of sneezing, by which they are driven from the nose $(\$ 342)$. Hence this action may be excited by an irritating agent (such as snuff) after the olfactory nerve has been divided, if the branches of the fifth pair be entire; whilst it does not take place when the fifth pair is paralysed, even though the sense of smell may be retained. This sense loses much of its acuteness, however, when the branches of the fifth pair supplying its organ can no longer discharge their functions ; for the membrane then becomes dry from the want of its proper secretion, and the odoriferous particles are consequently not properly applied to it.

509. Among animals that live in water, the olfactory organs cannot act to the like advantage; and we do not find much provision made for this sense. In the Whale tribe, the nostrils serve as the channels by which the water is expelled, that has been drawn-in through the mouth (\$185); they are situated at the top of the head, and are known as blow-holes. In Fishes, the nasal cavity has no posterior opening; but the surface of its lining membrane is very much extended by its arrangement in folds, which are sometimes disposed in a radiated manner around a centre, and sometimes parallel like the teeth of a comb. There are many Invertebrated Animals, from whose actions it may be judged that they possess a delicate sense of smell, although the precise seat of it cannot be assigned. This is the case especially with Insects, Crustacea, and the higher Mollusca. The lining membrane of the airtubes of Insects appears to be delicately sensitive to irritating vapours ( $\$ 443)$; but we have no evidence that it ministers to the sense of Smell properly so called.

\section{Sense of Hearing.}

510. By this sense we become acquainted with the Sounds produced by bodies in a certain state of vibration. The vibrations which sonorous bodies undergo, are communicated by 
them to the air, producing in it a series of undulations or waves, by which the sound is conveyed to a great distance. These undulations spread more widely as they become more distant from the sounding body, just like the ripples produced on the surface of the water when we throw a stone into it; and in proportion as they spread, they become less powerful. This is the reason why Sound becomes less intense as the sounding body is more distant. Although: air is the usual conducting medium for the sonorous undulations, liquids or solids may answer the same purpose. Thus if a person hold his head under water, whilst two stones are struck together, also under water, even at a considerable distance, he will hear the sound produced by the blow with extreme distinctness, and even with painful force. Or if the ear be laid against one end of a long piece of timber, whilst a scratch with a pin be made on the other, or a watch be laid upon it, even the faint sounds thus produced will be heard very distinctly. That a medium of some kind is necessary to convey the sonorous vibrations, is proved by the fact, that if a bell be made to ring in the receiver of an air-pump from which the air has been exhausted, no sound is heard, though its ringing becomes audible as soon as the air is allowed to re-enter.

511. It. is a fact of much importance, in regard to the action of the organ of Hearing, that sonorous vibrations which have been excited and are being transmitted in a medium of one kind, are not imparted with the same readiness to others. The following conclusions have been drawn from experimental inquiries on this subject. I. Vibrations excited in solid bodies may be transmitted to water without much loss of their intensity, although not with the same readiness that they would be communicated to another solid. II. On the other hand, vibrations excited in water lose something of their intensity in being propagated to solids; but they are returned, as it were, by these solids to the liquid, so that the sound is more loudly heard in the neighbourhood of those bodies, than it would otherwise have been. III. The sonorous vibrations of solid bodies are much more weakened by transmission to air; and those of air make but little,impression on solids. IV. Lastly, sonorous vibrations in water are transmitted but feebly to air ; and those which are taking place in air are with difficulty communicated to water; but the con- 
munication is rendered much more easy by the intervention of a membrane extended between" them.

512. The Auditory nerve, or nerve of Hearing, is adapted to receive and transmit to the brain the sonorous undulations produced in the surrounding medium by vibrating bodies. Now, it is obvious that it may be affected by these in various ways, especially in animals that inhabit the water. The vibrations excited in the liquid will be transmitted to the solid parts of the head, and thence to the nerve contained in it, without much interruption; and this independently of any special apparatus of hearing. Indeed, the simplest form of this apparatus is only designed to give increased effect to the vibrations thus excited in the solid parts of the head; for it consists merely of a cavity excavated in their thickness, which cavity is flled with fluid, and is lined by a membrane whereon the auditory nerve is minutely distributed. This is the condition of the organ of hearing in the Mollusca, where any such exists ; and also in many of the Crustacea. In those of the latter class which chiefly inhabit air, however, this cavity is excavated in the surface of the shell covering the head, and is shut-in by a membrane which is exposed to the surrounding medium. According to the principle (Iv.) mentioned in the last paragraph, the liquid contained in the chamber will be thrown into undulation by vibrations in air, as well as by those of water; so that those animals which possess this kind of apparatus are able to hear much better in air, than are those in which the cavity is completely shut-in by stony walls. Of the degree in which sonorous vibrations may be communicated to our own auditory nerves through the solid parts of the skull, we may easily satisfy ourselves by closing the ears carefully, and placing any part of the head against a solid body which communicates with the one in vibration. In this manner we may hear the sounds produced by the latter with considerable distinctness, though accompanied by an unpleasant jarring. A deaf gentleman was once agreeably surprised to find that, when smoking his pipe, with the bowl resting on his daughter's pianoforte, he could distinctly hear the music she was producing from it; and many deaf persons may be made to hear conversation, by holding a piece of stick between their own teeth, and placing it against the teeth of the person speaking. 
513. In animals which have the organ of hearing constructed upon the simple plan just described, the force of the vibrations of the fluid contained in the cavity is increased by several minute stony concretions suspended in it : these act according to the second principle just stated ( $\$ 511)$. They are termed otolithes, or ear-stones; and some traces of them may be found even in Man and the higher animals. ${ }^{1}$

514. We see, then, that a cavity excavated in the solid walls of the head, covered-in externally by a membrane, having the auditory nerve distributed upon its walls, and filled with fluid, is the simplest form of the organ of hearing; and may be regarded as including all that is essential to the exercise of this function. No more complicated apparatus is to be found in any of the Invertebrata; and even in the lowest Fishes there is but little variation from this type. On the other hand, in Man and the higher Vertebrata we find a very complex structure, adapted to render the faculty much more perfect; enabling us to receive impressions which make us.aware, not only of the presence of a sounding body, but of its nature, its direction, the pitch and peculiar quality of the sound; and also, it is probable, taking cognizance of sounds much fainter than those which would be perceptible to the lower animals. Yet even in the most complicated forms of the organ of hearing, we shall find that the essential part is still the same as that which forms the whole organ in the lower tribes; and also that the faculty is retained, though in an inferior degree, when by disease or injury the accessory parts are prevented from acting.-To the structure of the Ear of Man we shall now proceed.

515. The organ of hearing in Man may be divider into three parts - the external, the middle, and the internal ear. The former is the fibro-cartilaginous appendage placed on the outside of the head, to receive and collect the sounds which are to be transmitied to the interior; the two latter divisions are excavated in a bone of remarkable solidity, the petrous (stony) portion of the temporal bone. The uses of the different

1 Vesicles containing otoliths which are kept in rapid movement within them by ciliary action, are found in immediate contiguity with the cephalic ganglia of the lower Mollusks, or are even imbedded in their substance; and these seem to constitute the most rudimentary form of an organ of hearing. 
hollows and elevations on the surface of the external ear of Man are not very apparent; but it is probable that they direct the sonorous undulations towards the entrance of the canal which leads to the middle ear. The form of the external ear in many Quadrupeds evidently adapts it to this purpose; and there are several which have the power of changing its direction by muscular action, in such a manner as to enable it to cutch most advantageously the faintest sounds from any quarter. This is especially the case with animals that are naturally timorous, such as the Hare or the Deer; these have usually very large external ears. But it is among the Bat tribe-whose residence in the dark recesses of caverns and excavations makes their eyes of comparatively little use to them, and causes them to depend greatly for guidance in their movements upon the sense of hearing-that we find the greatest development of the external ear (fig. 201).

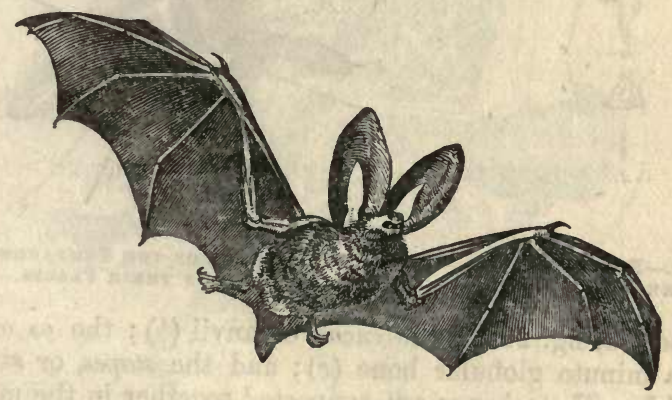

Fig. 201.-LONG-EARED BAT.

516. The canal hollowed-out in the temporal bone ( $d$, fig. 204) into which the external ear collects the sonorous vibrations, passes inwards until it is terminated by a membrane stretched across it, which is called the membrana tympani, or membrane of the drum of the ear $(g)$. This forms the outside wall of a cavity $(h)$ which constitutes the middle ear, and which is bounded on the inside by a bony wall that separates it from the internal ear. The cavity of the tym. panum is not one of the essential parts of the organ; for nothing analogous to it exists either in Fishes or in the lower Reptiles. It contains air; and communicates with the back 
of the nasal cavity ( $n$, fig. 200) by a canal termed the Eustachian tube ( $k$, fig. 204). The partial or complete closure of this tube, occasioned either by swelling of its lining membrane or by the viscid secretion from it, produces the slight deafness common among those who are suffering from "colds." Within the cavity of the tympanum, there is a very curious apparatus of small bones and muscles, which serves to establish a connexion between the membrane of the drum and the small membrane covering the entrance to the internal ear. These bones are four in number; and are termed the malleus or

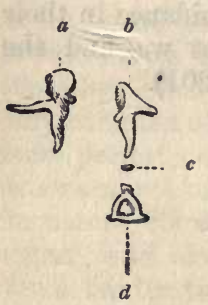

Fig. 202.-Boves or THE EAR.

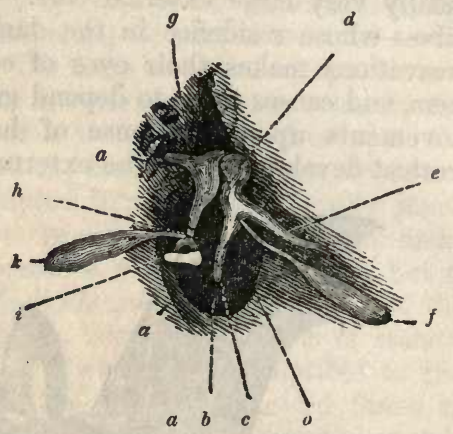

Fig. 203.-Cavity of THE TrMPaNUM, with the Bones in their Places.

hammer (a, fig. 202); the incus, or anvil (b) ; the os orbiculare, a minute globular bone $(c)$; and the stapes, or stirrupbone $(d)$. These bones are connected together in the manner represented in fig. 203 ; where $a$ a represents the wall of the tympanic cavity; $b$, the membrana tympani; $c$, one of the long processes of the malleus, which is attached to the membrane; $d$, the head of the malleus, which articulates with the incus; $e$, the other long process of the malleus, which is acted-on by the minute muscle $f$, that serves to tighten the tympanum ; $g$, the incus, of which one leg is in contact with the wall of the cavity, whilst the other is connected with the orbicular bone $h$; $i$, the stapes, of which the upper end is connected with the orbicular bone, whilst the lower (which is of an oval form) is attached to the membrane that covers the entrance to the internal ear; and $k$ is a small muscle which 
acts upon this bone in such a manner as to relax the tympanum.

517. The use of this apparatus is evidently to receive the sonorous vibrations from the air, and to transmit them to the membrane forming the entrance to the internal or essential part of the organ of hearing; in such a manner, that the sonorous vibrations excited in the latter may be much more powerful than they would be if the air acted immediately upon it. The usual state of the membrane of the tympanum. appears to be rather lax or slack; and when in this condition,

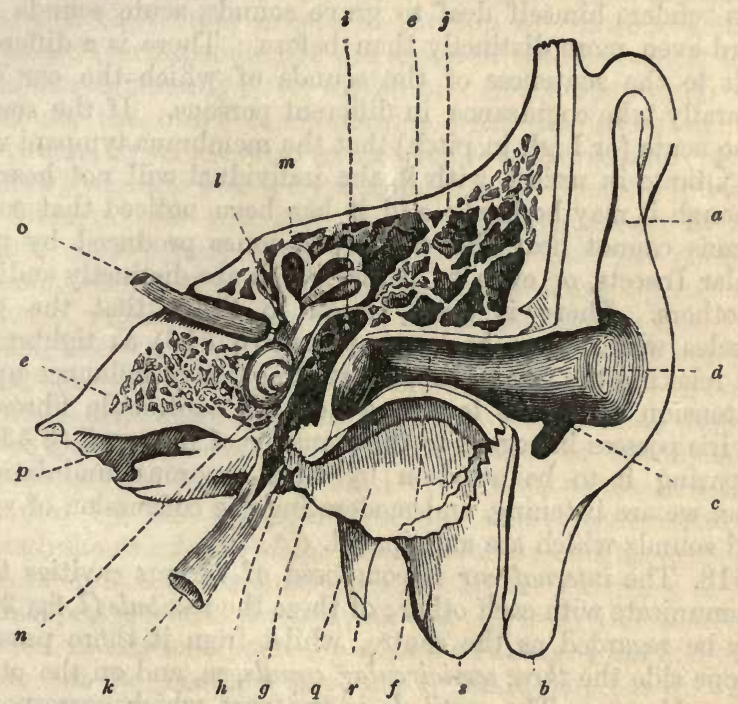

Fig. 204.-Verticat Section of the Organ of Hearting in MaN.

The internal portions are proportionately enlarged to make them more evident: $a, b, c$, the external ear; $d$, entrance to the auditory canal $f ; e, e$, petrous portion of the temporal bone, in which the internal ear is excavated; $g$, membrane of the tympanum; $h$, cavity of the tympanum, the chain of bones being removed; $i$, openings from the cavity into the cells $j$ excavated in the bone; on the side opposite the membrana tympani are seen the fenestra ovalis and rotunda ; $k$, Eustachian tube ; $l$, vestibule ; $m$, semicircular canals ; $n$, cochlea ; 0 , auditory nerve ; $p$, canal by which the carotid artery enters the skull; $q$, part of the glenoid fossa which receives the head of the lower jaw; $r$, styloid process of the temporal bone.

it vibrates in accordance with grave or deep tones. By the action of a small muscle lodged within the Eustachian tube, it 
may be tightened, so as to vibrate in accordance with sharper or higher tones; but it will then be less able to receive the impressions of deeper sounds. This state we may artificially produce either by holding the breath and forcing air into the Eustachian tube, so as to make the membrane bulge-out by pressure from within; or by exhausting the cavity by an effort at inspiration with the mouth and nostrils closed, which will cause the membrane to be pressed inwards by the external air. In either case the hearing is immediately found to be imperfect; but it will be observed that while the experimenter thus renders himself deaf to grave sounds, acute sounds are heard even more distinctly than before. There is a different limit to the acuteness of the sounds of which the ear can naturally take cognizance, in different persons. If the sound be so acute (or high in pitch) that the membrana tympani will not vibrate in unison with it, the individual will not hear it, although it may be loud; and it has been noticed that some persons cannot hear the very shrill tones produced by particular Insects, or even by Birds, which are distinctly audible to others. There is good reason to think that the two muscles which have been mentioned ( $\$ 516)$ as tightening and relaxing the tympanum, exert a regulative influence upon its tension analogous to that which the contractile fibres of the iris possess in regard to the diameter of the pupil ( $\$ 534)$; preparing it to be acted-on by faint sonorous undulations when we are listening, and moderating the concussion of very loud sounds which are anticipated.

518. The internal ear is composed of various cavities that communicate with each other; of these the vestibule ( $l$, fig. 204) may be regarded as the centre, whilst from it there pass-off on one side the three semicircular canals, $m$, and on the other the cochlea, $n$. The vestibule is the part which corresponds with the simple cavity that constitutes the whole organ of hearing in the lower animals ( $\$ 512)$, and the others may be regarded as extensions of it for particular purposes. It communicates with the cavity of the tympanum by a small orifice in the bony wall that separates them, termed the fenestra ovalis (oval window); but this orifice is closed by a membrane, to which the lower end of the stapes is attached. The three semicircular carals are passages excavated in the solid bone, and lined by a continuation of the same membrane as that 
which lines the vestibule; each passes-off from the vestibule and returns to it again. The cochlea, $n$, also is a cavity excavated in the hard bone, and lined by a continuation of the same membrane; its form is almost precisely that of the interior of a snail-shell (whence its name), being a spiral canal which makes about two turns and a half round a central pillar. This canal is divided into two, however, by a partition that runs along its whole length; which partition is partly formed by a very thin lamina of bone, and partly (in the living state) by a delicate membrane. The two passages do not communicate with each other except at the top or centre; at their lower end (corresponding to the mouth of the snail-shell) they terminate differently; for whilst one freely opens into the vestibule, the other communicates with the cavity of the tympanum, by an aperture termed the fenestra rotunda (round window), which is closed by a membrane. ${ }^{1}$ Thus the internal ear communicates with the cavity of the tympanum by two minute orifices only, - the fenestra ovalis and the fenestra rotunda,- both of them closed by membranes, against the former of which the stapes abuts, whilst the latter is free.

519. The whole internal ear is lined by a delicate membrane, on which the auditory nerve (o, fig. 204) is very minutely distributed, especially on the membranous portion of the partition between the two passages of the cochlea. The cavities are completely filled with fluid, which is set in vibration by the movements of the stapes, communicated through the membrane of the fenestra ovalis; and these vibrations are probably rendered more free by the existence of the second aperture- the fenestra rotunda. It is by the influence of these undulations upon the expanded fibrils of the auditory nerve, that the sensation of sound is produced; but in what way the different parts of the labyrinth (as this complex series of cavities is not unaptly called) contribute to the performance of this function, is not yet known. In all Fishes but the lowest, the three semicircular canals exist; they have, however, no vestige of a cochlea. In the true Reptiles, a rudiment of the cochlea may be generally discovered. In Birds, this cavity is more completely formed, though the passage is not spiral, but is nearly straight; of its real

1 There is a double spiral staircase constructed exactly on this plan in Tamworth church. 
character, however, there can be no doubt, from its being divided, like the cochlea of Mammals and of Man, by a membranous partition on which the nerve is spread out.

520. From the circumstance that in almost every instance in which the semicircular canals exist at all, they are three in number, and lie in three different directions, corresponding to those of the bottom and two adjoining sides of 'a cube, it has been supposed (and with much probability) that they assist in producing the idea of the direction of sounds. It has been also supposed that the cochlea is the organ by which we judge of the pitch of sounds; and this would seem to be not improbable, especially when we compare the development of the cochlea in different animals, with the variety in the pitch of the sounds which it is important they should hear distinctly, especially the voices of their own kind. The compass of the voice (that is, the distance between its highest and its lowest tones) is much greater in Mammals than in Birds; as is also the length of the cochlea. In Reptiles, which have little true vocal power, the cochlea is reduced to its lowest form; and in the Amphibia, it disappears altogether.

521. That the Vestibule, and the passages proceeding from it, constitute even in Man the essential part of the organ of hearing, is evident from the fact, that when (as happens not unfrequently) the membrana tympani has been destroyed by disease, and the chain of bones has been lost, the faculty is not by any means abolished, though it is deadened. In this state, the vibrations of the air must act at once upon the membrane of the fenestra ovalis, as in the lower animals which possess no external or middle ear; instead of striking the membrane of the tympanum, and being transmitted along the chain of bones.

522. It. has been stated ( $\S 510)$ that the sensation of hearing is produced by the successive undulations or vibrations communicated to the Ear from the sonorous body, either by the air, or by a liquid or solid medium. This is the case with all continuous sounds or tones; but single momentary sounds, such as those produced by the díscharge of a pistol, the blow of a hammer, the ticking of a watch, or the beat of a clock, make their impression on the ear by a single shock. All continuous tones are in fact caused by a succession of such shocks, communicated to the ear with sufficient rapidity 
for the interval between them not to be distinguished. Thus, if we cause a tight string to vibrate by pulling or striking it, we occasion, not one vibration only, but a long succession of vibrations (Mechan. Philos. \$ 187); every one of which gives a new impulse to the air, and produces a new impression on the organ of hearing. These vibrations we can see, when they are sufficiently extensive ; and we can always feel them, by placing the finger on the string. In the same manner, the vibrations of a bell or of a tuning-fork continue long after the first blow; and these, though we cannot see them, may be readily felt with the finger. It is, in fact, in their power of continuing to vibrate after they have been struck, that the peculiarity of these resonant bodies consists. In other instances in which continuous tones are produced, the vibrations are kept-up by the continued application of the original cause, and cease as soon as it is withdrawn : this is the case, for instance, in the string of the violin when set in vibration by the bow, and in the flute and organ-pipe when caused to sound by the passage of air through them.

523. In all these cases, then, the continuous tones are due to a succession of impulses given by the sounding body to the air; and according to the rapidity with which the impulses succeed one another, will be the pitch of the sound. It is not difficult to ascertain by experiment the number of such impulses required to produce particular tones. The lowest note $(\mathrm{C})$ given by any musical instrument (that which is produced by an open organ-pipe of 32 feet long, or by a stopped pipe of 16 feet) requires 16 impulses per second ; ${ }^{1}$ but continuous tones have been produced by impulses occurring at the rate of only 7 or 8 per second; so that the impression produced upon the ear by each must have lasted at least 1-7th or 1-8th of a second. On the other hand, it has been ascertained that the ear can appreciate tones produced by 24,000 impulses in a second; so that the limit already adverted to ( $(517)$ must be above this tone, the pitch of which is about 4 octares above the highest $F$ of the pianoforte.

524. The strength or loudness of musical tones depends

I A backward as well as forward vibration must take place with every impulse ; consequently the number of the vibrations is twice that of the impulses. 
upon the force and extent of the vibrations communicated by the sounding body to the air. Thus, when we draw the middle of a tight string far out of the straight line, and then let it go, a loud sound is produced, and we can see that the space through which the string passes from side to side is considerable. As the extent of the vibrations of the string diminishes, the sound becomes less powerful; and when we can no longer see the vibrations, but can only feel them, the sound is faint. The length of the undulations in the air corresponds with that of the vibrations in the sounding body; and consequently they will strike upon the tympanum with more or less force, according as these are longer or shorter. The cause of the differences in the timbre or quality of musical tones, - such, for instance, as those which exist between the tones of a flute, a violin, and a trumpet, all sounding a note of the same pitch,-are unknown; but they probably depend upon the different form of the vibrations.

525. The faculty of hearing, like that of sight, may be very much increased in acuteness by cultivation; but this increase depends rather upon the habit of attention to the faintest impressions made upon the organ, than upon any change in the organ itself. This habit may be cultivated in regard to sounds of some one particular class ; all others being heard as by an ordinary person. Thus the watchful North American Indian recognises footsteps, and can even distinguish between the tread of friends or foes, whilst his companion who lives amid the busy hum of cities is unconscious of the slightest sound. Yet the latter may be a Musician, capable of distinguishing the tones of all the different instruments in a large orchestra, of following any one of them through the part which it performs, and of detecting the least discord in the blended effects of the whole,-effects which would be, to his coloured companion, but an indistinct mass of sound. In the same manner, a person who has lived much in the country is able to distinguish the note of every species of bird which lends its voice to the general concert of Nature; whilst the inhabitant of a town hears only a confused assemblage of shrill sounds, which may impart to him a disagreeable rather than a pleasurable sensation.-Of the direction and distance of sounds, our ideas are for the most part formed by habit. Of the former we probably judge, in great degree, by the 
relative intensity of the impressions received by the two ears; though we may form some notion of it by either singly ( $\$ 520)$. Of the distance we judge by the intensity of the sound, comparing it with that which we know the same body to produce when nearer to us. The Ear may be deceived in this respect as well as the eye ( $\$ 566)$; thus the effect of a full band at a distance may be given by the subdued tones of a concealed orchestra close to us; and the Ventriloquist produces his deceptions by imitating, as closely as possible, not the sounds themselves, but the manner in which they would strike the ear.

\section{Sense of Sight.}

526. By the faculty of Sight we are made acquainted, ir the first place, with the presence of light; and by the medium of that agent we take cognizance of the forms of surrounding bodies, their colours, dimensions, and positions. It is desirable that a short account should be here given of the laws of the transmission of light; since, without the knowledge of them, the beautiful action of the Eye cannot be understood.

527. The rays of light uniformly travel in straight lines, so long as they traverse the same medium (air, water, or glass, for instance), without obstruction. When issuing from a single luminous point into space, they diverge or separate, in such a manner as to cover a larger and larger surface as they proceed ; and the intensity of the light diminishes in the same proportion. But when the rays pass from one medium to another either more or less dense, they are bent out of their straight course, or refracted; unless they should happen to pass from the one to the other in a direction perpendicular to the plane which separates them. This may be made evident by a very simple experiment. Place a coin or any heavy body ( $a$, fig. 205) at the bottom of a basin, and then

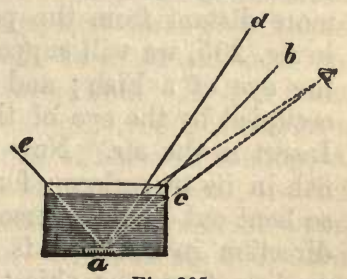

Fig. 205. retreat from it until the coin is hidden by the edge of the basin ; if water be then poured-in, up to the level c, the coin will again become visible, although neither its own place nor that of the observer has undergone any change. The reason of this 
is, that the rays of light, as they pass out of the water, are bent downwards, or from the perpendicular; so that they reach the eye of the observer when situated at a lower point than that at which the rays would have arrived if they had proceeded in a straight line. Thus the eye, situated at the end of the line $a c$, could not see the coin in a straight line, because rays passing in that line would be interrupted by the opaque sides of the basin; but it receives the ray which was passing through the water in the direction $a d$, and which was bent downwards at the moment of quitting it. If the eye had been placed directly over the coin, however, so that the ray passing through the latter to it would have emerged from the water in a direction perpendicular to its surface, no change in the apparent place of the object would have been made by pouring-in the water; since a ray that passes from one medium to another, however different, in a direction perpendicular to the surface which separates them, is not refracted. Those rays which pass-out most nearly in this direction are refracted least, whilst those which pass-out most nearly in the horizontal direction are refracted most.

528. The general law of refraction then is,-that all rays passing from a dense to a rare medium are refracted from the perpendicular, the degree of change being less as they are near the perpendicular, and greater as they depart from it. On the other hand, when rays pass from a rare medium into a dense one, they are bent towards the perpendicular; and this in a greater or less degree, according as their direction is more distant from the perpendicular, or nearer to it. Thus, in fig. 205, we will suppose the point $a$ to be the position of the eye of a Fish; and the end of the line $a c$ (previously occupied by the eye of the observer) to be the position of an Insect in the air. Now this insect will not be seen by the fish in its true place; for a ray passing from it to $c$ would be so bent out of its course as not to reach the point $a$. The direction in which it is really seen is $a d$; for the ray proceeding from the object to the surface of the water, there undergoes such a refraction that it is bent downwards to $a$; and, as we always judge of the place of an object by the direction in which the rays last come to the eye, the insect is seen by the fish at $d$, that is, considerably above its real place (§ 476). 
529. When the surface which separates the two media is not flat, but is either convex or concave (bulging or hollowedout), a very important alteration is produced in the direction

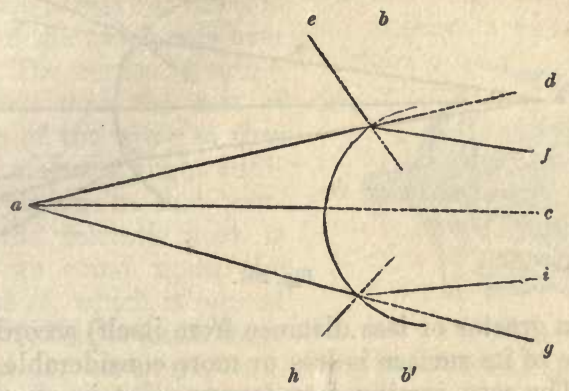

Fig. 206.

of the rays that fall upon it. Thus we shall suppose that three diverging rays, issuing from a point, $a$ (fig. 206), and traversing the air, strike upon a convex surface of glass, $b b^{\prime}$. The central ray $a c$ falls upon the glass in a direction perpendicular to its surface at that point, and passes-on unchanged in its course. But the ray a $d$ falls upon the surface very obliquely; and consequently in entering the glass it will be bent towards the line $e$, which is perpendicular to the surface at the point where it enters, and will pass onwards in the direction $f$. In the same manner, the ray $a g$ will be refracted into the direction $i$. Hence these rays, now converging, would be found, if prolonged, to meet each other again; and the point at which they meet is termed the focus. To this point all the other rays which fall upon the convex surface, at a moderate distance from the central ray, will also be conducted.

530. On the other hand, if the surface of the glass, instead of being convex, is concave, the diverging rays which fall upon it will be made to diverge still more. Thus in fig. 207, let $a$ be the point whence the rays issue, and $b b$ the surface of the lens; the central ray $a c$ will pass-on unchanged as before; but the ray $a d$ will be bent towards the perpendicular $e$, so as to pass-on in the direction $f$; and the ray $a j$ will be bent towards the perpendicular $h$, into the direction $i$. It is easy to understand that the change of direction will be greater, as 
the curvature of the lens is more considerable. Thus a convex lens has a long focus or a short focus (that is, brings rays to a

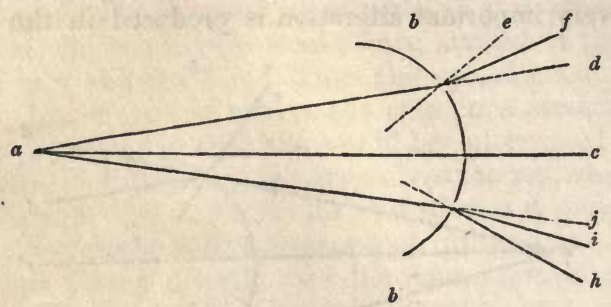

Fig. 207.

focus at a greater or less distance from itself) according as the curvature of its surface is less or more considerable.

531. The rays issuing from every point in an object, and falling upon a convex lens, are brought to a focus on the other side of the lens; and thus a distinct image or picture is formed upon any screen placed at the proper distance to receive it (as is seen in a Camera Obscura or a Magic Lantern), every point in that image being the representative of the corresponding point in the object, but this image being inverted.

532. Now, the Eye, in its most perfect form-such as it possesses in Man and the higher animals -is an optical instrument of wonderful completeness, designed to form an exact picture of surrounding objects upon the expanded surface of the optic nerve, by which its impression is conveyed to the brain. As it is in the most perfect form of this instrument that we are best able to judge of the uses of its different parts, it will be preferable to consider this in the first instance, and then to advert to the less complete forms which we meet with in the lower animals.

533. The Eye of Man, like that of all Vertebrata, has a nearly globular form. The walls of the sphere are composed of three coats; whilst in its interior are found three humors of a more or less fluid character. The outer coat, named the Sclerotic (s s', fig. 208), is tough and fibrous, and is destined to support and protect the delicate parts which it contains. It does not cover the whole globe, however; but gives place in the front of the eye to a transparent lamina of cartilaginous 
structure $c$, which is termed the Cornea. The manner in which this cornea is set upon the sclerotic coat, so as to serve as the continuation of it, may be compared to that in which a watchglass is made to serve as the continuation of the watch-case over the dial. The cornea is rather more convex than the rest of the sphere of the eye; so that the globe makes a slight additional projection in that part. -When the sclerotic coat is removed, we come upon the second coat $c h$, which is termed the Choroid; this is much more delicate in its structure, consisting almost entirely of bloodvessels and nerves; and it has a deep black hue, owing to its being lined with a thick layer of black pigment, which consists of cells that have the power of

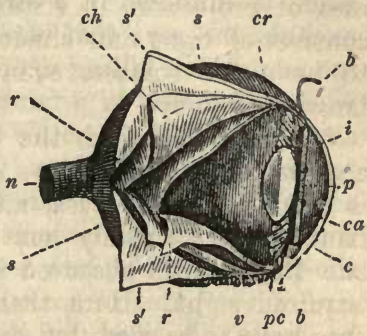

Fig. 208.-INTERIOR OF THE EYE.

$c$, cornea; $s$, sclerotic; $s^{\prime}$, portion of the sclerotic turned back to show the parts beneath; $c h$, choroid; $r$, retina; $n$, optic nerve; $c a$, anterior chamber; $i$, iris; $p$, pupil; $c r$, crystalline lens ; $p c$, ciliary processes; $v$, vitreous humor; $b b$, conjunctiva.

secreting a black granular matter in their interior. ${ }^{1}$ This coat also changes its character in the front of the eye; being there continuous with the Iris, or coloured portion, $i$, which forms a sort of curtain that hangs-down behind the cornea. The surface of the iris is flat, or nearly so; and there is consequently a space between it and the cornea, like that which intervenes between the dial-plate and the glass of a watch; this space is termed the anterior chamber of the eye. The iris is perforated in its middle by an aperture $p$, termed the Pupil. This aperture is always round in Man; but in animals whose range of vision is required to extend widely in a horizontal direction (such as the Ruminants, and others which feed upon herbage), it is an ellipse with the long diameter horizontal ; whilst in animals which rather seek their food above or below them (such as the Cat and other Carnivora which naturally live among trees and high places), the pupil is an ellipse whose long diameter is vertical.

1 Similar pigment-cells, having great variety in their form, are to be found composing the black spots on the skin of the Frog, Water Newt, \&c. 


\section{CONTRACTION AND DILATATION OF THE PUPIL :-RETINA.}

534. By the contraction and relaxation of certain fibres in the Iris, the size of the Pupil is changed according to the degree of light to which the eye is exposed; the aperture being made to diminish in a strong light, in such a manner as to exclude the rays that would be superfluous, and to prevent too many from falling upon the expansion of the optic nerve; whilst it dilates in a faint light, so as to admit as many rays as possible. If we notice the pupil of a Man who is looking towards the mid-day sun, we shall see that it is contracted to a small round speck; but the pupil of a Sheep would be contracted, in similar circumstances, into a horizontal slit; and the pupil of a Cat into a vertical one. The alteration in the size of the pupil in accordance with the degree of light, may be easily observed by stationing oneself at a window provided with shutters, and holding a looking-glass in the hand: if the light be at first strong, the pupil will be seen in a contracted state; but if the shutters be gradually closed, so as to diminish the amount of light that falls upon the eye, the pupil will be seen to enlarge; and it will diminish again when the shutters are re-opened. The blackness which the pupil always presents, in the healthy state of the eye, is due to our seeing the black lining of the back of the eye through it. In many Quadrupeds, the black pigment is replaced, in one portion of the eye, by a layer of a blueish colour, having an almost metallic lustre; and from this we see the light brilliantly reflected, when we look at their eyes in certain directions.

535. On turning back the choroid coat, we come to the third, $r$, of the layers of which the wall of the eye is composed: this is an extremely delicate film, chiefly consisting of nerve-cells and nerve-fibres that spread-out from the optic-nerve, $n$; and it is called the Retina (or net). It advances nearly as far as the iris ; but it is deficient in the front of the eye. The part of the retina which lines the globe at the point exactly opposite to the centre of the pupil, is distinguished from the rest by a peculiar yellow opacity, which causes it to be designated "the yellow spot." In this spot, visual sensibility is more acute than elsewhere. On the other hand, the part of the retina that covers the entrance of the optic-nerve (which is below "the yellow spot," and nearer to the nose) is so much less sensitive to light than the rest, that under 
certain circumstances the image that falls upon it may not be perceived at all ( $\$ 554)$.

536. The cavity of the globe is occupied by three humors of different consistence-the Aqupus, Vitreous, and Crystalline. The aqueous humor is nearly pure water, being nothing else than the serum of the blood very much diluted : it occupies the anterior chamber $c a$, and a small space behind the iris, in front of the crystalline lens. The vitreous humor $v$ resembles thin jelly in consistence, and occupies the greater part of the globe of the eye behind the iris. The crystalline humor $\mathrm{cr}$ is of much firmer consistence, resembling very thick jelly or soft gristle; it has the form of a doubleconvex lens, the posterior surface of which is more convex than its anterior; and hence it is commonly known as the crystalline lens. It is suspended in its place by a set of little bands $p c$, proceeding from the choroid coat, and known as the ciliary processes.

537. The cornea is covered externally by a membrane $b b$, termed the Conjunctiva. This membrane is perfectly transparent where it covers the cornea, and seems like an outer layer of it; the front of the sclerotic also is covered by it, but it is there semi-opaque, having a whitish colour. The membrane does not pass back over the globe of the eye, however, but bends forward again, as seen at $b b$, so as to form the lining of the eyelids, at the edge of which it becomes continuous with the skin. Thus the smooth surfaces of the eye and of the under side of the lids are both formed by this membrane; the mucous secretion from which serves to diminish the friction of one upon the other. But the smallest particle of any hard substance, which may become interposed between these surfaces, produces great irritation. It cannot pass far backwards, however, on account of the bend of the membrane at $b b$; and thus it may be always removed (if loose) with little difficulty. The lower lid can be easily drawn down, so as to expose the membrane as far as this bend; and any loose particle that is lying upon its surface may thus be detected and removed. But the upper lid, being longer, cannot be drawn out sufficiently for this purpose; and it is necessary to evert it, or turn it inside-out. As the knowledge of the mode of performing this very simple operation will often save a good deal of suffering, it will be here described. Nothing E E 2 
more is necessary than to close the upper lid-not forcibly, however; next to make pressure upon its upper part with a pencil, bodkin, knitting-needle, or other hard body of small diameter; and then, taking hold of the eyelashes, to draw the lower edge of the lid forwards and upwards. By a dexterous movement of this kind, the lid may be everted without any pain, a little temporary discomfort being all that the displacement occasions; its lining membrane is then exposed, and any offending particle may be readily removed.

538. The globe of the eye is moved by six muscles, which are lodged within the bony cavity or crbit, hollowed-out in the skull. All these muscles, except one, originate at the back of

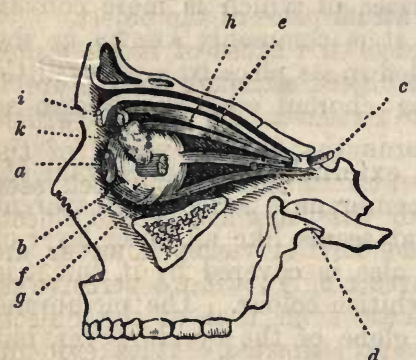

d the orbit, and are inserted into the sclerotic coat, near its front, by broad thin tendons. Four of them are termed recti or straight muscles. One of these, the superior rectus ( $e$, fig. 209 ), is inserted at the upper part of the eye, and consequently byits contraction rolls the globe upwards; another, $d$, the inferior rectus, pro-

Fig. 209.-Verticax Section of the OrBIT.

Showing the globe of the eye and its appendages ; $a$, cornea; $b$, sclerotic ; $c$, optic nerve; $d$, inferior rectus muscle; $e$, superior rectus ; $f$, cut extremity of the external rectus; $g$, end of the inferior oblique; $h$, superior oblique ; $i$, elevator of the upper lid; $k$, lachrymal gland. mentdownwards. A third, the internal rectus (which could not be shown in this figure), rolls the globe inwards, or towards the nose; whilst a fourth, the external rectus (the cut extremity of which is seen at $f$ ), turns it outwards. Besides these, there is a remarkable muscle, $h$, the superior oblique, which originates at the back of the orbit, comes forwards to the front, where its tendon passes through a pulley, and then turns backwards to be inserted into the sclerotic coat, at a point considerably behind the pulley. The sixth muscle, $g$, termed the inferior oblique, does not arise, like the rest, from the back of the orbit, but from its lower border. The action of the two oblique muscles (which act in antagonism the one to the other) appears to be to rotate the eyeball upon its axis ; as is done when the eyes are kept steadily fixed upon any 
object, whilst the head is inclined to one side or the other.Of these muscles, the superior, inierior, and internal recti, together with the inferior oblique, and also the elevator muscle, $i$, of the upper eyelid, are supplied with motor nerves by the third pair ( $\$ 459)$; whilst the superior oblique has a nerve to itself, the fourth; and the external rectus has another nerve to itself, the sixth.

539. There is this very peculiar circumstance attending the movements of the two eyes, - that although they are harmonious, they are seldom symmetrical. Thus, when we direct our eyes towards an object on one side of us, they move harmoniously, that is, with a common purpose; but their movement is not symmetrical, for one globe is rolled inwards by the internal rectus, whilst the other is rolled outwards by the external rectus. These two different actions seem to be instinctively connected, and to be guided by the sensations which are received through the two eyes respectively $(\$ 478)$. They are performed without any consciousness on our own part, when, having fixed our gaze upon any object, we rotate the head from side to side in the horizontal plane, the eyeballs executing a corresponding rotatioin in the opposite direction.

540. The eyebrows, eyelids, and eyelashes, serve in various ways for the protection of the eyes. In Birds and Reptiles there is a third eyelid, which is drawn across the eye by a muscle that passes through a loop in it. This nictitating membrane, as it is termed, is semi-transparent; and it serves to protect the eye from the too-powerful rays of light, without destroying the power of vision. The upper and lower eyelids of Mammals, and the nictitating membrane of Birds and Reptiles, are very frequently drawn over the front of the globe during the waking state, for the purpose of sweeping from it dust and other accidental impurities which would otherwise be injurious.

541. Beneath the upper eyelid, in the upper and outer portion of the orbit, is situated the lachrymal gland ( $k$, fig. $209)$; this is continually pouring-out a watery secretion over the globe of the eye, which serves to wash from it these impurities and to keep it moist. It is only, however, when the quantity of this secretion is increased by mental emotion or by irritation in the eye itself, so as to produce a flow of 
tears, that we become conscious of its existence. It is ordinarily drawn-off as fast as it is formed, by a curious apparatus situated at the inner border of the eye. If the edges of the lids be carefully examined, there will be seen upon each of them, close to the inner corner of the eye, a minute spot which is the entrance to a small canal termed the lachrymal duct. The two ducts, one commencing at the corner of the upper lid and the other at that of the lower, soon unite into one canal, which swells into a sort of reservoir, the lachrymal sac, that lies upon the side of the upper part of the nose; and from this reservoir a canal passes down through the bones of the nose into its cavity. By this apparatus, the fluid which is poured by the lachrymal gland over the exterior of the eye, is drawn-off at the interior after washing its surface; whence it is carried into the nose, to be got rid of by the current of air that passes through its cavity in breathing. The edges of the lids meet in such a manner, when they are closed, as to form a sort of gutter or channel, along which the lachrymal secretion flows from their outer to

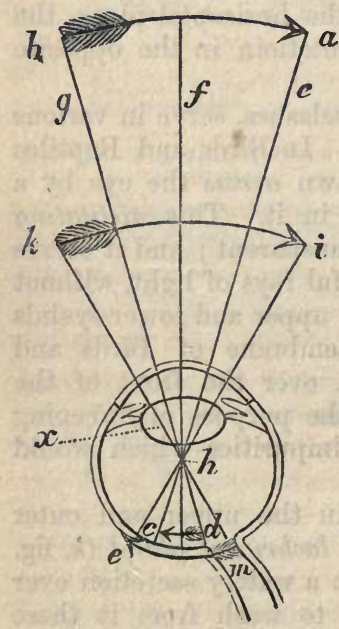

Fig. 210. their inner corner during sleep.

542. Having thus described the structure of the Eye, and the general actions of the parts by which it is adapted to the performance of its remarkable function, we shall examine into the details of this function; in other words, into the nature of vision.

543. The rays of light which diverge from the several points of any object, and fall upon the front of the cornea, are refracted by its convex surface whilst passing through it to the eye, and are made to converge slightly. They are brought more closely together by the crystalline lens, which they reach after passing through the pupil ; and its refracting influence, together with that produced by the vitreous humor, is such as to cause the rays that issued from each point to meet 
in a focus on the retina. As every point is thus represented in its proper position relatively to others (except that those which were above are now below, and vice versa), a complete inverted image or picture of the object is formed upon the retina. This is shown in fig. 210 ; where, for the sake of convenience, three rays only are represented as issuing from the centre and the two extremities of an object $a b$. These rays cross each other at $h$, in the middle of the eye ; so that those from $a$ being brought to a focus at $c$, and those from $b$ at $d$, and all the other rays being refracted in the same manner, a complete inverted picture of the object is formed at the back of the eye.

544. That this is really the case, may not only be inferred, but proved. If the eye of a Rabbit be removed from its socket, and cleansed of the muscles, fat, \&c., adherent to its back part, and a candle be then brought in front of it, the transparency of the sclerotic coat will allow the image of the candle that is formed upon the retina to be distinctly seen. Or, if we take the eye of a Sheep or an $\mathrm{Ox}$, and after cleansing it in the same manner, we cut out from the back of it. a portion of the sclerotic and choroid coats, covering the part of the retina thus left bare with a piece of tissue-paper (for the purpose of keeping-in the vitreous humor, without interrupting oux view of the image), a distinct but inverted miniature picture of all the objects in front of the eye will be seen at its back. It is necessary in these experiments that the eyes should be taken from animals recently killed; as the cornea and humors soon lose their transparency, and the distinctness of the picture is consequently impaired.

545. The black pigment, which is situated immediately behind the retina, - that is, in contact with its external surface, - is destined to absorb the rays of light immediately that they have passed through the retina; so as to prevent them from being reflected from one part of the interior of the globe of the eye to another, which would cause a great confusion and indistinctness in the picture. Hence it is that in those individuals (both among Man and the lower animals) in whose eyes this pigment is deficient, vision is extremely imperfect. The eyes of such individuals (termed Albinos) derive from the absence of pigment a very peculiar appearance. The iris does not possess its ordinary colour; but, 
owing to the large quantity of minute blood-vessels which it contains, it presents a bright red hue. The choroid coat, seen through the pupil, has exactly the same aspect; so that the pupil is not readily distinguished. During the day the vision of these Albinos is very indistinct, and the glare of light is painful to them; and it is only when twilight comes-on, that they can see clearly and without discomfort.

546. The eye is much more remarkable for its perfection as an optical instrument, than we might be led to suppose from the cursory view we have hitherto taken of its functions ; for by the peculiarities of its construction certain faults and defects are avoided, to which all ordinary optical instruments are liable. One of these, termed spherical aberration, results from the fact, that rays falling upon the central and the outer portions of an ordinary convex lens, whose surfaces form part of a sphere, are not brought to meet in one point, - the focus of the central portion being rather more distant than that of the outer part. This is shown in fig. 211, where $\mathrm{L} \mathrm{L}$ is the lens,

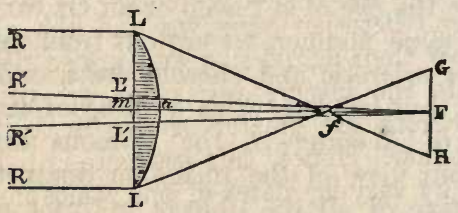

Fig. 211.

$\mathbf{R} L, \mathbf{R} L$, are rays falling upon its circumference, and $R^{\prime} L^{\prime}$, $\mathbf{R}^{\prime} \mathbf{L}^{\prime}$, are rays falling near its centre. The former set of rays meet in $f$; whilst the latter pass-on to $\mathrm{F}$, before they meet in a focus. This may be shown by covering the central and the outer portions of the lens, alternately, with some opaque substance, which shall stop all the rays of light proceeding through either. When the central portion is covered, a distinct image will be formed at $f$ by the rays falling upon the outer portion; and when the outer portion is covered, a distinct image will be formed at $\mathrm{F}$ by the rays that have passed through the central portion. But when the whole lens is employed, no distinct image is formed anywhere; for if a screen be held at $f$, it will receive, not only the rays which are brought to a focus at that point, but also the rays which are going-on to meet at F; whilst, on the other hand, if the 
screen be held at $\mathrm{F}$, it will receive, not only the rays which are brought to a focus there, but also those which, having met at $f$, have crossed and passed-on to $\mathrm{G}$ and $\mathrm{\mu}$.

547. Now this indistinctness is ordinarily got over in practice, by employing only the central portion of the lens; so that only those rays which correspond to $R^{\prime} L^{\prime}, R^{\prime} L^{\prime}$, shall pass. through it. This we observe in ordinary Microscopes and Telescopes;-a stop or perforated partition being interposed behind the lenses, so as to allow the light to pass through only a small aperture in their centre. By this plan a great deal of light is cut off, so that the image is rendered dark. The spherical aberration may be completely corrected, however, by a certain adaptation of two or more lenses whose surfaces have different curvatures; the effect of which is, to bring all the rays that have passed through every part of this compound lens to the same focus. Now this very adjustment is made in the eye, by the arrangement of the curvatures of the cornea and of the two surfaces of the crystalline lens; and in the well-formed eye it is so perfect as to produce complete distinctness in the image or picture thrown upon the retina. The only case in which this would not occur, is when an object is brought very near the eye; for the rays then diverge from each other at so wide an angle, that those which fall upon different parts of the lens would not be all brought to the same focus. This error is corrected by the contraction of the pupil, which takes place involuntarily when an object is brought very near the eye, and thus cuts-off the rays that would otherwise render the picture indistinct.

548. But there is another imperfection to which ordinary optical instruments are liable, that is completely corrected in the eye. If we look through a common Microscope, especially when a high power is employed, by the light of a lamp or candle, we see that the edges of the image are bordered by coloured fringes, which very much impair its distinctness, and prevent it from being seen in its true aspect. This is the result of what is termed chromatic aberration; and it results from the fact, that the rays of different colours, which are all blended in ordinary colourless light, are refracted by the same lens in different degrees, so as to be brought to a focus at different points. Thus we will suppose that the lens 


\section{CHROMATIC ABERRATION :-ACHROMATISM OF THE EYE.}

L L (fig. 211) has been corrected for spherical aberration ; and that $\mathrm{R} L, R \mathrm{~L}$, are violet rays falling upon it, whilst $\mathrm{R}^{\prime} \mathrm{L}^{\prime}, \mathrm{R}^{\prime} \mathrm{L}^{\prime}$, are red rays. The former are capable of being refracted in a much higher degree than the latter; so that they are brought to a focus at $f$, whilst the others do not meet until $\mathrm{F}$. Hence if a screen be placed to receive the image at $f$, the picture will be formed by the violet rays only, and will be surrounded by red fringes occasioned by the red rays which are passing on to their focus at F; whilst, on the other hand, if the screen be placed at F, the picture will be chiefly formed by the red rays, and will be surrounded by violet fringes produced by the violet rays, which, having met in $f$, have crossed and passed-on to $G$ and H. Now as from each point of almost every object proceed rays in which the different colours are blended, the refraction of an ordinary lens produces a separation of these, and a consequent indistinctness and false colouring in the picture. This is particularly the case with regard to the rays that pass through the outer portion of the lens; for, as these are subject to greater change in their direction than are those which pass through its centre, the separation of the differently-coloured rays of which they are composed is more considerable.

549. In practice, this error is got over, like the preceding, by very much contracting the aperture of the lens; so that only the central rays, in which the colours are but little separated, are allowed to pass. But it may be perfectly corrected by combining lenses formed out of different materials, which possess a different refracting power; the errors of these being made to counterbalance one another. Such lenses, which are termed achromatic, are now employed in all superior Telescopes and Microscopes; but no artificial combination can surpass that which exists in the Eye, the different density of whose humors is adjusted in such a manner as completely to answer this purpose. The contraction of the pupil which takes place when we look at a very near object, prevents the only imperfection which could occur; and thus the picture on the retina, in a healthy eye, is always rendered free from false colours. It is said that the first idea of uniting glasses of different kinds, so as to produce an achromatic lens, was taken from the Eye; and this is not at all improbable. In this, as in many other instances, Nature 
has served as a guide to Art; or, in other words, the Divine Artificer has thus condescended to teach the human workman.

550. There is another wonderful arrangement in the healthy Eye, which the optician can only imitate in his instruments in a very bungling manner. It is that by which the eye adapts itself to view objects at different distances from it, with equal distinctness. If we look at a near object with a Telescope, adjusting the instrument so as to see it distinctly, and then turn it towards a remote object, we shall not see the latter with equal clearness until the instrument has been again adjusted. If we then turn it back to the nearer object, we shall find that the change in the adjustment occasions the representation of it to be now indistinct; and in order to bring back the image to its former clearness, it is requisite to re-adjust the instrument to its first condition. This is a necessary consequence of the optical law, that the distance of the image from the lens which forms it, varies with that of the object,- - being increased as the ubject is brought nearer, and diminished as it recedes. If the Eye were constructed in the same manner, we should not be able to see distinctly, without the aid of artificial assistance, at any other distance than that for which it is adjusted. Hence if a perfect picture of an object situated at twelve inches' distance from the eye, were formed upon the retina, we should not be able to see it clearly when brought to the distance of six inches, nor when removed to the distance of six feet; because in the first of these cases the rays would not be brought to a focus upon the retina, but at a point behind it (if they were allowed to pass on unchecked); whilst in the second, they would be brought to a focus at a point nearer than the retina, and would consequently begin to separate again before they reach it.

551. But the healthy eye possesses a power of perfect adjustment to the viewing of objects situated at different distances; and this without any effort or intention on our parts, but, as it were, by an instinctive operation. That such a change really takes place, we may readily convince ourselves, by looking at a near and at a distant object placed in the same line, - a pencil-case, for instance, held up at a few inches from the eye, and a chimney half a mile off. We shall find that no effort of attention will enable us to see them both 
distinctly at the same time; but that, on whichever of the two objects we fix our eyes, we shall see it clearly, whilst the other will become indistinct. Recent observations have conclusively shown that this adjustment is brought about by an alteration in the curvature of the crystalline lens; its convexity being increased when a near object is looked at, so as to act more powerfully in bringing its diverging rays into convergence; and being diminished when the gaze is turned towards a distant object.

552. In advanced life, however, from the diminution in the convexity of the cornea and in the refracting power of the humors, the eye can no longer accommodate itself to near objects ; their rays not being brought to a focus by the time that they reach the retina. But as it is still able to see distant objects clearly, it is said to be long-sighted. By the use of a convex glass, however, adapted to supply that additional amount of refraction which is required, near objects may be distinctly seen.-A contrary state of the eye not unfrequently exists, in which the cornea is too convex, and the refracting power of the humors is too high; from which it happens that the rays proceeding from distant objects are brought to a focus too soon, so as to cross each other before they reach the retina. But as such an eye can form a very distinct picture of a near object, it is said to be near-sighted. This imperfection is remedied by interposing a concave lens between the object and the eye, by which its refracting power is diminished to the necessary degree.

553. In the choice of spectacles or eye-glasses for these purposes, particular care should be taken that they are not too powerful ; since great mischief is frequently done to the eye, by the employment of lenses of too great a curvature. A person who in youth and middle age has enjoyed good sight, very commonly finds it necessary to employ spectacles for assistance in reading and writing, as his age advances towards fifty years; and he should be very cautious, when first availing himself of their assistance, to employ those of the longest focus. As his age advances, it will be necessary to substitute more powerful lenses for these; but this should be done very gradually; and in no instance should a glass be employed that produces any apparent enlargement in the object, its proper use being only to render the object distinct. The evil 
influence of using spectacles of too high a power, soon manifests itself in the strained feeling which the eyes experience for some time; but this feeling at last subsides, in consequence of the eye having adapted itself to the glasses, and having thus undergone a change which it might otherwise take years to produce. In this manner the eyes of a person at sixty may be brought to the state which, under more careful management, might have been deferred ten or fifteen years longer.-Similar remarks apply to the use of concave lenses by short-sighted persons. . They should never be employed of a higher power than is requisite to see objects with distinctness, when at a moderate distance ; and on no account should any glasses be used that diminish their apparent size. As age advances, the eyes of short-sighted persons usually become more flattened, and are then able to adapt themselves to objects at a variety of distances; so that persons who have been short-sighted when young, are not unfrequently able to see distinctly at an advanced age, without the assistance of convex glasses.

554. The power of receiving and transmitting visual impressions is by no means uniform over the whole retina. In the whole field of vision which at any time lies before the eye, we only see with perfect distinctness that part to which its axis (namely, that diameter of the sphere which passes through the centre of the pupil) is directed, and of which the image, therefore, is formed upon "the yellow spot" ( $\$$ 535) which lies at the posterior pole of the axis. Nevertheless we have a sufficiently distinct perception of the remainder of the field, to enable us to judge of the general relations of its objects to each other and to those which we distinctly see: thus, whilst reading or writing, we can only recognise letters and words at any one moment within a spot which a sixpence or a shilling would cover, but we may distinguish the lines over the whole area of the page; and can plainly see the position of the book or paper upon the desk or table, together with the position of this in the apartment. In the act of reading or writing, as in surveying the different parts of a landscape or a picture, or in examining any solid object that is brought under our notice, we direct the axis of the eye successively to one point after another, until we have satisfied ourselves that we have gained a distinct view of every part, 
as well as of its relations to the rest. It will be presently shown that when we employ both eyes at once, their axes meet in the object, and that the degree of their convergence affords us a very important means of judgment as to their distance $(\$ \S 563,564)$. - The part of the retinal surface which lies over the entrance of the optic nerve, is remarkable for the imperfection of its power of receiving impressions; as is made apparent by the following experiment. Let two black spots be made upon a piece of paper, about four or five inches apart; then let the left eye be closed, and the right eye be strongly fixed upon the left-hand spot; if the paper be then moved backwards and forwards, so as to change its distance from the eye, a point will be found at which the right-hand spot disappears, though it is clearly seen when the paper is brought nearer or removed further; and it can be shown that in this position of the eye and the object, the rays from the righthand spot fall upon the point in question.

555. The degree in which the attention is directed to them, has a great influence on the readiness with which very minute or distant objects can be perceived; and there is a much greater variation in this respect amongst different individuals, than there is in regard to the absolute limits of vision. Many persons can distinctly see such objects, when their situation is exactly pointed-out to them, who cannot otherwise distinguish them. There must be few who have not experienced this, in regard to a balloon or a faint star in a clear sky, or a ship in the horizon; we easily see them after they have been pointed-out to us; but if we withdraw our eyes for a few minutes we search in vain for them, until they are again pointed-out to us by some one who has been watching in the interval. The faculty of rapidly descrying such objects much depends upon the habit of using the eyes in search of them; thus a seaman will distinguish land, when to the landsman there is no appearance more distinct than that of a faint cloud on the horizon presenting no definite outline; or he will recognise the course and rig of a distant ship, which to the landsman appears but as a white speck on the ocean : yet the landsman, placed before a piece of delicate machinery, might be able to astonish the seaman by distinguishing the forms and movements of minute parts, which to the latter appear only as a confused mass. 
556. The picture formed upon the retina closely resembles that which we see in a camera obscura. It represents the outlines, colours, lights and shades, and relative positions, of the objects before us; but these do not necessarily convey to the mind the knowledge of their real forms, characters, or distances. It would appear, from the actions of the lower animals, that many of them have the power of intuitively or instinctively determining the latter from the former, from the moment when they come into the world; thus a Fly-catcher just come out of its egg, has been seen to make a successful stroke with its bill at an insect which was near it. If it were not so, those animals which are thrown from the first upon their own resources, would perish almost inevitably; being starved by want of food during the time required for them to learn how to obtain it. But this is well known not to be the case in regard to Man. The infant is educating his senses long before any indications of mind present themselves. By the combination, especially, of the sensations of sight and touch, he is learning to judge of the shapes and surfaces of objects, as they would be felt, by the appearance they present,- to form an idea of their distance, by the mode in which his eyes are directed towards them ( $\$ 563)$, - and to estimate their size, by combining the notions obtained through the picture on the retina, with those he acquires by the movement of his hands over their different parts. A simple illustration will show how closely the ideas excited by the two sets of sensations are blended in our minds. The idea of smoothness is one which has reference to the touch, and yet it constantly occurs to us on looking at a surface which reflects light in a particular manner. On the other hand, the idea of polish is essentially visual, having reference to the reflection of light from the surface of the object; and yet it would occur to us from the sensation conveyed through the touch, even in the dark.

557. That this combination is not formed intuitively in Man, but is the result of experience, is particularly evident from cases in which the sense of sight has been wanting during the first years of life, and has then been obtained by an operation. Several such cases are now on record. The earliest, which still remains the most interesting, is one which occurred to Cheselden, a celebrated surgeon at the beginning of the last century. The youth (about twelve years of age), for 
some time after tolerably distinct vision had been obtained, saw everything flat as in a picture, the impression made upon his retina being simply transferred to his mind; and it was some time before he acquired the power of judging, by his sight, of the real forms, characters, and distances of objects around him. Thus, among other interesting circumstances, it is mentioned that he was well acquainted with a Dog and a Cat by feeling, but could not remember their respective characters when he saw them; one day, when thus puzzled, he took up the Cat in his arms and felt her attentively, at the same time looking steadfastly at her, so as to associate the two sets of ideas; and then, setting her down, said, "So, puss, I shall know you another time." A similar instance has come under the Author's own knowledge ; but the subject of it was scarcely old enough to present facts of so striking a character. One curious circumstance, however, may be mentioned, as fully bearing out the view here given. The lad had been accustomed to find his way readily about his father's house by the use of his hands, and he continued to do the same for some time after his sight was tolerably clear, being evidently puzzled, rather than assisted, by the impressions conveyed through his new sense; but, when learning a now locality, he employed his sight, and evidently perceived the increase of facility which he derived from it. Hence, we can have little hesitation in deciding upon the question which has perplexed many able reasoners, whether a person born blind, who was able by the sense of touch to distinguish a cube from a sphere, would, on suddenly obtaining his sight, be able to recognise these bodies by the latter sense. This question was answered in the negative by the celebrated mental philosopher, Locke, and with perfect justice.

558. We shall now inquire into the mode in which we form our notions of the nature, sizes, distances, \&c., of external objects, from their pictures impressed upon our retina. The first question is one on which a vast amount of discussion has taken place, with very little satisfactory result. It is,-why are the objects which we see, represented to our minds in their true erect position, their images upon the retina being inverted? Various replies to this question have been proposed at different times; and, amongst others, it has been actually assumed that the Infant really does see objects 
inverted, and that this error is only corrected by experience. The cases alluded-to in the last paragraph, however, satisfactorily prove this assumption to be incorrect; since, although the individuals saw everything upon the same plane, as in a picture, the representation was erect from the first. The fact now appears certainly to be, that we have an intuitive sense. of direction, which guides us in our appreciation of the actual situations of objects and parts of objects; so that, when a visual impression is made upon any part of the retina, we see the point from which the rays proceed, in the direction of a line drawn from the affected spot of the retina through the common centre (fig. $210, h$ ) through which all the rays pass, this line serving as a true guide to the actual place of the object.

559. The same may be said of the cause of single vision, that is, of our seeing but one object, although its picture is double, being formed upon both retinæ. In animals which, like Man, look straight forwards, the field of vision of the two eyes is nearly the same; so that most of the objects seen with one eye will be seen with the other also. The objects at the right and left sides of the field of vision, however, are seen with the right and left eyes singly; yet we perceive no difference in the aspect of these from that of the objects towards which both our eyes are directed. It is evident, then, that the pictures formed on the two retinæ are blended, as it were, by the mind, into a single perception. This seems to be, in part at least, the effect of habit. When the images do not fall upon parts of the two retinæ which are accustomed to act together, double vision is the result. Thus if, when looking steadily at an object, we press one of the eyeballs sideways with the finger, the object is seen double. In the same manner, if an affection of the nerves or muscles of one eye (as happens temporarily in intoxication) prevent it from being directed to the same point with its fellow, double vision of all objects is the result. This, when it does not soon pass away, is a not unfrequent symptom of serious disorder within the brain. If it continue long enough, however, the individual becomes accustomed to the double images, or rather ceases to perceive that they are double, probably because the mind becomes habituated to receive them, or rather perceives only one of the impressions on the two parts of the retinæ which now act together. For if, after the double vision has 


\section{COMBINATION OF RETINAL PICTURES :- STEREOSCOPE.}

passed away, the conformity of the two eyes be restored (as by the operation for the cure of squinting), there is double vision for some little time, although the two parts of the retinæ, which originally acted together, are now brought to do so again.

560. That the combination of the two images must be effected by an operation of the mind, is evident from another circumstance. It is easy to show that no near object is seen by the two eyes in exactly the same manner. Thus, let the reader hold up a thin book, in such a manner that its back shall be exactly in front of his nose, and at a moderate distance from it; he will observe, by closing first one eye and then the other, that his view of it is very different, according to the eye.with which he sees it. With the right eye he will see its back and right side, the latter very much foreshortened, but none of the left side; whilst with the left eye he will see its back and left side, the latter also foreshortened, but none of the right side. Hence if he were to draw a perspective view of the object as seen by each eye, the two delineations would be very different. But on looking at the object with the two eyes conjointly, there is no confusion between these pictures, nor does the mind dwell upon either of them singly; the union of the two gives us the idea of a solid projecting body - such an idea as we could have only acquired otherwise by the exercise of the sense of touch.

561. That this is really the case, has been proved by experiments with the very ingenious instrument (invented by Professor Wheatstone) known as the Stereoscope. In its original form this consisted of two plane mirrors, inclined with their backs to one another at an angle of $90^{\circ}$, the point of meeting being opposite to the middle of the forehead. Two drawings representing the different perspective views of any solid object, as seen by the two eyes, being placed before these mirrors, in such a manner that their images are reflected to the two eyes respectively, and are made to fall on corresponding parts of the two retinæ as the two images formed by the solid object itself would have done, so that their apparent places are the same,-the mind perceives not one or other of the single representations of the object, nor a confused union of the two, but a body projecting in relief, the exact counterpart of that from which the drawings 
were made. In the small portable instrument which has of late become so extensively popular, the like effect is produced by a particular arrangement of convex lenses, devised by Sir David Brewster, which also has the advantage of magnifying the pictures.

562. It is, then, by the combination which is effected through a mental process, based on the consentaneous perception of the two dissimilar pictures formed on the two retinæ, that these are made to blend into one representation, which gives the idea of projection. When we look at a distant object, our judgment is based on less positive data, the two pictures being then almost precisely the same; and hence it is impossible (without moving the head) to distinguish with certainty between a well-painted picture, in which the proportions, lights and shades, \&c. are well preserved, and the objects it is intended to represent, if we are prevented from knowing that it is a picture. Some admirable illusions of this kind have been effected in the Diorama. But a slight movement of the head suffices to dispel the doubt; since by this movement a great change would be effected in the perspective view of a solid object, - a little of the side of a projecting buttress or column being seen, for instance, where only the front was perceived before,-whilst the image formed by a picture is but slightly affected. The same indecision is experienced when we look with a single eye at certain near objects, which the mind can apprehend either as projecting or as receding, with equal, or nearly equal, readiness ; such, for example, as a metal plate stamped-out into a figure which stands-forth in relief on one side and is counter-sunk on the other. And the idea of the object which is the reverse of the reality may present itself most forcibly, if it should happen to be the one most familiar to the mind; thus if we look with one eye at the interior of a mask that has been coloured to the semblance of a human face, it will seem to rise into the likeness of the exterior; whilst the actual projecting surface of the mask will never seem to exhibit the concavity of the interior. ${ }^{1}$ In looking with a single eye,

1 In making these and similar experiments, it is necessary to take care that the whole of the projecting or receding surface is equally illuminated; since the presence of any shadow proceeding from a known source of light, destroys the illusion, by forcing the mind to recognise the real figure of the object. 
moreover, we are deprived of that power of measuring the relative distances of near objects, which we derive from the conjoint use of both eyes ( $\$ 563)$; and thus a well-painted picture, still more a photograph, may so strongly suggest the idea of projection, in virtue of its exact perspective and its contrast of light and shadow, that it is difficult to believe it to be a flat surface, even though it be within but arm's length of the eye.

563. Our idea of the distance of objects is evidently acquired by experience. An infant, when a bright object is held before its eyes, attempts to grasp it with its little hands, but obviously has no certain idea of its situation; and the same is observed in persons who have but recently acquired sight. Here, then, the impressions made upon the eyes have to be corrected by those received through the touch, before the power of judging of distances is acquired; but when it has been once acquired, we can accurately estimate the relative distances of near objects without using our hands. This we do chiefly by the interpretation we have learned to make, of the sensations which are occasioned in the muscles of the eyes by their various actions. When we fix both our eyes upon a distant object, their axes are nearly parallel to each other; but when we direct them to a near object, the axes of the eyes meet in the point at which we are looking. This is very easily seen by watching the eyes of another person, when fixed upon an object, first held at arm's length, and then brought nearer and nearer to the middle point between the eyes; the two corneæ are at first directed nearly straightforwards; but they gradually turn inwards as the object is brought nearer, and at last a very decided inward squint is produced, which disappears as soon as the object is removed. Thus, for objects within a moderate distance, the degree of convergence of the axes of the eyes, and the muscular sensations thereby produced, afford us sufficient means of judgment.

564. We perceive this in another, as well as in ourselves; for by observing his eyes, we can judge, not only of the direction, but of the distance, of the object he is looking at. Thus when a person A sees a friend B looking towards him, he can at once tell, by the appearance of B's eyes, whether he is looking at him, or at an object nearer or more 
remote; or whether, being in a contemplative mood, his eyes are fixed upon no definite object, but are looking into space. In the latter case, as in the case of blind persons in whose eyes there is no other indication of loss of sight, the peculiar vacant expression is due to the want of any convergence between the axes of the eyes, such as would indicate that they are fixed upon an object. The assistance which the joint use of both eyes affords in the estimation of distance, is evident from the fact, that, if we close one eye, we are unable to execute with certainty many actions which require a precise appreciation of the distance of near objects, - such as threading a needle, or snuffing a candle. Instances are not unfrequent in which persons have first become aware, by experiencing this difficulty, that they had lost the sight of one of their eyes.

565. In regard to distant objects, our judgment is chiefly founded upon their apparent size, if their actual size be known to us, and also upon the extent of ground which we see to intervene between ourselves and the object. But if we do not know their actual size, and are so situated that we cannot estimate the intervening space, we principally form our judgment from the greater or less distinctness of their colour and outline. Hence, this estimate is liable to be very much affected by varying states of the atmosphere; a distant ridge of hills, for example, sometimes appearing to be more remote, at other times to be comparatively near, according as the air is hazy or peculiarly clear.

566. Our notion of the size of an object is closely connected with that of its distance. It is founded on the dimensions of the picture formed by the object upon the retina ; but it is corrected by the known or supposed distance of the object itself. Thus, I hold up a book at a certain distance from my eye, and it covers the whole of the opposite window ; the apparent size of both pictures, therefore, is just the same; but knowing that the book is much nearer than the window, I infer that it is much smaller. When we know their respective distances, the estimate of their real sizes is very easy: but this is not the case when we only guess-at their distances. Hence our estimation of the size of objects even moderately distant, is much influenced by states of the atmosphere. Thus, if we walk across a common in a fog, 
a child approaching us appears to have the size of a man, and a man seems like a giant; for the indistinctness of the outline excites in the mind the idea of distance; and the same picture, if supposed to be that of a more remote object, will give rise to the idea of greater size. The want of innate power in Man to form a true conception of either size or distance, is well shown by the effect produced on the mind unprepared for such illusions, by a skilfully-painted picture, the view of which is so contrived that its distance from the eye cannot be estimated in the ordinary manner; the objects it represents being invested by the mind with their real sizes and respective distances, as if their real images were formed upon the retina. This illusion has been extremely complete in some of those who have seen the panoramic view of London in the Colosseum.

567. When a number of luminous impressions are made upon the retina at short intervals, they become blended into one, - the intervals being undistinguishable. Thus, when we rapidly move the end of a lighted stick in a straight line or circle, the impression produced is that of a band or ring of light; for the impression made by the light, as it passes each point, remains for some time subsequently. If the stick be whirled round with sufficient rapidity for it to reach any point a second time, before the impression made by its previous passage has departed, an unbroken circle of light is produced. By experiments made in this manner, we may determine the longest interval during which visual impressions remain on the sensorium; for if we find that a hot coal, whirling round at the rate of six times in a second, produces a continued circle of light, but that the circle is broken when it turns round only five times in a second, we know that the length of the impression is 1-6th of a second. By experiments of this kind, it has been found that the duration varies in different individuals, and in the same individual at different times, from 1-4th to 1-10th of a second. On this principle several very ingenious toys have been constructed, in which two or more images are combined, by the rapid revolution of a wheel on which they are painted.

568. Some persons, whose sight is perfectly good for forms, distances, \&c., are unable to discriminate colours. This is 
particularly noticed in regard to the complementary ${ }^{1}$ colours, especially red and green; so that such persons are not able to distinguish ripe cherries amongst the leaves of the tree, except by their form.

569. When the retina has been exposed for some time to a strong impression of some particular kind, it seems less susceptible of feebler impressions of the same kind; just as the ear, when it has been exposed to a series of very loud sounds (as the discharge of cannon in a naval engagement), is for some time deaf to fainter ones. Hence several curious visual phenomena result. If we look at any brightly luminous object, and then turn our eyes on a sheet of white paper, we shall perceive a dark spot upon it; the portion of the retina which had been affected by the bright image, not being affected by the fainter rays reflected by that part of the paper. If the eye has received a strong impression from a coloured object, the spot afterwards seen exhibits the complementary colour; thus, if the eye be fixed for any length of time upon a bright red spot on a white ground, and then be suddenly turned so as to rest upon the white surface, we see a green image of the spot. The same explanation applies to the curious phenomenon of coloured shadows. It may be not unfrequently observed at sunset, that, when the light of the sun acquires a bright orange colour from the hue of the clouds through which it passes, the shadows cast by it have a blue tint. Again, in a room with red curtains, the light which passes through these produces green shadows. In both instances, a strong impression of one colour is made upon the general surface of the retina; and at any particular spots from which the coloured light is excluded, that particular hue is not perceived in the faint light that remains, and its complement only is visible. The correctness of this explanation is proved by the fact, that, if the shadow be viewed through a tube, in such a manner that the coloured ground is excluded, it seems like an ordinary shadow.

1 White, or colourless light, is spoken of as composed of three primary colours, red, blue, and yellow. By the complementary colour is meant that which would be required to make white light, when mixed with the original. Thus, red is the complement of green (which is composed of yellow and blue); blue is the complement of orange (red and yellow); yellow of purple (red and blue); and vice vers $\hat{A}$, in all instances. 
570. Upon these properties of the eye are founded the laws of harmonious colouring; a full knowledge of which should be possessed by artists of every kind who are concerned with contrasts of colour, whether in pictures, architectural decorations, or even in dress. All complementary colours have an agreeable effect when judiciously disposed in combination; and all bright colours which are not complementary have a disagreeable effect, if they are predominant: this is especially the case in regard to the simple colours (red, blue, and yellow), strong combinations of any two of which, without any colour that is complementary to either of them, are extremely offensive. Painters who are ignorant of these laws, introduce a large quantity of dull grey into their pictures, in order to diminish the glaring effects which they would otherwise produce; but this benefit is obtained by a sacrifice of the vividness and force which may be secured in combination with the richest harmony, by proper attention to physiological principles.

571. The Eye is endowed with common sensibility ( $\$ 487$ ) by the fifth pair of nerves; and when this is paralysed, all parts of it are completely insensible to the touch, although the power of vision may remain unimpaired. It seldom preserves its healthy condition in this state, however; for the lachrymal and mucous secretions which protect its surface, are no longer formed as they should be ; and inflammation, often terminating in the destruction of the eye, is the result.

572. The visual sensations obtained through the Eye have numerous and varied purposes among the lower animals. That they chiefly serve to direct their movements, is evident from observation of these movements; and from the fact, that, when the eyes are covered or destroyed, most animals make little attempt at determinate motions, though they frequently exhibit a great deal of restlessness. There are a few Vertebrata, however, which do not possess perfectly-formed eyes, and which are consequently guided in their movements by other senses. This is the case with the Mole, which spends its whole life in burrowing beneath the ground; and also with the Proteus, and some others of the lower Amphibia, which inhabit the dark recesses of subterranean lakes and channels.

573. In the Articulated series of animals, we meet with 
eyes of a kind entirely different from those which have been previously described. In most Insects we notice a large black or dark-brown hemispherical body, situated on either side of the head (fig. 212); and in Crabs, Lobsters, \&c., we find spherical bodies of similar appearance mounted on short footstalks, which are capable of some degree of motion. When these are examined with the microscope, their surface

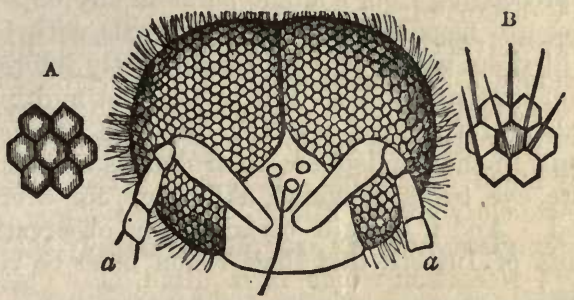

Fig. 212.-Head and Exes of the Bee, showing the Division into Facets. $a, a$, antennæ; A, facets enlarged; $\mathbf{B}$, the same with hairs growing between them.

is seen to be divided into a vast multitude of hexagonal (sixsided) facets. In a species of Beetle (Mordella) upwards of 25,000 of these have been counted; in a Butterfly, above 17,000 ; - in a Dragon-fly, more than 12,500; and in the common House-fly, 4,000. Every one of these facets may be regarded as the front of a distinct eye, which, however, instead of being globular, is conical in its form; the front being the base of the cone, and the apex or point being directed towards the optic nerve, which swells-out into a bulbous expansion that fills a large part of the interior of the hemisphere. Each individual eye consists, therefore, of its facet, which (being convex on both surfaces) acts as a lens; of the transparent cone behind this, which may be compared to the vitreous humour; and of the fibre which passes from the bulbous expansion of the optic nerve to the point of this cone. The several fibres are separated from one another by a considerable quantity of black pigment, which also fills up the spaces between the cones; and it is to this that the black appearance of the whole compound eye is due.

574. We must thus regard each of the cones, which, united together, constitute the hemispherical or globular mass, in the light of a distinct eye; but the entire aggregate seems to 
correspond in function with the single eye of the Vertebrate animal. For no rays except those which correspond in direction with the axis of each cone, can reach the fibre of the optic nerve at its apex; all others being stopped by the layer of black pigment which surrounds it. Hence it is evident that each separate eye must have an extremely limited range of vision, being adapted to receive but a very small collection of rays proceeding from a single point in any object; and as these eyes are usually immoveable, animals with but a small number of them would be very insufficiently informed of the position of external things. But by the vast multiplication in the number of the eyes, and the direction of their axes to every point in the hemisphere, their defects are compensated; a separate eye being provided, as it were, for every point to be viewed. And it is quite certain, from observation of the movements of Insects, that their vision must be very perfect and acute. ${ }^{1}$

575. Although these Compound Eyes exist in all Insects and in most Crustaceans, Spiders and Centipedes, they are in general not the only organs of vision which these animals possess. Most of them are also furnished with several simple eyes, analogous in their structure to those of higher animals, but les complex and perfect in their organization; these, which are for the most part disposed on the back of the head, are largest in Spiders. The larvæ of some Insects possess the simple eyes without the compound; the latter being only developed at the time of the last metamorphosis. The simple eyes of Insects do not appear to be nearly so efficient as instruments of vision, as are their compound ones ; for when the latter are covered, the animals seem almost as perplexed as if they were perfectly blinded. Simple eyes, closely resembling those of Insects in structure, are found in most of

1 It is commonly believed that each of these compound eyes produces its own image of the same external object, as do our two eyes; but from the description here given of their separate directions when united, it is evident that in no two of them can an image of the same object be formed at the same time. The membrane formed of all the lens-like corneæ united together, when separated from the other parts of the eye, and flattened-out, has the properties of a multiplying-glass, each lens forming a distinct image of the same object; but this is not the case when they are arranged in their natural position, because no two of them have the same direction. 
the Mollusca which possess a head-namely, in the Gasteropods, Pteropods, and Cephalopods; those of the last class present an evident approach to the eyes of Fishes, in the greater completeness of their structure, and in their adaptation for distinct vision. In many of the lower Mollusca, as in the Rotifera and several Annelida, and also at the end of the arms of the Star-fish, red spots may be seen, which appear to be rudiments of eyes; but no distinct organs of vision can be seen in the Zoophytes and lowest Mollusca ; although many of them appear very sensible to the action of light.

\section{that vill CHAPTER XII.}

ANIMAL MOTION, AND ITS INSTRUMENTS.

576. THE different modifications of the faculty of Sensation which have been described in the preceding chapter, enable Man and other Animals to become acquainted with what is going-on around them. But their connexion with the external world is not confined to this faculty ; for if they possessed it alone, they would be nearly as passive as are Plants,-experiencing, it is true, pain and pleasure from their sensations, but not having the power of avoiding the one or of procuring the other. They are endowed, however, with another faculty, that of spontaneous movement; which serves the double purpose of enabling them to act upon the inanimate world around them, and of communicating to each other their feelings and ideas. Thus, if we find ourselves scorched by a flame, we either withdraw our bodies from it, under the direction of the instinct which leads us to aroid suffering, or we set about to extinguish the fire by an act of the will, founded upon our rational knowledge of its injurious tendency. The Plant, even if it had sensation (which some naturalists have supposed), could do neither of these things. Again, it is entirely by the movements concerned in speech, by those giving expression to the countenance, and by the gestures of the body, that we convey to beings like ourselves a knowledge of what is passing in our own minds; of this power we know that plants are entirely destitute, and it is possessed in a very limited degree by the lower Animals. 


\section{MOVEMENTS NOT DEPENDENT ON MENTAL DIRECTION.}

\section{Contractile Tissues:-Muscular Contractility.}

577. When we examine into the nature of the movements of the lower tribes of Animals, however, we find that they bear a much closer analogy to those of Plants, than they do to those which are the expressions of the self-determining power of higher Animals. Among the simplest Protozoa, it seems as if the change of form of the single cell of which each individual is composed, were the sole means of movement which it possesses ( $\$ 129)$; and this change of form often appears rather to be due to the nutrient actions taking place within the cell, than to occur in respondence to any impression made upon its exterior. In such movements it is impossible to suppose with any probability that consciousness can participate. So, again, among Infusory Animalcules, all the moyements of the body are effected by the action of citia ( $\$ 133)$, which we know in our own experience to be entirely removed from any mental direction, and which we see to be exhibited under a no less remarkable aspect by the "zoospores" or motile buds of the Algæ (BotaNy, § 767).

578. Although the movements of the Hydra (\$121) and other Zoophytes may appear to indicate the existence of a selfdetermining power, yet it is very doubtful whether such an endowment is really possessed by these animals. For their contractile tissue is of the simplest possible character, resembling that which is found in the early state of newly-forming parts of higher Animals ; and when the movements executed by it are carefully compared with our own, it becomes obvious that they are analogous, not to those of the Human body and limbs generally, but to those of the muscular coat of the alimentary canal and of the muscles concerned in deglutition, which not only take place without any voluntary determination on our parts, but may even be performed without our consciousness. In like manner, the rhythmical movements of the umbrella-like discs of the Medusce ( $\$ 120)$, by which many species of them are propelled through the water, bear a much closer analogy to the rhythmical movements of the heart of higher Animals, than they do to any other of their actions; and are probably performed, like these, without any exercise of will, and even without the guidance of consciousness.

579. In proportion, however, as we ascend the scale, we 
find a peculiar tissue, the Muscular ( $\$ \S 55-59$ ), distinguished from the rest; in which the general contractility of the body becomes, as it were, concentrated. In proportion to the development and complexity of this muscular apparatus, it supersedes the more feeble contractility diffused through the fabric of the lower tribes. It now, moreover, becomes in great degree subjected to the Nervous System; by which all those parts of it which are not connected with the functions of C:yanic life merely, are rendered subservient to the Will, and thus become its instruments in determining the place and the various actions of the body. Still we find that the ordinary actions of those portions of the muscular apparatus which are most immediately subservient to the functions of organic life, are essentially independent of nervous influence, and are very little under its control; as we see in the case of the alternate contraction and relaxation of the heart, and the peristaltic movements of the alimentary canal.

580. The peculiar contractility of muscular fibre may be called into action by various means. As in certain vegetable tissues (Veger. Phys. § 390 ), contraction may be excited by a mechanical stimulus directly applied to the muscle itself. Thus, if the heart of an animal recently killed be touched with a pointed instrument, it will contract and then dilate, as if performing its ordinary action; and this may be repeated several times. In the same manner, if the walls of the intestinal canal be pricked or pinched, they will re-commence and continue for a short time their peristaltic movement. And if any part of an ordinary muscle be irritated in the same manner, that particular bundle will contract, but the rest will not be affected. Now these actions are analogous to those performed by the Sensitive Plant, Venus's Fly-trap, and many other plants, some part of whose tissues contracts in like manner when an irritation is applied to it, causing-it may be - extensive and important motions. It appears to be in this manner that the contractions of the heart, and of the alimentary tube from the stomach to the rectum, are ordinarily excited in the living body.

581. But there must be some other cause for the continuance of the rhythmical movements of the heart, as well as of some other organs; for the heart of many cold-blooded animals will continue to contract and dilate many hours after 
it has been removed from the body, when it neither receives nor propels blood. ${ }^{1}$ In the same manner, the peristaltic motions of the intestine continue to propel its contents for some time after the general death of the body; and may even take place when the whole tube has been removed from it, and has been completely emptied. There is strong reason, in fact, for attributing to certain kinds of muscular tissue an inherent motility, in virtue of which it moves of itself without any external stimulation; the changes which are concerned in its nutrition developing a force which must manifest itself in action; just as a Leyden jar, which is receiving a continuous charge from an electrical-machine, discharges itself whenever its electricity attains a certain tension.

582. But the muscles of the trunk, limbs, \&c., are not called into action in this manner; for, as just now stated, a stimulus applied to any one part of these does not excite contraction in the whole muscle (as it does in the case of the heart), but only in the individual bundle of fibres irritated. These muscles are all of them supplied with nerves ( $\$ 63$ ) from the Cerebro-spinal system, or the nervous centres that correspond to them in Invertebrated animals ; and it is only by a stimulus transmitted to them along these trunks, that all the bundles of which the muscle is composed can be called into action at once.

583. When the trunk of a nerve supplying a muscle is irritated by pricking, pinching, \&c., in the body of a living animal, or in one recently dead, the whole muscle is thrown into contraction; and this contraction is peculiarly strong when a current of Electricity is transmitted along the nerve. In cold-blooded animals, whose muscular fibre retains its vital properties for a much longer time after death than that of warm-blooded, this contraction may be excited by a very feeble current of electricity, even in a limb which has been separated from the body for twenty-four hours or more; and it was owing, in fact, to this circumstance, that the peculiar form of electricity which is now termed Galvanic or Voltaic was discovered. The wife of Galvani, who was Professor of Medicine

1 There is an instance on record, in which the heart of a sturgeon, that had been removed from the body and had been inflated with air, continued to beat until the auricle had become so dry as to rustle during its movements. 
at Bologna, being about to prepare some soup from frogs, and having taken off their skins, laid them on a table in his study, near the conductor of an electrical machine which had been recently charged; and she was much surprised, upon touching them with the scalpel (which must have received a spark from the machine), to observe the muscles of the frog strongly convulsed. Her husband, on being informed of the circumstance, repeated the experiment; and found that the muscles were called into action by electricity communicated through the metallic substance with which the limb was touched.

584. The experiment was repeated in various ways by Volta, who was Professor of Natural Philosophy at Pavia; and he found that the effects were much stronger when the connecting medium through which the electricity was sent, consisted of two metals instead of one ; and from this circumstance he was led to the discovery that electricity is produced by the contact of two different metals-a discovery which has since been so fruitful in most important results. The following simple experiment puts this in a striking point of view. If the skin of the legs of a Frog recently killed be removed, and the body be cut across, above the origin of the great (sciatic) nerve going to the legs, - if the spine and nerves be then enveloped in tin-foil, and the legs be laid upon a plate of silver or copper,-convulsive movements in the muscles will be excited every time that the metals are made to touch each other so as to complete the electric circuit.

585. Similar experiments have been tried with the Voltaic battery, upon the dead bodies of criminals recently executed. If one wire be placed upon the muscles which it is desired to call into action, and the other upon the part of the spine from which the nerves proceed, movements of every kind may be produced. No agent more effectually imitates the natural action of the nerves, in exciting the contractility of muscles, than Electricity thus transmitted along their trunks; and we have already seen ( $\$ 489)$ that Electricity, transmitted along the sensory nerves, excites the peculiar changes in the brain by which sensations are produced. Hence it has been supposed by some philosophers, that electricity is the.real force by which the nerves act upon the muscles; more especially since it is certain that, in those animals which generate large quantities of electricity, the nerves have a great share in this 
peculiar operation $(\$ 423)$. But there are many objections to such a view ; and it appears more correct to regard Electricity and Nerve-force as correlated,-that is, as each capable, under certain conditions, of exciting an equivalent measure of the other,-than to consider them as identical.

586. The power, whatever be its nature, by which the Nerves act upon the Muscles in the living body, originates in the central organs, or ganglionic masses, of the nervous system; and is propagated from these, through the nervous fibres, to the muscles, in a mode precisely analogous to that in which the electric power, called-forth by the action of an electrical machine or galvanic battery, is transmitted to any distance through conducting wires. If the conductor be divided, no action at the centre, however powerful, can produce any change at its extremities; and in this manner, by division of the nervous trunk, the muscle supplied by it is palsied. The muscle itself does not thereby lose its contractility; for it may still be made to contract by a stimulus transmitted through the part of the trunk that remains attached to it, - as, for instance, by pricking or pinching the cut extremity, or by passing an electric current along it; but it is completely withdrawn from the dominion of the nervous centres under which it previously was; and cannot be called into action either by the will, by an emotion, or by a reflex impulse. The part of the trunk in connexion with it soon loses its power of conveying irritations ; and the muscle itself, being thrown into disuse, in time loses its contractility.

587. From this last fact it has been supposed that the contractility of muscular fibre depends upon its connexion with the nervous system, and is not an endowment peculiar to itself. But this idea is disproved by a number of circumstances. Thus the contractility of the heart and intestinal tube is exhibited, long after these parts have been separated from their nerves. The contractility of other muscles may be exhausted by repeated excitement, so that even the stimulus of galvanism will not produce movement in them; and yet it may be recovered after the nervous trunks have been divided. And it has been ascertained that if the muscles be frequently exercised, as by the application of galvanism once or twice a day, they will retain their contractility for any length of time. This exercise is further found to have the effect of preventing 
the wasting-away of the muscles which otherwise takes place ; and thus we see that the preservation of this peculiar property is dependent upon the due nutrition of the muscle, whilst the loss of the property results from its want of nutrition, - as we find to be the case in regard to other tissues. Further, the activity of the nutrition of muscles depends in great part upon the use that is made of them; and thus we find that any set of muscles in continual employment undergoes a great increase in size and vigour; whilst those that are disused, even though their nervous connexions remain entire, lose their firmness and diminish in bulk, until, if the inaction be continued long enough, almost all trace of proper muscular substance disappears, and the contractility of the part is lost.

588. But a muscle may be palsied by some change taking place in the central organs, which shall prevent the nervous influence from being excited there. Thus by an effusion of blood in a certain part of the brain, the arm, leg, or the whole of one side may be paralysed to the influence of the will. But the muscles which are thus withdrawn from the power of the will, may sometimes be moved by an emotional or instinctive impulse, or by reflex action; their connexion with the parts of the nervous centres, in which these actions respectively originate, remaining unimpaired (Chap. x.). Thus a completely paralytic arm has been seen to be violently shaken, when the emotions of the patient were strongly excited by the approach of a friend. The muscles of the shoulder, in a case of complete paralysis of one side, were called into contraction in the reflex movement of yawning ( $\$ 341$ ). And the muscles of the legs, when their communication with the brain, - and consequently the control of the will over them,- -has been completely cut off, have been made to act energetically when the feet were tickled, although the patient was not conscious either of the irritation or of the motion. When the muscles are thus aroused to occasional activity, their nutrition is not so much impaired, and their contractility does not depart nearly as completely as when they are thrown into entire disuse by division of their nerves.

589. Muscles are commonly divided into voluntary and involuntary, according as they act in obedience to the will, or are not under its dominion. But this is not a correct division; since, whilst nearly all the muscles of the body are 
more or less under the control of the will, they may all at times have an involuntary action. The heart and the muscular coat of the alimentary canal, with the muscles concerned in swallowing and in one or two other actions of a similar character, are the only muscles which the will cannot either set in action, or control when in action. There are several muscles whose usual movements are of a reflex and therefore involuntary character, and are yet capable of being, to a certain extent, controlled and governed by the will. Such are the movements of respiration; which will continue to take place after the brain has been removed, and which go on regularly during the profoundest sleep and the most complete withdrawal of the attention from them. In the Invertebrated animals these motions are probably not influenced by the will ; but in the air-breathing Vertebrata they are placed in a certain degree under the dominion of the will, in order that they may be made to contribute to the production of the vocal actions of speaking, singing, \&c., which are restricted to these classes. We can hold the breath for a certain time by a voluntary effort, or we can expel or draw it in more quickly than usual; but no voluntary effort can cause the breath to be held for more than a few moments; for the uneasiness which is then felt, and which is continually increasing, causes an involuntary action of the muscles, by which action it is relieved.

590. But again, there are other muscles, whose ordinary actions are voluntary; but which are occasionally made to act independently of the will, or even against its direction. Such are those which are excited by the emotions, as in laughing, crying, sobbing, \&c. We may have the strongest desire to check these actions, owing to the unfitness of the time and place for their manifestation; and yet we may be unable to do so. And lastly, muscles whose action is usually voluntary may be occasionally called into powerful contraction, which the will cannot in the least degree control or prevent; this is the case in cramps, convulsions, \&c., of various kinds. - All these facts are readily accounted-for by the knowledge we now possess, as to the functions of the different parts of the nervous centres from which the muscles receive their stimulus to action (Chap. x.).

591. The vigorous action of the muscular structure is de- 
pendent upon several conditions. In the first place, it requires an active nutrition of the muscles themselves. Firm, plump, and high-coloured muscles act with greater force than those which are pale and flabby, even though the size of the latter may be greater. Again, in all those animals whose activity is greatest, a constant supply of oxygen is requisite for muscular vigour. This, like the nutritive material, is conveyed, in Birds and Mammals, by the blood ( $\$ 235)$; in Insects, on the other hand, it actually enters the muscular tissue in the state of atmospheric air ( $\$ 321$ ). In Reptiles, again, the blood goes to the tissues very imperfectly oxygenated; and their movements are comparatively slow and feeble. But it is a remarkable circumstance, that in the dead bodies of the latter, or in parts separated from the living body, the property of contractility does not depart nearly so soon as it does in similar parts of warm-blooded animals. By experiments on Mammals it has been found that the muscles of the trunk cannot be caused to contract by galvanism for more than two or three hours after death, though the auricles of the heart retain their contractility for some hours later. The muscles of Birds (whose respiration is more active, and whose temperature is higher) lose their contractility yet sooner; but those of Reptiles sometimes retain the power of contracting for several days. When venous or imperfectly-aerated blood is made to circulate through the vessels of warm-blooded animals, it acts like a poison upon them, diminishing or even destroying their contractility. ${ }^{1}$

592. Further, the energy of muscular contraction depends in great degree upon the power of the stimulus which is transmitted to it through the nervous system. We often have the opportunity of observing this, in the case of persons who are under the excitement of violent passion or of insanity; a delicate female becoming a match for three or four strong men, and even breaking cords and bands that would hold the most powerful man in his ordinary state. The strength in such circumstances seems almost preternatural ; but it is not

1 Other substances do this with even greater rapidity; thus a strong solution of nitrate of potass (nitre) injected into the blood-vessels, and conveyed by them to the heart, causes the immediate cessation of its action,- the poison finding its way, through the vessels of the organ itself, into the capillaries of its muscular structure. 


\section{FATIGUE. - ENERGY AND RAPIDITY OF MOVEMENT.}

greater than that which we see manifested in convulsive actions, where the movements depend only upon the reflex activity of the spinal cord. Thus a slender girl affected with a spasmodic affection of the muscles of the spine, which threw the back into an arch of which the head and the heels were the two resting-points, has been known to raise a weight of $900 \mathrm{lbs}$. laid on the abdomen with the absurd intention of straightening the body.

593. The sense of fatigue, which comes-on after prolonged muscular exertion, is really dependent upon a change in the brain, though usually referred by us to the muscles that have been exercised. For it is felt after voluntary motions only; and the very same muscles may be kept in reflex action for a much longer time, without any fatigue being experienced. Thus, we never feel tired of breathing; and yet a forced voluntary action of the muscles of respiration soon causes fatigue. The voluntary use of the muscles of our limbs, in walking or running, soon occasions weariness; but similar muscles are used in Birds and Insects, for very prolonged flights, without apparent fatigue; and as we find that the actions of flight may be performed, after the brain, or the ganglia that correspond to it in Insects, have been removed ( $\S \S 444,465)$, we may regard them as of a reflex character ; and the absence of fatigue is thus accounted-for.

594. The energy of muscular contraction appears to be greater in Insects, in proportion to their size, than it is in any other animals. Thus a Flea has been known to leap sixty times its own length, and to move as many times its own weight. The short-limbed Beetles that inhabit the ground have an enormous power, which is manifested both in their movement of heavy weights, and in the resistance they, overcome with their jaws. Thus the Dung- or "shard-borne" Beetle can support uninjured, and even elevate, a weight equal to at least 500 times that of its body. And the Stag-Beetle has been known to gnaw a hole of an inch diameter, in the side of an iron canister in which it had been confined. The rapidity of the movements of Insects is also most extraordinarily great, and is especially seen in the vibrations of their wings. It would be impossible to form an estimate of the time occupied by these, were it not for the musical tones they produce; and it may be calculated from these that the wings of 
many Insects strike the air several hundred times,-and those of some of the smaller Insects many thousand times,in a second of time.

\section{Applications of Muscular Power:-Bones and Joints.}

595. Muscular contraction performs an important part in nearly every one of the functions of which we have already treated. Thus the reception of the food, and its propulsion along the alimentary canal, forming part of the function of Digestion, are accomplished through its means. The Circulation of the blood, again, depends mainly on the agency of a contractile organ, the heart. The Respiration cannot be kept up, in the higher animals at least, without the aid of certain movements which are accomplished by the muscles. With the processes of Nutrition and Secretion it is not so closely connected ; but the latter is dependent upon it so far as this, that its products are carried out of the body by the aid of muscular contraction. And even in Sensation, the peculiar endowment of muscular tissue comes into use; by giving to the organs of sense those movements which enable them to take a wider range, and to apply themselves most perfectly to the objects before them. But we have now to study its applications in those general and partial movements of the body, on which depend the locomotion (or change of place) of animals, their attitudes, and a number of other important actions, entirely of a mechanical nature.

596. The organs by which these are effected, may be conveniently divided into the active and passive. The active are those which have peculiar vital powers within themselves, and which exert these in giving motion to other parts. To this class, therefore, we refer the Muscles, whose peculiar endowments have been just considered. The passive organs, on the other hand, are those which perform no action of themselves, which have no power but that of yielding a simply mechanical support, and which consequently perform no movement but such as they are made to do by the muscles. Of this kind are the hard parts which form the skeleton or solid framework of the body, whether this be internal or external.

597. In the lower tribes of animals, the muscles are all inserted in the soft and flexible membrane which covers the body; and it is by acting upon this, that they can change the 
form of the body in such a manner as to cause it to move, either altogether or in part. This is the case, for example, in the Leech, Earth-worm, and other Annelids; which are furnished with two sets of muscular fibres, one running along the body, and the other passing round it in rings. By the contraction of the former, the two ends are drawn together, so that the body is shortened; whilst by that of the latter, its diameter is lessened, so that it is necessarily lengthened. By these two movements, which take place alternately, the progression of the animal is accomplished; and by varying the contractions of one part or another, almost any form and direction can be given to the soft and flexible body.

598. But in the higher animals we find the apparatus of movement to consist, not only of muscles, but also of a framework of solid pieces, which serves to augment the precision, the force, and the extent of the movements; whilst, at the same time, it determines the general form of the body, and protects the viscera against injury from without. This solid framework, or skeleton, to which the muscles are attached, may be, as we have seen, either internal or external. In the Vertebrated classes, the hard skeleton is internal; in the Articulated series it is external; in the Mollusks it is external, but does not afford fixed attachments to muscles, except to such as draw together its valves, or connect it with the soft body of the animal ; and in the Radiata its position is variable, being sometimes external as in the Echinodermata, and sometimes internal as in the stony Corals.

599. The skeleton of Vertebrata differs from that of all the Invertebrated classes in the much higher character of its organization, which enables it to grow with the growth of the body generally, not merely in virtue of the additions it receives, but by the successive removal of its previously formed parts as occasion may require; so that the skeleton of the adult has been entirely substituted for that of the child, probably no part of the latter being contained in the former. The skeletons of the Invertebrata, where they are not formed of horny matter alone, are consolidated by carbonate of lime, which in some instances (as the shells of many Mollusks, and the Stony Corals) bears so large a proportion to the animal basis, that the latter can scarcely be detected. The growth of these skeletons takes place entirely by additions to 
the parts already formed; there is nothing like that "interstitial" change which we see in bone, and which is performed by the agency of the blood conveyed through the Haversian canals that traverse its substance; and where the skeleton is external, it must either be adapted by such additions to the augmenting bulk of the body it incloses, or must be cast-off and replaced by another. The latter method is that which is followed in the Crustacea ; of the former we have examples in the shell-bearing Mollusks, whose shells receive successive additions at their free margins, and in the Echinodermata, whose box-like envelopes are made to increase equally in all directions, by additions to the edges of the numerous separate pieces of which they are composed ( $\$ 118)$.

600 . The different portions of the skeleton are articulated, or united by joints to one another, in such a manner that they can move with greater or less freedom. This we see both in the Vertebrated and in the Articulated classes. In the latter, the joints are for the most part very simple in their construction. The different rings or pieces are held together by a flexible membrane passing from one to the other; this seems to be little else than a portion of the integument originally covering the body, which has remained unconsolidated whilst the rest has been hardened. And sometimes they are made to adhere to each other by a kind of "soldering," so as to be altogether immovable. But in the internal skeletons of the Vertebrata we find a more complex mode of union, fitted to afford scope for the greater variety of motions which their parts perform. Here, too, we find some parts immovably united to each other, where support and protection alone are required. These immovable articulations, of which there are several kinds, will be first considered.

601 . All the bones of the head and face (with the exception of the lower jaw), in Man and the higher Vertebrata, have their edges in immediate contact with each other; so that they hold together in the dry skull, as well as during life. Those bones of the skull, which inclose and protect the brain, are very firmly united by what are termed sutures, ${ }^{1}$ which are mostly formed by the interlocking of the jagged edges of one bone into corresponding notches of the adjoining one; though in some this kind of union is incomplete, while

${ }^{1}$ From the Latin sutura, a seam. 
in others it is replaced by a bevelling of the edges that are in contact, or by the reception of a ridge of one bone into a groove in the other. So firmly are the bones united in this manner, that it is difficult to separate them without breaking away some of their projecting parts; and in the skulls of old persons, the sutures are almost obliterated by the complete union between the adjacent bones. In the infant, on the other hand, the bones of the skull are only united to each other by a membranous substance; and there is a point at the top of the head, which is not even covered by a bony layer for some time after birth. It is only as the age advances, and ossification becomes more complete $(\$ 52)$, that firm bony union is effected.

602. In several other articulations, the bones do not come into direct contact with each other, but are connected by an intervening layer of cartilage, and also by ligaments and other fibrous membranes encircling the articulations. The adjacent surfaces of the bones are flat, and have a slight gliding movement over one another; but the extent of motion permitted is very small. This kind of articulation exists between the bodies of the vertebræ of Man and the higher Vertebrata, between the bones of the pelvis, and some other parts.

603. The proper movable articulations, by which the limbs are connected with the trunk, and their different divisions to each other, are those to which we commonly give the name of joints. In these, the surfaces of the adjacent bones are not united in any other way than by the ligaments and muscles which surround them; and they have a free gliding movement over each other. They are covered, it is true, by cartilage; this, however, does not pass from one bone to the other, as in the previous case, but forms a thin layer over the end of each, and presents a very smooth surface, which is secreted from the synovial membrane that envelops the joint lubricated by the fluid ( $\$ 44)$. The beautiful smoothness of the surfaces of the joints, and the manner in which the bones are held together by the muscles and ligaments, is well seen by examining the knuckle-joint at the lower end of a leg of mutton (before being cooked), and the joint which connects it: with the bones of the haunch. These two joints are examples of the two principal varieties of freely-movable 
articulations, - the hinge-joint, and the ball-and-socket joint. In the first of these, the surfaces of the bones are so formed that the movement, though free as regards its extent, is very limited in its direction; being in fact restricted to a backward and forward action in the same line, just like that given by a common hinge. In the second, the end of one bone is formed into a rounded head or ball, and this is received into a corresponding socket or cup in the other, the edge of which is usually deepened by cartilage; in this manner the bone which carries the ball is enabled to move upon the other in any direction, unless restrained by external checks. Of the hinge-joint we have examples in the elbow, the knee, and the joints of the fingers and toes. Of the perfect ball-and-socket joint we have in Man only two examples, - the shoulder, and the hip. In the former the socket is much shallower than in the latter, and the motions of the arm are consequently more extensive than those of the thigh : both, however, are unchecked in regard to their range and direction, except when the limb is brought against the body or against its fellow. The wrist and the ankle-joint are of an intermediate character; the former more resembling the ball-and-socket, and the latter the hinge-joint.

604. All these joints are more or less subject to dislocation, by violence of different kinds. This takes place by the slipping-away from each other of the two surfaces, which ought to be in contact. Thus the head of the humerus (or arm-bone) may slip over the edge of its socket, so as to lie entirely on the outside of it ; and this, in consequence of the shallowness of the cup, happens not unfrequently. The head of the thigh-bone, also, may slip out of its socket; but this accident is more rare, on account of the deepness of its cup. The elbow and knee-joints, as also those of the wrists, ankle, fingers, and toes, may be dislocated by the slipping of one surface on the other, either forwards or backwards, to one side or to the other. One of the most common dislocations is that of the thumb, the lowest articulation of which has rather the character of the ball-and-socket (with a very shallow cup), than of the hinge-joint. But in proportion to the liability of any joint to dislocation, is usually the ease with which it may be brought into place again.

605. Of the attachments of muscles $t$, the skeleton, one is 
usually called the origin, and the other the insertion,-the origin being in the part that is most fixed, and the insertion in that which moves upon it. Thus the muscle chiefly concerned in bending the elbow, has its origin at the shoulder and its insertion in the bones of the fore-arm; its general detion being to move the latter, while the former is fixed or nearly so. But its attachment to the fore-arm may really become its origin, and its other attachment its insertion; for, when a person is hanging by his hands from a beam or cord, and raises his body by bending his elbows, the fore-arm is the fixed point, and the shoulder is moved upon it. In like manner, the muscular mass at the bottom of the back, having one attachment to the bones of the pelvis and another to the thigh-bone, serves to draw the latter backwards when the former is made the fixed point, as when we rise-up from the sitting posture; but it is also continually serving to keep the body upright upon the thighs, the latter being the fixed point, and brings it back into this position when it has been bent forwards as in stooping.-Muscles are seldom directly attached to the hard parts, but are united with them by means of the fibrous bands which are called tendons (\$29). Sometimes the tendon is long and round; this is the case with several of those that move the hand and fingers (fig. 228), which arise from the muscles forming the fleshy part of the forearm, and may be felt at the wrists as hard round cords. In other instances, however, the tendon is a broad flat band; of such we find several within the shell of the body and limbs of the Crab or Lobster, when we have removed the muscle or flesh.

606. The action of any muscle, in producing a change in the position of a novable bone on which it acts, is determined in the first place by the nature of the movement of which the bone is capable; and in the second, by the direction in which the power of the muscle is applied to it. Having now considered the former of these conditions, we proceed to the latter. The contraction of a straight muscle, which is attached to a fixed point at one end, and to a movable point at the other, will obviously tend to draw the latter towards the former. Thus the muscles which bend the fingers lie in the palm of the hand and on the corresponding side of the fore-arm; whilst those that straighten the 
fingers are situated on the opposite side. But we often find that the direction of a muscle's action is changed, by the passing of its tendon through a pulley-like groove or loop; so that it draws the movable bone in a direction different from that of its fixed attachment. This is the case, for example, with some of the muscles that bend the toes; these being situated in the calf of the leg, would draw the toes upwards, were it not that their tendons are carried beneath the bones of the heel, working in smooth pulley-like channels hollowed-out in them (fig. 233) ; hence, when the muscle contracts, the tendons draw the ends of the toes towards the heel, and thus bend them.

607 . We generally find that even movements of a simple character are performed by the combined action of several muscles; of which some may be considered as the principal, and others as assistants. Those which are principals in one movement may become assistants in another; and vice versa. Thus, if we wish to bend the wrist directly downwards upon the fore-arm, we put in action, not only certain muscles whose tendency would be to produce this movement, but others which, acting by themselves, would produce a different motion. One of these would draw the wrist towards the thumb-side of the fore-arm, and the other towards the little-finger-side, and they become the principal muscles in these movements respectively; but when they act together, their several tendencies to draw the wrist to opposite sides counterbalance one another, and they simply assist the principal muscles in bending the wrist downwards upon the fore-arm.

608. Almost every muscle in the Human body has its antagonist, which performs an action precisely opposite to its own. Thus by one set of muscles, termed flexors, the joints are bent; by a contrary set, the extensors, they are straightened. One set of muscles draws the arm or leg outwards, or away from the central line of the body; another draws the limbs inwards. One set, again, closes the jaws ; and another opens it. But we find an economy of muscular substance in some of the lower animals, where parts are to be usually kept in a particular position, which has only to be changed occasionally and for a short time; the antagonism being then supplied by yellow elastic tissue (\$ 29).

609. We commonly find that, in order to preserve the 
necessary form of the animal body, Muscles are applied at a great mechanical disadvantage as regards the exercise of their power; that is, a much larger force is employed than would suffice, if differently applied, to overcome the resistance. But we generally find that, in this as in other forms of lever action, what is lost in power is gained in time; and thus a very slight change in the length of a muscle is sufficient to produce a considerable movement.

610. The first source of disadvantage results from the direction in which the muscle is attached to the bone. This is rarely at right angles to it ; and consequently a considerable part of the power is lost (see Mechan. Philos., § 299). Thus if the muscle $m$ (fig. 213), whose force we shall suppose equal

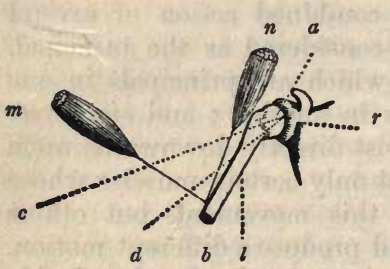

Fig. 213. to 10 , be fixed at right angles to the bone $l$, whose extremity $a$ is movable upon the point of support $r$, its force of contraction will be most advantageously applied to overcome the resistance, and will draw the bone from the position $a b$ into the direction a $c$, making it traverse a space which we shall also represent by 10 . But if this muscle act obliquely on the bone, in the direction of the line $n b$ for example, it will be quite otherwise; for it will then tend to draw the bone in the direction $b n$, and will consequently make it approach the articular surface $r$. But as this bears upon an immovable socket, and as the bone can move in no other way than by turning upon the point $r$ as upon a pivot, the contraction of the muscle to the same amount as before will carry the bone no further than into the direction $a d$; three-quarters of the force employed will thus be lost, and the resulting effect will be no more than one-fourth of that which the same power applied perpendicularly to the bone would have produced.

611. We usually find that the muscles are inserted so obliquely, that their power is applied at a great disadvantage; but this disadvantage is rendered much less than it would have otherwise been, by a very simple contrivance,-that very enlargement of the bones at the joints which is necessary to give them the required extent of surface for working over 
each other. Thus, let $r$ and $o$ (fig. 214) be two bones connected by a joint; and let the muscle $m$, which moves the lower bone upon the upper, be attached to the former at $i$. Now as this muscle acts almost precisely in the line of the bones themselves, almost all its power will be expended in drawing the lower bone against the upper. But by the enlargement of the ends of the bones, as seen in fig. 215, the direction of the tendon of the muscle $m$ is so changed, near its insertion $i$, that

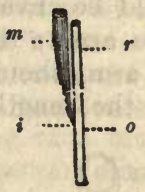

Fig. 214.

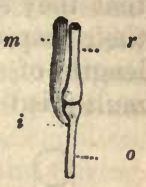

Fig. 215. the contraction of the muscle will cause the lower bone to turn upon the upper one with comparatively little loss of power. In the knee we find a still greater change of direction effected, by the interposition of a movable bone, the patella or knee-pan, in the substance of the tendon.

612. But the advantage or disadvantage with which the muscles act upon the bones, depends in great degree upon the relative distances of their point of attachment from the fulcrum on which the bone moves, and from the point at which the resistance is applied. Every bone acted-on by muscles may be regarded as a lever, having its fulcrum or point of support in the joint, its power where the muscle is attached to it, and its weight where the resistance is to be overcome; and the distances of the fulcrum from the power and the weight respectively are termed the two arms of the lever. Now, on the mechanical principles fully explained elsewhere (MECHAN. PHILOS., § 287), the relative length of these two arms determines the force which is necessary to overcome a given resistance. Thus in the Steelyard (fig. 216), the beam is divided into two arms of unequal length at the point of support or fulcrum $\alpha$;

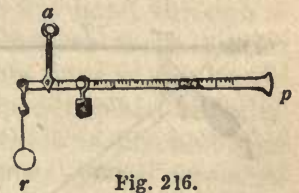
at the end of the short arm $r$, hangs the body whose downward pressure we wish to determine; and on the other $p$ there slides a weight, which will balance a greater or less amount of pressure at the opposite extremity $r$, according as it is made to hang from a point which is more distant from the fulcrum or nearer to it, - that is, according as the length 
of the power-arm of the lever is increased or diminished, that of the weight-arm remaining the same.

613. Now in order that there may be an equilibrium, or balancing between the power and the weight, it is necessary that they should be inversely proportional to the lengths of their respective arms ; that is, the power multiplied by the length of its arm, should be always equal to the weight multiplied by the length of its arm. Thus, to balance a

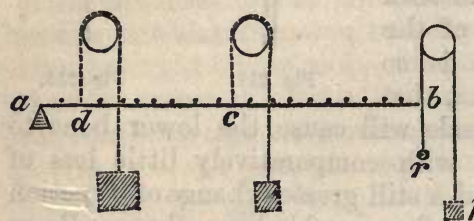

Fig. 217. certain resistance $r$, equal to 10 , and applied at the end of a lever $a b$ (fig. 217), whose length we shall call 20 , it is necessary that a force $p$, applied at the same point, and consequently at the same distance from the fulcrum $a$, should also be equal to 10 ; but, if the power be applied at the point $c$, which is at only half the distance from the fulcrum $a$, it must be doubled in amount, or equal to 20 ,since it must be sufficient, when multiplied by its distance 10 from the fulcrum, to make 200 , which is the product of the resistance 10 and its distance from the fulcrum 20; and in like manner, if the power be applied at $d$, where its distance from the fulcrum is only 2 , its amount must be 100 , in order that its product with the distance at which it is applied may be equal to 200. Hence, when a muscle is applied near the fulcrum, while the resistance is at a distance from

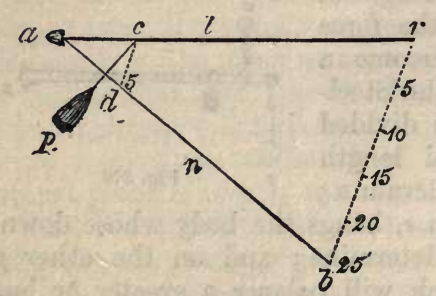

Fig. 218. it, so that the bone becomes a lever of the "third order," its force must be proportionably greater.

614. But this arrangement greatly increases the rapidity of the motion which is the consequence of the muscular action.

For let us suppose that the muscle $p$ (fig. 218) acts upon the lever $a r$, in such a manner that its point of insertion $c$ tra- 
verses a space equal to 5 in one second; the extremity $r$ of the lever will traverse a space equal to 25 in the same time, its distance from the fulcrum $a$ being five times as great as that of the point $c$ from the fulcrum. Hence, although, to raise a given weight at $r$, a power more than five times its amount must be applied at $c$, that power will raise the weight through a space five times as great as that through which itself passes in the same time. Thus, what is lost in power is gained in time; and the shortening of a muscle, small in amount, but effected with sufficient power, causes the raising of a weight through a considerable space.

615. We shall find that this is the case in regard to most of the muscular actions in the animal economy. Thus, the fore-arm (fig. $219, b, c$ ) is bent upon the arm $a$ by a muscle $d$,

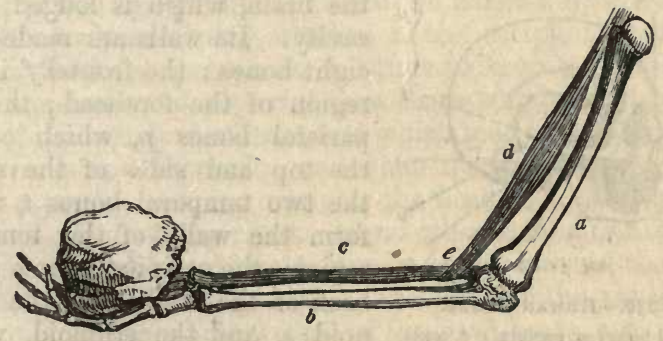

Fig. 219.

which arises from the top of the latter, and which is inserted at $e$, a short distance from the elbow-joint. Hence its contraction to a very slight extent will raise the hand through a considerable space; but a proportional increase in its power will be required to overcome any resisting force in the hand. -The arm is straightened again by an antagonist muscle, which lies on the back of the arm, and which is attached to a short projection made by one of the bones of the fore-arm behind the elbow : this muscle also operates at a similar disadvantage in regard to power, and advantage in point of time, in consequence of its point of attachment being so near to the fulcrum. In responding to its action, however, the bones of the fore-arm constitute a lever of the "first order ;" the elbowjoint, which serves as the fulcrum, being now between the power and the resistance. 
Motor Apparatus of Man:-Skeleton and Muscles.

616. Before entering upon the examination of the various movements of the lower animals, and of the means by which these are effected, it will be useful to acquire a general knowledge of the structure of the Human Skeleton, and of the uses of its several parts. The skeleton, which is formed by the union of about 200 bones, is divided like the body into head, trunk, and members. The bones of these parts will now be separately described.

617. The Head is composed of two parts, the cranium or skull, and the face. The cranium (fig. 220) is a bony case of oval form, occupying the upper and back part of the head,

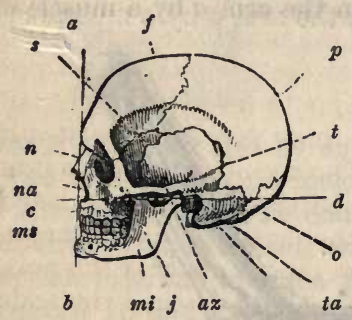

Fig. 220.-HUMAN SkULL.

$f$, frontal bone; $p$, parietal; $t$, temporal; 0 , occipital ; $\delta$, sphenoid; $n$, nasal; ms, superior maxillary ; $j$, malar or cheek bone; $m i$, inferior maxilla; $n a$, anterior opening of the nose; $t a$, auditory aperture; $a z$,zygomatic arch ; $a, b, c, d$, lines indicating the facial angle. and serving for the protection of the brain, which is lodged in its cavity. Its walls are made-up of eight bones : the frontal $f$ in the region of the forehead; the two parietal bones $p$, which occupy the top and sides of the skull; the two temporal bones $t$, which form the walls of the temporal region; the occipital bone 0 at the back of the head; and the sphenoid $s$, and the ethmoid, which assist in forming the floor of the cavity. These bones are firmly united to each other by sutures, the character of which varies in different parts of the cranium, so that they are the better able to resist external violence. Thus, a blow upon the top of the arch formed by the parietal bones will tend to separate them from each other and from the frontal bone, and to force asunder their lower borders. Both these effects are resisted by the peculiarity of the suture which unites different parts of the parietal bone to its neighbours; for at the top of the skull the bones are firmly held together by the interlocking of the projections of each, whilst the lower edge of the parietal bone is prevented from being driven outwards by the overlapping edge of the temporal bones, which form, as it were, a buttress to the arch. This same contrivance 
prevents the temporal bone from being driven inwards, as it might have otherwise been, by a blow on the side of the head.

618 . In the base or floor of the cavity of the cranium are seen a number of apertures, which serve for the passage of the blood-vessels that supply the brain, and of the nerves that issue from it. One of these apertures, much larger than the rest, and situated in the occipital bone, gives passage to the Spinal Cord ; and on each side of this aperture there is a large bony projection from the under surface, termed the condyle, by which the skull rests on the vertebral column, and is enabled to move forwards or backwards upon it. The head is nearly balanced upon this pivot; nevertheless, the portion situated in front of the joint is more heavy than that which is situated behind it, and is consequently not altogether counterpoised by the latter. Hence the muscles which, arising from the back and being attached to the occipital bone, tend to draw the head backwards, and thus to keep it upright, are more numerous and powerful than those which are situated in front of the vertebral column, and which tend to draw the head downwards and forwards ; and when the former are relaxed, as in a person sleeping upright, the head has a tendency to fall forwards upon the chest. In no other animal is this joint situated so far forwards as in Man. As we descend the scale, we fird it nearer and nearer to the back of the skull; and consequently the whole weight of the head bears, not directly upon the spine, but upon the muscles and ligaments by which it is attached to the vertebral column.

619. On each side of the base of the cranium, we observe. a large rounded projection, termed the mastoid. To this projection (which we feel behind the lower part of the ear) is attached on either side a powerful muscle, the sterno-mastrid $\left({ }^{23}\right.$, fig. 227), which passes downwards and towards the central line; so that the two muscles nearly meet at the bottom of the neck, where they are attached to the upper edge of the breastbone. These muscles, asting together, serve to draw the head forwards; but either of them acting separately will turn it to one side or the other. In front of these two projections of the skull, we notice the opening $t a$ of the external ear; which, like the different chambers of the internal ear, is excavated in a portion of the temporal bone which is termed petrous from its very dense and stony character. 
620. The face is formed by the union of fourteen bones; and presents five large cavities, which serve for the lodgment and protection of the organs of sight, smell, and taste. All the bones of the face, with the exception of the lower jaw, are completely immovable, and are firmly united to each other and to the bones of the cranium ( $\$ 617)$. The two principal are the superior maxillary ( $m s$, fig. 220), which form nearly the whole of the upper jaw, and are connected with the frontal bone in such a manner as to contribute to the formation of the orbital cavities in which the eye is lodged, and of the nasal cavities which form the interior of the nose ; they also constitute the front of the roof of the mouth; on the sides of the face, they articulate with the malar or cheek-bones $j$; whilst they are united behind with the palate-bones which form the back part of the roof of the mouth, and which in their turn are united to the sphenoid.

621 . The orbits, as we have already seen ( $\$ 538$ ), are two deep cavities, of a conical form, - the base of the cone being directed forwards, and its apex or point backwards ; the roof of these cavities is formed by a portion of the frontal bone, and their floor chiefly by the superior maxillary. Their inside wall is formed by the ethmoid bone, and by the small bone termed the lachrymal, in which is the canal for the passage of the tears into the nose ( $\$ 541)$; and the outside wall is formed partly by the cheek-bone and partly by the sphenoid,- - the latter also bounding the cavity at its deepest part, and containing the apertures which serve for the passage of the optic and other nerves that enter the orbit from the cranium. In the roof of the orbit, on its outer side, there is a broad shallow pit or depression, in which the lachrymal gland is lodged.

622. The greater part of the nose is formed by cartilages; so that, in the bony skull, the anterior opening of the nasal cavity ( $n a$, fig. 220 ) is very large ; and the bony portion of the nose, formed by the two small bones $(n)$ termed nasal, projects but slightly. The nasal cavity, divided in the middle by a vertical partition into two fossce or excavations, is very extensive ( $\$ 506)$; at the upper part it is hollowed-out into the ethmoid bone, the whole interior of which is made-up of large cells; its floor is formed by the arch of the palate, which separates it from the mouth; behind it extends as far 
as the back of the mouth, and communicates with the pharynx by two apertures termed the posterior nares (fig. $200, c)$. The partition between the fossæ is formed at the upper part by a plate that projects downwards from the ethmoid bone, and at the lower by a distinct bone called the vomer (or ploughshare) from its peculiar form; to the front edge of this last is attached a cartilage, which continues the partition forwards into the soft projecting portion of the nose. It is through the thin horizontal plate of the ethmoid bone, which separates the nasal cavity from that of the skull, that the olfactory nerves make their way out from the former into the latter : they descend in numerous branches, for the passage of which through the roof of the nose this plate is perforated by a number of small apertures, which give it a sieve-like aspect; whence it is called the cribriform $^{1}$ plate of the ethmoid.

623. It is in the superior maxillary bone that all the teeth of the upper jaw are implanted in Man; but in the embryo this bone is composed of several pieces; and one of these pieces, termed the intermaxillary bone (im, fig. 221), remains

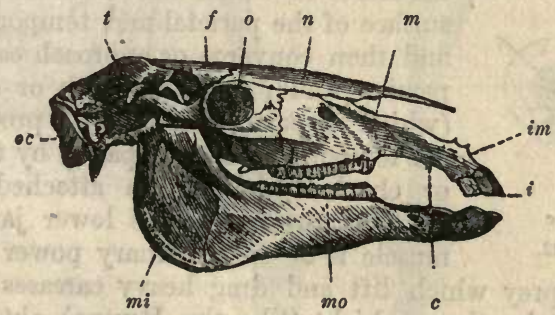

Fig. 221.-SKULL OF HORse.

oc, occipital bone; $t$, temporal ; $f$, frontal ; $n$, nasal ; $m$, superior maxillary; in, intermaxillary; $m i$, inferior maxillary; 0 , orbit; $i$, incisor teeth; $c$, canines; mo, molars.

permanently separate in most of the lower animals. The lower jaw of adult Man, also, is composed but of a single piece ; though this is divided in the infant on the central line, and the two halves remain separate in many of the lower animals. This bone has a general resemblance in form to a horse-shoe with its extremities turned up considerably; it is

1 From the Latin, cribrum, a sieve. H $\mathrm{H} \mathbf{2}$ 
articulated with the temporal bones by a condyle or projecting head with which each of these extremities is furnished; and this head is received into what is called the glenoid ${ }^{1}$ cavity on the under side of the temporal bone. In front of the condyle is another projection, or process, termed the coronoid (a, fig. 92), which serves for the attachment of one of the principal muscles that raise the jaw. These muscles are all attached near the angle of the jaw (or the point at which it bends upwards), and they consequently act at a small distance from its fulcrum, whilst the resistance is applied at the furthest point $(\$ 180)$. We are continually reminded of the loss of mechanical power which results from this, by our inability to exercise the same force with our front teeth that we can employ with the back. Thus, when we wish to crack a nut, or to crush any hard substance between the teeth, we almost instinctively carry it to the back of the jaws, so as to place it nearer the joint, where it may receive more of the power of the muscle.

624. The general arrangement of the chief muscles of the face is seen in fig. 222. The largest is the temporal muscle, $t$,

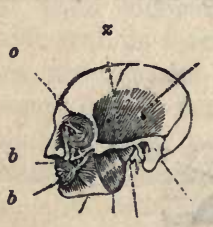

$j \mathrm{~m} c$

Fig. 222.

the fibres of which arise from an extensive surface of the parietal and temporal bones, and then converge or approach each other, passing under the bony arch or zygoma, $z$ (which is partly formed by a process from the temporal bone, and partly by the malar or cheek bone), to be attached to the a coronoid process of the lower jaw. This muscle is of extraordinary power in those beasts of prey which lift and arag heavy carcases in their jaws; and in those which (like the Hyæna) obtain their support by crushing the bones that others have left. It is assisted by the masseter muscle $m$, which passes from the zygomatic arch and cheek-bone to the angle of the lower jaw, and also by other muscles. Besides these, the figure shows the ring-like muscle or sphincter $o$, which surrounds the opening of the eye, and serves by its contraction to close the lids; and also the similar muscle $b b$, which surrounds the

I The term condyle is applied to most of the projecting surfaces of articulation, in different parts of the body; and the term glenoid to the cavities into which these are received. 


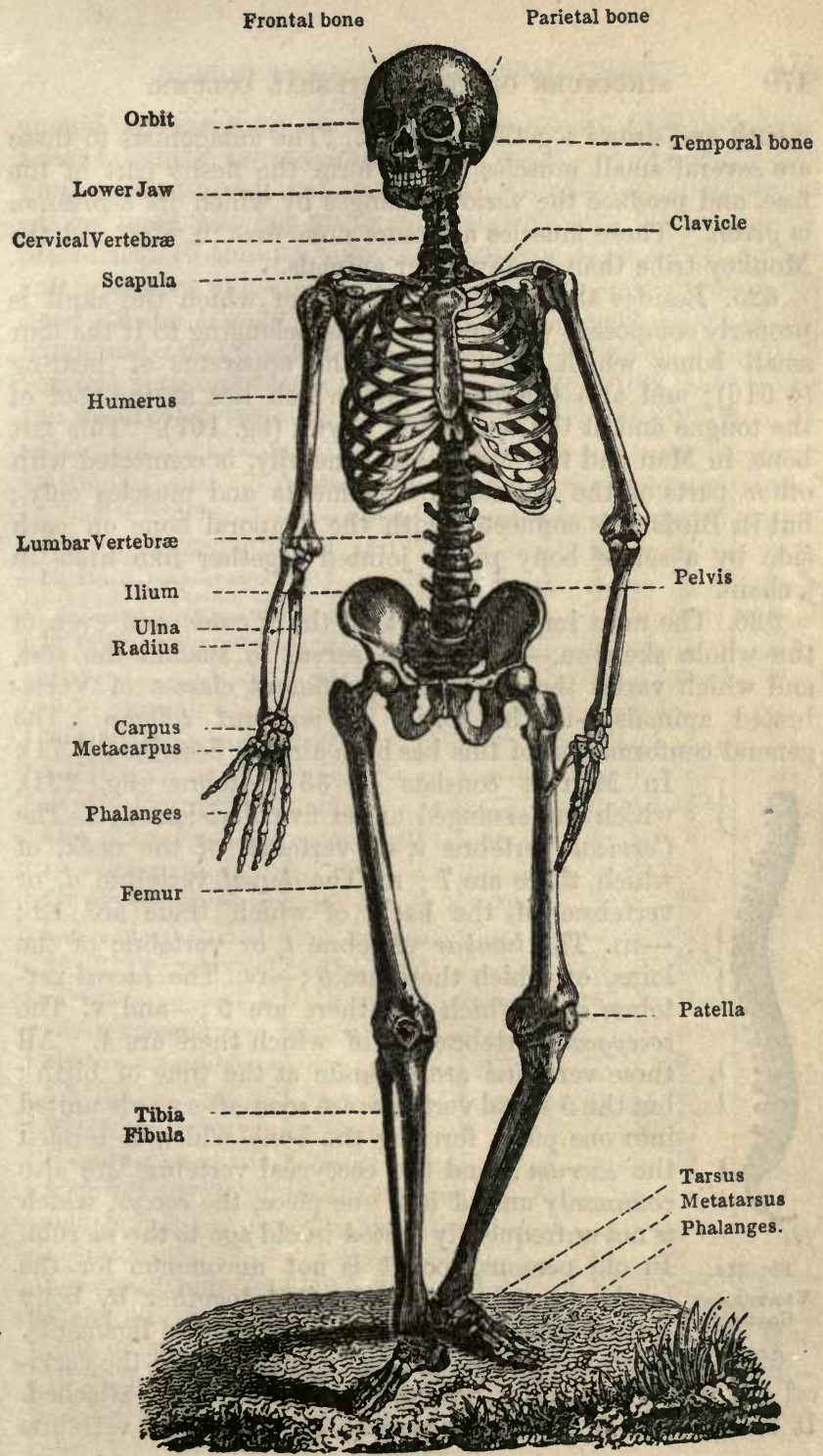

Fig. 223, -SKELETON OF MAN. 
mouth and draws together the lips. The antagonists to these are several small muscles which form the fleshy part of the face, and produce the various changes by which its expression is given. These muscles are more numerous in Man and the Monkey tribe than in any other animals.

625. Besides the twenty-two bones of which the skull is properly composed, we may reckon as belonging to it the four small bones which form part of the apparatus of hearing (\$ 516); and also the hyoid bone, which lies at the root of the tongue and at the top of the larynx (fig. 107). This last bone, in Man and the Mammalia generally, is connected with other parts of the skeleton by ligaments and muscles only; but in Birds it is connected with the temporal bone on each side by a set of bony pieces jointed together like links in a chain.

626. The most important part of the Trunk, and even of the whole skeleton, - that which serves to sustain the rest, and which varies the least in the different classes of Vertebrated animals, -is the spinal or vertebral column. The general conformation of this has been already described ( $\$ 71)$.

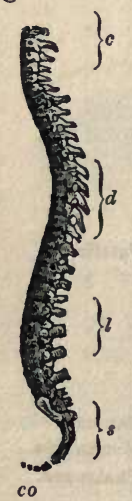
In Man it consists of 33 vertebræ (fig. 224), which are arranged under five divisions;-I. The Cervical vertebræ $c$, or vertebræ of the neck, of which there are 7 ; Ir. The dorsal vertebræ $d$, or vertebræ of the back, of which there are 12 ; -III. The lumbar vertebræ $l$, or vertebræ of the loins, of which there are 5 ;-rv. The sacral vertebræ $s$, of which also there are 5 ;-and v. The coccygeal vertebræ $c 0$, of which there are 4 . All these vertebræ are separate at the time of birth; but the 5 sacral vertebræ are soon afterwards united into one piece, forming the bone which is termed the sacrum: and the coccygeal vertebræ are also commonly united into one piece, the $\operatorname{coccy} x$, which is not unfrequently united in old age to the sacrum. Fig. 224. In old persons, too, it is not uncommon for the Vertemas lumbar vertebræ to be united together by bony Columa. matter deposited in their cartilages and ligaments. 627. The dorsal vertebræ are distinguished from the cervical and lumbar, as being those to which the ribs are attached. It is remarkable that the number of the cervical vertebræ 
should be the same in all the Mammalia; even the longnecked Giraffe having only seven, while the Whale, whose head seems to be joined to its body without the intervention of any neck, also has seven cervical vertebræ, although they are almost as thin as a sheet of paper. It is owing to the small number of joints in its neck, that the movements of the head of the Giraffe are far less graceful than those of the Swan and other long-necked Birds, in which the number of cervical vertebræ is much greater. The following table shows the number of vertebræ in animals of different groups.

\begin{tabular}{|c|c|c|c|c|c|c|}
\hline MaMMALIA. & Cervical. & Dorsal. & Lumbar. & Sacral. & Coccygeal. & Total. \\
\hline Man ........................... & 7 & 12 & 5 & 5 & 4 & 33 \\
\hline Long-tailed Monkey...... & 7 & 12 & 7 & 3 & 31 & 60 \\
\hline Lion ............................ & 7 & 13 & 7 & 3 & 26 & 56 \\
\hline Long-tailed Opossum ... & 7 & 16 & 6 & 2 & 36 & 67 \\
\hline Long-tailed Ant-Eater... & 7 & 16 & 3 & 6 & 40 & 72 \\
\hline Elephant ..................... & 7 & 20 & 3 & 4 & 27 & 61 \\
\hline Giraffe ......................... & 7 & 14 & 5 & 4 & 18 & 48 \\
\hline 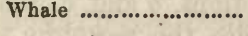 & 7 & 15 & 9 & 1 & 27 & 59 \\
\hline BIRDs. & & & & & & \\
\hline Vulture................. & 15 & 7 & - & 13 & 6 & 41 \\
\hline Swallow ....................... & 13 & 7 & - & 10 & 7 & 37 \\
\hline Turkey .......................... & 14 & 7 & - & 15 & 6 & 42 \\
\hline 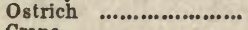 & 18 & 9 & - & 19 & 9 & 55 \\
\hline Crane & 17 & 10 & - & 15 & 6 & 48 \\
\hline Swan............................. & 23 & 11 & - & 16 & 8 & 58 \\
\hline REPTILEs. & & & & & & \\
\hline Tortoise ..................... & 9 & 10 & - & 3 & 20 & 42 \\
\hline Monitor (Lizard) .......... & 6 & 21 & 2 & 2 & 115 & 146 \\
\hline Python (Boa) ................ & - & 320 & - & - & 102 & 422 \\
\hline Rattle-Snake ............... & - & 171 & - & - & 36 & 207 \\
\hline Land Salamander .......... & 1 & 14 & - & 1 & 26 & 42 \\
\hline 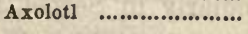 & 2 & 18 & - & - & 42 & 62 \\
\hline Fishes. & & & & 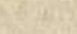 & (7) & \\
\hline Perch ....................... & - & 21 & - & - & 21 & 42 \\
\hline Mackerel ...................... & - & 15 & - & - & 16 & 31 \\
\hline Trichiurus ................... & - & 60 & - & - & 100 & 160 \\
\hline Salmon & - & 34 & - & - & 22 & 56 \\
\hline Cod $\quad \ldots \ldots \ldots \ldots \ldots \ldots \ldots . . . . . . . . . . .$. & - & 19 & - & - & 34 & 53 \\
\hline Conger Eel ..................... & - & 60 & - & - & 102 & 162 \\
\hline Electric Eel ................. & - & - & - & - & -7 & 236 \\
\hline Shark ........................ & - & 95 & - & - & 270 & 365 \\
\hline
\end{tabular}

We see from the above table, that it is by the multiplication of the coccygeal vertebræ, that the tail is prolonged in those animals which possess it. In fact, it is only in Man, and in those of the Ape tribe which approach nearest to him, that the number of these vertebræ is as low as 4. 
628. It has been already noticed ( $\$ 71$ ) that an ordinary character of the vertebrce consists in their being perforated by an aperture (fig. 225), which, when several vertebræ are united.

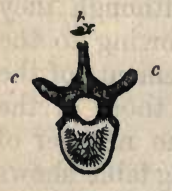

$a$ together, forms a continuous tube or canal for the lodgment of the spinal cord. This character is usually lost, however, in the coccygeal vertebræ; which are so much contracted and simplified as to contain no aperture. The purpose of the division of the spinal column into so large a number of separate Fig. 225.-SINGLE bones, is obviously to allow of considerable VERTEBra. freedom of motion by a slight shifting amongst the individual parts; whilst any such sudden bend as would be injurious to the spinal cord, is avoided. Each vertebra consists of a solid "body" a, which is situated in front of the spinal canal in Man, but below it in animals whose back has a horizontal position, and which serves to give solidity to the structure,-and of "processes" or projections, $b$ and $c$, that serve to form the spinal canal, and to unite the vertebræ to each other. In Man and other warm-blooded animals, the two surfaces of the "body" are nearly flat and are parallel to each other; and they are united to the corresponding surfaces of the neighbouring vertebræ by a disk of fibro-cartilage ( $\$ 47$ ), which extends through the whole space that intervenes between them, and which, being firmly adherent to both, prevents them from being far separated from each other:

629. But in Reptiles and Fishes, a different plan is adopted. In the animals of the former class, particularly in Serpents, we find one surface of each vertebra convex or projecting, and the other concave or hollowed-out; and the convex surface of each vertebra fits into the concave surface of the next, in such a manner that the whole spinal column becomes a series of ball-and-socket joints, and is thus endowed with that flexibility which is essential to the peculiar movements of these animals. In Fishes both surfaces are concave, and between each vertebra there is interposed a bag containing fluid, and having two convex surfaces, over which those of the vertebræ can freely play. Extreme facility of movement is thus given to the spinal column; but its strength is proportionally diminished. It is to be remembered, however, that strength is not required in the bony framework of animals, whose bodies, 
instead of being supported upon fous fixed points, are buoyed up in every part by a liquid of nearly the same density with themselves. The extreme flexibility of the spine of Fishes, enables them to propel their bodies by the movements of the hinder portion and tail from side to side ; their members, or pectoral and ventral fins (fig. 243), being but little used except for influencing the direction of their motion. And thus we see that in the lowest Vertebrata, as in the lower Articulata (such as the Leech and Earth-worm), the propulsion of the body being accomplished by the movements of the trunk itself, its skeleton (internal in the one case, external in the other) is left in the soft condition which it has in all at an early period: whilst in the higher classes of both series, - Birds and Insects for example,-the extremities being so developed, and being furnished with muscles so powerful that the function of locomotion is entirely committed to them, the skeleton of the body undergoes great consolidation, its various pieces being so knit together as to make the trunk almost immovable.

630. This knitting-together is partly accomplished by means of projections or processes from the several vertebræ, which are united to one another by muscles and ligaments. Of these processes there are seven in Man from each vertebra. One of these, termed the spinous process (b, fig. 225), projects directly backwards; and thus is formed the prominent ridge on the back, in which the ends of these projections can be distinguished. The spinous processes serve in Man to give attachment to the muscles, by which the trunk and head are kept erect; in Animals whose spine is horizontal, they are generally much longer, in order to give firm attachment to the muscles and ligaments which support the head (fig. 229, vc, vd). And in Fishes they are greatly prolonged (fig. 243), so as to increase the surface by the stroke of which from side to side the body is propelled through the water. On each side of the vertebra is a process ( $c$, fig. 225) which is called transverse; this serves for the attachment of the ribs to the vertebra. And lastly, from the upper and under side of each vertebra, two articulating processes project, which lock against each other in such a manner as to prevent the movements of the vertebræ from being carried to an injurious extent. These processes are peculiarly long in Birds, where they almost 
completely check the movements of the dorsal vertebræ; thereby giving to the trunk that firmness which is required for the attachment of the muscles of the wings. The portions of bone which pass backwards from the body of each vertebra to its transverse processes, and thus form the side-wall of the spinal canal, are called the arches of the vertebræ. These are the parts first formed. On the under edge of each there is a notch which corresponds with one in the upper side of the next, in such a manner that, when two vertebræ are placed together, a complete foramen or aperture is formed, which serves for the passage of the nerves that are given-off from the Spinal Cord ( $\$ 457$ ).

631. The vertebral column of Man is disposed in a double curve, as seen in fig. 224 ; the effect of this is to diminish the shock that would be produced by a sudden "jar," - such as when a man jumps from a height upon his feet. If the vertebral column had been quite straight, this jar would have been propagated directly upwards from the pelvis to the head, and would have produced very injurious effects upon the brain; but by means of the double curvature, and the elasticity of the ligaments, \&c. which hold together the vertebræ, it is chiefly expended in increasing for a moment the curves of the spine, which thus acts the part of a spring. The constant pressure of the head and upper part of the trunk has a tendency to increase these curves permanently, and thus to diminish the height of the body. The elasticity of the intervertebral substance, however, causes it to recover, during the time when the body is in the horizontal posture, the form it had lost by pressure in the upright position; and thus a man is taller by half an inch or more when he rises in the morning, than he was when he lay down the night before.

632. The first vertebra of the neck, termed the atlas, is much more movable than the rest, and differs considerably from them in its form. It is destitute of body; but it has a broad smooth surface on either side, on which rest the "condyles" of the occipital bone of the skull ( $\$ 618)$, in such a manner that the head is free to nod backwards and forwards. The atlas itself turns upon a sort of pivot, formed by an upward projection from the next vertebra, which is termed the axis; this projection, called from its form the processus 
dentatus (or tooth-like process), occupies the place of the body of the atlas; and by the rotation of the atlas around it, the movements of the head from side to side are accomplished. Wherever great freedom of motion is permitted, displacement or dislocation is necessarily more easy; and accordingly we find that the atlas and axis can be more easily separated from each other, than can any other two vertebræ. This dislocation may be produced by violence of different kinds; thus if the head be suddenly forced forwards while the neck is held back, the tooth of the axis may be caused to press against the spinal cord, and thus to interrupt or completely check its functions. Or, again, if the weight of the body be suspended from the head, and especially if it be thrown upon it with a jerk, the two vertebræ are liable to be dragged asunder, and the spinal cord to be stretched or broken. This is sometimes the immediate cause of death in hanging; and it has not unfrequently occurred when children have been held in the air by the hands applied to the head,-a thing often done in play, but of which the extreme danger should prevent its ever being practised. Any serious injury of the spinal cord in this region must be immediately fatal, for the reason formerly stated ( $\$ 470)$,- - that it causes the suspension of the motions of respiration.

633. The number of the ribs which are attached to the bodies and transverse processes of the dorsal vertebræ, is, in the Human species, twelve on each side. ${ }^{1}$ The number in different animals may be judged-of by that of the dorsal vertebræ in the table already given ( $\$ 627)$; since it is the attachment of the ribs that makes the essential difference between the dorsal vertebræ and the cervical or lumbar. The other extremity of each rib is connected with a cartilage, which is a sort of continuation of it; in Birds, the cartilages of the ribs are ossified or converted into bone. The cartilages of the first seven ribs (in Man), which are termed the true ribs, are united to the sternum or breast-bone, which forms the front wall of the thorax (fig. 163). The cartilages of the five lower ribs are not directly connected with this, and they are hence called false ribs ; those of three of them, however, are

1 It is scarcely necessary here to state, that the common notion respecting the deficiency of a rib on one side of the body of Man is a popular error. 
connected with the cartilage of the seventh rib; while the other two ribs, being completely unattached at the anterior ends, are termed floating ribs. The sternum or breast-bone is flat and of simple form in Man; but it is much larger in many other animals. In those which have need of great strength in the upper limbs, such as Birds, Bats, and Moles, it is not only increased in breadth, but is furnished with a projecting keel or ridge for the attachment of powerful muscles (fig. 250). In the Turtle tribe, on the contrary, it is very much extended on the sides, so as to afford, with the ribs, a complete protection to the contained parts ( $\$ 83)$.

634. We have next to consider the structure of the members or appendages which are attached to this central framework. These are spoken-of as superior and inferior, when we are treating of Man, whose erect posture places one pair above the other: but when the ordinary Quadrupeds are alluded to, they are termed anterior and posterior, one pair being in front of the other. Each member consists of a set of movable bones, which serve as levers; but the socket in which the first of these works, is formed by a bony framework, which is connected more or less closely with the spinal column. This framework, in the upper extremity, consists of the Scapula or blade-bone, and the Clavicle or collar-bone. In the lower extremity, it is formed by a set of bones, the union of which with the sacrum completes the Pelvis or bason at the bottom of the spinal column (fig. 223).

635. The Scapula is a large flat bone, which occupies the upper and external ${ }^{1}$ part of the back. Its form is somewhat triangular; and at its upper and outer angle is a broad but shallow cavity, destined to receive the head of the humerus or arm-bone. Above this cavity is a large projection, termed the acromion-process, which is united by ligaments, \&c., with the external end of the clavicle, and thus forms the bony eminence that we feel at the top of the shoulder. A little internally to this we find another process, the coracoid, which only serves in Man for the attachment of certain muscles, but which in Birds is developed into a distinct bone ( $\$ 668$ ). The hinder surface of the scapula is divided into two by a projecting ridge or keel, which gives a more extensive and

1 The term external is continually used in Anatomy, to describe the parts furthest removed from the central or median line of the body. 


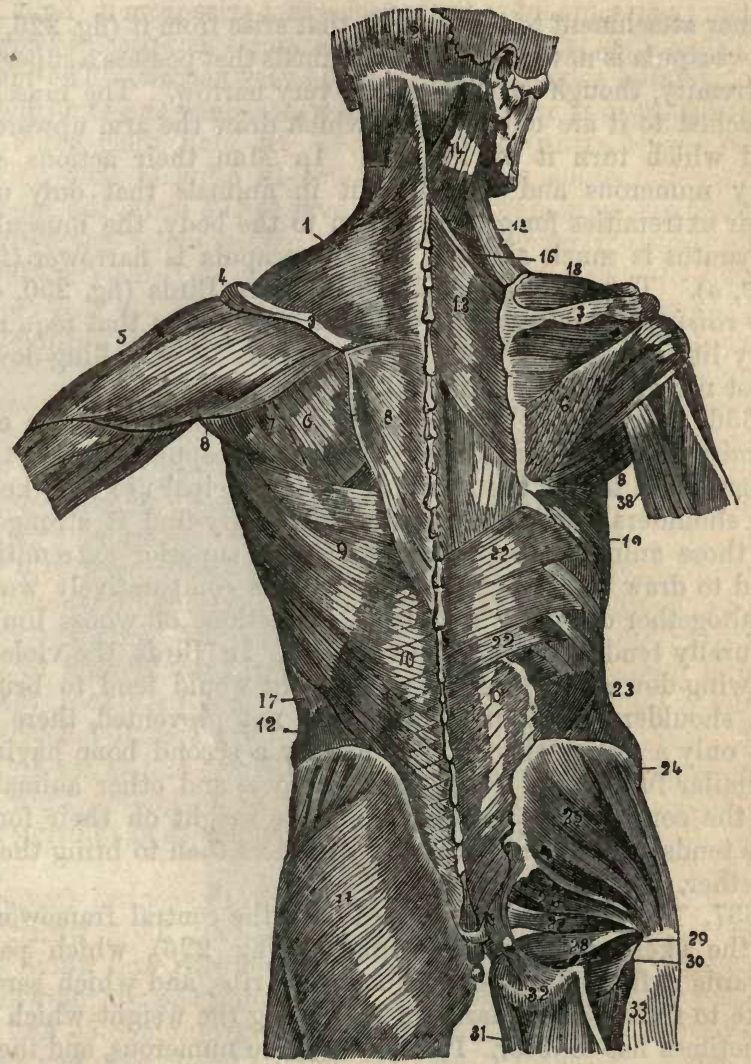

Fig. 226-MUSCles of THE Back OF The TRUNK, THF. SUPERTICIAL. LAYER BeING SHOWN ON THE LEFT SIDE, THE MIUDLE LAYER ON THE RIGHT.

1. Trapezius; 2, occipital bone; 3, spine of the scapula; 4, acromion process; 5 , deltoid; 6 , infraspinatus; 7 , teres minor ; 8 , teres major; 9 , latissimus dorsi; 10 , its aponeurosis; 11 , glutæus magnus; 12 , space between the latissimus dorsi and external oblique; 13 , rhomboideus major; 14 , splenius; 15 , angularis scapulæ; 16 , rhomboideus minor; 17 , external oblique; 18 , supraspinatus; 19 , serratus magnus; 22 , inferior serratus minor; 23 , internal ob'ique; 24 , crest of the iliac bone; 25 , glutæus medins; 26 , pyramidalis; 27 , gemellus superior; 28 , obturator internus; 29 , gemellus inferior; 31 , quadratus femoris; 31 , semi-membranosus ; 32 , tuberosity of the ischium; 33 , insertion of the glutæus maximus; 38 , long head of the triceps extensor of the arm. 
firmer attachment to the muscles that arise from it (fig. 226, ${ }^{3}$ ). The scapula is never deficient in animals that possess a superior extremity, though sometimes it is very narrow. The muscles attached to it are chiefly those which draw the arm upwards, and which turn it on its axis. In Man, their actions are very numerous and varied; but in animals that only use their extremities for giving motion to the body, the muscular apparatus is much simpler, and the scapula is narrower (fig. 229,0 ). This is particularly the case in Birds (fig. 250, o), the raising of whose wings in flight is an action that requires very little power, though for their depression or pulling-down great muscular force is needed.

636. The Clavicle is a rounded bone, attached at one extremity to the acromion-process of the scapula, and at the other to the top of the sternum. Its principal use is to keep the shoulders separate; and we accordingly find it strongest in those animals, the actions of whose superior extremities tend to draw them together; whilst it is comparatively weak or altogether deficient in animals, the actions of whose limbs naturally tend to keep them asunder. In Birds, the violent drawing-down of whose wings in flight would tend to bring the shoulders together if they were not prevented, there is not only a strong clavicle, but usually a second bone having a similar function ( $\$ 668)$. In the Horse and other animals, on the contrary, the bearing of whose weight on their forelegs tends rather to separate the shoulders than to bring them together, the clavicle is deficient.

637. The Scapula is connected with the central framework of the skeleton by various muscles (fig. 226), which pass towards it from the spinal column and ribs, and which serve alike to fix it, and to assist in sustaining the weight which it sometimes has to bear. In Man these are numerous, and their actions are various; since the scapula is left very movable in him, that the actions of the arm may be more free. In Quadrupeds it is generally more fixed; and the trunk is slung from it, as it were, by a muscle (the serratus magnus, ${ }^{9}$ ) of moderate thickness in Man, but in these animals of great strength, which passes from the scapula to be attached to the ribs.

638. The superior or anterior member itself is divided into three principal portions, - the arm, fore-arm, and hand. The arm is supported by a single long and cylindrical bone, which 
is called the lumerus; this has a large rounded head, which is received into the glenoid cavity of the scapula ; whilst its lower end is rather flattened, so as to articulate with the two bones of the fore-arm in the hinge-joint of the elbow. The

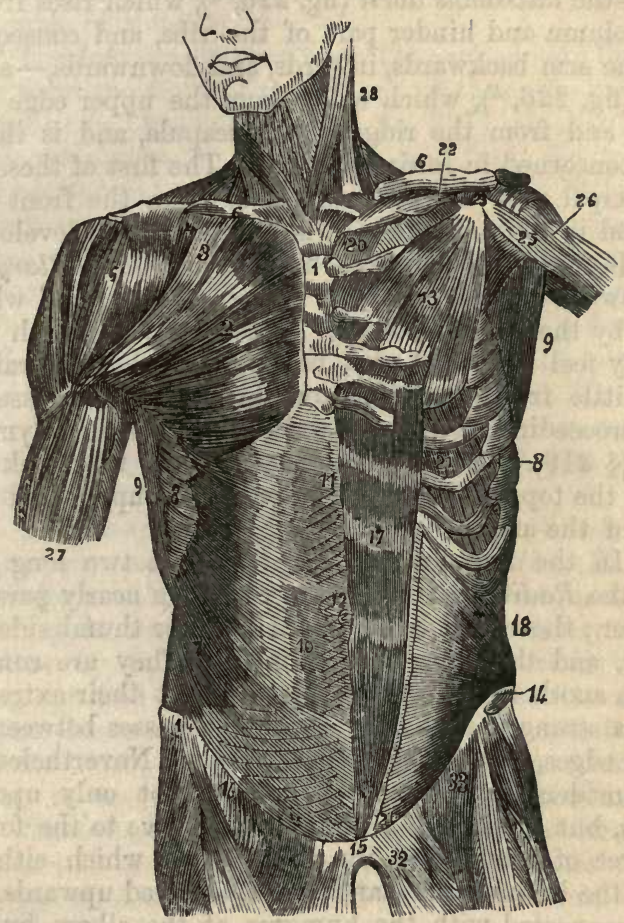

Fig. 227.-Muscles of the Front of the Trunk, the Superficial Later BeING SHOWN ON THE LEFT SIDE OF THE FIGURE, THE MIDDLE LAYER ON THE RIGHT.

1 , sternum; 2, sternal portion of the pectoralis major; 3 , clavicular portion of the same muscle; 4 , space between the deltoid and pectoral muscles; 5 , deltoid; 6,6 , clavicles; 7 , external oblique; 8 , digitations of the serratus magnus; 9,9 , latissimus dorsi ; 10, aponeurosis of the external oblique; 11 , linea alba ; 12 , umbilicus ; 13 , pectoralis minor; 14,14 , crests of the iliac bone; 15 , symphysis of the pubis; 16 , crural arches; 17 , rectus abdominis; 18 , inferior oblique; 20 , internal intercostal ; 21, external intercostal; 22, infra-clavicular; 23, coracoid process; 24 , in. ferior border of the internal oblique and transversalis muscles; 25 , short head of the biceps; 26 , long head of the biceps; 27 , biceps; 28, sternomastoid; 32 , adductor of the thigh; 33 , rectus femoris. 
muscles which move it are for the most part attached to its upper third ; and the chief of them are the pectoralis major (fig. 227, ${ }^{2},{ }^{3}$ ) which rises from the sternum and cartilages of the ribs, and consequently draws the arm forwards, inwards, and downwards, - the latissimus dorsi (fig. 226, ${ }^{9}$ ), which rises from the spinal column and hinder part of the ribs, and consequently draws the arm backwards, inwards, and downwards, - and the deltoid (fig. 226, ${ }^{5}$ ), which arises from the upper edge of the clavicle, and from the ridge of the scapula, and is the chief muscle concerned in raising the arm. The first of these forms the principal part of the fleshy mass upon the front of the chest, and is the muscle which is so remarkably developed in Birds. It forms also the front boundary of the axillary space, or hollow of the arm-pit, the hinder boundary of which is formed by the second muscle. This space, of which we can distinctly feel the front and back walls when we raise the arm a little from the side, contains the large vessels and nerves proceeding to the arm, and also a number of lymphatic glands (§219). The deltoid muscle forms the thick fleshy mass on the top of the shoulder and on the upper part of the outside of the arm.

639. In the fore-arm of Man there are two long bones, termed the Radius and the Ulna, which lie nearly parallel to each other; the radius being on the outer or thumb side of the fore-arm, and the ulna on the inner. They are connected with one another, not only by ligaments at their extremities, but by a strong fibrous membrane that passes between their adjacent edges, along their entire length. Nevertheless they have considerable freedom of motion, not only upon the humerus, but upon each other; so as to give to the fore-arm the power of rotation on its own axis, by which either the palm or the back of the hand may be turned upwards. The ulna is connected with the humerus, at the elbow, by means of a hinge-joint, into which the radius does not enter; but it is the radius with which the hand is connected at the wrist, by a kind of ball-and-socket joint, the ulna having no direct share in this articulation: hence, while both bones move together in bending or straightening the elbow, we can make the radius roll round the ulna, carrying the hand with it. This movement is one of very great importance, in rendering the hand capable of a great variety of uses to which it would 
be otherwise inapplicable. It is only among the higher orders of Quadrupeds, however, that it can possibly be executed ; for in the lower, the two bones are united more or less completely into one, or are articulated in such a manner as to be incapable of rotation.

640. The fore-arm is bent upon the arm, chiefly by muscles that lie upon the front of the latter; of these the principal is the biceps or two-headed muscle (", fig. 227), which arises from the coracoid process of the scapula, and from the top of the glenoid cavity, and is inserted into the radius a little in front of the elbow, forming a great part of the fleshy mass in front of the arm (fig. 219). The arm is straightened again by a large muscle, the triceps or three-headed muscle, which arises from the back of the humerus and scapula, and passes down to be inserted into a projection of the ulna behind the elbow-joint, forming the fleshy mass at the back of the arm. The museles which rotate the fore-arm arise from the lower end of the humerus, or from one of its own bones, and pass obliquely across to the other.

641. The Hand is anatomically divided into three portions, - the carpus, metacarpres, and phalanges (fig. 223). The carpus, which is the portion nearest the wrist-joint, is composed of eight small short bones, which are firmly united to each other by ligaments, but yet have a certain degree of motion permitted them; these are arranged in two rows, of which one has a rounded surface, and enters into the formation of the wrist-joint; whilst the other has a series of shallow pits, to receive the rounded heads of the metacarpal bones. These last almost precisely resemble the bones of the fingers, and in the skeleton might be mistaken for their first joints ; but with the exception of that of the thumb they are all united to each other by ligaments and muscles, so as to form the compact framework which gives support to the palm of the hand. The metacarpal bone of the thumb is much more free in its movements; and it is chiefly by an alteration in its direction, that the thumb can be opposed to the fingers. The thumb and fingers are formed by a series of small bones which are termed the phalanges; of these there are only two in the thumb, whilst there are three in the fingers. They are bent on each other chiefly by the action of the muscles that occupy the front of the fore-arm; and they are extended or 
straightened by others that lie along its back. These terminate in long tendons, which are bound down at the wrist by a fibrous band that stretches between the bony projections on

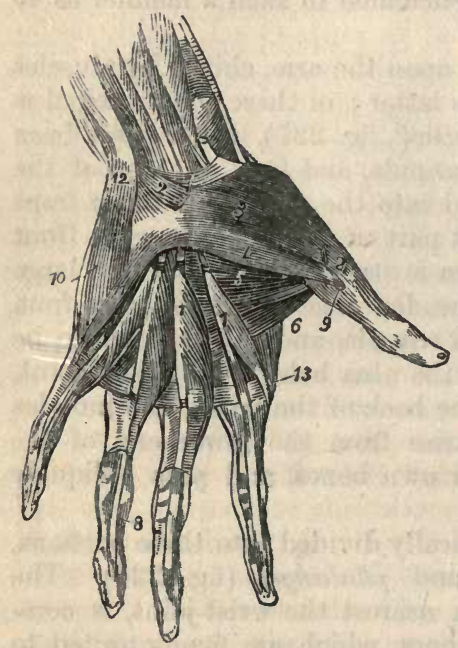

Fig. 228.-Muscles of the Palm of THE HaNd (SUPERPicial LaYeR)

1 , anterior annular ligament of the carpus ; 2,2 , extremities of the short abductor of the thumb, the intermediate body of the muscle having been removed; 3 , opposing muscle of the thumb; 4, short flexor of the thumb; 5 , adductor of the thumb; 6 , lower border of the same muscle; 7,7 , lumbricales; 8 , one of the tendons of the deep flexor of the fingers, passing-on to insert itself in the bone of the third pha$\operatorname{lan} x$, after perforating the tendon of the superficial flexor; 9, tendon of the long fiexor of the thumb; 10 , adductor of the little finger; 11, short flexor of the little finger; 12 , pisiform bone; 13, first dorsal interosseous muscle. it is desired to grasp ; whilst the long levers formed by the arm and fore-arm, allow the place of the entire hand to be rapidly changed to a considerable extent. It is principally by the movements of the humerus upon the scapula, that the direction of the limb is given ; the bending or straightening of the limb regulates its length; whilst the moveligament (fig. 228). The tendons then spread asunder in the hand, and pass-on to be inserted into the bones of the several fingers, being reinforced by a set of small muscles that arise from the hand itself.

642. When we consider the superior extremity of Man as a whole, we remark that the several levers which are joined end-to-end to form it, diminish progressively in length. Thus the arm is longer than the fore-arm; the latter is longer than the wrist ; and each of the phalanges is longer than the one which succeeds it. The purpose of this arrangement is very evident. The numerous joints, in the neighbourhood of each other, which we see towards the extremity of the limb, permit its several portions to change their place in various ways, so as to accommodate themselves to the form of the body which

either side, and is termed
the annular (or ring-like) 
ments of the thumb and fingers are concerned in its particular applications.

643. The hand of Man is distinguished from the extremity of most Quadrupeds by its possession of an opposable thumb, - that is, of a finger which can be made to act in a direction opposite to that of the rest. But among the Apes and Monkeys, we find this peculiarity not only in the superior extremity, but also in the inferior; whence these animals are said to be quadrumanous or four-handed, whilst Man is bimanous, possessing two hands only. It must not be supposed, however, that Apes and Monkeys are superior in this respect to Man; for they possess this distinguishing character in a much less striking degree than he does. All the four extremities of Apes and Monkeys possess the power of grasping, but they are all used also for support; and we find that in consequence of the shortness of the thumb and great toe, the grasping power is very inferior to that which Man possesses. But of the four extremities of Man, one pair is specially adapted for support, and the other for prehension or grasping; and this by the length and mobility of the thumb, which is capable of being brought into exact opposition to the extremities of all the fingers, whether singly or in combination. But even in those Quadrumana which most nearly approach Man, the thumb is so short and weak, and the fingers so long and slender, that their tips can scarcely be opposed to each other, and then with only a slight degree of force; hence, although completely adapted for clinging round bodies of a certain size,- such as the small branches of trees, \&c. - the extremities of the Quadrumana can neither seize very minute objects with that precision, nor support large ones with that firmness, which is essential to the-dexterous performance of a variety of actions for which the hand of Man is admirably suited. Hence they may be more appropriately termed claspers than hands.

644. In many of the inferior Mammalia, whose extremities are adapted for support only, we find each row of phalanges consolidated into two bones, or even into one. This is the case, for example, in the Ruminant Quadrupeds, as the Camel (fig. 229), and in the Horse (§652). Such an arrangement obviously increases the firmness of the limb, though it altogether deprives it of prehensile power. In other inI 12 
stances, we find the number of bones in the hand increased, but all of them enclosed in one envelope, so that the fingers are not separate. This is the case with many aquatic animals

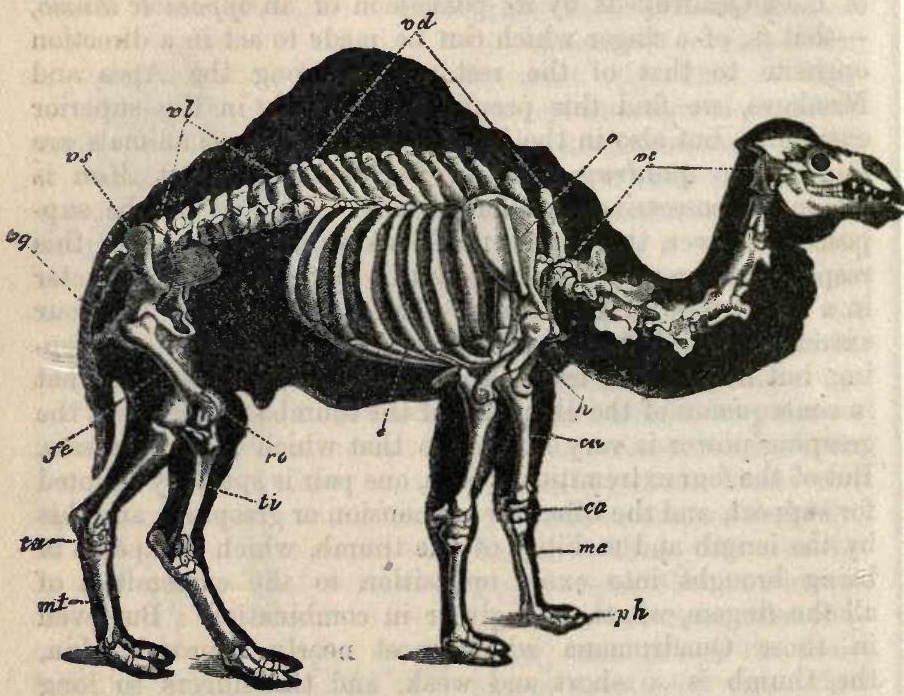

Fig. 229.-SKeleton of the CAMEL.

$v c$, cervical vertebræ ; $v d$, dorsal vertebræ ; vl, lumbar vertebræ ; vs. sacral vertebræ; $v q$, caudal vertebræ; $s$, scapula; $h$, humerus; $c u$, ulna; $c a$, carpus ; mc, metacarpus; $p h$, phalanges; fe, femur; ro, patella ; $t i$, tibia ; $t a$, tarsus ; $m t$, metatarsus.

- such as the Whale tribe among Mammals, Turtles among Reptiles, and Fishes in general,-in which the hand is made to serve as a fin or paddle. In most of these, the bones of the arm are very short; and the movements of the extremity are chiefly confined to the wrist-joint.

645. The structure of the lower extremities has a very great analogy to that of the upper; and the principal differences to be remarked between them, are such as are necessary to give to the former more solidity at the expense of freedom of motion, and to make them organs of locomotion instead of organs of prehension. Here, too, we have a bony framework, for the purpose of connecting the limb itself with the spine; and as the weight of the body is constantly thrown upon the 
lower extremities, this framework is much more firmly attached to that of the trunk, than is the case with that which supports the arms. It consists, on each side, of a bone which in the adult state is single, though at an early age it is composed of three distinct pieces ; and this is closely connected with the sacrum behind, while it meets with its fellow in front in such a manner as to form a sort of bason termed the Pelvis. The spreading sides of this, formed by the iliac bones (Fig. 213), afford support above to the viscera contained in the abdomen; and they give attachment by both surfaces to large muscles by which the thigh-bone is moved, and by their edges to large expanded muscles that pass upwards to the ribs and sternum, and form the walls of the abdomen. Below this spreading portion, we find the articular cavity of the thighbone, which is so deep as almost to form a hemispheric cup when it is completed by its cartilaginous border. The movements of the thigh-bone are consequently more limited than those of the arm; but it is much less liable to displacement.

646. The thigh, like the arm, contains but a single bone, which is named the Femur. Its upper extremity is bent at an angle; and its rounded head is separated from the rest by a narrow portion which is termed its neck. At the point where this neck joins the shaft of the bone, there are two large projections termed trochanters, one on the outer side and the other on the inner; these serve to give attachment to the muscles by which the thigh is moved. Of these muscles, one descends from the lumbar vertebræ, and passes-down with another that rises from the upper expanded surface of the pelvis, over the front border of the pelvis, to be attached to the smaller and interior of the projections just mentioned; these with the assistance of other muscles raise or draw forwards the thigh, - an action which does not require in Man to be performed with any great force. The muscles which draw back the thigh, on the other hand, arise from the under surface and back of the pelvis, where they form a very thick fleshy mass $\left({ }^{11},{ }^{25}\right.$, fig. 226$)$; and they pass to the larger and external projection, and to a ridge which runs from it down the thigh-bone. Other muscles which arise from the lower border of the pelvis, serve to rotate the thigh upon its axis. The lower end of the thigh-bone spreads into two large condyles, on which the principal bone of the leg moves backwards and 
forwards. The knee is a good example of a pure hinge-joint, all its movements being restricted to one plane.

647. The leg, although containing two bones like the forearm, does not in Man possess the peculiar movement which characterises it. One of these bones, called the Tibia, is much larger than the other which is called the Fibula; and it is the former alone on which the thigh-bone rests, and which itself rests upon the foot, so that no movement of rotation is permitted in the leg. In fact, the fibula, which is a long slender bone running nearly parallel with the tibia (fig. 223), looks like a mere appendage or rudiment, and serves only for the attachment of muscles. The upper end of the tibia is broad, and has two shallow excavations, in which the condyles of the femur are received. Upon the front of the knee-joint we find a small separate bone, the patella or knee-pan ; the purpose of this is to change the direction of the tendons that come down from the front of the thigh to be attached to the tibia; in such a manner as to enable them to act more advantageously, upon the principle formerly stated (\$. 611). In the elbow-joint, this change was not required; since the ulna projects sufficiently far backwards to afford advantageous attachment to the tendon of the extensor muscle. - The very powerful muscles which tend to straighten the knee-joint, arise from the front of the pelvis and from the femur itself; and they form the fleshy mass of the front of the thigh. On the other hand, those which bend the knee arise from the lower border of the pelvis and from the back of the thigh-bone, and pass downwards to be inserted into the sides of the tibia and fibula a little below the knee, their tendons forming the two strong cords known as the hamstrings. The articulating surface at the lower extremity of the leg, which enters into the ankle-joint, is principally formed by the tibia; but its outer border is formed by the fibula, which there makes a considerable projection that can be felt through the skin.-In the Quadrumana, and in a less degree in some other Mammals, the two bones of the leg resemble those of the fore-arm ; and are so articulated as to give to the foot a power of rotation corresponding with that of the hand.

648. The Foot is composed, like the hand, of three distinct portions, which are called the tarsus, metatarsus, and phalanges. There are seven bones in the tarsus, all of which are larger 
than those of the earpus, and some of them of considerable size. The articulation with the leg is formed by one of these only, the astragalus, which projects above the rest, and is imbedded between the projecting extremity of the tibia (which forms the inner boundary of the ankle-joint) and that of the fibula. The astragalus rests on the os calcis or bone of the heel, which projects considerably backwards, and is connected in front with the other bones of the tarsus. In front of the tarsus we find the metatarsus, composed of five long bones, which in man are all attached to each other, but of which one is separate in the Quadrumana, in order to give freer play to the great toe, the action of which resembles that of the thumb. The toes, like the fingers, are composed of three phalanges (with the exception of the great toe, which has only two); these are in Man much shorter than those of the hand, and are evidently not adapted for prehension; but in many of the Quadrumana, their length is nearly equal to that of the fingers, and the great toe is as opposable as the thumb. The foot is far from being thus converted, however, into a perfect hand; but it becomes a very useful instrument for clasping the small branches and twigs of the trees among which these animals live. The foot of Man is distinguished from theirs, by

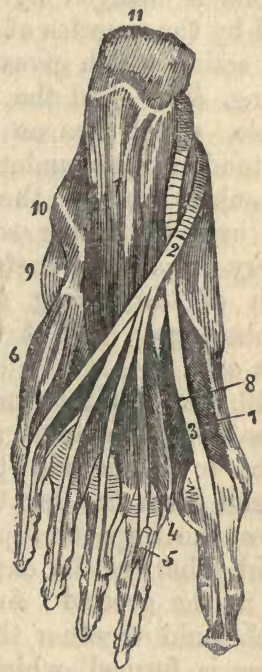

Fig. 230,-Muscles of The Sole of THE FOOT (MIDDLE LAYER).

1 , accessory of the long flexor of the toes; 2, tendon of the long flexor issuing from its sheath; 3 , tendon of the long flexor of the great toe; 4 , first lumbricalis ; 5 , tendon of the superficial flexor, divided behind its perforation; 6 , short flexor of the lit tle toe; 7 , short flexor of the great toe; 8 , portion of the oblique abductor of the great toe; 9 , posterior extremity of the fifth metatarsal bone; 10 , sheath of the long peroneal; 11, os calcis, or bone of the heel.

its power of being planted flat upon the ground, and thus affording a firm basis of support. Even the Chimpanzee and the Orang, when they attempt to walk erect, rest upon 
the side of the foot; and the absence of a projecting heel causes them to be very deficient in the power of keeping the leg upright upon it. For it is to this projection that the strong muscles of the calf of the leg are fixed, by which the heel is drawn upwards or the leg drawn back upon it. Other muscles at the side and back of the leg, the direction of whose tendons is changed by a sort of pulley at the ankle-joint, aided by the muscles of the foot itself, serve to bend the toes, -an action which gives great assistance in walking, running, leaping, \&c. And the toes are straightened by an extensor muscle, which lies on the front of the leg, and of which the tendon runs under an annular ligament that encircles the ankle, and is then divided and spread-out to the toes, over the upper surface of the foot. The great toe is a very important instrument in the act of walking, since much of the spring forwards is given by the bending of its phalanges; and it is provided with two flexor muscles of its own.

649. On the internal side of the foot, the bones of the tarsus and metatarsus form a kind of vault or arch, which serves to lodge and protect the vessels and nerves that descend from the leg towards the toes. This arch further serves the important purpose of deadening the shock that would otherwise be experienced every time that the foot is put to the ground; for, by the elasticity of the ligaments which hold together the bones that compose it, a sort of spring is formed, which yields for a moment to the shock, and then recovers itself. We feel the difference which this makes, when we jump from a height upon our heels ; the jar is then propagated directly upwards from the heel to the leg, thence to the thigh, and thence to the spinal column, and if it were not from the peculiar manner in which this is constructed ( $(631)$, a severe shock of this kind might produce fatal effects by concussion (or shaking) of the brain. In animals which walk upon four extremities, the difference of direction in which the legs are connected with the spine prevents a jar from being propagated along the latter to a similar degree. But in those which are destined to obtain their food by sudden and extensive leaps, such as the animals of the Cat tribe (the Lion, Tiger, \&c.), we find an arrangement of the bones of the foot, well adapted to diminish the 
shock produced by the sudden descent of the body upon the ground.

Of the Attitudes of the body, and the various kinds of Locomotion.

650. A small number of Vertebrated animals,--Serpents, for instance,- - bear habitually on the whole length of their bodies, which rest entirely on the ground; and their only movements are effected by undulations of the spinal column. But the rest are supported upon their extremities; and we give the name of standing to that position in which the animal rests supported by its limbs upon the ground or on any firm horizontal basis. In maintaining this position, the extensor muscles, by which the joints are straightened, must be in continual action, since the limbs would otherwise bend beneath the weight of the body. Now as the sense of fatigue, in any set of muscles, depends in great degree upon the length of time during which they have been in continuous action, the maintenance of the standing posture for a long period is, in most animals, more fatiguing than walking; since in the latter exercise the action of the flexors alternates with that of the extensors.

651. But this condition is not the only one essential to steadiness in the standing posture; for in order that the body may rest firmly upon the members, it must be in equilibrium. It has been shown (Mechan. Philos. Chap. Iv.) that equilibrium exists, - or in other words, that a body remains at rest in its position,-not only when it bears upon the whole of a broad surface, but also when it is so placed that the tendencies of its different parts to descend or gravitate towards the earth counterbalance each other. This is the case when its centre of gravity is supported,that is, when a line drawn

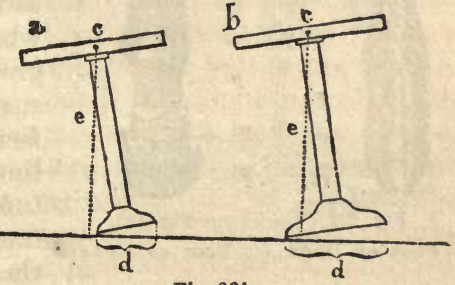

Fig. 231. perpendicularly from that centre falls within the base. In order, then, that an animal may rest in equilibrium on its legs, it is necessary that the vertical line from its centre of gravity (or line of direction) should fall within the space 
which its feet cover and inclose between them; and the wider this space, in proportion to the height of the centre of gravity, the more stable will the equilibrium be, since the body may be more displaced without being upset. Thus in fig. 231 the table $a$ must be upset; because the line of direction $e$ from the centre of gravity $c$ falls outside the base of support $d$; whilst the table $b$, although equally inclined, will not be upset but will return to its proper place, because the line of direction $e$ from its centre of gravity $c$ falls within its base $d$. Hence an animal which is supported upon four legs will stand much more firmly than one which rests on two only; since its real base is the whole space included between its four points of support. And again, an animal is more firm when standing upon two legs, than when resting upon one only.

652. Moreover when an animal rests upon four legs, the extent of its base is but little influenced by the size of the

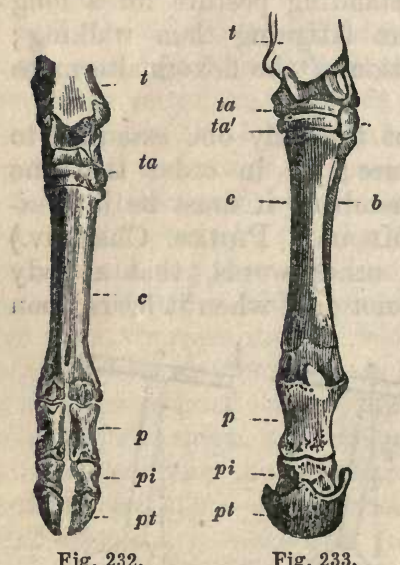

Fig. 232. feet; and thus to render them broad would be to increase their weight without adding much to their use as supports. This is easily understood by comparing a quadruped to a four-legged table; if the legs are sufficiently strong to support the weight that rests upon them, it matters little in regard to the steadiness of the table, whether they bear upon the ground by mere points or by flat surfaces ; since it is the large surface that would be enclosed by lines joining them, which constitutes the real base. Hence we FOOT OF DEER.

Fig. 233.
Foot of Horse: find that, in most quadrupeds, the limbs only touch the ground by slightly-dilated extremities; and the number of fingers is reduced more and more, without diminishing their effect as instruments of locomotion. Thus in Ruminant animals, as the Deer, the number of toes is reduced to two in each foot, as seen in fig. 232, where $t$ represents the tibia, ta the bones of 
the tarsus, $c$ the bone of the metatarsus termed the canon (in which the trace of a division into two pieces can be seen), and $p, p i, p t$, the three phalanges of the toes, of which the last is enveloped in the hoof, which is nothing else than a large nail inclosing the whole extremity of the toe. In the Horse this consolidation is carried still further than in the Ruminants, for it has only one toe in each foot (fig. 233); but we see the rudiment of an additional bone in the metatarsus $b$, which is commonly termed the splint bone.

653. But when an animal is supported upon two feet only, whatever may be their degree of separation from each other, the base of support cannot have sufficient extent, unless the extremities touch the ground by a considerable surface. This is the case with the foot of Man, and still more with that of many Birds which habitually stand upon one leg (fig. 234). In order that an animal may hold itself in equilibrium upon a single limb, it is necessary that the foot should be placed vertically beneath the centre of gravity of the body; and that its muscles should be so arranged as to permit it to keep this limb inflexible and immovable. Man can accomplish this, for the centre of gravity of his body is at about the middle of the pelvis ; and to place this vertically over one foot, it is sufficient for him to bend himself a little from the side which is not supported. But the greater number of Quadrupeds are destitute of the power of doing this; and a large part of them cannot even raise themselves on their hind legs, on

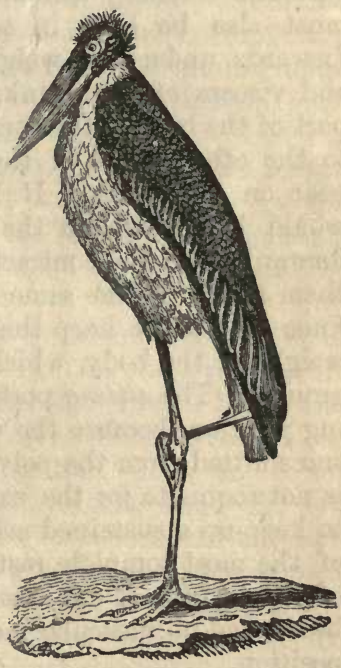

Fig. 234. account of the direction of these members relatively to the trunk; or if they can do so for an instant, they cannot maintain themselves in this position. The reason of this is very simple. The base of support, on account of the smallness of the feet, is very narrow, and the centre of gravity of 
the body is placed near the front; hence the body must be entirely changed in its position by a violent and not sustainable action of the muscles which connect it with the hind legs; and, when thus reared up, it cannot rest with firmness on account of the narrowness of the base.

654. There are some Quadrupeds, however, which are able to raise themselves occasionally into this position; this is the case, not only with the Quadrumana, but also with the Bear, Squirrel, and other animals whose habits require them to ascend and live among trees, - as well as in the Kangaroo, and animals constructed upon the same plan, whose peculiar organisation will be presently considered (\$ 661). In standing upright, the muscles of the back part of the neck are kept in a contracted state, to retain the head in equilibrium on the vertebral column; and the extensor muscles of that column must also be kept in action, to prevent it from bending forwards under the weight of the head, upper extremities, and viscera of the trunk. The whole weight of the upper part of the body is thus transmitted to the sacrum, and thence to the other bones of the pelvis. by which it is brought to bear on the femur. If left to themselves, the thigh-bones would bend beneath the pelvis, and the trunk would fall forwards ; but the contraction of their extensor muscles keeps them firm. In the same manner, the extensor muscles of the knee and ankle keep these joints from yielding beneath the weight of the body, which is thus at last transmitted to the ground. The sitting posture is less fatiguing than the standing position, because the weight of the body is then directly transmitted from the pelvis to the base of support, so that it is not requisite for the extensor muscles of the lower limbs to keep-up a sustained action. But the lying posture is that of the most complete rest; because the weight of every part of the body is at once transmitted to the surface on which it bears, and no muscular movement is requisite to keep it in its position.

655. This difference in muscular effort, is the cause of a well-marked variation in the pulse, according to the position in which the body is at the time. From a considerable number of observations it has been found that the average pulse of an adult man is about 81 when standing, 71 when sitting, and 66 when lying; so, that the difference between 
PULSE IN DIFFERENT POSTURES :-ACT OF WALKING. 493

standing and sitting is 10 beats or 1-8th of the whole, whilst the difference between sitting and lying is 5 beats or 1-13th of the whole. In the female, the pulse is quicker in each position by from 10 to 14 beats per minute; but the differences occasioned by position are nearly the same. It will be observed that the difference between standing and sitting is greater than that between sitting and lying; and this closely corresponds with the relative amounts of muscular exertion required in these positions respectively. At the moment when the posture is changed, the pulse is considerably quickened, in consequence of the muscular effort required for the purpose, which acts especially on the veins, and forces the blood more rapidly back to the heart ( $\$ 279)$; but this increase in rapidity is temporary only.

656. All that has been said of the positions of Vertebrated animals applies equally well to those of the Invertebrata, which like them have the body raised from the ground upon extremities. This is the case in the higher Articulata, such as Insects, Crustacea, Arachnida, and Myriapoda. But the lower Articulata crawl, like Serpents, upon the whole length of their bodies; or, being aquatic, are buoyed-up by the element they inhabit. And among the Mollusca and Radiata, there are none that have members upon which they can be said to stand.

657. The progressive movements by which the bodies of Man and other animals are made to change their places, are accomplished by means of the alternate contractions and extensions of those limbs, which we have hitherto considered only as supporting them in a rigid position. It is easy to see that when a joint is straightened after being bent, the two ends of the levers which form it must be separated from each other, and that motion must thus be given to the parts against which one or both of them bear. Now in the ordinary movements of progression, one of these levers bears against the ground, which is immovable ; and the whole motion produced by straightening the joint must consequently be communicated to the body. In the ordinary act of walking, one of the fect is planted in front, whilst the other is extended or carried backwards beneath the leg, by the action of the muscles of the calf aided by those of the toes (\$. 648). Its length is thus increased; and as it bears upon the resisting soil, this 
elongation acts through the thigh upon the pelvis, and thus carries forward the whole body. At the same time, the pelvis makes a slight turn upon the femur of the other side on which it is resting; and the limb which was at first behind the other, is now drawn forward by a flexion of its joints, and is plantcd on the ground in front of the other, so as to serve for the support of the body in its turn; whilst the other, by extending itself, gives a fresh forward impulse to the body. Thus each limb is alternately made to support the whole weight of the body, just as it would do in standing on one leg; while at the same time the other is engaged in urging it forwards. Hence the centre of gravity must vibrate a little from side to side in the act of walking, so that it may be brought alternately over each foot; and this movement from side to side is the more obvious, in proportion as the pelvis is wider, and the limbs more separated from each other. Hence it is more seen in women than in men, on account of the greater proportional breadth of the hips in the former.

658. In all the higher animals, as in Man, there are members which serve for locomotion; but the nature of these movements varies greatly; and there is a corresponding difference in the structure of the instruments by which they are performed. The manner in which the Creator has made the same organs answer a variety of different purposes, in accordance with the habits of the animals to which they belong, is a most interesting object of study; for we see the most varied results attained, without the least departure from the general plan which has been adopted in the construction of the various species of the same group; and this solely by slight changes in the forms and proportions of some of the instruments whose union makes-up the entire body. The organs of locomotion in the Mammalia furnish us with obvious examples of this principle. This class includes not only the quadrupeds which run or bound along the surface of the ground,-but animals which are destined to live solely in water like fishes,-others which sometimes swim through that element and sometimes inhabit the land, - others which possess wings that enable them to fly through the air like birds,-and others which only employ their anterior members for grasping or feeling; yet in all these animals, these organs are constructed of the same parts, In the paddles of a Seal 
(fig. 240), the wing of a Bat (fig. 251), and the fore-paw of a Squirrel or a Mole, we find the same bones as in the arm of Man (fig. 223). And even in the fore-legs of the Ruminant Quadrupeds, and in those of the Solidungula, or single-toed animals (such as the Horse), we can usually perceive traces of the existence of three or four toes, whose bones are more or less completely united.

659. From what has already been stated as to the influence of the length of the levers on the quickness of the movement of the extremities (§.614), it is easy to see that animals which have the most rapid progression must necessarily have long members; since, the quickness with which the extensor muscles act remaining the same, the change of place in the free extremity of the lever will be greater, in proportion as that extremity is more distant from the point of insertion of the muscles that move it, and from the fulcrum on which the lever works. But in proportion to the elongation of this arm of the lever, must be the increase in the power of the muscles that move it, in order to overcome the same resistance; according to the general principle that what is gained in velocity is lost in power. Hence, in order to endow an animal with great agility, it is only necessary to lengthen its limbs, and to render its muscles capable of exerting a proportional power.

660. We have seen that in walking, the body is sustained upon one limb (in quadrupeds, upon one pair of limbs), whilst it is pushed onwards by the other; so that it never ceases to bear upon the ground. In munning, however, the body of Man momentarily quits its support at intervals; the foot in advance not being planted on the ground by the time that the hinder one quits it. In this action, the Ostrich and its allies probably surpass all other animals ; as they can outstrip the fleetest horse at full gallop, or the swiftest greyhound at its greatest speed. The amble of Quadrupeds is a pace which resembles the walk or run of bipeds, the two legs on one side being moved together, whilst the body rests upon the other. This pace is peculiar to the Giraffe, and to horses which have been trained to execute it. The trot, however, is a step of a different and much more secure nature. The forefoot of one side is raised and advanced with the hind foot on the other side ; and when these are set down, the other fore 
and hind feet are raised and advanced together. Now, if we consider the fore-feet of a horse as constituting the four angles of a parallelogram, it is easy to see that the base of support, when the feet are thus raised, will be one of its diagonals; and as the feet are alternately advanced, the weight will alternately be thrown upon these two lines. But the centre of gravity in the horse, especially when carrying a rider, is in a point almost exactly above that at which the two diagonals cross ; so that it is always supported either by the one or the other. The gallop of greatest speed is a run performed on the same plan as the trot; - that is, the right fore and left hind feet leave and reach the ground together, and then the left fore and right hind feet are advanced. The canter is a kind of step altogether different. The four legs strike the ground successively, the left hind foot reaching it first, the right hind foot second, the left fore foot third, and the right fore foot fourth.-The celebrated race-horse Eclipse, when galloping at liberty and with his greatest speed, passed over the space of 25 feet at each stride or leap; this he repeated $2 \frac{1}{3}$ times in a second, so as to pass over 58 feet in that time, which was at the rate of nearly 4 miles in six minutes and two seconds. But this performance was completely surpassed by that of Flying Childers, who was computed to have passed over $82 \frac{1}{2}$ feet in a second, or nearly a mile in a minute.

661. In leaping, the body is projected into the air by the sudden extension of the joints, especially those of the hinder part of the body which had been previously bent; and having traversed a greater or less distance, the body comes again to the ground and may be again projected. This is a kind of motion usually practised by many animals whose structure is expressly adapted to it. Thus among Mammals we find several in which the hind legs are enormously elongated, for the purpose of giving greater quickness to the motion of the body; and their muscles are developed to an extraordinary degree in order to supply the necessary force. This is the case among most of the animals of the order Rodentia, such as the Hare, Rabbit, Squirrel, \&c.; but particularly in the Jerboa or Jumping Rat, and in the Kangaroo and its allies. In these animals the fore feet, which are little used for progression, are comparatively small; and in the last they are 
ACTS OF RUNNING AND LEAPING:-KANGAROO.

less than half the length of the hinder limbs (fig. 235). The feet, as well as the legs, of the Kangaroo are very long (fig.

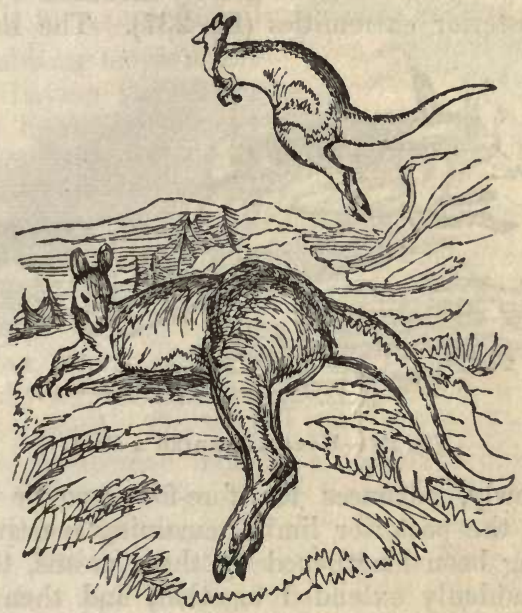

Fig. 235.-KANGARoos.

236), so as to afford (in conjunction with the tail) a firm support to the animal when preparing to leap. Quadrupeds in

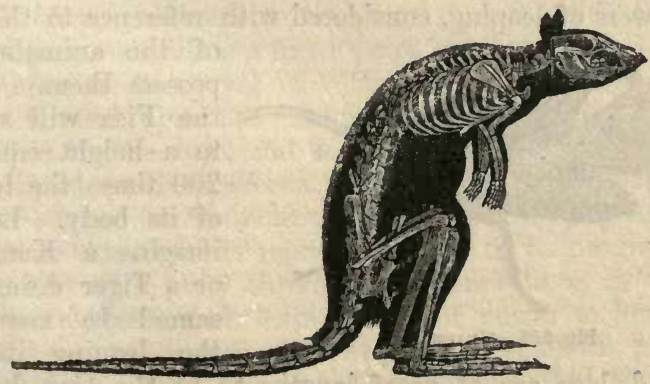

Fig. 236.-SKeLETON OP KANGAROO,

which the length of the posterior extremities greatly predominates over that of the anterior, are observed to descend hills with difficulty at a rapid pace, since the forward inclination 
of their bodies places them in continual danger of oversetting; they therefore take a zig-zag course. In ascending a hill, however, their progression is greatly favoured by the length of their posterior extremities (fig. 237). The Rabbit, when

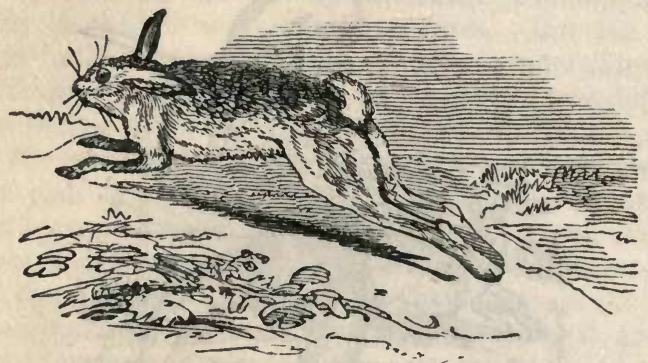

Fig. 237.-HARE ASCENDING A HILL.

moving slowly, advances the fore-feet two or three steps alternately, the posterior limbs remaining inactive; and the body having been lengthened by these means, the posterior legs are suddenly extended together, and then drawn forwards: thus the rabbit walks with the fore and leaps with the hind pair of legs. The Frog moves in a very similar manner.

662. It is among Insects that we find the most extraordinary powers of leaping, considered with reference to the size

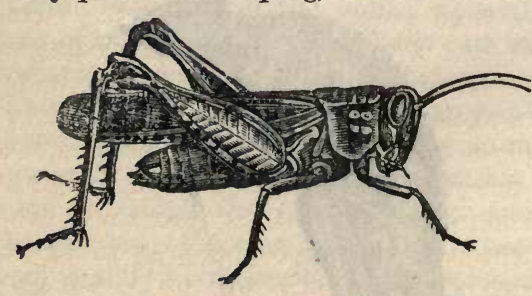

Fig. 238.-CRICKET.

of the animals that possess them. Thus the Flea will spring to a height equal to 200 times the length of its body. Let us imagine a Kangaroo or a Tiger doing the same! In many of the leaping insects, the hind legs are of great length, as in the Grasshopper and Cricket tribe (fig. 238); and in one curious family, that of the Poduras or spring-tails, the leap is accomplished by the sudden extension of the tail, which is ordinarily bent under the body (fig. 239). A very remarkable kind of leap is 
executed by the Beetles of the family of Elateridoe; the larva of one species of which devours the roots of wheat, and is known under the name of the "wire-worm ;" whilst other species inhabiting tropical climates, and having the power of emitting light, are termed "fire-flies" (§ 397). The legs of these insects are very short; so that when they are laid on

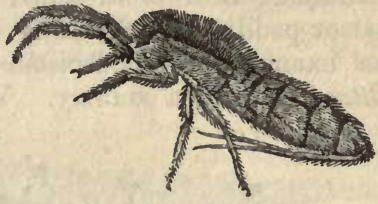

Fig. 239.-PODURA. their backs they cannot by means of them recover their natural position. This they are enabled to do, however, by their power of jerking backwards the head and upper part of the thorax, which causes the body to be projected vertically into the air, whence it usually descends with the feet towards the ground. The leap of the Crickets, Locusts, Froghoppers, \&c. is executed more in a horizontal direction; and it is assisted by the wings, which bear-up the body whilst it is moving onwards through the air. In this manner a Locust can traverse 200 times its length, and a Frog-hopper 250 times; which is as if a Man were to take a quarter of a mile at one leap.

663. Swimming and Flying are movements which have much resemblance to each other; both being executed in a fluid medium, which to a certain extent buoys-up the body, which offers resistance to its progress, and which also offers something resembling a fixed point against which the members may act to propel it. The chief differences between them depend upon the nature of the medium; this being liquid in the one case, and aeriform or gaseous in the other. The liquid medium affords more support to the body, and a firmer surface for the action of its propelling organs; but at the same time it offers more resistance to its progress. The movement of a body through the atmosphere, as in flight, requires a considerable expenditure of power to keep it up; and the yielding nature of the element prevents the propelling organs from acting against a firm surface; but the onward movement, in consequence of the slight resistance, is easily. accomplished.

664. When the feet of a Quadruped are to serve both as walking and swimming organs, the end is accomplished by K K 2 
the spreading-out of the fingers, and their union by means of a fold of skin which is stretched over them; as the web of a swimming Bird is stretched over its toes, so as to make an oar or paddle of sufficiently wide surface. This is the ease, for example, in the Ornithorhyncus of Australia, and in the Otter of our own country. When the members are intended

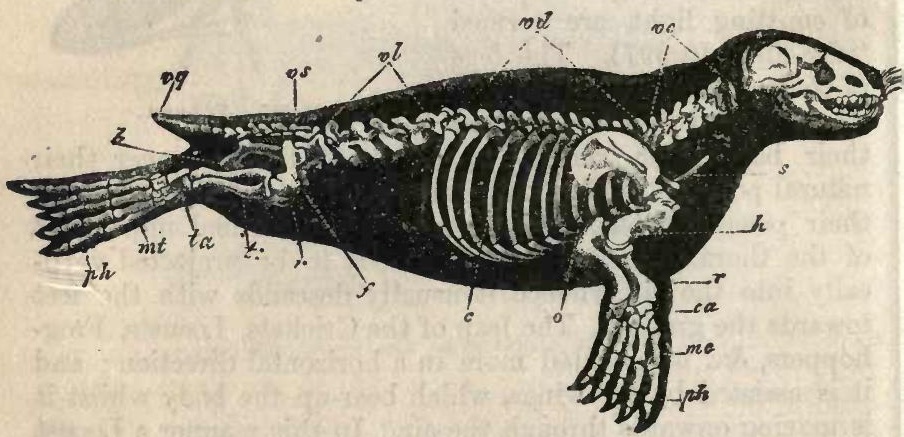

Fig. 240.-SKeleton of Seit.

$v c$, cervical vertebræ, $v d$, dorsal vertebræ; $v l$, lumbar vertebræ; $v s$, sacral vertebræ; $v q$, caudal vertebræ; $b$, pelvis ; $s$, sternum; $h$, humerus; $r$, radius ; $c a$, carpus ; $m c$, metacarpus ; $p h$, phalanges ; $o$, scapula ; $c$, ribs ; $f$, femur; $r$, patella ; $t$, tibia ; $t a$, tarsus ; $m t$, metatarsus; $p h$, phalanges.

exclusively for swimming, however, they undergo more considerable modifications in structure. The parts corresponding with the arm and fore-arm are very short, and the movements of the hand are thus limited, but they can be accomplished with all the more force. But the bones of the hand are large and spread asunder, and are enclosed in a firm integument which may even cover their extremities. Sometimes the number and arrangement of these bones are precisely the same as in the hand of Man; this we see in the Seal (fig. 240), where their extremities are furnished with separate claws that project beyond the integument. Sometimes the number of phalanges in the fingers is considerably increased, as in the Whale; and in other instances, the fingers are replaced by a multitude of small rods of bone, enclosed within a continuous skin, such as we see in the fins of Fishes (fig. 243).

665. In the Seal, which does not depart widely in its general construction from land quadrupeds, the hind feet are formed upon the same plan as the fore; but they are carried 
far backwards, so as almost to occupy the position of the tail. In the Whale and its allies, on the other hand, the posterior extremities are almost entirely wanting, and the tail is greatly prolonged and expanded at its extremity (fig. 241). This

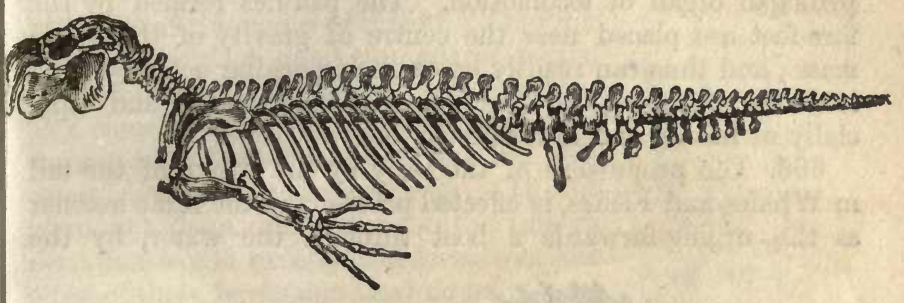

Fig. 241.-SKELETON of Dugong.

expansion, however (which is in the horizontal direction, fig. 242), is not supported by bones, except in its centre ; but it consists internally of cartilages and tendons, which last are prolonged from a set of very powerful muscles that are attached to the spine, and give to this organ an enormous force

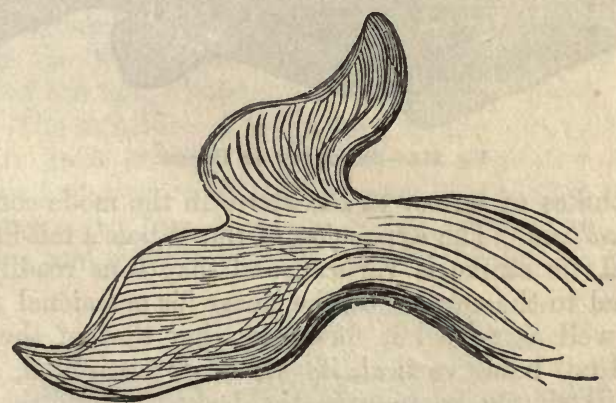

Fig. 242.-TAIL-Fin of WHALE.

and great variety of motion. The texture of the portion of it by which the blow is usually given, is such that it can hardly be injured; it is so tough that it cannot be torn, and so free from feeling, that a stroke of it against a hard substance gives no pain to the animal. If it strike a boat across the middle with its edge, the boat is cut asunder as clean and suddenly as if by one stroke of a giant axe ; whereas, if it strike with 
the flat surface, the boat is driven to the depth of many fathoms with the swiftness of an arrow. Hence this tail is a most efficient instrument for the propulsion of the bulky body of the Whale through the water; and it is, in fact, its principal organ of locomotion. The paddles formed by the fore-feet are placed near the centre of gravity of the whole mass ; and thus can readily exert their peculiar action, which is that of changing the direction of the movement, and especially of raising and lowering the body.

666. The propulsion of the body by the stroke of the tail in Whales and Fishes, is effected precisely in the same manner as the urging-forwards a boat through the water, by the

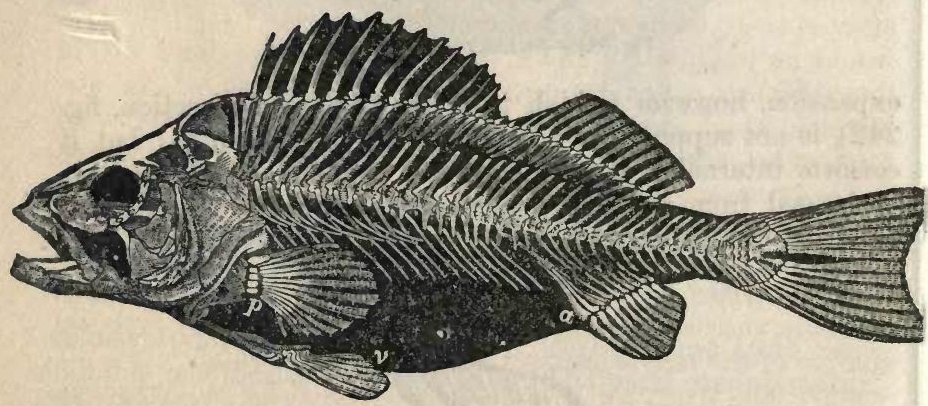

Fig. 243.-Skeletor of Perch.

lateral strokes of an oar at the stern, in the mode commonly termed sculling. The expansion of the Whale's tail-fin being horizontal, its stroke is vertical, and may thus readily bring the animal to the surface of the water for occasional respiration, as well as propel it forwards; but that of the Fish's body and tail being vertical, its stroke is horizontal, and its action will simply be to urge the body through the water. The power of ascending and descending, as well as of changing the direction of the motion, is principally due to the side-fins, which represent the arms and legs. The direction of the surface and stroke of these side-fins varies in different species. In the Cod, Halibut, and others, their action appears to be principally directed towards keeping the body in its right position in the water; since, without such an action, the body would be liable to turn over, in consequence of the position 
ACTION OF THE FINS OF FISHES :-FLYING FISH. 503

of its centre of gravity. In other instances, the pectoral and ventral fins move in such a manner as to assist the action of the tail. In the Rays, the pectoral fins are developed to an enormous extent (fig. 244); and being directed horizontally, their action is vertical like that of the wings of a bird. They are furnished with a great number of joints, by which they are rendered very flexible; and their surface may be thus increased during the down-stroke of the fin, and diminished during the up-stroke. If this were not done, the action of the fins in elevation would exactly counterbalance the effect of their depression; and no movement

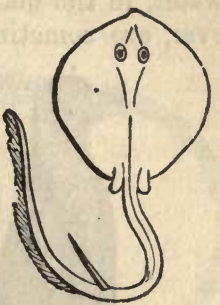

Fig. 244,-RAY. would be produced. The great power of the pectoral fins of these Fishes seems connected with their want of an airbladder, which causes them to require a constant exercise of force to keep them up in the water. Their propulsion forwards is chiefly accomplished, as in other Fishes, by the action of the tail. But sometimes the Rays change their position and swim sideways, making horizontal strokes with the pectoral fins (whose surface is then vertical), by which they are moved through the water, and sustaining themselves by vertical strokes of the tail, whose surface is then horizontal.

667. The structure of the organs adapted for movement in air bears great analogy to that of such expanded fins; and there are instances in which the same instruments may serve both purposes. Thus there are Fishes which are able to quit the water, and execute leaps of considerable length, supported

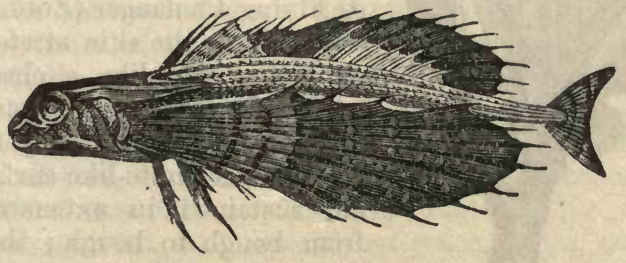

Fig. 245,-FLYiNG-Fish.

upon their wing-like pectoral fins. These are known as Flying-Fish (fig. 245); but it is not correct to speak of their 
504 ORGANS OF FLIGHT:-FLYING FISH, PENGUIN, ETC.

movement as one of flight, since it does not appear that they have any power of propelling themselves in the air; the impulse being given at the moment of their quitting the water, in the manner of a leap. From 50 to 100 yards, however, are sometimes traversed by the Fish at one leap; and

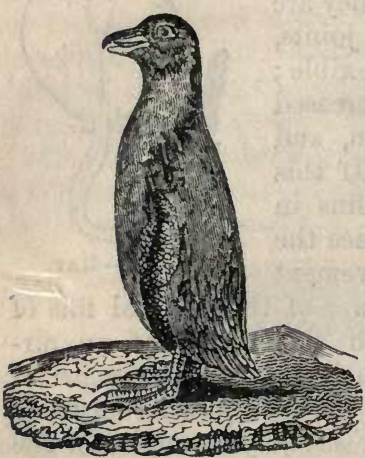

Fig. 246.-PENGUIN.

the height to which it rises from the surface of the water is occasionally such as to carry it over the deck of a ship. On the other hand, there are several among the diving Birds which use their wings as instruments of progression beneath the water-in other words, as fins. The most remarkably constructed of all these is the Penguin (fig. 246 ), in which the wings are so short as to be incapable of answering any other purpose; but there are several species in which they may be used as organs of flight in the air, without losing their fin-like power in the water.There are several animals that can sustain themselves for a

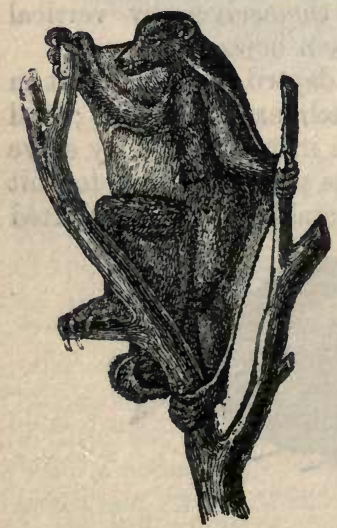

Fig. 247.-GALEOPITHECUS. short time in the air, by the aid of an expanded surface formed by an extension of the skin and serving as a parachute. This is the case, for instance, with the Galeopithecus, or Flying Lemur (fig. 247), the Flying Squirrel, and the Petaurus, or Flying Phalanger (ZooL. § 314), which have the skin stretched out on either side like a cloak, supported by the anterior and posterior extremities and by the tail. By this parachute-like surface they are sustained in extensive leaps from bough to bough; though it does not enable them to support themselves in the air for any length of time. In the Draco Volans (fig. 248), a little animal which lives among the trees of tropical forests, the body is furnished 
with a wing-like appendage on either side, formed by an expansion of the skin over six lengthened ribs. These appendages serve as a kind of parachute, on which this little

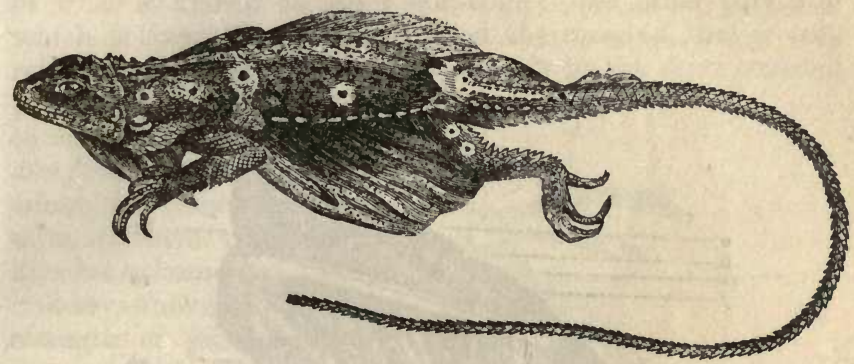

Fig. 248.-Draco Volans.

animal, not more than a few inches long, flutters from branch to branch in search of its insect prey, or shoots, like the flying squirrel, from tree to tree. They cannot be made to strike the air, and therefore are not true wings; but they can be folded up and extended at the will of the animal.

668. True wings, or instruments of propulsion as well as of support in the air, are found in some members of all classes of air-breathing Vertebrata; but they are especially characteristic of the class of Birds, in which the absence of them is the exception to the general rule, whilst in Mammals and Reptiles it is their presence which constitutes the exception. These wings are universally formed by some modification of the anterior extremities, which renders them unfit to be used as instruments of progression on the ground; but the nature of this modification varies considerably. In the Bird, the required extent of surface is chiefly given by the feathers; these are supported upon an anterior member, of which the arm and fore-arm (especially the latter) constitute the largest part, the hand being contracted and consolidated. The general structure of the Bird's skeleton, the whole of which is modified with special reference to the actions of flight, is shown in fig. 249, which represents that of the Vulture. The head is supported upon a very flexible neck, of which the vertebræ $v c$ are often very numerous. The vertebræ of the back and loins, however, are usually few in 
number, and are connected together very firmly, so as to form a nearly inflexible column; and this, again, is closely united to the sacrum vs. The vertebræ of the tail $v q$ are few in

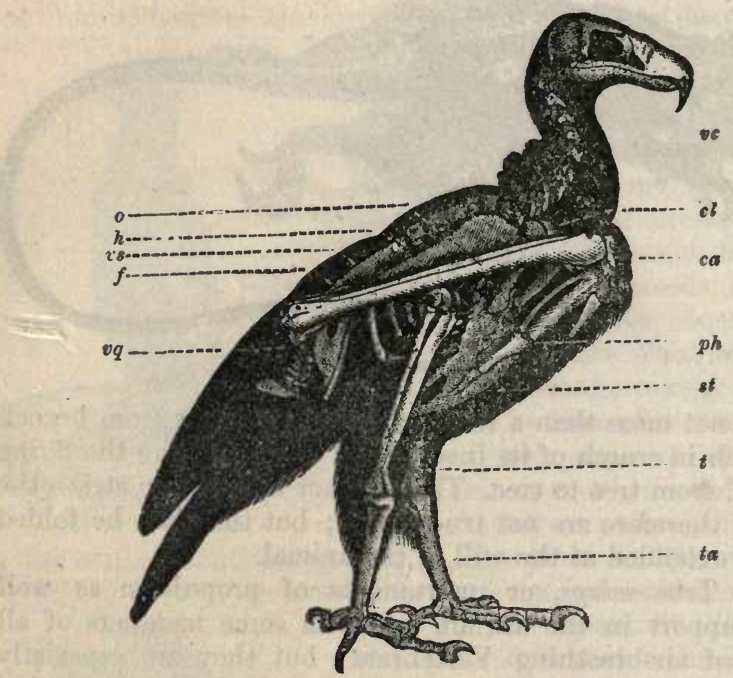

Fig. 249.-SKeleton of VuLtura.

$v c$, cervical vertebræ; $v s$, sacral vertebræ; $v q$, caudal vertebræ; $c l$, clavicle; $h$, humerus ; 0 , fore-arm; $c a$, carpus; $p h$, phalanges ; st, sternum; $f$, femur; $t$, tibia ; $t a$, tarsus.

number, and possess little motion. The ribs are very strongly connected to each other and to the vertebræ, and are united to the sternum st by bony instead of cartilaginous prolongations. Thus the whole bony apparatus of the trunk is very strongly knit together; and the purpose of this is evidently to give as firm an attachment as rossible to the muscles which move the wings. The sternum is raised into a high keel or ridge (as is better seen in fig. $250, b$ ), for the attachment of the powerful pectoral muscles which draw down the wings; and the degree of this projection is proportioned to the power of flight which the species possesses,-the sternum being flat (as in Mammals) in birds which, like the Ostrich, have the wings undeveloped. The scapula (fig. 250,0 ), to which are 
attached the muscles that raise the wings, is very narrow in Birds, in accordance with their small demand for muscular power in this direction. This narrow scapula forms one part of what is known as the "side-bone;" the other part $c$ of which is formed by a bone termed the coracoid, that is only represented in Man and other Mammals by the short coracoid process of the scapula (§ 635). The two clavicles $f f$ are united together where they join the sternum, to form the forklike bone known as the " merry - thought," the strength of which, like the projection of the keel of the sternum, serves to indicate the power of flight, by the degree of resistance which it is capable of affording to the drawing-together of the shoulder-joints by the action of the pectoral muscles. The bones of

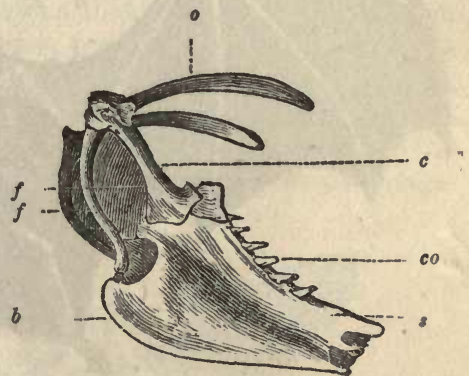

Fig. 250.-BONES OF THE SHOULDER AND BREAST OF BIRDS.

$o$, scapula; $c$, coracoid bone; $f$, clavicles united at their junction with the summit of the keel $b$ of the sternum $\delta$, which is connected with the ribs by the ossified costal cartilages $c o$. the pinion consist of the humerus (fig. 249, $h$ ), the two bones of the fore-arm $o$, the bones of the wrist ca (which are here scarcely developed), and the bones of the fingers $p h$, each joint of which shows indications of being made up of two or three separate bones united together. In no bird are these bones ever separated into distinct fingers, since they are never required for any other purpose than that of supporting the wing-feathers.-The leg is connected with the spinal column by a pelvis, of which the iliac bones are greatly lengthened and firmly attached to the spine, but which is not completed into a ring by the junction of the bones in front, as in Mammals; such a completion would have prevented the passage of the bulky eggs deposited by these animals ( $\$ 755)$. In the hinder extremity we find the femur or thigh-bone (principally concealed in the figure by the bones of the wing), the two bones of the leg $t$, which are commonly united in part of their length, the shank or ancle-bones $t a$, which are peculiarly 
elongated in the wading birds, and the four separate toes, by the spread of which the body is firmly supported, though resting only on two feet.

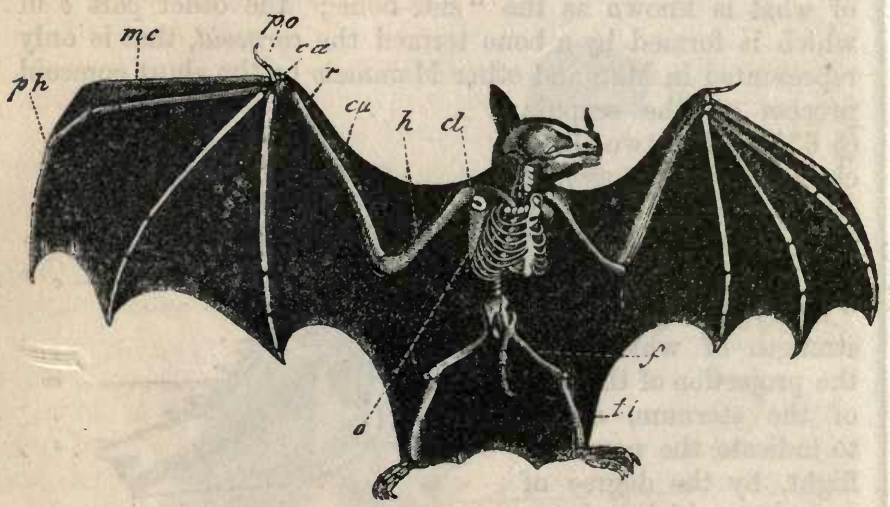

Fig. 251.-SKeLETON OF BAT. (References as in Fig. 229.)

669. In the Bat (fig. 251), however, the plan is very different. We have here no long stiff feathers, by the projection of which from the limb itself the surface may be increased to almost any extent; but the wing is formed by an expansion

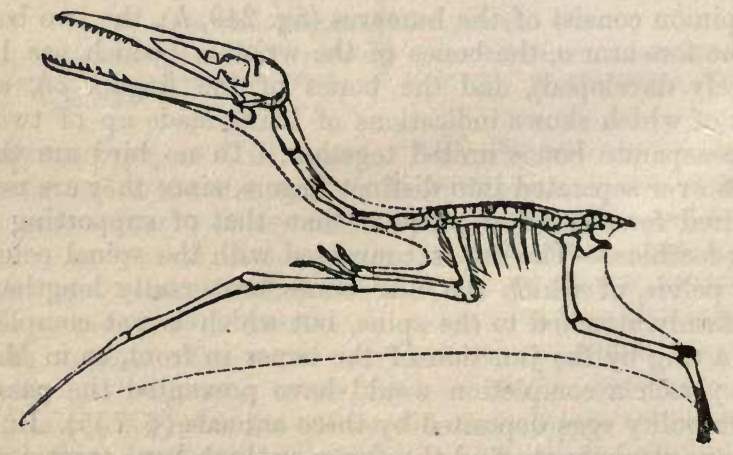

Fig. 252.-SKeleton of Pterodactyle.

of soft and delicate skin over a framework of bones, which must consequently be made to support it to its very edge. 
This is accomplished by the enormous extension of the bones of the hand, especially the metacarpal $m c$, which are here separate; and the membrane is further sustained by the legs and tail. The thumb po is not included in the wing, but serves as a hook by which the animal can suspend itself.The only true flying Reptile is (or rather was) the Pterodactyle, a kind of winged lizard, which does not now exist, but of whose character the skeletons that are found imbedded in the earth afford most convincing proof. The structure of its wing differed from that of either Birds or Bats ; for it appears, from the conformation of its anterior member (fig. 252), that the animal could have used it for resting or walking, the framework of the wing being formed by the enormous elongation of one finger only.

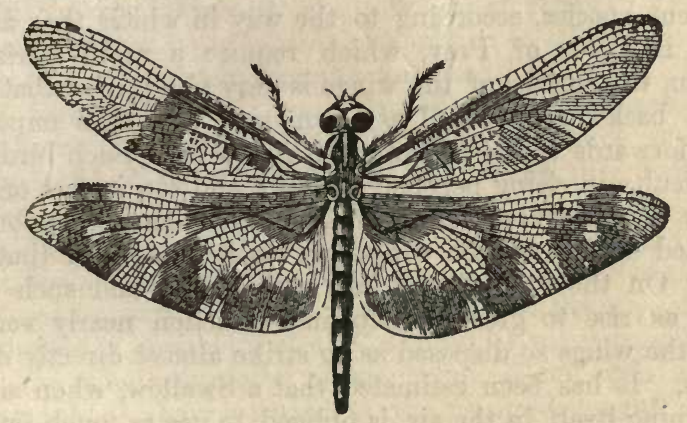

Fig. 253.-DRAGON FLY.

670. The wings of Insects (fig. 253) have no correspondence whatever with those of Vertebrata, except in serving for the like use, and in being composed of an expanded surface of membrane, stretched upon a firm framework. This framework is not composed of solid pieces jointed together, but is merely an extension of the air-tubes and vessels within the body, which are strengthened by a continuation of its hard envelope. Their only action is a hinge-like movement at the point where they are united to the body; and this is accomplished by powerful muscles contained within the thorax.

671. In all instances, the action of the wings must be such, that the air is struck with less force during the up-stroke than 
during the down-stroke; otherwise the effect of the former would neutralise that of the latter. This is partly accomplished by the great velocity of the down-stroke compared with the up-stroke, which causes the resistance of the air to be much greater against the former than against the latter. ${ }^{1}$ But it is by the alteration in the surface of the wing, as it acts upon the air, that the chief difference is made in Birds ; the arrangement of their great feathers being such, that they strike the air with their flat sides, but present only their edges in rising. What is called "feathering the oar" in rowing, is a similar operation, performed with the same intention, and deriving its name from this resemblance.

672. The degree in which the wings act in raising the body or in propelling it through the air, varies considerably in different species, according to the way in which they are set. Thus in Birds of Prey, which require a rapid horizontal motion, the surface of the wings is very oblique, so that they strike backwards as well as downwards, and thus impel the body forwards whilst sustaining it in the air. Such birds find a difficulty in rising perpendicularly ; and can in fact only do so by flying against the wind, which then acts upon the inclined surface of the wings just as it does upon that of a kite. On the other hand, the Lark, Quail, and such other birds as rise to great heights in a direction nearly vertical, have the wings so disposed as to strike almost directly downwards. It has been estimated that a Swallow, when simply sustaining itself in the air, is obliged to use as much force to prevent its fall, as would raise its own weight to a height of about twenty-six feet in a second. Hence, we may form some idea of the enormous expenditure of foree which must take place, when the body is not only supported, but raised and propelled through the air. The Eider-duck is said to fly 90 miles in an hour, and the Hawk 150. The Swallow and Swift pass nearly the whole of the long summer days upon the wing, in search of food for themselves and their

1 This resistance varies as the square of the velocity of the stroke. Hence, if the down-stroke be made three times as fast as the up-stroke, the resistance it experiences will be nine times as great. But as this only operates during one-third of the time, it is in effect equal to three times that which operates against the up-stroke, and which would tend to lower the Bird in the air. 
helpless offspring; and the rapidity of their flight is such, that they can scarcely traverse less than seven or eight hundred miles in that time, although they go but a short distance from home. The flight of Insects is even more remarkable for its velocity in proportion to their size; thus a Swallow, which is one of the swiftest-flying of Birds, has been seen to chase a Dragon-fly for some time without success ; the Insect always keeping about six feet in advance of the Bird, and turning to one side and the other so instantaneously, that the Swallow, with all its powers of flight, and its tact in chasing Insects, was unable to capture it.

673. If the preceding estimate of the power expended by a Bird in sustaining itself in the air be correct, it may be easily proved that it would be impossible for a Man to sustain himself in the air by means of his muscular strength alone, in any manner that he is capable of applying it. It is calculated that a man of ordinary strength can raise $13 \frac{1}{4} \mathrm{lbs}$. to a height of $3 \frac{1}{4}$ feet per second; and can continue this exertion for eight hours in the day. He will then exert a force capable of raising $\left(13 \frac{1}{4} \times 60 \times 60 \times 8\right) 381,600$ lbs. to a height of $3 \frac{1}{4}$ feet; or one-eighth that amount, namely $47,700 \mathrm{lbs}$., to the height of twenty-six feet, which, as we have seen, is that to which the Bird would raise itself in one second, by the force it is obliged to exert in order to sustain itself in the air. Now if we suppose it possible that a Man could by any means concentrate the whole muscular power required for such a day's labour, into as short a period as the accomplishment of this object requires, we might find the time during which it would support him in the air, by simply dividing this amount by his weight, which we may take to be 150 lbs. The quotient is 318 , which is the number of seconds, during which the expenditure of a force that would raise $47,700 \mathrm{lbs}$. to a height of twenty-six feet, will keep his body supported in the air ; and this is but little more than five minutes. There is no possible means, however, by which a Man could thus concentrate the force of eight hours' labour, into the short interval in which he would have to expend it while supporting himself in the air. And we have elsewhere seen (MECHanics, § 285), that by no combination of mechanical powers can force be created; as these only enable force to be more advantageously applied. Hence, the problem of human flight will never be 


\section{2}

USE OF PREHENSILE ORGANS IN LOCOMOTION.

solved, until some source of power shall be discovered, far surpassing that which his muscular strength affords, and so portable in its nature as not materially to add to his weight.

674. The only other organs of locomotion which we have to cônsider, are those of prehension. Of these, the principal have been elsewhere noticed, with reference to their use in laying hold of food and conveying it to the mouth ( $\$ 172)$, and with regard to the differences between the hand of Man and the claspers of the Quadrumana ( $\$ 643$ ). The hand of Man is seldom employed to assist in his locomotion, except in swimming (where it serves the purpose of a fin), and in climbing; neither of which kinds of movement can be said to be natural to him. But the claspers of the Quadrumana

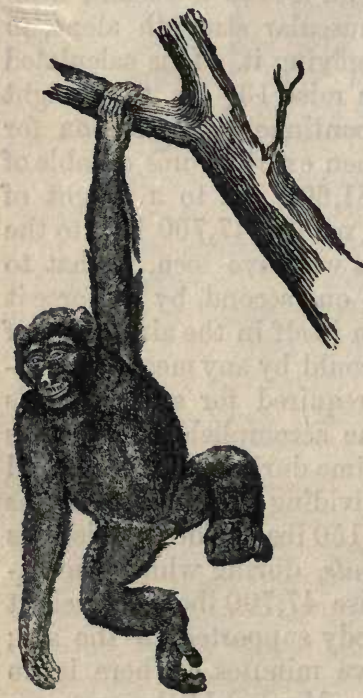

Fig. 254.-СhIMPANZEE. (fig. 254) are most efficient instruments of locomotion; enabling them not only to grasp the branches of the trees which they climb to despoil them of their fruit, but also to catch hold of them at the end of a long leap. This they do with the most wonderful agility; as all who have seen Monkeys in circumstances at all like those of their natural habitations, must have observed. The Gibbons, or long-armed Apes of the East Indies, are probably the most remarkable in this respect. The Author has seen the Ungkaputi leap round and round a room of about fifteen feet square, catching at each side by some small support attached to the wall; and taking its next leap (if such it could be called) by merely swinging itself from this, without touching anything solid with its

feet. There are many of the Monkey tribe, however, especially in the New World, whose hands are less efficient as instruments of prehension; and these are furnished with a prehensile tail; that is, a tail which can be coiled round the branch of a tree, and by which the animal can suspend itself 
(fig. 255). A similar tail is possessed by some of the Opossum tribe; and by the Chameleon among Reptiles.

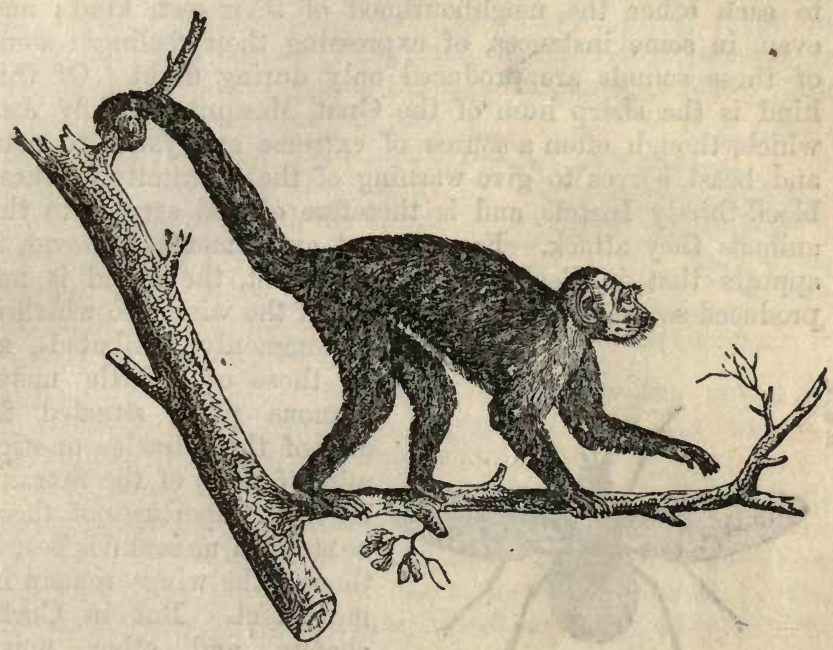

Fig. 255.-White-throated SAJOU.

\section{CHAPTER XIII.}

OF THE PRODUCTION OF SOUNDS : VOICE AND SPEECH.

675. IT is not by their movements alone, that Animals are enabled to influence one another. Were it so, their communication would be restricted to the small amount which can be effected by signs and gestures. This, however, is necessarily the case amongst aquatic animals in general; since they are prevented by the nature of the medium they breathe from producing sounds through its means. Some of them appear to have the power of communicating with each other by the vibrations which they can excite in the water; of this we have already noticed an example among the Whale tribe ( $\$ 491)$; and there is reason to believe that certain Mollusks possess a similar means of communication. 
676. Many Insects have the power of producing a continuous sound, which probably serves the purpose of intimating to each other the neighbourhood of their own kind; and even, in some instances, of expressing their feelings : some of these sounds are produced only during flight. Of this kind is the sharp hum of the Gnat, Mosquito, Gad-fly, \&c., which, though often a source of extreme annoyance to man and beast, serves to give warning of the proximity of these blood-thirsty Insects, and is therefore of real service to the animals they attack. From recent experiments, however, it appears that in Bees and Flies, at least, the sound is not produced so much by the vibrations of the wings (to which it

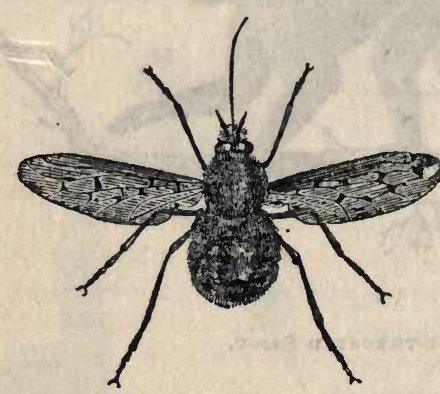

Fig. 250.-BомвтLIUs. is commonly attributed), as by those of a little membranous plate, situated in one of the spiracles or stigmata ( $\$ 321$ ) of the thorax; for if the apertures of these be stopped, no sound is heard, though the wings remain in movement. But in Cockchafers, and other noisy Beetles, Butterflies, \&c., no such apparatus can be discovered. Other sounds are produced while the insect is feeding; that occasioned by the armies of Locusts, when incalculable millions of powerful jaws are in action at the same time, has been compared to the crackling of a flame of fire driven by the wind. Certain two-winged Flies, distinguished by a long proboscis (fig. 256), make a humming sound whilst sucking honey from flowers; and the same is the case with some of the Hawk-moths.

677. Some Insects are remarkable for a peculiar mode of calling, commanding, or giving an alarm. The neuters or soldiers among the White Ants make a vibrating sound, rather shriller and quicker than the ticking of a watch, by striking against hard substances with their mandibles; this seems intended to keep the labourers, who answer it by a hiss, upon the alert and at their work. The well-known sound termed the "death-watch" is produced by a small beetle termed Anobium (fig. 257), that burrows in old timber; and 
it is occasioned by the striking of its mandibles upon the wood. The sound is evidently intended by the animal as a means of communication with its fellows; for if it be answered it is continually repeated, whilst if no answer be returned the animal repeats the signal in another place. The noise exactly resembles that produced by tapping moderately with the nail upon the table; and, when familiarised, the insect will very readily answer this imitation.-The most remarkable example of the production of sounds for the purpose of autho-

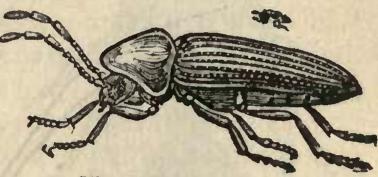

Fig. 257.-Aмовгим.

Natural size and magnified. rity, is that of the Queen-Bee; which has the power of influencing the whole hive, especially about the time of swarming, by the peculiar notes she produces.

678. Many Insects have the power of expressing their passions, also, - as fear, anger, sorrow, joy, or love,-by the sounds they can generate. The most curious of those given out under the influence of alarm is that produced by the Splinx A tropos or Death's-head Hawk-moth (fig. 258); which

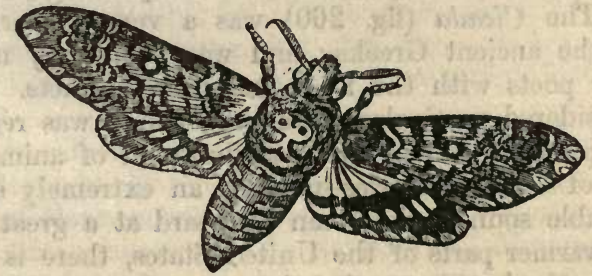

Fig. 258.-Sphixx Atropus.

when confined, or taken into the hand, sends forth a strong and sharp cry, resembling, some say, that of a mouse, but more plaintive and even lamentable. The means by which this cry is produced, have not yet been certainly ascertained. The influence of anger, sorrow, and joy, in modifying the tone of the hum of Bees, is well known to those who have studied their habits; the first is particularly evident in the sharp angry tone which is heard when the hive has been disturbed, especially if some of the Bees have been killed; the second

\section{L $\mathrm{L} 2$}


is manifested in a low plaintive tone which is given-out when the queen has been taken away; and the cheerful humming which is immediately heard when the sovereign is restored, is an evident indication of the last. Of all the Insects inhabiting this country, the most noisy are the Crickets; whose

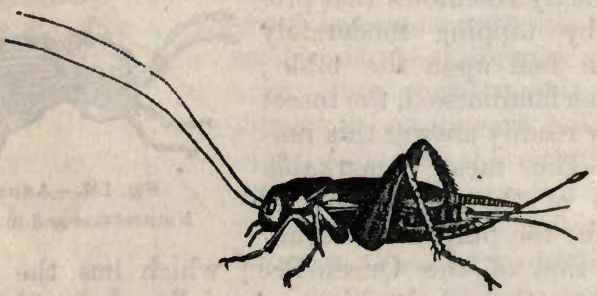

Fig. 259.-House-CricKet.

sound, which seems to be their expression of love, is produced by the rubbing of the elytra or wing-covers one against the other. In several species it may be distinctly seen that a very strong nervure on one of these has a jagged surface like that of a file; and that this works against a collection of smaller nervures, which resemble so many strings.

679. The Cicada (fig. 260) was a very favourite insect among the ancient Greeks; and was frequently mentioned by their poets with the most endearing epithets. Its song was considered particularly musical ; and it was regarded as the happiest as well as the most innocent of animals. The Cicadæ of other countries produce an extremely shrill and disagreeable sound, which can be heard at a great distance. In the warmer parts of the United States, there is a species which, in the hotter months of summer, is a very troublesome and impertinent neighbour. The Cicadæ of Brazil are said to be audible at the distance of a mile : this is as if a man of ordinary stature, supposing his powers of voice increased in the ratio of his size, could be heard all over the world. The organs by which the sound is produced are placed on the under side of the body, between the base of the hind legs and the abdomen, and consist externally of a pair of large flattened plates of a horny texture, varying in form in the different species. When these are raised, they are found to conceal a large cavity partially covered with a membrane of a 
much more delicate nature than the external covering, with a horny plate in the middle, which lies along the bottom. Still more internally are two bundles of muscles, which are the real agents in producing the sound; for, when they are pulled and suddenly let go, even in a dead specimen, the sound is produced as well as though the insect were alive. They draw-in and force-out, by their alternate and rapid contraction, a horny drum or membrane, stretched in such a manner as to vibrate readily; the sound occasioned by the movements of which passes out through an aperture resembling the sound-holes of a violin. The Fulgoro, also, have considerable sound-producing powers, but exert them in the night, whilst the Cicadæ perform in the day. The Great Lantern-

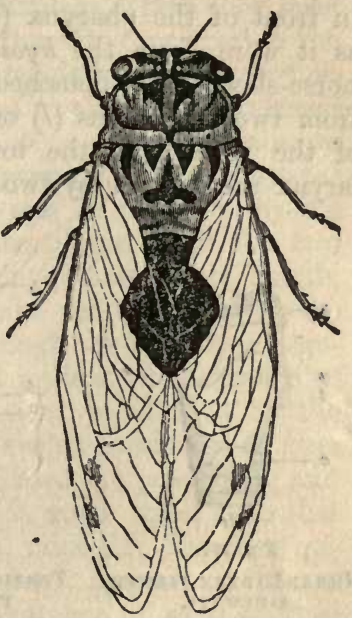

Fig. 260.-CicanA. fly of Guiana ( $\$ 400$, fig. 175) begins regularly at sunset; and its noise, resembling that of a razor-grinder at work, is so loud, that the insect is called "scare-sleep" by the Dutch colonists.

680. In all air-breathing Vertebrata, the production of sound depends upon the passage of air through a certain portion of the respiratory tube, which is so constructed as to set the air in vibration. In Reptiles and Mammals, it is at. the point where the windpipe opens into the front of the pharynx, that this vibrating apparatus is situated. Few of the animals of the former class, however, can produce any other sound than a hiss, occasioned by the passage of air through the narrow chink by which the trachea communicates with the pharynx; but this sound, owing to the great capacity of their lungs ( $\$ 325)$, is often very much prolonged. Among Mammals, on the other hand, there are few, if any, which have not some vocal sound; but the variety and expressiveness which can be given to it differ considerably in the several tribes of this class, being by far the greatest in 
Man. This sound is produced by the apparatus termed the larynx, which is situated beneath the base of the tongue, and in front of the pharynx ( $\$ 192$, fig. 107). It is suspended, as it were, from the hyoid bone ( $h$, fig. 261), - a bone of a horse-shoe form, detached from the rest of the skeleton; from two projections $(l)$ on the upper side of which, several of the muscles of the tongue originate. The sides of the larynx are formed by two large cartilages ( $t$, fig. 261), which

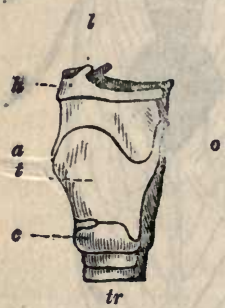

Fig. 261.

HUMAN LARYNX, VIEWED SIDEWAYS.

$h$, hyoid bone; $l$, point of origin of muscles of the tongue; $t$, thyroid cartilage; $a$, projection in front, commonly called Adam's apple; $c$,cricoid cartilage; $t r$, trachea ; 0 , posterior side of the larnyx, in contact with the osophagus.

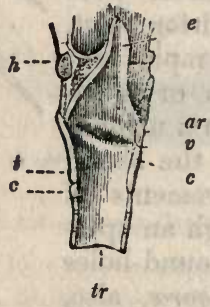

Fig. 262.

Verticat Section op THE LARYNX.

ar, arytenoid cartilages; $v$, ventricle of the glottis ; e, ep glot tis;- the other references as before.

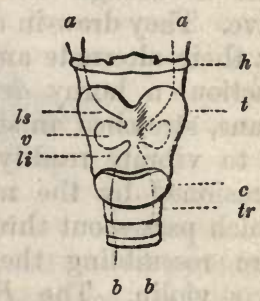

Fig. 263.

FroNt VIEW OP THE LARYNX.

The interior wall is marked by the lines $a, a, b, b$; $-l i$, inferior ligaments of the glottis, or vocal cords; $l_{s \text {, superior liga- }}$ ments; - the other references as before.

are termed the thyroid cartilages; where these meet on the middle line a projection is formed, which is particularly prominent in Man, and has received the name of Pomum Adami, or Adam's apple (a). The thyroid cartilages rest upon another, termed the cricoid $(c)$; this has the form of a ring, much deeper behind than in front, and surmounts the trachea, with the upper ring of which its lower edge is connected by a membrane. Upon the upper surface of the back of the cricoid cartilage, where there is an open space left between the two thyroid cartilages, are mounted two small cartilaginous bodies, the arytenoid (ar, fig. 262). These are movable to a certain extent; and their position may be changed in various directions by several muscles which act upon them. 
681. To these arytenoid cartilages are attached two ligaments of elastic fibrous substance ( $\$ 23$ ), which pass forwards to be attached to the front of the thyroid cartilage, where they meet in the same point. These are the instruments concerned in the production of sound, and also in the regulation of the aperture by which air passes into the trachea; and they are termed the vocal cords or ligaments (fig. 263, li). By the meeting of these ligaments in front and their separation behind, the usual aperture has the form of a V; but it may be narrowed by the drawing-together of the arytenoid cartilages, until the two vocal ligaments touch each other along their whole length, and the aperture is completely closed. In this manner, the amount of air permitted to pass through the larynx is regulated; and a protection is afforded against the entrance of solid substances. An additional guard is afforded by the doubling of the lining membrane, in such a manner as to form a second pair of folds ( $l s$, fig. 263), above the preceding; and over the space between these (which is much wider than that between the vocal cords) there is a valve-like flap, the epiglottis (e, fig. 262), which is pushed-down upon it in the act of swallowing, so as to prevent the entrance of solid or fluid particles into the space beneath, which is called the glottis. From the causes formerly mentioned ( $\$ 193$ ), such particles are occasionally drawn into the glottis ; and they excite, by a reflex action, an involuntary and extremely violent cough, which tends to expel them again. Sometimes, however, solid bodies of no inconsiderable size find a lodgment in the wide spaces ( $v$, fig. 263) between the upper and lower pair of ligaments, which are termed the ventricles of the larynx; and occasionally they pass through the opening between the vocal cords, which is termed the rima glottidis or fissure of the glottis, into the wind-pipe.

682. In the ordinary acts of inspiration and expiration, the arytenoid cartilages are wide apart, so that the aperture is as large as possible; but for the production of vocal sounds, it is necessary that the aperture should be narrowed, and that the flat sides, rather than the edges, of the rocal ligaments should be opposed to one another. This is accomplished by a peculiar movement of the arytenoid cartilages, occasioned by the contraction of certain muscles. When these ligaments are thus brought into position, the air in passing through the 
larynx sets them in vibration, in a manner very much resembling that in which the reed of a Hautboy or Clarionet, or the tongue of an Accordion or Harmoninm, is set in vibration by the current of air that is made to pass beneath them. The rapidity of the vibrations, and consequently the pitch of the sound ( $\$ 523$ ), depends on the degree of tension or tightness of the vocal ligaments; and this is regulated by muscles which act upon the thyroid and arytenoid cartilages. If the thyroid cartilage be drawn forwards, and the arytenoid cartilages backwards, the two ends of the vocal cords will be further separated from each other, and they will consequently be tightened; by the contrary movements they will be relaxed.

683. It is on account of the greater length of the vocal cords, that the pitch of the voice is much lower in Man than in Woman; but this difference does not arise until the end of the period of childhood, the size of the larynx being about the same in the Boy and Girl up to the age of 14 or 15 years, but then undergoing a rapid increase in the former, whilst it remains nearly stationary in the latter. Hence it is that Boys, as well as Girls and Women, sing treble; whilst Men sing tenor which is about an octave lower than the treble, or bass which is lower still.

684. The cause of the variations of timbre or quality in different voices, is not certainly known; but it appears to be due, in part, to differences in the degree of flexibility and smoothness in the cartilages of the larynx. In women and children these cartilages are usually soft and flexible, and their voices clear and smooth; whilst in men, and in women whose voices have a masculine roughness, the cartilages are harder, and are sometimes almost completely ossified. The loudness of the voice depends in part upon the force with which the air is expelled from the lungs; but the variations in strength of voice which exist among different individuals, are in some measure due to the degree in which its resonance is increased by the vibration of the other parts of the larynx and of the neighbouring cavities. In the Howling Monkeys of America, there are several pouches which open from the larynx, and are destined to increase the volume of tone that issues from it; one of these is excavated in the substance of the hyoid bone itself, which is very greatly enlarged; and to this bony drum seems due the mournful plaintiveness which characterises the 
tone of these animals. Although the largest of the American Monkeys, these Howlers are of inconsiderable size; yet their voices are louder than the roaring of lions, being distinctly audible at the distance of two miles; and, when a number are congregated together, the effect is terrific.

685. In Birds, the situation of the vocal organ is very different. The trachea opens into the pharynx, as in Reptiles, by a mere slit; the borders of which have no other movement than that of approaching one another, so as to close the aperture when necessary. But at the lower extremity of the trachea, just where it subdivides into the bronchial tubes, there is a sort of larynx or vocal organ, which is of very complex construction, especially in the singing-birds. The external surface of this larynx is represented in fig. 264 ; its muscles, $m m^{\prime}$, being left $t$ in their places on one side, and removed on the other. At $t$, is seen the trachea ; at the lower extremity of which, $t$, is a sort of bony drum, $l$, divided at its lower part by a partition of the same material (o, fig. $b$ $265)$, which is surmounted by a semilunar membrane (c). This drum communi-

cates below with the two Fig. 264.-LARYNX or Fig. 265.-VERTrCAX
A ROok. bronchial tubes, $b b^{\prime}$ (fig.

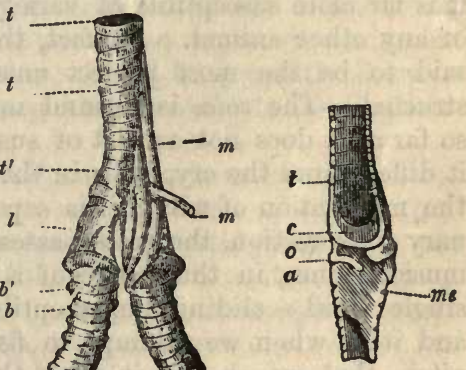
264), each of which has its own glottis and vocal cords; the inner lip of one of these is seen at $a$ (fig. 265); and at me is shown a drum-like membrane, forming the inner wall of the bronchial tube, which probably increases the resonance of the voice. These parts are acted-on by several muscles, the number of which varies according to the compass and flexibility of the voice in the different species; being very considerable in the most esteemed of the singing-birds, and being reduced to a small amount in those which have no vocal powers. In some, indeed, they are altogether absent; and the state of the glottis can be influenced only by those muscles which raise and lower the whole trachea.

686 . The vocal sounds produced by the action of the larynx 
are of very different characters; and may be distinguished into the cry, the song, and the ordinary or acquired voice.The cry is generally a sharp sound, having little modulation or accuracy of pitch, and being usually disagreeable in its timbre or quality. It is that by which animals express their unpleasing emotions, especially pain or terror ; and the Human infant, like many of the lower animals, can utter no other sound.-In song, by the regulation of the vocal cords, definite and sustained musical tones are produced, which can be changed or modulated at the will of the individual. Different species of Birds have their respective songs; which are partly instinctive, depending upon the construction of their larynx; and are partly governed by their education. In Man, the power of song is entirely acquired; but, when once acquired, it is far more susceptible of variety and expression than that of any other animal. In fact, the larynx of Man may be said to be the most perfect musical instrument ever constructed.-The voice is a sound more resembling the cry, in so far as it does not consist of sustained musical tones; but it differs from the cry, both in the quality of its tone, and in the modulation of which it is capable by the will. In ordinary conversation, the voice passes through a great variety of musical tones, in the course of a single sentence, or even a single word,-sliding imperceptibly from one to another; and it is when we attempt to fix it definitely to a certain pitch, that we change it from the speaking to the singing tone.

687. It is to the wonderful power that Man possesses, of producing articulate sounds, which form a medium whereby he can communicate ideas of any kind to his fellows, - that much of his superiority to other animals is due. Nevertheless, it is not to this alone that we must attribute it ; for many animals, especially Birds, can produce, by imitation, sounds as articulate as those of Man; but the mind which originates them, and which uses them as expressions of its ideas and desires, is deficient.

688. All spoken language is made up of a certain number of elementary sounds, which are combined into syllables, words, and sentences. It may be easily shown, upon arithmetical principles, that from 20 or more of these elementary sounds, an almost infinite variety of combinations may be produced; 
and from such an inexhaustible store there is no difficulty in deriving new combinations, to represent any new ideas that we may desire to express. These simple or elementary sounds ought to be represented by an equal number of single letters; this is the case, however, in but few languages. Our own is particularly faulty in this respect; for there are many simple sounds that can be only represented by a combination of letters, whilst others may be represented by more than one single letter, and in some instances a single letter represents a composite sound. Thus the sounds of $a u$ and th are really simple ones, and ought to be represented by single letters. Again, the sound of $k$ is represented also by the hard $c$, as in the first syllable of concert; and the sound of $s$ by the soft $c$, as in the second syllable of the same word, where the $c$ is sounded exactly as the $s$ in consent. And the letter $i$ (as usually pronounced in English) does not represent a simple sound, but a combination of two, as will be presently shown. Most of the Continental languages are superior to the English in this respect.

689. Vocal sounds are divided into Vowels and Consonants; the true distinction between which appears to be, that the Vowel sounds are continuous tones, modified by the form of the aperture through which they pass out; whilst in giving utterance to Consonants, there is a partial or complete interruption to the breath in its passage through the organs in front of the larynx. Hence all true Vowels may be prolonged for any length of time that the breath is supplied from the lungs ; whilst the sound of many Consonants is momentary only. It is easy for any one to convince himself that the Vowel sounds are governed simply by the form of the cavity of the mouth, and by that of the aperture of the lips; by passing, in one continued tone, from one of the following Vowel sounds to another:-

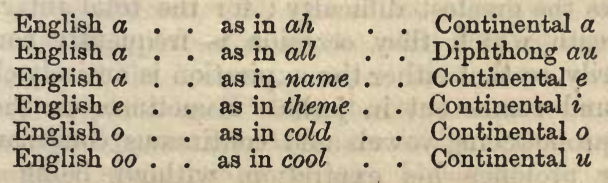

The short Vowel sounds, as $a$ in fat, $e$ in met, $o$ in pot, \&c., are not capable of being prolonged; as they are formed in 
the act of preparation for sounding the succeeding consonant. The sound of the English $i$ is a compound one, being formed in the act of transition from that of $a$ in $a h$, to that of $e$ as in theme; hence it cannot be prolonged; and it is the very worst vowel sound upon which to sing a long note, since it is impossible to prevent its being heard as one of the sounds of which it is composed. Much discussion has taken place withr eference to the true characters of the letters $w$ and $y$, when employed to commence words, as wall, yawl, wet, yet. A little attention to the state of the mouth in pronouncing them will show, however, that they are then really vowel sounds, rapidly transformed into the succeeding ones; for the sound of $w$ in this situation is 00 ; and that of $y$ is the long $e$; so that wall might be spelt ōoall, and yawl eaul.

690. Consonants are naturally divided into those which require a total stoppage of the breath at the moment previous to their being pronounced, and which cannot therefore be prolonged; and those in pronouncing which the interruption is partial, and which can be prolonged like the vowels. The former are termed explosive consonants; the latter continuous. The explosive consonants are $b$ and $p, d$ and $t$, the hard $g$ and $k$. All the others are continuous; but the sound is modified by the position of the tongue, palate, lips, and teeth ; and also by the degree in which the air is permitted to pass through the nose.

691. The study of the mode in which the different consonants are produced, is of particular importance to those who labour under defective speech, especially that difficulty which is known as stammering. This very annoying impediment is occasioned by a want of proper control over the muscles concerned in articulation; which are sometimes affected with a kind of spasmodic action. It is in the pronunciation of the consonants of the explosive class, that the stammerer usually experiences the greatest difficulty; for the total interruption to the breath which they occasion is frequently continued involuntarily, so that either the expiration is entirely checked, or the sound comes out in jerks. Sometimes, on the other hand, in pronouncing vowels and continuous consonants, the stammerer prolongs his expiration, without being able to check it. The best method of curing this defect (where there is no malformation of the organs of speech, but merely a want 
of power to use them aright), is to study the particular defect under which the individual suffers; and then to make him practise systematically the various movements concerned in the production of the sounds in question, at first separately, and afterwards in combination, until he feels that his voluntary control over them is complete.

\section{CHAPTER XIV.}

OF INSTINCT AND INTELLIGENCE.

692. IT will be remembered that, when the Nervous System was described (Chap. xI.), it was shown to be the instrument of three classes of operations, each of which seems to be performed by a distinct portion of the apparatus.-I. The first of these is the class of simply Reflex actions, which are executed only in respondence or answer to impressions made upon the nerves proceeding to a ganglionic centre ; as when a Dytiscus, whose head has been cut off, executes swimming movements immediately that its feet come in contact with water. These movements evidently take place without any choice or direction on the part of the animal, which, in executing them, seems like a mere machine adapted to perform certain actions when certain springs are touched; and it has been shown that they may be called-forth even without its consciousness. Of these reflex movements, the Spinal Cord of Vertebrata, and in Invertebrata the ganglia corresponding to it (in regard to their connexions with the organs of locomotion, respiration, \&c.), are the instruments.-II. The second class comprehends those Instinctive actions, which differ from the preceding in being dependent on the sensations received by the animal, and in being, therefore, never performed without its consciousness. Nevertheless, the animal in executing them is not guided by any perception of the object to be attained, or by any choice of the means by which it is to be accomplished; but acts blindly and involuntarily, in accordance with an irresistible impulse, implanted in it by its Creator for the purpose of causing it to do that, without or even against its Will, which it would not have chosen or devised by its very imperfect intelligence. The actions of this class are most 
wonderful in the Invertebrata, which possess the least Intelligence; and, on the contrary, they are fewest and least remarkable in Man, whose Intelligence is highest. From the constant proportion they bear to the size of the ganglia of sensation, which form nearly the whole nervous mass in the head of Insects, \&c., and a large part of that of the lower Vertebrata, but which are comparatively small in the Mammalia and especially so in Man, there seems good reason to regard these organs as their chief instruments.-III. The third and highest class of actions, is that in which Intelligence is the guide, and the Will the immediate agent. The animal receives sensations, forms a notion of their cause, reasons upon the ideas thus excited, perceives the end to be attained, chooses or devises the means of accomplishing it, and voluntarily puts those means into execution. These actions are seen, in their highest and most complete form, in Man; but they are not confined to him ; for, as will be shown hereafter, true reasoning processes are performed by many of the lower animals. There can be no doubt that the Cerebral Hemispheres, which form the Brain properly so called, constitute the instrument by which these actions are executed; for we find that their size and development bear a very regular proportion to the degree of Intelligence which the animal possesses.

693. It follows, then; that the lower we descend in the scale of Animal life, the larger is the proportion of the movements of any particular species which we are to attribute to the Reflex and the Instinctive classes; whilst the proportion which is due to Intelligence and Will diminishes in a like degree. Thus we have seen that the ordinary movements of locomotion, which Man performs in the first instance by voluntary effort, are reflex in Insects ( $(445)$; and there can be no reasonable doubt that the movements of the tentacula of the Hydra, by which it entraps its prey and draws it to the entrance of its stomach ( $\$ 121)$, are of a reflex, rather than a voluntary or instinctive character, since they are obviously analogous to those movements of the pharyngeal muscles, by which the food is grasped and carried into the alimentary tube of the highest animals ( $\$ 195)$. There is one curious fact, which would seem to indicate a difference between them, but which is really a strong argument in favour of their analogy. It is 
continually observed that when the stomach of the Polype is full, its arms do not make any attempt to seize objects that touch them; so that small worms, insects, \&c., which would at other times be entrapped, may now come near them with impunity. It has been supposed that this results from an act of choice on the part of the animal, and that its choice is influenced by its consciousness that its stomach is supplied with food. It must seem improbable that an Animal which so nearly resembles Plants in its general habits, and in which the nervous system is so obscure that it has not yet been discovered, should possess mental endowments of so high a character; and we may find, in studying our own functions, a circumstance exactly parallel to that just mentioned. For when we commence eating, with a good appetite, we may notice that the muscles of Deglutition act very readily; but when we are completely satisfied, it is often difficult to excite these muscles to contraction, so as to swallow another morsel, even though, for the gratification of our palate, we may desire to do so. Thus we see how much better a guide we find in Nature, for the amount of food we require, than in our own pampered tastes.

694. The first class, that of Reflex movements, has been already considered in sufficient detail ; but it is intended, in the present chapter, to offer some examples of those of the second and third classes,- those actions, namely, which are guided by Instinct and Intelligence respectively. These actions may be usually distinguished by the two following tests :1. Although, in most cases, experience is required to give the Will command over the muscles concerned in its operations, no experience or education is required, in order that the different actions which result from an Instinctive impulse may follow one another with unerring precision. 2. Instinctive actions are performed by the different individuals of the same species, nearly, if not exactly, in the same manner; presenting no such variation of the means applied to the objects in view, and admitting of no such improvements in the progress of life, or in the succession of ages, as we observe in the habits of individual Men, or in the manners and customs of nations, which are for the most part adapted to the attainment of particular ends, by voluntary efforts guided and directed by reason.-Where, as in the examples hereafter to be men- 
tioned ( $\$ 717)$, we find individual animals "learning wisdom by experience," and acquiring the power of performing actions which do not correspond with their natural instincts, we cannot do otherwise than regard them as possessed of a certain degree of Intelligence, by which they are rendered susceptible of education.

695. The amount of Intelligence displayed in such acquirements, can only be judged-of, however, by carefully examining the circumstances under which they are made. If the new habits are gained-like the talking of a Parrot-by imitation simply, no great degree of intelligence is manifested; but if it spontaneously result from a reasoning process on the part of the animal, our idea of its sagacity is raised. There may be a combination of both these conditions; as in the following curious circumstance, related to the Author by a friend who has repeatedly witnessed it. Some horses kept in a paddock were supplied with water by a trough, which was occasionally filled from a pump,-not, however, as often as the horses seem to have wished; for one of them learned, of his own accord, to supply himself and his companions, by taking the pump-handle between his teeth, and working it with his head. The others, however, appear to have been less clever, or more lazy; and finding that this one had the power of supplying their wants, they would teaze him, by biting, kicking, \&c., until he had pumped for them, and would not allow him to drink until they were satisfied. That this was not a mere act of imitation, appears from the circumstance that the horse did not attempt to imitate the movement of the man, but performed the same action in a different manner,-evidently because it had associated in its mind the motion of the pumphandle with the supply of water.

696. The Instincts of Animals may be shown to have immediate reference, probably in every instance, to the supply of the wants of the individual, or to the continuance of the race. Thus we have Instincts which guide in the selection and acquirement of food ; others which govern the construction of a habitation for the individual, and of a receptacle for the eggs,-and these may influence a number at once, in such a manner as to unite them into a society; and others which direct their migrations, whether in search of food, for the deposit of their eggs, or for other purposes. Of these, some examples will now be given. 
697. Among the instincts which direct animals in the acquirement of their food, few are more remarkable than those possessed by the larva of the Ant-lion (fig. 266), a small insect allied to the Dragon-fly. This larva (fig. 267) is destined to feed

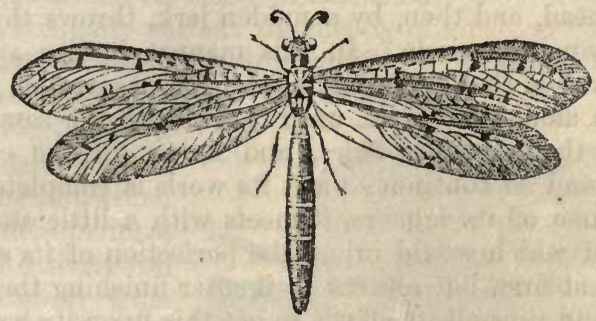

Fig. 266.-Ant Lion IN PERfect state.

upon ants and other small insects, whose juices it sucks ; but it moves slowly and with difficulty, so that it could scarcely have obtained the requisite supply of food, if Nature had not guided it in the construction of a remarkable snare, which entraps the prey it could not acquire by pursuit. It digs in fine sand a little funnel-shaped pit (fig. 268), and conceals

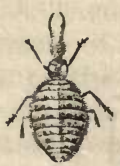

Fig. 267. LARVA or THE ANT-LION.

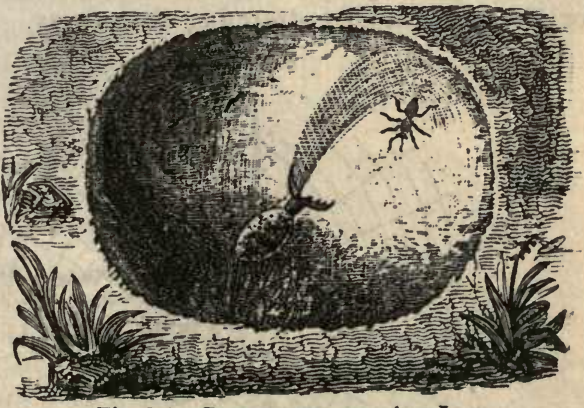

Fig. 268.-Pitfali of the ANt-Li:x.

itself at the bottom of this, until an insect falls over its edge; and if its victim seeks to escape, or stops in its fall to the bottom, it throws over it, by means of its head and mandibles, a quantity of sand, by which the insect is caused to roll down the steep, within reach of its captor. The manner in which 
the Ant-lion digs this pit is extremely curious. After having examined the spot where it purposes to establish itself, it traces a circle of the dimensions of the mouth of its pit; then, placing itself within this line, and making use of one of its legs as a spade, it digs out a quantity of sand, which it heaps upon its head, and then, by a sudden jerk, throws this some inches beyond its circle. In this manner it digs a trench, which serves as the border of its intended excavation, moving backwards along the circle, until it comes to the same point again; it then changes sides, and moves in the contrary direction, and so continues until its work is completed. If, in the course of its labours, it meets with a little stone, the presence of which would injure the perfection of its snare, it neglects it at first, but returns to it after finishing the rest of its work, and uses all its efforts to get this upon its back, and carry it out of the excavation; but if it cannot succeed in doing so, it abandons its work and commences anew elsewhere. When the inclination of the walls of the pit has been altered by any slip, as almost always happens when an insect has fallen-in, the Ant-lion hastens to repair the damage.

698. Snares of a still more singular character are constructed by many Spiders, which spin webs of the finest silk, for the purpose of entrapping their prey. The arrangement

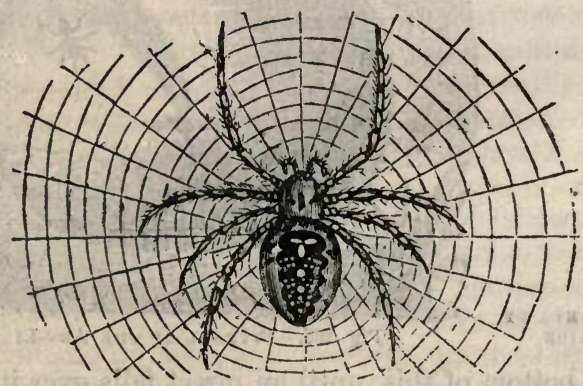

Fig. 269.-Ereira Diatema.

of these toils varies according to the species, and sometimes does not present any regularity; but in several instancos it is 
of extreme elegance; and no one can watch the labours of a common garden spider, as, for instance, the Epeira diadema (fig. 269), without being struck with the marvellous sagacity which it displays in the execution of its work, and the perfection with which its web is constructed.

699. An equally curious instinct is often displayed in the construction of the habitations which the animal designs for its abode. Thus the Hamster (fig. 270), a small rodent animal allied to the Rat, which is met with in most cultivated districts on the Continent from Alsace to Siberia, and which is very injurious to agriculture, constructs a burrow in the

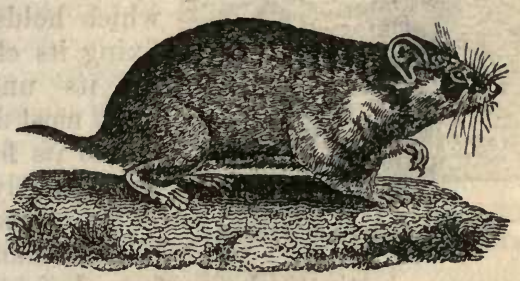

Fig. 270.-HAMsrer. soil which has always two openings,- - one in an oblique direction, which serves the animal for casting out the earth it has dug away,- the other perpendicular, which is the passage by which it enters and makes its exit. These galleries lead to a regular series of circular excavations, which communicate with each other by horizontal passages ; one of these cavities, furnished with a bed of dried herbage, is the abode of the Hamster ; while the others serve as magazines for the provisions which it collects in large quantities.

700. There are certain Spiders known to Zoologists under the name of Mygale, which perform operations analogous to those of the Hamster, but still more complicated; for not only do they excavate in the ground a large and commodious habitation, but they line it with a silken tapestry, and furnish it with a door regularly hung upon a hinge (fig. 271). For this purpose, the Mygale digs, in a clayey soil, 'a sort of cylindrical pit, about 3 or 4 inches in length; and plasters its walls with a kind of very consistent mortar. It then constructs, of alternate layers of earth, and of threads woven into a web, a trap-door exactly adapted to the orifice of its hole, and only capable of opening outwards ; and it attaches this by a hinge of the same thread to the tapestried lining of its chamber. The outside of this trap-door is covered with 
materials resembling the soil around; and so little does it differ from this, as to be with difficulty distinguished, even

by a person seeking to discover

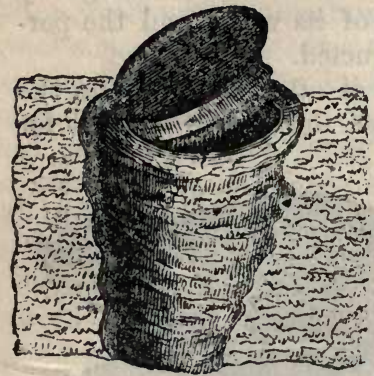

Fig. 271. Nest of MYgale. the Spider's habitation. If an attempt is made to lift it, when the animal is within its excavation, the movement is resisted by the whole force of the Spider, which holds down the door, by fixing its claws into small holes on its under surface at the point most distant from the hinge, where its force may be most advantageously applied.

701. Among Insects, we find

a great number of very curious processes instinctively performed in the construction of their habitations. Many Caterpillars form for themselves a protection, by rolling together portions of leaves, and attaching these by threads. In almost every garden, we may observe (at the proper season) nests of this kind, on the leaves of the Lilac or Gooseberry ; and a similar one, represented in fig. 272 , is constructed in the leaves of the oak, by the caterpillar of a small nocturnal Butterfly, the Tortrix viridissima. The Larva of the little Clothes-moth, again, forms a sort of tubular sheath, composed of the filaments it detaches

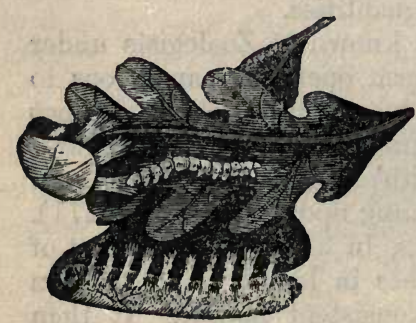

Fig. 272. $-\mathrm{N}$ LST of Tortrix. from the stuff through which it excavates its galleries; this sheath it is continually prolonging at one extremity; and when, in consequence of the growth of the larva, its tube becomes too small for its comfortable residence, it slits it down and lets-in a piece. The aquatic Larvæ of the Caddiceflies (fig. 273, c), which are commonly known as Caddiceworms, house themselves in straws, pieces of hollow stick, rushes, \&c.; and those of some species glue together a number of minute stones, pieces of stick, small shells, \&c., 
so as to make a tube $(A)$, in which the animal creeps along the bottom and sides of the brook it inhabits, and sometimes rows itself on the surface of the water. When fullgrown, the larva attaches its case by threads to some large stone; and then covers its mouth with an open net-work of threads (B), sufficiently close to prevent the entrance of insects, but with meshes permitting the water to pass through. In this way it undergoes its metamorphosis into the Pupa state; and a short time before its

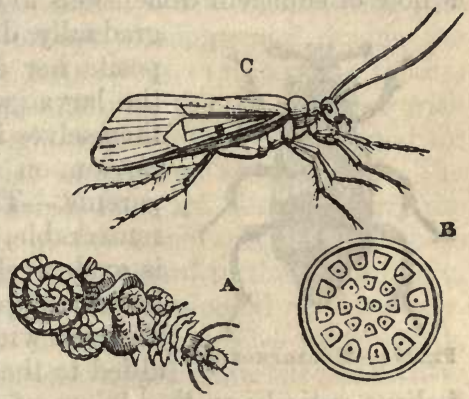

Fig. 273.-C, PhRYGANFA OR CADDICE-FLY A, tube formed by its larva; B, network at the entrance of the tube.

last change it cuts the threads of the network, by means of two hooks with which its head is furnished, and creeps out of the water; soon after which it changes into the perfect insect.

702. It is scarcely possible to point to any actions better fitted to give an idea of the nature of Instinct, than those which are performed by various Insects when they deposit their eggs. These animals never behold their progeny, and cannot acquire any notion from experience, therefore, of that which their eggs will produce; nevertheless they have the remarkable habit of placing, in the neighbourhood of each of these bodies, a supply of aliment fitted for the nourishment of the larva that is to proceed from it; and this they do, even when they are themselves living on food of an entirely different nature, such as would not be adapted for the larva. They cannot be guided in such actions by anything like reason, since the data on which alone they could reason correctly are wanting to them; so that they would be led to conclusions altogether erroneous, if they were not prompted by an unerring instinct to adopt the means best adapted for the attainment of the required end.

703. Of this kind of instinct, the Necrophorus (fig. 274), a kind of Beetle not uncommon in our fields, offers a good example. When the female is about to lay her eggs, she 
seeks for the dead body of a mole, shrew, or such other quadruped; and having found one, she excavates beneath it a hole of sufficient dimensions to contain the body, which she

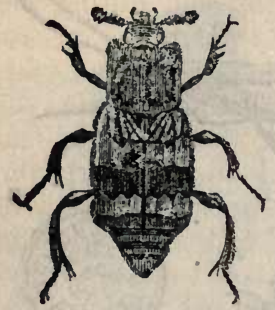

Fig. 274. -NECrophorUs. gradually drags into it; she then deposits her eggs in the carcase, so that the larvæ, when they come forth, find themselves in the midst of a supply of carrion, on which they feed like their parents. - This instinct is still more remarkable, when an Insect whose diet is exclusively vegetable prepares for its larva a supply of animal food. Such is the case with the Pompilus, an Insect allied to the wasp. In its perfect state it lives entirely on the juices of flowers; but the larvæ are carnivorous ; and the mother provides for them the requisite supply of the food they require, by placing in the nest, by the side of the eggs, the body of a spider or caterpillar which she had previously killed by means of her sting. The Xylocopa,

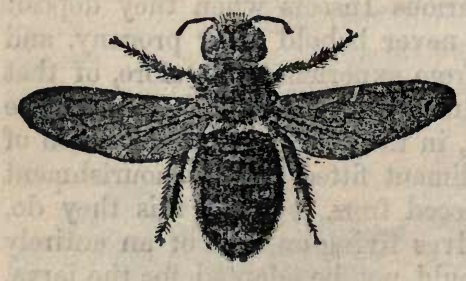

Fig. 275.-XYLOCOPA.

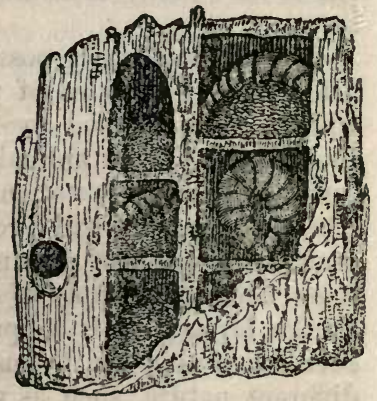

Fig. 276.-Nest of XrLocopa.

or Carpenter-bee (fig. 275), has very analogous habits; the female makes long burrows in wood, palings, \&c., in which she excavates a series of cells (fig. 276); and in every one of these she deposits an egg, with a supply of pollen-paste.

704. The instinct of support and protection to the young and helpless offspring, is seen in all animals in which it is needed; and it is particularly observable in Birds. The nests 
which they construct are destined much more for the reception of their eggs, and for the protection of the young, than for their own residence; for there are few Birds which pass much time in their nests, except at night, and during the period of incubation. It is impossible to watch the process of their construction, without admiring the perseverance with which the materials are brought together that are destined for their erection, and the art with which these are arranged. The form and structure of the habitations are always nearly the same among the individuals of the same species; but there is necessarily a certain latitude in regard to the materials of which they are composed, since the same could not be everywhere procured. The nests of different species vary greatly, however, both as to form, structure, and materials; and these are admirably adapted to the particular circumstances in which the young families are respectively destined to live. Sometimes these habitations are constructed of earth, the particles of which are united by the viscid saliva of the Bird into a tenacious mortar ; and they are then commonly built against the sides of a rock or wall. But, in general, they are com-

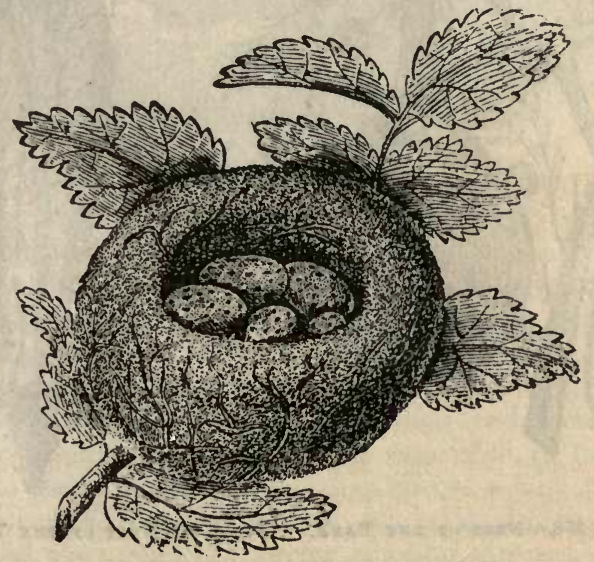

Fig. 277. -Nest of Goldrinch.

posed of sticks, straws, and other vegetable substances ; and are placed either on the ground, or among the branches of 
trees. The greater number of them have a somewhat hemispherical form, resembling a little round basket; and their interior is lined with moss and down (fig. 277).

705. But sometimes the arrangement is much more complicated, so as to avert some particular danger, or to answer some special purpose. Thus the nest of the Baya, a little Indian bird allied to our Bulfinch, has the form of a bottle; and it is suspended from a twig of such slenderness and flexibility, that neither monkeys, serpents, nor squirrels can reach it (fig. 278). It is rendered still more secure against the attacks of its numerous enemies, by the formation of the entrance of the nest on its under side, so as to be only reached by even the bird itself with the aid of its wings. This curious habitation is constructed of long grass ; and several chambers are found in its interior, of which one serves for the female
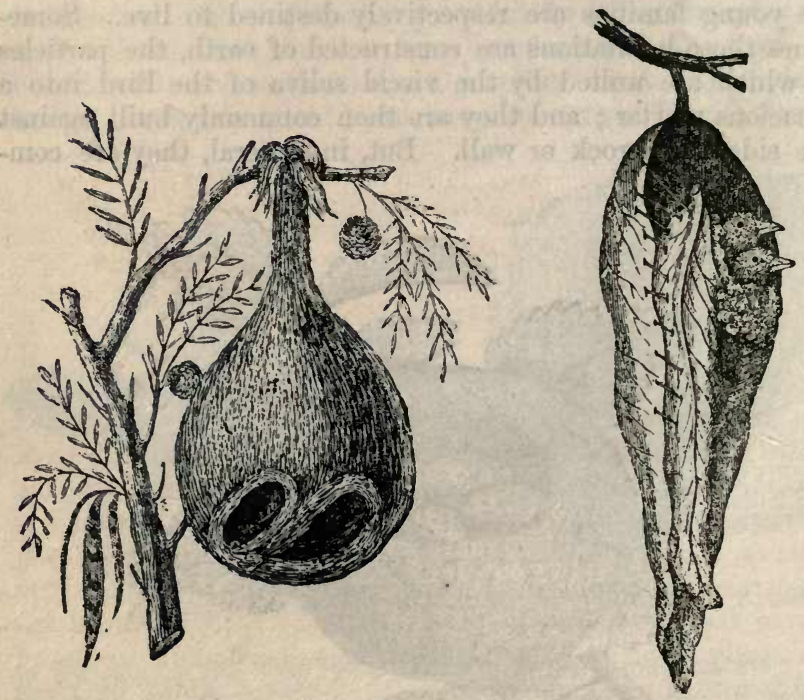

Fig. 278.-Nest G the Baya. Fig. 279.-Nest of the Tailor-Bird.

to sit on her eggs, whilst another is occupied by the male, who solaces his companion with his song, whilst she is occupied in maternal cares. Another curious nest is that of the 
Sylvia sutoria, or Tailor-bird, a little eastern bird allied to our linnet; which, by the aid of filaments of cotton drawn from the cotton-plant, sews leaves together with its beak and feet, in such a manner as to conceal the nest which they enclose from the observation of its enemies (fig. 279).

706. The association of a number of individuals of a certain species, for the performance of labours in which they all unite to one common end, is anuther most remarkable example of the operation of instinct. Several Mammals exhibit this tendency in a greater or less degree; but the most interesting of all, in this point of view, is the Beaver (fig. 280), which is

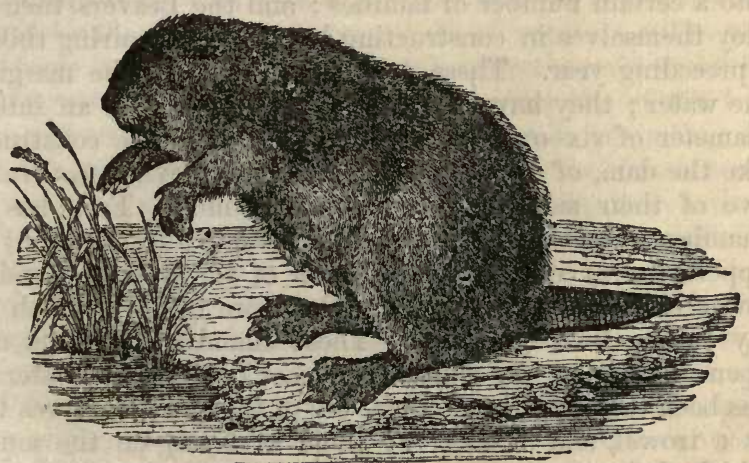

Fig. 290.-BEAvER.

now chiefly found in Canada, though it formerly abounded on the Continent of Europe. During the summer it lives solitarily in burrows, which it excavates for itself on the borders of lakes and streams; but as the cold season approaches, it quits its retreat, and unites itself with its fellows, to construct, in common with them, a winter residence. It is only in the most solitary places that their architectural instinct fully developes itself. Having associated in troops of from two to three hundred each, they choose a lake ur river which is deep enough to prevent its being frozen to the bottom; and they generally prefer running streams, for the sake of the convenience which these afford in the transportation of the materials of their erection. They begin by constructing a sloping dam, whereby the water is kept-up to 
a uniform height; this they form of branches interlaced one with another, the intervals between them being filled with stones and mud, with which materials they give a coat of rough-cast to the exterior also. When the dam passes across a running stream, they make it convex towards the current; by which it is caused to possess much greater strength than if it were straight. This dam is usually eleven or twelve feet across at its base, and it is enlarged every year ; and it frequently becomes covered with vegetation, so as to form a kind of hedge.

707. When the dam is completed, the community separates into a certain number of families ; and the Beavers then employ themselves in constructing huts, or in repairing those of a preceding year. These cabins are built on the margin of the water; they have usually an oval form, and an internal diameter of six or seven feet. Their walls are constructed, like the dam, of branches of trees; and they are covered on two of their sides with a coating of mud. Each has two chambers, one above the other, separated by a floor; the upper one serves as the habitation of the Beavers; and the lower one as the magazine for the store of bark which they lay up for their provision. These chambers have no other opening than one by which they pass out into the water. It has been said that the flat oval tail of the Beavers serves them as a trowel, and is used. by them in laying-on the mud of which their erections are partly composed; but it does not appear that they use any other implements than their incisor teeth and fore-feet. With their strong incisors they cut-down the branches and even the trunks of trees which may be suitable; and by the aid of their mouths and fore-feet they drag these from one place to another. When they establish themselves on the banks of a running stream, they cut-down trees above the point where they intend to construct their dwellings, set them afloat, and, profiting by the current, direct them to the required spot. It is also with their feet that they dig-up the earth they require for mortar, from the banks or from the bottom of the water. These operations are executed with extraordinary rapidity, although they are only carried-on during the night. When the neighbourhood of Man prevents the Beavers from multiplying to the degree necessary to form such associations, and from possessing the 
tranquillity which they require for the construction of the works now described, they no longer build huts, but live in excavations in the banks of the water.

708. The building instinct shows itself, even when the Beaver is in captivity, and under circumstances in which it could be of no use. A half-domesticated individual, in the possession of Mr. Broderip, began to build as soon as it was let out of its cage and materials were placed in its way. Even when it was only half-grown, it would drag along a large sweeping-brush or warming-pan, grasping the handle with its teeth, so that the load came over its shoulder; and would endeavour to lay this with other materials, in the mode employed by the Beaver when in a state of nature. "The long and large materials were always taken first; and two of the longest were generally laid cross-wise, with one of the ends of each touching the wall, and the other ends projecting out into the room. The area formed by the cross-brushes and the wall, he would fill up with hand-brushes, rush-baskets, books, boots, sticks, cloths, dried turf, or anything portable. As the work grew high, he supported himself upon his tail, which propped him up admirably; and he would often, after laying-on one of his building materials, sit up over against it, appearing to consider his work, or, as the country people say, 'judge it.' This pause was sometimes followed by changing the position of the material judged; and sometimes it was left in its place. After he had piled up his materials in one part of the room (for he generally chose the same place), he proceeded to wall-up the space between the feet of a chest of drawers which stood at a little distance from it, high enough on his legs to make the bottom a roof for him; using for this purpose dried turf and sticks, which he laid very even, and filling up the interstices with bits of coal, hay, cloth, or anything he could pick up. This last place he seemed to appropriate for his dwelling; the former work seemed to be intended for a dam. When he had walled-up the space between the feet of the chest of drawers, he proceeded to carry-in sticks, cloths, hay, cotton, \&c., and to make a nest; and when he had done, he would sit up under the drawers, and comb himself with the nails of his hind feet."

709. We see, in this account, a very interesting example of the irrational character of Instinct. If the animal were 
guided in its ordinary building operations by such an amount of intelligence as would lead it to choose and execute its various movements with a design to accomplish certain ends, the same intelligence would direct it to leave these actions unperformed when the purpose no longer required it; instead of which, we see that the animal is impelled by an internal impulse to construct erections as nearly resembling those which it would build-up on the banks of its native streams, as the materials and circumstances will permit.-Other animals are, in like manner, occasionally conducted by their natural instincts to the performance of actions equally irrational, and quite incapable of answering the purpose which the particular instinct is destined to serve. Thus the Hen will sit upon an egg-shaped piece of chalk, as readily as upon her own egg, being deceived without difficulty by the general similarity of its appearance; and the Flesh-fly lays its eggs in the petals of the carrion-flower, whose odour so much resembles that of tainted meat as evidently to furnish the same attraction to the insect.

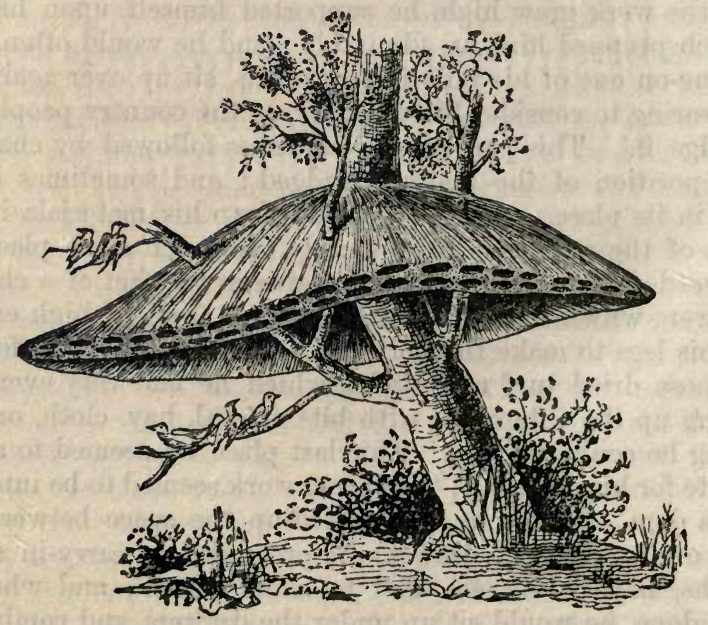

Fig. 281.-Nest of Republican Grosbeak.

710. Societies like those of the Beaver are rare among Birds, whose associations are usually less perfect. There is 
a small species, however, termed the Republican Grosbeak (Loxia Socia), which lives in numerous societies in the neighbourhood of the Cape of Good Hope, and constructs its nest under a sort of roof which is common to the whole colony (fig. 281).

711. It is among Insects that we find the most remarkable examples of this kind of social instinct; and the structures which are produced by the united labours of a large number, working together in harmony, are extremely interesting. The nests of Wasps are constructed in this manner. In order to

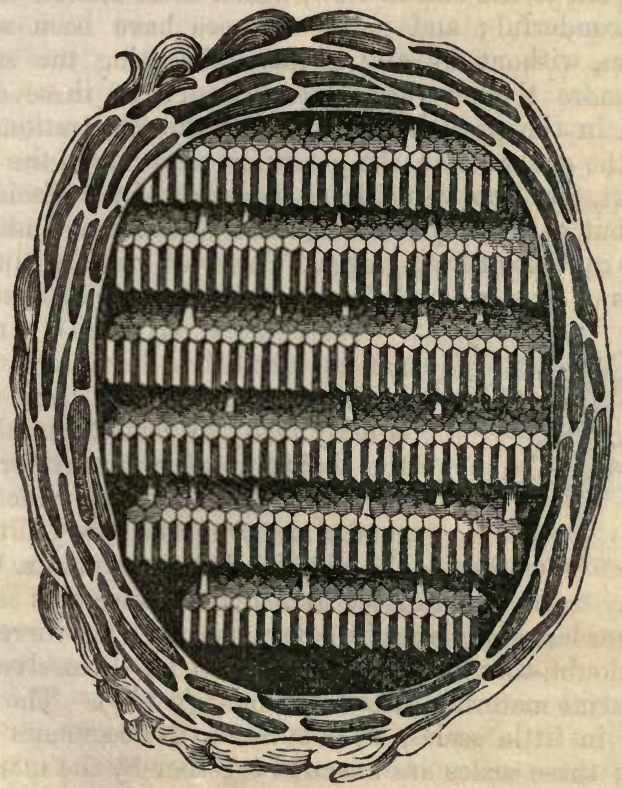

Fig. 282,-Nest of WAsP.

form the materials for building them, these Insects detach with their mandibles the fibres of old wood, which they convert by mastication into a sort of pulp that hardens into the consistence of pasteboard; of this substance they construct several ranges of hexagonal cells; and the combs thus formed are 
arranged parallel to each other at a regular distance, and are united at intervals by little columns which serve to suspend. them (fig. 272). The whole is either hung in the air, lodged in the hollow of a tree, or buried in the ground; and it is sometimes enclosed in a general envelope, sometimes left uncovered, according to the species.

712. The same community of labour is observed in the ordinary Hive-Bees, which present to the intelligent observer a source of interesting occupation that scarcely ever fails. The number and variety of instincts, each of them most perfectly adapted to the end in view, which these Insects exhibit, is must wonderful; and many volumes have been written upon them, without by any means exhausting the subject. Nothing more than a very general sketch of these can be attempted in the present treatise ; but the illustrations they afford of the general remarks heretofore made upon the nature of Instinct, are too valuable to be passed-by. Each Hive contains but a single queen; and she is the only individual ordinarily capable of laying eggs. There are usually from 6 to 800 males or drones; and from 10,000 to 30,000 neuters or

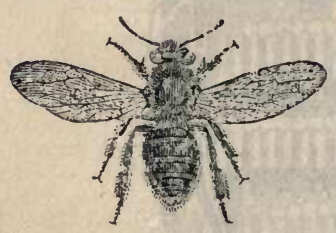

Fig. 283.-WorkiNg BeE. "working-bees" (fig. 283). In their natural condition Bees live in the hollows of trees; but they appear equally ready to avail themselves of the habitations prepared for them by Man. The cells of which their combs are composed, are built-up of the material that we term wax. Of this a part may be obtained direct from Plants, since it is secreted in greater or less abundance by several species ; but there seems to be no doubt, that Bees can elaborate it for themselves from the saccharine materials of their aliment ( $\$ 155)$. The wax is separated in little scales, from between the segments of the abdomen; these scales are kneaded-together by the mandibles of the Insect, and are then applied to the construction of the cells. It is easy to understand that the hexagonal form is that which enables the cells to be best adapted to the purposes for which they are built, whilst the least amount of material is expended. As they are intended not only to contain a store of honey, but also to serve as the residence for the larvoe (fig. 284) and pupoe (fig. 285), it is evident that their form 
must approach near to that of the cylinder, in order that there may be the greatest economy of space; but it is also evident that if their walls were circular, a large quantity of

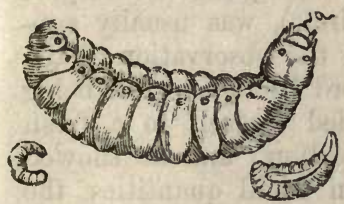

Fig. 284.-LARv正 of BEE.

(Natural size and Magnified.)

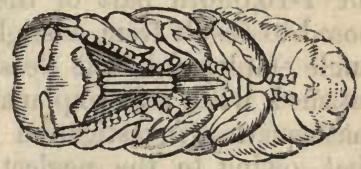

Fig. 285.-PUPA OF BEE.

(Magnified.)

material would be required to fill up the interspaces left between them; whilst, by giving the cells the hexagonal form, their walls everywhere have the same thickness, and their cavity is sufficiently well adapted to the forms of the larva and the pupa.

713. Every comb contains two sets of cells, one opening on each of its faces. The cells of one side, however, are not exactly opposite to those of the other, for the middle of each cell abuts against the point where the walls of three cells meet on the opposite side ; and thus the partition that separates the cells of the opposite sides is greatly strengthened. This partition is not flat, but consists of three planes, which meet

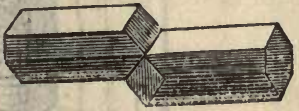

Fig. 286.-Hexagonat Cencs. (Showing the manner of their union at the Base.) each other at a particular angle, so as to make the centre of the cell its deepest part. Of the three planes which form the bottom of each cell, one forms part of the bottom of each of the three cells against which it abuts on the opposite side, as shown in the accompanying figure. Now it can be proved, by the aid of mathematical calculation of a very high order, that, in order to combine the greatest strength with the least expenditure of material, the edges of these planes should have a certain fixed inclination; and the angles formed by them were ascertained by the measurement of Maraldi to be $109^{\circ} 28^{\prime}$, and $70^{\circ} 32^{\prime}$ respectively. By the very intricate mathematical calculations of Koenig, it was determined that the angles should be $109^{\circ} 26^{\prime}$, and $70^{\circ} 34^{\prime}$, - a coincidence between the theory of the Mathematician and the practice of the Bee (untaught, save by its Creator!', which has been ever 
regarded as truly marvellous, and as affording one of the most remarkable examples of the operation of instinct. The very small discrepancy, amounting to only two minutes of a degree (or 1-10,800th part of the whole circle), was usually supposed to result from a slight error in the observation of the angle employed by the Bees ; until Lord Brougham, not being satisfied with this explanation, applied himself to a fresh mathematical investigation of the question; and he showed that, owing to the neglect of certain small quantities, the result previously obtained was erroneous to the exact amount of two minutes; so that the Bees proved to be right, and the Mathematician wrong. ${ }^{2}$

714. The ordinary cells of the comb are of two sizes; one for the larvæ of the working-bees, and the other for those of

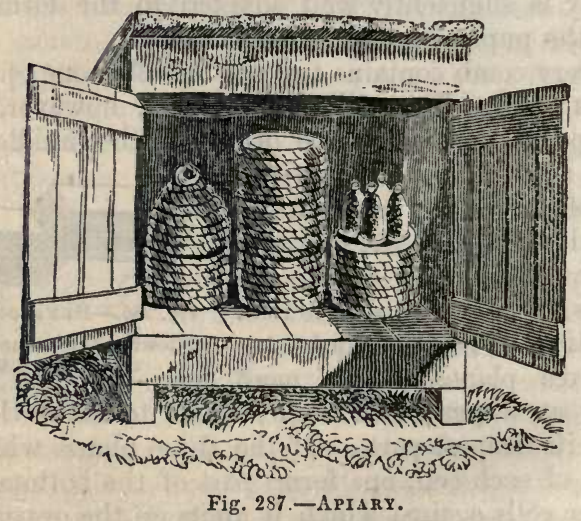

the drones. Both of these may be used for laying-up a store of food, either for themselves or their progeny; but it is ob-

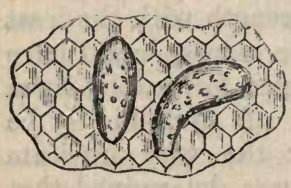

Fig. 288.-Royal Cexls. served that in the breeding season the central portion only of each comb is tenanted by the young Bees, this being the part of the hive where they will most constantly obtain the warmth requisite for their development (\$ 411). The deposition of the eggs in these cells only, therefore, is another remark-

1 See his Supplement to New Edition of Paley's Natural Theology. 
able instinct on the part of the Queen; and this is further manifested in the fact, that she never deposits eggs in the comb which fills the glasses that are sometimes placed on the top of a hive, as in fig. 287, the temperature of these glasses being necessarily lower than that of the interior of the hive.-The "royal cells," as they are termed, in which the larvæ of the young queens are reared, are different in form from the rest (fig. 288); sometimes they lie in the midst of them; but most commonly they project from the sides or edges of the comb.

715. The food which the Bees collect is of two kinds,the honey of flowers for themselves, and the pollen for their larvæ. The honey, which they suck-up by means of their proboscis-like tongues (fig. 289), seems to undergo some change

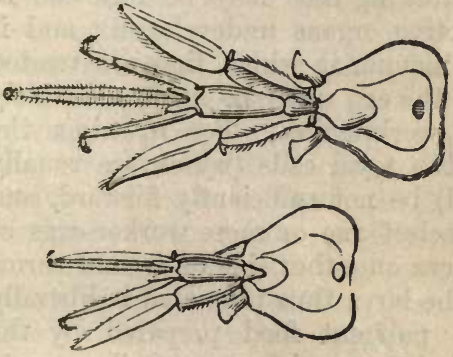

Fig. 289.-BEE's Mouth.

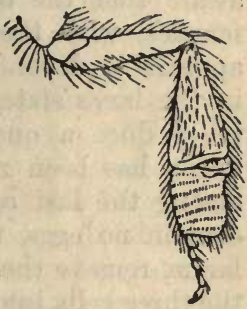

Fig. 290.

HIND LEG OF WORKER.

in their digestive cavity ; and the part not required for nourishment is afterwards returned from the stomach, and deposited in one of the cells, which, when filled, is sealed with a covering of wax. The pollen is gathered by rubbing the body either against the anthers, or against other parts of the flower over which it may have been scattered by their bursting; and when the surface of the body has been sufficiently dusted with its fine particles, these are collected from it by little brushes with which the feet of the Bee are furnished, and are worked-up into small pellets, which the Insect carries home in basket-shaped hollows, of which there is one on each hind-thigh (fig. 290). The pollen or farina thus collected is worked-up with honey in a mass, to which the name of "bee bread" has been given; and with this the larvæ are 
nourished, until the time when they are about to pass into the pupa state. The mouth of the cell is then sealed by a waxen cover; and the larva spins a delicate silken cocoon, within which it undergoes its metamorphosis. In the chrysalis state it remains quite inactive for some days; and during the latter part of this period, when it is rapidly approaching the condition of the perfect Insect, its development is aided by the heat supplied by the "nurse-bees," whose remarkable instinct has been already described ( $\$ 411)$.

716. One of the most curious features in the whole economy of Bees is the manner in which they manufacture new Queens, when from any cause (as by the intentional removal of her from the hive) their sovereign has been lost. In order to understand the process, it is necessary to be aware that the ordinary working-bees may be regarded as females, with the reproductive organs undeveloped; and it appears to depend on the manner in which they are treated in the larva state, whether the egg shall be made ultimately to produce a queen or a working-bee. For if, when the queen has been removed, the royal cells (which are usually among the last constructed) be not sufficiently forward, and contain no eggs, the bees select one or more worker-eggs or larvæ, remove the egg or larva on either side of it, and throw the three cells into one. The larva thus promoted is liberally fed with "royal jelly," a pungent food prepared by the working-bees for the exclusive nourishment of the queen larvæ; and in due time it comes forth a perfect queen. This change is doubtless owing to the peculiar effect of the food; and it is remarkable that it should operate, not only in developing the reproductive organs, but also in altering the shape of her tongue, jaws, and sting, in depriving her of the power of producing wax, and in obliterating the hollows just referred-to, which would otherwise have been formed upon her thighs.

\section{Manifestations of Intelligence.}

717. The amount of reasoning power possessed by some among the lower animals, may be considered as very much upon a par with that exhibited by an intelligent child, about the time when it is learning to speak. One of its first exercises is in the connexion or association of ideas, which is the 
source of the faculty of Memory, and thus becomes the foundation of that power of profiting by experience, which is manifested in the actions of animals that are distinguished for Intelligence. Such a power is well shown in the following instance, related to the Author by an eye-witness. A Wren built its nest in the slate-quarries at Penrhyn, in such a situation as to be liable to great disturbance from the occasional explosions. It soon, however, learned to quit its nest and fly to a little distance, on the ringing of the bell which warned the workmen. This action, having been noticed, was frequently shown to visitors, the bell being rung when there was not to be an explosion; so that the poor bird suffered many needless alarms. It seems gradually to have learned, however, that the first notion it had formed, by the association of the ringing of the bell with the explosion, was liable to exceptions, and to have formed another more correct ; for it was observed, after a time, that the wren did not leave its nest, unless the ringing of the bell was followed by the moving-away of the workmen.-A similar process of association, carried rather further, but still quite simple enough to be readily believed, is shown in two Dogs, which have been taught by their master to play at dominoes, and which go through the game with another person (under circumstances which render the idea of collusion with their master impossible) with the utmost regularity and correctness; not only playing rightly themselves, but watching to see that their adversary does so too. This, also, is a feat which a very young child might be taught to perform.-A third instance has reference to the patient endurance of bodily pain, in opposition to the instinctive tendency to struggle against the infliction of it, and evidently occasioned by a voluntary effort on the part of the animal, made by it in obedience to the dictates of its reason. Dr. Davy mentions having seen an Elephant, in India, that was suffering under a deep abscess in its back, which it was necessary to lay open in order to effect a cure. "He was kneeling down, for the convenience of the operator, not tied; his keeper was at his head. He did not flinch, but rather inclined towards the surgeon, uttering a low suppressed groan. He seemed conscious that what was doing was intended for his good; no human being could have behaved better; and so confident were the natives that he N N 2 
would behave as he did, that they never thought of tying him." It were much to be wished, that all human beings would imitate this docile Elephant's self-control. It is sometimes manifested, however, even in Infancy; the painful operation of lancing the gums being often sustained without a cry, from the consciousness of the benefit derived from it.

718. It has been stated that the relative amount of Intelligence in different animals bears a pretty constant proportion to the size and development of the Cerebral hemispheres (\$ 452). That size alone, however, does not produce the difference, is evident from a number of facts. As we advance from the lower to the higher Vertebrata, we observe an obvious advance in the complexity of the structure of the brain. In proportion to the increase in the number and depth of the convolutions by which its surface is extended ( $\$ 456)$, do we find an increase in the thickness of the layer of grey or vesicular matter ( $\$ 61)$, which seems to be the real centre of all the operations of the organ. The arrangement of the white or tubular tissue ( $\$ 60)$, which forms the interior of the mass, also increases in complexity; and as we ascend from the lower Mammalia up to Man, we trace a great increase in the number of the fibres which establish communications between different parts of the surface. Still there can be no doubt that the size of the Cerebrum, compared with that of the Spinal Cord and of the Sensory Ganglia at its summit, usually affords a tolerably correct measure of the intelligence of the animal ; and that, even in comparing together different Men, we shall find the same rule to hold good, when due allowance has been made for the comparative activity of their general functions, such as is expressed by the word temperament. Thus, two men, having brains of the same size and general conformation, may differ greatly in mental vigour, because the general system of one performs its functions much more actively and energetically than that of the other. For the same reason, a man of small brain, but whose general habit is active, may have a more powerful mind than another whose brain is much larger, yet whose system is inert, his perceptions dull, and his movements languid. But of two men alike in these respects, and having the same general configuration of head, it cannot be doubted that the one with the larger brain will surpass the other. It is a striking fact, that 
almost all those persons who have been eminent for the amount of their acquirements, or for the influence they have obtained by their talents for command over their fellow-men, have had large brains: this was the case, for example, with Newton, Cuvier, and Napoleon.

719. The size of the brain, and especially of its anterior lobes (which seem particularly connected with the higher reasoning powers), as compared with that of the face, may be estimated pretty correctly by the measurement of the facial angle; as proposed by Camper, an eminent Dutch naturalist. This is done by drawing a horizontal line ( $c d$, figs. 291 and 292), between the entrance to the ear and the floor of the nose, so as to pass in the direction of the base of the skull ; this is met by another line $(a, b)$ which passes from the most prominent part of the forehead to the front of the upper jaw. It is evident that this last will be more inclined to the former, so as to make a more acute angle with it, in pro-

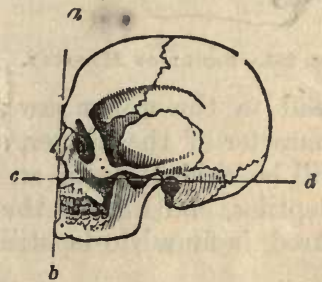

Fig. 291.-SKult of EURopeax. portion as the face is more developed and the forehead more retreating; whilst it will approach more nearly to a right angle, if the forehead be prominent, and the muzzle project but little. Hence this facial angle will indicate, with tolerable correctness, the proportion which the brain bears to the face,- - the instrument of intelligence, to the receptacle of the organs of sense.

720. Of all animals, there are none in which the facial angle is so open as in Man; and great variations exist in this respect, even among the different human races. Thus, in European heads, the angle is usually about $80^{\circ}$ (fig. 291). The ancient Greeks, in those statues of Deities and Heroes to which they wished to give the appearance of the greatest intellectual power, made it $90^{\circ}$, or even more, by the projection they gave to the forehead. On the other

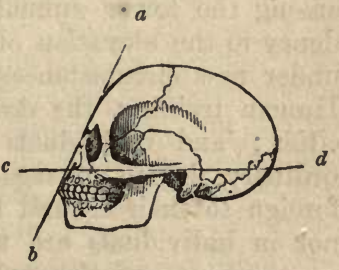

Fig. 292.-SKULL OE NEGRO. hand, in the Negro races, it is commonly about $70^{\circ}$ (fig. 292); 
in the different species of the Monkey tribe, it varies from about $65^{\circ}$ to $30^{\circ}$ (fig. 293) ; and as we descend still lower, we find it still more acute. In the Horse and Boar, for example, it becomes impossible to draw a straight line from the fore-

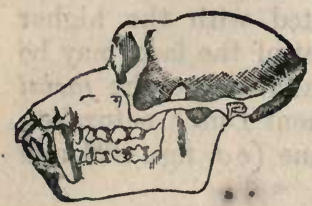

Fig. 293.-SkUlL of Macacus.

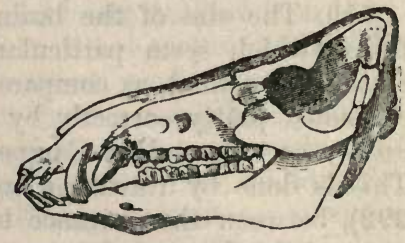

Fig. 294. -SKULL of BOAR.

head to the upper jaw; in consequence of the retreating character of the former, and the projection of the nose ; this will be evident from an examination of fig. 294. In Birds, Reptiles, and Fishes, the facial angle, when it can be measured, is found to be still further diminished.

721. It appears, then, that the mind of Man differs from that of the lower animals, rather as to the degree in which the reasoning faculties are developed in him, than by anything peculiar in their kind. Among the more sagacious Quadrupeds, it is easy to discover instances of reasoning as close and prolonged as that which usually takes place in early childhood; and it is only with the advance of age and the maturity of the powers, that the superiority of Man becomes evident. The foundation of this superiority lies in the power of self-direction and self-improvement which Man possesses. No race among the lower animals ever exhibits a spontaneous tendency to the elevation of its mental powers. When placed under new circumstances, and especially when subjected to Human training, the domesticable races acquire new capacities; and individuals frequently display a very extraordinary degree of sagacious appreciation of matters quite foreign to their natural habits of life. But neither in races nor in individuals are these powers transmitted from one generation to another, when, left to themselves, they return to anything like a state of nature. In Man, on the other hand, the power which every rightly-constituted and rightly- 
trained individual possesses ( $\$ 525$ ) of fixing his attention upon any particular object of consciousness, to the exclusion of all others, becomes the source of the highest and most enduring intellectual advancement, and of all moral improvement. It is in virtue of this power, that he is not only enabled to profit largely by the acquired knowledge of others, but that he comes to possess a moral responsibility for the use he makes of his faculties, which cannot be predicated of beings whose succession of ideas is entirely determined by impressions made from without.

722. There is another attribute, moreover, by which Man seems to be distinguished from all other animals ; namely, that disposition to believe in the existence of an unseen but powerful Being, which seems never to be wanting (under some form or other) in any race or nation, although (like other natural tendencies) it may be defective in individuals. It requires a higher mental cultivation than is commonly to be met with among savage races, to conceive of this Power as having a spiritual existence ; but it appears, from the reports of Missionaries who have laboured to spread Christianity amongst the Heathen, that an aptitude or readiness to receive this idea is rarely wanting; so that the faculty is obviously present, though it has not been called into operation.-Closely connected with this tendency to believe in a Great unseen Power, is the desire to share in His spiritual existence, which seems to have been implanted by the Creator in the mind of Man, and the existence of which is one of the chief natural arguments for the immortality of the soul, since it could. scarcely be supposed that such a desire would have been implanted, if it were not in some way to be gratified. Such views tend to show us the true nobility of Man's rational and moral nature, and the mode in which he may most effectually fulfil the ends for which his Creator designed him. We learn from them the evil of yielding to those merely animal tendencies,-those "fleshly lusts which war against the soul," - that are characteristic of beings far below him. in the scale of existence, and tend to degrade him to their level ; and the dignity of those pursuits, which, by exercising his intellect, and by expanding and strengthening the higher part of his moral nature, tend to raise him towards the perfection of the Divine Being. 


\section{CHAPTER XV.}

\section{OF REPRODUCTION.}

723. There is no one of the functions of living beings, that distinguishes them in a more striking and evident manner from the inert bodies which surround them, than the process of Reproduction. . By this function, each race of Plants and Animals is perpetuated; whilst the individuals composing it successively disappear from the face of the earth, by that death and decay which is the common lot of all.-A very unnecessary degree of mystery has been spread around this process. It has been regarded as one altogether inscrutable, whose real nature could not be unveiled, even by the scientific inquirer, and whose secrets the uninitiated should never seek to comprehend. But so much light has been thrown upon it by recent investigations, that we now know at least as much of this, as of almost any other function; and the Author's experience has led him to believe that such knowledge may be communicated to the general reader, without the least infringement of the purest delicacy of feeling. In his own judgment, indeed, it is far better to afford a legitimate satisfaction to the curiosity which naturally exists upon the subject, than, by refusing all information, to drive the inquirer into objectionable methods of gratifying it.

724. It has been elsewhere shown (VEget. Phys., Chaps. IX., XII.), that, in the Vegetable Kingdom, there are two distinct modes by which the propagation of Plants may take place; - the extension of the parent structure into new portions, which, being independent of it and of each other, can maintain their lives when separated from it;-and the origination of a new being by the concurrent action of two sets of cells set apart for this special function, and designated "sperm-cells" and "germ-cells." The bodies of the first class are known as leaf-buds or gemmoe in the Flowering Plants, and sometimes also among Cryptogamia, some of which last, as the Marchantia (VEgEt. Phys. § 757), are furnished with a peculiar means of producing them; and it appears from recent investigations that the "spores " of Ferns, 
Mosses, and Hepaticæ, as well also the "zoospores" of Algæ, belong to the same class of reproductive bodies. The genmæ of Phanerogamia may be developed in connexion with the parent structure, and may continue to form a part of it ; or they may be removed from it (as in the processes of budding, grafting, \&c.), and may be developed into new individuals.On the other hand, the bodies of the second class are known as seeds among Flowering Plants; among the Cryptogamia they present a variety of forms. From the very first, these are destined to produce new individuals ; and although they are often assisted in the early stage of their development by the parent, they are its true offspring, rather than (like gemmæ) extensions of itself. Both these modes of Reproduction, namely, gemmation and sexual generation, exist in the Animal Kingdom; but the former is confined to its lower tribes, among which we often find it exercised in very remarkable modes.

\section{Gemmiparous or Non-Sexual Reproduction.}

725. Among Infusoria ( $\$ 133$ ) we find the process of gemmation, or of fission, which is a modification of it, to be almost the only ostensible means of propagation which the beings composing that wonderful group possess. The former may be continually witnessed by the microscopic observer in the common Vorticella, a bell-shaped-animalcule attached by a stalk

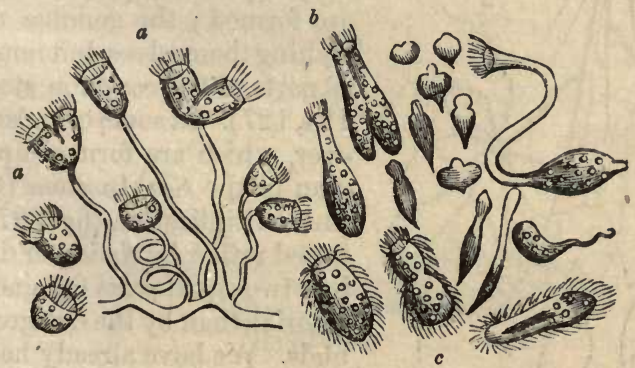

Fig. 295.-VARious Forms of ANivaxcules, some of them undergoing spontaneous fission.

(fig. 295, a, a), and abundant in almost every pool in which aquatic vegetables grow, especially clustering around the stems of Duckweed; and its various stages closely resemble those 
which have been already described ( $\$ 122)$ in the Hydra. But not unfrequently in this species, and ordinarily in many others, the body divides into two equal parts, in each of which we see a mouth and other parts resembling those of the original. This division is gradual. A narrowing of the body along or across its middle (for the fission or cleavage sometimes takes place lengthways, as at $b$, sometimes transversely, as at $c$ ), is first seen; the indentation at the edge becomes gradually deeper, and at last the two parts hold together by but a narrow band, which finally breaks, and they become free.-The same method of multiplication is observed among the simple Rhizopoda (\$ 129); but when the gemmæ remain connected with each other, as in Zoophytes, we have such composite fabrics as are presented to us in the classes of Foraminifera (\$131) and Sponges (\$136).

726. Reproduction by Gemmation is most characteristically seen among the Radiated classes; and in none better than in the Hydra already so frequently referred-to. Although this interesting little animal sometimes reproduces itself by true sexual generation ( $\S 734)$, yet its usual mode of propagation is by buds (\$122), as shown on the left hand side of the accompanying figure (fig. 296). And, as already explained, it

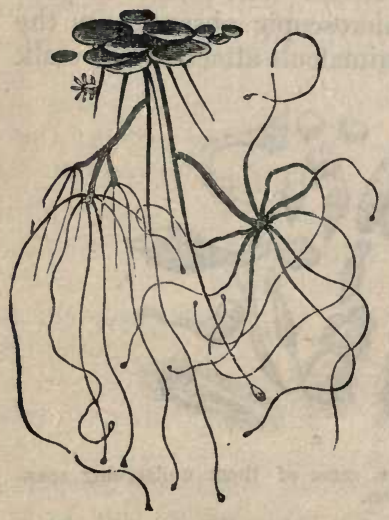

Fig. 296.-HYDR weed): one of them developing buds. is by this same process of gemmation that the arborescent structures of the Composite Zoophytes are formed; the gemmæ not detaching themselves, but remaining as parts of the common stock ( $\$ \S$ $124,127)$. In some of those, however, which are formed upon the plan of the Sea Anemone (\$ 126), the multiplication (fig. 297) is effected rather by fission or division into two equal parts (as among Infusoria), than by the out-growth of buds. We have already had occasion to notice $(\$ 125)$ the very remarkable form of gemmation that takes place among Zoophytes, giving origin to independent beings which seem to belong to a class altogether different, but which 
are in reality the representatives of the flower-buds of Plants, distinguished by their capability, not only of living and enduring, but of obtaining their own nutriment, after their spontaneous detachment from the stock that bore them. Among the Medusae we occasionally meet with instances of propagation by buds that resemble the stock from which they proceed, and that are thrown off in due time so as to lead independent lives; but this kind of gemmation seems limited to the lower members of the group. In a large proportion of it, however, a very extraordinary kind of multiplication by gemmation takes place at an early period of development ( $(740)$. - - In the highest Radiata, the class of

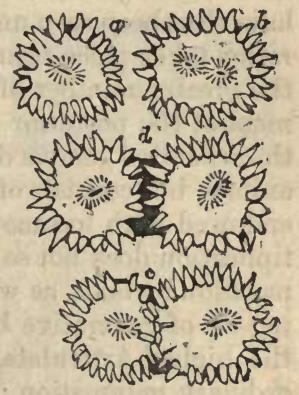

Fig. 297.-PoLYPES of Astrea,

Undergoing fission; $a, b, c$, $d$, successive stages.

Echinodermata, we take leare of multiplication by gemmation altogether; for although the bodies of these creatures possess a very extraordinary reproductive power, so that the result of very severe injuries may be repaired ( $\$ 389)$, we do not find that they either spontaneously produce independent buds, or that they have the capacity for being multiplied by artificial division.

727. Among several of the lower Articulata, detached segments of the body appear to be capable of reproducing the whole; and there are some whose ordinary propagation is

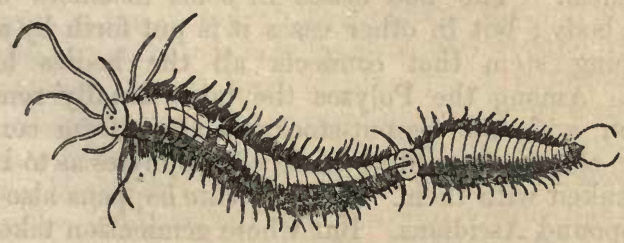

Fig. 298.-Nereis Prolifera.

accomplished by an exercise of this power. Thus in the Nais, an aquatic worm allied to the Earth-worm, the last joint of the body gradually extends and increases to the size of the rest of the animal; and a separation is made by a narrowing of the 
preceding joint, which at last divides. Previously to its separation, however, the young one often shoots out another from its own last joint, in a similar manner ; and three successions have thus been seen united. In some species of Nereis, the separation takes place nearer the middle of the body (fig. 298). In the greater number of cases, however, in which such a detachment of the posterior part of the body of Annelids takes place, the separated gemma does not contain the structure of the entire animal, but consists of little else than the generative apparatus, endowed with locomotive organs; so that this process of multiplication does not so much correspond with the ordinary propagation by buds, as with the peculiar development and throwing-off of generative buds to be presently described.-Among the higher Articulata, we do not meet with any instances of ordinary gemmation; but the non-sexual production, which is now known to take place not only in the Aphides ( $\$ 746$ ) but in many other Insects, as well as in Rotifera (WheelAnimalcules) in Entomostracous Crustacea (Water Fleas, \&c.), and probably in some higher Crustacea, must be regarded as a peculiar form of the same process ; the offspring being produced from eggs, which have the power of self-development without sexual fertilization, and which must therefore be accounted internal gemmoe.

728. In the Molluscous series, the power of multiplying by gemmation appears to be limited to the Tunicata (\$114) and the Polyzoa ( $\$ 115$ ); being restricted in the first of these classes to a section of the group; whilst in the second, which closely follows the habit of Zoophytes, it seems to be universal. The bud arises in some instances directly from the body; but in other cases it is put forth by a stolon or creeping stem that connects all the bodies together (fig. 63). Among the Polyzoa the buds usually remain in connexion with the parent-stock, so as to form composite fabrics so closely resembling those of Zoophytes as to be commonly ranked with them. And the like happens also among the Compound Ascidians. But where gemmation takes place among the solitary Tunicata, the bud becomes detached, and maintains a perfectly independent existence. There is a very curious case of internal gemmation among the Salpoe (a tribe of Tunicata which are not attached, but float over the waves); for the buds are developed, not from the exterior of the 
body, but from a kind of stolon within it; and they differ from their parent-stock in having organs of attachment to each other, whereby they hold together in long

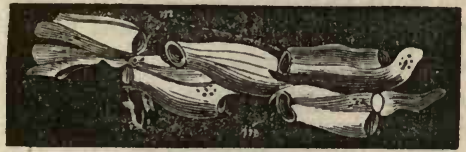

Fig. 299.-Aggregate SALPE.

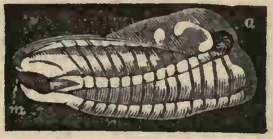

Fig. 300.-SOLITARY SALPA.

chains (fig. 299). These, in their turn, being furnished with true generative organs, give origin to the solitary Salpx (fig. 300 ) by ordinary sexual reproduction; whilst the solitary Salpa never comes to possess any sexual apparatus, but merely continues the race by gemmation from its internal stolon.

729. In the Vertebrated classes, as in the higher Mollusca, we lose all trace of propagation by gemmation as an ordinary method of multiplication. Yet there is evidence that this power is not altogether extinguished, even in Man. For we not unfrequently hear of "monstrosities by excess," that is, of cases in which the body possesses a superfluity of some of its parts ; the simplest cases being those of double thumbs or of six fingers on each hand, and the gradation being so continuous from these to cases like that of the Siamese twins (in which there are two complete bodies united only by a cross band), as to make it evident that they are all referable to one common principle. And although it has been commonly believed that monsters with two heads and one body, or with two bodies and one head, or with supernumerary legs or arms, are results of the partial "fusion" of two distinct germs at an early period, yet there is now far stronger reason to believe that they proceed from a kind of attempt at multiplication by fission or gemmation, that is sometimes made by a single germ at a time when its grade of development corresponds with that of a Hydra or Planaria; which attempt, under peculiarly favourable circumstances, may proceed to the full length of production of two complete bodies. (See § 390.)

\section{Sexual Reproduction, or Generation.}

730. We now have to notice the most important features of the proper Generative process, which differs from the 
preceding in exactly the same manner as the flowering and fruiting of Plants differ from their extension and propagation by leaf-buds.- In all save the very lowest tribes of Animals, we meet at particular seasons with two peculiar sets of cells, termed sperm-cells and germ-cells; these are sometimes borme by the same individuals, which then correspond as regards their reproductive apparatus with the generality of Flowering Plants ; but they are more commonly separated, as in dioecious Plants (Veget. Physiol., § 409); the individual bearing the "sperm-cells" being then designated as the male, and the individual bearing the "germ-cells" as the female.

731. The "sperm-cells" very closely resemble those contained within the antheridia of Cryptogamia (VEGET. PHysior. $\S 399$; Botany, $\$ \$ 737,776)$. When mature, each cell is

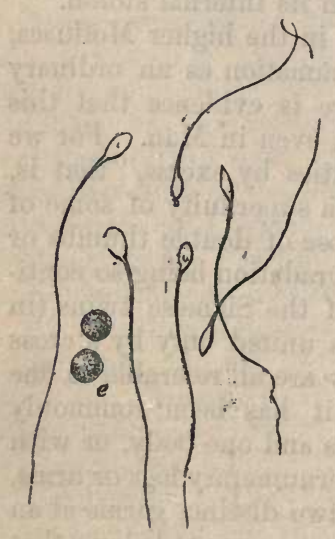

Fig. 301.-Spermatozoids : $e$, immature sperm-cells. found to contain one or more spirally coiled filaments (fig. 301), which, when set free by the bursting of the cell, have an active spontaneous movement lasting for some time like ciliary action. These filaments were formerly regarded as true Animalcules; but since other examples of independent movement have been discovered in what are certainly nothing else than detached parts of the organism, and more especially since moving filaments of a precisely analogous character have been discovered in Plants, all idea of their independent animality has been laid aside, and they are now known as spermatozoids. - The use of their motor activity is obviously to bring them into contact with the germ-cells, when both have been set free from the interior of the bodies within which they were formed. When the sperm-cells are developed in a special or distinct organ, as happens in all save the lowest types of Animal structure, this organ usually more or less resembles the ordinary glands in structure ( $\$ \$ 356,357)$, and is termed the Testis.

732. The "germ-cells" are not so clearly distinguished 
from other cells by the nature of their contents, though they are usually recognisable by the peculiar nuclei they present; each cell is known as the germinal vesicle (fig. $302, d$ ), whilst its nucleus $(e)$ is designated the germinal spot. - The act of fertilization appears to consist in the contact of one or more spermatozoids with the exterior of the germinal vesicle; the spermatozoids, ceasing to move, undergo a sort of liquefaction ; and the product of their dissolution, being received by absorption into the interior of the germinal vesicle, mingles with its contents, to form with them the basis of the new structure.When, as usually happens, the germ-cells are developed in a

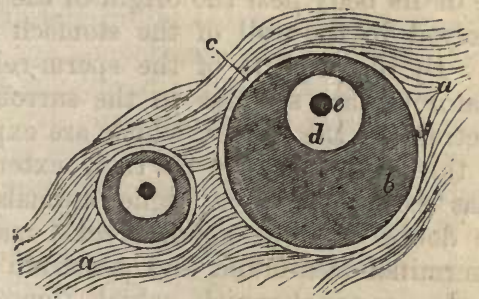

Fig. 302.-SEction OF OVARIUM OF FowL:

$a$, fibrous substance of the ovary; $b$, yolk; $c$, yolk-bag; $d$, germinal vesicle; $e$, germinal spot.

special and distinct organ, this organ, which is termed the Ovary, has very commonly among the lower animals a glandular character, the mature ova being discharged by the oviduct, just as the products of secretion pass-off through the ducts of their respective glands: but among the Vertebrata the ovary has a much more solid texture, and the germ-cells, developed in the very midst of its fibrous tissue (fig. 302, a), have to find their way to its surface, and to burst forth from it; being then received into an oviduct, whose trumpet-shaped mouth embraces the ovary, so as to prevent the liberated germs from falling (as they would otherwise do) into the visceral cavity of the body.

733. With the "germ-cell" there is always associated in Animals, as in Flowering Plants, a store of nutriment that serves for the early development of the germ; this consists of a mixture of albuminous and oily matter, known as the yolk (fig. 302,b); and it is inclosed in a membranous envelope $c$, 
termed the yolk-bag. The yolk-bag and its contents, namely the yolk and the germinal vesicle, constitute the ovum. The eggs of many animals (as of Birds) contain an additional store of liquid albumen, the "white," enveloping the yolk-bag and destined to be gradually drawn into it, so as to replace the albumen of the yolk as it is progressively used-up in the development of the embryo ; and the "shell" is a subsequent formation around this $(\$ 755)$.

734. The Hydra presents us with a very apposite illustration of the simplest mode in which the generative function is performed. Sperm-cells are developed at certain periods in the substance of its body near the origin of the arms; whilst ovules are evolved in the wall of the stomach nearer to the foot or base. By the rupture of the sperm-cells, their contained spermatozoids are set free in the surrounding water, and they penetrate to the ovules, which are exposed to their influence by the thinning-away of their exterior covering. From what has been observed in higher animals, there seems no reasonable doubt that the spermatozoids make their way through the germinal membrane, and penetrate into absolute contact with the germinal vesicle, which then lies near the surface of the ovule. What is the precise change effected by fertilization, has not yet been fully ascertained; the germinal vesicle, however, disappears; and it would seem as if its contents, with the product of the liquefaction of the spermatozoids, were diffused through the yolk, which soon begins to undergo changes of a very remarkable nature.

735. Before going further, it may be well to notice the remarkable antagonism which exists between the processes of Gemmation and Generation, as regards the conditions by which they are respectively favoured. For we see that in the Hydra, as in Plants, the extension of the body into buds is promoted by warmth and a copious supply of food; so that, as it would appear, if these be afforded, this mode of multiplication may be protracted indefinitely. On the other hand, if the supply of food be limited and the temperature lowered, the production of buds ceases, and the formation of sperm-cells and of germ-cells begins. The result is that ova or eggs are produced, which have a very firm horny covering, and possess a great power of resistance to cold; and thus provision is made for the continuance of the race through a winter tem- 
perature that might be fatal to the Hydræ themselves. The same thing is observable among the Rotifera; for, as has long been known, two kinds of eggs are produced by them, the ordinary and the "winter eggs ;" and it now appears that the ordinary eggs, being evolved without any generative process, and with a rapidity proportional to the favouring influences of food and warmth, are really to be regarded as internal gemmæ; whilst the "winter eggs," which are produced in the autumn by the concurrent action of males and females, and have a peculiarly dense horny investment, are the only true ova. Among the Aphides ( $\$ 746)$, again, it has been experimentally shown that the non-sexual multiplication may be indefinitely protracted by warmth and food; whilst a reduction in the temperature and in the supply of nutriment causes this at any time to give place to sexual generation.

736. The first obvious change that presents itself in the Ovum, after its fertilization, is the "segmentation," or division of the yolk-mass into two halves, by the formation of a sort of hour-glass contraction, which gradually deepens, until it produces a complete separation. Another segmentation of these two halves soon follows in the opposite direction, so that the yolk-mass becomes divided into four segments ; each of these in its turn undergoes the like subdivision; and this duplicating process is repeated, forming successively 8,16 , $32,64, \& c .$, segments, until a "mulberry-mass" is produced, which is composed of an aggregation of an immense number of minute yolk-spherules. Up to this stage, the developmental process takes place on essentially the same plan in all animals, save that in some the process of segmentation does not extend to the entire mass of the yolk, but only to a small proportion of it, which is distinguished as the "germ-yolk," whilst the remainder, which is applied to the nourishment of the more advanced embryo through an entirely different channel, is known as the "food-yolk" (\$ 754).

737. It appears, among some of the simplest Worms, as if the "mulberry-mass" gradually shaped itself into the body of the animal, without the intervention of any intermediate structure ; but in almost all Animals, the first stages in development tend to the production of a membranous expansion that may be likened to the "cotyledon " of Flowering Plants,with this important difference, however, that whilst the latter 00 
spreads itself out so as to come into contact with the "albumen" of the seed by its external surface, the "germinal membrane" of the Animal forms itself around the yolk, and thus constitutes as it were a temporary stomach, within which the nutrient material is stored-up, and through the walls of which it is drawn into the embryo. This is accomplished in the following manner. The spherules of the outer layer of the mulberry-mass which are in immediate contact with the yolk-bag become invested with walls of their own, and thus become converted into proper cells; these are somewhat flattened and of a polygonal shape, very much resembling those of the epithelium of serous membrane (fig. 10). Another layer is afterwards formed within this, the cells of which retain more of their original globular form. But the spherules of the internal portion of the mulberry-mass, instead of becoming converted into cells, undergo dissolution and return to the condition of a liquid yolk; so that the ovum, in this stage of its development, consists of two layers of cells, constituting what are known as the "serous" and the "mucous" layers of the germinal membrane, enclosing a mass of nutritious matter on which a change has been worked that seems to predispose it to become organized.

738. The development of the Polypes seems to advance but little beyond this point. The covering of the ovum bursts, and the contained embryo is set at liberty as soon as the germinal membrane has been formed around the yolk. In this state it becomes clothed with cilia, and is termed a gemmule; and it-swims about freely in water for some time. Its form gradually becomes more elongated (fig. 303),

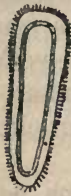
tapering away at one end, which attaches itself after a time to some solid body; and its development into the polype-form soon commences. In the group of which the Hydra is an example, this change takes place in the following simple manner. The germinal membrane gradually thins away at the point furthest Fig. 303. removed from the attached base, and at last an aperture is formed, which becomes the mouth; from around this aperture the tentacula or arms shoot forth, a single row being first formed, and others being afterwards added in those species in which they are numerous. Thus the two layers of the germinal membrane enter into the permanent structure of 
the animal ; the outer one constituting the external integument, and the inner becoming the lining of the stomach. The arborescent fabric of the composite Hydrozoa ( $\$ 124)$ is gradually evolved by continuous gemmation from the original Polype; and whilst in some of them the sperm-cells and ova are developed within peculiar capsules not ostensibly differing (except in size) from the ordinary polype-cells, there are others in which they are the product of peculiar buds having the form and structure of Medusa, which buds in many instances become detached, and henceforth live as independent zooids, their sexual apparatus being only evolved after they have separated themselves from the parent stock. The sperm-cells and the ova are developed within different Medusa-buds ; but both kinds of buds may (in many cases at least) be put forth from the same Polype-stock, as in moncecious Flowering-Plants.

739. Although the two layers of the germinal membrane remain united in the Hydra and other Zoophytes formed upon its simple plan, they separate from each other at certain points in the Sea Anemone and its allies, so that a series of chambers is formed between then ; and these chambers are afterwards set apart for the production of sperm-cells and germ-cells (\$126). We do not meet in this group of Anthozoa with any example of that detachment of the sexual apparatus in the form of separate zooids, which is so remarkable a feature of the Hydrozoa.

740. The development of the Medusce, as elucidated by recent discoveries, presents several features of extraordinary interest. The sexes are distinct in these animals; spermcells being developed in some individuals, and ova in others, within the four chambers that surround the stomach ( $\$ 120)$. When the ova have received the fertilizing influence, their first products are ciliated gemmules resembling those of Hydraform Polypes (fig. 304, a). These, after moving about for some time in the ovarial chambers of their parent, make their exit by the orifices of these, and then swim freely through the water. Gradually, however, they undergo the usual elongation, and fix themselves by one extremity $(e)$; at the opposite extremity a depression appears in the middle, which is to become the mouth, as seen at $b$, and an elongation of the four corners $(c, f)$ gives origin to the first tentacula, 002 
which are afterwards increased by the addition of many others (g). In this manner a true polype is formed, which leads the life of a Hydra, and, like it, propagates its kind by the formation of polype-buds, which detach themselves and lead independent lives ; and thus from a single Medusan egg there may arise a whole colony of polypes multiplied by gemmation. These differ entirely from Hydræ, however, in regard to their sexual apparatus, which is detached (as in the composite Hydrozoa) under an entirely different form, that of a Medusa. The body of

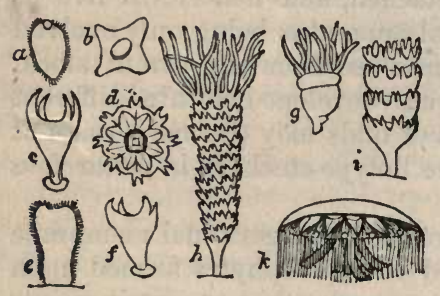

Fig. 304.-DEVELOPMENT OF MEDUSE. the polype undergoes a great lengthening, and seems as if divided by transverse bands, which gradually deepen, so as to make the whole body almost resemble a pile of saucers with divided edges $(h)$; for beneath the lowest of these saucer-like disks, a new set of polype-arms makes its appearance; and after the detachment of the whole pile of disks, the polype-body remains at their base, and may continue to lead its former life, and to propagate itself in the polype-form. The disks progressively enlarge, those at the summit of the pile increasing most rapidly, and then detaching themselves from the pile $(i)$; when thus detached, they swim about freely in the water after the manner of the smaller and simpler Medusæ, to which they closely correspond in form $(d)$; and they gradually enlarge and acquire the structure of their original parents $(k)$. It is not correct to represent (which is commonly done) the pile of Medusa-disks as being formed" by the subdivision of the polype-body. The Medusa-disks are in reality sexual buds, resembling those of the composite Hydrozoa (§ 125); and the only essential difference between the two cases lies in the fact, that among the latter it is the Zoophytic form which ostensibly constitutes the animal (the Medusan buds being thrown-off only at certain times for a special purpose), whilst the former are only known (save to such as search-out the history of their polypoid development) in the Medusan stage of their lives.

741. The recent researches of Professor Müller and others 
have brought to light a most remarkable set of facts in regard to the developmental history of the Echinodermata. The details of this history vary so greatly in the different sections of the group, that all which can be here attempted is a general notice of its most important features. The embryonic mass of cells, which is produced in the ordinary way from the egg, is usually converted, not (as in Insects) into a larva which is subsequently to attain the perfect form by a process of metamorphosis, but into a peculiar being, destined to a merely temporary existence, whose function seems to be to give origin to the real Echinoderm by internal gemmation, to obtain and prepare for it the materials of its development, and to carry it to a distance from its fellows, so as to prevent the spots inhabited by the several species from being overcrowded by the accumulation of their progeny. These larval zooids present many points of resemblance to the larvæ of certain Annelids; their bodies have a bilateral not a radial symmetry, the two sides being exactly alike; each side

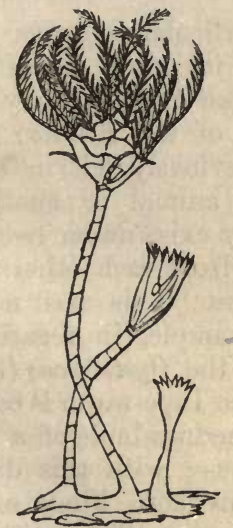

Fig. 305.-Pentacrisoid Larva of Comatula.

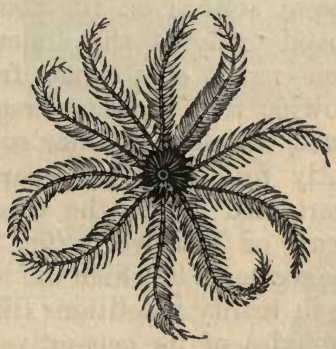

Fig. 306.-Coмntura.

is furnished with a ciliated fringe along the whole or the greater part of its length; and the two fringes are united by an upper and a lower transverse ciliated band, between which the mouth of the zooid is situated. Although the adult Star-fish and Sand-stars have neither intestinal tube nor 
anal orifice, their larval zooids, like those of other Echinoderms, always possess both. It is from the side of the intestinal canal, that the young Echinoderm is usually budded-off. In some instances it separates itself completely from the zooid, when it has attained a certain stage of development, no part of the latter entering into its composition; this seems to be the case in the Comatula, which present this further remarkable feature, that the young Echinoderm at first attaches itself to some fixed object by a footstalk (fig. 305), so as to resemble the fossil Encrinites in every essential particular, but afterwards becomes detached, and henceforth remains free (fig. 306). In the Starfish and Echinus, the only part of the larval zooid which is retained in the Echinoderm, is a portion that is (as it were) pinched-off from the stomach and intestines. In the Holothuria (fig. 67), on the other hand, which has a much closer confurmity to the type of the Annelids, a much larger part of the larva is retained in the adult, and the process of development more nearly resembles an ordinary metamorphosis.

742. Passing-on now to the Articulated series, we find that the developmental history of its lower forms presents phenomena not less remarkable than those already noticed. Recent studies on the propagation of the Entozoa have removed many of the difficulties previously felt-in regard to their mode of passage from one animal to another; by showing that the same creature may exist under two or more forms, which may differ so greatly from each other as apparently to belong to separate orders. This has now been ascertained to be the case, for example, in regard to the Toenia or Tape-worm (fig. 53) and the Cysticercus (fig. 307). The segments of which the body of the Tape-worm is composed, are in reality repetitions (like the medusa-buds of a Hydroid zoophyte) of its generative apparatus; with this difference, however, that each segment contains both kinds of sexual organs, so that the eggs it contains are fertilized without any extraneous assistance. These segments, when mature, detach themselves one by one ; and being voided from the intestine, fall to the ground, over which the eggs they contain become disseminated by various agencies. Being swallowed with the herbage or the water ingested by herbivorous animals, the eggs are conveyed into their stomachs, where the little 
embryos escape from them. These embryos, which are small vesicles furnished with six minute hooks or spines, make their way through the walls of the stomach into the substance of other viscera ; and by getting into the current of the circulation, they are sometimes carried to remote parts of the body. Nourished by the juices which it absorbs, the vesicle swells: and a head resembling that of the Tape-worm (fig. $307, a$ ), begins to bud from its wall into its cavity. In this condition the product of the egg of the Tape-worm has long been known as the Cysticercus (its presence in large numbers in the flesh of the Pig giving to it that diseased appearance which is known as "measly"), without its relationship to its parent being in the least suspected; and it undergoes no further change until the flesh of the animal it inhabits is devoured by some other, so that the Cysticercus is conveyed into the intestinal canal of the latter. The head which was previously turned into the vesicle, now protrudes from its exterior (fig. 307), and attaches itself to the intestine of its new host by means of the hooks and suckers with which it is furnished $(a)$; the vesicle is then cast off, and its place is taken by the series of generative segments successively budded-forth from the head, which constitutes the body of a new Tape-worm; and from the ova which these produce there springs a new generation, which

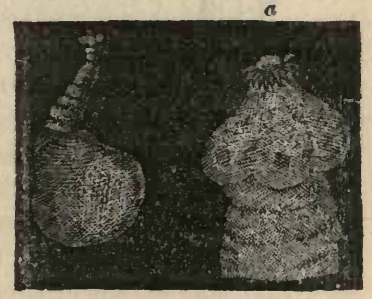

Fig. 307.-Crstrcercus; $a$, head greatly enlarged.

repeats the same curious cycle.-Numerous other parasites present a history that resembles the preceding in all its essential features, whilst varying in details (see Zoology, §§925, 926).

743. The Trematode Entozoa, of which the Distoma (known under the name of fluke) that infests the livers of Sheep is a characteristic example, undergo a yet longer succession of changes ; these have been especially studied in a species which infests the Lymnceus, one of the Water-snails, and which is represented in fig. 308. From the egg

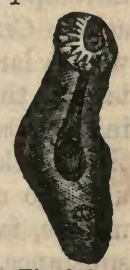

Fig. 308. DistoMA OF LYM X XOUS. deposited by this Distoma is produced a long flat-bodied embryo 
(fig. 309) having a sac-like body clothed with cilia and capable of motion ; within the anterior part of which is to be observed an elongated stomach $s$, whilst the posterior portion of the cavity is occupied by a number of bodies $a$, having a general resemblance to itself, which are produced from it by a process of internal gemmation. These, when mature, burst forth from the containing cyst, and develope themselves into worms (fig. 310) not very unlike the preceding in general form and organization, though differing from them in some important particulars; and in their turn they produce, by internal gemmation, a fresh brood of bodies much more dissimilar to themselves. These, when set free in due time,

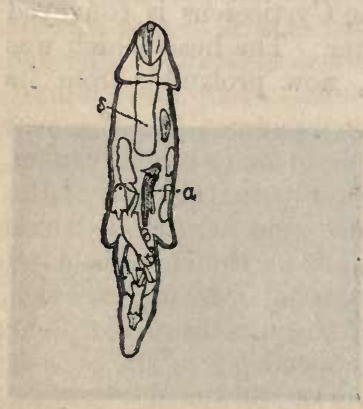

Fig. 309.

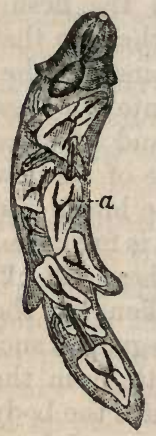

Fig. 310.

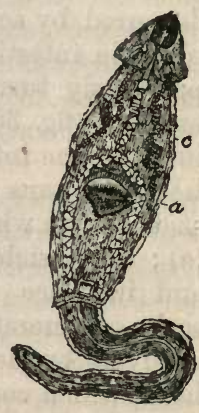

Fig. 311.

Grand-Nurse of Cercaria.

Nurse of Cercaria.

develope themselves into a form which has long been known as the Cercaria (fig. 311), and which has a tadpole-like body with a large sucker $a$ in its middle, a triangular head, a long tail by the motion of which it swims, and various viscera $c$ in its interior. The Cercariæ attach themselves by means of their sucker to the bodies of the Lymnæus, and then begin to undergo an important metamorphosis. The tail, being now useless, falls off ; the animal becomes invested with a mucous substance, within which it lies encysted like a chrysalis within its cocoon; and, on its emergence from this it presents itself as a Distoma, which is ready by the performanee of the true generative process, to set in renewed operation the whole 
succession of these changes.-Thus between every act of generation there intervene two sets of gemmations, by which a single embryo may produce a multitude of Cercariæ; and the conversion of the Cercaria into the Distoma involves, in addition, a metamorphosis not less complete than that of Insects. The body (fig. 310) within which the Cercariæ are developed, and which is the second remove from the Distoma, has been called their "nurse ;" and that (fig. 309) from which the nurses themselves are developed, and which is the first remove from the Distoma, has been called their "grandnurse."

744. Among the Annelida, or Worms properly so called, there is considerable variety in the history of development; some of them, as the Leech and Earth-worm, coming forth from the egg in a nearly perfect state; whilst in most of the marine worms that state is not attained until long after the embryo has begun to lead an independent life. This embryo, on its first emersion from the egg, very commonly has an oval or roundish body, furnished with one or more bands of cilia, by the agency of which it swims freely in the water; the body then gradually becomes elongated, and additional bands of cilia make their appearance; and after a time a mouth and intestinal canal are formed, indications of eyes and of a segmental division show themselves, and the cilia disappear, their place being usually taken by bristly appendages. The body is gradually elongated by the production of additional segments, sometimes to the number of several hundred; each new segment being formed between those which were previously the last and the last but one. Thus the formation of the body of the Worm is really accomplished by a process of continuous gemmation; and it is therefore the less surprising that some of these worms should be capable of producing independent buds ( $\$ 727$ ), which buds, however, not unfrequently resemble the segments of the Tape-worm, in containing nothing else than the generative apparatus, save locomotive organs for the purpose of dispersing its products.

745. It was in the class of INSECTs that the phenomena of metamorphosis were first studied; and notwithstanding the familiarity of the leading facts of the case, it is desirable to recapitulate them here, for the sake of showing their relationship to those we have been already considering. There are 
some Insects (such as the Grasshopper and the Cricket) which come-forth from the egg in a form so nearly resembling that which they are ultimately to present, that the deficiency of wings is their principal difference. Such are said to undergo an incomplete metamorphosis; the fact being, however, not that these finally attain a less elevated condition than other Insects, but that they make a much nearer approach to it in that part of their embryonic state which they pass within the egg. In the tribes of Beetles, Butterflies, Bees, and Flies, on the other hand, the embryo comes-forth from the egg in the condition of a Worm; and only acquires either the form or structure of an Insect after a complete netamorphosis, in which every part of its organization undergoes important modifications. The larva, sometimes known as a "maggot," sometimes as a "caterpillar" or "grub," is in many instances completely destitute of legs; and where it does possess feet by which it can crawl, these are not jointed members, but mere fleshy protuberances. The segments are all nearly equal and similar, both externally and internally; they are never more than thirteen in number, counting the head as one; and having been all formed in the first instance by the subdivision of the original yolk-mass, they undergo no subsequent augmentation but that of size. The voracity of the larva is its most extraordinary characteristic ; and its increase in bulk is proportional, the full-sized larva being estimated in some instances to weigh no less than 72,000 times as much as it did when it came-forth from the egg ( $\$ 141)$. During this rapid increase, its skin is several times thrown off; a new one being first formed within this, better adapted to its augmented size. Very little change takes place in the structure of the larva, until after the completion of its growth ; it then ceases to eat, and frequently forms some protection to itself, either by spinning a silken cocoon, or by gluing bits of stick, straw, \&c. into a case, or it may bury itself in the ground. The last larva-skin hardens into a firm case around the body, which, diminishing in size, shrinks away from its interior. The creature, now known as a chrysalis or pupa, remains for some time without food and apparently inert; important changes, however, are taking place within its body, which tend towards the formation of the organs of the perfect insect; and these are produced at the expense of the mass of nutrient material that 
had been stored-up within the body of the larva. When the development of the wings, legs, \&c. has been completed, the Imago or perfect insect bursts-forth from its pupa-case, and enters upon the life of activity for which it is destined. In this condition alone does it possess proper generative organs; and the business of rearing the larvæ, or of preparing a habitation in which they shall find a store of food laid-up for them, seems generally one of the principal objects of its existence. In many instances, indeed, as in the Silkworm, the Imago takes no nourishment whatever, and dies as soon as the generative act has been completed, and the fertilized eggs have been deposited.-It is scarcely possible to find a greater contrast than that which exists between the footless Maggot, almost destitute of the power of movement, and having no capacity but that of gorging itself with the nourishment provided for its sustenance, and the active Bee, almost constantly on the wing, darting from flower to flower in search of the honied sweets which it is now content to sip, and coming home to toil in the construction of that wonderful edifice which human skill could have scarcely rivalled, certainly not surpassed. And it is evident that the true way of looking at the metamorphoses of Insects, is, to consider the chrysalis condition as a continuation of the developmental process. which takes place within the egg; the larva being adapted to come forth into the world for a time in a very immature condition, that it may obtain for itself such a supply of nutrient material as could not have been stored up within the egg, without adding so greatly to its bulk, as to render impossible that enormous multiplication in the number of eggs which is so characteristic a phenomenon in the history of this class.

746. A remarkable departure from the method of sexual propagation common among Insects, has long been known to occur in the tribe of Aphides, or "plant-lice" (ZooL. § 785); which multiply themselves while yet in a state of development that may be considered as larval, without any proper generative act. No distinction exists between males and females, but every individual is formed upon the ordinary female type ; and eggs are produced from an ovarium, which are hatched within the body, so that the young come forth alive. These in their turn repeat the same process within a 
very short time; and one viviparous brood succeeds another, so long as adequate warmth and food are supplied. Ten or twelve are thus commonly put-forth in a single season; and as each brood may consist of a hundred individuals, it may be easily calculated that no fewer than ten thousand million million of Aphides may thus be produced in one summer from a single individual. With the advance of autumn, however, the last brood of larval Aphides, instead of continuing to propagate after this fashion, is developed into the perfect sexual form ; distinct males and females present themselves ; the true generative process is performed; and, as its result, eggs are deposited, that are capable of resisting the cold of winter, which would be fatal to the viviparous larvæ. From these eggs, larval Aphides are hatched in the spring, which repeat the same curious series of phenomena.-Recent inquiries have shown that this method of propagation is by no means confined to the Aphides, but is common to many other Insects. Thus, it appears that the various species of Cynips or gall-fly (ZooL. § 755) are for the most part known only under the female type, and that they can propagate without any male ; the eggs which they deposit producing larvæ, which are developed into the likeness of their parents without receiving any fertilization. It may be surmised that, as among Aphides, males make their appearance under certain conditions, and that a proper generative act occasionally intervenes between the successive productions of non-sexual broods. Comparing these phenomena with those of the gemmation of Salpæ (\$ 728), and with other cases of like nature, it might be supposed that the non-sexual or agamic ${ }^{1}$ production of Aphides, Cynipidae, \&c. is only another case of the same kind. But there is this peculiarity about it,- that the young are produced, not from buds, but from bodies having all the characters of ordinary eggs. And it would seem, from the facts next to be mentioned, that in certain cases the same ovum may develope itself either with or without fertilization; its product in the two cases, however, being different.

747. Among Bees, Wasps, Ants, and other social Insects, the generative process is performed in a very peculiar manner. By far the larger proportion of their communities are neuters, that is, are incapable of reproduction; the continuance of the 
race being effected by a comparatively small number of individuals. Thus, among the common Hive-Bees, the queen is the only perfect female; the drones are the males; and the workers are neuters. But these neuters are undeveloped females, as is shown by the curious fact already mentioned with regard to the capacity of their larvæ for being developed under certain conditions into queens (\$ 716). In the case of the "queen-bee," - as in the still more remarkable case of the queen Termes (Zoor. § 740), which will lay 80,000 eggs in twenty-four hours, and will continue to do so at the same rate for many weeks, - a single generative act suffices for the fertilization of a long succession of ova, though a large proportion of these may have been undeveloped at the time of its occurrence; for the spermatic fluid is stored-up in a little receptacle opening-off from the oviduct of the female, so that a minute portion of it may come into contact with the eggs, as they descend one after the other. But it appears from recent inquiries, that the worker-eggs alone undergo this fertilization, and that the drone-eggs are deposited without receiving it; and there is strong reason to believe that the very same eggs may be developed either into workers or into drones, according as they do or do not receive the influence of the spermatic fluid. When engaged in depositing her eggs, the queen moves over the cells of the comb, apparently without any order, dropping an egg into each; and it seems to be determined by the size of the cells (those prepared for the drone-eggs being of larger diameter than those destined for the worker-eggs), whether or not this fertilizing act shall be performed as the eggs descend. It has long been observed, that queen-bees will occasionally deposit eggs without having left the hive for the "nuptial flight" with the male ; and the eggs thus deposited always prove to be drones. It has also been observed that eggs are occasionally laid by workers; and of these also the products are always drones. Hence, it seems certain that the drones are always developed from agamic or unfertilized eggs ; and it would appear that these very eggs, if fertilized by the male spermatic fluid, would produce workers. This is one of the most curious discoveries yet made in the physiology of Bees; and it remains to be determined how far the same thing is true among other tribes of Insects. 
748. In the class of Crustacea there is great variety as to the degree of netamorphosis undergone by the young after their emersion from the egg ; for whilst there are several instances in which either the mature form, or one closely resembling it, is presented from the first, the more common fact is that the early or larval condition is extremely dissimilar to that of the parent, and that a succession of changes has to be gone-through before the latter is attained. This is in no instance more remarkable than in that of the common $\mathrm{Crab}$, whose larva (fig. 48) was long known under the name of Zoea, and was supposed to belong to a type altogether different. No instance of agamic reproduction by eggs is as yet known among the higher Crustacea; but in the Entomostracous division there are probably many examples of it. Thus in the little Daphnia (Zoor. \$ 879), one of the commonest of the "water fleas," the ordinary eggs seem to be always "agamic ;" whilst the eggs which are formed within the peculiar case termed the ephippium, and which seem enabled by its protection to endure a degree of cold that is fatal to the ordinary eggs as well as to the parents, are the products of sexual action. The Cyclops, again, has been found, like the Aphis, to produce many

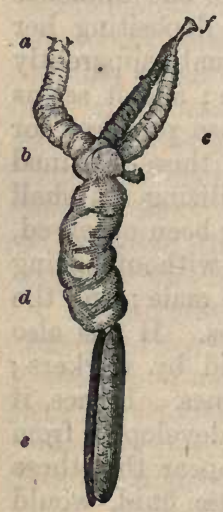
successive broods, which broods repeat the like mode of propagation, without the appearance of a male. - Among the examples presented by this class, of entire change of form in the progress of development, none are more remarkable than those which are met with among the suctorial tribes, which live as parasites upon the exterior of other animals, especially Fishes. As an example of this change we may refer to the Lernoca (fig. 312), an animal which is not unfrequently found clinging to the eyes and gills of fish, the anterior part of its body being commonly imbedded in the substance of the part to which it attaches itself. This creature Fig. 312.-LerNas. is characterized by the size of its large suctorial trunk $a$, and by that of its single pair of legs $c$, which terminate together in the sucker $f$; and by the immense development of the abdominal portion of its body $d$, 
as well as of the two large egg-capsules $e$, which are attached to it. Yet in its larval condition (fig. 313) it is an active little creature, resembling in all essential particulars the larvæ of Entomostraca generally; and from this type the males do not depart nearly so much as the females, the former retaining the general plan of structure, as well as the activity of habits, that prevail among the Entomostraca, whilst the latter lose their instruments of movement, acquiring the apparatus of suction and prehension.

Fig. 313.

LARVA OF LERNAA.
749. A still more remarkable example of metamorphosis is presented by the tribe of Cirrhipeds ( $\$ 102)$, which, notwithstanding that they were long ranked as Mollusks, on account of their shelly investment and their immovable attachment to solid bodies, are now known to be so closely related to Crustacea, as in the opinion of many naturalists to rank merely as a subdivision of that group. The young, alike of the Lepas or "barnacle," and of the Balanus or "acorn-shell," very much resemble those of the ordinary Entomostraca ; they possess eyes and several pairs of motor appendages in this state; and they swim freely through the water, after the manner of water-fleas. Before their last change, they are enclosed in a bivalve carapace like that of Cypris; and they then possess a pair of large four-jointed antennæ, which are well furnished with muscles; and it is by these antennæ that the animal finally attaches itself, by means of a peculiar cement which is poured out from ducts running up into them from the body. In the anterior of the recently-attached larva, the young Cirrhiped may be detected with its valves and cirrhi, like the embryo insect in its pupa-case ; and the carapace and integuments of the larva being thrown-off like a pupa-case, the perfect form is disclosed. In most of these animals, as in many mollusks, the sexes are united in the same individuals; but when they are separate, the males are minute imperfectly-formed creatures, which lead a sort of parasitic life upon the surface of the females; and in some of the hermaphrodite species "supplemental males" are also provided.

750. Among the Rotifera or Wheel-Animalcules, the double mode of reproduction by agamic and by sexual eggs seems to be the ordinary rule. The former may go on at a prodigious 
rate; so that from the known rate of propagation in a Hydatina, it is calculated that nearly seventeen millions might be produced from a single individual within twenty-four days," although not more than three or four ova are being brought forward at once. The latter takes place only at certain seasons, most frequently before the winter; the sexual eggs, in these as in other cases, being endowed with a special power of resisting cold. It is only occasionally, therefore, that males are to be met with; and it is a remarkable circumstance that they are in many instances destined for so brief an existence, as not even to be furnished with any digestive apparatus ; so that their development is completed, and their reproductive function performed, at the sole expense of the nutriment which was furnished by the egg.

751. In the class of Arachrida there is nothing that corresponds with the metamorphosis of Insects; for Spiders and Scorpions attain to the full development of their organs within the egg, so that the young come-forth from it differing in scarcely any respect from their parents, except in size; and among the Acaridoe or "mites," the only important difference lies in the deficiency of one of the four pairs of legs, which is supplied after the first moult. This completion of the process of development within the egg could scarcely take place, but for the large supply of nutriment afforded to the embryo by the yolk. The Arachnida deposit a far smaller number of eggs than Insects do ; and thus, as each egg can be made of much greater size, there is no necessity for that early emersion of the embryo in search of the material for its continued development, which has been shown to be the real purpose of the larva-life of Insects ( $\$ 745)$.

752. In the Molluscous series the generative function presents few such peculiarities as have been noticed among Articulata. Save in the lowest members of the series, the Tunicata and the Polyzoa ( $\S \S 114,115)$, we have no example of reproduction by gemmation; and no instance of reproduction by agamic ova is yet known. The union of the two sexes in the same individual is much more common in this series than among Articulata; and thus the fertilization of the eggs is secured without the exercise of locomotive power. It is remarkable, however, that the embryos even of such Mollusks as are destined to remain almost motionless when they attain 
their adult condition, are adapted to move actively through the water: those of the Polyzoa swimming forth as ciliated gemmules; those of the Tunicata having a tadpole-like tail, formed by an outgrowth from the "mulberry mass," which propels them by its lateral strokes ; and those of the Gasteropods having two large eiliated lobes, much resembling the "wheels" of Rotifera, placed one on either side of the mouth. It is further remarkable that all Gasteropods possess a shell in their early condition, whether or not they are to possess one ultimately. Among some of the Pectinibranchiata, which constitute the highest order of this class (ZooL. \$ 985), a very remarkable provision has been observed for carrying-on the development of the embryo to a more advanced stage than is attained in other instances within the egg. In addition to the "germ-yolk," which undergoes the usual processes of fission and of conversion into the mulberry-mass ( $\$ 736)$, by the subsequent metamorphoses of which the body of the embryo is formed, we find a quantity of "food-yolk" stored-up with each embryo, which may be likened to the supply that is provided by many Insects for the nutrition of their larvæ on their first emersion from the egg ( $\$ 703)$; this store is greedily devoured by the embryos, as soon as they have a mouth to swallow it and a stomach to hold it; and it is at the expense of this, that all their later development is carried on.

753. The class of Cephalopods, in which the sexes are always separate, presents us with some extremely curious provisions for bringing the products of the sperm-cells into contact with the eggs. Whilst passing through the duct that conveys them forth from the glandular organ within which they are formed, the spermatozoids cluster together ; and these clusters become invested with peculiar casings, which, when immersed in water, have a peculiar movement that enables them to advance through it, and causes them, when they meet with an obstacle, to mupture and set free their contents. In this mode it is that the spermatozoids find their way into the midst of the large grape-like clusters of eggs which have been deposited by the females, and which thus receive the fertilizing influence of the male. - In the Argonaut or " paper-nautilus" (ZooL. $\S 962$ ) there is a still more remarkable provision for the same end. All the individuals of this species that form the beautiful paper-like shell from which it derives 
its name, are females; and it now seems clear that the essential purpose of the shell is the protection, not of the animal (which is not in any way attached to it), but of the eggs. The male is of such comparatively insignificant size, that, not being provided with a shell, it was not recognised until recently as belonging to the same species. The spermatic duct passes through one of its arms, which is extended into a long whip-like appendage ; and in this duct are found bundles of spermatozoids, all contained within one casing, which does not possess any self-moving power. At a certain epoch, this arm detaches itself entirely from the body, and moves freely through the water by means of the apparatus of nerves and muscles with which it is endowed, until it comes into contact with a female of its own species, whose eggs are fertilized by its contents which are then set free. In this detached condition, the arm was long since observed within the shell of the Argonaut, and was supposed to be a parasitic Worm ; subsequent inquiry showed it to possess, in its suckers and its nervo-miscular apparatus, the characteristic structure of the Cephalopod, and it was at first supposed to be itself the male, destitute (like the male of some Rotifera, § 750) of any nutritive apparatus. Its true history, as now elucidated, is one of the most curiously-exceptional phenomena in the whole of this department of physiology.

754. Having at last arrived at the Vertebrated series, we shall take a general survey of the history of Development as presented to us in the case most familiar to every one, the formation of the Chick within the egg of the Bird ; pointing out, as occasion arises, the principal points of difference between the mode in which the process is there carried-on, and the corresponding phenomena presented by other classes.The ovum, as formed within the ovary, has neither "white" nor "shell," but consists of the yolk-bag and its contents alone. Under the influence of domestication, which affords a more constant supply of food and warmth than the Fowl would obtain in its natural condition, a much larger number of eggs are produced by the hen than she would produce in her wild state; so that, instead of laying four or five at a time, with long intervals between each deposit, she is continually evolving them. An enormous quantity of "foodyolk" is prepared, in addition to the "germ-yolk;" and thus the Bird's egg comes to acquire a far larger size in pruportion 
to that of the parent, than we see in any other animals. The appearance of the ovary of the Fowl in process of laying is shown in fig. 314 ; its surface is rendered uneven or knobby

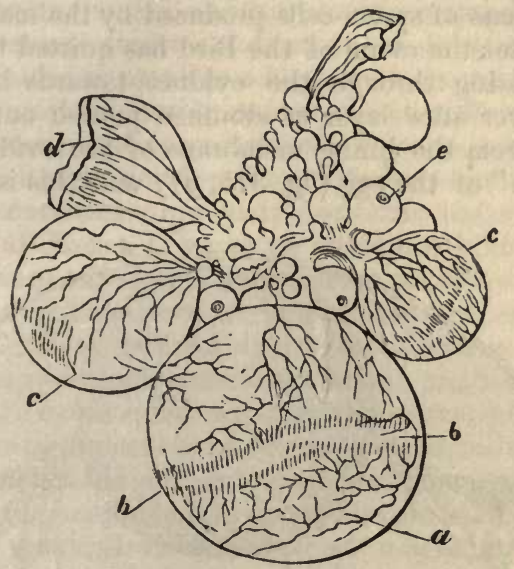

Fig. 314.-OVARx of THE Fown, with ova in various stages of development:

$a$, mature ovum within its calyx, which is about to rupture along the non-vascular streak $b b ; c c$, less advanced ova; $d$, a calyx from which the ovum has escaped; $e$, still younger ova.

by the protrusion of the ova in various stages of enlargement, those which are most mature $(a, c)$ forming pear-shaped projections which only hang-on by a narrow stalk; but each ovum is still included within an extension of the fibrous substance of the ovary (fig. 302), termed the calyx, through which blood-vessels are conveyed over its surface; and when the ovum escapes by the rupture of this, along a line $(b)$ from which the vessels have previously retreated, the calyx remains as an open cup $(d)$. The ovarium of such Mammals as produce several young at once, presents a corresponding appearance as each set of ova is approaching maturity; save that, as the mass of yolk is comparatively small (no "food-yolk" being provided), the ova do not project so much from its surface. In Fishes, on the other hand, we find an enormous number of ova produced at once; the whole ovarium, when they are approaching maturity, being so crowded with them that its. P P 2 
own substance is scarcely distinguishable. Not only thousands but tens of thousands of eggs are often produced by a single individual, their aggregate forming what is known as the "hard roe;" whilst the "soft roe" or "milt" is the corresponding mass of sperm-cells produced by the male.

755. After the ovum of the Bird has quitted the ovarium, and is passing through the oviduct towards its outlet, it receives layer after layer of albumen poured out in a viscid condition from the lining membrane of the oviduct, forming the "white" of the egg (fig. $315, g$ ); and this is inclosed in

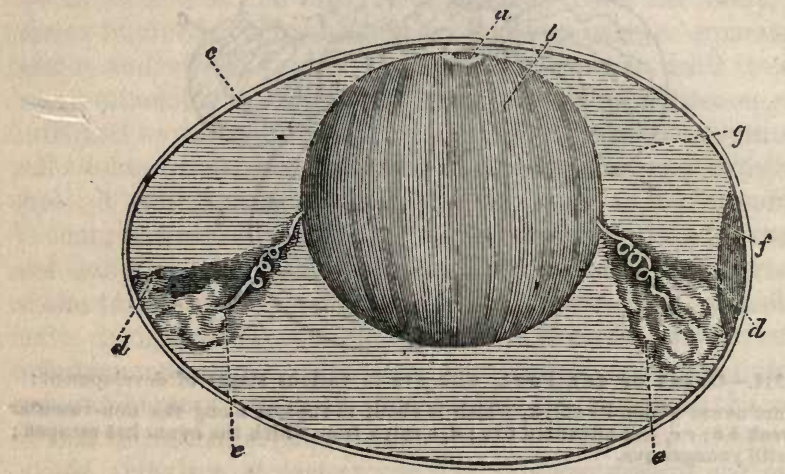

Fig. 315.-Section of Fowl's EGG:

$a$, cicatricula; $b$, yolk-bag ; $c$, membrane lining shell; $d$, attachment of chalazæ; $e$, chalazæ; $f$, air-space; $g$, albumen.

a double membrane composed of a network of fibres, which is formed by the consolidation of a plastic exudation ( $\$ 391$ ), poured out after the albuminous exudation has been completed. The outer layer of this membrane is consolidated by: the deposit of calcareous particles in the interspaces of its fibrous matting, so as to form the "shell" of the egg; an arrangement that gives the necessary protection, without cutting-off the contents of the shell from that communication with the atmosphere which is requisite for the development of the embryo. The inner layer, which forms a lining to the shell, separates into two laminæ at the large end of the egg; and, inclosed between these, there is a bubble of air $(f)$, which serves to give the young bird, just before it is hatched, the 
power of filling its lungs with air. The yolk-bag floats within the albumen, and always tends to take the highest place, being the lighter of the two ; but it is kept nearly to one place by two cords $(e, e)$ termed the chalazoe, which seem formed of peculiarly viscid albumen, and connect the yolk-bag with the lining membrane at the two ends of the shell $(d, d)$. In this manner the yolk-bag is always kept at the part of the shell where it can most favourably receive the warmth imparted to it by the mother; and the cicatricula or germ-spot (which is the mass of cells first developed from the germ-yolk) is made, by a similar contrivance, always to rise to the highest point.In the eggs of Fishes there is no additional albumen; and in those of Frogs the albumen is common to the general mass of ova, constituting the peculiar "gelatinous envelope," which forms long necklace-like strings of "spawn," within which the black yolk-bags are disposed at tolerably regular intervals. In Mammals, each ovum receives a separate investment of a jellylike substance in its passage along the oviduct into the uterus ; and around this there is formed a fibrous membrane termed the Chorion, which is destined to take a very important share in the subsequent nutrition of the embryo ( $\$ 761$ ).

756. In the eggs of Frogs, as in those of Mammals, the whole of the yolk undergoes the process of segmentation already described ( $\$ 736$ ), and takes a share in the formation of the "mulberry mass." But in the eggs of Fishes, Birds, and the higher Reptiles, this process is limited to that small portion of the yolk which is distinguished as the germ-yolk; and the formation of the germinal membrane takes place after a different fashion. The mass of cells that immediately results from segmentation, flattens itself out on the surface of the yolk, forming the minute semi-opaque whitish spot, which is known as the cicatricula, germ-spot, or "tread;" and by a further extension it constitutes the "germinal membrane," which gradually spreads itself over the food-yolk; at the same time dividing itself into two layers, between which a third is afterwards interposed. Thus the "germinal membrane," which may be compared to the seed-leaves or cotyledons of Plants, forms a sort of temporary stomach round the inass of nutriment prepared for the sustenance of the embryo; the whole of which nutriment, as will be presently seen, is absorbed into the body of the embryo through its instrumentality. 
757. The first indication of the permanent fabric in all Vertebrated animals, consists of a delicate longitudinal streak, termed the "primitive trace" (fig. 316, $b$ ), that is observable in the midst of a pellucid area, which is again surrounded by

a ring of more opaque as-

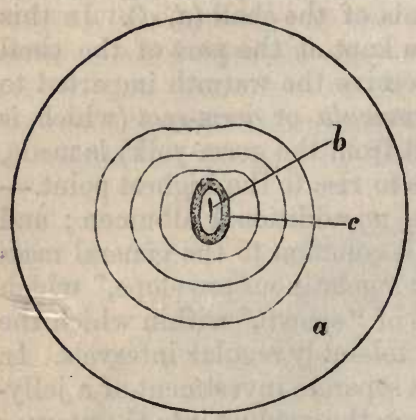

Fig. 316.-York-BAg of Fown's EgG, after twelve hours' incubation :

$a$, yolk; $b$, primitive trace surrounded by pellucid area; $c$, more opaque ring, the commencement of the vascular area.

pect $(c)$. This primitive trace is the foundation of the Vertebral Column. It is in the first instance a mere furrow in the outer layer of the germinal membrane; but the sides of this furrow, known as the dorsal laminoe, rise up and arch-over, so as gradually to meet and convert the furrow into a canal. The meeting first takes place in what is afterwards to become the middle of the back; and here we find the first distinct rudiments of the vertebral column, in the condition of a series of small square plates (figs. 317, $c, c$, $325, l, l)$ on either side, which are the representatives of the arches of as many vertebræ. The furrow widens-out in the situation of the head, so as to form the receptacle $(d)$ for the series of large ganglionic masses that is to constitute the brain (fig. $323, d, e, f$ ); and though its sides do not there close-in for some time longer, it receives a special hood-like covering from a peculiar fold of the germinal membrane, the edge of which is seen at $e$, fig. 317 . The cells, of which the parts of these laminx that bound the bottom and sides of this furrow are composed, appear to furnish the rudiments of the nervous centres that are afterwards to occupy the canal; but beneath its deepest part there lies a continuous rod of peculiar nucleated cells $(f)$, the chorda dorsalis, which marks-out the situation afterwards to be taken by the bodies of the vertebræ. This remains the only representative of the vertebral column in the Lamprey and other Fishes of a low grade, the development of whose bony skeleton is checked so early that it never advances beyond this simple embryonic type ( $\$ 53)$. 
758. During the progress of this change, another very important one is taking place, which is destined for the nourishment of the embryo during its further development. This is the formation of vessels in the substance of the germinal membrane; which vessels serve to take up the nourishment supplied by the yolk, and to convey it through the tissues of the embryo. The space over which these vessels spread

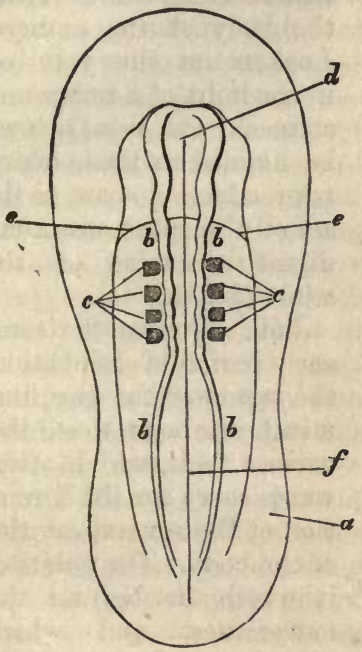

Fig. 317,-More advanced EMBRYO OF Fow L, much enlarged:

$a$, pellucid area; $b \quad b$, dorsal laminæ; $c c$, rudiments of vertebral arches; $d$, dilatation for brain; $e e$, cephalic hood; $f$, chorda dorsalis.

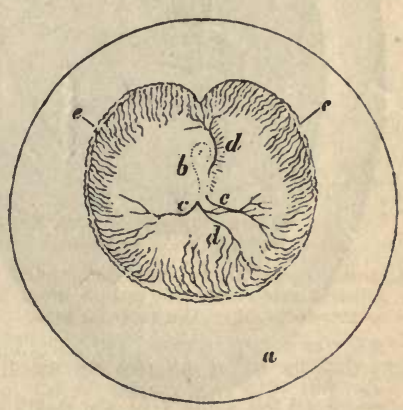

Fig. 318.-YoLK.BAg or Fow's EgG, at the beginning of the third day of incubation :

$a$, yolk; $b$, embryo; $c c$, arteries of vascular area; $d d$, veins ; $e$ e, terminal sinus.

themselves, is called the Vascular Area ; it makes its appearance during the second day of incubation in the Fowl's egg (fig. 316,c), and soon spreads itself over the surface of the yolk (figs. 318, 319). Islets or points of a dark colour first appear in it; these unite in rows; and at last continuous vessels are formed. The heart makes its appearance at the twenty-seventh hour of incubation, as a simple dilatation of the trunk into which the blood-vessels unite (fig. $320, h$ ). Its wall is at 


\section{DEVELOPMENT OF VESSELS AND DIGESTIVE CAVITY.}

first formed by a layer of cells ; and no muscular structure is seen in it, until after its regular pulsations have commenced. It is in these vessels that the first blood is formed; and the same process appears to be continued through the whole period of incubation, the yolk being progressively converted into

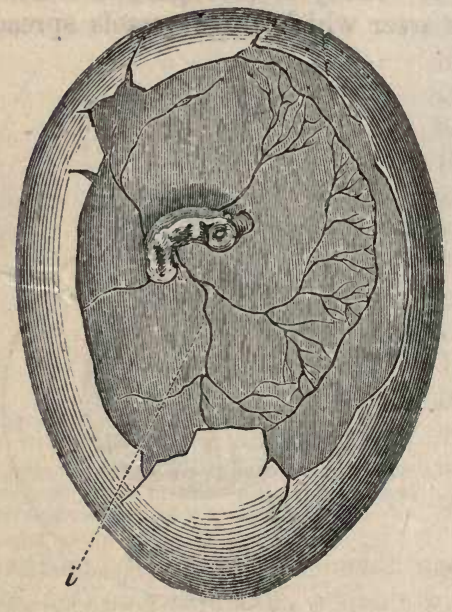

Fig. 319.-EMBRYo of BIRd, with THE Vessels, $i$, of the vascular area, after four days' incubation.

spread-out from the sides of the embryo, are doubled-in so as to make a depression upon the yolk; and their folded edges

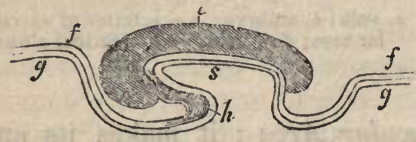

Fig. 320.-Diagram of the Formation of the Digestive Cavity:

$e$, embryo ; $f, g$, layers of germinal membrane; $h$, heart; $s$, stomach. conveyed by the great trunks which collect it into the body of the embryo. Looking at the yolk-bag in the light of a temporary stomach, its vessels may be likened to those which take so large a share in the act of absorption from the digestive cavity of the adult ( $\$ 218)$.

759. During the same early period of incubation, the layers of the germinal membrane begin to exhibit various folds, which afterwards serve for the formation of the several cavities of the body. The points of it which lie beyond the extremities, and which gradually approach one another under the abdomen, which lies next the interior of the egg. In this manner is formed the permanent digestive cavity ; which is first a simple pouch communicating with

blood, and this blood being the yolk-bag, by a wide opening, as seen at $s$, fig. 320 ; but which is gradually separated from it by the narrowing of this orifice (fig. 322 ), the connecting portion being elongated into a duct (fig. 321,6$)$. Thus we may 
say that the digestive cavity in Vertebrata is formed by the pinching-off (as it were) of a small portion of the general sac of the yolk. In the Mammalia, the remainder of the yolk-bag is completely separated from this by the closure of its narrow orifice, and is afterwards thrown off; so that only a very small portion of the germinal membrane is received into the permanent structure. But in Birds and other oviparous animals, the whole of the yolkbag is ultimately drawn into the abdomen of the embryo; the former gradually shrinking as its contents are exhausted; and the latter enlarging, so as to receive it as a little pouch or appendage. In Fishes, the hatching of the egg very commonly takes place before this process has been completed; so that the little Fish swims about with the yolk-bag hanging from its body.

760. The embryo, like the adult, has need of Respiration; partly that its own heat may be kept up; and partly that the

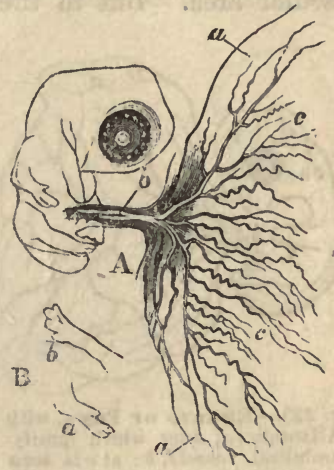

Fig. 321.-A, MORE ADVANCED EMBRYo OF Fow the vitelline duct $b$, with the yolkbag $a \quad a$, over which are distributed the blood-vessels $c$ c; $\mathrm{B}$, early form of the anterior extremity $a$, and of the posterior extremity $b$. carbonic acid liberated in the various processes of nutrition, may be set free. Owing to the peculiar structure of the membrane covering the albumen and forming the basis of the

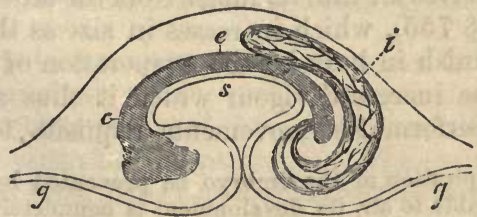

Fig 322.-Diagram of the Formation of the Altantors, $i$. (The other references as in fig. 320.)

shell ( $\$ 755)$, the outer air is enabled to gain access to the interior of the egg; and at first its action upon the blood, 
whilst circulating in the vascular area, is sufficient. In Fishes, no further provision is made for this process; since, by the time it would be required, the egg is hatched; the young animal comes forth into the medium it is permanently to inhabit, its own gills come into play, and the air contained in the water can act directly upon the blood circulating in the vascular area. But in the higher oviparous animals, whose development proceeds further

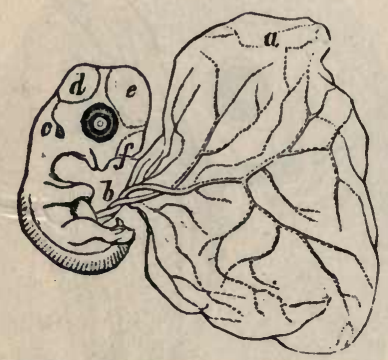

Fig. 323.-ЕмвRчo or Fow $x$, with the Allantois, $a$, over whicl ramify the umbilical vessels, $b$; at $c$ is seen the indication of the place of the externai ear; $d, e, f$, rudiments of the cerebellum, optic ganglia, and cerebrum respectively. it lies in close proximity with the membrane of the shell, it is very advantageously situated for receiving the influence of the air. It thus serves as the temporary respiratory apparatus of the Chick, up to the time when it is preparing to quit the egg. ${ }^{1}$ There is reason to believe that the bird then receives air into its lungs, from the air-space formerly mentioned $(\$ 755)$, which increases in size as the contents of the egg diminish in bulk by the evaporation of their watery part. By the increased vigour which it thus acquires, it is enabled to perform the movements requisite for extricating

1 If the respiration of the embryo be prevented by rendering the shell impermeable to air, its development is completely checked. No means of accomplishing this is so effectual, as smearing the shell with oil or grease of any kind. Hence the effect of the well-known practice of buttering the surface of the egg, in preventing the chick from being reared; and the same operation, if performed when the egg is quite fresh, will preserve it for some time fit for eating, its decomposition being prevented by the complete exclusion of the air. 
DEVELOPMENT AND NUTRITION OF MAMMALIAN EMBRYO. 587

itself from its shell ; which it does entirely by its own exertions. When it thus becomes independent of the allantois, the circulation through the latter diminishes; and almost the whole sac is separated from the body by the contraction of the connecting foot-stalk, which at last gives way.

761. The formation of the yolk-bag and the allantois takes place in Mammals (fig. 324) almost exactly on the same plan
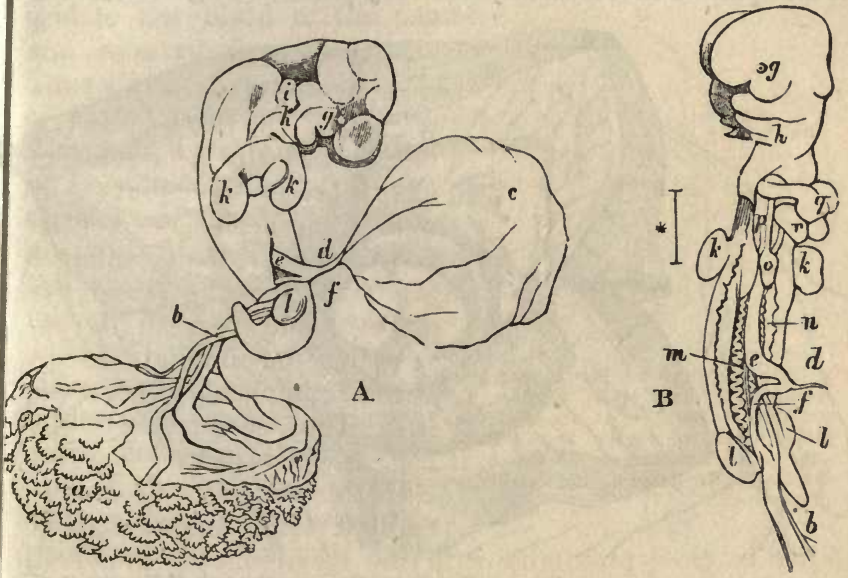

Fig. 324.-ЕмвRYо OF MOLE:

$A$, entire; $\mathbf{B}$, with the abdomen laid open:- $a$, chorion; $b$, footstalk of allantois and umbilical vessels; $c$, yolk-bag; $d$, vitelline duct; $e$, upper portion of intestinal canal ; $f$, lower portion; $g$, eye; $h$, indication of branchial arches ; $i$, auditory vesicle; $k$, anterior, and $l$, posterior extremity: $m, n$, Wolffian bodies or rudimentary kidneys; $o$, rudimentary lung; $p$, trachea; $q$, ventricle of heart; $r$, atrium of heart.

as in Birds ; but on account of the absence of food-yolk, these sacs are comparatively small ; and the function of both is superseded, at an early period of the development of the embryo, by a new and remarkable contrivance. The ovum, in passing through the oviduct, has been already stated to receive a new envelope, analogous to that which forms the membrane of the shell in Birds ; this is termed the Chorion. It is then received into the cavity of the Uterus, a receptacle within which it is delayed for a considerable period, and continually supplied with nourishment drawn from the blood of the parent. From 
the whole surface of the chorion, a number of little tufts shoot out (fig. 325), which come into contact with the lining membrane of the uterus; and this is furnished with a multitude of glandular follicles, which secrete a nutritious fluid that is absorbed by the tufts of the chorion, and by them communicated to the embryo. When the allantois is formed, it serves to carry the blood-vessels of the embryo to the inner surface of one part of the chorion ; and they shoot through this, so as

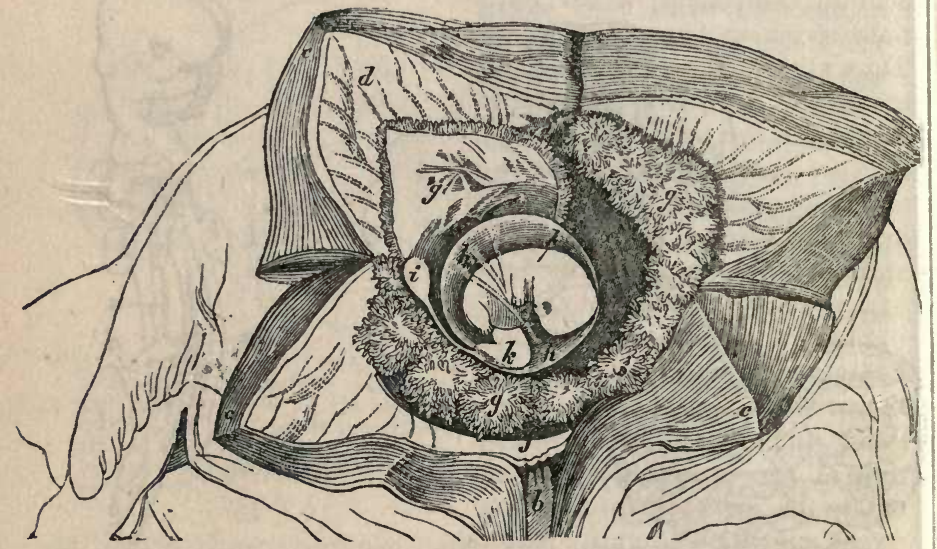

Fig. 325.-Interior of Human Uterus at the seventh week of Pregnancy: $b$, outlet of the uterus, of which the walls $c, c, c, c$, laid open by incision, are turned back to display its contents; $d d$, its lining membrane; $g$, tufted surface of the chorion ; $g^{2}$, its internal aspect; $h, h$, amnion; $i$, yolk-bag; $k$, umbilical cord; $l$, embryo.

to dip-down, as it were, into large expanded vessels that extend outwards from the walls of the uterus. In this manner is formed, in all the higher Mammalia, the important organ termed the Placenta; which essentially consists of the ramifications of the foetal blood-vessels contained in the Umbilical Cord or "navel-string," ensheathed by prolongations of the large vessels of the maternal uterus. In the Marsupials and Monotremes (Zoology, $§ \S 309-320$ ), no placenta is ever formed, the embryo coming into the world in a stage scarcely more advanced than that represented in fig. 325. In either case, the vessels of the embryo are enabled to absorb from the blood contained in those of the parent, through the thin 
walls of both, the materials requisite for its growth; but there is no direct communication between the two. The same means serve for the aeration of the blood of the embryo; for this, being brought from its body in the venous condition, is exposed to the influence of the arterial blood of the parent, through the thin walls of its vessels, - just as the venous blood of aquatic animals is aerated in their gill-tufts, - and passes back to the embryo in the arterial condition, having imparted its carbonic acid to the blood of the parent, and received from it oxygen.Thus all but the very early stages of development are performed in Mammals, by means of which we scarcely find a trace in Oviparous animals; yet the ova of both are originally formed on the same plan, and the first changes which they undergo are exactly analogous.

762. It would not be consistent either with the design or with the limits of this work, to enter in much detail into the consideration of the processes of development, although they present many points of the highest interest. The general history of the evolution of the Circulating apparatus and of the Nervous centres may, however, be noticed, as characteristic examples of the mode in which the evolution of the several organs of the body takes place.The Heart, in Man and other Mammals, as in the Bird ( $\$ 758)$, is at first a simple tube, resembling the pulsatile trunk that remains as

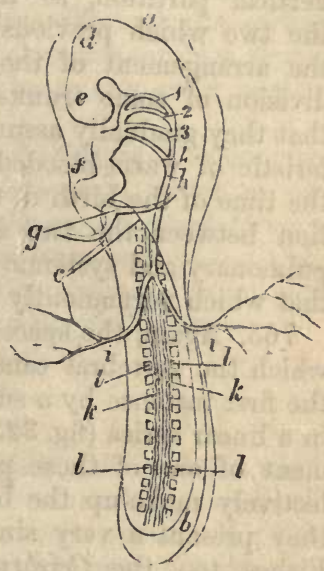

Fig. 326.-ЕмвRYo of the FowL, from the Ovum shown in fig. 318 , greatly enlarged:

$a, b$, folds of germinal membrane, enveloping head and tail; $c$, lateral folds; $d, e$, rudiments of optic ganglia and cerebrum; $f$, heart; $g$, dilated termination of venous trunk, forming atrium of heart; $h$, aorta ; $1,2,3,4$, branchial arches; $i$, $i$, vessels of vascular area; $k, k$, dorsal laminæ; $l, l$, rudiments of vertebraI arches. the sole organ of impulsion in the lowest forms of circulating apparatus. After a time this tube is doubled upon itself, and two cavities are formed, an auricle and a ventricle ; in this condition, it strongly resembles the heart of the Fish (\$286). The circulation too is, at an early period, that of the Fish; for the 
arterial trunk that springs from the ventricle, divides into a set of arches on each side (fig. 326), which closely resemble the branchial arches of Fishes and Tadpoles. Although no gills are present, yet there is a series of clefts on each side of the neck, passing through to the pharynx, which are analogous to the branchial apertures of the cartilaginous Fishes ( $\$ 317)$. After a time, however, the auricle and ventricle of the heart are each divided by a vertical partition, so that four cavities are formed, out of the two which previously existed; and at the same period, the arrangement of the vessels undergoes a change, by the division of some trunks, and the obliteration of others, so that they gradually assume the distribution which is characteristic of warm-blooded animals ( $\$ 281$ ). But even up to the time of the birth of the Mammalia, there is a communication between the two sides of the heart, and between the pulmonary and systemic vessels, which is closely analogous to that which permanently exists in the Crocodile ( $\$ 283)$.

763. Again, the space within the head of the embryo, into which the vertebral canal widens-out ( $\$ 757)$, is occupied in the first instance by a succession of vesicles or bags, arranged in a linear series (fig. $323, d, e, f$ ) ; each of which is the rudiment of one of those principal ganglionic masses, that collectively make-up the brain of the Fish ( $\$ 453$ ), in which they present a very similar aspect (fig. 192). As in many Fishes, too, the Cerebrum is inferior in size to the Optic ganglia, and only comes to surpass and finally (as it were) to overpower them ( $\$ \$ 455,456)$ in the later periods of embryonic development.

764. The true representation of these and similar facts is not, as was maintained when they were first brought into view, that the several organs of the higher animals go through a series of forms which remain permanent in the lower; but that the development of all animals formed upon the same general plan commences in the same manner, their special differences manifesting themselves as development proceeds. Thus, as we have seen, the foundation of the Vertebral column is laid in all Vertebrata in precisely the same method (\$ 757); in some of the lowest Fishes, the evolution of this structure is checked at so early a period that it never advances beyond the embryonic type ( $\$ 53)$; but the fully-formed spine has a 
characteristically-different structure in each of the classes of Vertebrata, which is not presented at any period in the history of the others. So, the evolution of the Circulating apparatus commences in all Vertebrata upon the same original plan ; and from this plan there is but little departure in the Fish; but the circulating apparatus of the early Human embryo, however like that of the adult Fish, differs from it in this essential particular, - the absence of gill-tufts receiving capillary vessels from the branchial arches $(\$ 286)$. The like is true in regard to the Nervous centres; for although the earliest condition of the Human brain very closely resembles that of the brain of the foetal Fish, it never bears any exact analogy to that of the adult Fish.

765. Hence the principal facts of Organic Development adnit of being stated in this general formula, which we owe to the sagacity of Von Baer,-that the more special forms of structure arise progressively out of the more general,-a principle than which there is none more comprehensive or more important in the whole range of Physiological Science.

766. The Unity of Plan which is visible through the whole Animal Kingdom, is nowhere more remarkable than in the function of which an outline has now been given. We have seen that, however apparently different, the essential character of the Reproductive process is the same in the highest Animal as in the lowest. It has been shown that the development of the highly-organized body of Man,- - though it is to serve as the instrument of those exalted faculties, by the right employment of which he is made "but a little lower than the Angels,"commences from the same starting-point with that of the meanest ereature living : for even Man, in all the pride of his philosophy, and all the splendour of his luxury, was once but a single cell, undistinguishable, by all human means of observation, from that which constitutes the entire fabric of the simplest Protozoon. And when the Physiologist is inclined to dwell unduly upon his capacity for penetrating the secrets of Nature, it may be salutary for him to reflect that,- - even when he has attained the furthest linits of his Science, by advancing to those general principles which tend to place it 
on the elevation which others have already reached,- - he yet knows nothing of those wondrous operations, which are the essential parts of every one of those complicated functions by which the life of the body is sustained. Why one cell should absorb,-why another, that seems exactly to resemble it, should assimilate,-why a third should secrete,-why a fourth should prepare the reproductive germs, - and why, of the two germs that seem exactly similar, one should be developed into the simplest Zoophyte, and another into the complex fabric of Man,-are questions that Physiology is not likely ever to answer. All our science is but the investigation of the mode or plan on which the Creator acts ; the Power which operates' is Infinite, and therefore inscrutable to our limited comprehension. But when Man shall have passed through this embryo state, and shall have undergone that metamorphosis by which everything whose purpose was temporary shall be thrown aside, and his permanent or immortal essence shall alone remain, then, we are encouraged to believe, his finite mind shall be raised more nearly to the character of the Infinite, all his highest aspirations shall be gratified, and neverending sources of delightful contemplation shall be continually opening to his view. The Philosopher who has attained the highest summit of mortal wisdom, is he who, if he use his mind aright, has the clearest perception of the limits of human knowledge, and the most earnest desires for the lifting of the veil that separates him from the Unseen. He, then, has the strongest motives for that humility of spirit and purity of heart, without which, we are assured, none shall see God. 


\section{N D E X.}

\section{N.B.-The numbers refer to the paragraphs.}

A.

Aberratron, chromatic, 548, 549 ; spherical, 546, 547; avoided in the eye, 549 .

ABSORPTION, nutritive, 171; in Vertebrata, through lacteals, 217 ; through blood-vessels, 218; in Invertebrata, 225 ; in non-vascular animals, 225; interstitial, performed by lymphatics, 219 ; from general surface, by veins, 220.

Abstinence, power of, 140, 141, 152.

ACALEPH $\mathbb{~ E , ~} 120$; luminousness of, 395 ; (see Medusce).

Acephalous Mollusca, 111, 113.

Actinophrys, 130.

Adipose tissue, 46.

Aeration of blood, 253, 303.

Aerating surface, $311,312$.

Agamic Reproduction, of Insects, 746, 747 ; of Entomostraca, 748.

Air, atmospheric, composition of, 300 ; need of in animals, 207; change of, by respiration, 300-302; effects of deprivation of, 297, 298, 310, 338 .

- bladder of Fishes, 324. cells of Birds, $80,326,327$.

- tubes and sacs of Insects, 320-322. Albinos, 545 .

Albumen, chemical composition of, 13, 14 ; use of in blood, 240.

Albuminous principles of food, 153 ; destination of, $158-164$.

Aliment, definition of, 132 (see Food).

Allantois, 760, 761 .

Ammonite, 111.

Amphibia, 85, 87 ; circulation in, 287289 ; (see Batrachia and Frog).

Amphioxus, 53.

Anabas, respiratory organs of, 318 .

Anastomosis, of arteries, 262-265; of nerves, 428.

Anemone, Sea, 126, 127; reproduction of, 726,739 .

Aneurism, 263.

Animal Life, functions of, $6,426,427$; nervous system of, 461.
Animals, distinctive characters of, 612: dependent on Plants for support, $144-147$.

Animalcules, structure of, 13, 136, 137 ; heat produced by, 404 ; movements of, 577 ; reproduction of, 135,725 .

ANNELIDA, general characters of, 104 ; circulation in, 294; respiration of, 314 ; luminosity of, 396 ; nervous system of, 440; muscular motion of, 597 ; reproduction of, 727,744 .

Aunular ligament, of wrist, 641 ; of ancle, 648 .

Anobium, sound of, 677 .

Ant-eaters, 186.

Antennæ of Insects, uses of, 498.

Anthozoa, 126; development of, 736 .

Ant-lion, instinct of, 697 .

Aorta, 258; valves of, 273.

Aphides, agamic reproduction of, 727 , $735,746$.

Aplysia, nervous system of, 438 .

Aquatic animals, respiration in, 298.

Aqueous humor, 536.

ARACINIDA, general characters of, 106 . circulation in, 293; respiration in, 323 ; development of, 751 .

Arachnoid membrane, 43.

Area pellucida, 757.

- vasculosa, 758 .

Areas, comparative, of arteries, 247.

Arenicola, 314.

Areolar tissue, structure and properties of, $24-27$.

A rgonaut, reproduction of, 753 .

Arm, bones and muscles of, $638-640$.

Arteries, 246 ; comparative areas of, 247 ; walls of, 248 ; pressure of blood in, 249 ; protection given to, 250 ; division of into capillaries, 251 ; general distribution of, in Man, 258, 259; in Bird, 260,261; peculiarities of distribution of, $262,264,265$; flow of blood through, 274-276; wounds of, 277.

ARticulAta, general structure of, 9396 ; skeleton of, $597-600$; norvous system of, $440-447$; eyes of, $573-575$. Articulate sounds, 687-691. 
Articulation of bones, different modes of, $600-604$.

Arytenoid cartilages, 680,681 .

Ascidia, 114; nervous system of, 435 .

Asphyxia, 280, 338 ; treatment of, 339 .

Assimilating glands, 223, 224.

Astræa, fission of, 726 .

Atlas-vertebra, 632.

Attention, effect of, on sense of touch, 494 ; on hearing, 525 ; on sight, 555 ; on mental power, 721 .

Auditory nerve, 512.

Auricles of heart, 257; action of, 270 .

Automatic movements, 479.

Axis vertebra, 632 .

Axolotl, 87.

Azotized principles of food, 153, 154; destination of, $158-165$; effects of excess of, 348.

Azotized compounds, excretion of, 346 -348 .

B.

Balancing of body, 480,481 .

Balanus, 102 ; development of, 749.

Ball-and-socket joint, 603.

Barnacle, 102; development of, 749 .

Basement-membrane, 31 .

Bat, wings of, 669 ; ear of, 515 ; hybernation of, 407 ; peculiar sensibility of, 494.

Batrachia, 86, 87; reparative power of, 390 ; development of egg of, 756 .

Baya, nest of, 705 .

Beat of heart, 269, 271.

Beaumont, Dr., his experiments upon gastric fluid, 207, 208.

Beaver, operations of, 706-708.

Bees, formation of wax by, 156; heat produced by, 410,411; use of antennæ in, 498 ; sounds of, 678 ; instincts of, $712-716$; reproduction of, 747 .

Beetle, digestive apparatus of, 202.

Bell, Sir C., his discoveries, 429, 451 .

Bicuspid teeth, 187.

Bile, chemical characters of, 364 ; use of, in digestion, 213; formed from venous blood, 266, 366; purposes of excretion of, $346-351,365$; effect of suspension of, 351 .

Bilious complaints, 350 .

Binary subdivision of cells, 33 .

BIRDS, general characters of, $78-80$; digestive apparatus of, 200 ; digestive powers of, 201; blood-dises in, 230 ; arterial system in, 260 ; circulation in, 281,282 ; respiration in, 326,327 ; heat of, 407,413 ; nervous system of, 455 ; intelligence of, 484, 717; wings of, 669 ; flight of, 672 ; larynx of, 685 ; nests of, 704, 705; social instinct in, 710 ; ovarium of, 754 ; structure of eggs of, 755; development of embryo of, $757-760$.
Bladder, gall, 362 ; urinary, 362.

Blastema, 33 ; production of cells from, 34 .

Blood of Vertebrata, general characters of, 226 ; arterial and venous, 227; general purposes of, 228, 239; composed of liquor sanguinis and corpuscles, 229 ; coagulation of, $236-239$; serum of, 238 ; buffy coat of, 236 ; flow of, means of checking, 277, 278; changes of, in disease, 233; assimilating power of, 242.

Blood of Invertebrata, character of, 235.

Blood Corpuscles, colourless, 35, 234. red, 35, 229-234; action of endosmose on, 231 ; composition of, 232; variety of proportion of, in different animals, 233; functions of, 235,241 ; change of, in respiration, 310 ; connexion of, with heat liberated, 413.

Blood-vessels, 244; formation of in new tissues, 393 ; in embryo, 758.

Bombylius, sound of, 676 .

Bone, structure of, 49,50 ; chemical composition of, 51 ; formation of, from cartilage, 52, 53; reparation of, 390.

Bones of head, $617-693$; of ear, 516; of spine, 626-632; of trunk, 623; of upper extremity, $635-644$; of lower extremity, $645-648$.

Bowerbankia, 115, 356.

Brain of Vertebrata, 72; not the only source of action, 465,466 ; comparative size of, 718, 719; development of, 763 ; (see Cerebrum and Cerebellum).

Branchial arches, 286-289; of embryo, 762.

Bronchial tubes, 326, 328.

Bryozoa, 115; (see Polyzoa).

Buds, reproduction by (see Gemmation).

Buffy coat of blood, 236.

Bulk required in food, 205.

Butter, 377.

C.

Caddice-worms, 701.

Calamary, 111.

Camel, stomach of, 198; skeleton of, 644 .

Campanularia, 124.

Cancelli of bone, 49 ; formation of, 52 .

Canine teeth, 181, 183.

Cantering, 660 .

Capillary vessels, 251; movement of blood in, 275, 280.

Carapace of Turtles, 83.

Carbon, modes of excretion of, 345-351, 365 ; combustion of, $157,305,306$, 412,413 .

Carbonic acid, set free in respiration, 301,303 ; mode of its production, 305, 306 ; quantity of, proportional to activity of animal, $307-309$; amount of, disengaged by Man, 334 ; deterioration of atmosphere by, $335-338$. 
Carnivorous tribes of animals, 148-151 ; nutrition of, 161; teeth of, 179, 182.

Carpenter-Bee, 703.

Cartilage, structure of, 47 ; nutrition of, 48 ; transformation of, into bone, 52.

Casein, 15.

Cat, electricity of, 418.

Cauda equina, 460 .

Cells, nature of, 32 ; multiplication of, 33 ; new production of, 34,393 ; independent condition of, 35 ; origin of all organisms from, 379 ; differentiation of functions of, 380 .

Cells of Bee, 712,713 ; royal, 714,716 .

Cementum of Teeth, 54 .

Centipede, 93, 103; reflex actions of, 443.

CEPHALOPODA, general characters of, 121 ; circulation in, 291; respiration in, 316 ; nervous system in, 448 ; reproduction of, 753 .

Cercaria-larva of distoma, 743.

Cerebellum, 449; development of, in differerit classes, 452-456; in Man, 458 ; functions of, 480,481 .

Cerebro-spinal nerves, 458-461.

Cerebrum, 449; development of, in different classes of Vertebrata, 452456; in Man, 458; development of, correspondent with intelligence, 452, 718 ; functions of, $483-485$; effects of removal of, 465 .

Cerumen of ear, 375.

Chætodon rostratus, 476.

Chalazæ of egg, 755.

Cheese, 377.

Chemical constitution of organized bodies, 4 ; of albumen, 13,14 ; of casein, 15 ; of syntonin, 16 ; of fibrin, 17,18 ; of gelatin, 19; of chondrin, 20 ; of bone, 51 ; of teeth, 54 .

Cheselden's case, 507 .

Childers, speed of, 660 .

Chimpanzee, 173, 674 .

Cholesterin, 364.

Cholic acid, 364 .

Chondrin, 20.

Chorda dorsalis, 53, 757.

Chorion, 761.

Choroid coat, 533.

Chromatic aberration, 548, 549.

Chrysalis, 97, 309, 745 .

Chyle, 171, 213, 222; change of, in lacteals, 222, 223 ; delivery of into bloodvessels, 221.

Chylification, 171, 212-215.

Chyme, 171, 211.

Cicada, sound of, 679 .

Cicatricula, 755,756 .

Cilia, 45, 143, 319, 329.

Ciliary processes, 536 .

Circulation, 253 ; purposes of, 228, 253, 254: complete double, 282; greater, 253 ; lesser, 253, 268; pecu- liarity of, in liver, 267; mechanism of, $269,270$.

Circulation, course of, in warm-blooded animals, 282 ; in foetus, 283,762 ; in Reptiles, 284,285; in Fishes, 286 ; in Amphibia, 287, 288; in Invertebrata, 289; in Mollusca, 290; in Cephalopoda, 291 ; in Crustacea, 292 : in Insects, 293; in Spiders, 293; in Worms, 291; in Tunicata and Echinodermata, 295; in Zoophytes and Sponges, 295.

Cirrhipeda, general structure of, 102 ; development of, 749 .

Claspers of monkeys, 643,674 .

Clavicle, 634, 636.

Climbing perch, 318 .

Coagulation of albumen, 14; of fibrin, 17; of blood, 236-238; of chyle, 222 .

Coagulable lymph, 391 .

Cochlea of ear, $518-520$.

Cockchafer, digestive apparatus of, 358 .

Cod, brain of, 453 .

Cod-liver oil, use of, 386.

Cœcum, 214.

Cold sustained by animals, Introd., 405.

Cold-blooded animals, temperature of, 403-406.

Coloured shadows, 569.

Colouring matter, formed by cells, 359 , $360,533$.

Colourless corpuscles of blood, 234.

Colours, wallt of power to distinguish, 468; complementary, $568-570$.

Comatula, development of, 741 .

Combustion in animal body, 157, 305, $306,412,413$.

Commissures of nervous system, 434, 458.

Complementary colours, 568-570.

Compound eyes of Articulata, $572-575$.

$$
\text { Polypes, 124, } 127 .
$$

Tunicata, 114.

ConchIfERA, 113; respiration in, 316 ; luminosity of, 396 ; nervous system of, 437 .

Conjunctival membrane, 537 .

Consonants, 689,690 .

Consumption, nature and treatment of, 386.

Contractility of muscular fibre, 579, 590 ; dependent on its nutrition, 591.

Contractions of heart, 269, 271, 581, 587. of muscles, energy of, 592 -594 ; use of, in organic functions, 595 ; in locomotion, 595.

Convex surfaces, influence of, on light, 529-531.

Convolutions of brain, $456,458$.

Convulsive movements, 473,474 ; energy of, 592 .

Cooiing effects of evaporation, 372, 373 .

Coral-forming animals, 131-134.

Cornea, 533 ; structure of, 46.

Corpora striata, 458.

Corpus callosum, 458. 
Corpuscles of blood, 35 (see Blood-corpuscles).

$$
\text { chyle, } 222 .
$$

Cortical substance, of brain, 430 .

Coughing, act of, 342.

$$
\text { kidney, } 357 .
$$

Crab, anatomy of, 99 ; metamorphosis of, 101 ; nervous system of, 447 .

Cranium, bones of, 617-623.

Crassamentum of blood, 236.

Cray-fish, 95.

Cricket, leaping powers of, 662 ; sound produced by, 678 .

Cricoid cartilage, 680 .

Crinoidea, 118.

Crocodile, 93.

Crusta petrosa of teeth, 54.

Crustacea, general characters of, 99101; formation of shell of, 170; teeth of, in stomach, 202 ; circulation in, 292 ; respiration in, 315 ; liver of, 356 ; limminousness of, 396 ; reproduction of claws in, 389 ; generation of, 748 ; development of, 101, 748 .

Crystalline lens, 536; reproduction of, 390 ; change of form of, 551 .

Cutis, structure of, 37 ; sensibility of, $490,491$.

Cuttle-fish, 111; ink of, 359.

Cynips, agamic reproduction of, 746 .

Cysticercus, development of, 742 .

D.

Daphnia, reproduction of, 748 .

Death, of the body, 67 ; apparent, 66 . of parts, continually taking place, 65,68 .

Death-watch, 677.

Death's-head Moth, 678.

Decay of dead animal matter, 54, 160.

Decay continually taking place in living body, 65,68 .

Deer, foot of, 652 .

Defecation, 171, 216.

Degeneration of tissues, from want of use, 30.

Deglutition, 171, 192-196, 470.

Dentine, 54 .

Development, first stages of in ovum, 736, 737; of Polypes, 738, 739 ; of $\mathrm{Me}-$ dusæ, 740; of Echinodermata, 741; of Entozoa, 742, 743; of Annelida, 744; of Insects, 745-747; of Crustacea, 748; of Cirrhipeda, 749; of Rotifera, 750; of Arachnida, 751; of Mollusca, 752; of Vertebrata, 756-762.

Diaphragm, 328; deficiency of, in Birds and Reptiles, 327 ; peculiar to Mammals, 330 ; action of, in respiration, $331-333$

Diet, natural, of Man, 163-165.

Differentiation of structure and function, 380.

Digestion, several stages of, 171 ; gastric, 204-211; intestinal, 212-215.
Digestive cavity, characteristic of Animals, 8; different forms of, 197-203 ; formation of, in embryo, 759 .

Direction of action of muscles, $606-611$. visual objects, 558 .

Dislocations, 604.

Distance, adaptation of the eye to, 550 , 551 ; mode of estimating, $563-565$.

Distoma, development of, 743 .

Division of labour in living organisms, 2. Dogs, intelligence of, 717 .

Doris, circulation in, 290; respiration in, 316.

Dorsal vessel of Articulata, 293.

Double vision, 559.

Draco volans, 668 .

Drowning, 338; treatment of, 339.

Drum of the ear, 516.

Duration of luminous impressions, 567.

Dytiscus, 444.

\section{E,}

Ear, simplest forms of, 512, 514; of Man, external, 515 ; middle, 516, 517; internal, $518-521$; bones of, 516 .

Earthworm, 104, 142, 389, 400, 404, 597.

Echinonermata, 118, 119 ; circulation in, 295; luminousness of, 396; development of, 741 .

Echinus, 118; teeth of, 189.

Egg of Birds, structure of, 755 ; shell of, its permeability to gases, 760 .

Elastic fibrous tissue, 23, 29.

Elasticity of arteries, 274, 275; of foot, 649 ; of vertebral column, 631 .

Elaterida, luminousness of, 397 ; leaping power of, 662 .

Electricity, animal, sources of, 416,417 ; in Cat, 418; in Fishes, 419-423; of muscle and nerve, $424 ;$ analogy of to nervous agency, 488,585 .

Electric organs, structure of, 421, 422; copiously supplied with nerves, 423 .

Elephant, trunk of, 172, 493; molar teeth of, 102 ; tusks of, 177 ; intelligence of, 717 .

Embryo, origin of, 737,757 ; development of, $757-762$; circulation in, 283 , 758,762 ; respiration in, 760 .

Emotions, 477, 478; influence of on muscles, 590 ; on organic functions, 461.

Enamel, structure and composition of, 54 ; arrangement of, 182.

Encrinites, 118.

Endosmose, action of, on blood corpuscles, 232.

Entomostraca, agamic reproduction of, $727,748$.

Entozoa, 105; development of, 742, 743.

Ephemera, 315.

Ephippial eggs of Daphnia, 748.

Epidermis, structure of, 38 ; use of, 492.

Epidermic appendages, 38 .

Epiglottis, 193, 681. 
-Epileptic fits, 473.

Epithelium, structure of, 40 ; action of, in secretion, $41,42,355$.

Eunice, 314 .

Eustachian tube, 516, 517.

Evaporation from surface, 370-373; cooling effects of, 372,373 .

Excretion, objects of, 345-351 ; materials of, formed in the blood, 351 ; effects of retention of, 351 .

Exhalation of moisture from the lungs, 343 ; from the skin, $371-374$; amount of, 374 .

Eye, an optical instrument, 532, 543553 ; structure of, in Man, 533-536; muscles of, 538 ; motions of, 538,539 ; aberration corrected in, 547,549 ; adaptation of to distance, 550,551 ; limits of vision by, 554 ; common sensibility of, 571 ; long and near-sighted, 552 , 553; peculiar structure of, in Articulata, 573-575; deficient in some Vertebrata, 572 ; rudimeuts of, in the lower animals, 575 .

Eyelids, uses of, 537 .

F.

Face, bones of, $690-623$; muscles of, 624.

Facial angle, 719, 720.

Fat, structure and uses of, 46,412; deposition of, 157, 162; of blood, 232, 241.

Fatigue, sense of, 595.

Fertilization of ovum, 732, 734, 736.

Fibre, muscular (see Muscular Fibre).

Fibres, of nerves (see Nerves).

Fibrin, composition and properties of, 17,18; uses of in blood, $236-240$.

Fibrous membranes, 29.

- tissues, general uses of, 12 ; formation of, 22 ; general characters of, $22-30$; nutrition of, 384,390 .

Fibro-cartilages, 47.

Fins of fishes, 666 .

Fire-flies, 397.

Frsнes, general structure of, 88-91; teeth of, 188 ; circulation in, 286 ; respiration in, 317, 318; air-bladder of, 324; luminousness of, 396 ; heat of, 405 ; electricity of, $419-424$; nervous system of, 453 ; organs of smell of, 509 ; vertebral column of, 629,630 ; movements of, 666,667 ; reproduction of, $754,755,759$.

Fission, multiplication of cells by, 33 ; of Infusoria by, 135, 725 .

Flea, leaping powers of, 594, 662 .

Flesh-fly, voracity of larva of, 141.

Flight, action of, $667-672$; impossible in Man, 673.

Flying Fish, 667 .

Lemur, 668 .
Flying Phalanger, 668. Squirrel, 668.

Follicles, of mucous membrane, 41. of glands, $42,355,356$.

Food of Animals, 7, 8; derived from Plants, 144-147; from Animals, 148150 ; chemical nature of, $153,154,164$; mineral ingredients of, 166,167 . of, 165 demand for, 140, 141; economy

Food-yolk, 736, 752, 754.

Foot, structure of, 648,649 .

Foraminifera, 128, 131.

Freezing of animal bodies, 67,405 .

Frog tribe, 86, 87 ; blood-discs of, 230 ; metamorphosis of, 86,87 ; change of circulating system in, 287-289; respiration of, 325 ; experiments on nervous system of, 466,468 ; eggs of, 755 ; development of, 756 .

Fulgorida, luminousness of, 400 ; sound emitted by, 679 .

Functions of living beings, 2; nutritive, 6 ; animal, 6 ; relation of organic and animal, $425-427$.

G.

Gall-bladder, 362 .

Galloping, 660 .

Galvanic electricity, discovery of, 583 .

Ganglia, 61 (see Nervous System).

of special sense, functions of, $475-479$.

- olfactive and optic, 453-456.

Gases, poisonous, 335, 344 .

GASTEROPODA, 107, 108, 112; palate of, 189 ; circulation in, 290 ; respiration in, 316 ; nervous system of, 438 ; development of, 752.

Gastric follicles, 204.

- juice, 204; properties of, 207210 ; artificial, 210.

Gavial, teeth of, 186 .

Gelatin, chemical compostion of, 19; use of as food, 159; present in blood, 380.

Gelatinous nerve-fibres, 60.

principles of food, 153 ; destination of, 159.

Gemmation, multiplication by, of Plants, 724 ; of Infusoria, 725 ; of Zoophytes, 726; of Medusæ, 726, 741; of Echirodermata, 726, 741; of Articulata, $7\llcorner 7$, 744 ; of Mollusca, 728; of Vertebrata, 729; antagonism of, to Generation, 735; (see Agamic Reproduction.)

Gemmules of polypes, 738 .

Generation, sexual, essential nature of, 730-733; anragonism of, to gemmation, 735; simplest form of, 734 .

Germ-cells of plants, 724; of animals, 732.

Germ-yolk, 736, 752, 754 . 
Germinal membrane, 737, 756.

$$
\text { spot, } 732 \text {. }
$$

vesicle, 732.

Gizzard, of Birds, 200, 201 ; of Insects, 202 ; of Bryozoa, 202; of Rotifera, 202. Glands, secreting, structure of, 355-358. Glaucus, 316 .

Globules of Blood (see Blood).

Globulin, 232.

Glosso-pharyngeal nerve, 459, 470.

Glottis, 193, 681.

Glow-worms, 398-401.

Gluten of bread, composition of, 153 .

Glycine, 364 .

Glycocholic acid, 364 .

Gnat, larva of, 321.

Goat-moth, larva of, 141.

Goldfinch, nest of, 704 .

Gout, nature and cure of, 348, 349 .

Granulation, repair of wounds by, 392 .

Gravel, 348, 367.

Gregory, Dr., case of, 349.

Grey substance of nerves, 430,431 .

Guiding Sensations, importance of, 478 .

Gymnotus, electricity of, $419-424$.

\section{H.}

Habitual actions, 479.

Hæmatin, 232.

Hairs, structure of, 38 .

Hall, Dr. Marshall, his treatment of asphy xis, 339.

Hanister, instinct of, 699.

Hands, use of, in prehension, 172, 173; in locomotion, 674 ; structure of, $641-$ 644.

Hare, leaps of the, 661 .

Hartz forest, devastated by Insects, 147.

Haversian canals of bone, 49,50 ; forma. tion of, 52.

Head, definition of, 111 ; bones of, $617-$ 623 ; muscles of, 624 .

Healing of wounds, 391, 392.

Hearing, sense of, $510-524$; improved by cultivation, 525 .

Heart, 245 ; structure of, in Man, \&c., 256,257 ; respiratory and systemic, 281 ; action of, $269,270,581,583$; valves of, 272,273 ; number of pulsations of, 271; development of, 758 , 762.

- structure of, in Reptiles, 284 ; in Fishes, 286; in Mollusca, 290; in Cephalopoda, 291 ; in Crustacea, 292 ; in Insects, 293.

Heat, sustainable by Animals, Introd., $372,373$.

- generated by Animals, 403-415; of Invertebrata, 404 ; of Fishes, 405 ; of Reptiles, 406; of Birds, 407 ; of Mammals, 407; of Man, 407; of young animals, 408, 409; of Insects, 410 , 111.
Heat, animal, dependent on combustion of carbon and hydrogen, 412,413 ; on supply of oxygen, 413 ; maintained by respiration, 414; influence of nervous system on, 415.

Hemispheres of Brain, 449.

Herbivorous animals, $144-147$; nutrition of, 162 ; teeth of, 179,182 .

Hiccup, 341.

Hinge-joint, 603.

Hippuric acid, 367.

Holothuria, 118, 119; reparative power of, 389 ; development of, 741 .

Horse, foot of, 652; intelligence of, 695 .

Howling Monkeys, 684 .

Humble-bee, heat produced by, 410 .

Hunger, sense of, 140, 205.

Hyalæa, 112.

Hybernation, 309.

Hydatina, multiplication of, 750 .

Hydra, 121; referred to, 131, 296, 577; propagation of, by buds, 122, 726; by artificial division, 122; by eggs, 123, 734 ; development of ovum of, 738 .

Hydrogen, combustion of, in animal body, 343.

Hydropathic system, 374 .

Hydrophobia, 474.

Hydrozoa, 124, 125; development of, 726, 738.

Hyoid bone, 625, 680 .

Hysteric disorder, 474.

I.

Iliac bones, 645 .

Images, formation of, by lenses, 531 ; on retina, 543, 544.

Imago or perfect insect, $\mathbf{7 4 5}$.

Immortality of the soul of man, 721, 722.

Impressions on nervous system, $\mathbf{4 3 2 , 4 8 6 .}$

Incisor teeth, 181, 183.

Infants, necessity of warmth to, 408, 409.

Infusoria, 133-135; multiplication of, 725.

Ink, of cuttle-fish, 359 .

Insalivation, 179, 190, 191.

In SECTs, general characters of, 97 ; digestive apparatus of, 202 ; circulation in, 293 ; respiration in, $308,321,322$; reparative powers of, 389 ; secreting apparatus in, 358 ; luminousness of, 397401 ; heat of, 410,411 ; nervous system of, 440-446; instincts of, 483, 484, 667 -716 ; antennæ of, 498,499 ; eyes of, 573-575; muscular power of, 594, 662 ; wings of, 670 ; production of sounds by, 676,679 ; reproduction of, 745,746 .

Instinctive actions, 692; jredominance of, in Articulata, 96; characters of, 694 ; examples of, 696-716; correspondence of, with ganglia of special sense, $475-479$; irrationality of, 709. 
Intelligence of Vertebrata, 73, 483-485, 694 ; examples of, 695,717 ; correspondence of, with development of the Cerebrum, 452, 692, 718-720.

Intervertebral substance, 631 .

Intestinal juice, 213.

$$
\text { tube, motion of aliment }
$$

through, 215; digestion continued in, 213, 214; relative length of, 213. 742,743 .

Invertebrata, 92; absorption in, 225; nature of circulating fluid in, 225, 235 ;

heat of, 404 ; skeletons of, 598,599 .

Involuntary movements, 589,590 .

Iris, 533, 534 .

Iron, a constituent of animal bodies, 166,

167; of red corpuscles of blood, 232.

Ivory, structure and composition of, 54 .

Iulus, 103.

\section{J.}

Jaw, motion of, in Quadrupeds, 138; in Man, 189 ; articulation of, 623 .

Jelly-fish, 120.

Joints, 603; dislocation of, 604.

\section{K.}

Kangaroo, leaping powers of, 661 ; skeleton of, 661 .

Kidneys, structure of, $357,358,368,369$; purposes of their excretion, $346-348$, 367.

L.

Labyrinth of ear, 519 .

Lachrymal apparatus, 540,

$$
\begin{aligned}
& -\quad \text { gland, } 540 . \\
& \text { sac, } 540 .
\end{aligned}
$$

Lacteals, $217,218$.

Lactic acid, $349,367$.

Lacunæ of bone, 50 .

Lamprey, 317; chorda dorsalis of, 53, 757.

Lampyridæ, 397-399.

Land-crabs, 315 .

Lantern-flies, 400.

Larva, of Cirrhipeds, 749; of Crab, 101; of Echinodermata, 741; of Entozoa, 742; of Insects, $97,141,745$; of Medusæ, 740.

Larynx, 192; structure of, 680,681 , action of, $682-684$; in Birds, 685 .

Laughing, act of, 341.

Leaping, 661, 662 .

Leech, 104, 105.

Leg, bones and muscles of, 647 .

Lepidosiren, 81; blood-discs of, 230 ; respiration of, 324.

Leverage of bones, $612-615$.

Life, maintained by continual change, 68.

Ligaments, structure of, 29. vocal, $681-684$.
Light, emitted by living animals, 394402 ; by dead bodies, 402 .

propagation of, 526 ; refraction of $527-532$.

Lime, amount of, in bones, 49 ; in teeth, 54 ; in egg-shell, 169; in shells of Mollusks, 169 ; in shells of Crustacea, 170.

- sources of, in animal bodies, 166 170.

Limulus, 100.

Lingual nerve, 500 .

Liquids, reception of, 173.

Liquor sanguinis, $229,232,236-241,385$, $387,391$.

Lithic acid, 346, 348, 367.

Liver, structure of, $356,358,363$; circulation in, 267, 363 ; assimilating action of, 224 ; secreting action of, $364-366$; formation of sugar by, 366 .

- objects of it 3 excretion, 346,350 .

Living beings, distinctive characters of, $1-5$.

Lizard tribe, 84 ; reparative powers of, 390.

Lobster, 100 ; circulation in, 292.

Lock-jaw, 473.

Locomotion, reflex movements of, 471; organs of, 596.

Locusts, voracity of, 146 ; multiplication of prevented, 149.

Long-siglited eyes, $552,553$.

Luminousıess, animal, 394-400 ; uses of, 401 ; from decomposition, 402.

Lungs, rudimentary in Fishes, 324 ; in Reptiles, 325 ; in Birds, 326, 327 ; in Mammals, 326-333.

Lymnæus, parasites of, 743.

Lymph, coagulable, 391 .

Lymphatics, 219, 220.

Mactra, 113.

M.

Madrepore, 127.

Malapterurus, electric, 419, 422.

Malpighian bodies of kidney, 369 .

MAMMALs, general structure of, 77 ; digestive apparatus in, 197-199; blooddiscs in, 229; circulation in, 281, 282 ; respiration in, $328-333$; heat of, 407 ; nervous system of, 456 ; reproduction in, 756,761 .

Mammary glands, structure of, 376.

Man, food of, 163; stomach of, 197 ; heart of, 256, 257 ; arterial system of, 258 ; quantity of air required by, 334 -337 ; reproduction of lost parts in, 390 ; repair of injuries in, $391-393$; heat of, 407 ; nervous system of, 456 462 ; peculiar characters of soul of, $721,722$.

Mantis, actions of, 444 .

Mantle of Mollusca, 107.

Marmot, hybernation of, 309 .

Marrow of bones, 49 .

- Spinal (see Spinal Cord). 
Mastication, 171, 174-189.

Mastodon, teeth of, 182.

Measles of pork, 742 .

Medulla oblongata, 450,460 .

Medusce, 120 ; development of, 125, 740 ; circulation in, 296 ; rhythmical movements of, 578; gemmation of, 726 .

Membrana Tympani, 516.

Membrane, basement or primary, 31 .

Membranes, fibrous, 29 ; serous, 28,43 ; mucous, 39-41.

Mesentery, 217.

Mesenteric glands, 217.

Metamorphosis of Frog tribe, 86, 87. Insects, 97, 745 .

Crustacea, 101.

Milk, different classes of aliment contained in, 158; chemical composition of, 377 ; influence of mind on, 353 .

Milk-teeth, 184.

Mineral ingredients required by Animals, $166-170$.

Mitral valve, 272.

Molar teeth, $181-183$.

MouluscA, general characters of, 106110 ; circulation in, 290 ; respiration in, 316,320 ; structure of liver in, 356 ; of kidneys, 358 ; luminousness of, 396 ; nervous system of, $435-439$; development of, 752 .

Monkey, interior of, 77 .

Monstrusities by excess, 729 .

Mortality under different circumstances, Introd.

Mucous membranes, general structure of, $39-41$.

Muiberry mass of ovum, 736, 737 .

Muscles, general purposes of, 10 ; general mode of action of, $605-615$; of eye, 538 ; of face, 624 ; of trunk, 637 ; of arm, 638,640 ; of hand, 641 ; of leg, 646,647 ; of foot, 648 .

Muscular Contraction, $58,59,579$; conditions of, 591 ; stimuli to, $580-586$; influence of electricity on, $583-585$; relation of, to nervous power, 586$588,592,593$; voluntary and involuntary, 589, 590; energy of, $592-594$; use of, in organic functions, 595 ; in locomotion, 605-615.

Muscular Fibre, structure of, $55-57$; contraction of, 58 ; alternates with relaxation, 58, 59 .

Musk, odour of, 504 .

Mygale, nest of, 700 .

MYRIAPODA, general structure of, 103 ; nervous system of, 440 .

\section{N.}

Nails, structure of, 38 .

Nais, spontaneous fission of, 727 .

Near-sighted eyes, 552, 553.

Necrophorus, instinct of, 703.

Negro, skin of, 375 .

Nemestrina, trunk of, 173.
Nepa, tracheal system of, 322 .

Nereis, 104, 314, 727.

Nerita, palate of, 189.

Nervous Sxstem, general structure of, 483 ; general objects of, $9,10,429-$ 432; form of, in Vertebrata, 72; in Articulata, 94; in Mollusca, 110; in Radiata, 116.

particular structure and actions of, in Radiata, 434; in Mollusca, 435-439; in Articulata, 440 -446 ; in highest Invertebrata, 447, 448; inVertebrata, $449-452$; in Fishes, 453; in Reptiles, 454; in Birds, 455 ; in Mammalia, 456; in Man, 457-462. Sympathetic, 461,462 . the instrument of the mind, 427; influence of, on secretion, 190,353 ; on muscular contraction, 584,585 ; on animal heat, 415 .

Nervous Tissue, white or fibrous substance of, 62, 63 ; distribution of, 63 ; grey or vesicular substance of, 61 .

Nests of Insects and Birds, 700-705, $710-714$.

Newt, 87 .

Nictitating membrane, 540 .

Nitrogen, absorption and exhalation of, 302.

Non-azotized constituents of food, 154 ; destination of, 155-157, 162-165; effects of excess of, 350 .

Nose, structure of, 506,507 ; common sensibility of, 508 .

Nurse-bees, 411.

Nurses of Cercariæ, 743.

Nutrition of tissues, increased by use, 242,589 ; dependent on liquor sanguinis, $240,241,385$; mode of, in different tissues, 384,385 ; share of blood in, 387 ; share of blood-vessels in, 388 ; share of tissues in, 387 ; imperfect forms of, 386 .

O.

Oak, caterpillars supported on, 145.

Octopus, 121.

Odours, 504, 505.

CEsophagus, 192.

Oleaginous principles, 153 ; destination of, $154-157,162$.

Olfactive ganglia, $453-456,458$. nerve, 507.

Optic ganglia, $453-456,458$.

- nerve, 459.

Organic Life, $6,425,426$; nervous system of, 461 .

Functions, relation of, to animal, 425-427; influenced by enrotions, 461.

Organized bodies, distinctive form of, 1 ; structure of, 2 ; consistence of, 3 ; chemical constitution of, 4 ; actions of, 5 . 
Organs of Sense (see Sensation, Organs of).

Ornithorhyncus, 186, 664 .

Otolithes, 513.

Ovarium, 732 ; of Bird, 754.

Ovum, structure of, 732,733 .

Oxygen, carried by blood-corpuscles, 235 ; by liquor sanguinis, 241 ; absorbed in respiration, $300-306,343$, 346 ; consumption of, dependent on nuuscular action, 307-309.

Oyster, 113, 316, 437.

\section{P.}

Palates of Gasteropods, 189.

Palpi of Insects, $172,503$.

Palsy of muscles, $586-588$.

Paludina, 112.

Pancreatic fluid, use of in digestion, 213.

Papillæ of skin, 37, 490; of tongue, 500.

Parotid gland, 356.

Paxy-waxy, 29.

Pecten, nerrous system of, 110,437 .

Pectinibranchiata, development of, 752.

Pedal ganglia, of Mollusks, 437, 438; of Articulata, 446.

Pelvis, 645.

Penguin, 667.

Pentacrinoid-larva of Comatula, 741.

Perch, skeleton of, 666.

Pericardium, 43.

Peristaltic movement of intestines, 215, 579.

Peritoneum, 43.

Perspiration, $371-374$.

Pharyngeal ganglia, of Mollusks, 438; of Articulata, 446.

Pharnyx, 192.

Phosphorescence of the sea, 394, 395 .

Phosphorus in animal bodies, 166; sources of, 167; light produced by, 402.

Pia rnater, 458 .

Pigment, black, of eye, 533 ; use of, 545

Pigment-cells, 533.

Pitch of sound, dependent on number of vibrations, 523,682 .

Placenta of Mammals, 761 .

Planaria, reparative power of, 389.

Plants, general comparisor of with Animals, 6-12 ; afford food to Animals, 144-147; resemblance of their life to organic life in Animals, 425, 426.

Plastron, of Turtles, 83.

Plethoric state of body, 233.

Pleura, 328.

Pneumogastric nerve, 459, 470.

Podura, leaping power of, 662 .

Poisonous gases, 344.

Polycystina, 132.

Polype, fresh-water (see Hydra).

Polypifera, 121-125 (see Zuophytes).

Polyzoa, 115; gizzard of, 202 ; circula- tion of, 295 ; gemmation of, 728 ; development of, 752 .

Pompilus, nest of, 703.

PORIFERA, 136, 137; circulation in, 296.

Portal system of blood-vessels, 267, 366 .

Poulp, 316, 448.

Primitive trace, $75 \%$.

Prehension, act of, 171-173, 643, 674.

Projection, idea of, to what due, $560-562$.

Proteus, blood-dises of, 230, 231.

Protozos, 128 ; movements of, 577.

Pseudopodia, 130, 131.

Pterodactylus, 669.

Pteropoda, 122.

Pulmonary circulation, 268.

Pulse, 276; influence of posture on, 655.

Pupa, of insect, 97.

Pupil, 553; dilatation and contraction of, 534 .

Pus, 393.

Q.

Quadrumana, extremities of, 643,648 , 674.

Queen-bee, 712, 714, 716, 747.

R.

Rabbit, teeth of, 177; movements of, 661 .

RADIATA, general characters of, 116, 117 ; stomach of, 203 ; nervous system of, 434 .

Radius, 639.

Ray, pecufîr swimming of, 666 .

- electric, 419.

Red Corpuscles of blood (see BloodCorpuscles).

Reflex actions, 195, 340, 430, 692; in Moliusca, 436, 439; in Articulata, 442 $-445,693$; in Vertebrata, 451; the spinal cord their instrument, 464474 ; dependent on stimuli, 466 ; not dependent on sensation, $467-469$.

Refraction of light, $527-532$.

Relief, perception of, $560-562$.

Rennet, action of, 15; nature of, 199.

Repair of injuries, $389-393$.

Reproduction (see Development, Gemmation, and Generation).

REPTILES, general characters of, 8187 ; teeth of, 187; blood-discs in, 430 ; circulation in, 284, 285; respiration in, 325 ; importance of skin, as respiratory organ in, 325 ; heat of, 406 ; nervous system of, 454 ; vertebral column of, 629 ; reproduction of, $756-760$.

Republican Grosbeak, 710.

Resistance, sense of, 496 .

REspiration, 299; use of blood-corpuscles in, 235 ; necessity for, 297 ; in aquatic animals, 298; changes produced by, in air, 300-302, 334-336; in blood, 303-306; related to nervomuscular activity, 307 ; energy of, in 
Birds, Mammalia, and Insects, 308; small amount of, in cold-blooded animals, 309, 310; no special provision for in lowest, 311 ; uses of cilia in, 319,329 ; an excreting process, 345 , 346 ; subservient to maintenance of heat, 412-414; in embryo, 760, 761.

Respiratory apparatus of Annelida, 314; of aquatic Insects, 315 ; of Crustacea, 315 ; of Mollusca, 316, 320 ; of Fishes, $317,318,324$; of Myriapoda, 320; of Insects, $321-322$; of Arachnida, 323 ; of Reptiles, 325; of Birds, 326,327; of Mammals, 328-333; of embryo, 760,761 .

- movements, 331-334, 340342.

surface, extension of, 312 ; prolongation of, externally, into gills, 313 ; internally into lungs, 313 .

- system of nerves, in Articulata, 446; in Mollusca, 437, 438; in Vertebrata, 450.

Rete mucosum, 38 .

Retina, 535; yellow spot of, 554; insensible spot of, 554 .

Rhizopods, 129 ; substance of, 64 .

Rhythmical movements, 578,581 .

Ribs, 633.

Rodentia, teeth of, 177.

Rooks, benefit of, 148 .

Rotifera, 105; reproduction of, 750 ; drying up of, 66 .

Ruminating Animals, stomach of, 198; foot of, 652 .

Running; act of, 66 .

Saccharine aliments, 153 ; destination of, $155-157,162$; conversion of, into oleaginous, 156.

Sacrum, 624,645 .

Salamander, 732.

Saliva, secretion of, 190 ; union of with food, 191.

Salpa, reproduction of, 728 .

Salt, use of, 166, 167.

Sandhopper, 100.

Sanguification, 222-224.

Sarcode, 128.

Saunderson, case of, 496 .

Scapula, $634,635,637$.

Sclerotic coat, 533.

Scurvy, 165.

Seal, 664,665 .

Sebaceous follicles, 38,375 .

SeCretion, general nature of, 245 ; act of, performed by cells, 42, 354; distinguished from excretion, 352 ; influence of mind upon, 353 ; transference of, 361.

Secreting follicles, 355,356 .

membranes, 355 .

tubes, $357,358$.

Segmentation of yolk, 736, 756 .
Semicircular canals, 518, 520 .

Semilunar valves, 273 .

Sensation, 432, 486; organs of, 9 ; general, 487 ; special, 488, 489 ; dependent on supply of blood, 63,487 ; modes of exciting, 487, 488.

Sensori-motor actions, 430.

Sensorium, 429, 486.

Sensory ganglia, 452 ; functions of, 475 479.

Serous membranes, structure of, 28 ; arrangement of, 43 .

Serpents, 85,203 ; lung of, 325 ; vertebral column of, 629 .

Serpula, 314 .

Serum of blood, 238.

Shark, teeth of, 188; brain of, 453.

Shell of Mollusca, 106; of Crustacea, 99,170 ; of Bird's egg, 755 .

Siamese twins, 729 .

Sighing, 341.

Sight, sense of, $526-575$ (see Vision).

Silk-worm, voracity of larva of, 141 .

Single Vision, 559.

Sitting posture, 654,655 .

Size, visual estimate of, 566 .

Skeleton, position of, in different animals, 598 ; internal, of Vertebrata, 71,599 ; external, of Articulata, 93, 598; of Mollusca, 106, 598; of Radiata, 118, $124,127,131,132,598$; of Man, 616 ; of Camel, 644; of Bird, 669; of Perch, 666 ; of Kangaroo, 661 ; of Seal, 664 ; of Dugong, 664; of Bat, 669 ; of Pterodactylus, 669 .

- articulation of pieces of, $601-605$.

Skin, structure of, 36-38; exhalation from, 370-374; secretions from, 375 ; sensory papillæ of, 37,490 ; sensibility of, $491-495$.

Skull, bones of, $617-619$.

Sloth, peculiar arterial distribution in, 264.

Slug, 106, 107.

Smell, sense of, 504-509; concerned in taste, 501 .

Snail, 106, 107; torpidity of, 67 ; respiration of, 320 ; reparative power of, 389 . Sneezing, 342, 508 .

Sobbing, 341,

Societies of animals, 706-711.

Song of animals, 686.

Soul of Man, 721, 722 .

Sounds, propagated by vibrations, $510-$ 512; produced by insects, $676-679$; by larynx, 682 ; pitch of, dependent on number of vibrations, 523,682 .

Spatangus, 142.

Spectacles, choice of, 553 .

Speech, articulate, 686-691.

Spermatozoids, 731 .

Sperm-cells, of plants, 724 ; of animals, $730,731$.

Spherical aberration, 546, 547.

Sphinx atropos, sound produced by, 678 . — ligustri, nervous system of, 441 . 
Spiders, 98 ; circulation in, 293 ; respiration in, 323 ; nervous system of, 447 ; instincts of, 698,700 .

Spinal column, 71, 626-632.

Spinal cord, 72, 451, 460 ; independent powers of, $464-474$; nerves, 451,457 , 460.

Spiracles of Insects, $320-322$.

Spleen, uses of, 224.

Sponge, 136, 137; circulation in, 296.

Stammering, 691.

Standing posture, $650-654$.

Star-fish, 116-119 ; reparative power of, 389 ; nervous system of, 434 ; development of, 741 .

Sternum, 633.

Sterenscope, 561 .

Stomach, need of in animals, 8 ; form of, 197 ; in Ruminants, 198, 199; movements of, 206.

Stomato-gastris system of nerves, 447 450.

Stork, 653 ,

Strychnia, action of, 474.

Sturgeon, continued action of heart of, 583.

Sucking, act of, 172,472 .

Suffocation, 338,339 .

Sugar, formation of by liver, 366 .

Sulphur in animal bodies, 166 ; sources of, 167.

Supra-renal capsules, 224.

Sutures, 602.

Swallowing, act of, 192-196.

Swimming, act of, $663-666$.

Symmetry of disease, 380 .

Sympathetic system of nerves, 60, 61, 461,462 .

Syncope, 271.

Synovial membranes, 44 .

Syntonin, 16.

T.

Tadpole, 95-97; circulation in, 287288.

Tailor-bird, nest of, 705 .

Tape-worm, 105; development of, 742.

Tardigrada, drying-up of, 66.

Taste, sense of, 499,503 .

Taurine, 364 .

Tauro-cholic acid, 368.

Teeth, structure of, 54; development of, 174 ; cutting of, 175 ; cessation of growth of, 176; continued growth of, 177 ; structure of, $178-180$; different kinds of, 181-183; first set of, 184; motion of, in mastication, 178-180.

Teething, convulsions of, $174,473,474$.

Tellina, 316.

Temperament, 718

Temperature, sense of, 497 .

Tendons, structure of, 29 ; attachment of muscles by, 605 .

Testacella, 106.
Testis, 731.

Tetanus, 380 .

Thalami optici, 458.

Thigh, bone and muscles of, 646 .

Thoracic duct, 221.

Thorax, 328; movements of, 332 .

Thumb, uses of, 643 ; reproduction of, 390.

Thunny, temperature of, 405 .

Thymus gland, 224.

Thyroid cartilage, 680 .

$$
\text { gland, } 224 .
$$

Timbre of Sounds, 524 .

Tissues, of Animals, distinctive pec11liarities of, 10-12; chemical composition of, 13-21; fibrous, 22-30 ; membranous, $37-45$; osseous, $49-54$; cellular, 32-36, 46-48; muscular, 5559 ; nervous, $60-63$; degeneration of, from want of use, 58 ; continual decay and renewal of, 67,68 ; self-formative power of, 382,387 ; reproduction of, 390.

Tongue, nerves of, 501; mechanical uses of, 503.

Torpedo, electricity of, $419,421$.

Torpidity of animals, 66, 309 .

Tortoise-shell, 92.

Tortrix, nest of, 701 .

Touch, sense of, $490-499$.

Tracheæ of Insects, 321, 322.

Tranference of secretion, 361 .

Transfusion of blood, 239.

Trematode Entozoa, 743.

Tricuspid valve, 272.

Tridacne, 119.

Tritonia, 316.

Trunk of Elephant, 172. Insects, 173.

Tubercle, nature of, 386 .

Tunicata, 114 ; circulation in, 295 ; respiration in, 316 ; luminousness of, 396 ; nervous system of, 435, 436; gemmiparous reproduction of, 728 ; development of, 752 .

Turbo, anatomy of, 108.

Turnip-fly, voracity of, 147.

Turtle tribe, 83 .

Tympanum, 516, 517.

U.

Ulna, 639.

Ungkaputi, 674.

Unity of Design, 261, 763.

Urea, $346,367$.

Ureters, 362.

Uric acid, 346, 348, 367.

Urinary apparatus, $368,369$.

— bladder, 362.

- excretion, purposes of, 346-

348 ; effects of suspension of, 351 ; composition of, 367 ; water discharged by, 369 .

Uterus, 761. 
v.

Valves of heart. 272, 273; of veins, 279.

Vascular area, 758.

Vegetative repetition of parts, 2.

$V$ eins, 246 ; structure of, 248 ; pressure of blood in, 249; arrangement of, 250, 266 ; flow of blood through, 277, 278; valves in, 279.

Vena cava, 266.

Vena portæ, 267, 366.

Ventilation, importance of, $336,337$.

Ventricles of brain, 458 .

Ventriloquism, 525 .

Vertebræ, structure of, 71, 628; classification of, 626 ; number of, 627 ; connexion of, in Reptiles and Fishes, 629 ; in Man, 630; in Birds, 630.

Vertebral column, 70, 71, 626-632; first development of, 757 .

VERTEBRATA, general characters of, 70 -76 ; nervous system of, $449-452$; skeleton of, 599 ; gemmation in, 729 ; embryonic development of, $757-762$.

Vessels, origin of, 393.

Vestibule of ear, 518,521 .

Vibrations, sonorous, $510-512$; pitch determined by number of, 523,682 .

Villi of mucous membrane. 41 ; absorption performed through, 41, 217.

$V$ ision, dependent on light, 526, 542 ; adaptation of eye to distinct, $543-553$; infuence of attention on, 555 ; inferences drawn from, $556-566$; duration of impressions, 567; distinction of colours by, 568-570; erect, though picture inverted, 558 ; single, with two eyes; 559 ; double, 559.

Vitality, independent, of parts of organism, 65 .

Vitreous humour, 536.

Vocal cords, 681 .

Voice, confined to Vertebrata, 680 ; how produced in larynx, 682 ; differences in pitch and quality of, 683,684 .

Voltaic electricity, discovery of, 583,584 .

Voluntary movements, 589,590 .

Vorticella, reproduction of, 725 .

Vowel sounds, 689,690 .

Vulture, skeleton of, $66 \mathrm{~S}$.
W.

Walking, act of, 657 .

Warm-blooiled animals, 407-409.

Wasps, nest of, 711 .

Waste of the system, $160,307,345$.

Water, passed off by kidneys, 369 ; exhaled from lungs, 343,344 ; from skin, $370-374$.

Water-Newt, 87.

Wax formed from sugar only, 155.

Webs of Spiders, 698 .

Whale, mouth of, 185 ; peculiar arterial distribution in, 265 ; sensibility of surface in, 491; blow-holes of, 509; propulsion of, in water, 665,666 .

Whalebone, 185.

Wheel-animalcules, (see Rotifera). ${ }^{\text {" }}$

White fibrous tissue, 23-29.

White of egg, 14, 755 .

Wings, of Birds, 78, 668; of Bat, 669 ; of Pterodactylus, 669 ; of Insects, 670 ; action of, $667-672$.

Winter eggs of Hydra and Rotifera, 735.

Wounds, healing of, $391-393$; of arteries, treatment of, 277.

Wren, intelligence of, 717.

$\mathrm{X}$.

Xylocopa, nest of, 703 .

Y.

Yawning, 341.

Yellow Fibrous tissue, 23-29.

Yellow spot of retina, 554 .

Yolk of egg, 733, 736, 754 .

Yolk-bag, 733, 754.

Young animals, heat of, 408, 409.

z.

Zoea, larva of Crab, 101.

Zoophytes, 117, 121; tissues of, 64,577 ; circulation in, 296; gemmiparous production of, 726 ; development of Medusæ from, 738, 740; generation of, $738,739$. 
\begin{tabular}{|r|}
\hline \\
$\mid$
\end{tabular} 



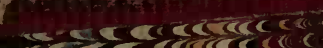

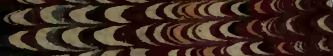
-cect acoses a

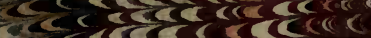

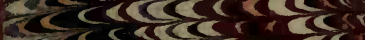
$\rightarrow<<<<1<$ कर

$\mathrm{c}+\mathrm{C}$
$\mathrm{C}$

U.C. BERKELEY LIBRARIES

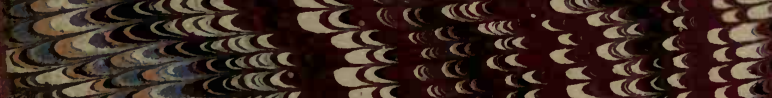

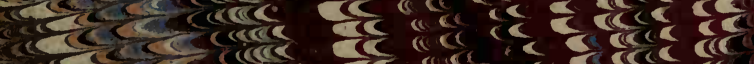

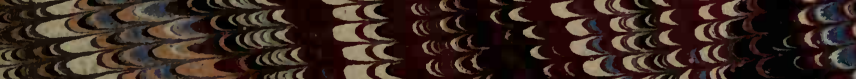

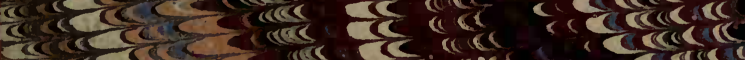

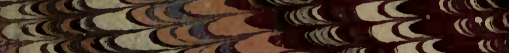

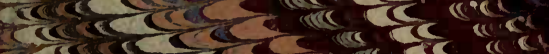

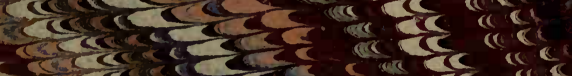

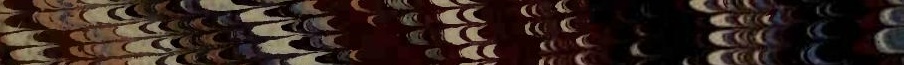

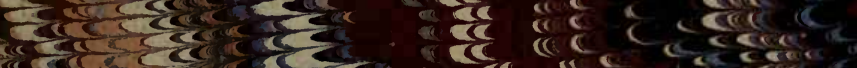

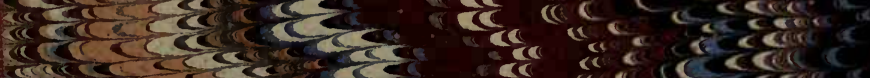

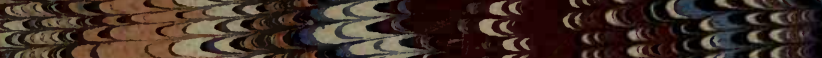

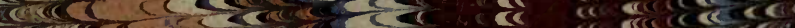
sc

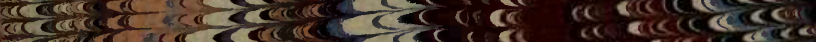

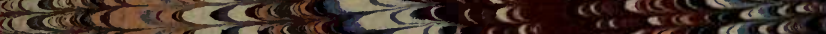

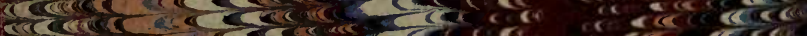

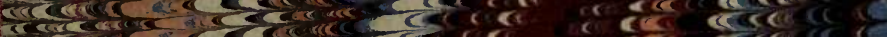

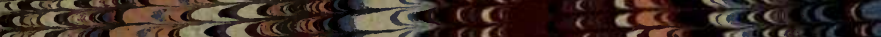

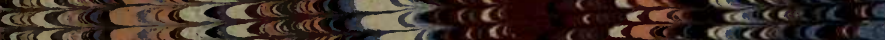

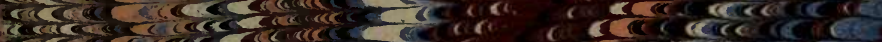

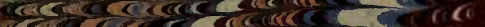

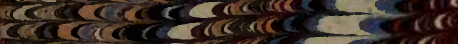

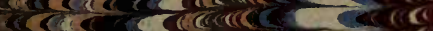

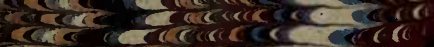

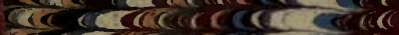

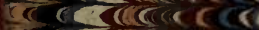

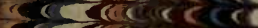
$\rightarrow \operatorname{coc}_{0} \cos$

$c<\cos c)$

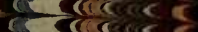

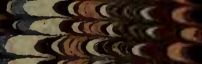

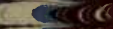

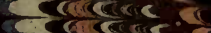

$\longrightarrow \frac{\operatorname{coc} c \cos 2}{2}$

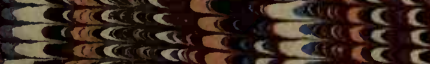

$-\sec \left(\frac{1}{6}\right)$

(c)

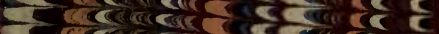

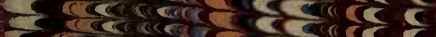

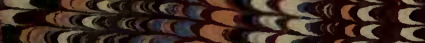

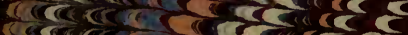

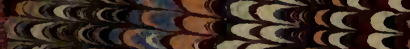

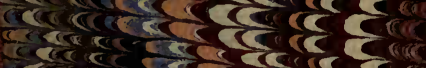

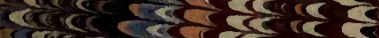

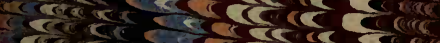

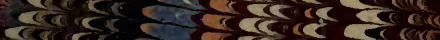

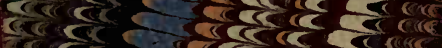

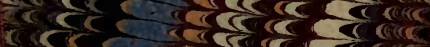

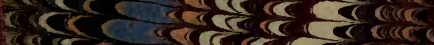

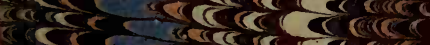

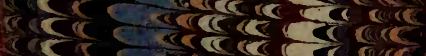

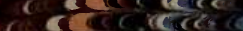
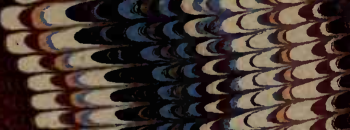

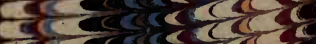

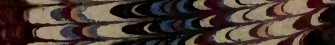

inc

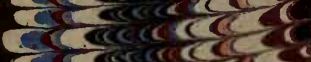

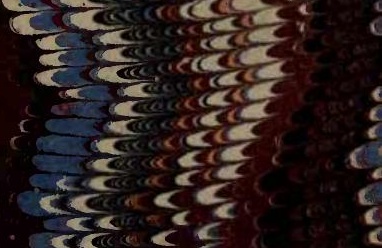


